\title{
Nondestructive Post-Irradiation Examination of Loop-1, S1, and B1 Rods
}

R. L. Bratton

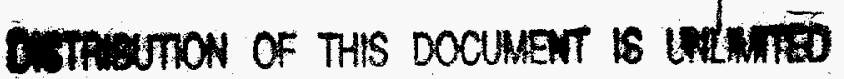

Published May 1997

Idaho National Engineering Laboratory Specialty Engineering and Science Department Lockheed Martin Idaho Technologies Company Idaho Falls, Idaho 83415

Prepared for the U.S. Department of Energy Assistant Secretary for the Office of Defense Programs Under DOE Idaho Operations Office Contract DE-AC07-94ID13223 


\section{DISCLAIMER}

This report was prepared as an account of work sponsored by an agency of the United States Government. Neither the United States Government nor any agency thereof, nor any of their employees, makes any warranty, express or implied, or assumes any legal liability or responsibility for the accuracy, completeness, or usefulness of any information, apparatus, product, or process disclosed, or represents that its use would not infringe privately owned rights. Reference herein to any specific commercial product, process, or service by trade name, trademark, manufacturer, or otherwise does not necessarily constitute or imply its endorsement, recommendation, or favoring by the United States Government or any agency thereof. The views and opinions of authors expressed herein do not necessarily state or reflect those of the United States Government or any agency thereof. 


\section{DISCLAIMER}

Portions of this document may be illegible electronic image products. Images are produced from the best available original document. 


\section{ABSTRACT}

As a part of the Pacific Northwest National Laboratory's Tritium Target Development Program, eleven tritium target rods were irradiated in the Advanced Test Reactor located at the Idaho National Engineering and Environmental Laboratory during 1991. Both nondestructive and destructive post-irradiation examination on all eleven rods was planned under the Tritium Target Development Program. Funding for the program was reduced in 1991 resulting in the early removal of the program experiments before reaching their irradiation goals. Post-irradiation examination was only performed on one of the irradiated rods at the Pacific Northwest National Laboratory before the program was terminated in 1992.

On December 6, 1995, the Secretary of Energy announced the pursuit of the Commercial Light-Water Reactor option for producing tritium establishing the Tritium Target Qualification Program at the Pacific Northwest National Laboratory. This program decided to pursue nondestructive and destructive post-irradiation examination of the ten remaining rods from the previous program. The Idaho National Engineering and Environmental Laboratory, who was assigned the project lead for this examination, contracted the services of the Hot Fuels Examination Facility at Argonne-West National Laboratory, located on their site, to perform the nondestructive examinations.

The ten rods comprise three experiments. The Loop-1 experiment irradiated eight target rods in a loop configuration for 217 irradiation days. The other two rods were irradiated in two separate irradiation experiments, designated as S1 and B1 for 143 effective full-power days, but at different power levels.

After the ten rods were transferred from the ATR Canal to the Hot Fuels Examination Facility, the following examinations were performed:

- Visual Examination and Photography

- Neutron Radiography

- Axial Gamma Scanning

- Contact Profilometry Measurement

- Bow and Length Measurements

- $\quad$ Rod Puncture and Plenum Gas Analysis/Measurement of Plenum Gas Quantity

- Void Volume Determination

- Internal Pressure Determination.

This report presents the data collected during these examinations. 


\section{CONTENTS}

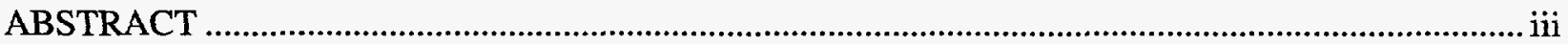

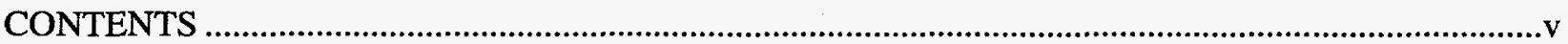

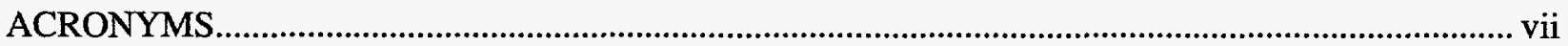

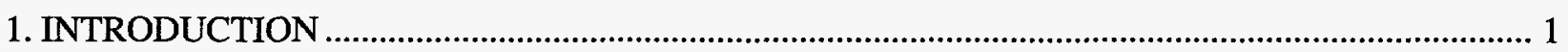

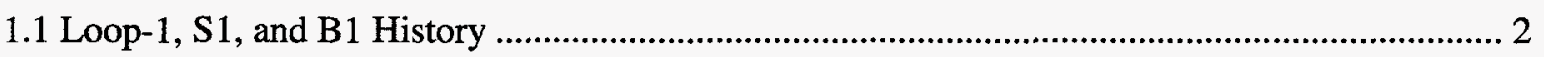

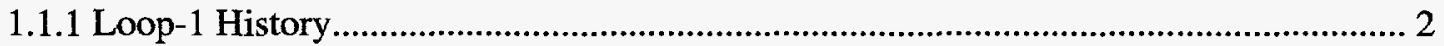

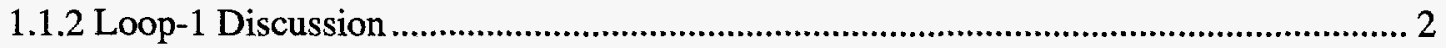

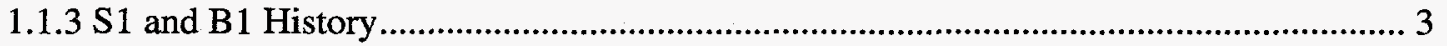

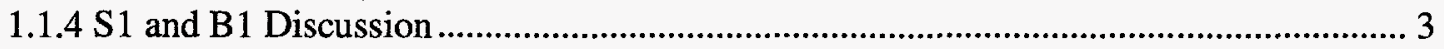

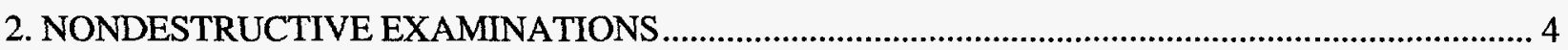

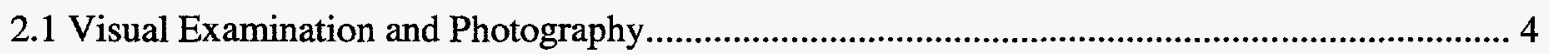

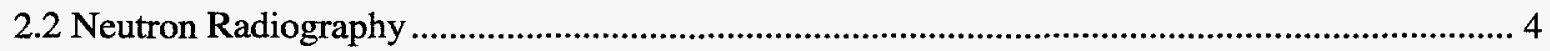

2.2.1 Operational Notes for Radiography ................................................................................ 4

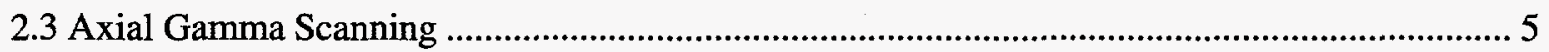

2.3.1 Operational Notes on Gamma Scanning...................................................................... 5

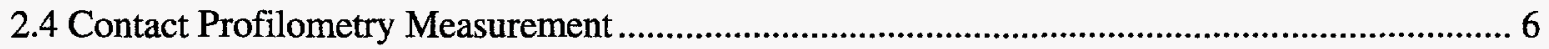

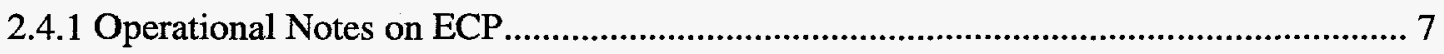

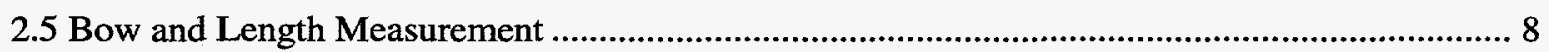

2.6 Gas, Analysis, Sample, and Recharged System .................................................................... 10

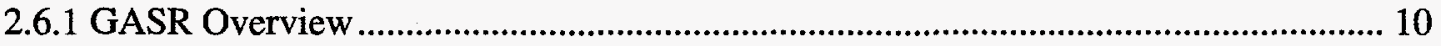

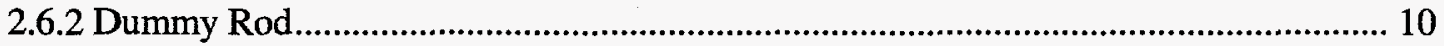

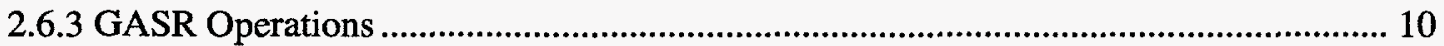

2.6.4 Rod Void Volume And Pressure Calculations ........................................................... 11

Appendix A-Letter Report on Loop 1 Cutting Problems

Appendix B-Photographs 
Appendix C-Neutron Radiography

Appendix D—Gamma Scan

Appendix E-Profilometry Plots

Appendix F-Bow and Length

Appendix G-Dummy Rod Data

Appendix H-Calibration Sheets and Puncture Data

Appendix I-Plenum Gas Analysis

\section{FIGURES}

Figure 1. Sample of a neutron radiograph.

Figure 2. Profilometry gauge for measuring axial diameter profiles of elements and capsules.

Figure 3. View looking down on top of element/capsule

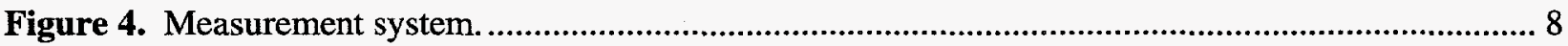

Figure 5. Elevation view of element as measured..................................................................................

\section{TABLES}

Table 1. Examinations performed for nondestructive PIE at the HFEF.

Table 2. Loop-1 chronology. 2

Table 3. S1 and B1 history. 3 


\section{ACRONYMS}

ANL

ASCII

ATR

CCW

CLWR

$\mathrm{dc}$

DOS

ECP

GASR

HFEF

ID

INEEL

LMITCO

PGS

PIE

PNNL

TTDP

UPG
Argonne-West National Laboratory

American Standard Code for Information Interchange

Advanced Test Reactor

counterclockwise

Commercial Light-Water Reactor

direct current

disk operating system

element contact profilometry

gas, analysis, sample, and recharge system

Hot Fuels Examination Facility

identification

Idaho National Engineering and Environmental Laboratory

Lockheed Martin Idaho Technologies Company

precision gamma scanner

post-irradiation examination

Pacific Northwest National Laboratory

Tritium Target Development Program

ultra-pure germanium 


\section{Nondestructive Post-Irradiation Examination of Loop-1, S1, and B1 Rods}

\section{INTRODUCTION}

Loop-1, S1, and B1 were removed from the Advanced Test Reactor (ATR) core in 1991 and stored in the ATR canal until 1996 when the Commercial Light-Water Reactor (CLWR) Project was funded to pursue the qualification of the CLWR target. Part of this project was assigned to the Idaho National Engineering and Environmental Laboratory (INEEL), which is operated by Lockheed Martin Idaho Technologies (LMITCO), to pursue the nondestructive post-irradiation examination (PIE) of the Loop-1, S1, and B1 rods at the Argonne-West National Laboratory (ANL) Hot Fuel Examination Facility (HFEF). ANL was a subcontractor to LMITCO for this work.

Requirements for nondestructive PIE were transmitted to LMITCO on December 19, 1995 by letter from J. L. Ethridge of Pacific Northwest National Laboratory (PNNL) to Dr. D. C. Mecham of LMITCO.

Table 1. Examinations performed for nondestructive PIE at the HFEF.

\begin{tabular}{|c|c|}
\hline Type & Explanation \\
\hline Visual Examination and Photography & $\begin{array}{l}\text { Visually inspect and photograph the } 10 \text { rods through the } \\
\text { periscope in the HFEF Cell. Construct a full length } \\
\text { photographic mosaic of rods WL-10 and WL- } 26 .\end{array}$ \\
\hline Neutron Radiography & $\begin{array}{l}\text { A procedure similar to } \mathrm{x} \text {-ray radiography, except neutrons } \\
\text { are used as the penetrating radiation. }\end{array}$ \\
\hline Axial Gamma Scanning & $\begin{array}{l}\text { Scan the entire length of the rod for isotopes. The two } \\
\text { isotopes present in detectable quantities are } \mathrm{Mn}^{54} \text { and } \\
\mathrm{Co}^{60} \text {. }\end{array}$ \\
\hline Contact Profilometry Measurement & Measure the diameter of each rod. \\
\hline Bow and Length Measurement & Measure the bow and free-hanging length of each rod. \\
\hline $\begin{array}{l}\text { Rod Puncture and Plenum Gas Analysis/ } \\
\text { Measurement of Plenum Gas Quantity }\end{array}$ & $\begin{array}{l}\text { Laser puncture each rod and sample the plenum gas for } \\
\text { tritium and other gas species. Measure the rod's void } \\
\text { volume and pressure prior to puncture. }\end{array}$ \\
\hline $\begin{array}{l}\text { Recovery of Flux Wires and Counting of } \\
\text { Flux Wires }\end{array}$ & $\begin{array}{l}\text { Work started on this effort, but was suspended because a } \\
\text { different method was identified that would provide a flux } \\
\text { measurement with less uncertainty. The measurement will } \\
\text { be performed during destructive PIE at PNNL. }\end{array}$ \\
\hline $\begin{array}{l}\text { Recovery of End-Plug Piece From Two } \\
\text { Rods }\end{array}$ & This operation was canceled before it was started. \\
\hline
\end{tabular}

The objective of this report is to only present data taken during the nondestructive PIE at ANL. No data analyses or interpretations will be presented in this report. The data analyses will be presented under a separate cover at a later time. 


\subsection{Loop-1, S1, and B1 History}

\subsubsection{Loop-1 History}

Table 2. Loop-1 chronology.

\begin{tabular}{cl}
\hline Date & \multicolumn{1}{c}{ Event } \\
\hline $10 / 05 / 91$ & $\begin{array}{l}\text { Loop-1 experiment removed from the ATR Core after 217 irradiation days and stored in } \\
\text { the ATR Canal. }\end{array}$ \\
$02 / 22 / 94$ & Unanticipated occurrence in the ATR Canal during cutting of the Loop-1 shroud. \\
$02 / 05 / 96$ & Loop-1 experiment shroud cut in the ATR Canal and Loop-1 assembly retrieved. \\
$07 / 17 / 96$ & Loop-1 loaded in T2 Cask and transferred to the ANL HFEF. \\
$10 / 07 / 96$ & T2 Cask unloaded. Loop-1 assembly placed in the HFEF Cells. \\
$10 / 11 / 96$ & Disassembly of the individual Loop-1 rods in the HFEF Cells. \\
\hline
\end{tabular}

\subsubsection{Loop-1 Discussion}

Prior to current CLWR activities, the New Production Reactor Light Water Reactor experiments were scheduled to be disposed of at the burial grounds. This required the experiment lengths to be reduced to a size amenable for disposal. During the cutting of Loop-1, an "Occurrence" was declared. The use of an underwater dc arc saw resulted in the formation of a hydrogen bubble that ignited while cutting the zircaloy contained in the Loop-1 capsule. The operation was suspended and the Loop-1 experiment returned to storage in the ATR Canal. All disposal efforts were suspended pending review of the conditions leading to the hydrogen bubble ignition. The root cause report is enclosed in Appendix A.

The Loop-1 experiment was laid horizontal in the ATR Canal and secured to a table to facilitate the cutting of the experiment's shroud by pneumatic band saw. After cutting, the experiment was righted vertically and the Loop- 1 assembly removed by a special tool. The Loop-1 assembly was loaded into a shipping container in the ATR Canal. The shipping container was lifted out of the ATR Canal and temporally left suspended above the ATR Canal to drain. The shipping container was then placed into a $2 \mathrm{R}$ basket inside the T2 Cask, in open air transfer. The $2 \mathrm{~T}$ Cask was transferred to the ANL HFEF for nondestructive PIE

Due to a HFEF shutdown, the facility could not unload the T2 Cask immediately after receiving it from ATR. After a three month delay, the assembly was unloaded from the T2 Cask and placed in the HFEF Cells to begin disassembly procedures.

During disassembly, it was noticed that the rods were loose within the assembly, with some variation in the relative axial displacement of the bottom ends. The rods were easily pulled (by manipulator) horizontally from the assembly grid structure. The visual appearance of the rods showed isolated areas of high luster in comparison to the remaining surface area of the rods. 


\subsubsection{S1 and B1 History}

Table 3. S1 and B1 history.

\begin{tabular}{|c|c|}
\hline Date & Event \\
\hline $07 / 21 / 91$ & $\begin{array}{l}\text { S1 and B1 experiments removed from the ATR Core after } 143 \text { effective full-power } \\
\text { days. }\end{array}$ \\
\hline $08 / 29 / 96$ & Shroud length reduction of $S 1$ in the ATR Canal. \\
\hline 09/03/96 & Shroud length reduction of B1 in the ATR Canal. \\
\hline $09 / 24-25 / 96$ & Disassembly of the S1 and B1 capsule in the ATR Hot Cells. \\
\hline $10 / 05 / 96$ & S1 and $\mathrm{B} 1$ rods loaded into the T2 Cask for transfer to ANL HFEF. \\
\hline $10 / 28 / 96$ & $\mathrm{~S} 1$ and $\mathrm{B} 1$ rods unloaded from $\mathrm{T} 2$ Cask and placed in the HFEF Cells. \\
\hline
\end{tabular}

\subsubsection{S1 and B1 Discussion}

The lengths of the shrouds for both experiments were approximately 15 feet. The transfer cask used to transport the experiments from the ATR to the ATR Hot Cells is limited to an internal height of five feet. This necessitated reducing the length of the shroud to five feet by using a pneumatic band saw in the ATR Canal and making cuts on both ends of the shroud. Once the two experiments were transferred to the ATR Hot Cells, additional cuts were made in the cell to expose the inner pressure capsule. A horizontal screw was used to push out the inner capsule from the shroud. Both rods were allowed to freely slide out of the inner pressure capsule onto a strongback. A catch basin was used during cutting to collect any internal water in the capsules. Little water was collected from the disassembly of $\mathrm{S} 1$, but water was collected in the basins during disassembly of B1. In addition, particulate matter came out of the inner pressure capsule as B 1 was slid out onto the strongback. Both rods were smeared for tritium after disassembly.

Results of the analysis on the collected B1 water sample indicated a below background reading for tritium. The radio-isotopic analysis of the $\mathrm{B} 1$ particulate indicated the presence of the isotope $\mathrm{Co}^{60}$. Chemical analysis of the B1 particulate appeared analogous to products of stainless steel corrosion.

Results of the smears from the ATR Hot Cells for S1 and B1 indicated a strong positive for the presence of tritium. Upon arrival from the ATR Hot Cells, the S1 and B1 rods were smeared again in the ANL HFEF Cell. The results indicated a high positive, but a second set of smears indicated below background readings. The difference between the readings could possibly be due to the method of taking the smears. ATR Hot Cells use a polystyrene "popcorn" bead as the smear medium. After smearing, the bead is directly immersed into the scintillation cocktail, where it completely dissolves, and the cocktail is then counted. HFEF uses a cotton material disk, which is immersed in distilled water (after swiping), as a leaching medium. The distilled water is transferred to the scintillation cocktail for counting. 


\section{NONDESTRUCTIVE EXAMINATIONS}

\subsection{Visual Examination and Photography}

The Loop-1, S1, and B1 rods were visually inspected along the entire axial length through the ANL HFEF periscope at medium magnification $(\approx 10 \mathrm{X})$. Smaller features were visually examined with high magnification $(\approx 25 \mathrm{X})$. After all the rods were visually inspected, each rod was remounted in the periscope and photographs were taken of unique features, except for WL-10 and WL-26. These rods were selected for a full length photo composite taken at four angles of rotation $0,90,180$, and 270 degrees. The photographs and their index are found in Appendix B.

\subsection{Neutron Radiography}

Neutron radiography is a procedure similar to x-ray radiography where neutrons are used instead of x-rays to expose metal foils instead of film. Four Loop-1 rods at a time or both S1 and B1 rods were inserted in stainless steel carrier tubes and positioned in front of the neutron shutter. Behind the stainless steel carrier tubes is a film pack consisting of dysprosium, cadmium, and indium foils. After a 30 minute neutron exposure the film pack is removed and disassembled. The dysprosium and indium foils have unexposed film placed in contact and held together in a light tight vacuum chamber overnight to expose the film. The exposed film is developed to normal background density. Due to the length of the film, several shots are required to exposed the entire length of the rods. The dysprosium exposed films represent the thermal radiographs and the indium exposed film represents the epithermal radiographs.

In addition to the films, "positives" of the films are made by exposing a high intensity light source through the films backed by black and white photographic film. This process provides photographs of the films. The photos, scanned and printed on $8 \times 17$ inch paper, are found in Appendix $C$.

\subsubsection{Operational Notes for Radiography}

When reading a neutron radiograph, orient the film so that the label is "right-reading," is located along the left side, and the ANL logo is in the bottom left corner. In this orientation, images appear as if the observer's eye were located at the source of neutrons.

The label (see the Figure 1) shows the Shot \# (for internal ANL use), the Subassembly or Test ID, the elevation of the radiograph, and the Element or Specimen ID. The Element or Specimen ID reads from top to bottom (when oriented as above) and corresponds to the elements or specimens from left to right in the image.

All duplicate negatives of the radiographs have small holes punched in their bottom comers. Original radiograph films and positive prints do not have holes punched in them.

The two types of radiographs can be readily distinguished by the relative contrast of the images. Thermal radiographs have a high contrast, and the image of any fuel in the radiograph is generally white on the film. Labels on thermal radiographs are usually clear and easy to read. Epithermal radiographs have less contrast, but show more image detail in the pellet areas. Labels on these radiographs are difficult to read and often show on the film as dark blocks. 
The background density of duplicate negatives is generally slightly darker that the originals because the duplicates are developed to match the density of the image areas of originals.

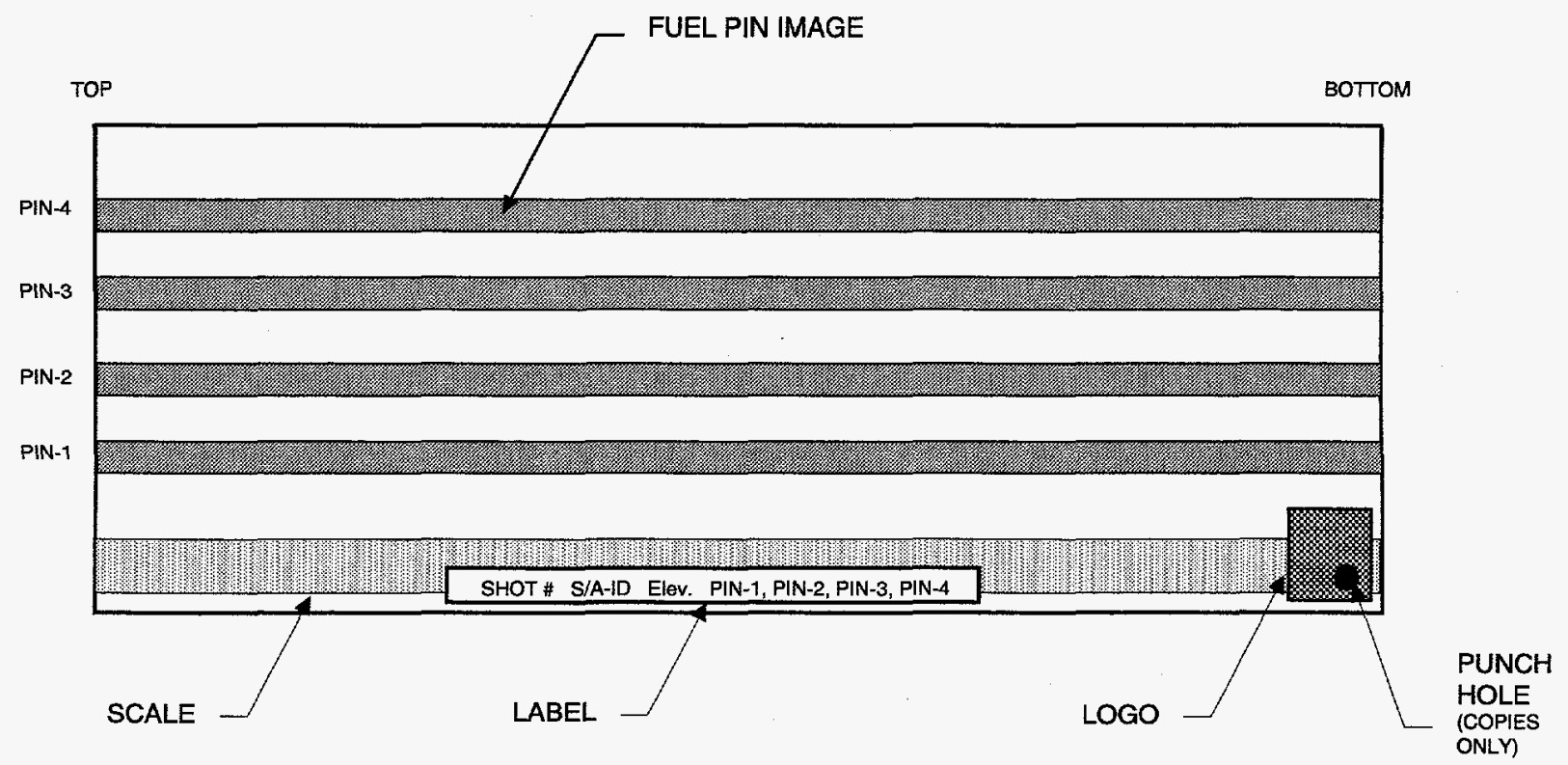

Figure 1. Sample of a neutron radiograph.

\subsection{Axial Gamma Scanning}

Gamma scans are presented in graphic form for each rod. Each rod has three scans associated with it, a gross scan, an $\mathrm{Mn}^{54}$ scan and a $\mathrm{Co}^{60}$ scan. These are found in Appendix D. Below are notes which describe the operation of the gamma scanning station.

\subsubsection{Operational Notes on Gamma Scanning}

The Precision Gamma Scanner-II (PGS-II) is designed to intermittently transverse rod-like objects in the form of a small, solid-angle collimator for collection of gamma spectra at a series of positions. Usually the increment of transverse is set equal to the collimator slit opening to provide complete object coverage.

The gamma-ray spectroscopy subsystem consists of an ultra-pure germanium (UPG) detector with a nominal efficiency of $10 \%$ (relative to a $2 \times 2$-in. NaI detector). Active Compton-suppression is accomplished by detecting the time-of-life delay between the UPG and the surrounding large-volume NaI guard detector. A background suppression factor of 6-10 (based on $\mathrm{Co}^{60}$ peaks) is normally achieved.

The PGS-II is designed and calibrated to position and report the location of the centroid of the collimator (with respect to the upper tip of the scanned object) to an accuracy of \pm 0.005 inches for each positional measurement.

Data was acquired in spectral mode, which maps gamma energies (in the range of 50 to $1,600 \mathrm{k}$ ) into 4,096 channels. 
The collimator slit is rectangular with a width of 0.880 inches and an adjustable height of 0.0 to 0.100 inches The slit opening was 0.100 inches for all measurements. Slit opening is reported in all data tables and charts. The distance between the rod and UPG is eight feet.

The live time of each positional count is infinitely adjustable, but is usually fixed for any given scan. The live time used for a scan is reported in all data and tables.

Because active isotopes $\mathrm{Mn}^{54}$ and $\mathrm{Co}^{60}$ have long decay times in comparison to the count times, there was no correction for decay during counting.

Gross, $\mathrm{Mn}^{54}$, and $\mathrm{Co}^{60}$ axial data along the length the rod is provided in digital form on a CDROM. The data are stored as plain ASCII data in DOS file format (i.e., records are terminated by $<\mathrm{CR}><\mathrm{LF}>$ pairs). The first 10 records contain self-explanatory header information. Following the header records, spectral data are stored in tab-delimited fields as follows:

$<$ Position $\rangle\langle t a b\rangle\langle$ Gross $\rangle<t a b<$ Isotope-1 $>\langle t a b\rangle \ldots<$ Isotope-N $\rangle<C R><L F\rangle$

\subsection{Contact Profilometry Measurement}

The element contact profilometry (ECP) is a high-throughput continuous-contact profilometry gauge for measuring axial-diameter profiles of elements and capsules. Horizontally-opposed linear transducers contact the element as it is pulled vertically through sapphire-tipped probes showing in Figure 2. Guide rollers are positioned three inches above and below the transducers to maintain the element vertical with respect to the horizontal transducers. Certified calibration standards are used for zero, mid-span, and full-span calibration. The data for ECP is found in Appendix E for angles, $\Phi_{c}$, of 0 , 45,90 , and 135. ECP was not performed on WL-10 or WL-26.

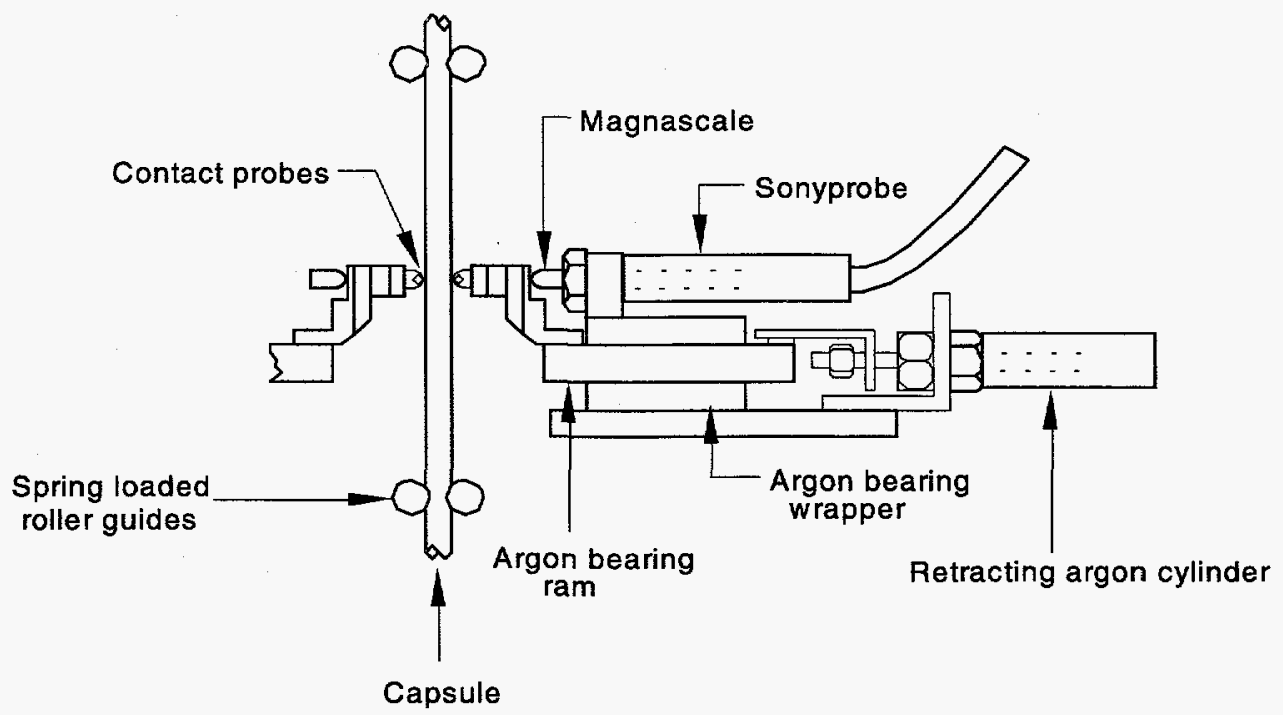

RED97 SG0082

Figure 2. Profilometry gauge for measuring axial diameter profiles of elements and capsules. 


\subsubsection{Operational Notes on ECP}

When fuel elements (pins) or capsules are significantly bowed, a situation can arise when the lower tip of the element or capsule exits the bottom guide roller near the end of the measurement. Depending on the direction and magnitude of the bow, the portion of the element or capsule below the upper guide roller can assume an angle with respect to the measuring probes causing an erroneously high reading. This is an inherent feature of the system that cannot be corrected; therefore, the resultant data must be interpreted with caution.

Full-length scans usually start as high as possible on the element or capsule (but no higher than the upper cladding weld) and end at the lower cladding weld. Because of interference from the upper roller and the collet applied to the element or capsule, the topmost four inches cannot be measured.

The "Z-Position" reported on ANL-supplied plots is the raw position $\left(Z_{s}\right.$, in inches) of the in-cell positioning stage and has no fixed or known offset from the top or bottom of the element or capsule. Note that the stage Z-Position is higher toward the bottom of an element or capsule.

The "Diameter" reported on ANL-supplied plots is the profile dimension (in inches) perpendicular to the azimuthal position, $\phi_{\mathrm{e}}$ (in degrees), as shown in the diagram below.

By convention, azimuthal positions increase counterclockwise (CCW) from the azimuthal reference feature when viewed from the top of the element or capsule as shown in Figure 3.

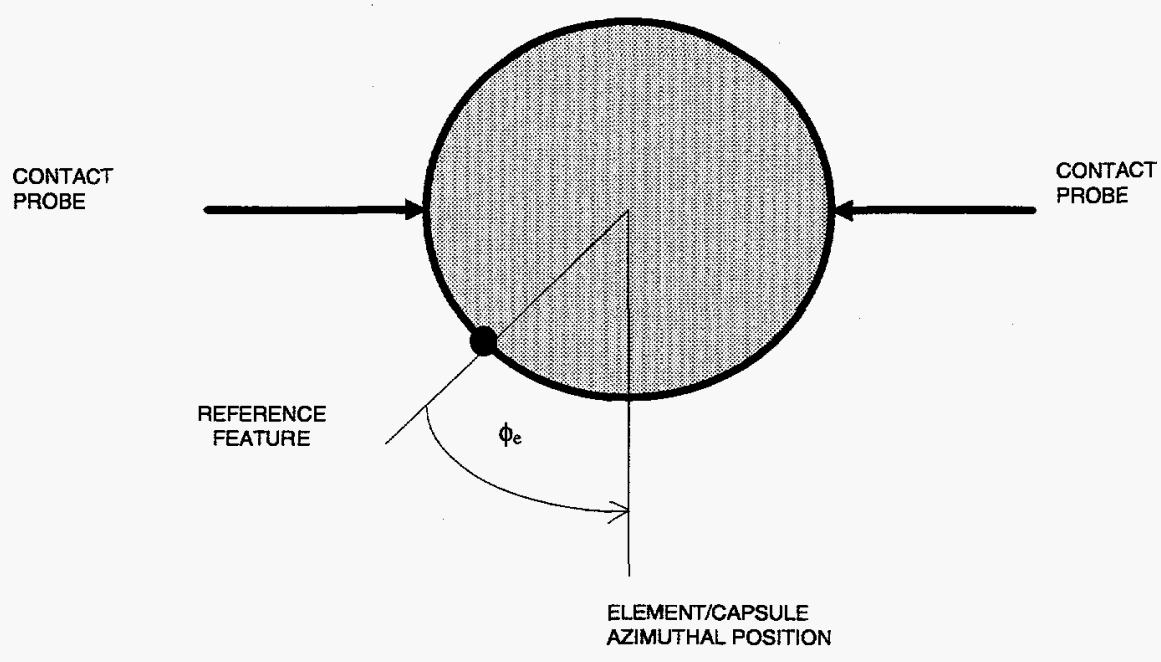

Figure 3. View looking down on top of element/capsule.

During measurement of the profile, recorded data are sometimes corrupted by electronic noise. Until the source of this noise can be discovered and corrected, obvious data errors may have been removed from the attached data reports. 
Copies of the profilometry data are provided on CD-ROMs. By convention, the data from each azimuthal angle is contained in a separate file with the name:

\section{filename ext}

where:

$$
\begin{aligned}
& \text { filename = Element/Capsule ID } \\
& \text { ext }=\text { Azimuthal Angle (e.g., 045) }
\end{aligned}
$$

Within each file, the data are recorded as plain ASCII text with each line terminated by a $<\mathrm{CR}><\mathrm{LF}>$. The first three lines of data contain descriptive text information (such as S/A ID and PIN ID) that is self explanatory. Below this are three columns of tab-delimited numeric data corresponding (left-toright) to: "Z-Position (in.)", " $\phi_{\mathrm{s}}\left({ }^{\circ}\right)$ and "Diameter (in.)". The end of data are sometimes flagged by large contrasting numbers, usually a string of nines (e.g., "999.999").

\subsection{Bow and Length Measurement}

The rod bow and length measurement system is mounted in conjunction with the rod contact profilometer on a standard examination stage. The rod hangs freely from a collet-style end fitting applied to its end plug. The end fitting is held rigidly in the gripper attached to the vertical carriage of the examination stage. The measurement system (see Figure 4) consists of two horizontal screw-driven flat bars, oriented at right angles to each other, on which an edge following light-beam device is mounted. When the light beam is interrupted, the screw drive stops the motion of the bar.

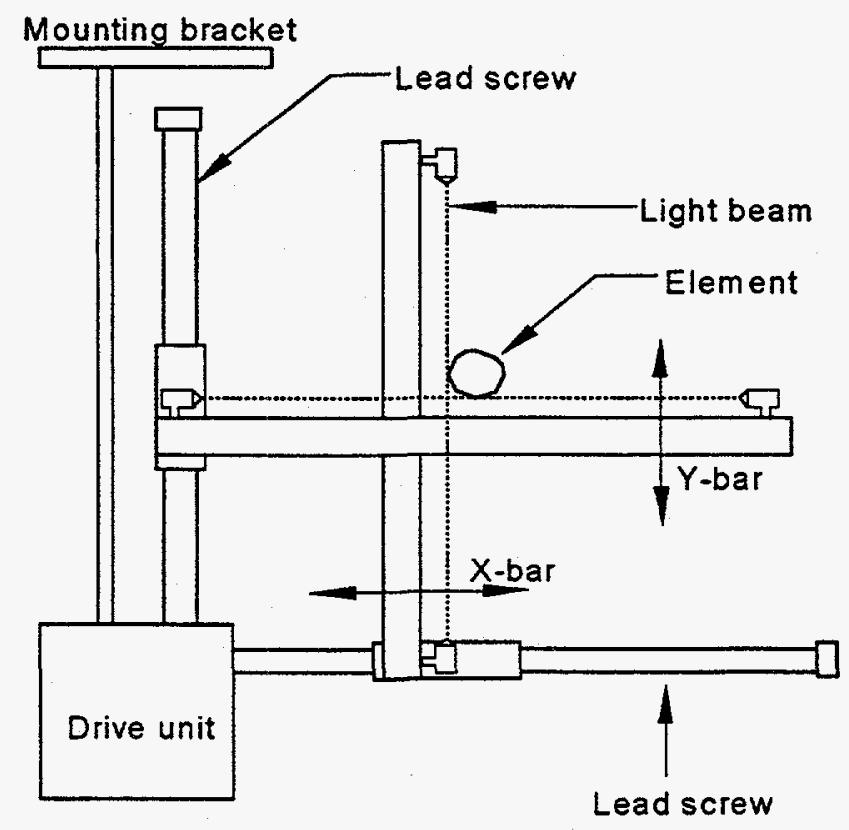

RED97 560083

Figure 4. Measurement system.

For each element, a series of $x-y$ coordinates is obtained at increments along the element $(Z)$ by running the bars toward the element until each light beam is interrupted by the edge of the element and 
converting the position into an $x-y$ coordinate pair. This sequence is repeated until a full set of $x, y, z$ coordinates is obtained.

The length of the element is determined and the positions of the element end points are extrapolated from the measured data. The reference, from which bow is measured, is an imaginary tip to tip centerline connecting the element position $\left(\mathrm{Z}_{\mathrm{i}}\right)$; the horizontal distance $\left(\mathrm{D}_{\mathrm{i}}\right)$ is calculated from this centerline $\left(\mathrm{X}_{\mathrm{ci}}, \mathrm{Y}_{\mathrm{ci}}\right)$ to the element $\left(\mathrm{X}_{\mathrm{i}}, \mathrm{Y}_{\mathrm{i}}\right)$. However, bow $\left(\mathrm{B}_{\mathrm{i}}\right)$ is defined as the perpendicular distance and, as shown in Figure 5 , the tip-to-tip centerline is offset from vertical by the angle $\theta$; therefore, the actual bow $\left(B_{i}\right)$ is calculated from the horizontal distance by correcting for the angle $\theta$. The hanging length of the element is measured by touching the bottom tip of the rod to a conductive plate on the bow and length gage assembly, and comparing that Z-position to the Z-position of a length standard similarly positioned.

All of the calculations of bow are performed by an online, data-reduction computer. The data recorded consists of a table of bow data for each rod, a table of maximum bow (and bow direction) for each rod, and a table of the corrected length of each rod. The bow measurements were made at 2-in. increments along the length of the rod. The corrected length of the rod is calculated by summing the 2-in. segment lengths (from tip to tip) between the calculated 3D positions of the element centerline (i.e., along the length of the bowed shape of the element). The data for the bow and length measurements are found in Appendix F.

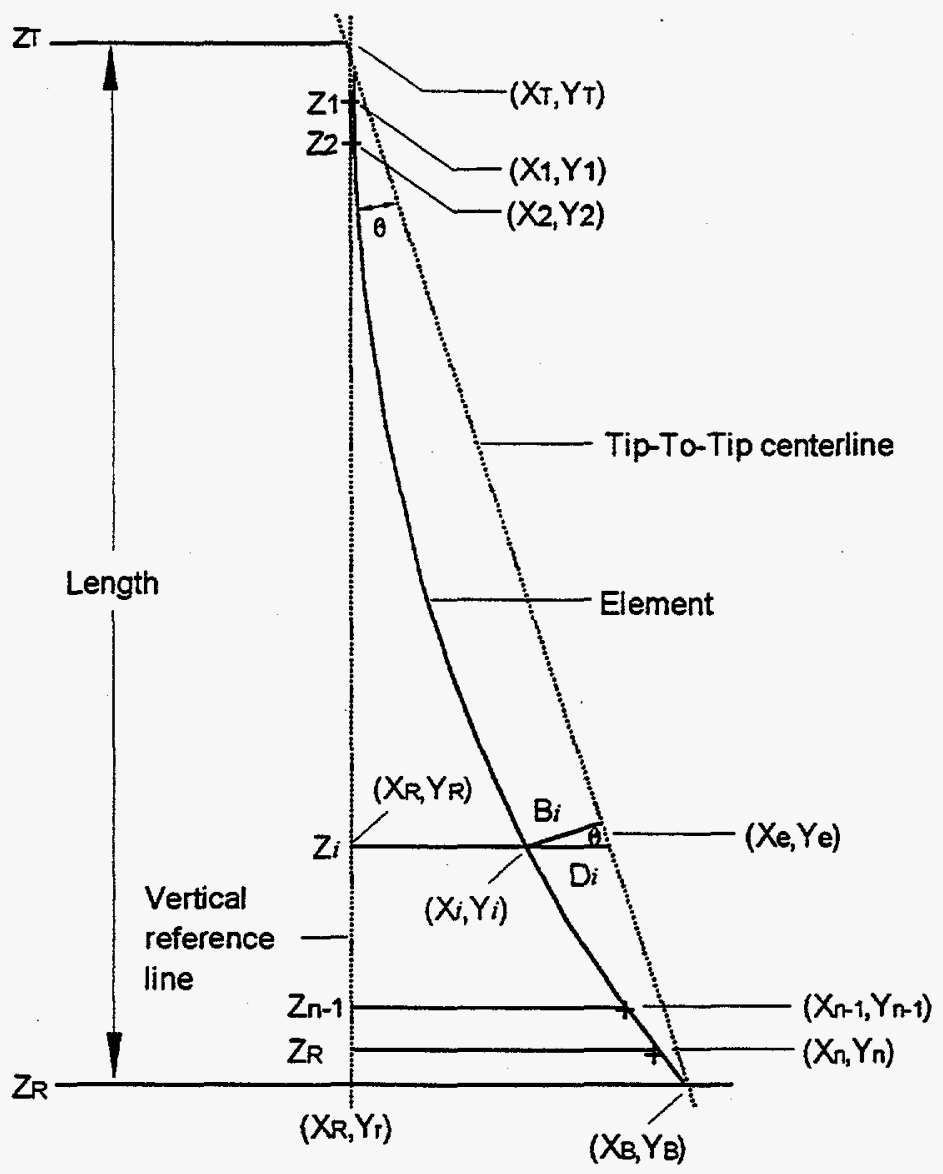

REDP7 SCO083

Figure 5. Elevation view of element as measured. 


\subsection{Gas, Analysis, Sample, and Recharged System}

\subsubsection{GASR Overview}

The Gas, Analysis, Sample and Recharge System (GASR) located in the ANL HFEF was used to obtain the rod internal pressure and void volume and plenum gas samples for analysis. The laser punctures the capsule in the plenum region and releases the rod gas into calibrated volumes. After the gas has reached equilibrium, it is further expanded into evacuated sample bottles out-of-cell for mass spectrometric and tritium concentration analysis. Then the capsule and seal-head chamber are repeatedly refilled with helium gas and reopened into known evacuated calibrated system volumes in order to calculate the capsule's volume. After calculating the volume of the capsule, the pressure of the capsule (prior to puncture) is calculated using the equilibrium pressure values measured after expansion into the calibrated volumes.

\subsubsection{Dummy Rod}

To provide assurances that the GASR system was measuring accurate rod void volumes and pressures, a rod was taken from the Critical Test- 2 assembly and modified by drilling through the upper end cap into the plenum and welding a valve on the upper end cap of the rod. The rod was pressurized with helium to a known pressure and temperature. The ANL was not told what the pressure was until the puncture and calculations were complete. The calculated pressure obtained from GASR data was within experimental accuracy of the measured pressure of the rod. Information on the dummy rod preparation and GASR puncture data is found Appendix G.

\subsubsection{GASR Operations}

Based on estimated ranges supplied by PNNL for Loop-1, S1, and B1 internal rod pressures, an auxiliary volume was needed to maintain the seal-head pressure, including the auxiliary volume, below 50 psia. This restriction prevents the resultant pressure from expansion into the manifold of being greater than atmospheric pressure. An interlock is in place which prohibits taking samples of gas outside the cell at pressures greater than atmospheric.

The rod is clamped against the saddle-shaped gasket in the seal head. The entire system, including the sample chambers, is evacuated to $<30$ mtorr. chambers.

The seal head, including the auxiliary volume, is isolated from the manifold and the sampling

The laser drills a small hole 5 to $10 \mathrm{~mm}$ in diameter in the capsule wall of the plenum region. The gas expands into the seal-head and auxiliary volume where the pressure is measured on a high-range meter $(\mathrm{P} 1)$.

When the pressure has equilibrated in the seal-head and auxiliary volume, a valve is opened to the manifold and the gas expands into the manifold, where the pressure is monitored on an accurate lowrange meter $(\mathrm{P} 2)$ and the capsule temperature is measured by an in-cell thermocouple. The assumption is made that all expansions are adiabatic, since no temperature increase is seen in the rod. 
Valves to the sample lines are opened to allow the gas to expand into the sample bottles. Isolation valves on the sample bottles are closed to capture the gas samples and the bottles are carried to the Analytical Laboratories. The pressure reading from $\mathrm{P} 2$ and a temperature reading from a thermometer inside the sampling box are recorded on the traveler accompanying the bottles to the analytical laboratory.

A series of backfill and expansion operations are performed to accurately determine the capsule void volume. The auxiliary volume is isolated from the system during all backfill and expansion operations. Helium is used to backfill the capsule to selected target pressure ranging from atmospheric to $45 \mathrm{psia}$, as monitored by P2. The capsule/seal head is isolated and the manifold is re-evacuated to $<30$ mtorr. The helium in the capsule/seal head is then expanded into the manifold where the pressure is monitored on $\mathrm{P} 2$.

The rod is backfilled with helium gas before rewelding. The laser beam is defocused and fired to create a puddle weld. Attempts to reweld all rods were not successful. On the first rod, WL-18, silicone was used to plug the hole, but this procedure was discontinued on the remaining rods.

\subsubsection{Rod Void Volume And Pressure Calculations}

Variables used to describe the GASR system are the seal-head volume, $\mathrm{V}_{\text {seal, }}$, the manifold volume, $\mathrm{V}_{\mathrm{man}}$, and the auxiliary volume, $\mathrm{V}_{\mathrm{aux}}$. The pressures are measured by two pressure gauges. The pressure gauge, $\mathrm{P} 1$, which measures the pressure in the seal-head and auxiliary volume, is denoted by $\mathrm{P}_{\text {seal }}$. The gauge has a range of 0 to 200 psia with an accuracy of \pm 0.1 psia. The pressure gauge in the manifold, $P 2$, measures the pressure, $P_{\operatorname{man}}$. $P 2$ has a range of 0 to 50 psia with an accuracy of \pm 0.01 psia. $P_{\text {cap }}$ and $V_{\text {cap }}$ are the rod's void volume and internal pressure prior to puncture. $P_{\text {seal }}$ is only measured once, upon initial puncture. This is the only measurement that $\mathrm{P} 1$ is used for due to its inherent inaccuracies. All other pressure measurements are made with the more accurate $\mathrm{P} 2$ pressure gauge.

Below is the mass balance equation describing the expansion from the capsule into the seal-head and auxiliary volume (denoted as the primary side) after puncture.

$$
\mathrm{P}_{\text {cap }} \mathrm{V}_{\text {cap }}=\mathrm{P}_{\text {seal }}\left(\mathrm{V}_{\text {cap }}+\mathrm{V}_{\text {seal }}+\mathrm{V}_{\text {aux }}\right)
$$

The expansion from the primary side into the manifold of the GASR system (denoted as the secondary side) is the second expansion. This expansion can be performed with the auxiliary volume open or isolated from the system. Except for one rod out of the ten, the auxiliary volume was open to the secondary side during expansion. Below is the mass balance equation for the expansion into the manifold with the auxiliary volume open.

$$
P_{\text {seal }}\left(V_{\text {cap }}+V_{\text {seal }}+V_{\text {aux }}\right)=P_{\text {man }}\left(V_{\text {cap }}+V_{\text {seal }}+V_{\text {aux }}+V_{\text {man }}\right)
$$

$P_{\text {cap }}$ can be obtained by substituting the above expression for $V_{\text {cap }}$ into the prior expression for $P_{\text {cap. }}$

$$
\mathrm{P}_{\text {cap }}=\mathrm{P}_{\text {man }}\left(\mathrm{V}_{\text {cap }}+\mathrm{V}_{\text {seal }}+\mathrm{V}_{\text {aux }}+\mathrm{V}_{\text {man }}\right) / \mathrm{V}_{\text {cap }}
$$


There was one rod where the auxiliary volume was isolated from the seal-head volume during expansion into the secondary side. In this case, the mass balance equation governing the expansion into the manifold is modified by eliminating the auxiliary volume on both sides of the equation:

$$
\begin{aligned}
& P_{\text {seal_mod }}\left(V_{\text {cap }}+V_{\text {seal }}\right)=P_{\text {man }}\left(V_{\text {cap }}+V_{\text {seal }}+V_{\text {man }}\right) \\
& P_{\text {seal_mod }}=P_{\text {man }}\left(V_{\text {cap }}+V_{\text {seal }}+V_{\text {man }}\right) /\left(V_{\text {cap }}+V_{\text {seal }}\right)
\end{aligned}
$$

Where $\mathrm{P}_{\text {seal_mod }}$ represents the pressure of only the gas in the seal-head volume and does not include the moles of gas in the auxiliary volume. $P_{\text {seal_mod }}$ is used in the expression for $V_{\text {cap }}$ replacing $P_{\text {seal }}$. This results in an expression to calculate the $\mathrm{P}_{\text {cap }}$ when the auxiliary volume is isolated during expansion:

$$
P_{\text {cap }}=P_{\text {man }}\left(V_{\text {cap }}+V_{\text {seal }}+V_{\text {aux }}\right)\left(V_{\text {cap }}+V_{\text {seal }}+V_{\text {man }}\right) /\left(V_{\text {cap }}\left(V_{\text {cap }}+V_{\text {seal }}\right)\right.
$$

During backfills, the auxiliary volume was isolated from the seal-head volume, so the expansion of the seal head and capsule expands directly into the manifold volume. The mass balance for this operation becomes:

$$
P_{\text {seal }}\left(V_{\text {cap }}+V_{\text {seal }}\right)=P_{\text {man }}\left(V_{\text {cap }}+V_{\text {seal }}+V_{\text {man }}\right)
$$

Solving for $\mathrm{V}_{\text {cap }}: \quad \mathrm{V}_{\text {cap }}=\left(\left(\mathrm{P}_{\text {man }}-\mathrm{P}_{\text {seal }}\right) \mathrm{V}_{\text {seal }}+\mathrm{P}_{\text {man }} \mathrm{V}_{\text {man }}\right) /\left(\mathrm{P}_{\text {seal }}-\mathrm{P}_{\text {man }}\right)$

From each backfill a value for $\mathrm{V}_{\text {cap }}$ can be obtained along with a value for $\mathrm{P}_{\text {cap. }}$. Data from the punctures is located in Appendix $\mathrm{H}$, including the calibration sheets, the data sheets, sample bottle table and volumes ANL's spread sheet for void volume and rod pressure, and MATHCAD work sheets used to verify the calculations.

Appendix I contains the results for the analysis of the plenum gas. 
Appendix A

Letter Report on Loop 1 Cutting Problems 


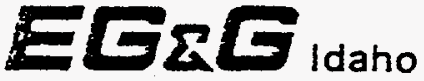

"Providing research and development services to the government"

\section{INTEROFFICE CORRESPONDENCE}

Date: $\quad$ March 7, 1994

To: $\quad$ L. R. Davison, MS 7101

From: $\quad$ L. B. Lundberg, MS 3890

Subject: ANALYSIS OF LIGHT WATER NEW PRODUCTION REACTOR LOOP-I

EXPERIMENTAL ASSEMBLY CUTTING PROBLEMS - LBL-06-94

On February 22, 1994, while attempting to cut off the end of the New Production Reactor (NPR) Light Water Reactor (LWR) Loop-1 Experiment Assembly under the water of the ATR can, an unusual "noise and vibration" were noted. The cutting operation was stopped, and an 'Occurrence' was declared. An Occurrence Report was written, entitled, "Noise and Vibration Observed During Underwater Cutting Operations in the Advanced Test Reactor Canal" (ID-EGG-ATR-1994-0004). The report states that the assembly was being cut with an underwater DC-arc saw which cuts metal by producing a plasma arc between the circumference of the rotating blade of the saw and the work piece. The arc saw cuts by melting through the work piece under the blade. The saw was cutting through a region of the assembiy that contained zircaloy captured inside a stainless steel collar. The cutting line is indicated in Figure 1 below.

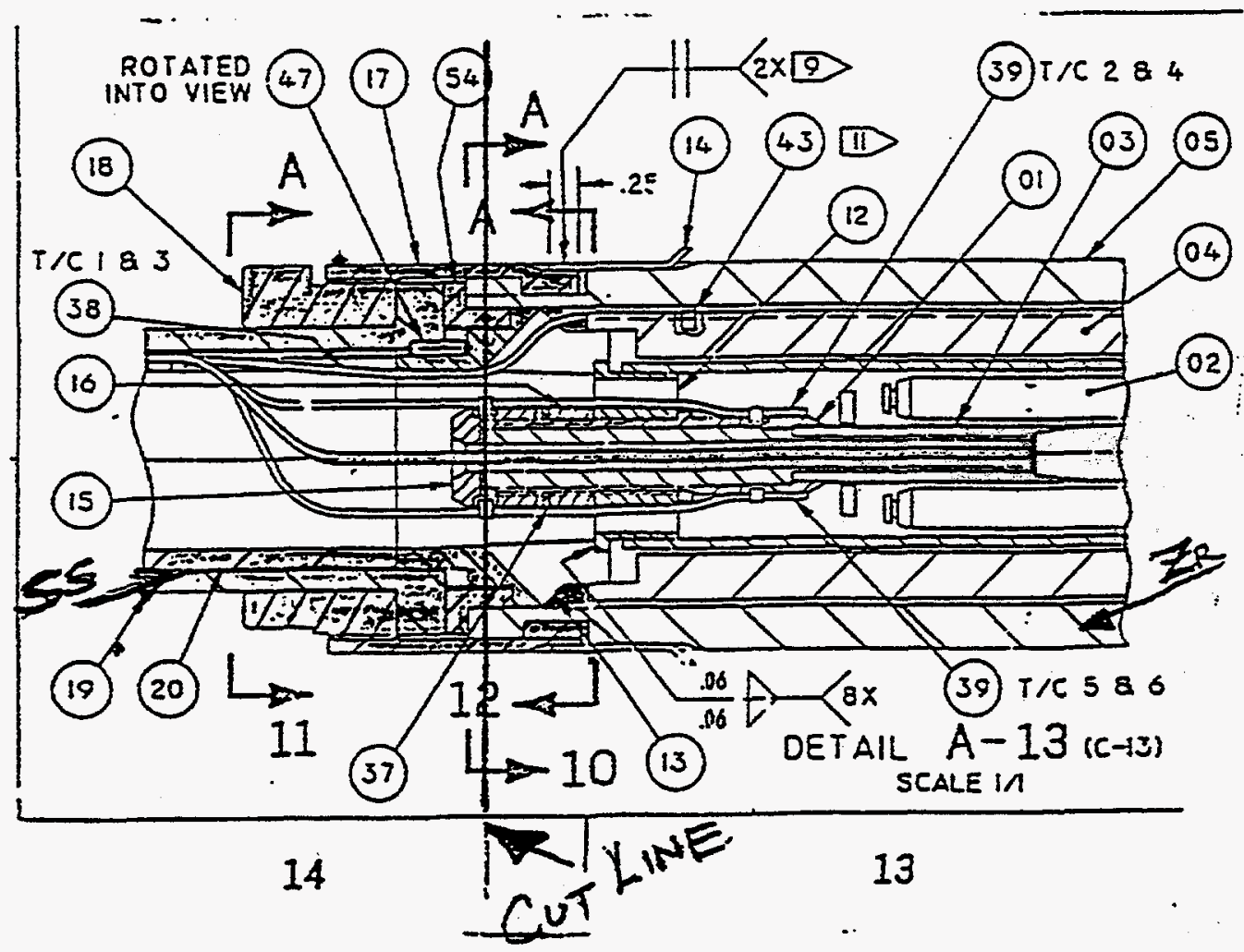

Figure 1. Cross-section of experimental assembly being cut with the arc saw in the ATR canal. A-1 
L. R. Davison

March 7, 1994

LBL-06-94

Page 2

In subsequent conversations with Lee Davison and Robert $0^{\prime}$ Connor, it was determined that the "vibrations" were actually "muffled thumps" which can be interpreted to have been caused by water hammers or small explosions. These small explosions were felt through the feet of individuals standing on the concrete floor near the canal. A reddish flash and a cloud of bubbles was al so observed to emanate from the cutting area. The resultant bubble cloud obscured the view of the cutoff saw. It was also stated that the cloud of bubbles was the size $f$ a "pie plate" when it reached the surface of the water.

The bubbles probably contained hydrogen that was generated by the reaction between zirconium and water: $\mathrm{Zr}+2 \mathrm{H}_{2} \mathrm{O} \Rightarrow \mathrm{ZrO}_{2}+2 \mathrm{H}_{2} \uparrow$. This type of chemical reaction applies to arc cutting of most metals under water. This reaction is expected to proceed at a very high rate because the rotating arc saw ejects molten metal droplets from the kerf into the surrounding water.

It is important to realize that the hydrogen cannot ignite under water, and the primary source of chemical energy is the reaction between molten metal droplets and the water. Electrolysis of the water due to the voltage between the saw blade and the work piece will produce an additional small quantity of hydrogen, but this source is expected to be insignificant compared to that generated by metal-water reactions.

An estimate was made for the amount of hydrogen generated in the observed explosion. It was estimated that a sufficient quantity of hydrogen was generated to fill a cylinder 10" in diameter by $10^{\prime \prime}$ high just below the surface of the water. Assuming that this volume is at standard temperature and pressure (STP: $298 \mathrm{~K}$ and 1 atmosphere pressure), it was calculated that about 0.6 moles of hydrogen were contained in the bubbles. This indicates that about 26 grams ( 0.3 moles) of zirconium reacted with the water to cause the explosion.

The maximum rate of metal ejection from the kerf was calculated from the highest electrical power input to the arc saw. Using enthalpy data for zircaloy, it was calculated that, for an arc saw cutting only zircaloy and operating at 27 volts and 3000 amperes, an upper limit melting rate of about $91 \mathrm{~g} / \mathrm{s}$ could be achieved. Oxidizing this amount of zirconium in water would generate up to $600 \mathrm{~kW}$ of thermal energy. The metal droplets will have to be small to ignite explosively in water at the melting temperature because it has been reported that bulk molten zirconium will not explosively react with water when the liquid metal is below $2873 \mathrm{~K}^{2}$. Explosive metal-water reactions are observed for metals that do not form a protective oxide at about 1.03 times the absolute melting temperature of the metal ${ }^{3}$. For small zirconium droplets, explosive reactions could be expected at metal temperatures as $10 \mathrm{~W}$ as $2194 \mathrm{~K}$, only $64^{\circ} \mathrm{K}$ above the melting point of 
L. R. Davison

March 7, 1994

LBL-06-94

Page 3

zirconium. The observed, chaotically occurring explosions probably resulted from the special conditions of ejected droplet size and number. For example, these explosions could have occurred as a result of bursts of metal droplets being ejected after a brief holdup in the kerf due to the high surface tension of molten zircaloy.

Steam bubbles generated from the chemically derived heat will also add to the problem. The steam bubbles will rapidly collapse after they are formed, and the force generated from the collapse will be significant and impulsive in nature. For example, we calculated that a $4-\mathrm{cm}$ diameter steam bubble collapses with a force of $127 \mathrm{kN}(28,500 \mathrm{lbf})$ under $138 \mathrm{kPa}$ (20 psia), $300 \mathrm{~K}$ $\left(80^{\circ} \mathrm{F}\right.$ ) water. (A description of the details of this calculation is attached.)

Hazards of cutting zircaloy with an arc saw are clearly indicated by this incident. Cutting zircaloy with an arc saw under water causes explosions, probabiy due to metal-water reactions. The potential severity of an explosion resulting from this procedure appears to be proportional to the power applied to the arc, i.e., the cutting rate. The ability of the work piece to hold up and eject, in bursts, large quantities of molten metal is chaotic, and therefore, the magnitude of an explosion resulting from contact with water cannot be predicted with any confidence.

The explosion hazard associated with cutting zircaloy under water would probably become negligible if the cutting method did not generate molten metal, especially if the cutting tool generated only large chips or turnings. A bandsaw or a circular saw with a toothed blade are likely candidates for cutting zircaloy with reasonably large chips and without generating molten metal.

\section{References}

1 SCDAP/RELAP5/M002 Code Manual, Vo1. 4: MATPRO - A Library of Materials Properties for Light-Water-Reactor Accident Analysis, J. K. Hohorst (Ed.), U.S. Nuclear Regulatory Commission Report No. NUREG/CR-5273, February 1990, p. 4.3-17.

2 P. Kuan and B. J. Buescher, "Ignition Threshold of Molten Aluminum in Water," in Heat Transfer - Minneapolis 1991, S. B. Yilmaz and B. G. Volintine (Eds.), AIChE Symposium Series, Vol. 87, No. 283, AIChE, NY (1991) pp. 177-181. 
L. R. Davison

March 7, 1994

LBL-06-94

Page 4

3 L. F. Epstein, "Correlation and Prediction of Explosive Metal-WaterReaction Temperatures," Nuclear Science and Engineering, 10 (1961) pp. 247-253

djm

Attachment:

As Stated

CC: R. C. Green, MS 4129

R. R. O'Connor, MS 7136

S. T. Polkinghorne, MS 3885

J. R. Wolf, MS 3890 q $x$

L. B. Lundberg Letter File 
Attachment 1

March 07, 1994

LBL-06-94

Page 1 of 3

Bubble Collapse Height and Force Estimates

Nomenclature

C sound speed

$c_{p}$ specific heat

$d$ initial bubble diameter

$F$ force

g acceleration of gravity

$\mathrm{H}$ height at collapse

$h_{\text {fo }}$ latent heat of condensation

$\mathrm{Ja}$ Jakob number (Equation 2)

$P_{\infty}$ liquid pressure

$\mathrm{Pr}$ Prandt 1 number

Ra Rayleigh number (Equation 3 )

$T_{\text {sat }}$ saturation temperature

$T_{\infty}$ liquid temperature

Greek symbols

$\checkmark$ kinematic viscosity

$\rho$ density

Subscripts

1 liquid property at $T_{\infty}$

$f$ liquid property at $T_{f}=\frac{1}{2}\left(T_{\text {sat }}+T_{\infty}\right)$

$\checkmark$ vapor property at saturation

\section{Collapse Height}

The height saturated steam bubbles rise in subcooled water before collapsing (i.e., condensing) can be estimated as follows:

$\mathrm{H} / \mathrm{d}=768 / \mathrm{Ja}^{3 / 5} \mathrm{Ra}^{1 / 4}$

where

$\mathrm{Ja}=\rho_{t} \mathrm{c}_{\mathrm{pf}}\left(T_{\mathrm{sat}}-T_{\infty}\right) / \rho_{\mathrm{v}} h_{\mathrm{fg}}$

$R a=\left[g\left(\rho_{1}-\rho_{v}\right) d^{3} / \rho_{f} v_{f}^{2}\right] \operatorname{Pr}_{f}$.

Solutions to Equation 1 for initial bubble radii ranging from 0.1 to $4 \mathrm{~cm}$ are shown below. The water pressure and temperature were assumed to be $138 \mathrm{kPa}(20 \mathrm{psia})$ and $300 \mathrm{~K}\left(80^{\circ} \mathrm{F}\right)$, respectively, and the bubbles were assumed to contain no noncondensable gas (air or $\mathrm{H}_{2}$ ). In addition, Equation 1 was assumed to be valid at low pressure (see Note).

\begin{tabular}{cc}
$\mathrm{d}(\mathrm{cm})$ & $\frac{H(\mathrm{~cm})}{0.17}$ \\
\hline 0.1 & 0.20 \\
0.2 & 0.25 \\
0.5 & 0.30 \\
1.0 & 0.36 \\
2.0 & 0.40 \\
3.0 & 0.43 \\
4.0 &
\end{tabular}


Attachment 1

March 07, 1994

LBL-06-94

Page 2 of 3

These results indicate that the steam bubbles would collapse almost immediately after being introduced into highly subcooled water. On the basis of these results, it appears unlikely that any steam reached the surface during underwater cutting operations in the ATR canal.

Note: The experiments reported in Reference 1 were performed for pressures ranging from 150 to 900 psia, for subcooling from 27 to $180^{\circ} \mathrm{F}$, and for initial bubble diameters of about $3 \mathrm{~mm}$. Collapse heights ranged from 2 to 15 times the initial bubble diameter, depending on operating conditions. The authors noted that collapse heights increase with increasing pressure because the latent heat per unit volume increases significantly with pressure, necessitating a longer bubble lifetime to transfer the heat liberated by condensation. The above results are consistent with this observation.

\section{Collapse Force}

The maximum force exerted on an "infinite" flat wall due to rapid bubble collapse can be estimated as follows: ${ }^{2}$

$F=4.5 d^{2}\left(\rho_{1} C_{1}\right)\left(P_{\infty} / \rho_{1}\right)^{1 / 2}$

Solutions to Equation 4 for bubble radii ranging from 0.1 to $4 \mathrm{~cm}$ are given below. The water pressure and temperature were again assumed to be $138 \mathrm{kPa}$ (20 psia) and $300 \mathrm{~K}\left(80^{\circ} \mathrm{F}\right)$, respectively.

$\begin{array}{ccc}\frac{d(\mathrm{~cm})}{0.1} & \frac{F(\mathrm{kN})}{0.0792} & \frac{F(1 \mathrm{bf})}{17.8} \\ 0.2 & 0.317 & 71.2 \\ 0.5 & 1.98 & 445 \\ 1.0 & 7.92 & 1780 \\ 2.0 & 31.7 & 7120 \\ 3.0 & 71.3 & 16000 \\ 4.0 & 127 & 28500\end{array}$

These results indicate that bubble collapse forces can be significant, which may explain the vibration and noise detected during underwater cutting operations in the ATR canal. 
Attachment 1

March 07, 1994

LBL-06-94

Page 3 of 3

Property Values at $138 \mathrm{kPa}$ and $300 \mathrm{~K}$

$$
\begin{aligned}
& T_{\text {set }}=382 \mathrm{~K}\left(228^{\circ} \mathrm{F}\right) \\
& T_{f}=341 \mathrm{~K}\left(154^{\circ} \mathrm{F}\right) \\
& \rho_{f}=980 \mathrm{~kg} / \mathrm{m}^{3} \\
& \rho_{t}=996 \mathrm{~kg} / \mathrm{m}^{3} \\
& \rho_{v}=0.798 \mathrm{~kg} / \mathrm{m}^{3} \\
& v_{f}=4.26 \times 10^{-7} \mathrm{~m}^{2} / \mathrm{s} \\
& c_{p f}=4180 \mathrm{~J} / \mathrm{kg} \cdot \mathrm{K} \\
& h_{f g}=2.23 \times 10^{6} \mathrm{~J} / \mathrm{kg} \\
& \mathrm{Pr}_{f}=2.64 \\
& C=1500 \mathrm{~m} / \mathrm{s}
\end{aligned}
$$

\section{References}

1. G. G. Brucker and E. M. Sparrow, "Direct Contact Condensation of Steam Bubbles in Water at High Pressure," Int. J. Heat Mass Iransfer, 20, 1977 , pp. $371-381$.

2. F. J. Moody, Introduction to Unsteady Thermofluid Mechanics, New York: John Wiley \& Sons, 1990, p. 570 . 


$$
\text { A-8 }
$$


Appendix B

Photographs 


\section{Appendix B}

\section{Table of Contents}

\section{Loop-1}

\begin{tabular}{|c|c|c|}
\hline PHOTO-ID & PIN-ID & Caption \\
\hline 9G1 & Loop Assembly & Top half of face \#1 (Bundle $0^{\circ}$ face) \\
\hline $9 \mathrm{G} 2$ & Loop Assembly & Bottom half of face $\# 1$ (Bundle $0^{\circ}$ face) \\
\hline 9G3 & Loop Assembly & Top half of face $\# 2$ (Bundle $90^{\circ}$ face) \\
\hline 9G4 & Loop Assembly & Bottom half of face $\# 2$ (Bundle $90^{\circ}$ face) \\
\hline 9G5 & Loop Assembly & Top half of face \#3 (Bundle $180^{\circ}$ face) \\
\hline 9G6 & Loop Assembly & Bottom half of face $\# 3$ (Bundle $180^{\circ}$ face) \\
\hline 9G7 & Loop Assembly & Top half of face \#4 (Bundle $270^{\circ}$ face) \\
\hline 9G8 & Loop Assembly & Bottom half of face $\# 4$ (Bundle $270^{\circ}$ face) \\
\hline 9G9 & Loop Assembly & Through-mirror view of bottom of bundle (face \#1 up) \\
\hline $11 \mathrm{G} 1$ & WL-01 & Shiny axial patch \\
\hline $11 \mathrm{G} 2$ & WL-01 & Low-luster surface (bottom of target) \\
\hline $11 \mathrm{G} 3$ & WL-01 & Light-to-dark transition \\
\hline $11 \mathrm{G} 4$ & WL-01 & Shiny surface (upper middle) \\
\hline $11 \mathrm{G} 5$ & WL-01 & Upper weld (shows end-fitting grip marks) \\
\hline $11 \mathrm{G} 6$ & WL-27 & General pitting (typical) \\
\hline $11 \mathrm{G} 7$ & WL-27 & Large pit (typical) \\
\hline $11 \mathrm{G} 8$ & WL-27 & Large pit (typical) \\
\hline $11 \mathrm{G} 9$ & WL-27 & Barrier transition (typical) \\
\hline $11 \mathrm{G} 10$ & WL-27 & Spacer grid marks (typical) \\
\hline $11 \mathrm{G} 11$ & WL-26 & General pitting \\
\hline $11 \mathrm{G} 12$ & WL-26 & Lower tip \\
\hline $11 \mathrm{G} 13$ & WL-22 & Typical barrier (bottom, front) \\
\hline $11 \mathrm{G} 14$ & WL-22 & Typical barrier (bottom, back) \\
\hline $11 \mathrm{G} 15$ & WL-22 & Typical barrier (center) - Rotational composite, $328^{\circ}$ \\
\hline $11 \mathrm{G} 16$ & WL-22 & Typical barrier (center) - Rotational composite, $28^{\circ}$ \\
\hline $11 \mathrm{G} 17$ & WL-22 & Typical barrier (center) - Rotational composite, $88^{\circ}$ \\
\hline $11 \mathrm{G} 18$ & WL-22 & Typical barrier (center) - Rotational composite, $148^{\circ}$ \\
\hline
\end{tabular}


Loop-1

\begin{tabular}{lll}
\hline PHOTO-ID & PIN-ID & \multicolumn{1}{c}{ Caption } \\
\hline $11 G 19$ & WL-22 & Typical barrier (center) - Rotational composite, 207 \\
$11 G 20$ & WL-22 & Typical barrier (center) - Rotational composite, $268^{\circ}$ \\
$11 G 21$ & WL-22 & Barrier islands \\
$11 G 22$ & WL-22 & Lower tip \\
$11 G 23$ & WL-18 & Footprints \\
$11 G 24$ & WL-18 & Bottom tip \\
$11 G 25$ & WL-10 & Dark pit \\
$11 G 26$ & WL-09 & Typical barrier (bottom) - Rotational composite, $119^{\circ}$ \\
$11 G 27$ & WL-09 & Typical barrier (bottom) - Rotational composite, $179^{\circ}$ \\
$11 G 28$ & WL-09 & Typical barrier (bottom) - Rotational composite, $239^{\circ}$ \\
$11 G 29$ & WL-09 & Typical barrier (bottom) - Rotational composite, $299^{\circ}$ \\
$11 G 30$ & WL-09 & Typical barrier (bottom) - Rotational composite, $359^{\circ}$ \\
$11 G 31$ & WL-09 & Typical barrier (bottom) - Rotational composite, $60^{\circ}$ \\
$11 G 32$ & WL-03 & Footprints \\
$11 G 33$ & WL-03 & Top End \\
$11 G 34$ & WL-03 & Bottom End \\
$11 G 35$ & WL-03 & Dark stain \\
\hline
\end{tabular}

WL-10 Photo Composite

$\begin{array}{lll}15 \mathrm{G} 1 & \text { WL-10 } & \text { Angle of } 270 \\ 15 \mathrm{G} 2 & \text { WL-10 } & \text { Angle of } 270 \\ 15 \mathrm{G} 3 & \text { WL-10 } & \text { Angle of } 270 \\ 15 \mathrm{G} 4 & \text { WL-10 } & \text { Angle of } 270 \\ 15 \mathrm{G} 5 & \text { WL-10 } & \text { Angle of } 0 \\ 15 \mathrm{G} 6 & \text { WL-10 } & \text { Angle of } 0 \\ 15 \mathrm{G} 7 & \text { WL-10 } & \text { Angle of } 0 \\ 15 \mathrm{G} 8 & \text { WL-10 } & \text { Angle of } 0 \\ 15 \mathrm{G} 9 & \text { WL-10 } & \text { Angle of } 90 \\ 15 \mathrm{G} 10 & \text { WL-10 } & \text { Angle of } 90 \\ 15 \mathrm{G} 11 & \text { WL-10 } & \text { Angle of } 90 \\ 15 \mathrm{G} 12 & \text { WL-10 } & \text { Angle of } 90 \\ 15 \mathrm{G} 13 & \text { WL-10 } & \text { Angle of } 180\end{array}$

B-ii 
Loop-1

\begin{tabular}{cll}
\hline PHOTO-ID & PIN-ID & Caption \\
\hline $15 G 14$ & WL-10 & Angle of 180 \\
$15 G 15$ & WL-10 & Angle of 180 \\
$15 G 16$ & WL-10 & Angle of 180 \\
\hline $15 G 17$ & & WL-26 Photo Composite \\
\hline $15 G 18$ & WL-26 & Angle of 270 \\
$15 G 19$ & WL-26 & Angle of 270 \\
$15 G 20$ & WL-26 & Angle of 270 \\
$15 G 21$ & WL-26 & Angle of 270 \\
$15 G 22$ & WL-26 & Angle of 0 \\
$15 G 23$ & WL-26 & Angle of 0 \\
$15 G 24$ & WL-26 & Angle of 0 \\
$15 G 25$ & WL-26 & Angle of 0 \\
$15 G 26$ & WL-26 & Angle of 90 \\
$15 G 27$ & WL-26 & Angle of 90 \\
$15 G 28$ & WL-26 & Angle of 90 \\
$15 G 29$ & WL-26 & Angle of 90 \\
$15 G 30$ & WL-26 & Angle of 180 \\
$15 G 31$ & WL-26 & Angle of 180 \\
$15 G 32$ & WL-26 & Angle of 180 \\
\hline & & Angle of 180 \\
\hline
\end{tabular}

B-iii 
S1 and B1 Photos

\begin{tabular}{|c|c|c|}
\hline PHOTO-ID & PIN-ID & Caption \\
\hline $96-659-1-30$ & WT-11 & ATR Hot Cell Disassembly \\
\hline $96-659-2-26$ & WT-12 & ATR Hot Cell Disassembly \\
\hline $96-659-2-24$ & WT-12 & ATR Hot Cell Disassembly \\
\hline $10 \mathrm{G} 1$ & WT-11 & Disassembly \\
\hline $10 \mathrm{G} 2$ & WT-12 & Disassembly \\
\hline $14 \mathrm{G} 1$ & WT-11 & Top end \\
\hline $14 \mathrm{G} 2$ & WT-11 & Bottom end \\
\hline 14G3 & WT-11 & Typical scratches (rotation composite), $29^{\circ}$ \\
\hline $14 \mathrm{G} 4$ & WT-11 & Typical scratches (rotation composite), $89^{\circ}$ \\
\hline 14G5 & WT-11 & Typical scratches (rotation composite), $150^{\circ}$ \\
\hline $14 \mathrm{G} 6$ & WT-11 & Typical scratches (rotation composite), $210^{\circ}$ \\
\hline $14 \mathrm{G} 7$ & WT-11 & Typical scratches (rotation composite), $269^{\circ}$ \\
\hline $14 \mathrm{G} 8$ & WT-11 & Typical scratches (rotation composite), $329^{\circ}$ \\
\hline $14 \mathrm{G} 9$ & WT-12 & Top end \\
\hline $14 \mathrm{G} 10$ & WT-12 & Top end \\
\hline $14 \mathrm{G} 11$ & WT-12 & Bottom end \\
\hline $14 \mathrm{G} 12$ & WT-12 & Typical scratches (rotation composite), $291^{\circ}$, elev. $46.3 \mathrm{in}$. \\
\hline $14 \mathrm{G} 13$ & WT-12 & Typical scratches (rotation composite), $351^{\circ}$, elev. 46.3 in. \\
\hline $14 \mathrm{G} 14$ & WT-12 & Typical scratches (rotation composite), $52^{\circ}$, elev. 46.3 in. \\
\hline $14 \mathrm{G} 15$ & WT-12 & Typical scratches (rotation composite), $112^{\circ}$, elev. 46.3 in. \\
\hline $14 \mathrm{G} 16$ & WT-12 & Typical scratches (rotation composite), $171^{\circ}$, elev. 46.3 in. \\
\hline $14 \mathrm{G} 17$ & WT-12 & Typical scratches (rotation composite), $232^{\circ}$, elev. $46.3 \mathrm{in}$. \\
\hline $14 \mathrm{G} 18$ & WT-12 & Typical scratches (rotation composite), $292^{\circ}$, elev. 16.8 in. \\
\hline $14 \mathrm{G} 19$ & WT-12 & Typical scratches (rotation composite), $352^{\circ}$, elev. $16.8 \mathrm{in}$. \\
\hline $14 \mathrm{G} 20$ & WT-12 & Typical scratches (rotation composite), $52^{\circ}$, elev. 16.8 in. \\
\hline $14 G 21$ & WT-12 & Typical scratches (rotation composite), $112^{\circ}$, elev. 16.8 in. \\
\hline $14 \mathrm{G} 22$ & WT-12 & Typical scratches (rotation composite), $172^{\circ}$, elev. 16.8 in. \\
\hline $14 G 23$ & WT-12 & Typical scratches (rotation composite), $232^{\circ}$, elev. $16.8 \mathrm{in}$. \\
\hline
\end{tabular}




\section{ARGONNE NATIONAL LABORATORY}

P. O. Box 2528, Idaho Falis, Idaho 83403-2528

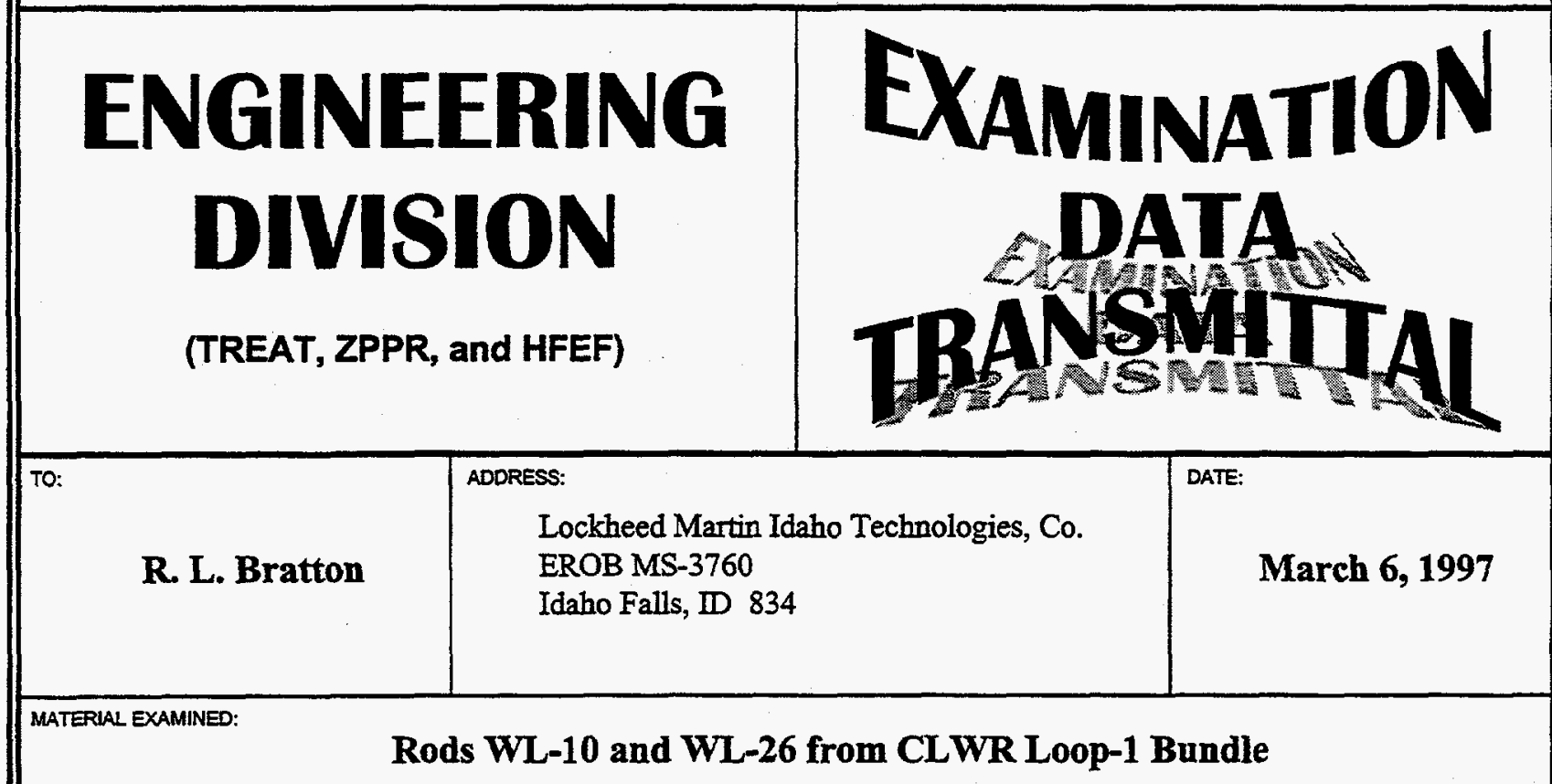

TYPE OF EXAMINATION:

Composite Photography

FORM OF DATA:

Photo Index and Mosaics (5)

NOTES:

CC: w/Attachments

Loop-1 file (unassembled)

CC: w/o Attachments

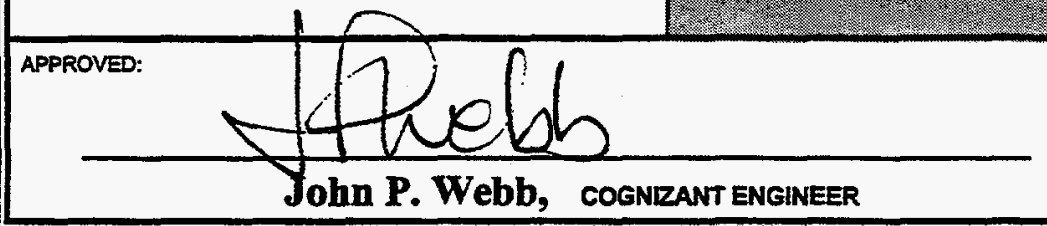

Questions regarding this data transmittal should be directed to the Cognizant Engineer at extension (208) 533-7773. 


\section{ARGONNE NATIONAL LABORATORY - WEST ENGINEERING DIVISION}

\section{INDEX OF PHOTOGRAPHS}

\begin{tabular}{|c|c|c|c|c|c|c|}
\hline FACILTY: & 口 TREAT & 口 ZPPR & 区 HFEF & 口 OTHER: & DATE: & $11 / 26 / 96$ \\
\hline ITEM: & $\begin{array}{l}\text { S/A or Test } \\
\text { Loop-1 }\end{array}$ & $\mathrm{Ca}$ & $\begin{array}{l}\text { WL-10/Elem } \\
\text { WLen }\end{array}$ & $\int^{\text {Subcap }}$ N/A & Other & \\
\hline SUBJECT & & & Comp & osite Photograph & & \\
\hline
\end{tabular}

\begin{tabular}{|c|c|c|c|c|}
\hline PHOTO ID & 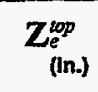 & $\begin{array}{c}\mathbf{Z}_{e}^{\text {bet }} \\
\text { (in.) }\end{array}$ & $\begin{array}{c}\phi_{e}^{*} \\
\left.0^{\circ}\right)\end{array}$ & DESCRIPTION \\
\hline 15G1 & 2.5 & 22.0 & 270 & \\
\hline $15 G 2$ & 17.0 & 38.0 & 270 & \\
\hline $15 G 3$ & 31.5 & 52.0 & 270 & \\
\hline $15 G 4$ & 45.5 & 60.0 & 270 & \\
\hline 15G5 & 2.5 & 22.0 & 0 & \\
\hline $15 G 6$ & 17.0 & 38.0 & 0 & \\
\hline 15G7 & 31.5 & 52.0 & 0 & \\
\hline $15 G 8$ & 45.5 & 60.0 & 0 & \\
\hline 15G9 & 2.5 & 22.0 & 90 & \\
\hline $15 G 10$ & 17.0 & 38.0 & 90 & \\
\hline 15G11 & 31.5 & 52.0 & 90 & \\
\hline 15G12 & 45.5 & 60.0 & 90 & \\
\hline $15 G 13$ & 2.5 & 22.0 & 180 & \\
\hline 15G14 & 17.0 & 38.0 & 180 & \\
\hline 15G15 & 31.5 & 52.0 & 180 & \\
\hline $15 G 16$ & 45.5 & 60.0 & 180 & \\
\hline & & & & \\
\hline & & & & \\
\hline & & & & \\
\hline & & & & \\
\hline & & & & \\
\hline & & & & \\
\hline & & & & \\
\hline & & & & \\
\hline & & & & \\
\hline & & & & \\
\hline
\end{tabular}

REMARKS:

* Arbitrary angular reference 


\section{ARGONNE NATIONAL LABORATORY - WEST ENGINEERING DIVISION}

\section{INDEX OF PHOTOGRAPHS}

\begin{tabular}{|c|c|c|c|c|c|c|}
\hline$\overline{\text { FACILTTY: }}$ & $\square$ TREAT & $\square$ ZPPR & 『 HFEF & 口 OTHER: & DATE: & $11 / 26 / 96$ \\
\hline TIEM: & $\begin{array}{l}\text { S/A or Test } \\
\text { Loop-1 }\end{array}$ & $\mathrm{Cz}_{\mathrm{z}}$ & $\begin{array}{l}\text { Wlem } \\
\text { WL-26 }\end{array}$ & ${ }^{\text {Subcap }}$ N/A & Other & \\
\hline \multicolumn{7}{|c|}{ Composite Photography } \\
\hline
\end{tabular}

\begin{tabular}{|c|c|c|c|c|}
\hline PHOTO ID & $\begin{array}{l}Z_{\text {(ep }}^{\text {top }} \\
\text { (in.) }\end{array}$ & $\begin{array}{l}\mathbf{Z}_{e}^{b o s} \\
\text { (nn.) }\end{array}$ & $\begin{array}{l}\phi_{e}^{*} \\
\left.{ }^{\circ}\right)\end{array}$ & DESCRIPTION \\
\hline $15 \mathrm{G17}$ & 2.5 & 22.0 & 270 & \\
\hline $15 G 18$ & 17.0 & 38.0 & 270 & \\
\hline 15G19 & 31.5 & 52.0 & 270 & \\
\hline $15 G 20$ & $\overline{45.5}$ & 60.0 & 270 & \\
\hline 15G21 & 2.5 & 22.0 & 0 & \\
\hline $15 G 22$ & 17.0 & 38.0 & 0 & \\
\hline $15 G 23$ & 31.5 & 52.0 & 0 & \\
\hline 15G24 & 45.5 & 60.0 & 0 & \\
\hline $15 G 25$ & 2.5 & 22.0 & 90 & \\
\hline $15 G 26$ & 17.0 & 38.0 & 90 & \\
\hline 15G27 & 31.5 & 52.0 & 90 & \\
\hline $15 G 28$ & 45.5 & 60.0 & 90 & \\
\hline $15 G 29$ & 2.5 & 22.0 & 180 & \\
\hline $15 G 30$ & $\overline{17.0}$ & 38.0 & 180 & \\
\hline 15G31 & 31.5 & 52.0 & 180 & \\
\hline $15 G 32$ & 45.5 & 60.0 & 180 & \\
\hline & & & & \\
\hline & & & & \\
\hline & & & & \\
\hline & & & & \\
\hline & & & & \\
\hline & & & & \\
\hline & & & & \\
\hline & & & & \\
\hline & & & & \\
\hline
\end{tabular}

REMARKS:

* Arbitrary angular reference 


\section{ARGONNE NATIONAL LABORATORY \\ P. O. Box 2528, Idaho Falls, Idaho $83403-2528$}

\begin{tabular}{|c|c|c|}
\hline \multicolumn{3}{|c|}{ (TREAT, ZPPR, and HFEF) } \\
\hline R. L. Bratton & $\begin{array}{l}\text { ADDRESS: } \\
\text { Lockheed Martin Idaho Technologies Co. } \\
\text { P. O. Box } 1625 \\
\text { EROB MS- } 3760 \\
\text { Idaho Falls, DD } 83415\end{array}$ & March 3, 1997 \\
\hline \multicolumn{3}{|c|}{ Two Rods from CLWR Test S1/B1 } \\
\hline \multicolumn{3}{|c|}{ Visual Examination } \\
\hline \multicolumn{3}{|c|}{ Data Sheets, Photo Index, Negatives (1) and Prints (5) } \\
\hline \multicolumn{3}{|l|}{ NOTES: } \\
\hline \multicolumn{3}{|l|}{$\begin{array}{l}\text { cc: w/Attachments } \\
\text { S1/B1 file (prints on }\end{array}$} \\
\hline \multicolumn{3}{|r|}{ 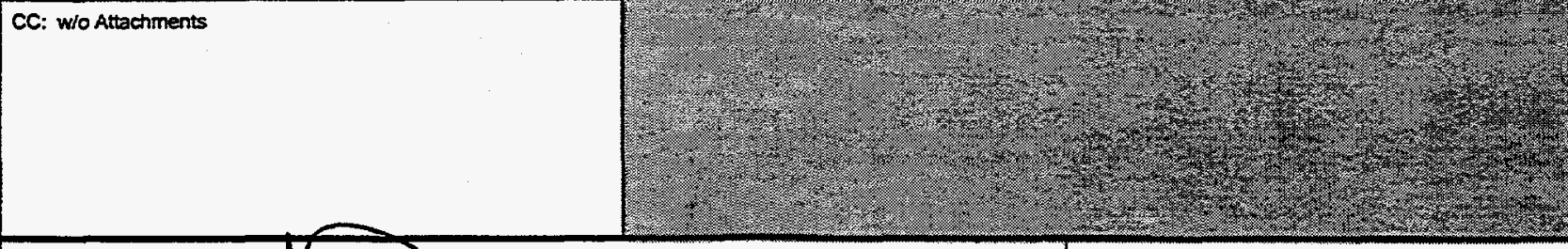 } \\
\hline APPROVED: & COGNIZANT E & $\begin{array}{l}\text { Questions regarding this data } \\
\text { transmittal should be directed to } \\
\text { the Cognizant Engineer at } \\
\text { extension (208) 533-7773. }\end{array}$ \\
\hline
\end{tabular}

Revised: 2/11/97 


\section{ARGONNE NATIONAL LABORATORY \\ Hot Fuel Examination Facility}

\section{INDEX OF PHOTOGRAPHS}

\begin{tabular}{|c|c|c|c|c|}
\hline FACILTY: & $\square$ FMF & X HFEF & HER: & DATE: \\
\hline TREM: & $\begin{array}{l}\text { S/A or Test } \\
\text { CLWR S1/B1 }\end{array}$ & $\begin{array}{l}\text { Cap/Elem } \\
\text { As noted }\end{array}$ & $\begin{array}{l}\text { Subcap } \\
\text { N/A }\end{array}$ & Other \\
\hline \multicolumn{5}{|c|}{ Visual examination of CLWR Target Rods } \\
\hline
\end{tabular}

\begin{tabular}{|c|c|c|c|c|c|}
\hline PHOTO ID & PIN ID & $\mathrm{z}_{\mathrm{e}}{ }^{1}$ & $\phi_{e}^{2}$ & MAG. & DESCRIPTION \\
\hline 14G1 & WT-11 & 48.6 & 287 & Med & Top end \\
\hline 14G2 & WT-11 & 0.7 & 189 & Med & Bottom end \\
\hline$\overline{14 G 3}$ & WT-11 & 28.0 & 29 & $\overline{\text { Med }}$ & Typical scratches (Rotational composite) \\
\hline$\overline{14 G 4}$ & $\overline{\text { WT-11 }}$ & 28.0 & 89 & Med & Typical scratches (Rotational composite) \\
\hline 14G5 & WT-11 & 28.0 & 150 & Med & Typical scratches (Rotational composite) \\
\hline$\overline{14 G 6}$ & WT-11 & $\overline{28.0}$ & 210 & $\overline{\text { Med }}$ & Typical scratches (Rotational composite) \\
\hline 14G7 & WT-11 & 28.0 & 269 & Med & Typical scratches (Rotational composite) \\
\hline $14 \mathrm{G8}$ & WT-11 & 28.0 & 329 & Med & Typical scratches (Rotational composite) \\
\hline$\overline{14 G 9}$ & $\overline{\text { WT-12 }}$ & 48.7 & 201 & Med & Top end \\
\hline 14G10 & WT-12 & $\overline{48.7}$ & 317 & Med & Top end \\
\hline 14 G11 & WT-12 & 0.5 & 354 & Med & Bottom end \\
\hline $14 \mathrm{G12}$ & $\overline{\text { WT-12 }}$ & 46.3 & 291 & Med & Typical (Rotational composite) \\
\hline 14G13 & WT-12 & 46.3 & 351 & Med & Typical (Rotational composite) \\
\hline 14 G14 & WT-12 & 46.3 & 52 & Med & Typical (Rotational composite) \\
\hline 14 G15 & WT-12 & 46.3 & 112 & Med & Typical (Rotational composite) \\
\hline $14 \mathrm{G16}$ & WT-12 & 46.3 & 171 & Med & Typical (Rotational composite) \\
\hline 14G17 & WT-12 & $\overline{46.3}$ & 232 & Med & Typical (Rotational composite) \\
\hline 14 G18 & WT-12 & 16.8 & 292 & Med & Typical (Rotational composite) \\
\hline 14G19 & $\overline{\mathrm{WT}}-12$ & 16.8 & 352 & Med & Typical (Rotational composite) \\
\hline $14 G 20$ & WT-12 & $\overline{16.8}$ & 52 & Med & Typical (Rotational composite) \\
\hline 14G21 & WT-12 & 16.8 & $\overline{112}$ & Med & Typical (Rotational composite) \\
\hline 14 G22 & WT-12 & $\overline{16.8}$ & $\overline{172}$ & $\overline{\text { Med }}$ & Typical (Rotational composite) \\
\hline 14 G23 & WT-12 & 16.8 & 232 & Med & Typical (Rotational composite) \\
\hline & & & & & \\
\hline & & & & & \\
\hline
\end{tabular}

REMARKS:

1. Approximate axial position of photo center above bottom tip (in inches).

2. Azimuthal angle of photo (in degrees) measured $\mathrm{CCW}$ from visual reference (index notch toward eye). 
VISUAL EXAMINATION DATA SHEET

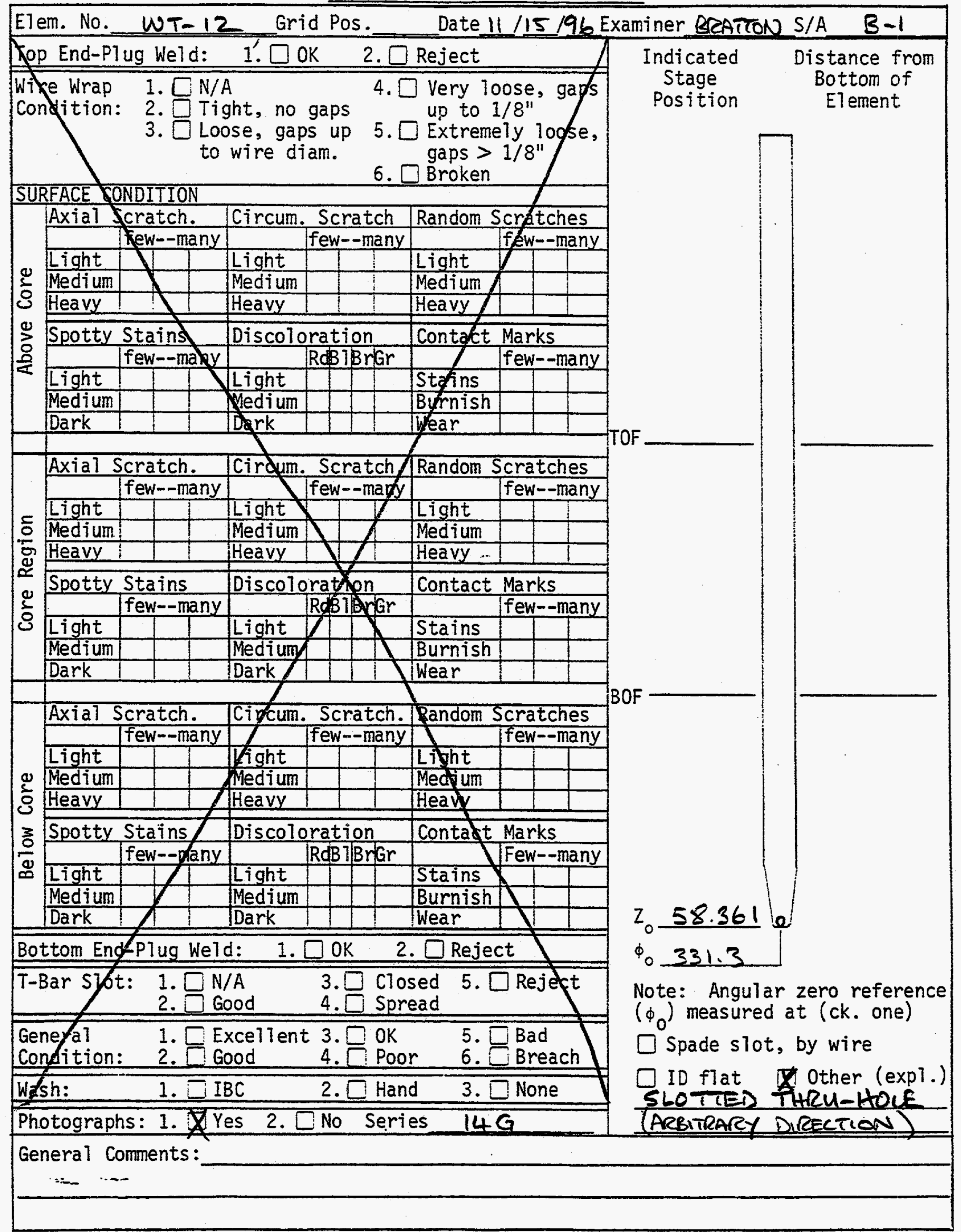

Note: $\dot{\theta}_{\mathrm{e}}=\phi_{j}-\phi_{0}$, if measured CCW; $\epsilon_{\mathrm{e}}=\Phi_{0}-\Phi_{i}$, if CW (looking down at top of element). 
VISUAL EXAMINATION DATA SHEET

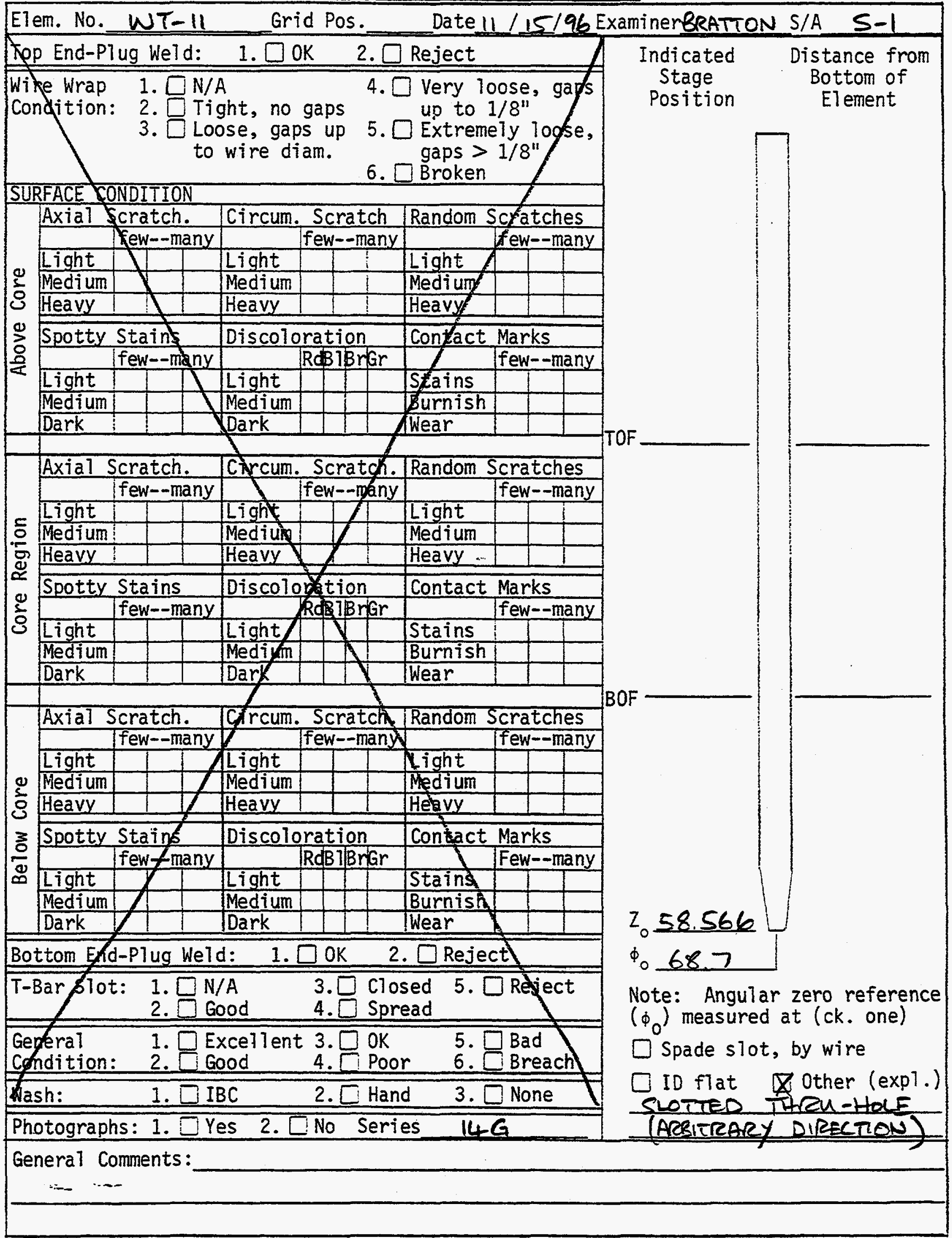

Note: $\dot{\theta}_{e}=\phi_{j}-\phi_{0}$, if measured $\mathrm{CCW} ; \epsilon_{e}=\Phi_{0}-\Phi_{j}$, if CW (looking down at top of element). 
B-8 


\section{Loop 1: Photographs}

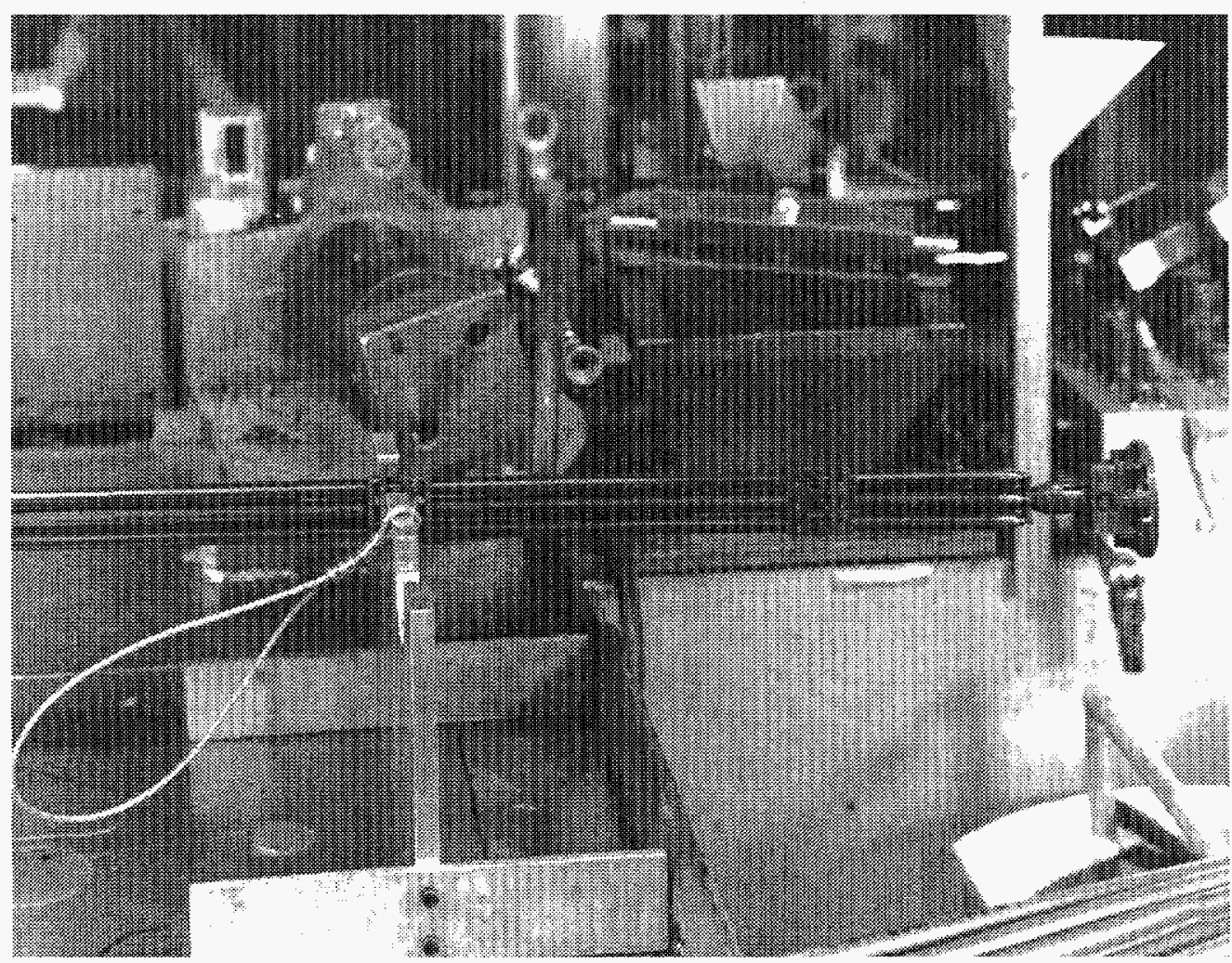

9G1. Loop Assembly, Top half of face \#1 (Bundle $0^{\circ}$ face)

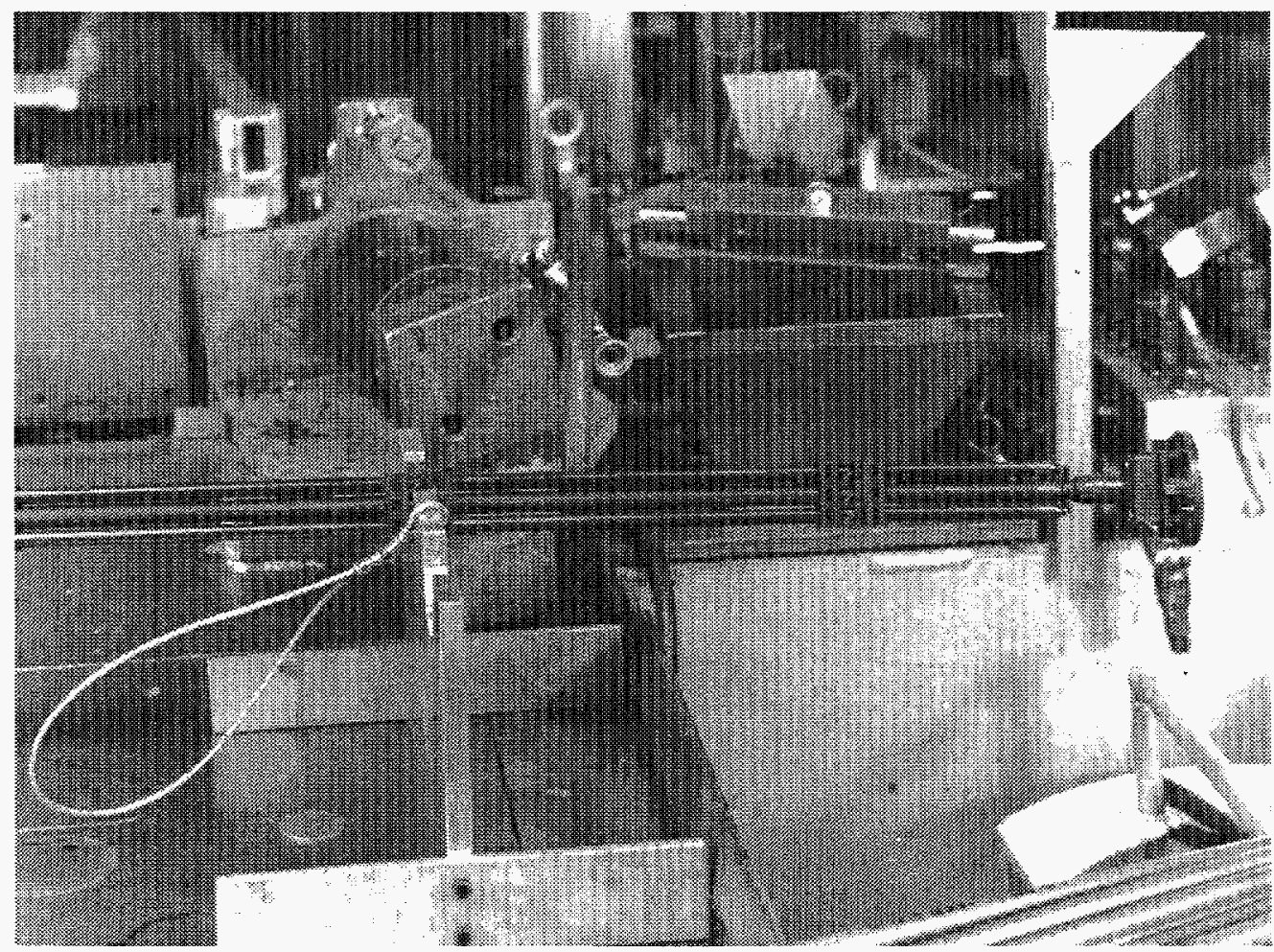

9G2. Loop Assembly, Bottom half of face \#1 (Bundle $0^{\circ}$ face) 


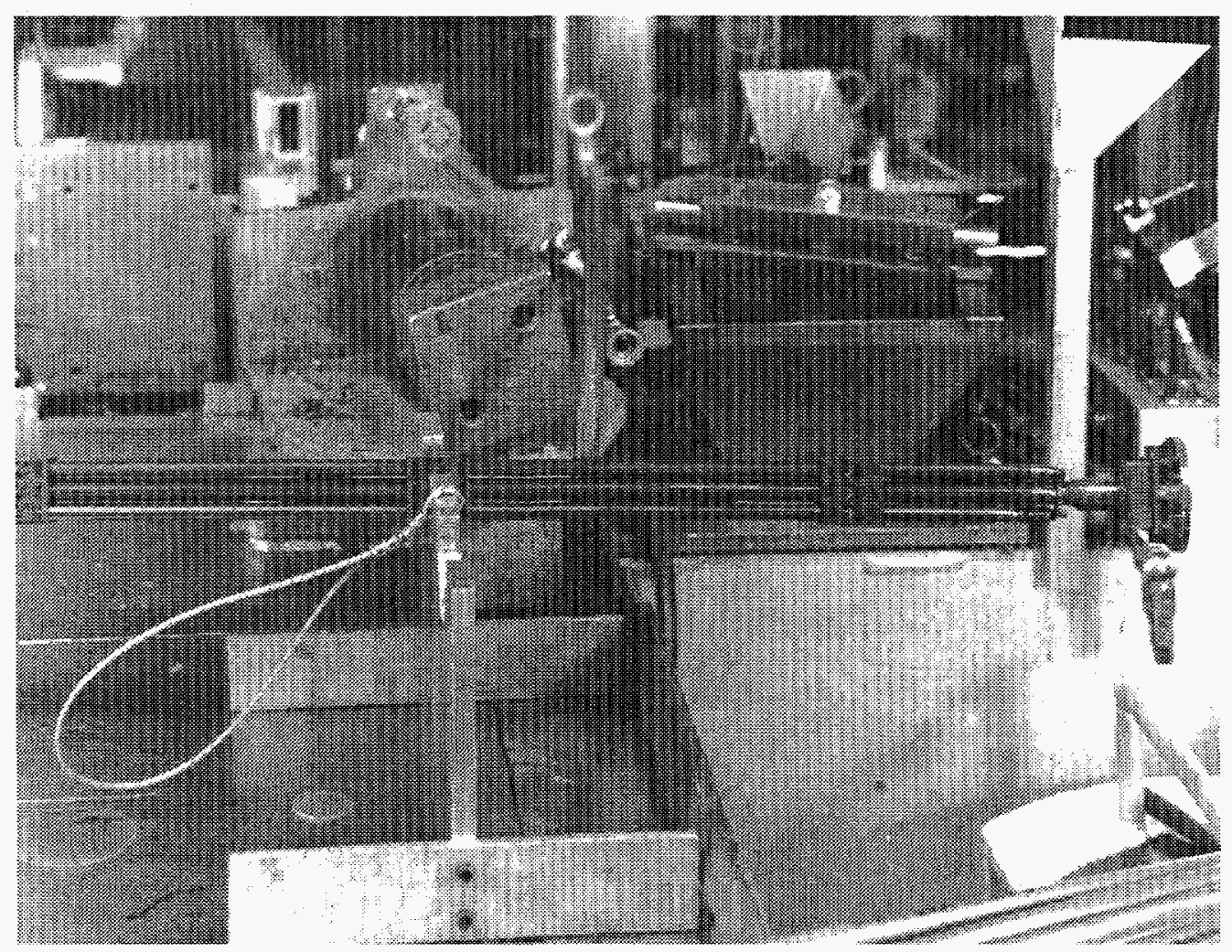

9G3. Loop Assembly, Top half of face \#2 (Bundle $90^{\circ}$ face)

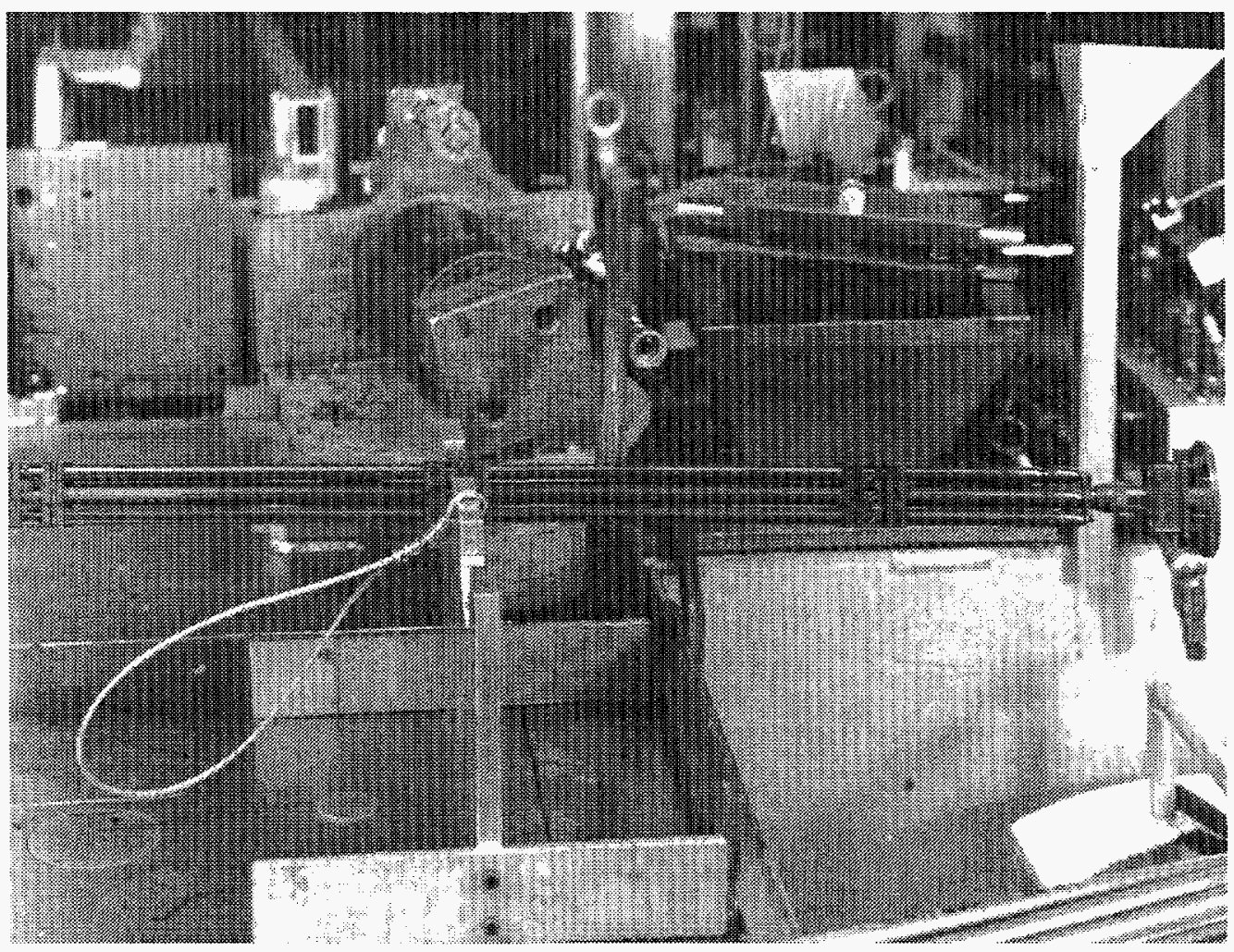

9G4. Loop Assembly, Bottom half of face \#2 (Bundle $90^{\circ}$ face) 


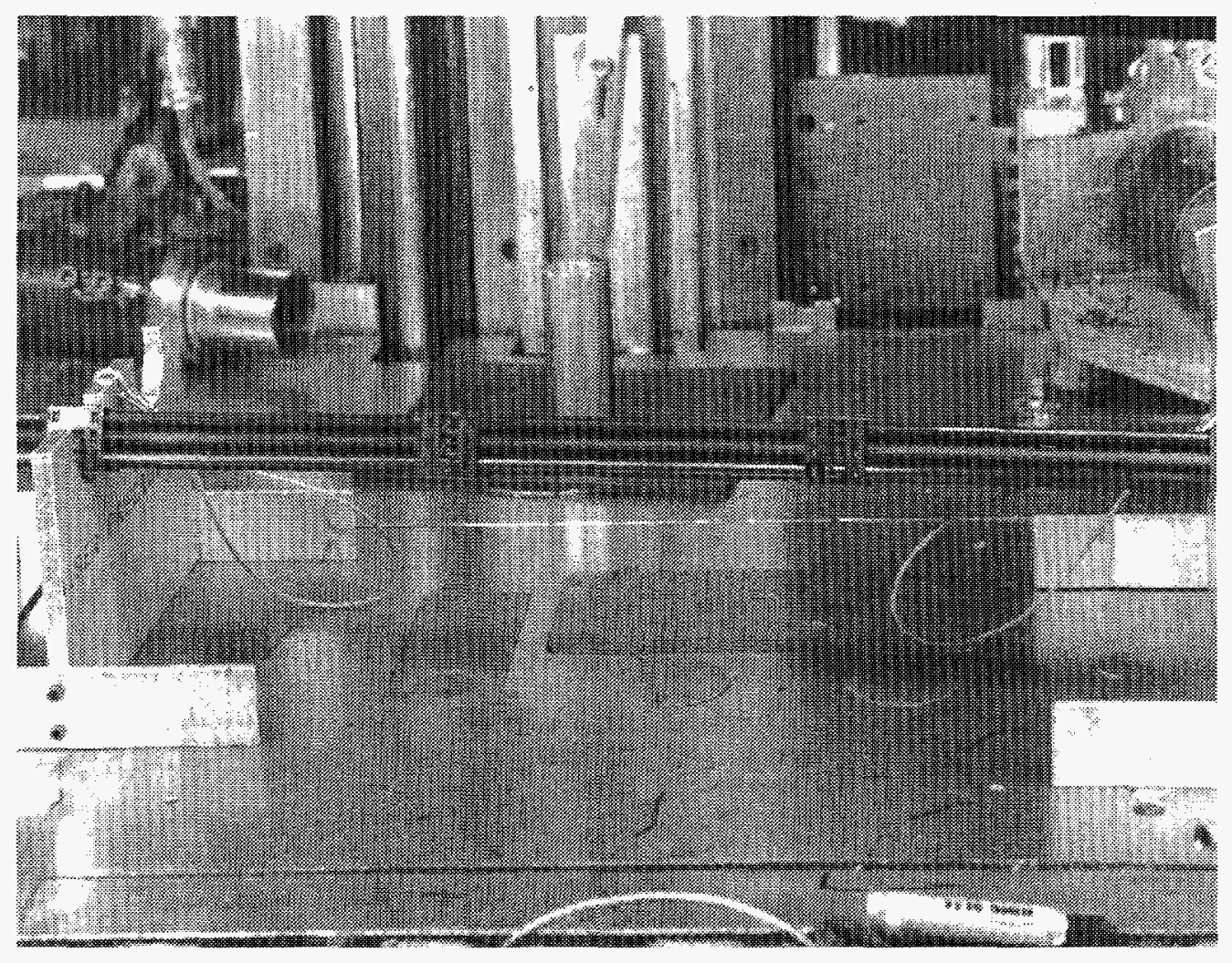

9G5. Loop Assembly, Top half of face \#3 (Bundle $180^{\circ}$ face)

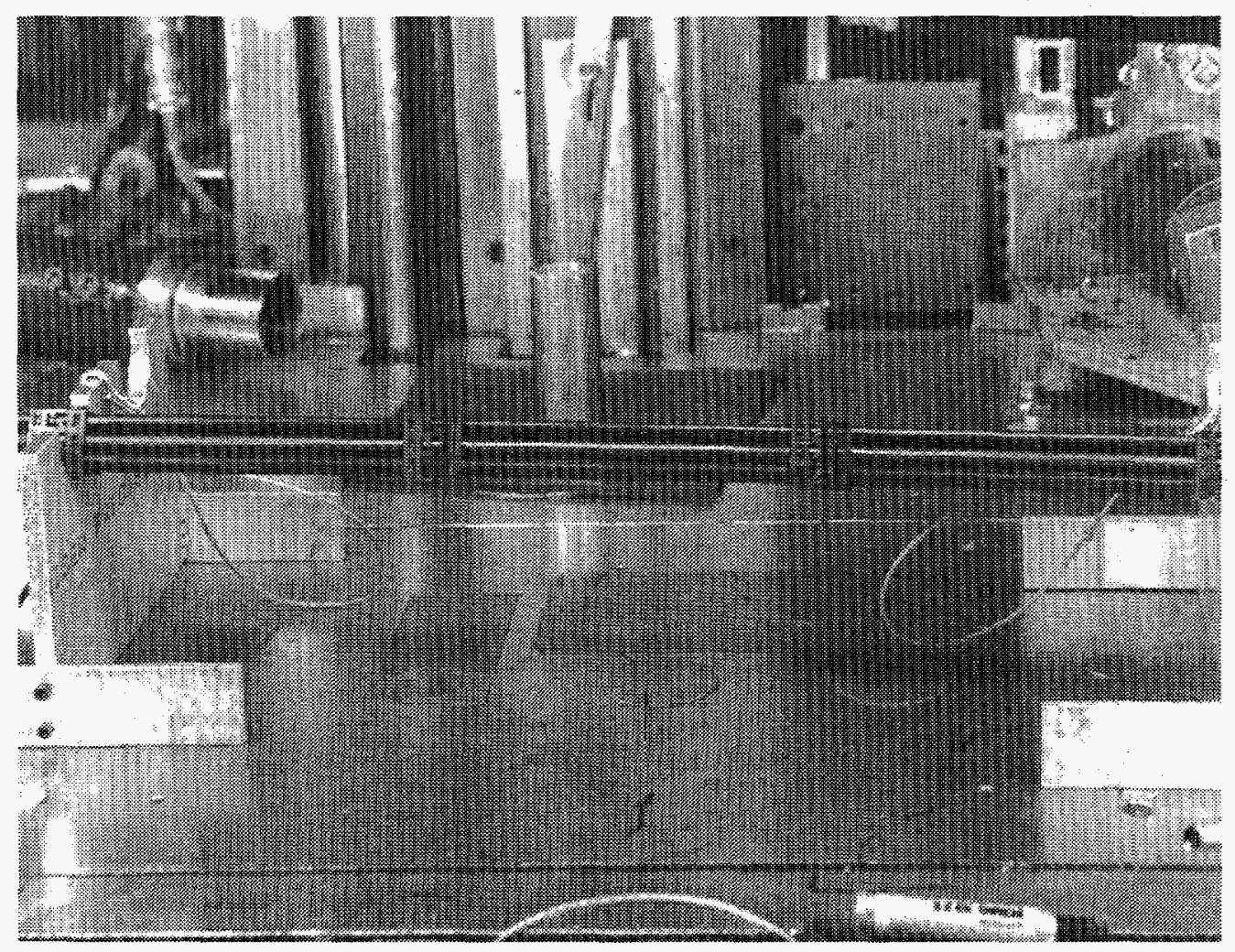

9G6. Loop Assembly, Bottom half of face \#3 (Bundle $180^{\circ}$ face) 


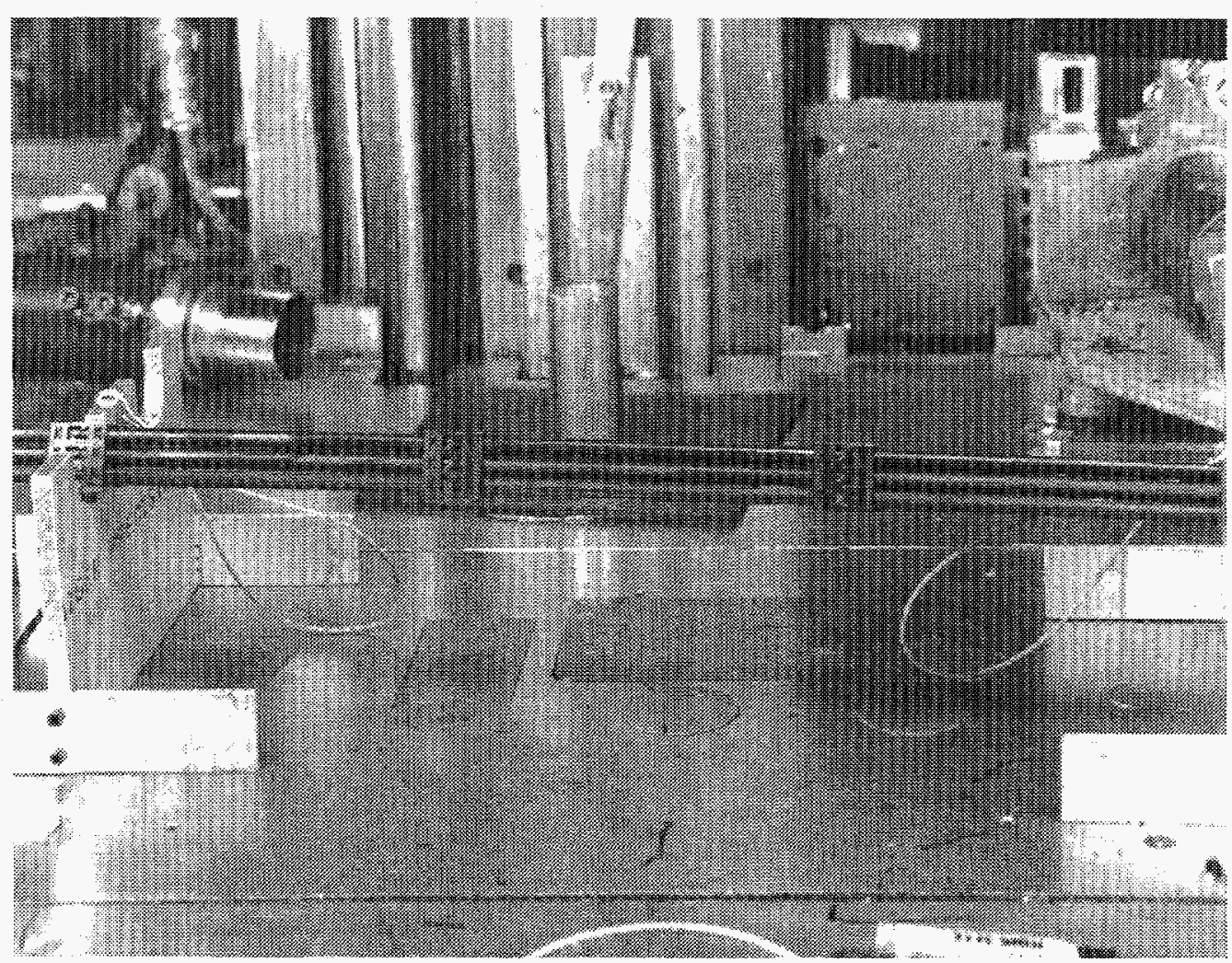

9G7. Loop Assembly, Top half of face \#4 (Bundle $270^{\circ}$ face)

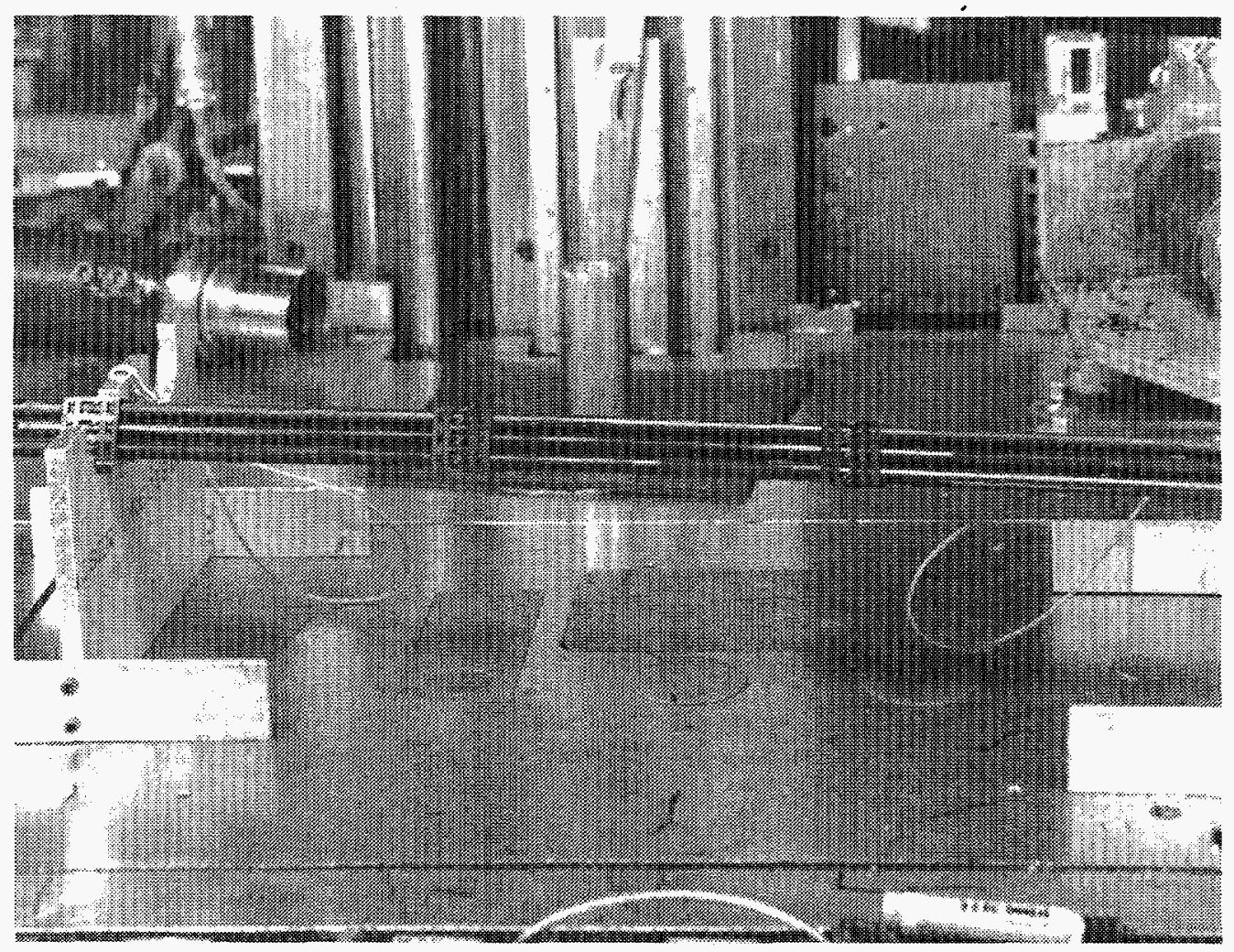

9G8. Loop Assembly, Bottom half of face \#4 (Bundle $270^{\circ}$ face) 


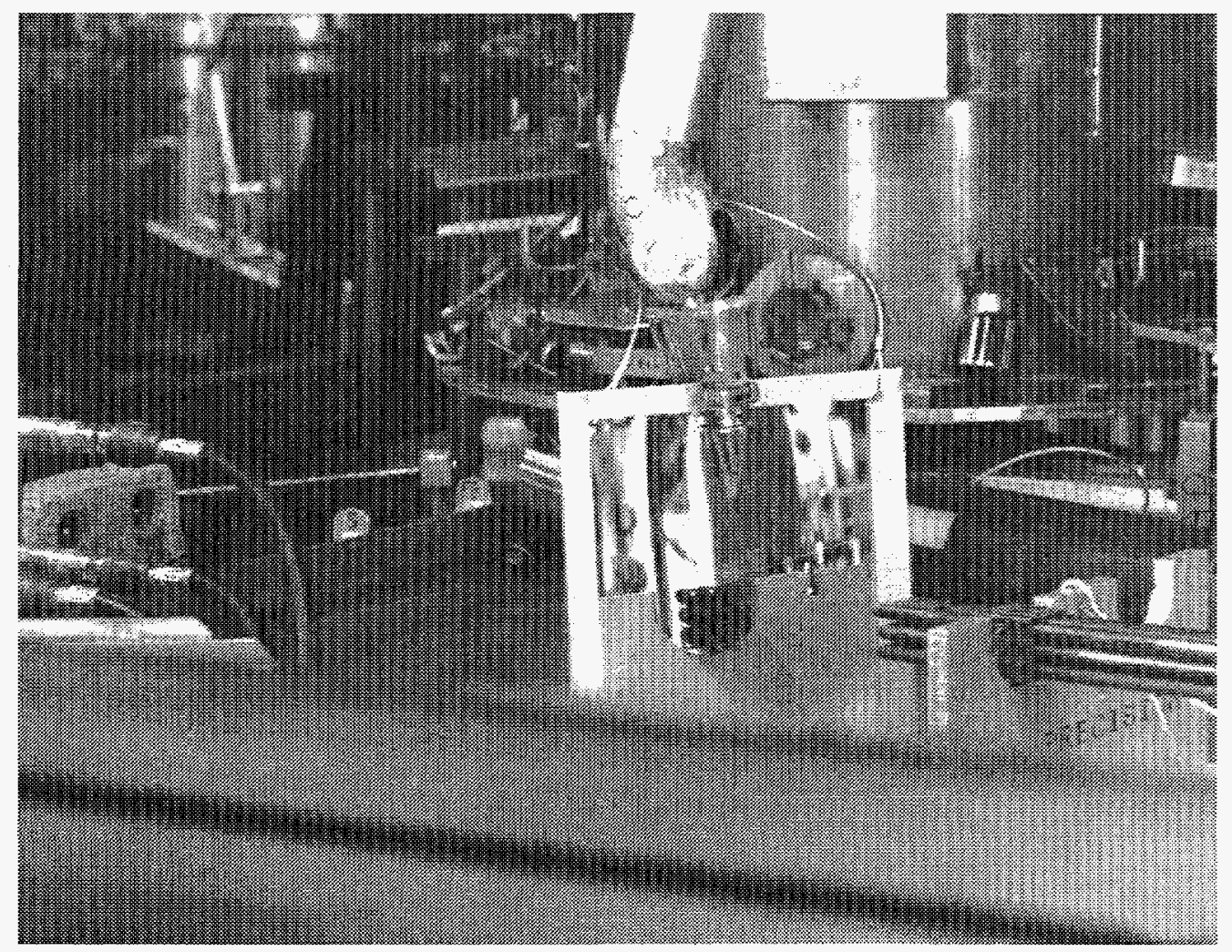

9G9. Loop Assmbly, Through-mirror view of bottom of bundle (face \#1 up)

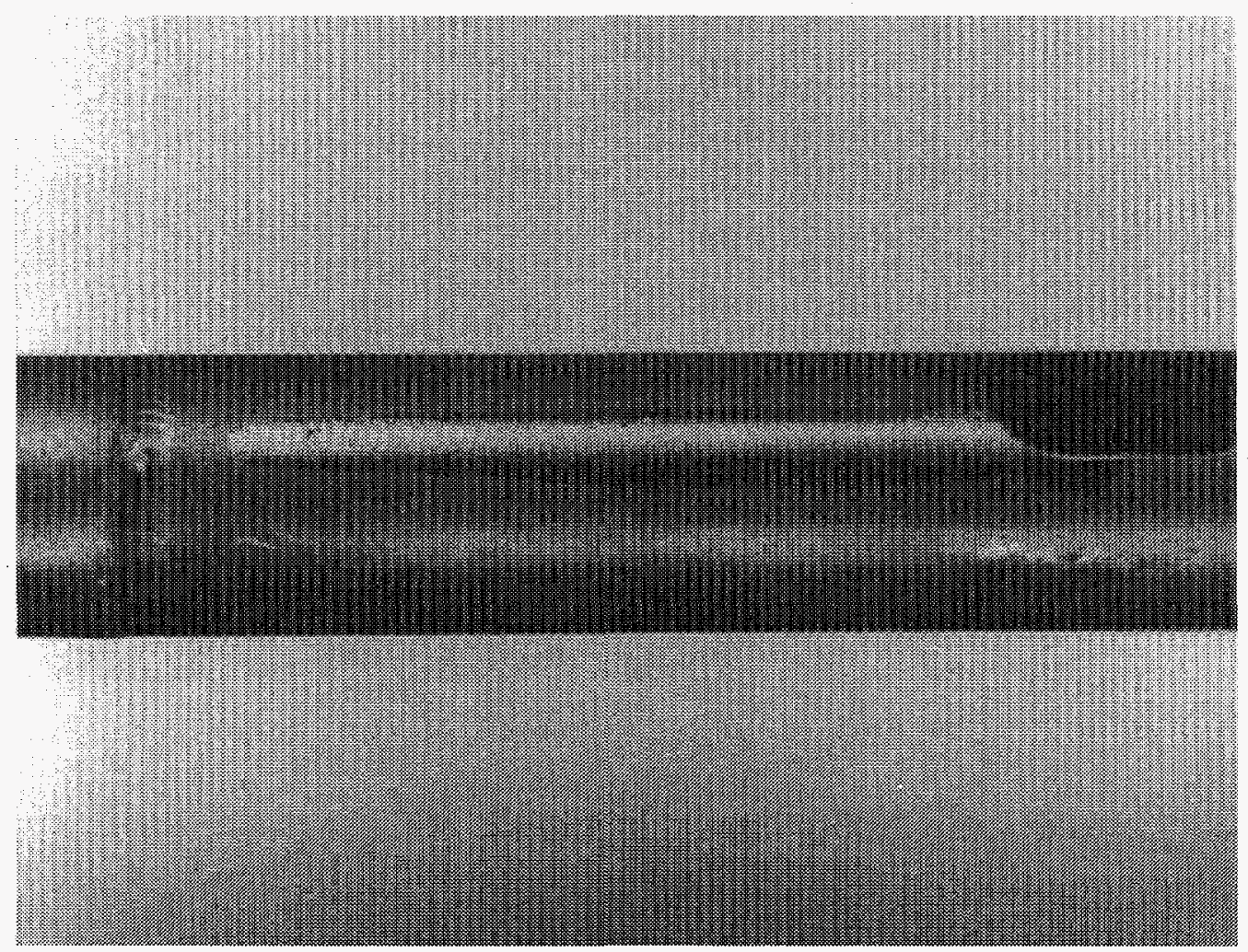

11G1. WL-01, Shiny axial patch 


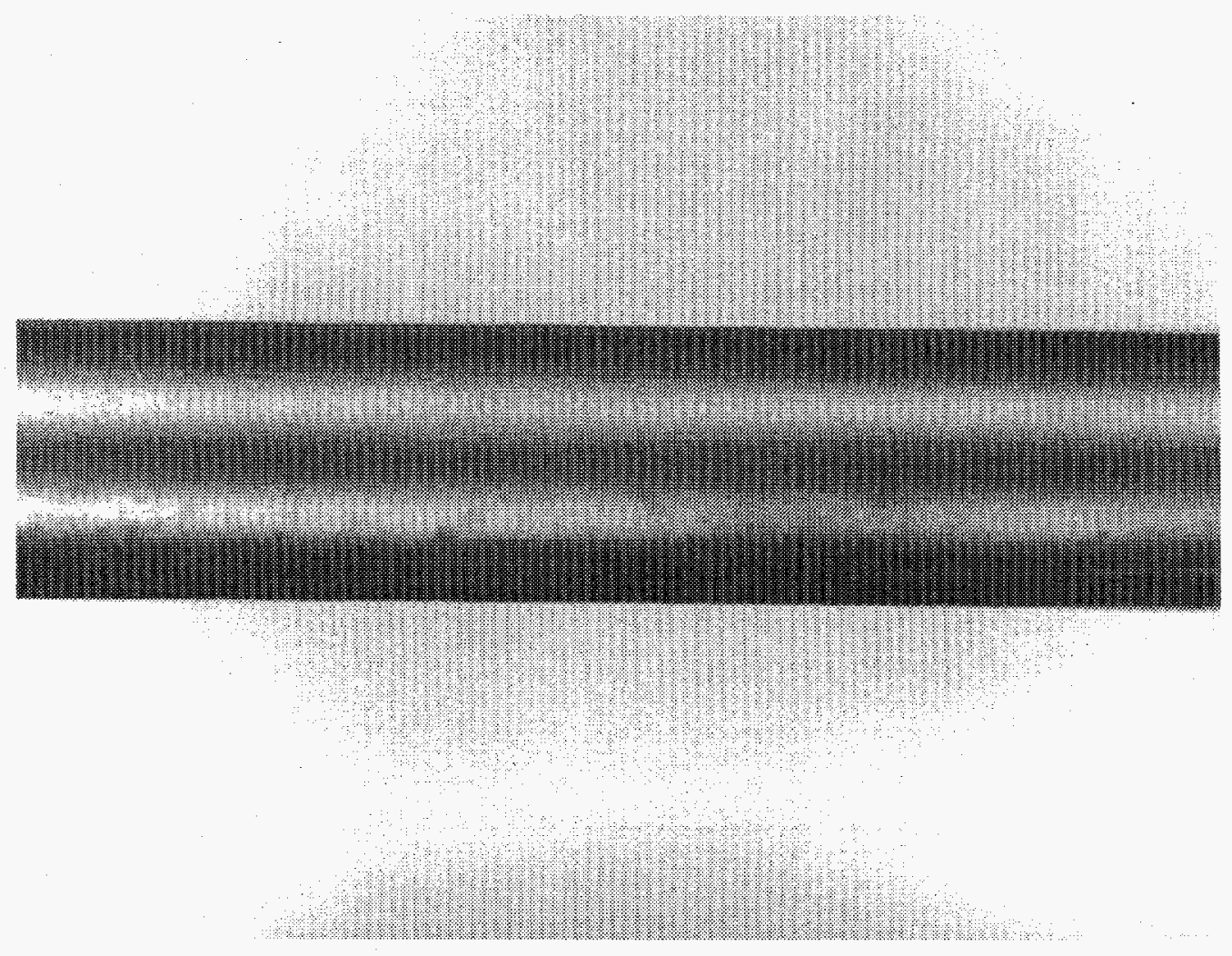

11G.2. WL-01, Low-luster surface (bottom of target)

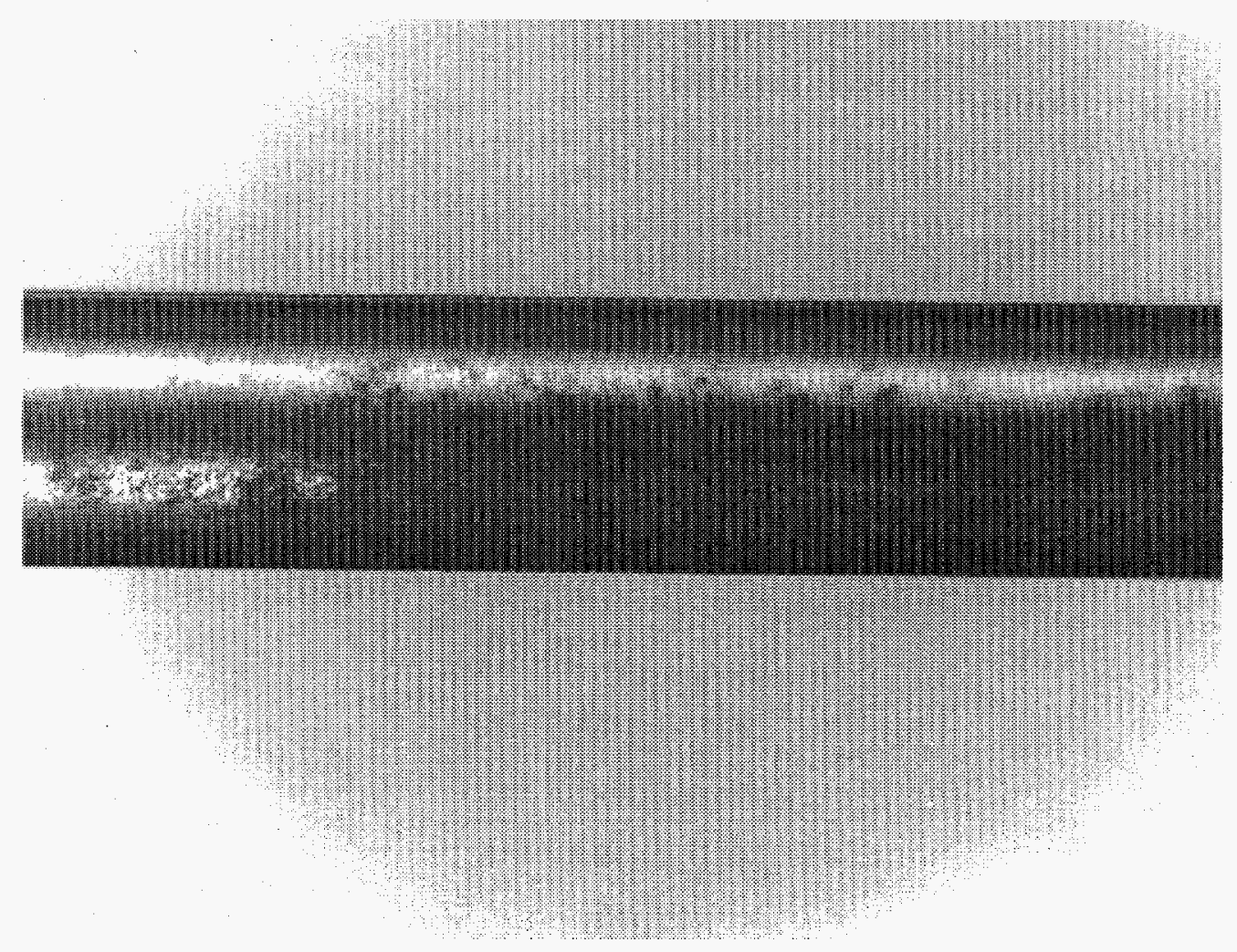

11G3. WL-01, Light-to-dark transition 


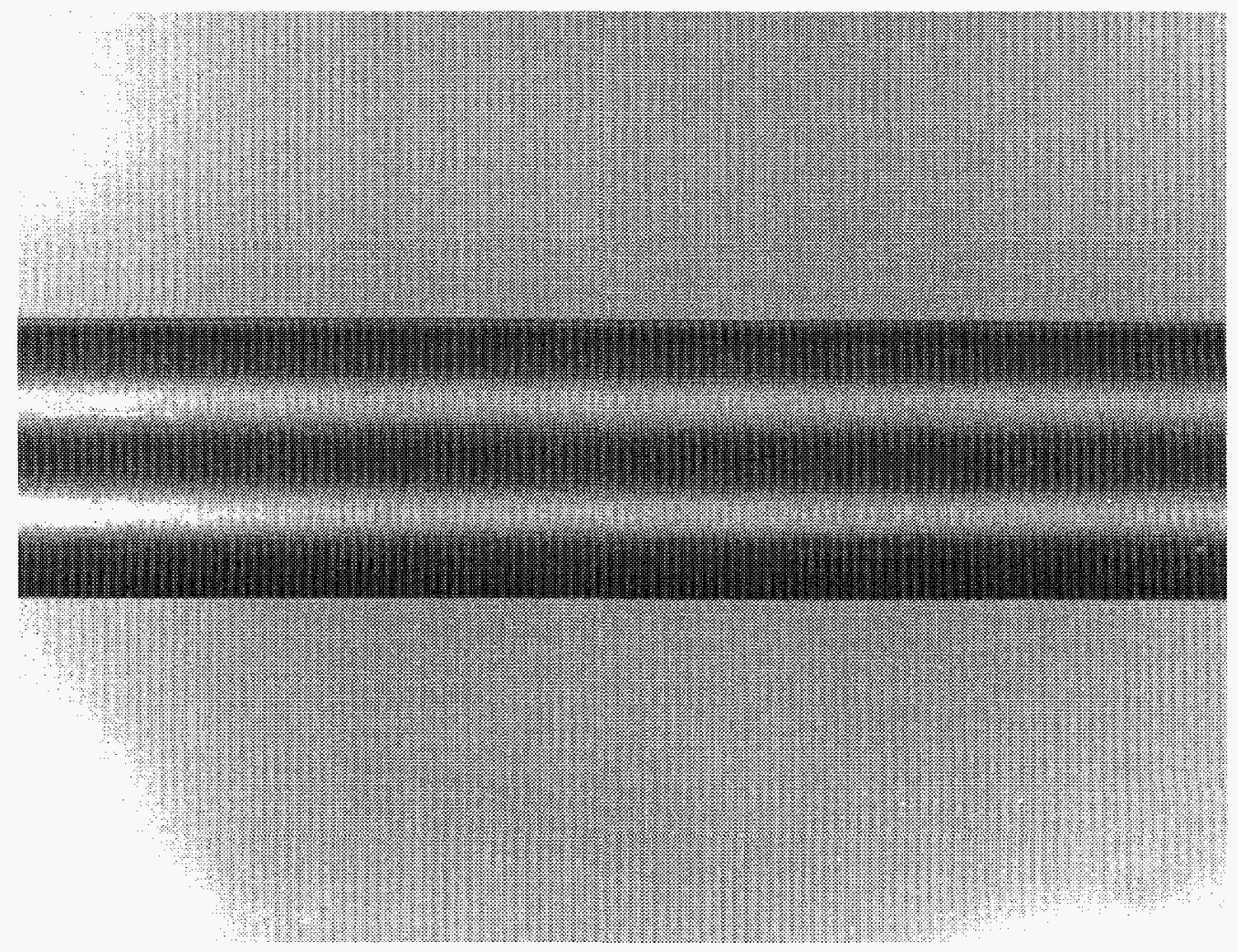

11G4. WL-01, Shiny surface (upper middle)

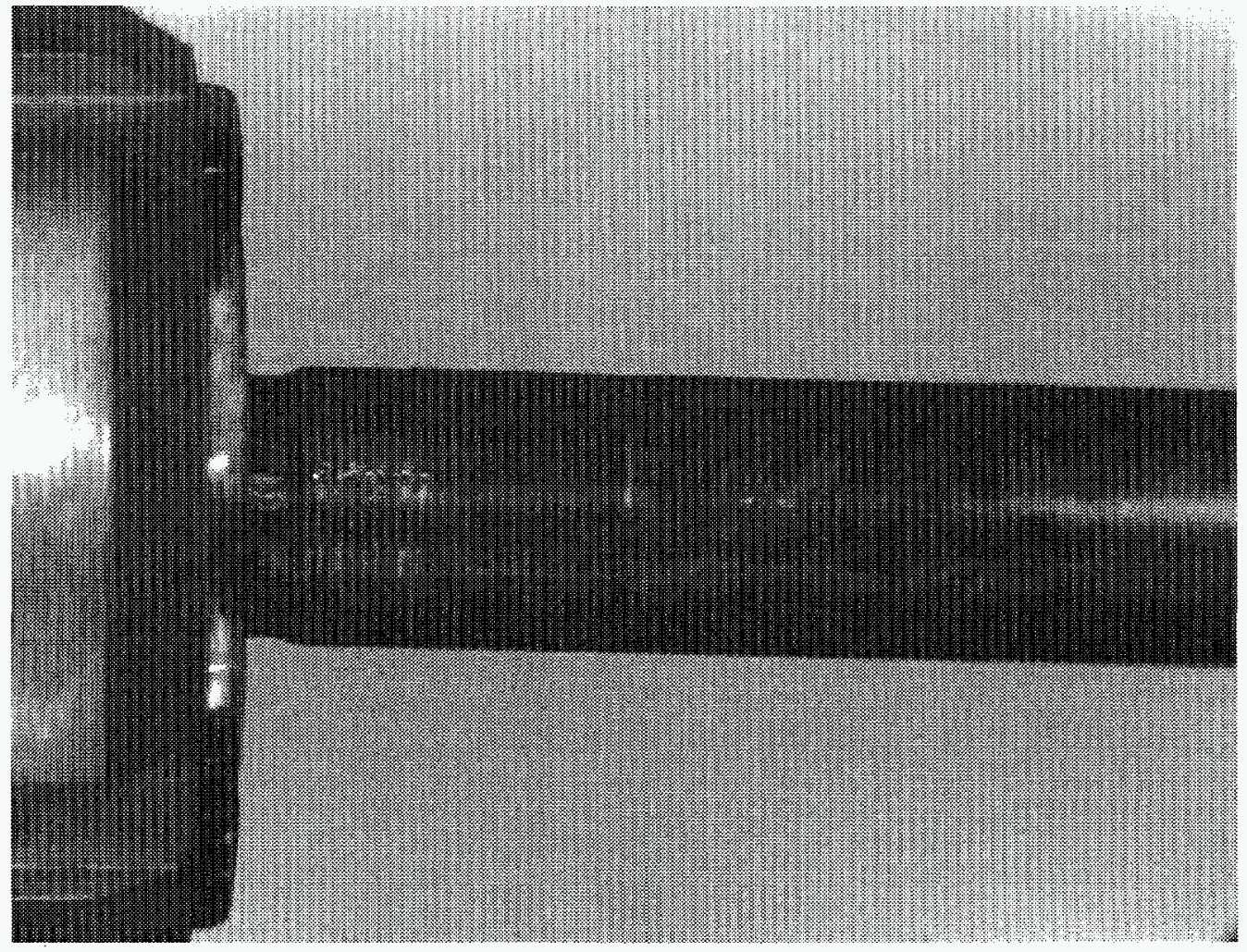

11G5. WL-01, Upper weld (shows end-fitting grip marks ) 


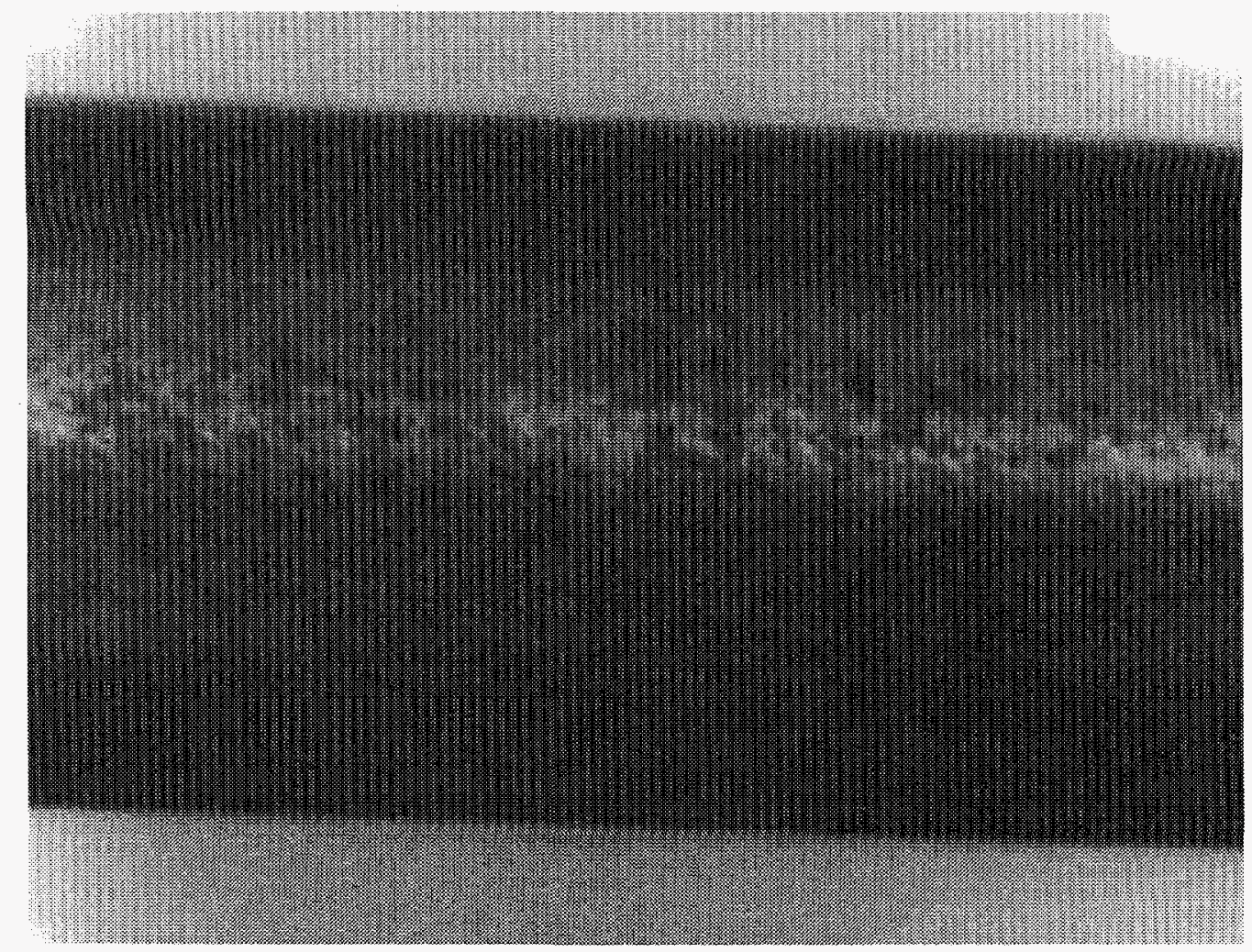

11G6. WL-27, General pitting (typical)

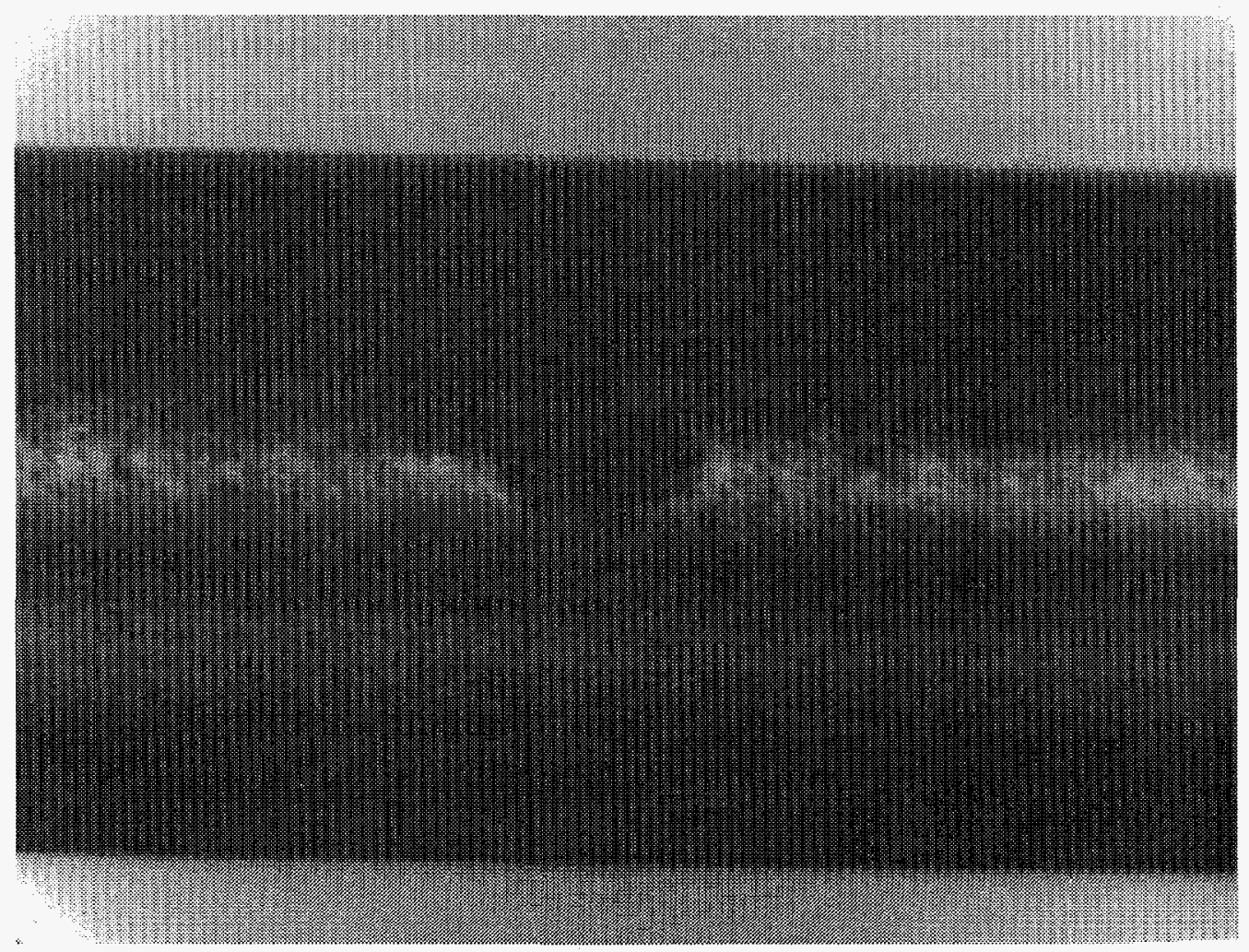

11G7. WL-27, Large pit (typical) 


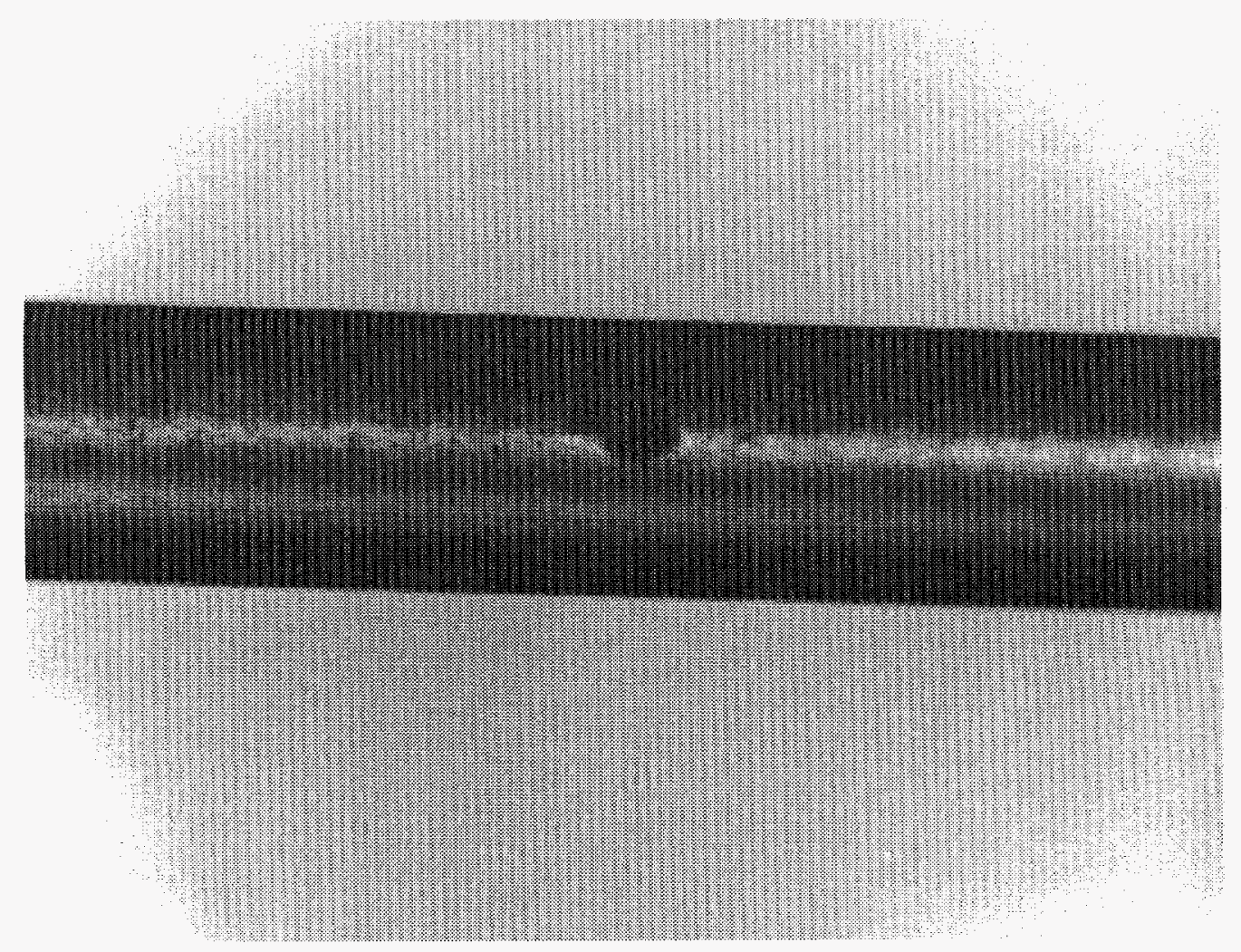

11G8. WL-27, Large pit (typical)
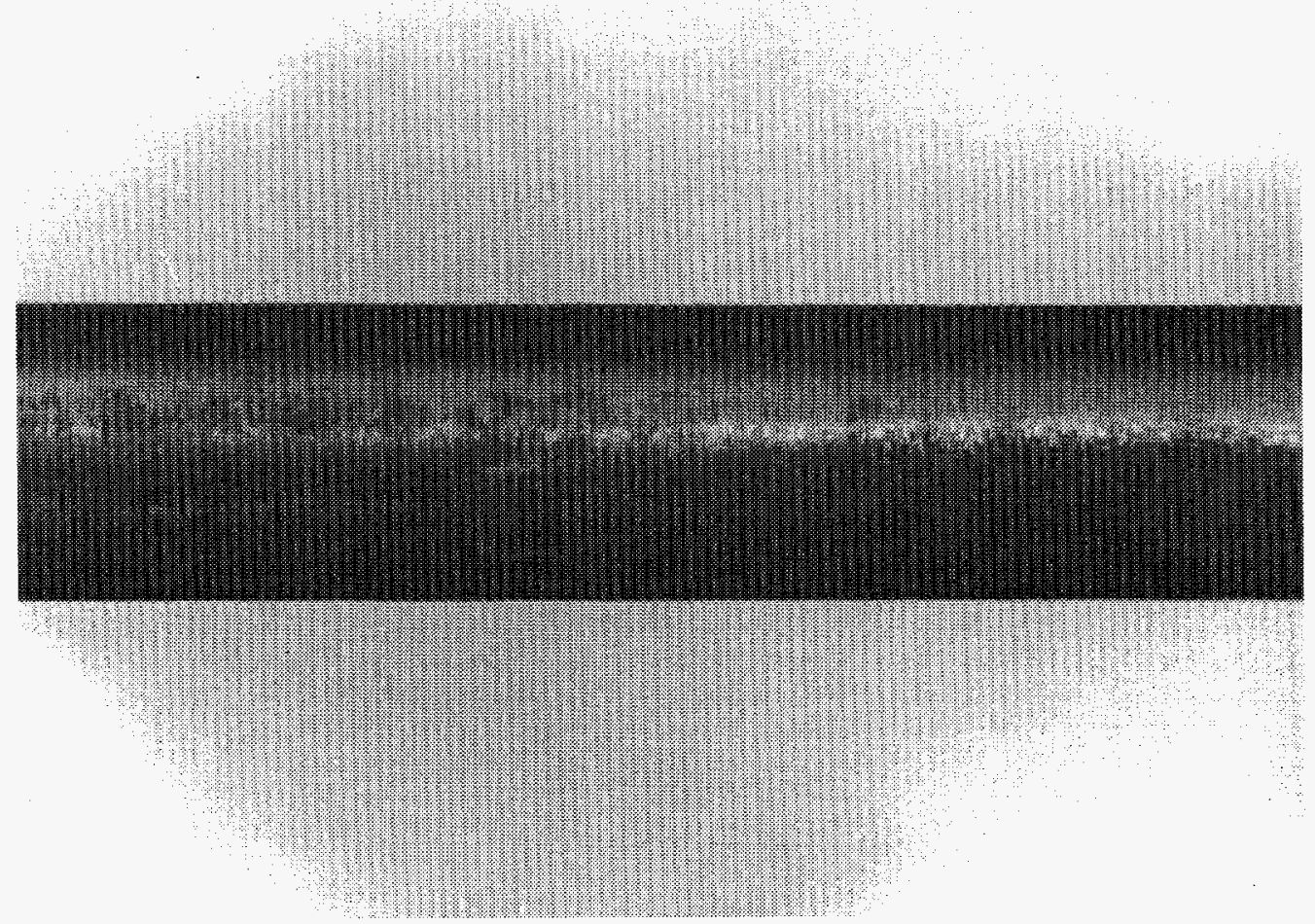

11G9. WL-27, Barrier transition (typical) 


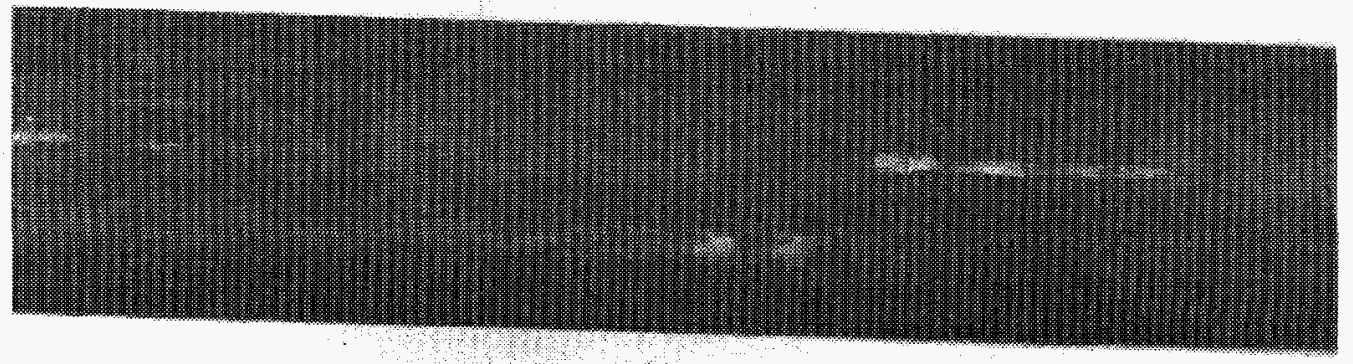

11G10. WL-27, Spacer grid marks (typical)

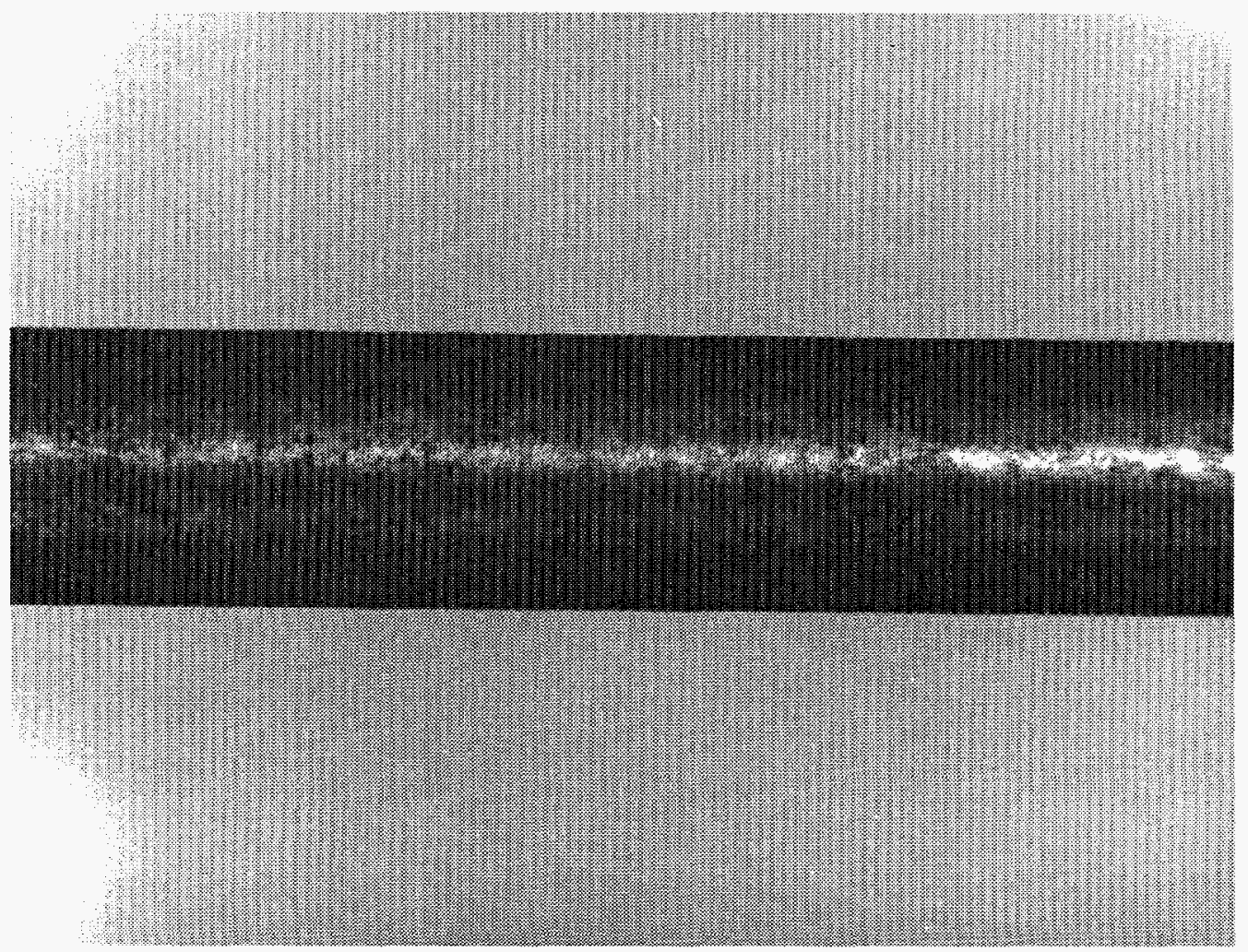

11G11. WL-26, General pitting 


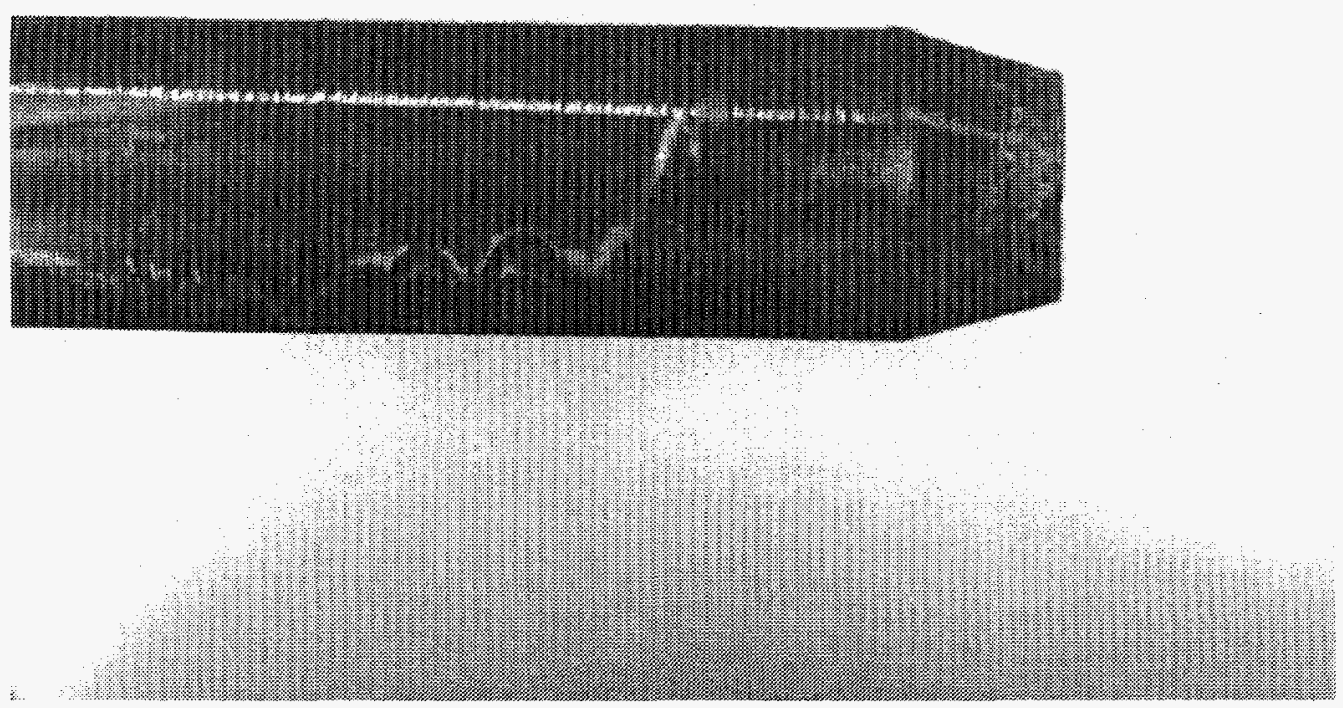

11G12. WL-26, Lower tip

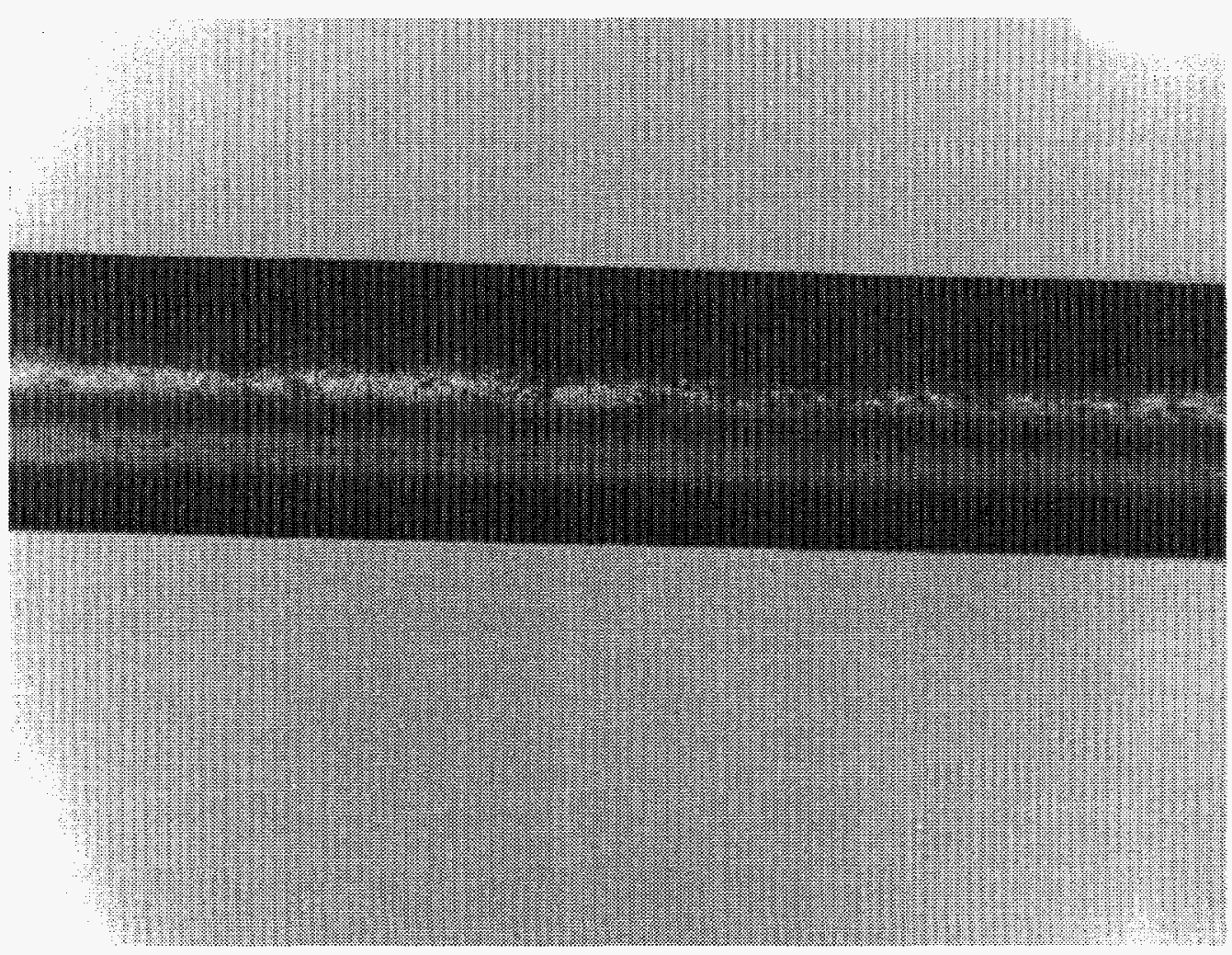

11G13. WL-22, Typical barrier (bottom, front) 


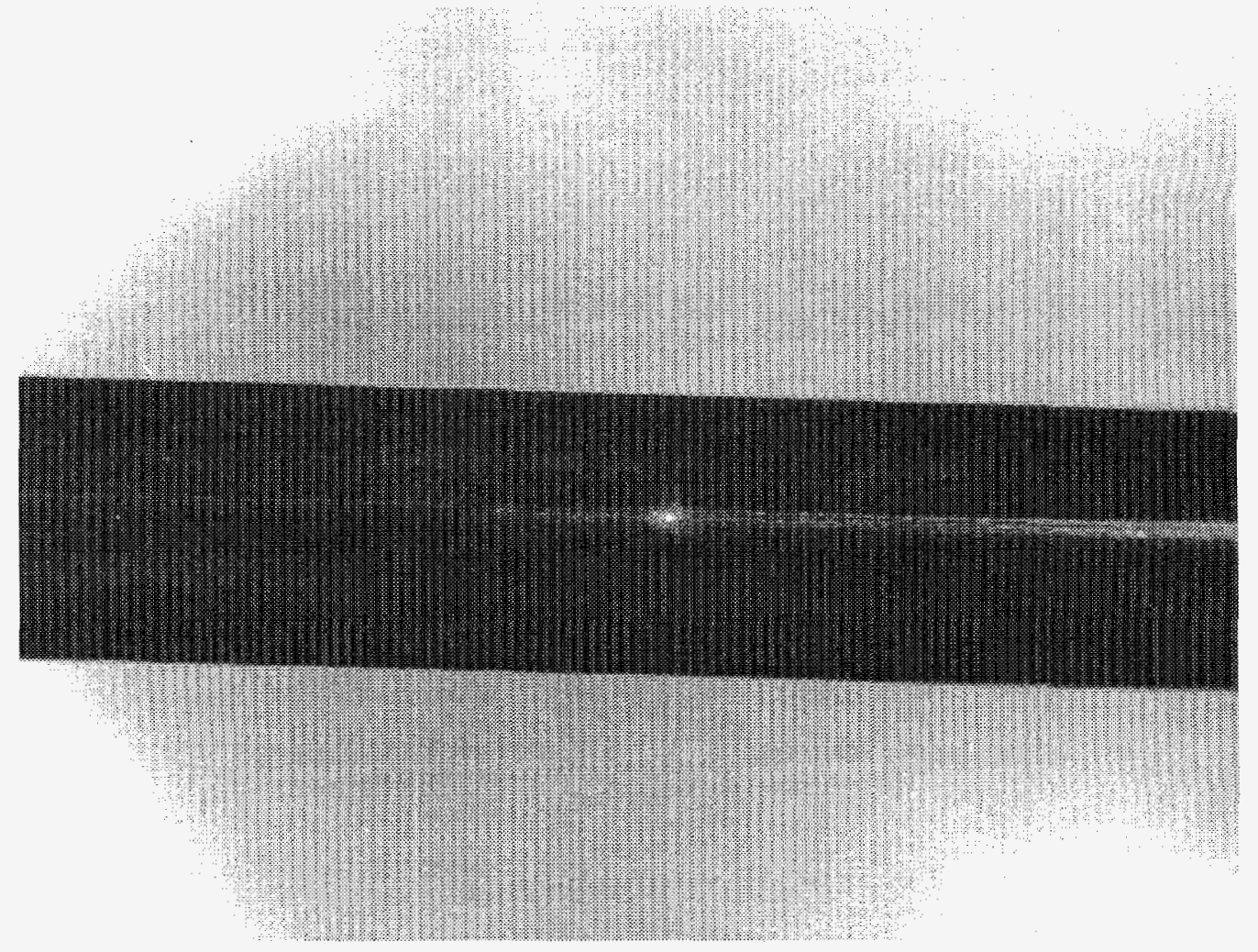

11G14. WL-22, Typical barrier (bottom, back)

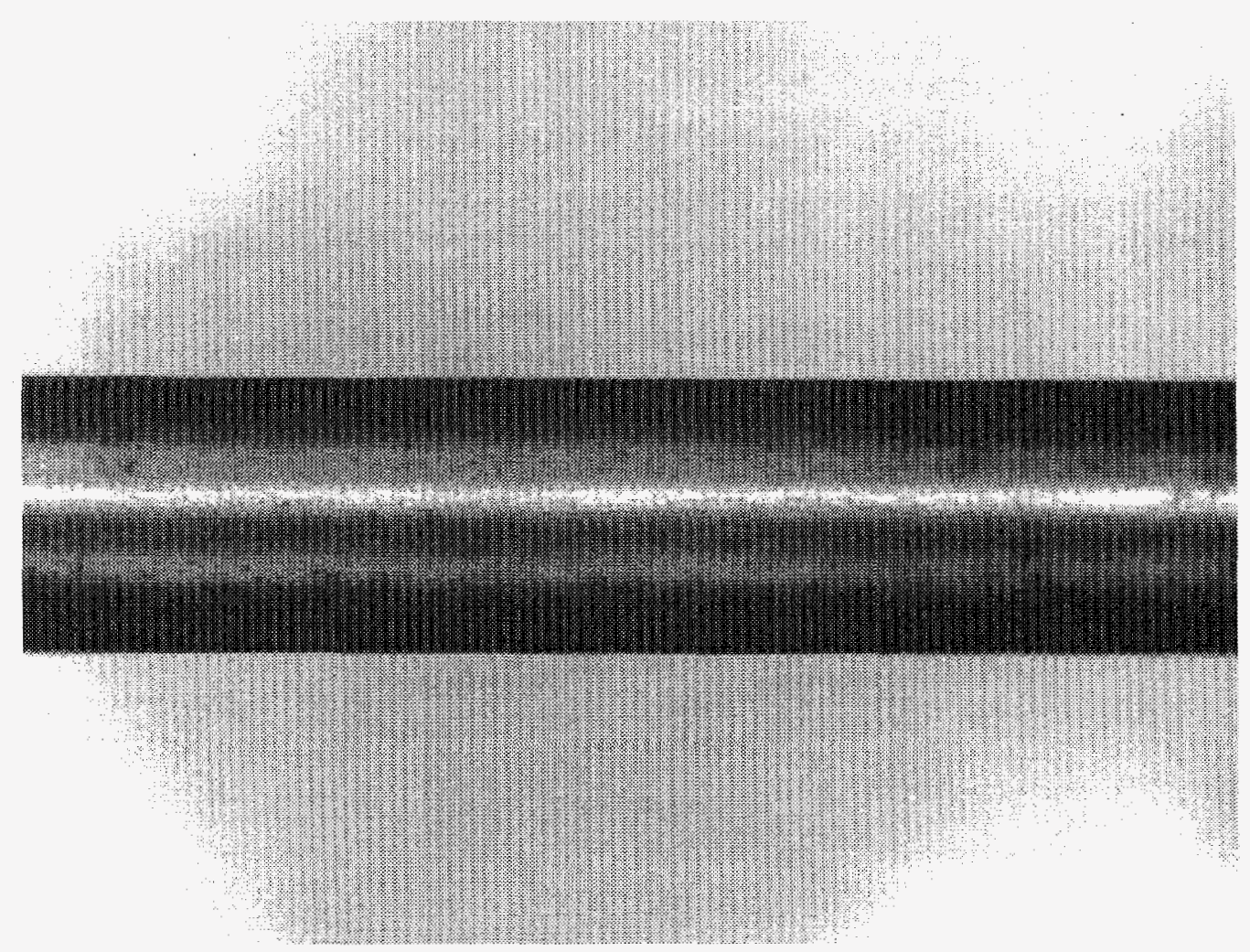

11G15. WL-22, Typical barrier (center) - Rotational composite, $328^{\circ}$ 


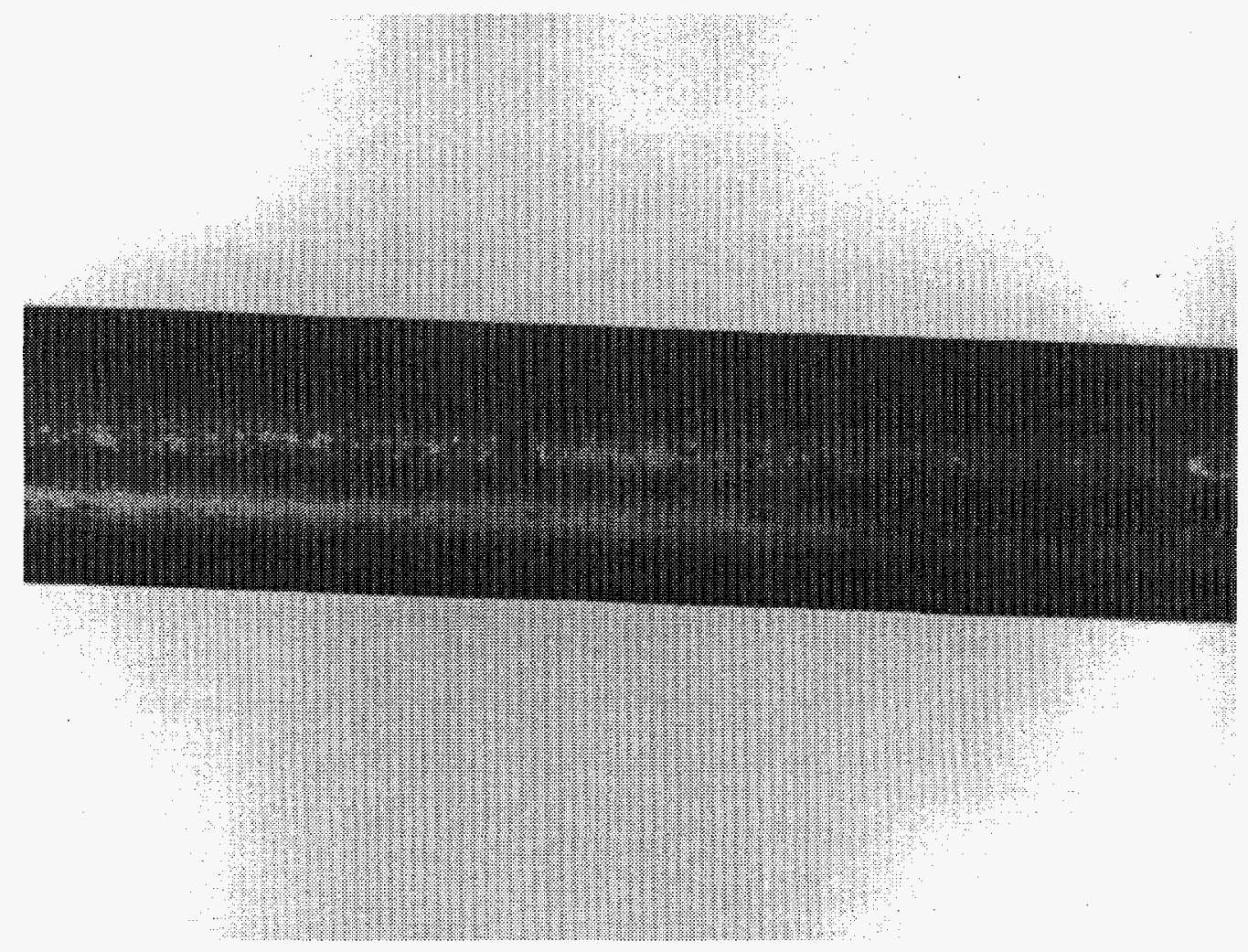

11G16. WL-22, Typical barrier (center) - Rotational composite, $28^{\circ}$

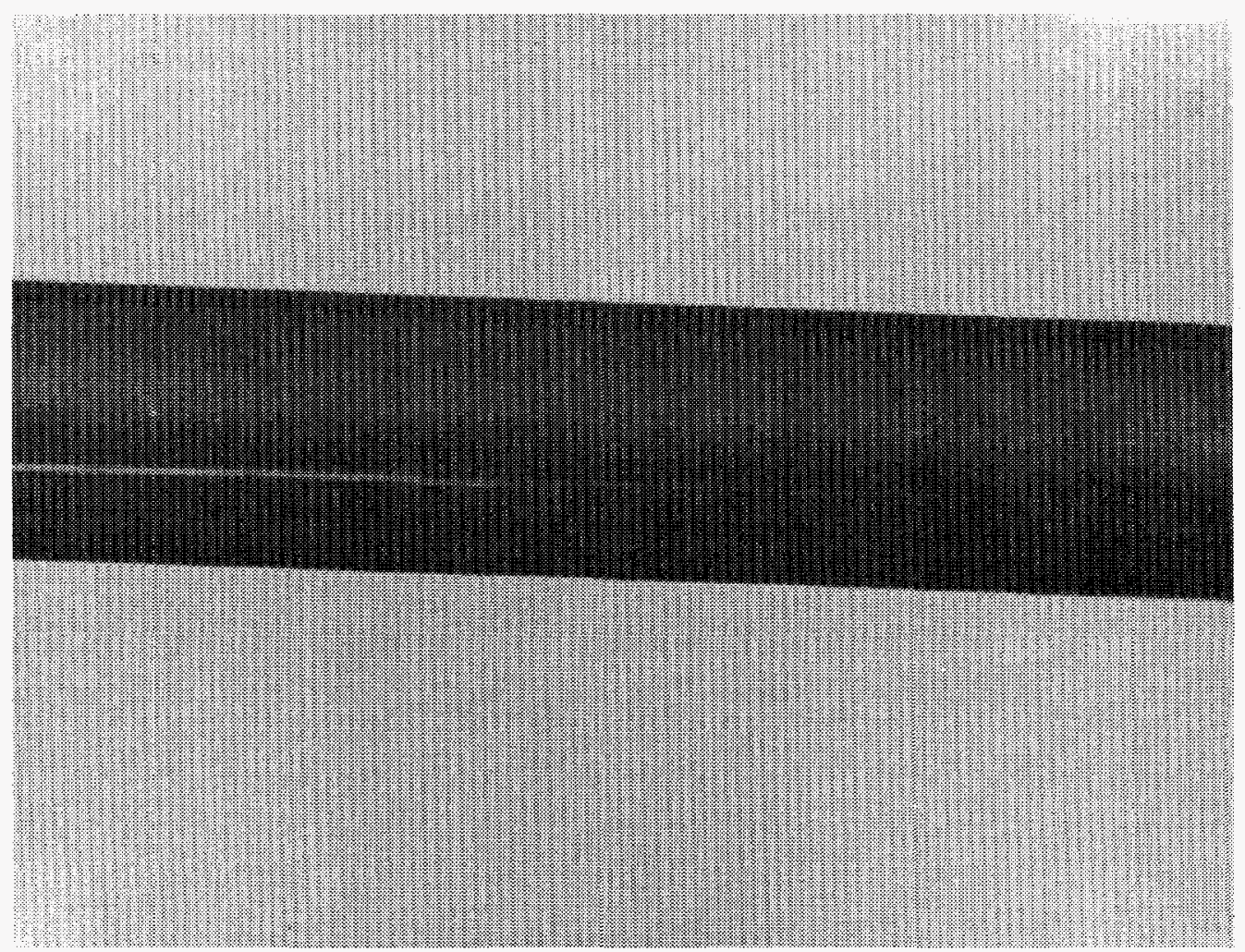

11G17. WL-22, Typical barrier (center) - Rotational composite, $88^{\circ}$ 


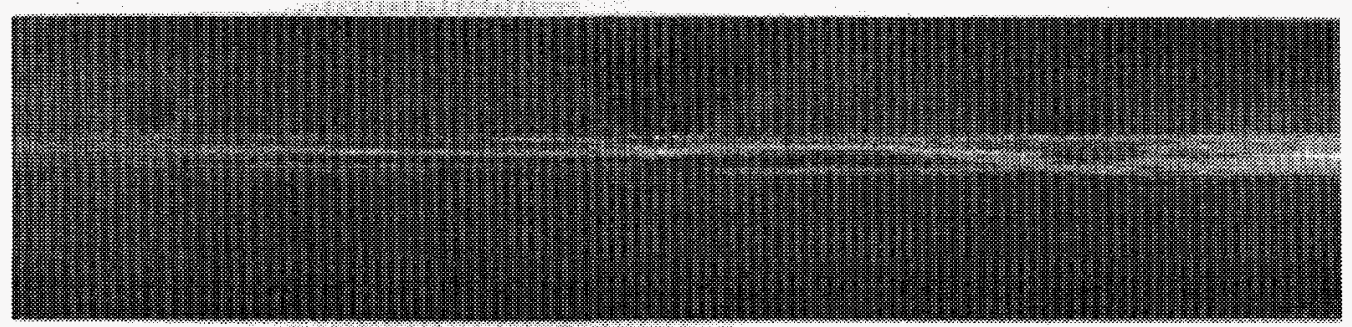

11G18. WL-22, Typical barrier (center) - Rotational composite, $148^{\circ}$

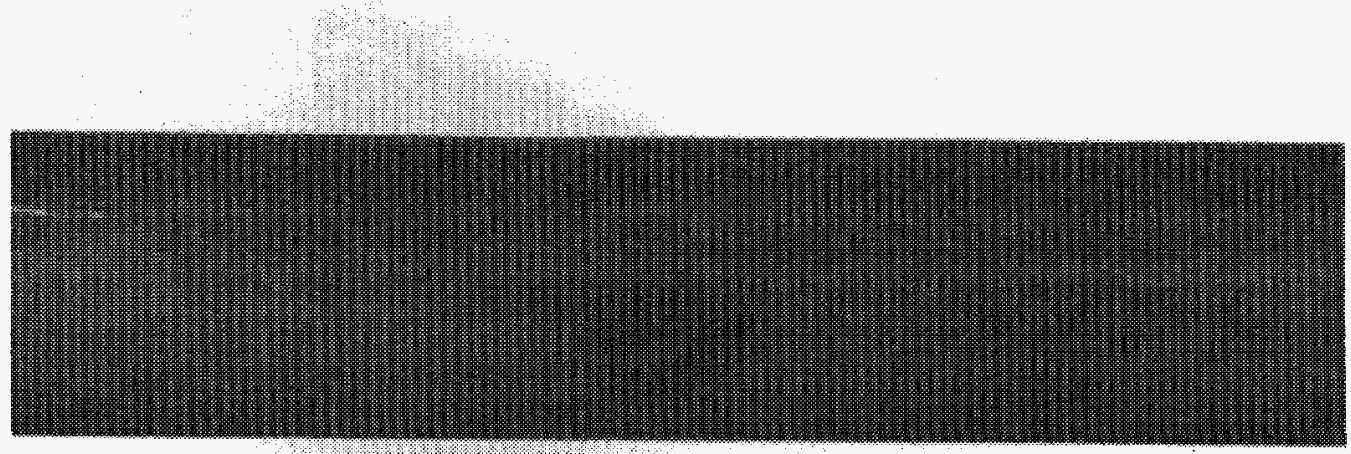

11G19. WL-22, Typical barrier (center) - Rotational composite, $207^{\circ}$ 


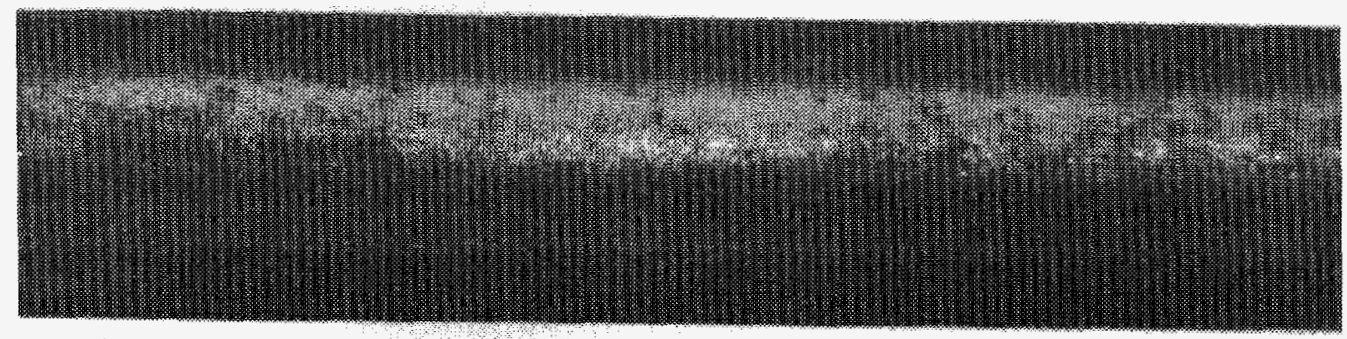

11G20. WL-22, Typical barrier (center) - Rotational composite, $207^{\circ}$

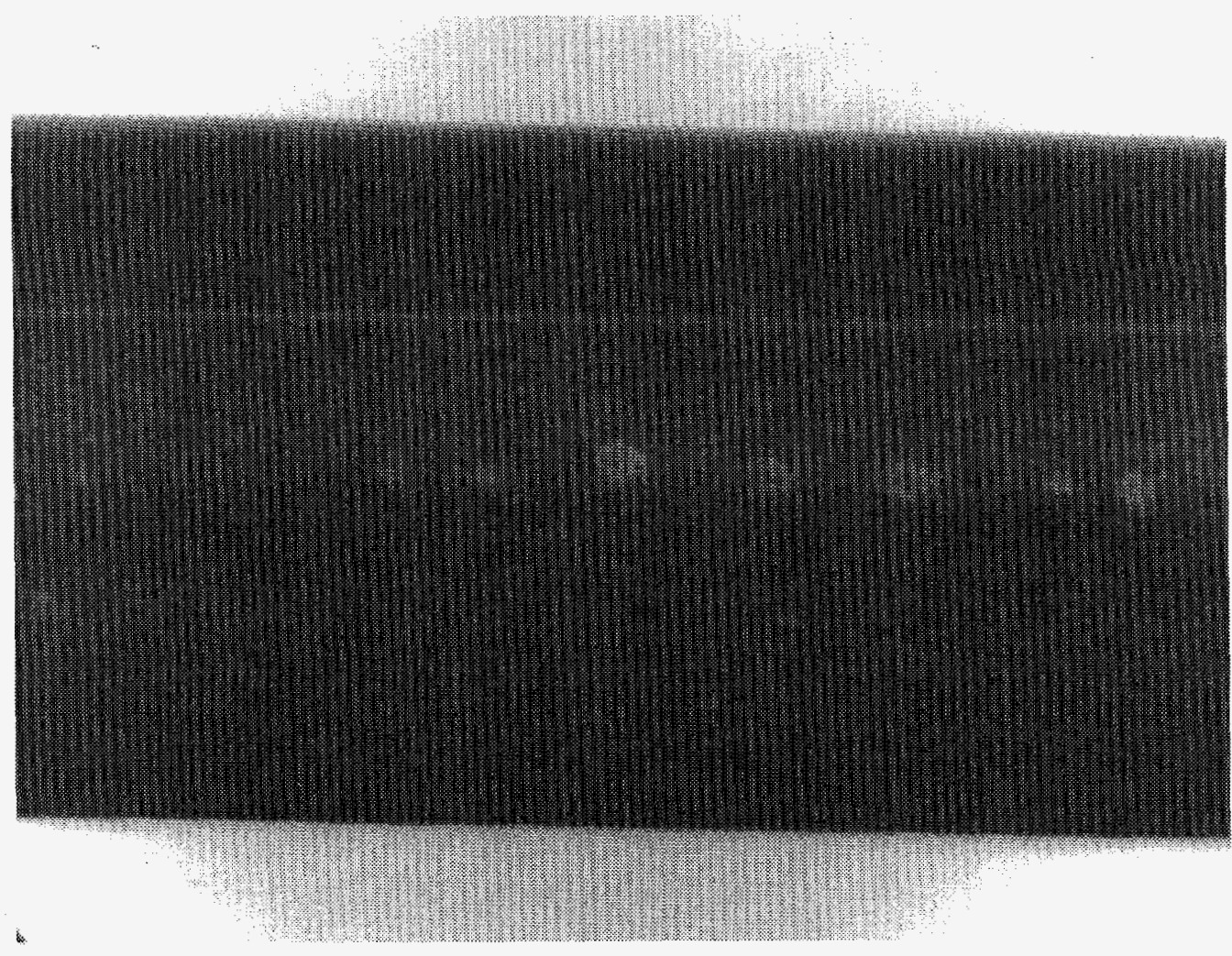

11G21. WL-22, Barrier islands 


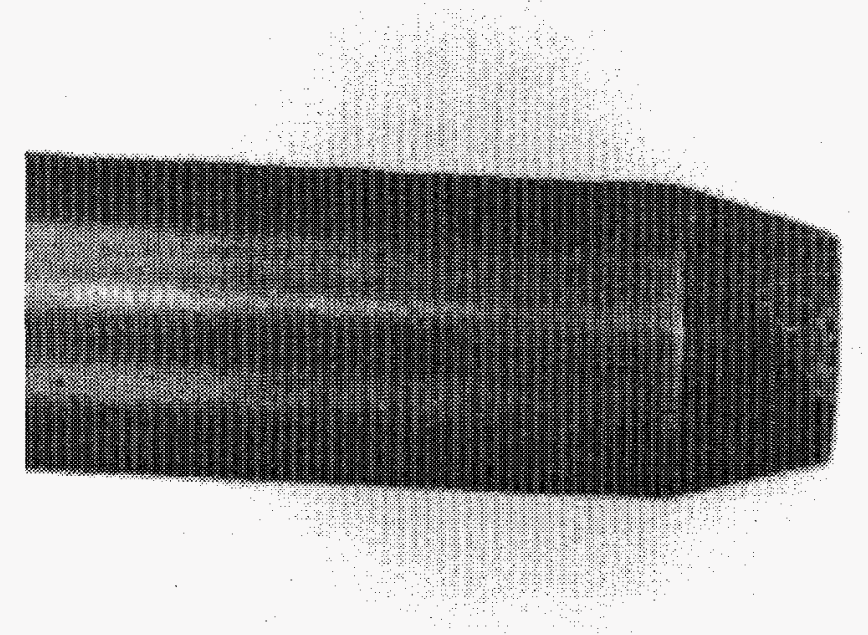

11G22. WL-22, Lower tip

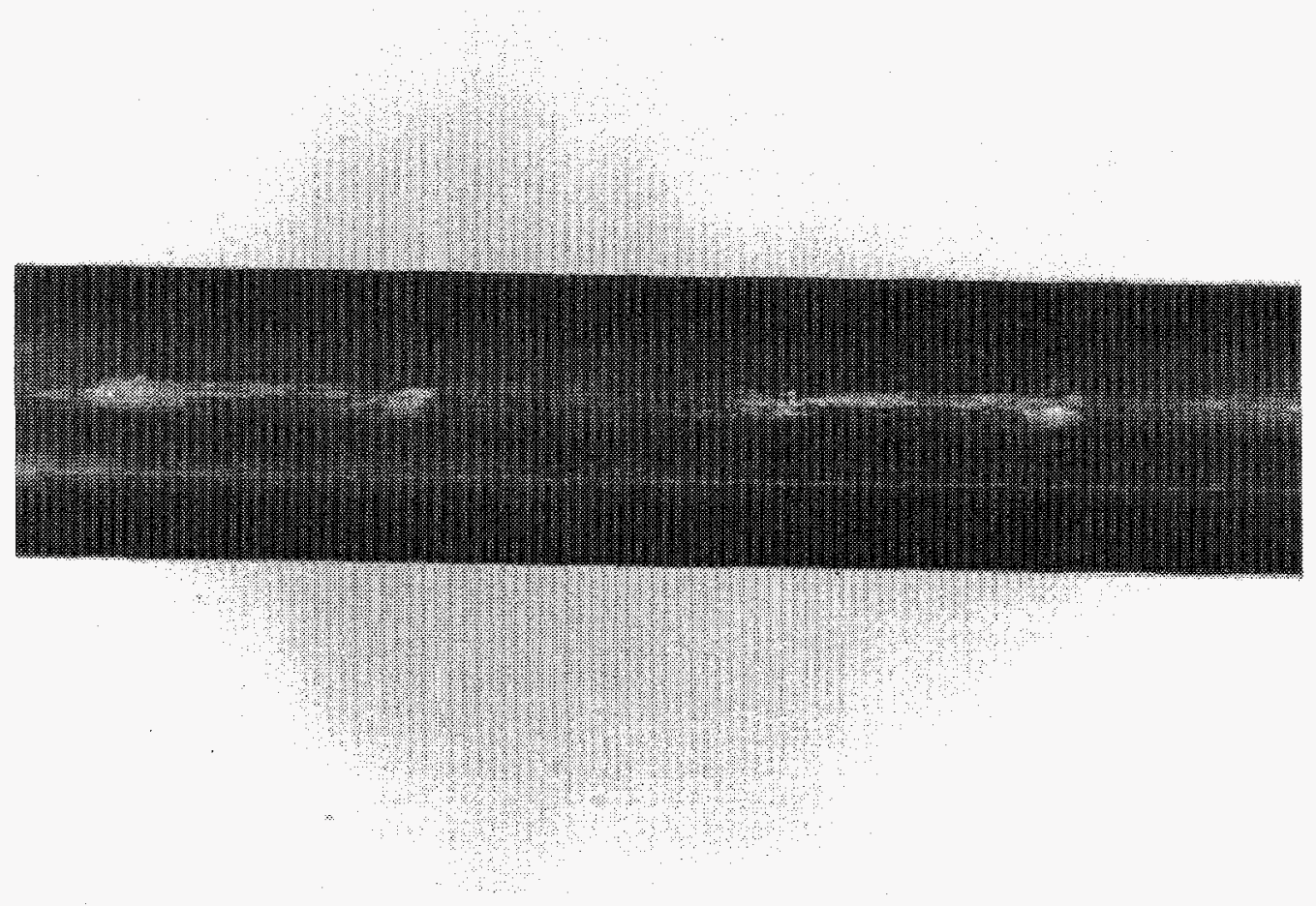

11G23. WL-18, Footprints

B-24 


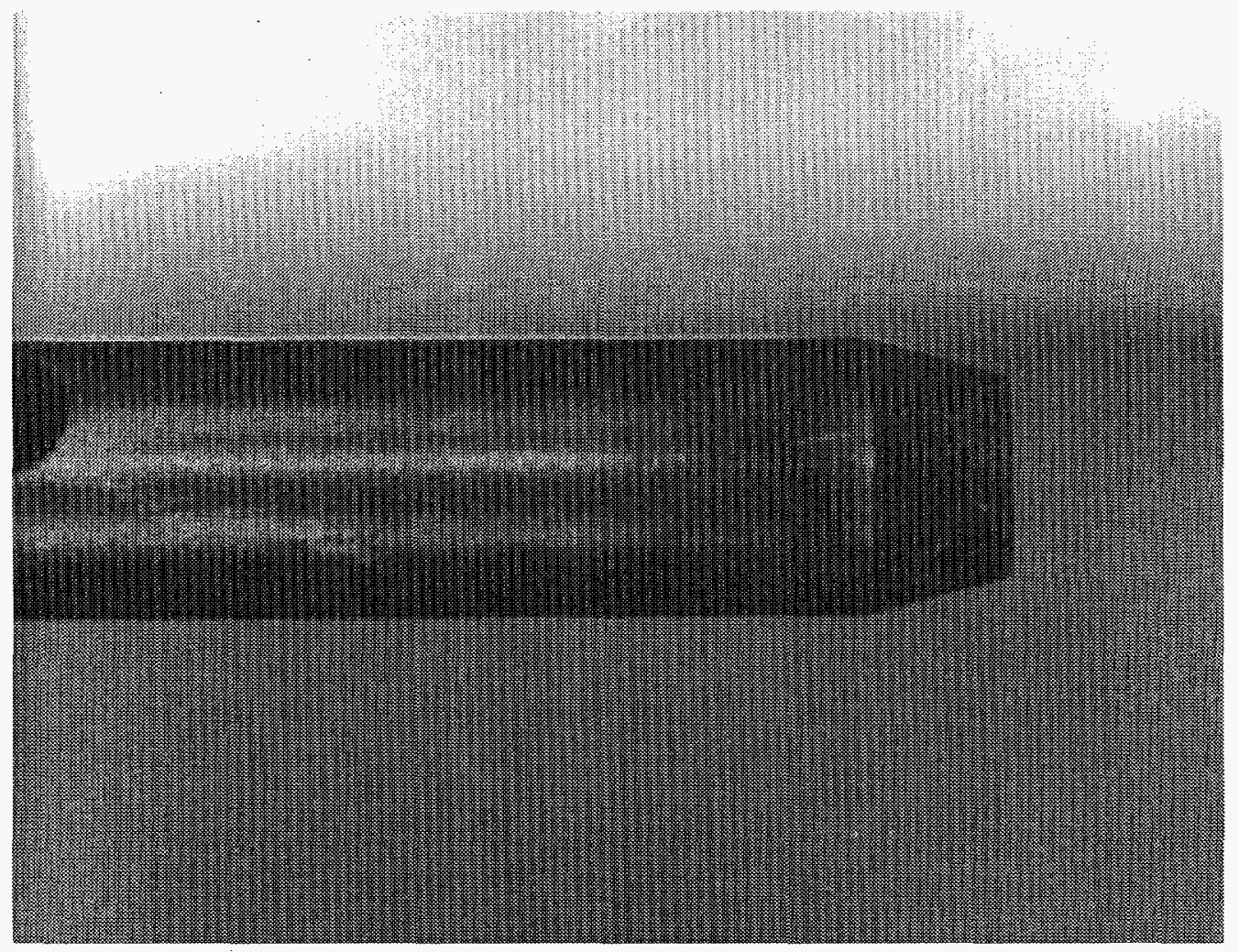

11G24. WL-18, Bottom tip

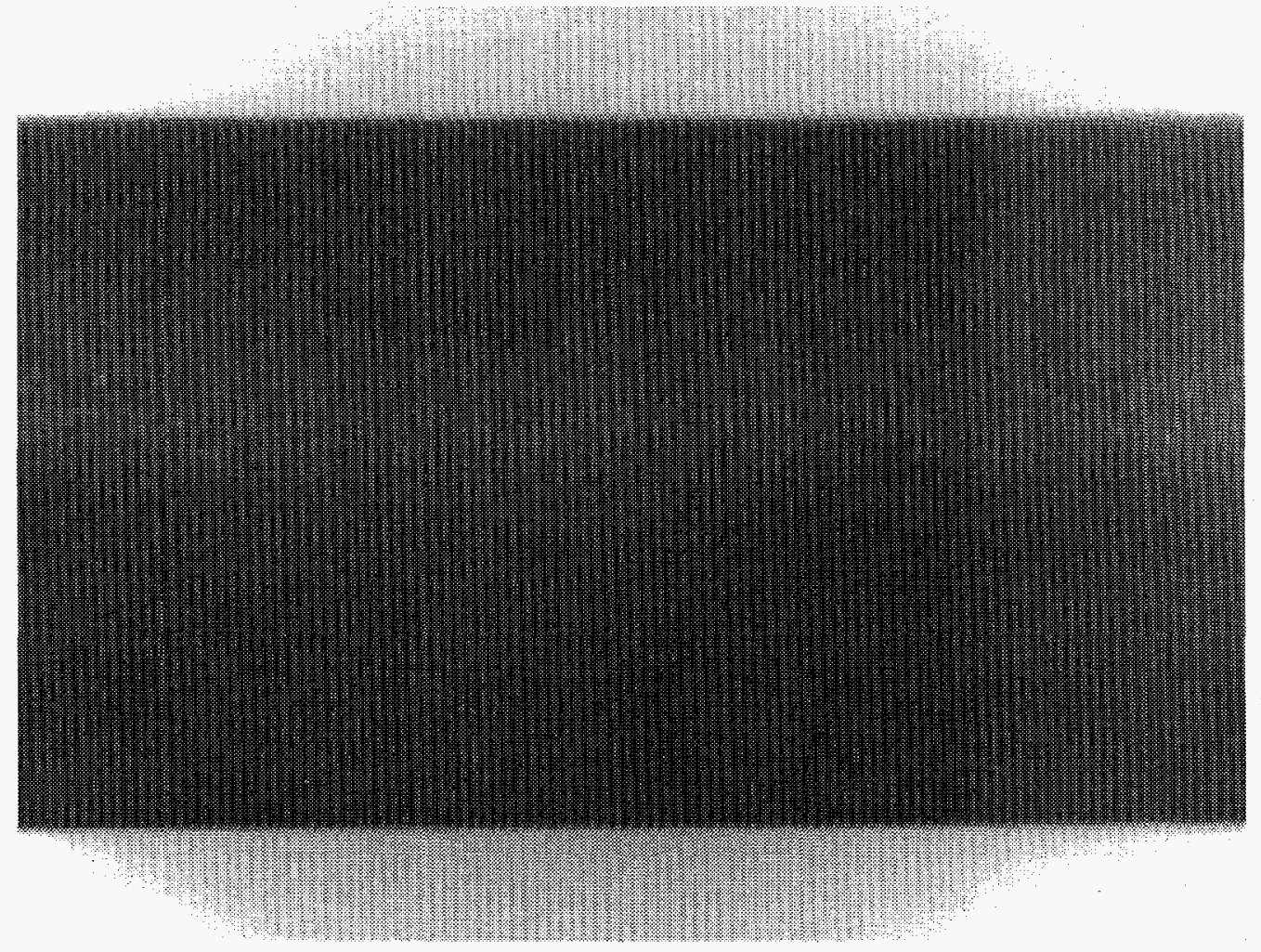

11G25. WL-10, Dark pit

B-25 


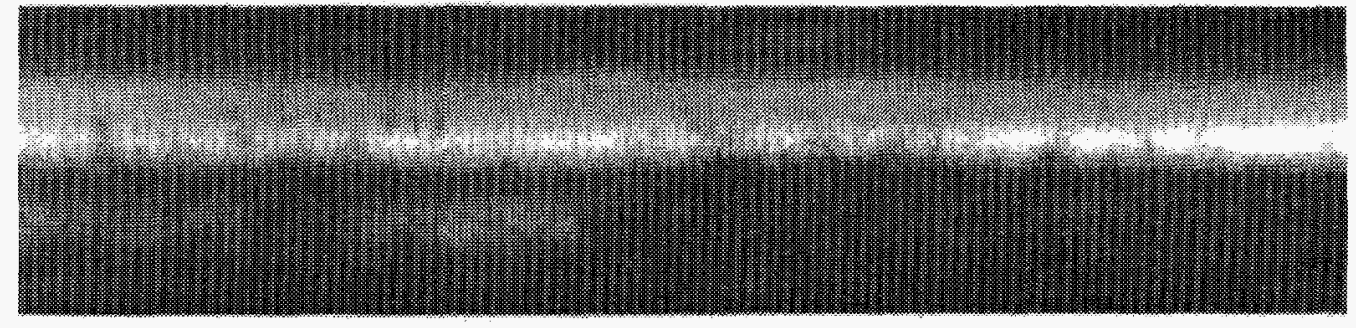

11G26. WL-09, Typical barrier (bottom) - Rotational composite, $119^{\circ}$

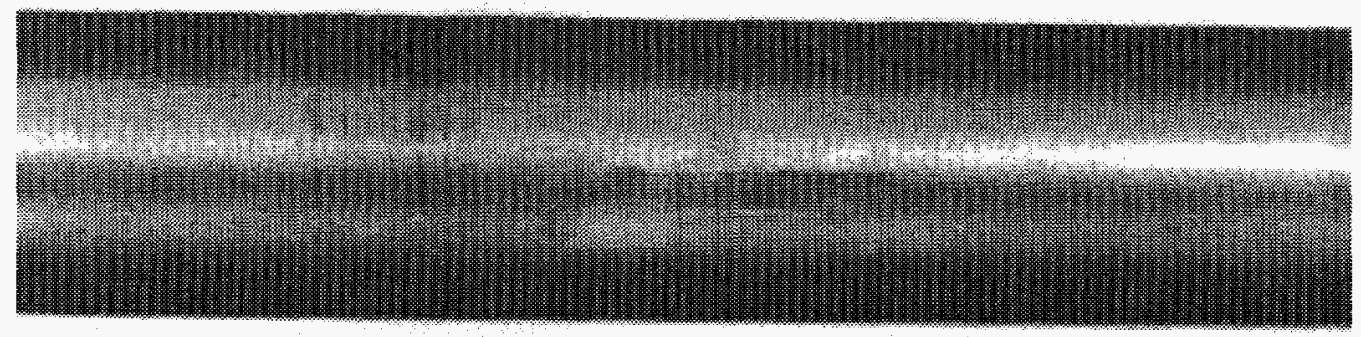

11G27. WL-09, Typical barrier (bottom) - Rotational composite, $179^{\circ}$ 


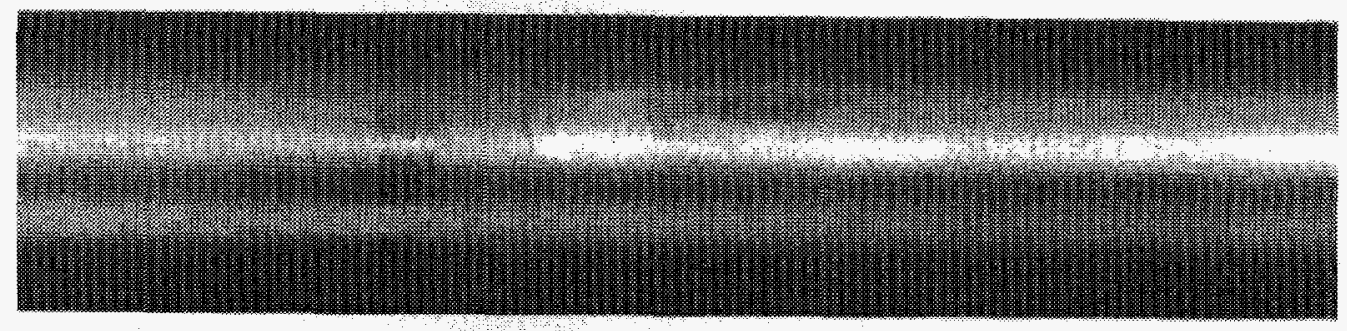

11G28. WL-09, Typical barrier (bottom) - Rotational composite, $239^{\circ}$

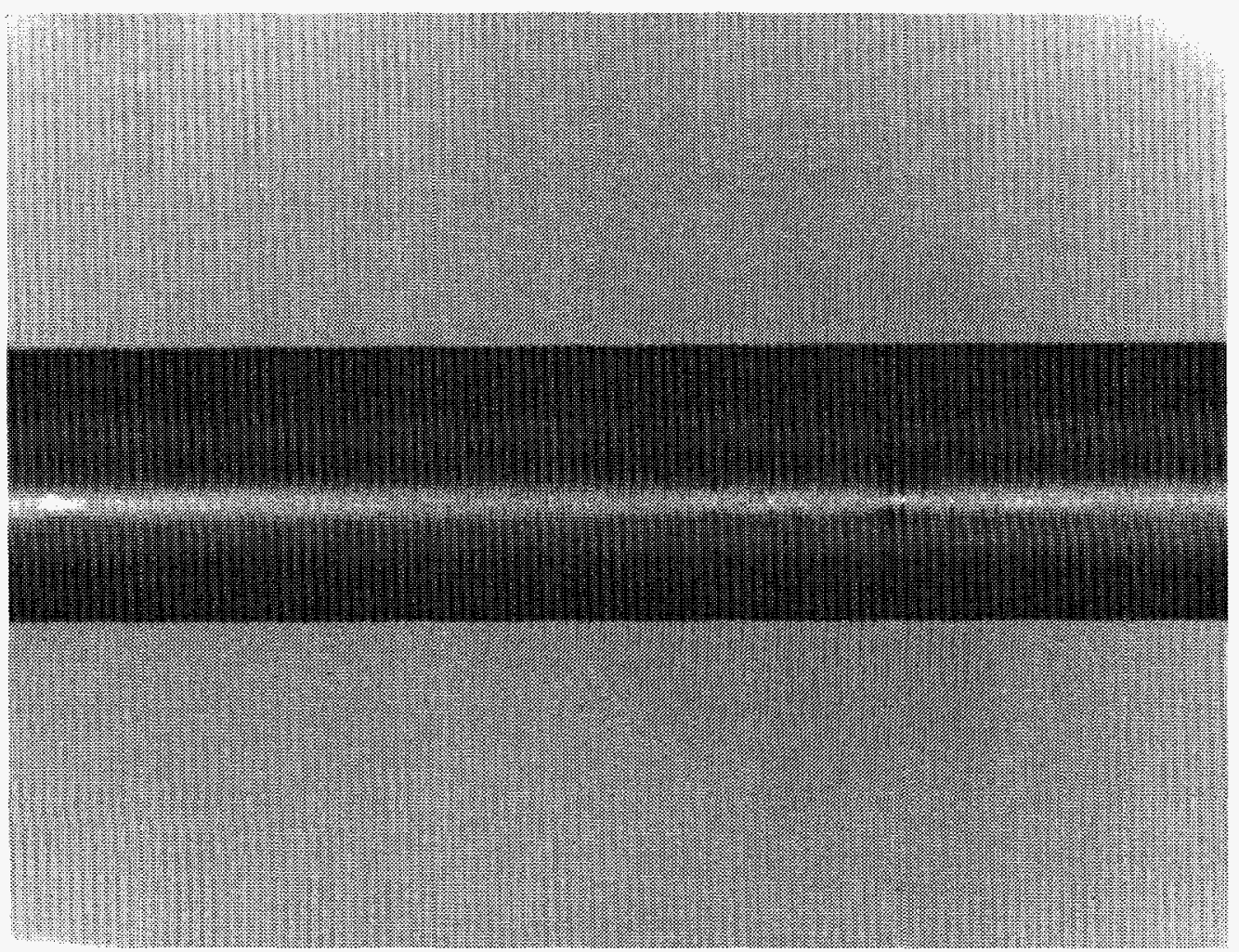

11G29. WL-09, Typical barrier (bottom) - Rotational composite, $299^{\circ}$ 


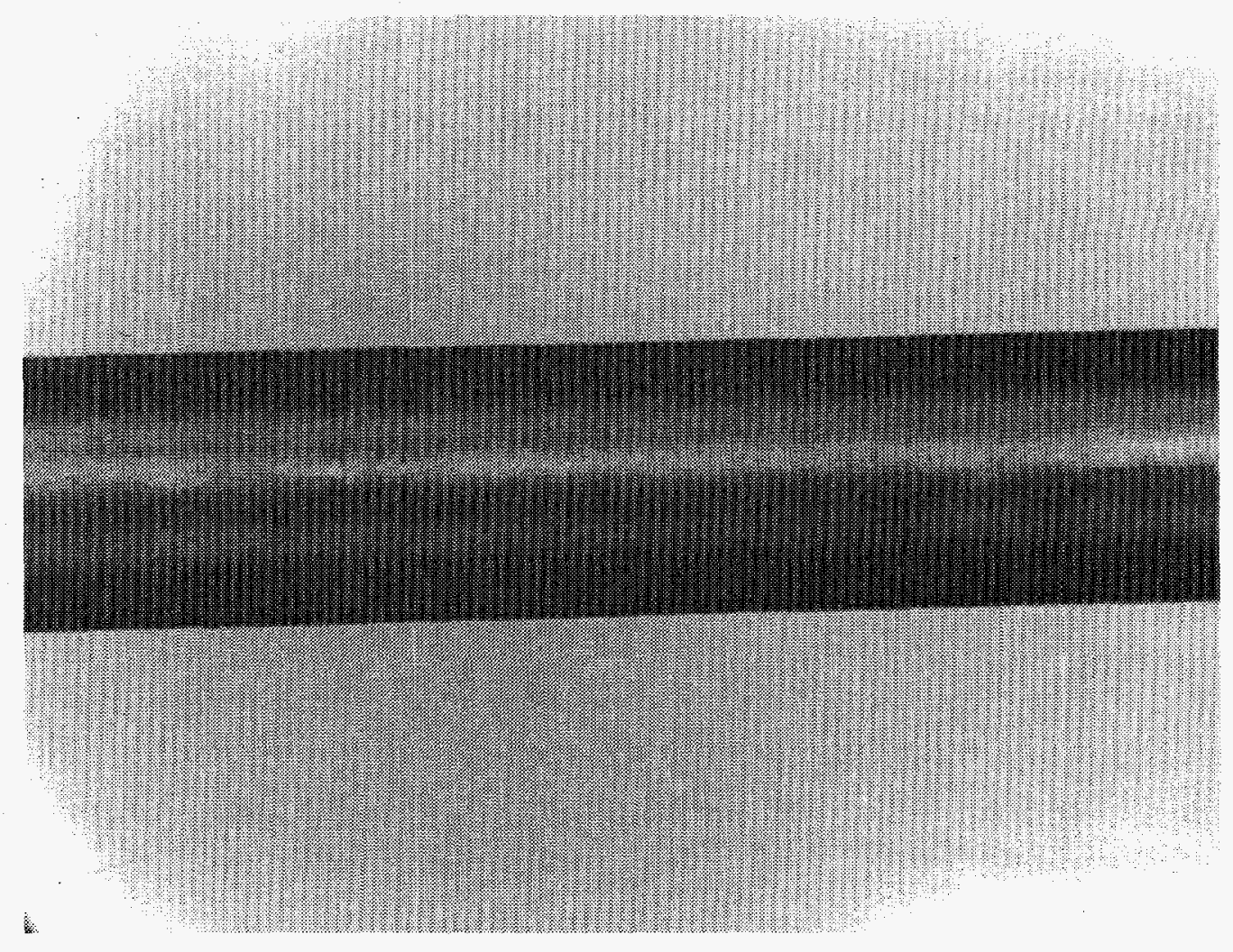

11G30. WL-09, Typical barrier (bottom) - Rotational composite, $359^{\circ}$
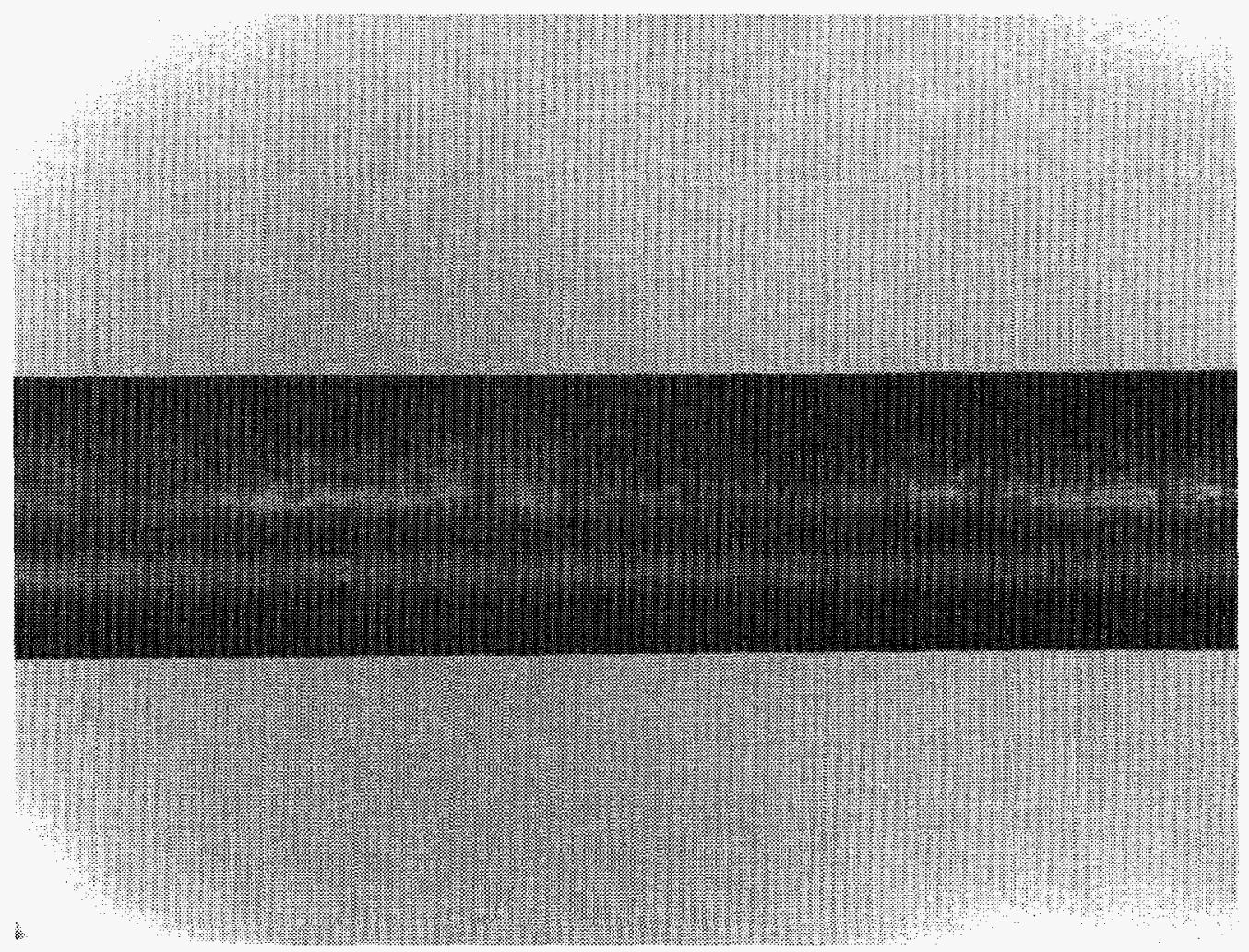

11G31. WL-09, Typical barrier (bottom) - Rotational composite, $60^{\circ}$ 


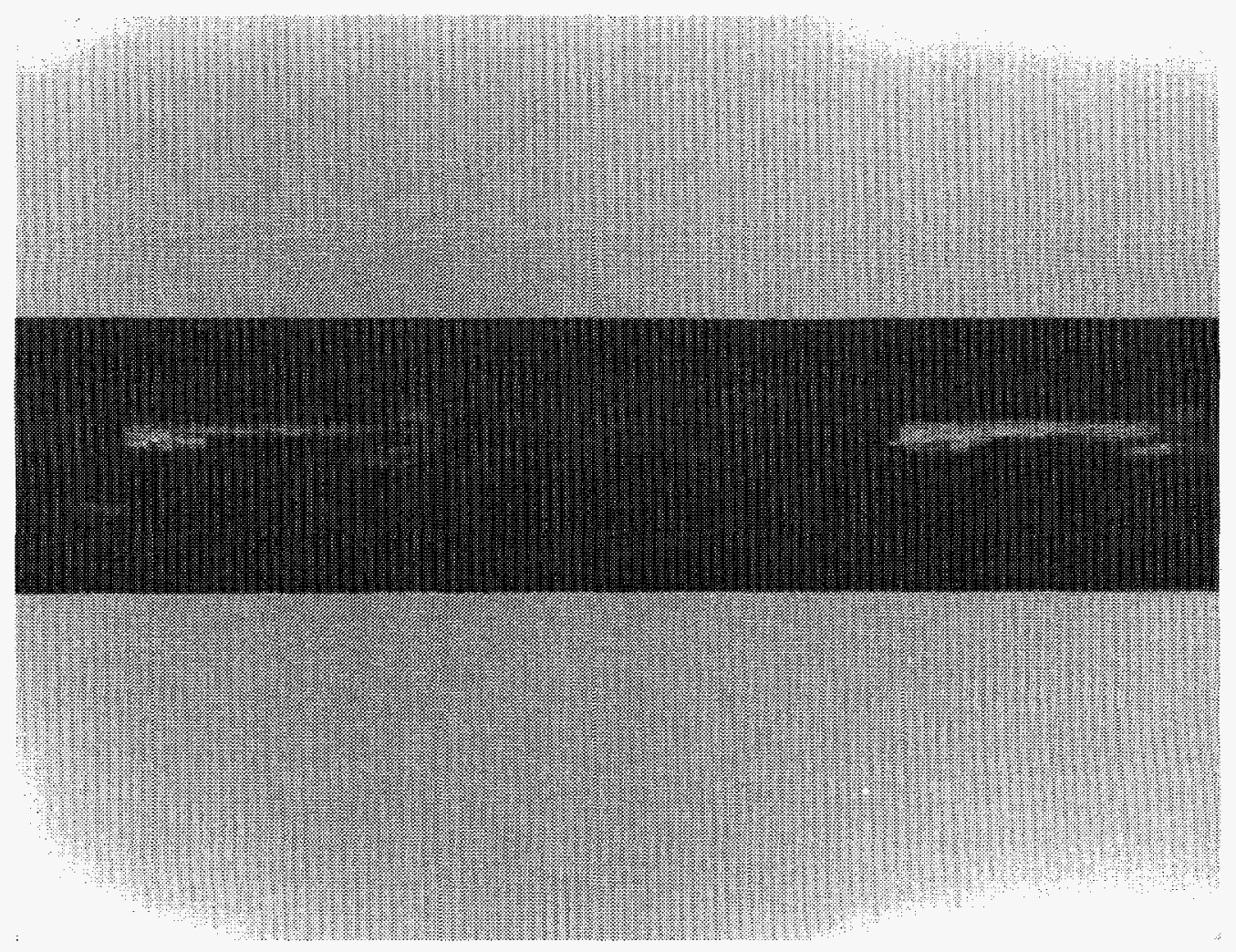

11G32. WL-03, Footprints

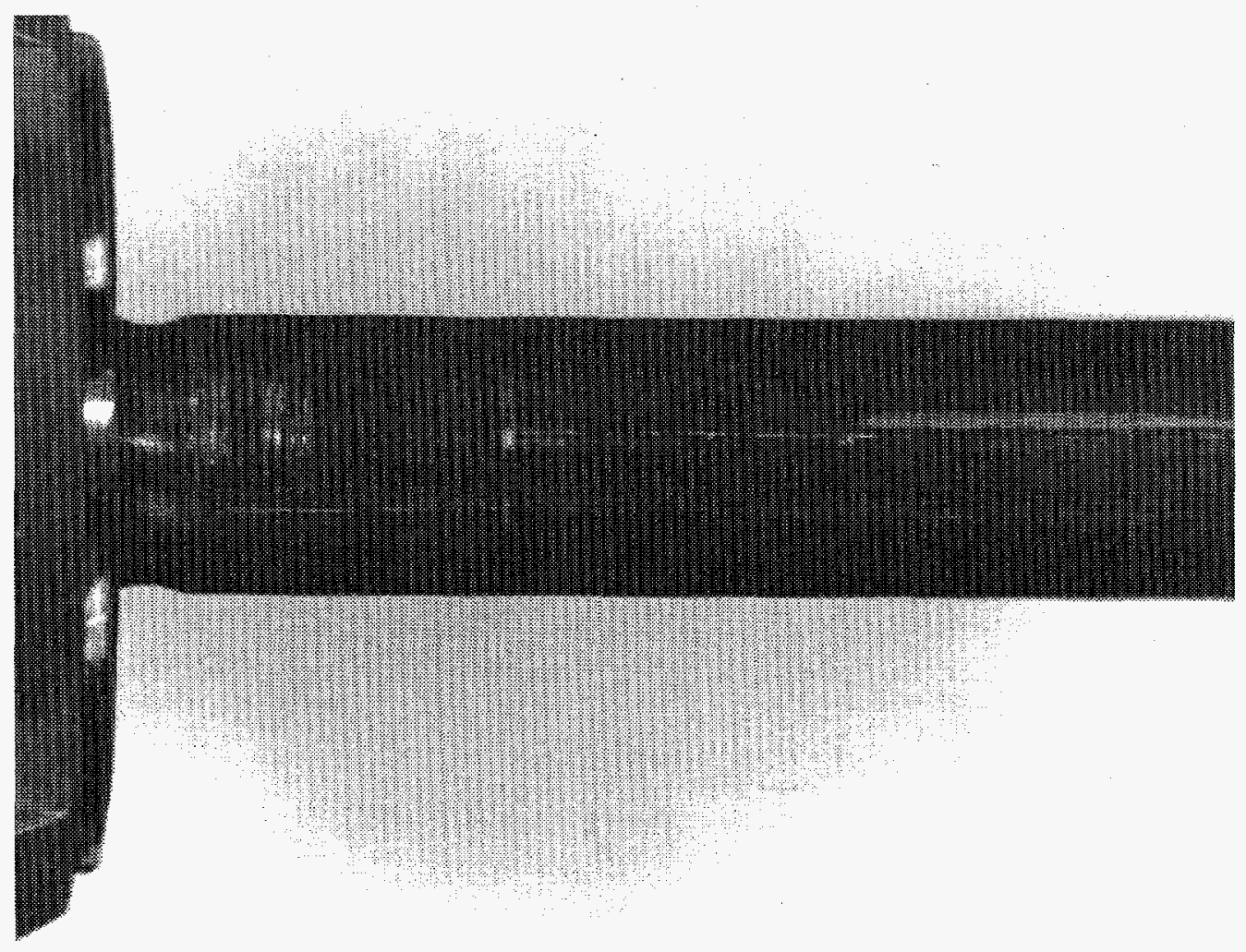

11G33. WL-03, Top End 


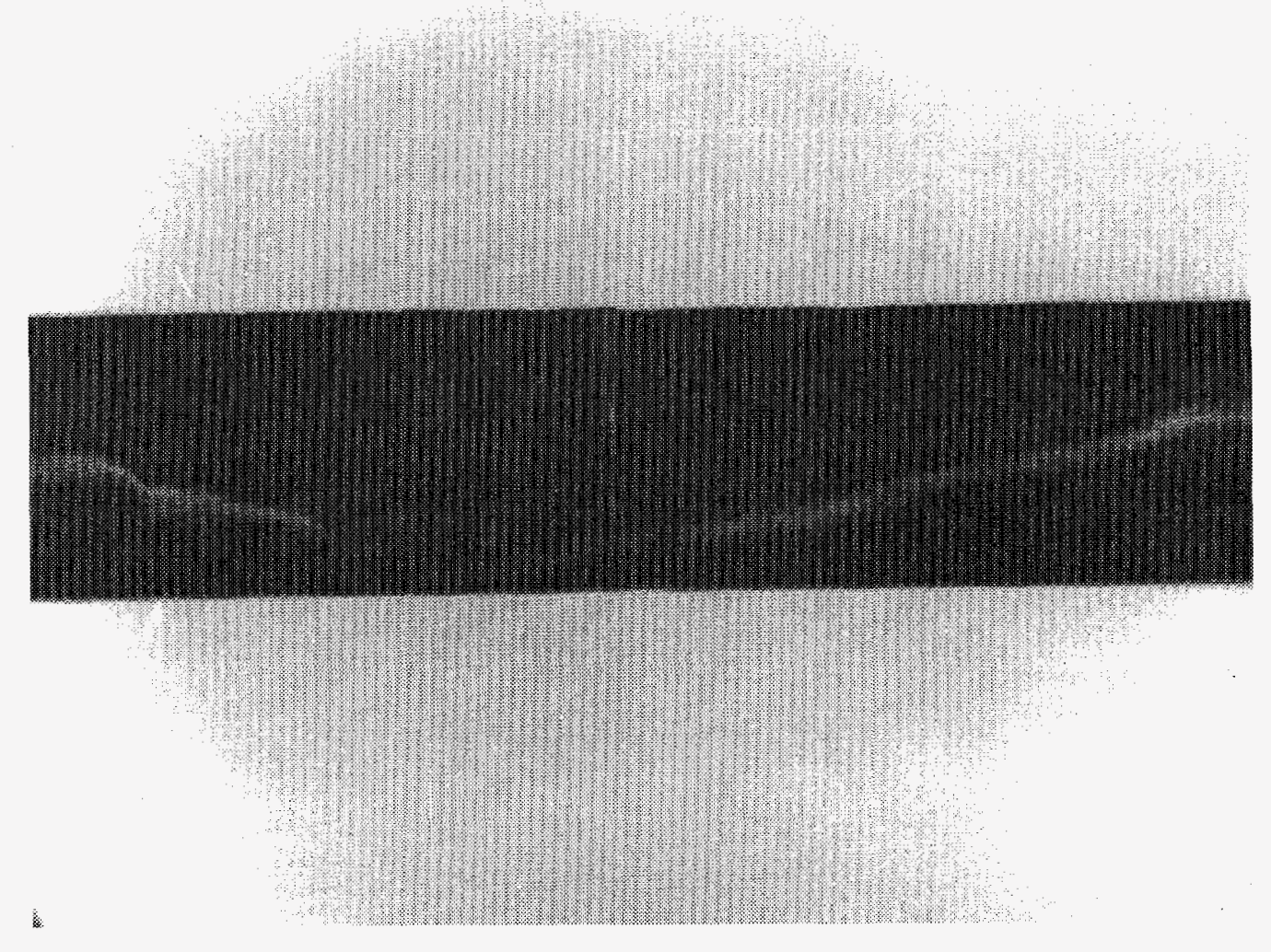

11G34. WL-03, Bottom End

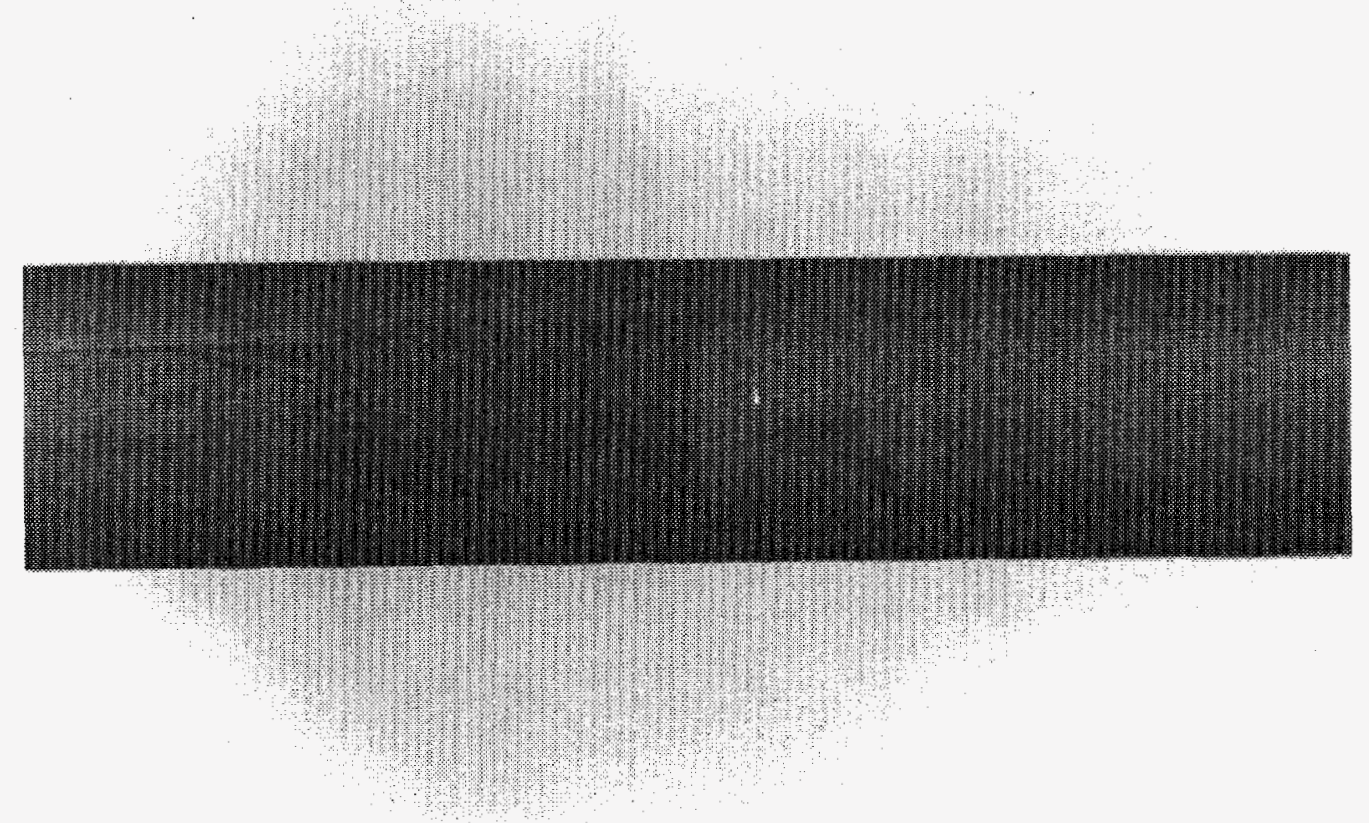

11G35. WL-03, Dark stain

B-30 


\section{WL-10 Photo Composite:}

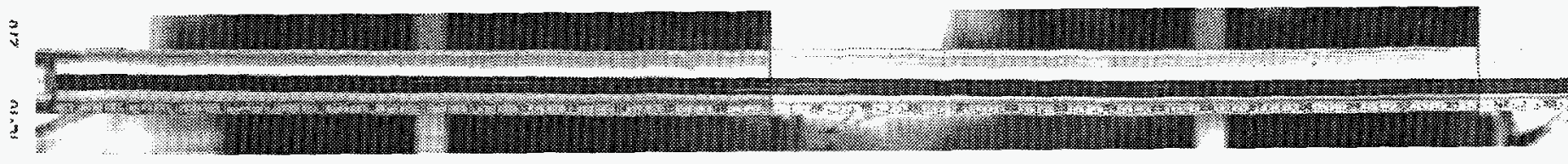

15G1-15G4. W

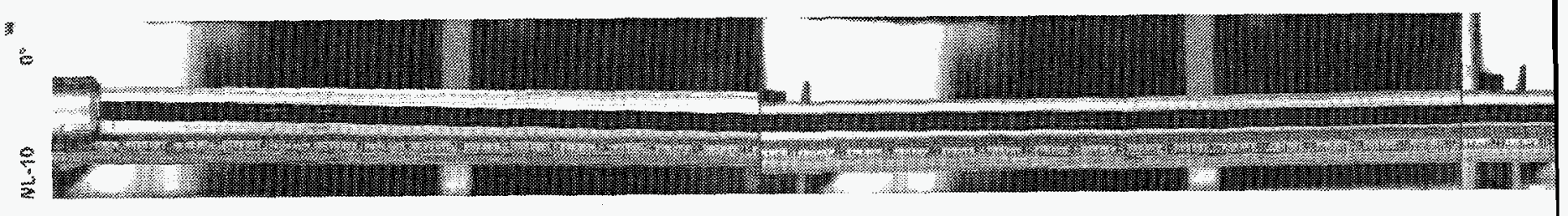

15G5-15G8. W

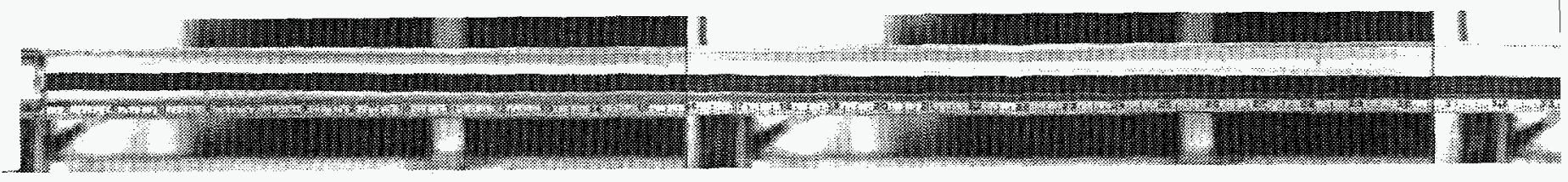

$15 \mathrm{G} 9-15 \mathrm{G} 12 . \mathrm{W}$

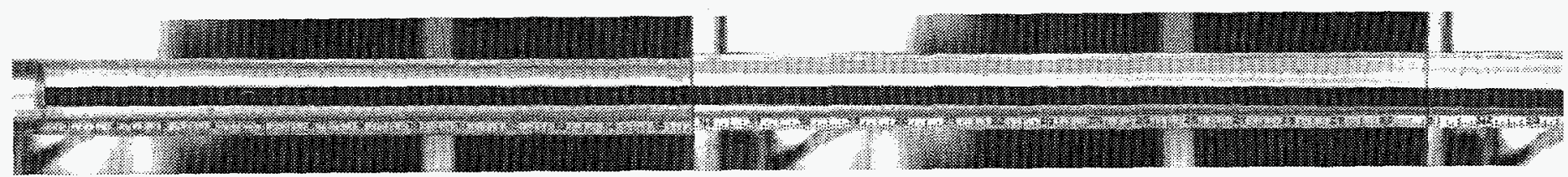

15G13-15G16. U 


\section{WL-26 Photo Composite:}

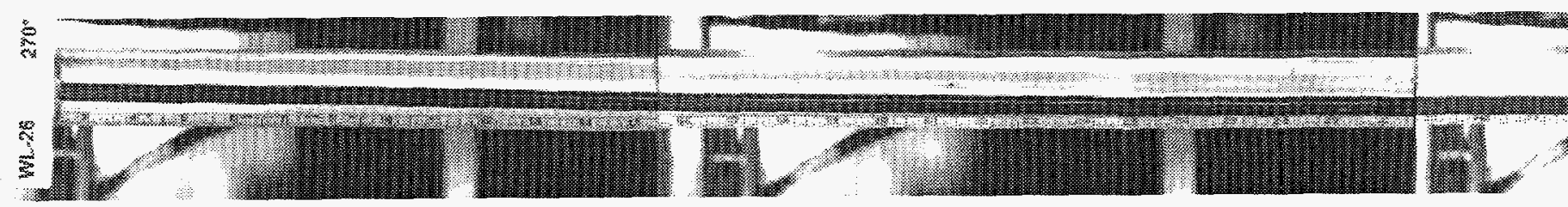

15G17-15G20.

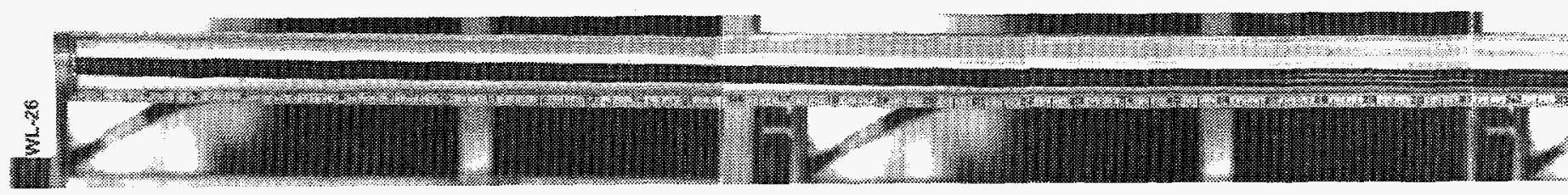

$15 \mathrm{G} 21-15 \mathrm{G} 24$.

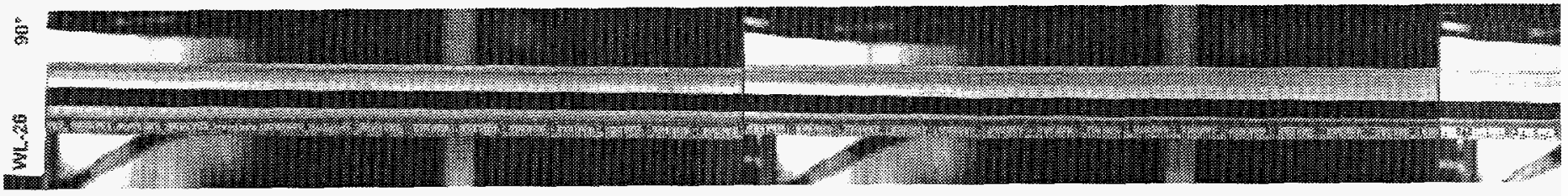

15G25-15G28. V

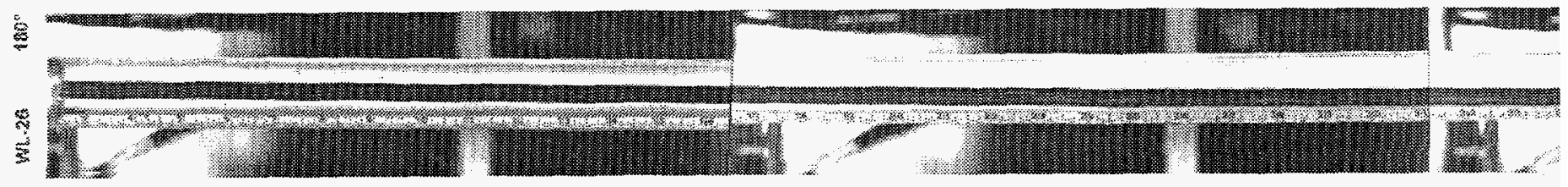

$15 \mathrm{G} 29-15 \mathrm{G} 32 . \mathrm{V}$ 


\section{S1/B1 Photos:}

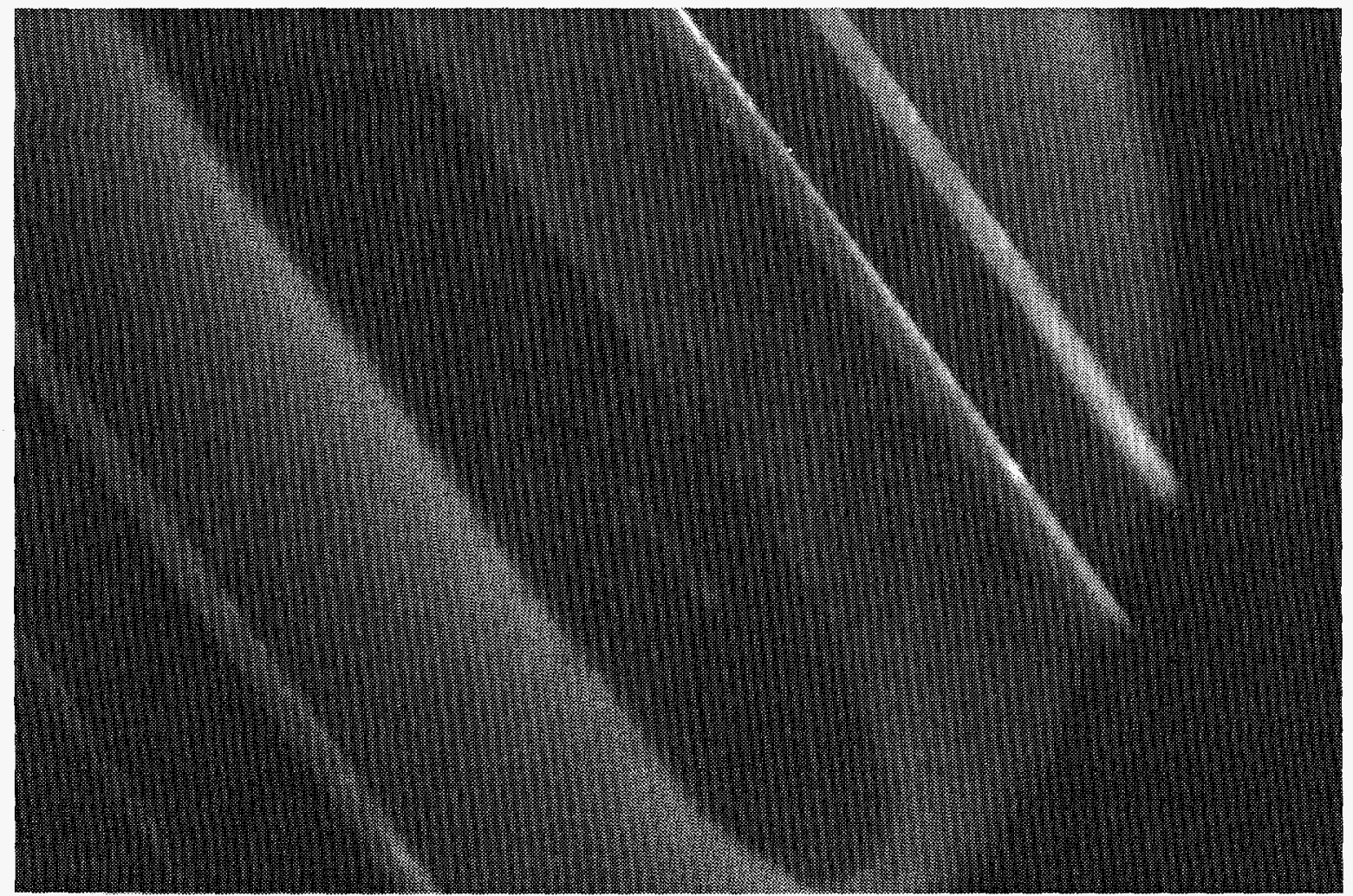

96-659-1-30. WT-11, ATR Hot Cell Disassembly

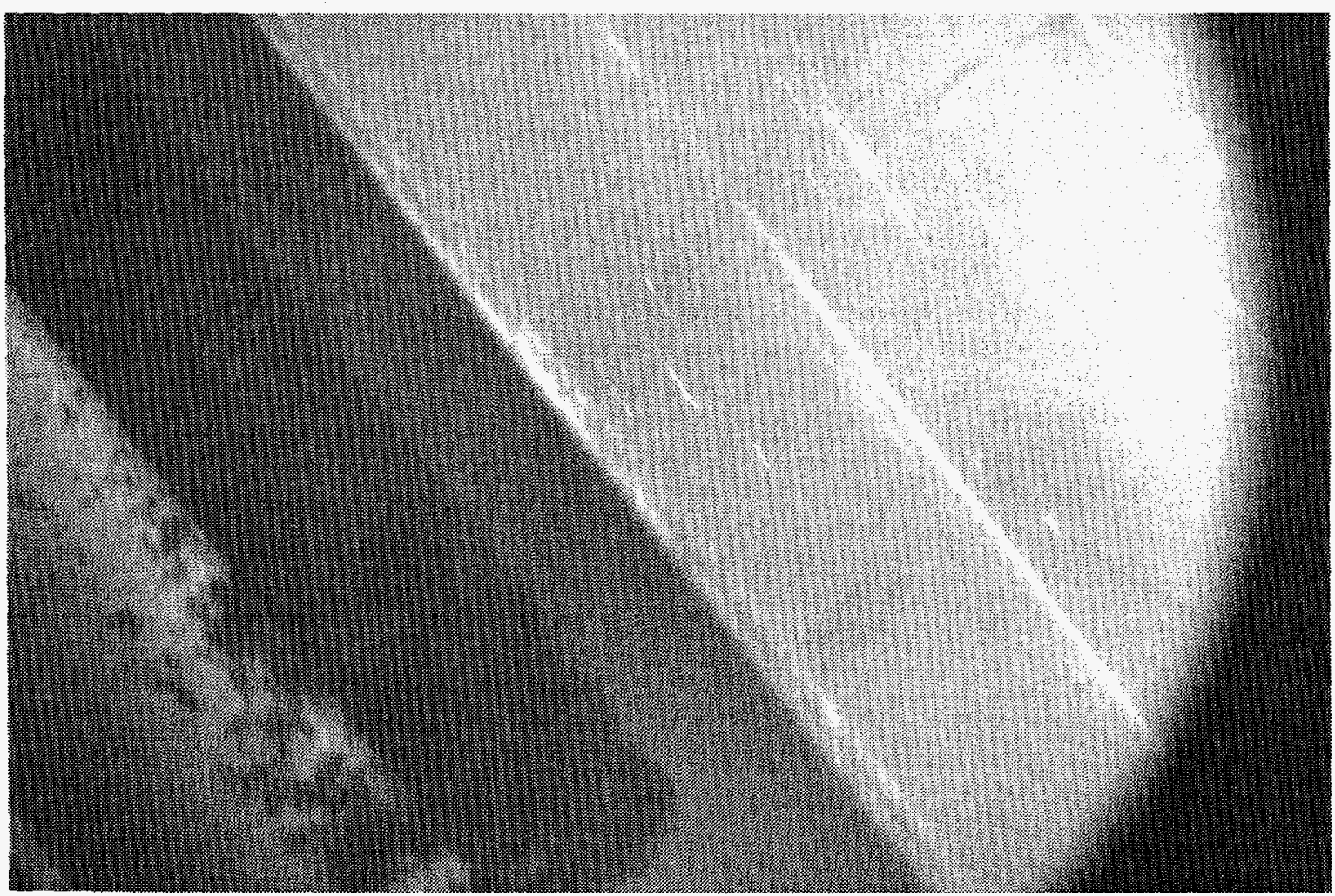

96-659-2-26. WT-12, ATR Hot Cell Disassembly 


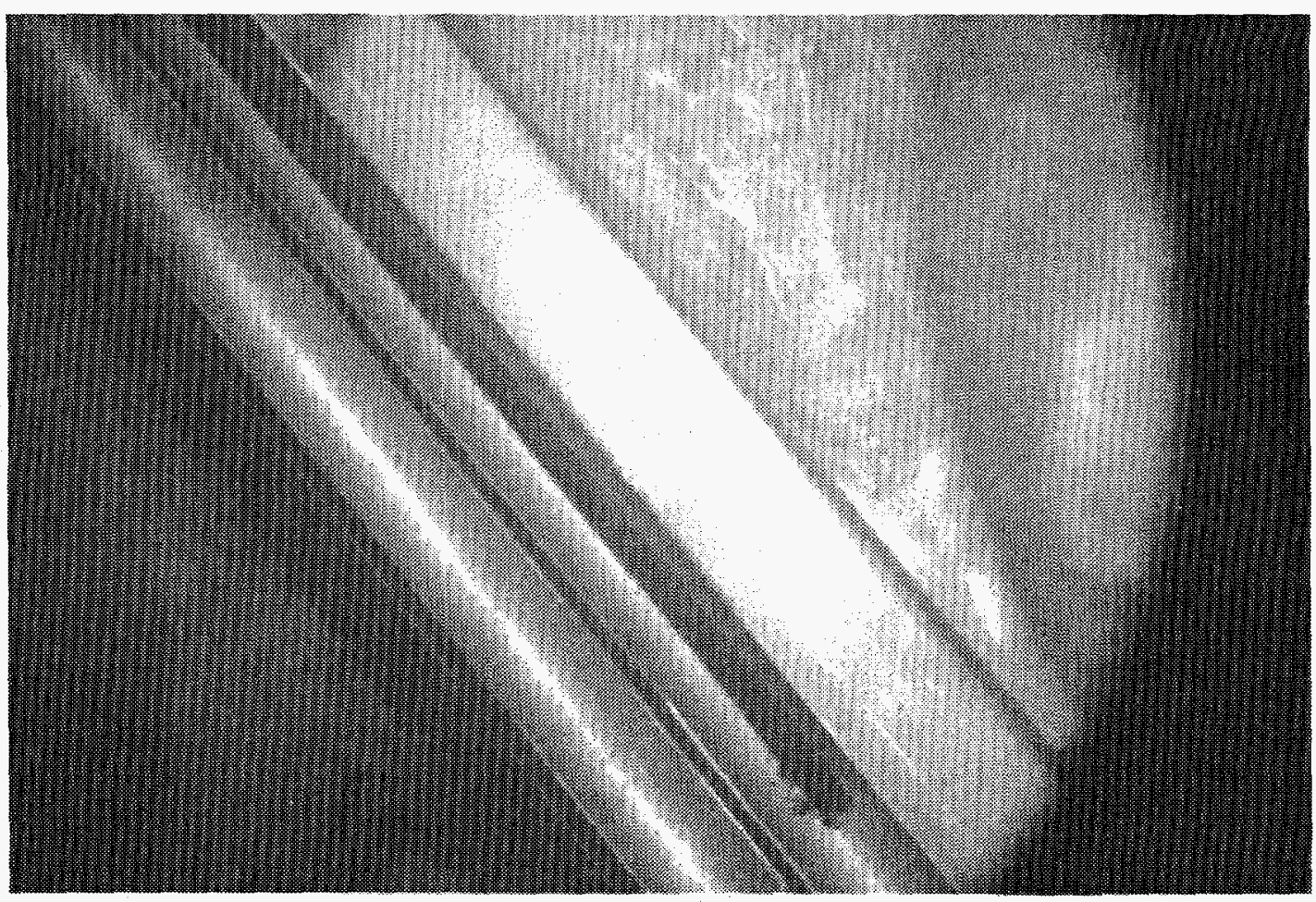

96-659-2-24. WT-12, ATR Hot Cell Disassembly

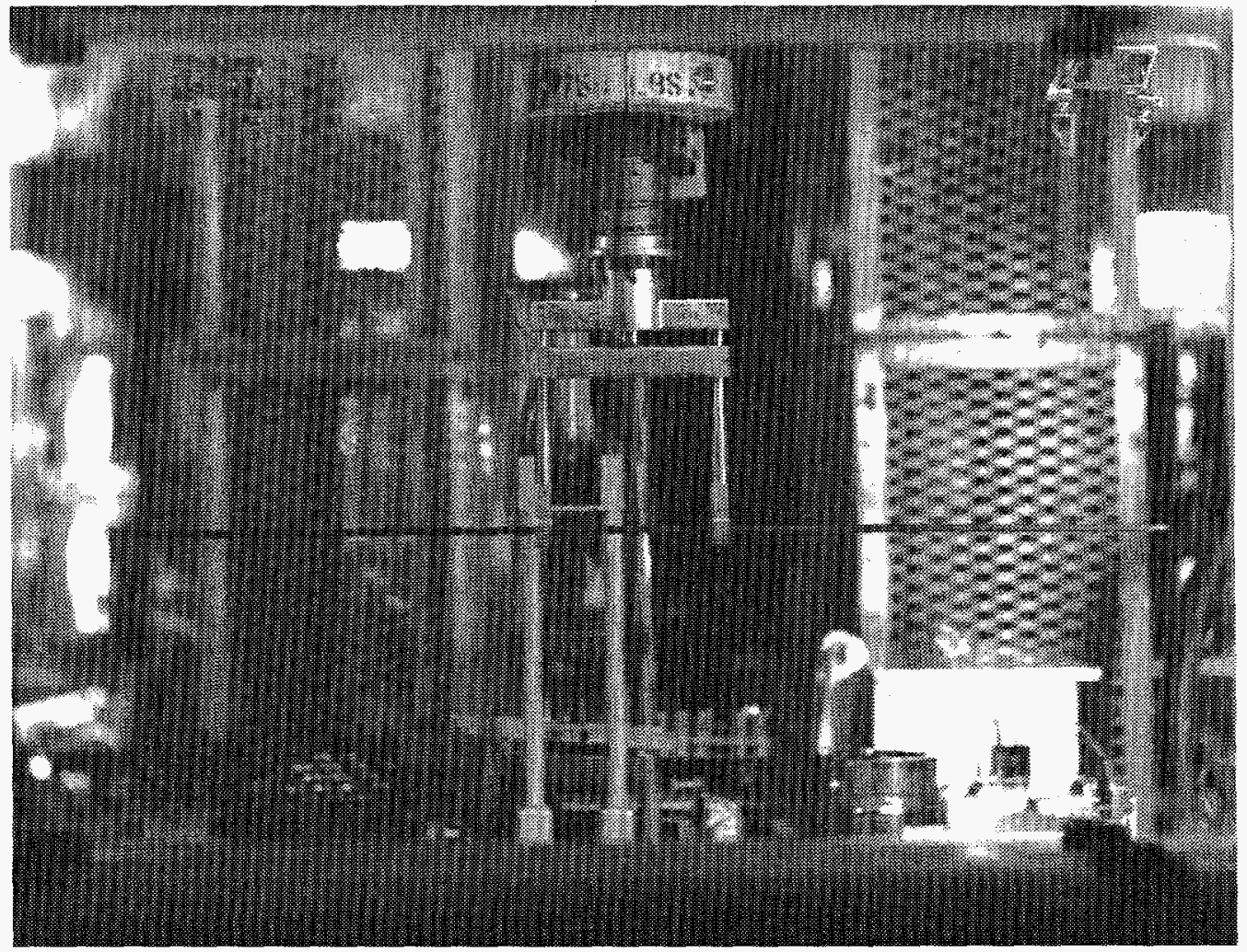

10G1. WT-11, Disassembly 


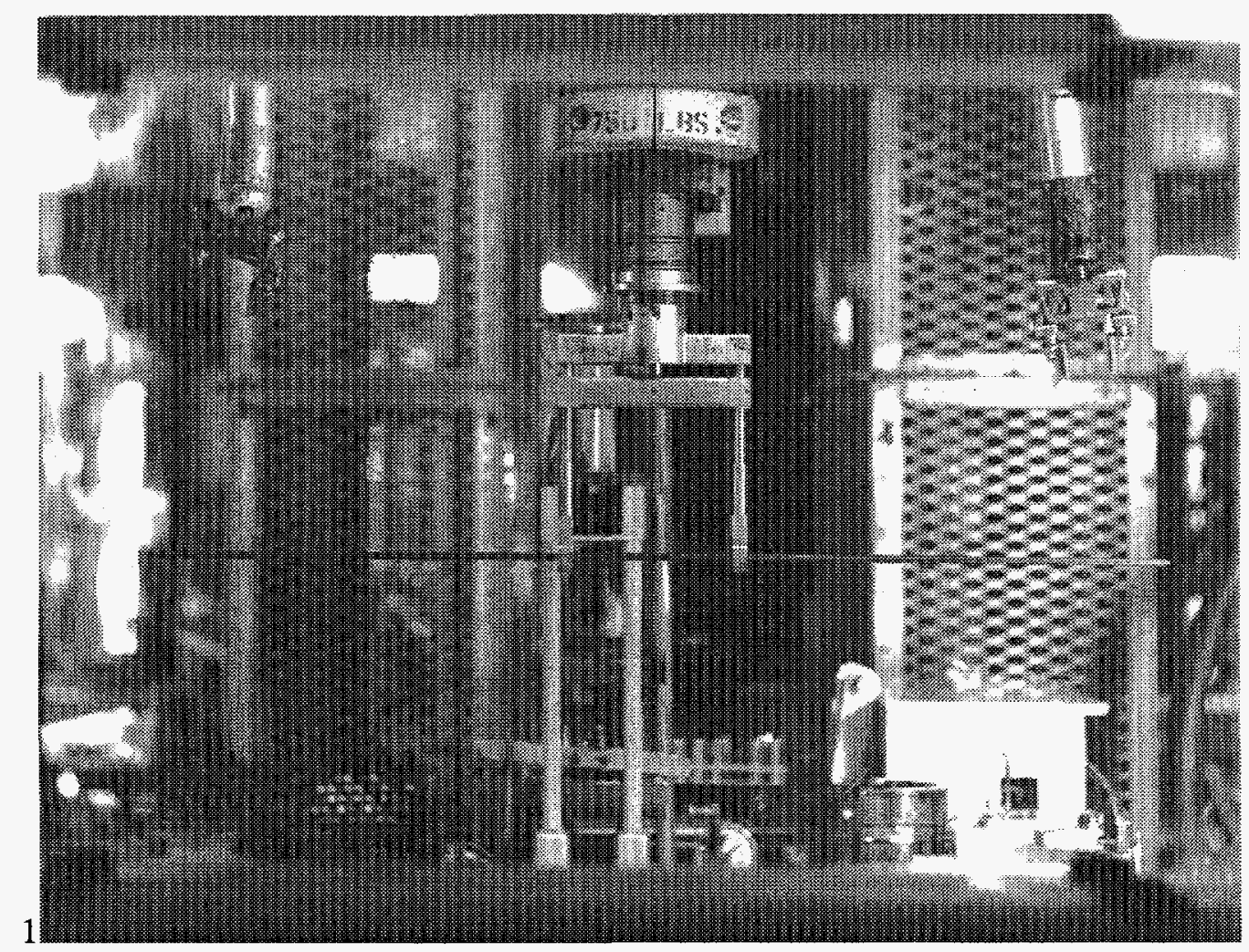

10G2. WT-12, Disassembly

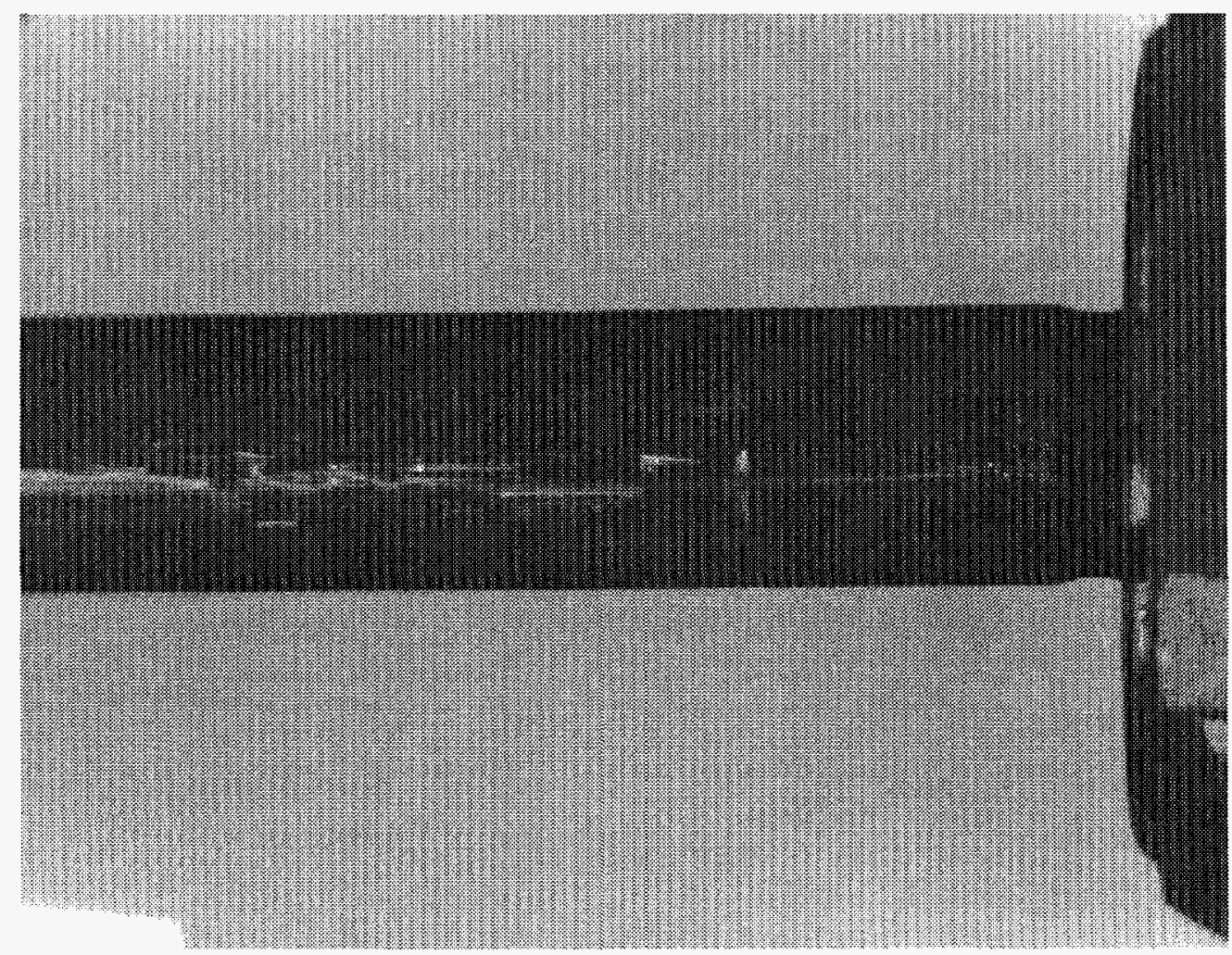

14G1. WT-11, Top end 


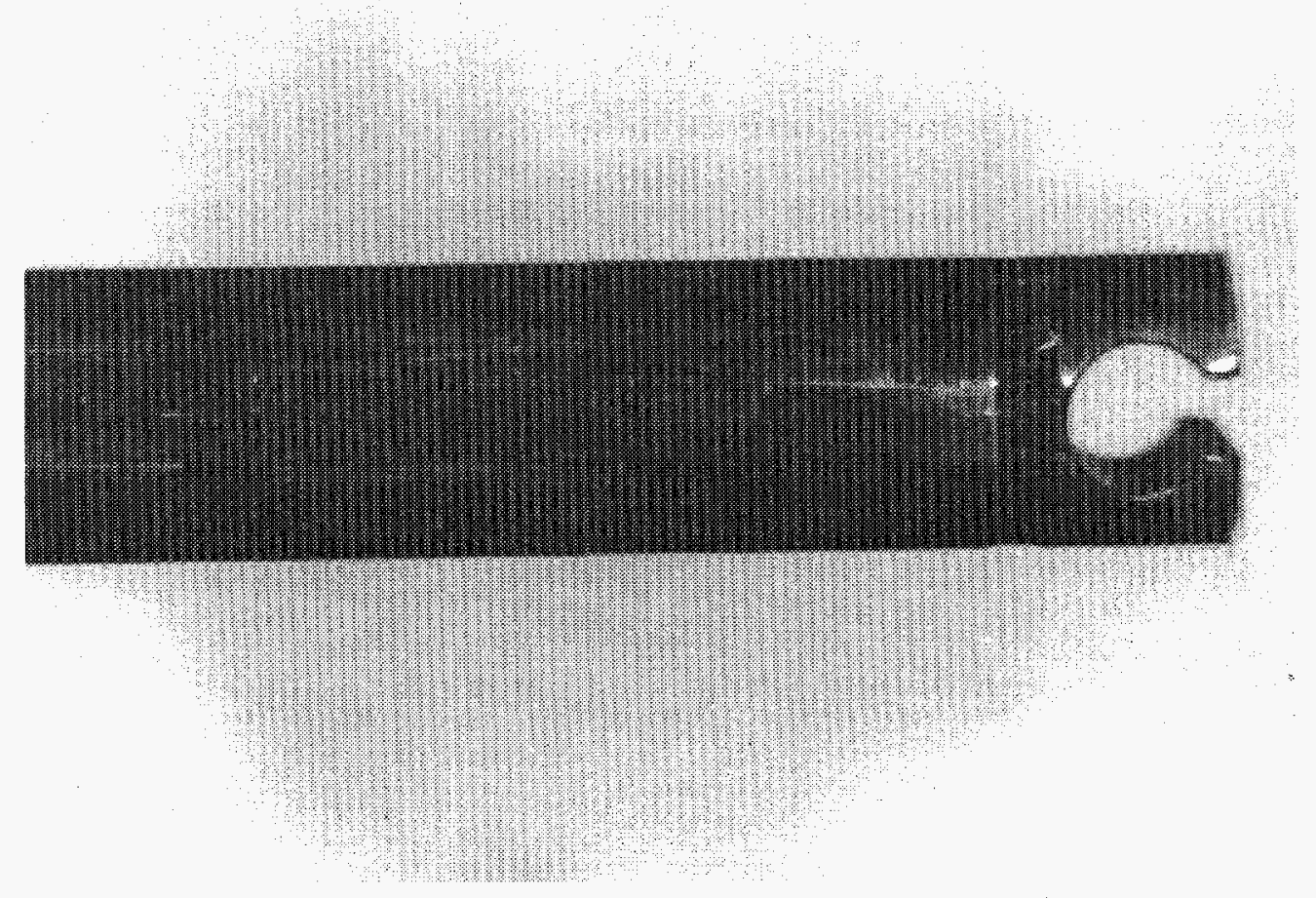

14G2. WT-11, Bottom end

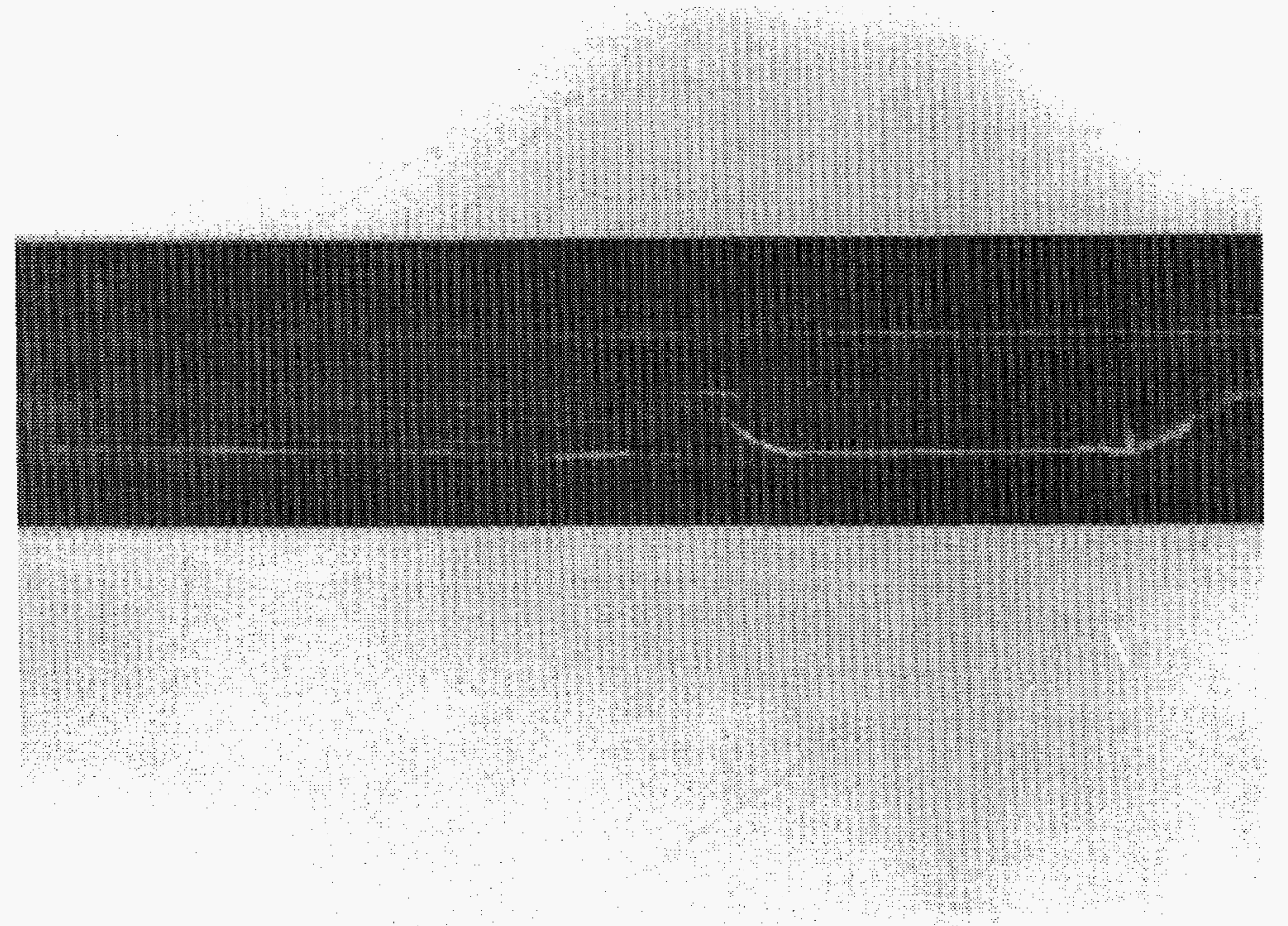

14G3. WT-11, Typical scratches (rotation composite), $29^{\circ}$ 


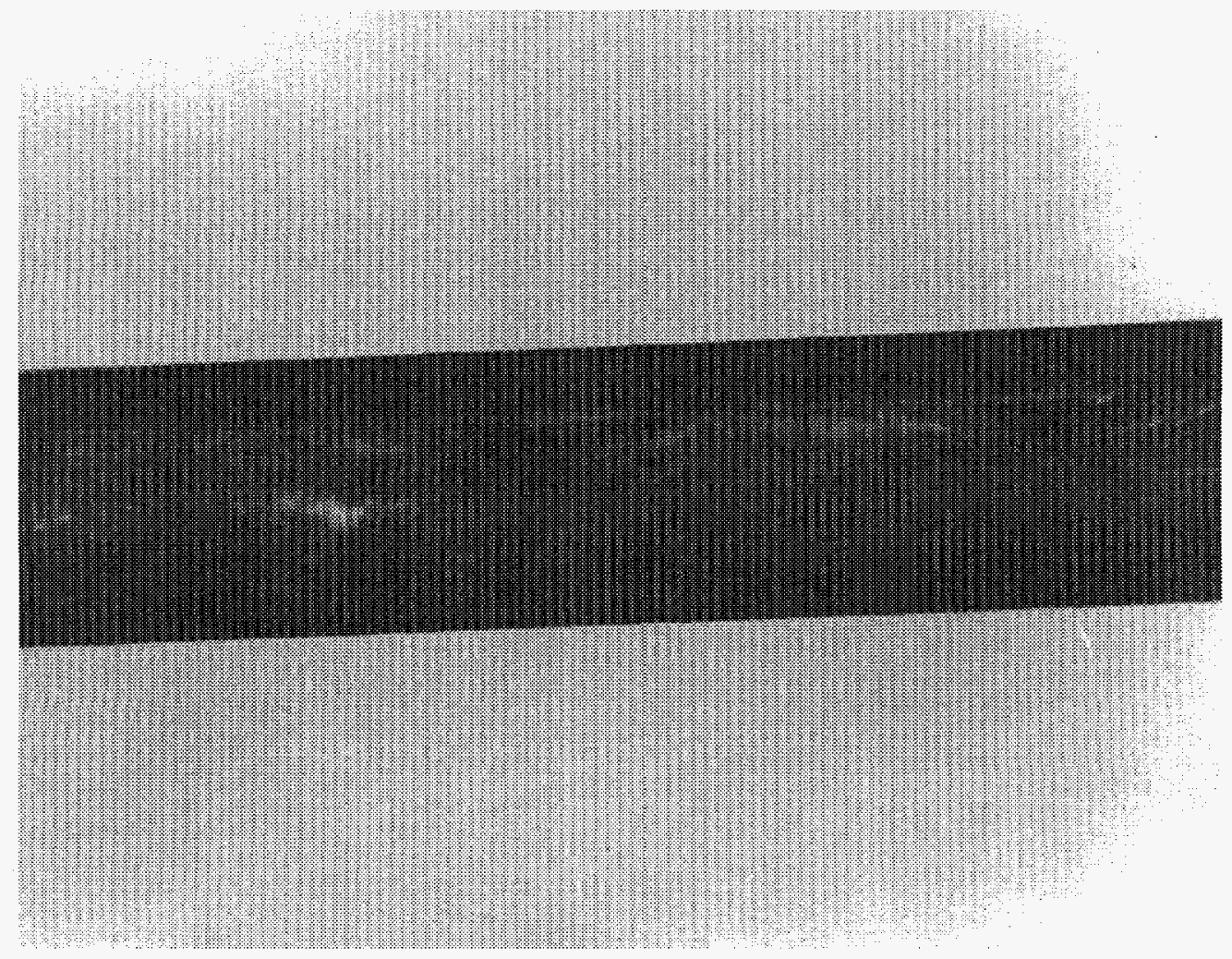

14G4. WT-11, Typical scratches (rotation composite), $89^{\circ}$

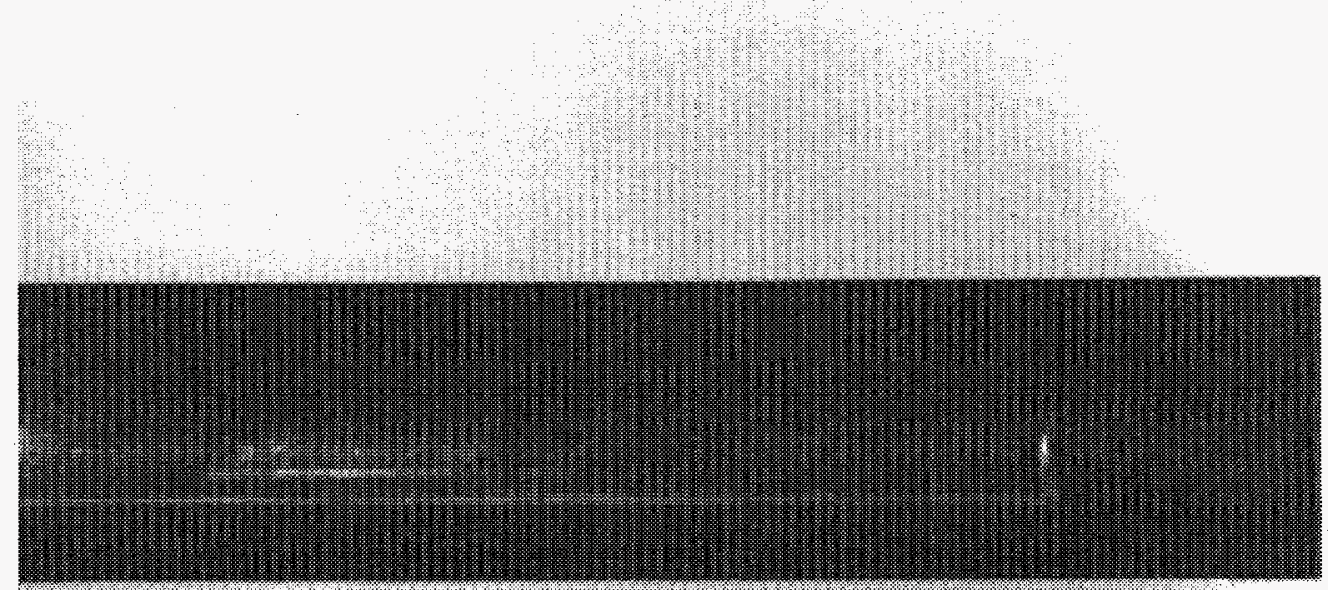

14G5. WT-11, Typical scratches (rotation composite), $150^{\circ}$ 
14G6. WT-11, Typical scratches (rotation composite), $210^{\circ}$

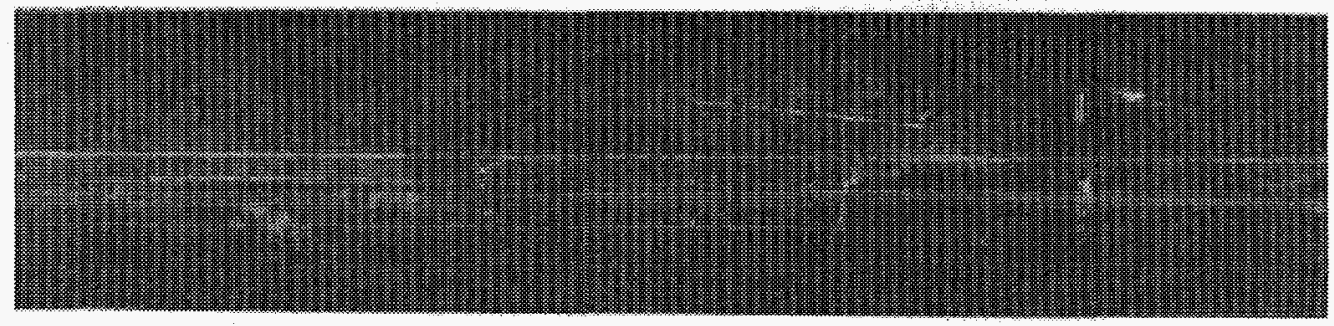

14G7. WT-11, Typical scratches (rotation composite), $269^{\circ}$ 


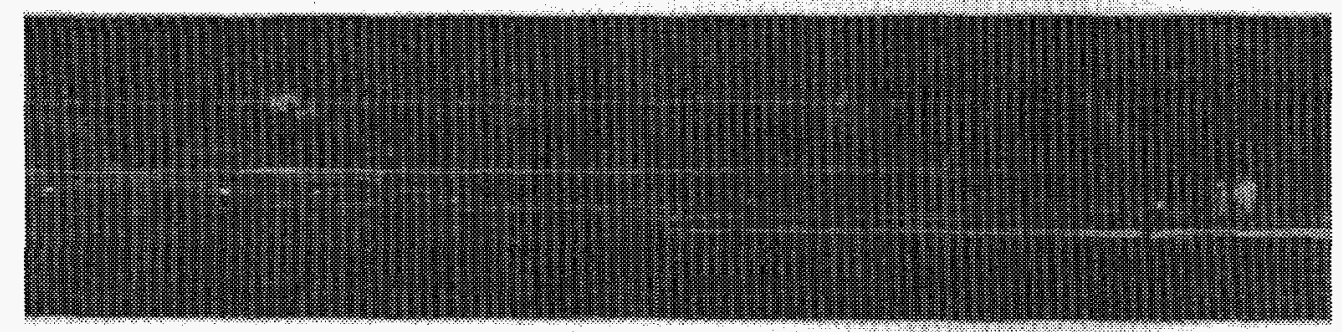

14G8. WT-11, Typical scratches (rotation composite), $329^{\circ}$

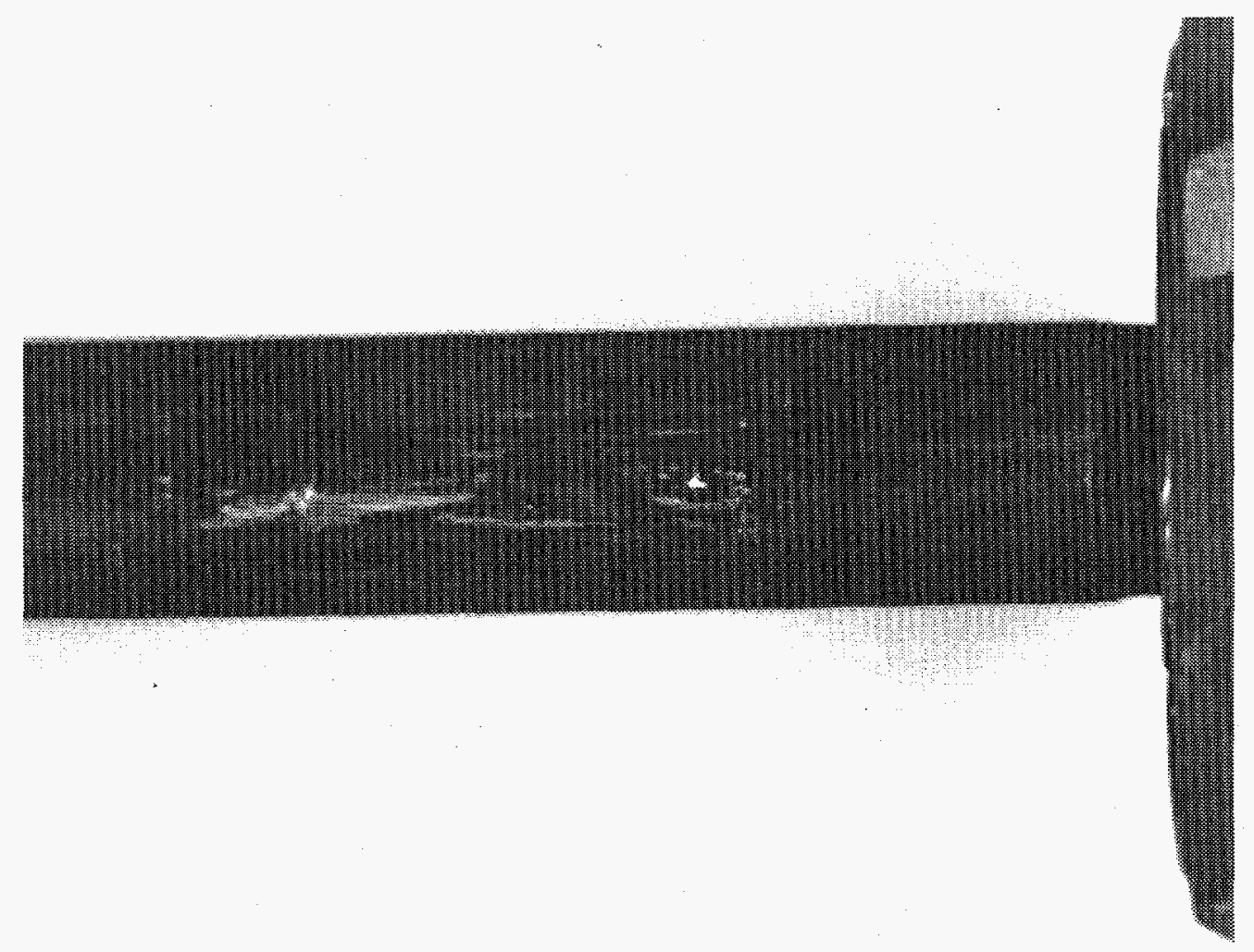

14G9. WT-12, Top end 


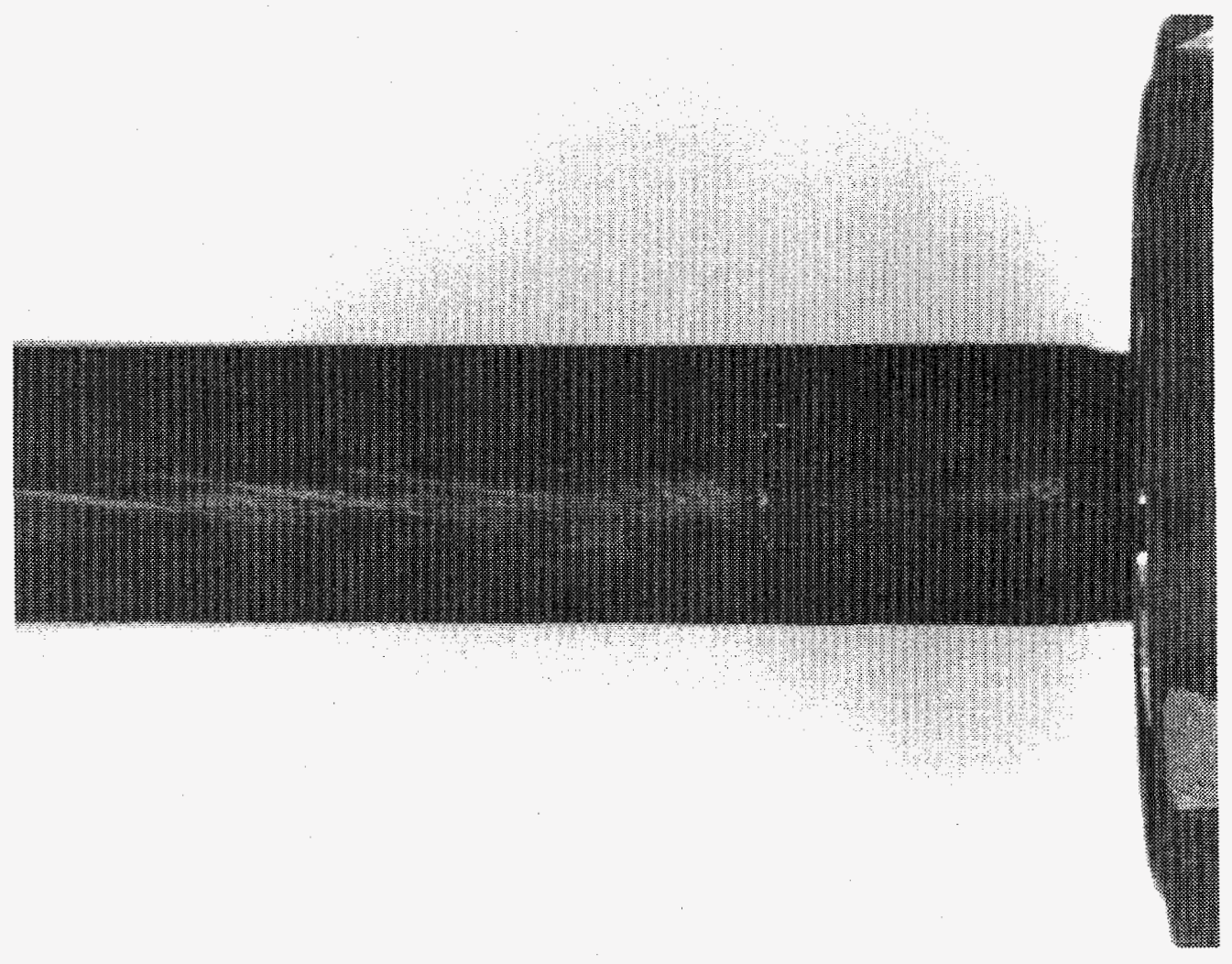

14G10. WT-12, Top end

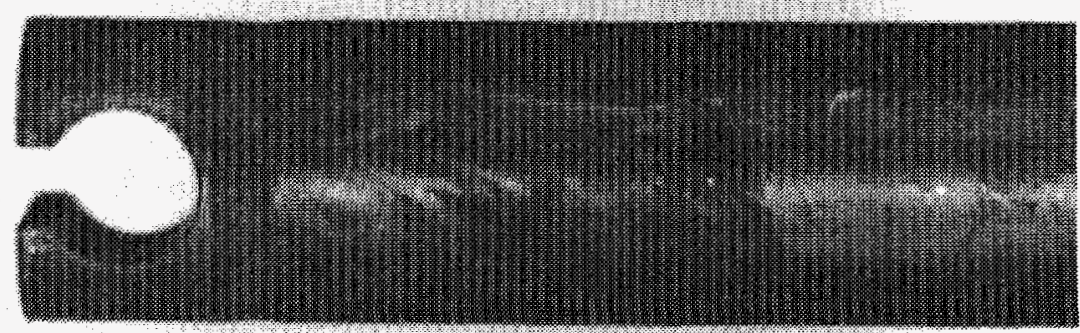

14G11. WT-12, Bottom end

$B-40$ 


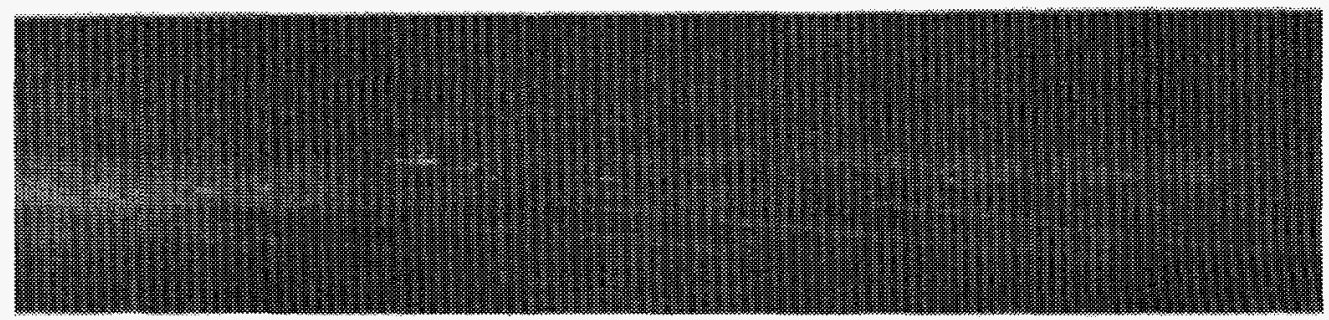

14G12. WT-12, Typical scratches (rotation composite), $291^{\circ}$, elev. 46.3 in.

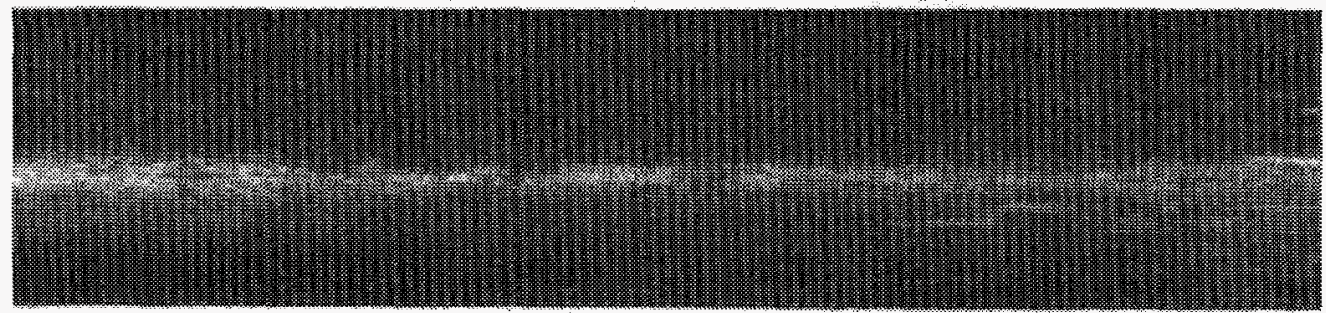

14G13. WT-12, Typical scratches (rotation composite), $351^{\circ}$, elev. $46.3 \mathrm{in}$. 


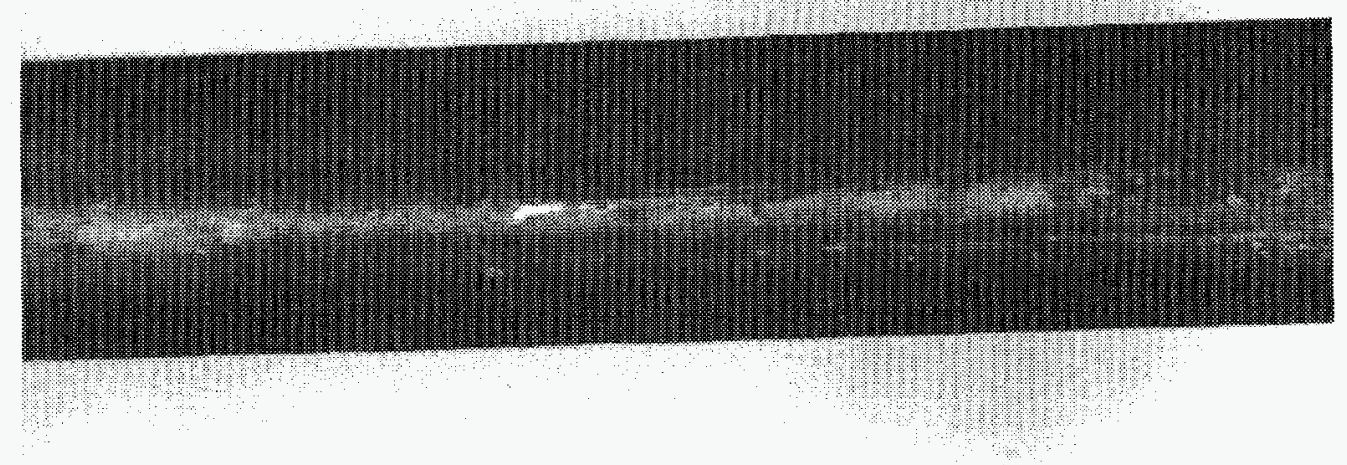

14G14. WT-12, Typical scratches (rotation composite), 52 ${ }^{\circ}$, elev. $46.3 \mathrm{in}$.

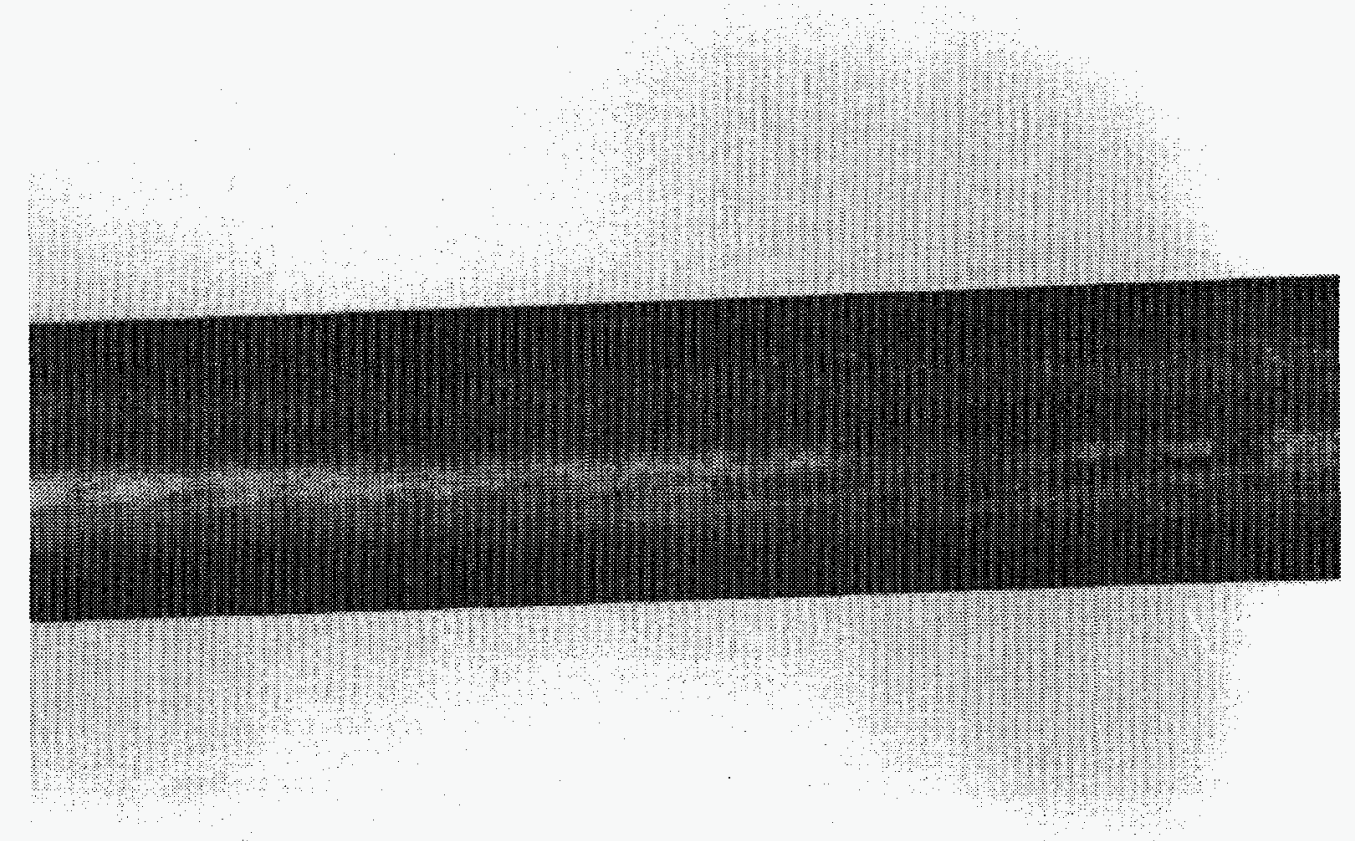

14G15. WT-12, Typical scratches (rotation composite), $112^{\circ}$, elev. $46.3 \mathrm{in}$. 
14G16. WT-12, Typical scratches (rotation composite), $171^{\circ}$, elev. $46.3 \mathrm{in}$.

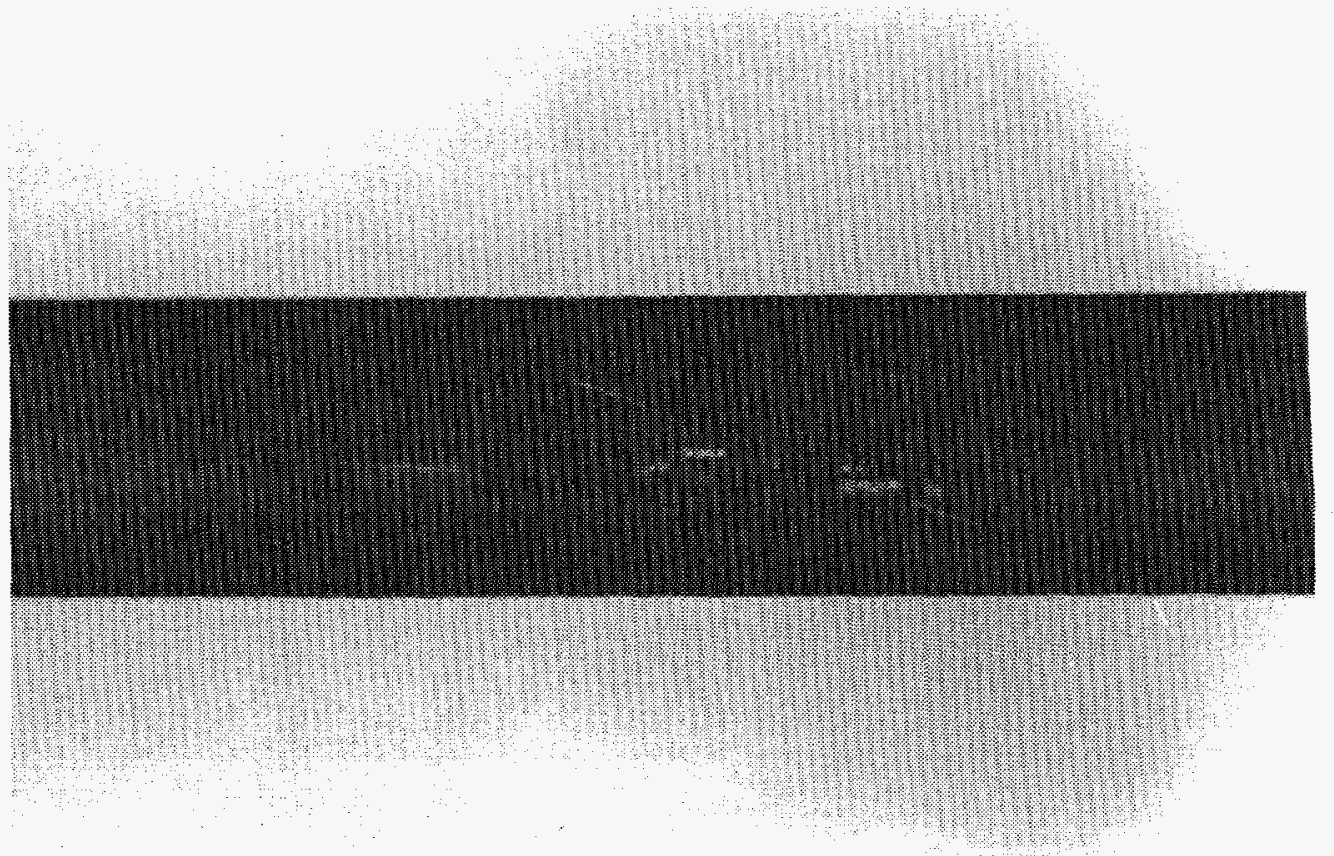

14G17. WT-12, Typical scratches (rotation composite), $232^{\circ}$, elev. $46.3 \mathrm{in}$. 


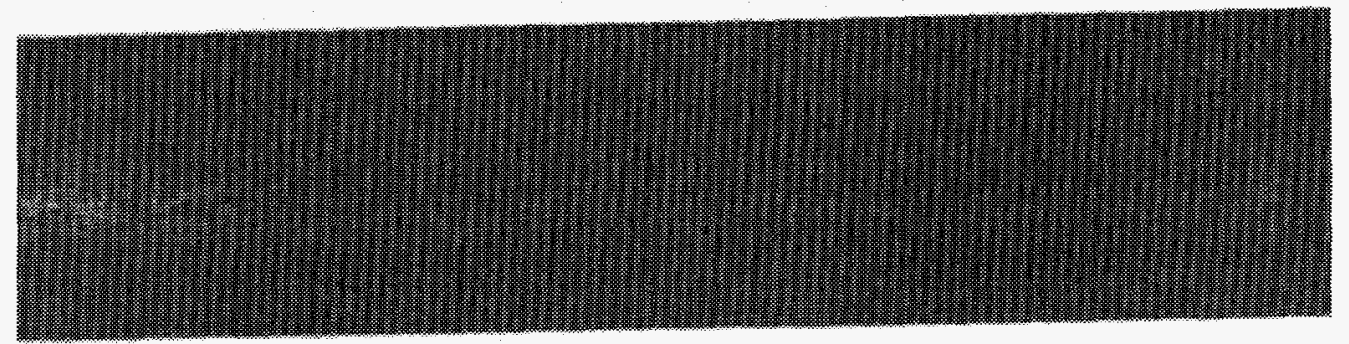

14G18. WT-12, Typical scratches (rotation composite), $292^{\circ}$, elev. $16.8 \mathrm{in.}$

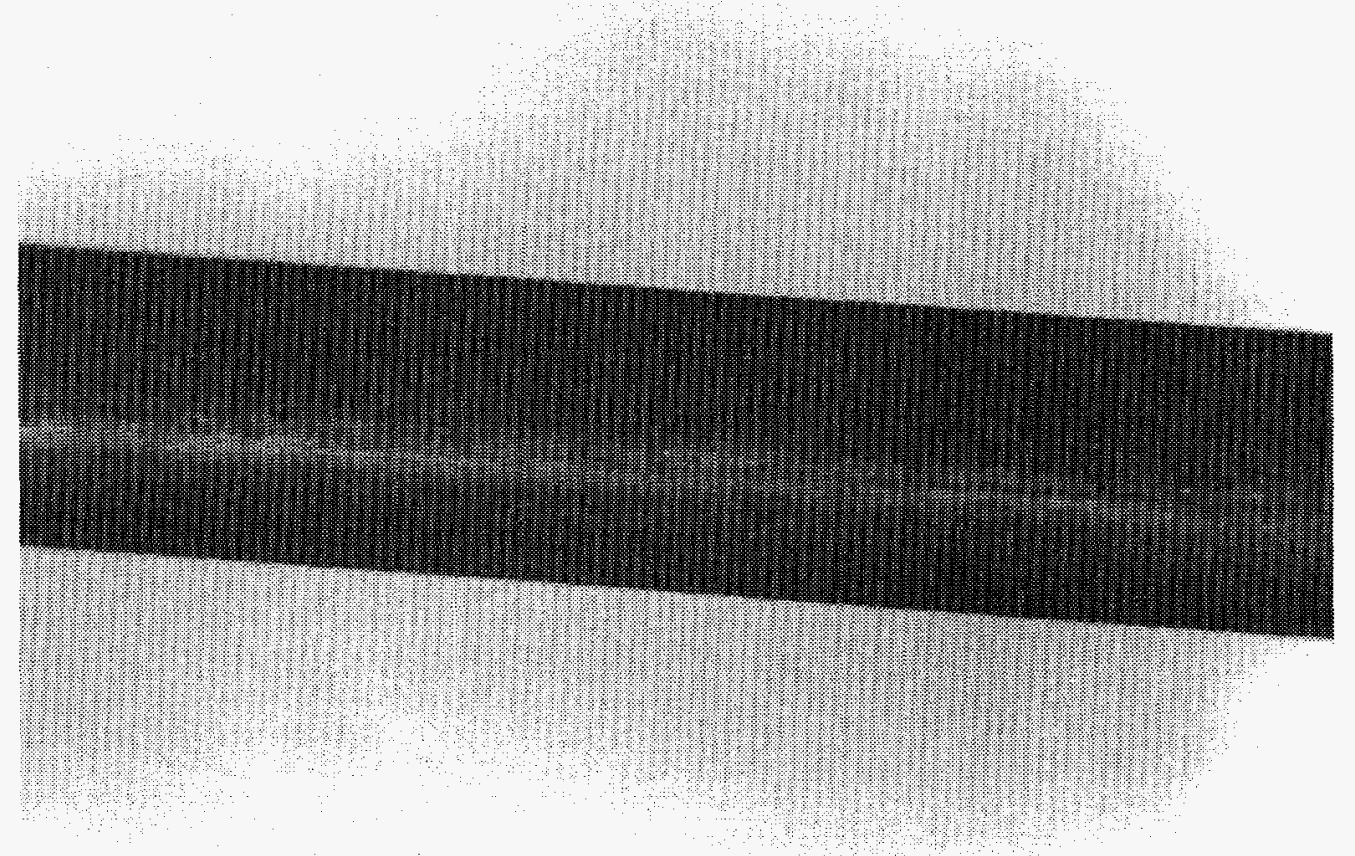

14G19. WT-12, Typical scratches (rotation composite), $352^{\circ}, 16.8$ in. 


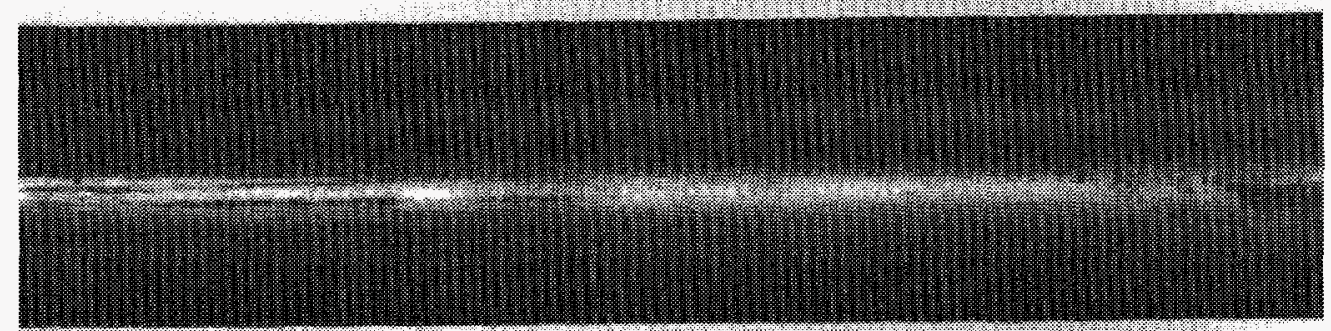

14G20. WT-12, Typical scratches (rotation composite), $52^{\circ}, 16.8 \mathrm{in}$.

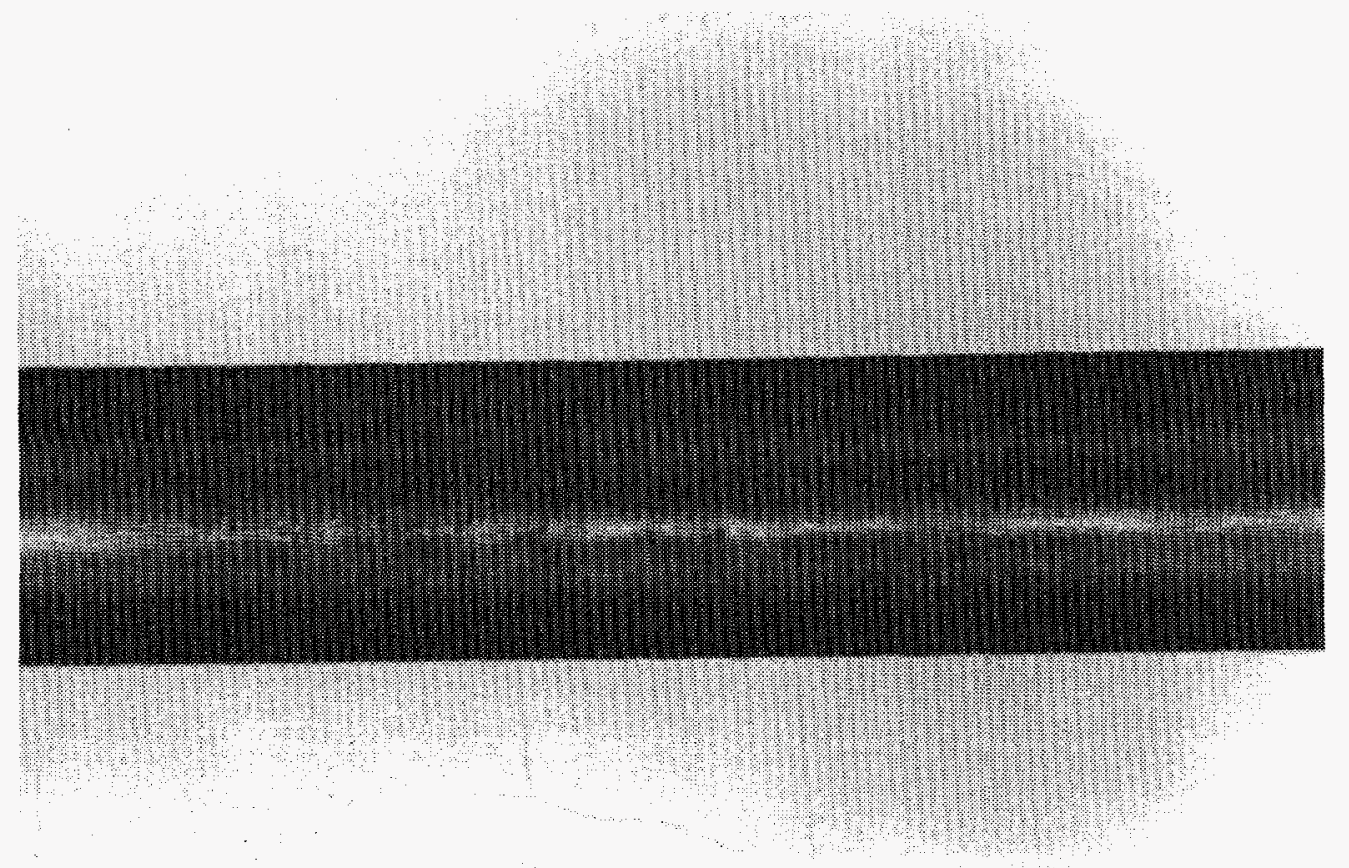

14G21. WT-12, Typical scratches (rotation composite), $112^{\circ}$, elev. $16.8 \mathrm{in.}$ 


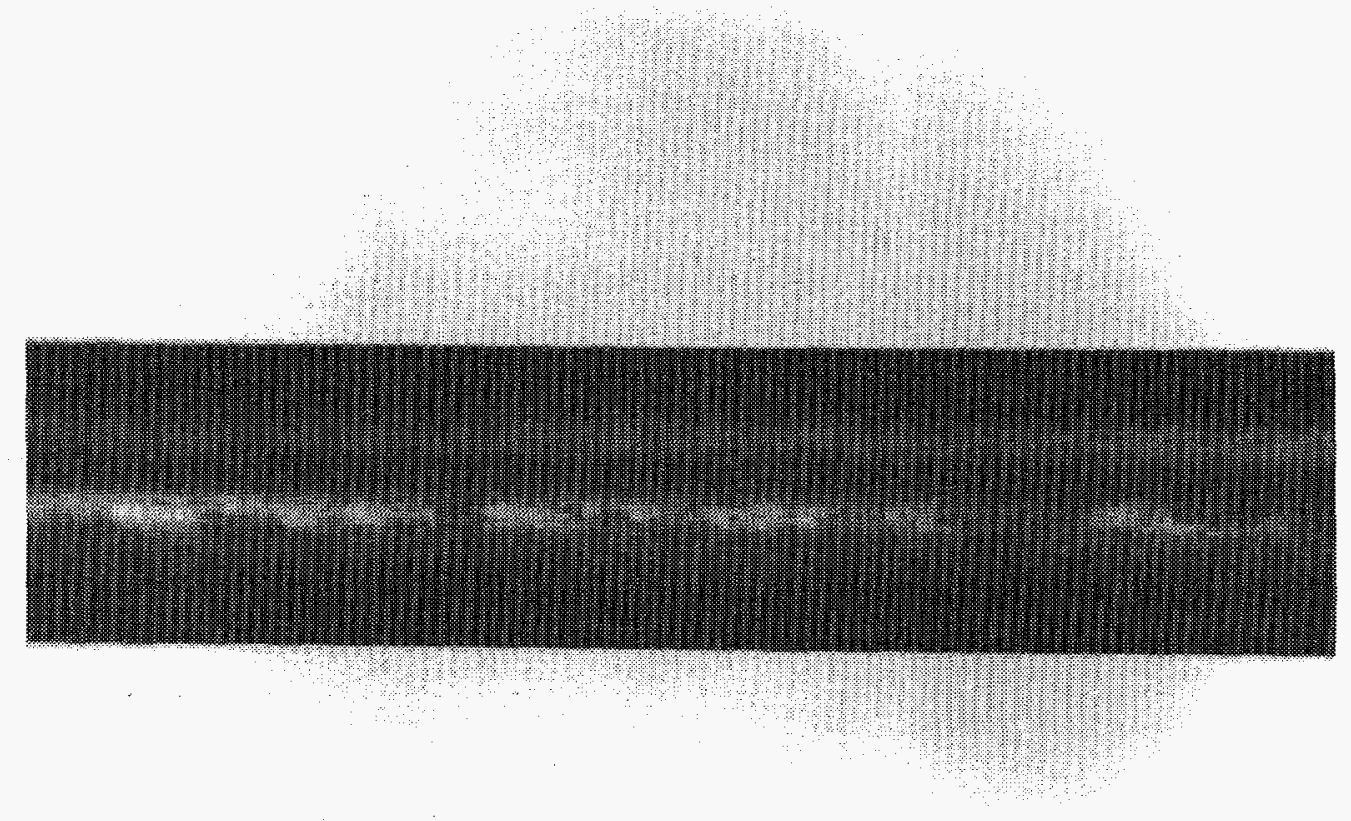

14G22. WT-12, Typical scratches (rotation composite), $172^{\circ}$, elev. $16.8 \mathrm{in}$.

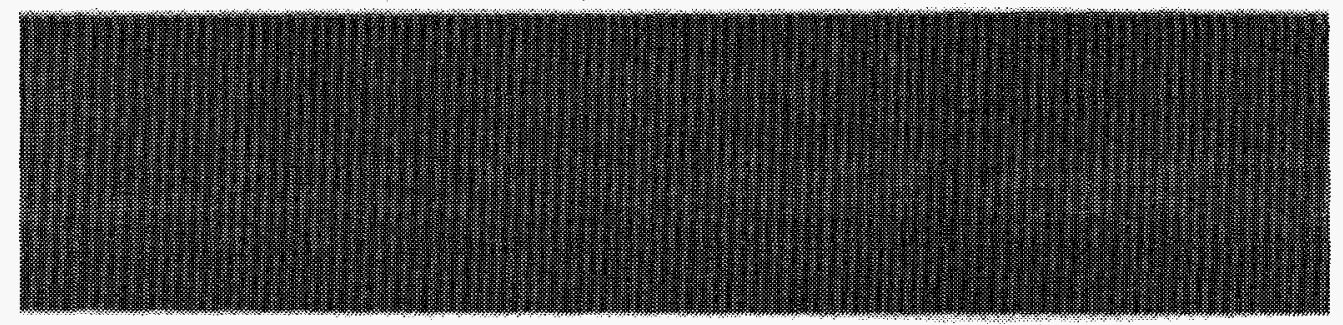

14G23. WT-12, Typical scratches (rotation composite), $232^{\circ}$, elev. $16.8 \mathrm{in}$. 
Appendix C

Neutron Radiography 


\section{Appendix C}

\section{Table of Contents}

\section{Loop-1}

\begin{tabular}{|c|c|c|}
\hline & Photograph & Orientation \\
\hline $96-148$ & $0^{\circ} \quad 0$ in. WL-26, 10, 01,03 & Thermal (ruler visible) \\
\hline $96-148$ & $0^{\circ} \quad 0$ in. WL-26, $10,01,03$ & Epithermal (ruler not visible) \\
\hline $96-147$ & $0^{\circ} 16$ in. $\mathrm{WL}-26,10,01,03$ & Thermal \\
\hline 96-147 & $0^{\circ} 16$ in. WL- $26,10,01,03$ & Epithermal \\
\hline $96-146$ & $0^{\circ} 32$ in. WL-26, 10, 01,03 & Thermal \\
\hline $96-146$ & $0^{\circ} 32$ in. WL-26, 10, 01,03 & Epithermal \\
\hline $96-145$ & $0^{\circ} 48$ in. WL-26, $10,01,03$ & Thermal \\
\hline $96-145$ & $0^{\circ} 48$ in. WL-26, 10, 01,03 & Epithermal \\
\hline $96-152$ & $90^{\circ} 0$ in. WL- $26,10,01,03$ & Thermal \\
\hline $96-152$ & $90^{\circ} \quad 0$ in. WL- $26,10,01,03$ & Epithermal \\
\hline $96-151$ & $90^{\circ} 16$ in. WL-26,10, 01,03 & Thermal \\
\hline $96-151$ & $90^{\circ} 16$ in. WL-26,10, 01, 03 & Epithermal \\
\hline $96-150$ & $90^{\circ} 32$ in. WL-26,10, 01, 03 & Thermal \\
\hline $96-150$ & $90^{\circ} 32$ in. WL-26,10, 01, 03 & Epithermal \\
\hline $96-149$ & $90^{\circ} 48$ in. WL-26,10, 01,03 & Thermal \\
\hline 96-149 & $90^{\circ} 48$ in. WL- $26,10,01,03$ & Epithermal \\
\hline $96-140$ & $0^{\circ} \quad 0$ in. WL-09, $18,22,27$ & Thermal \\
\hline $96-140$ & $0^{\circ} \quad 0$ in. WL- $09,18,22,27$ & Epithermal \\
\hline $96-139$ & $0^{\circ} 16$ in. WL-09, $18,22,27$ & Thermal \\
\hline $96-139$ & $0^{\circ} 16$ in. WL- $09,18,22,27$ & Epithermal \\
\hline $96-138$ & $0^{\circ} 32$ in. WL-09, $18,22,27$ & Thermal \\
\hline $96-138$ & $0^{\circ} 32$ in. WL-09, 18, 22, 27 & Epithermal \\
\hline $96-137$ & $0^{\circ} 48$ in. WL-09, 18, 22, 27 & Thermal \\
\hline $96-137$ & $0^{\circ} 48$ in. WL-09, $18,22,27$ & Epithermal \\
\hline $96-144$ & $90^{\circ} \quad 0$ in. WL-09, 18, 22, 27 & Thermal \\
\hline $96-144$ & $90^{\circ} \quad 0$ in. WL-09, $18,22,27$ & Epithermal \\
\hline $96-143$ & $90^{\circ} 16$ in. WL-09, $18,22,27$ & Thermal \\
\hline
\end{tabular}


Loop-1

\begin{tabular}{|c|c|c|}
\hline & Photograph & Orientation \\
\hline $96-14390^{\circ}$ & 16 in. WL-09, $18,22,27$ & Epithermal \\
\hline $96-14290^{\circ}$ & 32 in. WL-09, $18,22,27$ & Thermal \\
\hline $96-14290^{\circ}$ & 32 in. WL-09, 18, 22, 27 & Epithermal \\
\hline $96-14190^{\circ}$ & 48 in. WL-09, 18, 22, 27 & Thermal \\
\hline $96-14190^{\circ}$ & 48 in. WL-09, $18,22,27$ & Epithermal \\
\hline \multicolumn{3}{|c|}{ S1 and B1 Rods } \\
\hline & Photograph & Orientation \\
\hline $96-1710^{\circ}$ & 0 in. $B 1, S 1$ & Thermal \\
\hline $96-1710^{\circ}$ & 0 in. $B 1, S 1$ & Epithermal \\
\hline $96-170 \quad 0^{\circ}$ & 16 in. $B 1, S 1$ & Thermal \\
\hline $96-1700^{\circ}$ & 16 in. $B 1, S 1$ & Epithermal \\
\hline $96-1690^{\circ}$ & 32 in. $B 1, S 1$ & Thermal \\
\hline $96-1690^{\circ}$ & 32 in. $B 1, S 1$ & Epithermal \\
\hline $96-1680^{\circ}$ & 48 in. $\mathrm{B} 1, \mathrm{~S} 1$ & Thermal \\
\hline $96-1680^{\circ}$ & 48 in. $B 1, S 1$ & Epithermal \\
\hline $96-1750^{\circ}$ & 0 in. $B 1, S 1$ & Thermal \\
\hline $96-1750^{\circ}$ & 0 in. $\mathrm{B} 1, \mathrm{~S} 1$ & Epithermal \\
\hline $96-1740^{\circ}$ & 16 in. $B 1, S 1$ & Thermal \\
\hline $96-1740^{\circ}$ & 16 in. $B 1, S 1$ & Epithermal \\
\hline $96-1730^{\circ}$ & 32 in. $B 1, S 1$ & Thermal \\
\hline $96-1730^{\circ}$ & 32 in. $B 1, S 1$ & Epithermal \\
\hline $96-1720^{\circ}$ & 48 in. $B 1, S 1$ & Thermal \\
\hline $96-1720^{\circ}$ & 48 in. $\mathrm{B} 1, \mathrm{~S} 1$ & Epithermal \\
\hline
\end{tabular}

To set up appendix pages, scan at a 300 DPI resolution; put on $11 \times 17$-in. paper; set top and side margins at 1 inch and bottom margin at $1 / 2$ inch; center the page number at the bottom of each page starting with C-3; place two photos on each page, thermal above and epithermal below, keeping like numbered pairs together (i.e., 96-172 $0^{\circ} 48$ in.); separate pictures by 1 inch; and add the applicable caption at the bottom of each page (taken from the above list that includes both pictures, for example: 96-172 $0^{\circ} 48$ in. B1, S1 Thermal (above) and Epithermal (below). The scanned positives cannot be oriented in a right-reading mode since they are not films. 

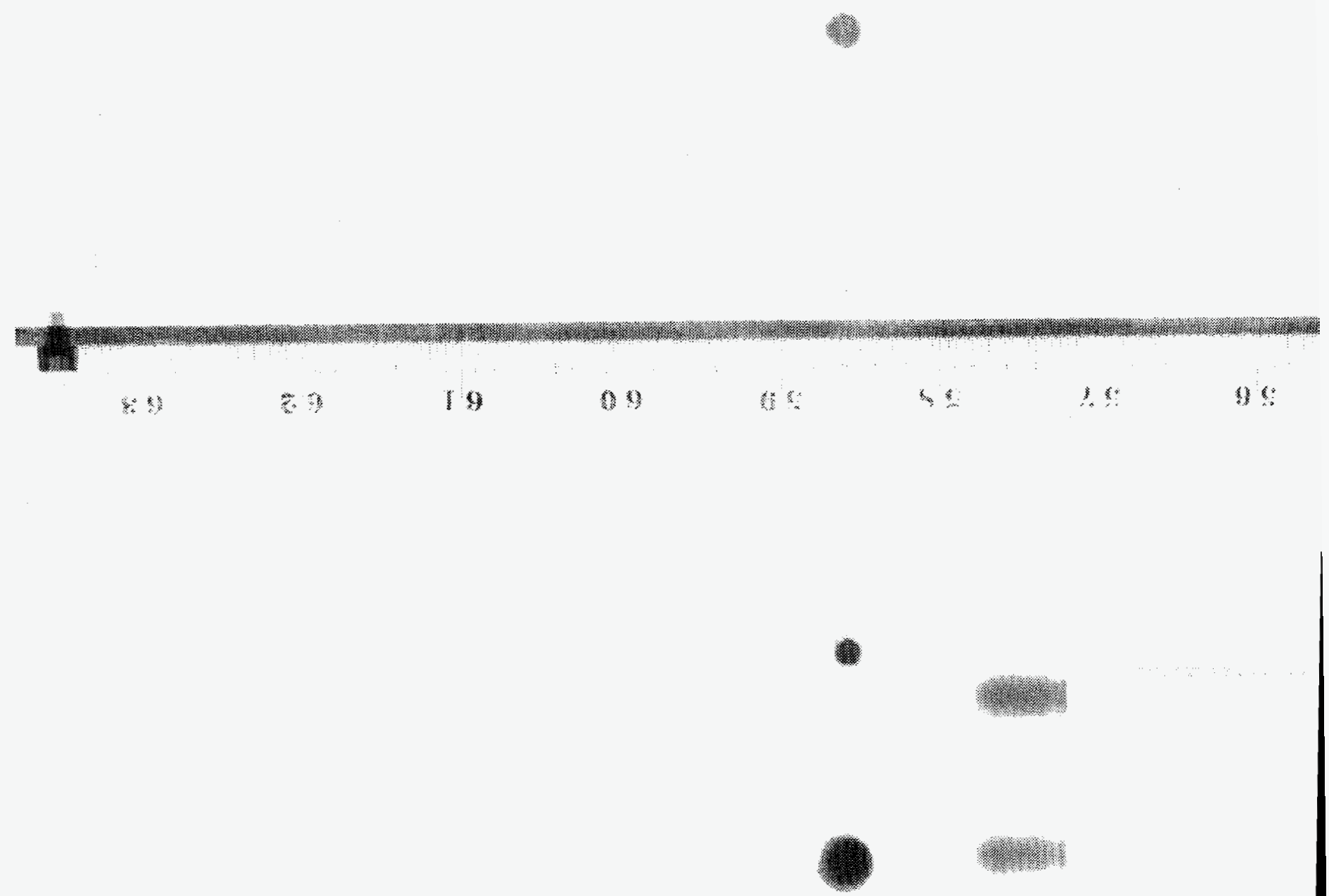

\%:

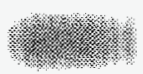

3.

96-148 $0^{\circ} \quad 0$ in. WL-26, 10, 01, 03 Thermal (above) and Epithermal (below) 

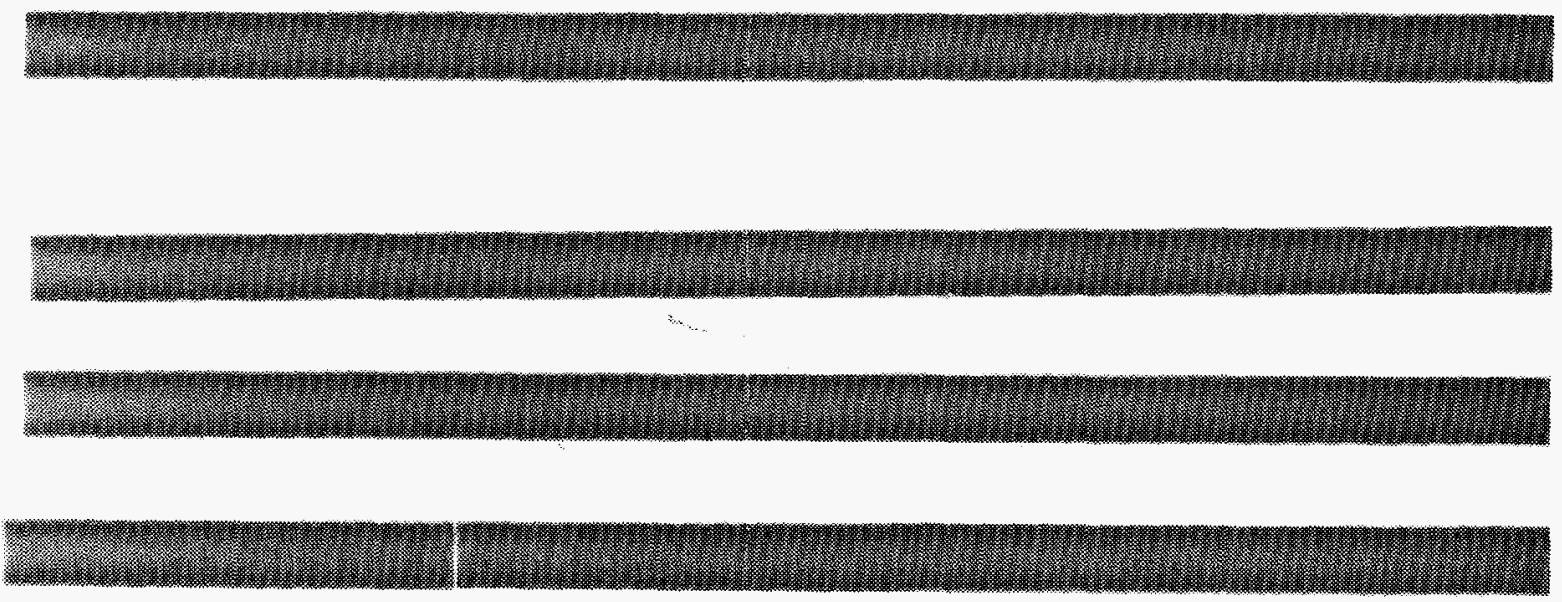

26.

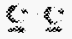
s:
管:
(...
15
n :
$\sqrt{3}$
i

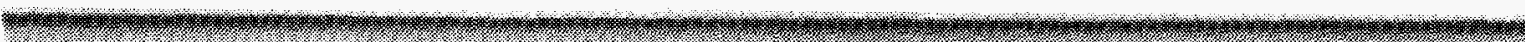

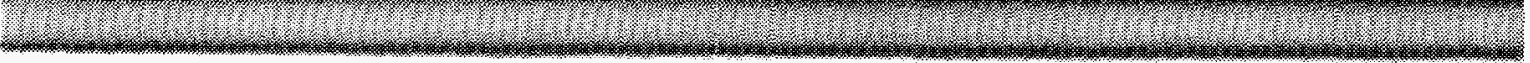

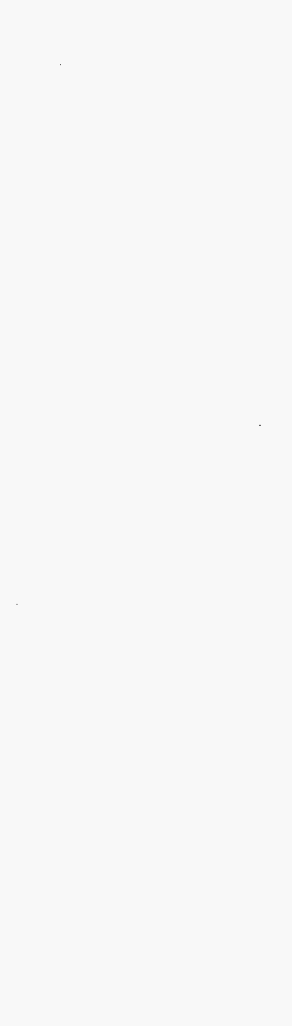

\%:-

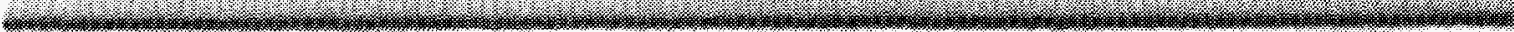

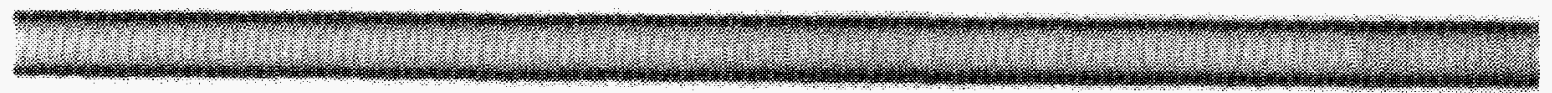

.

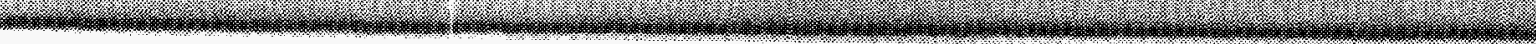



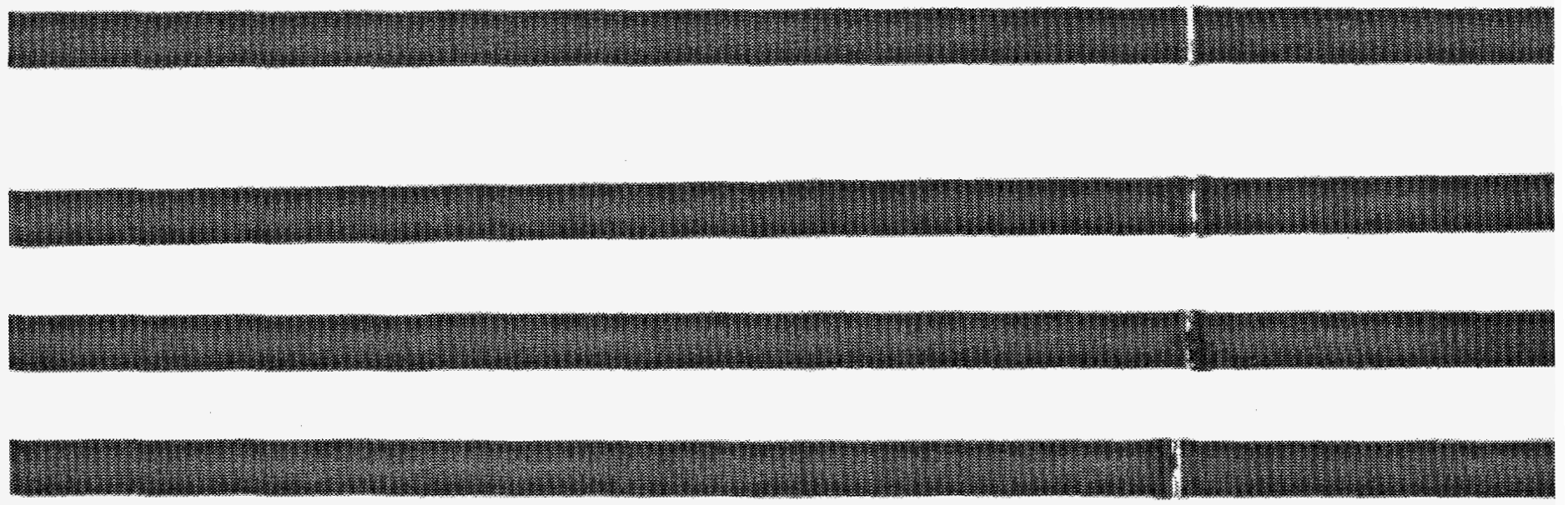

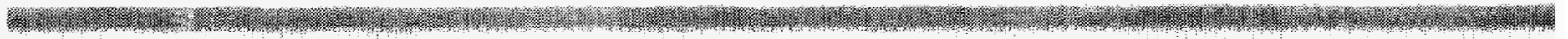
\&

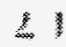
3
1.
\%
5.
6

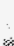

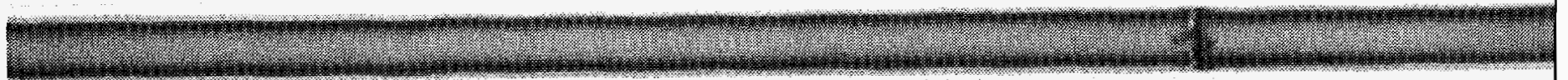

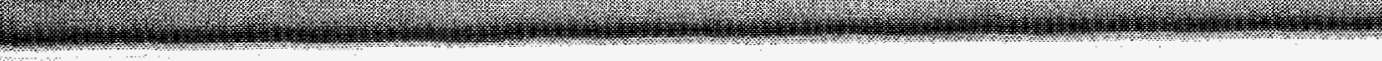

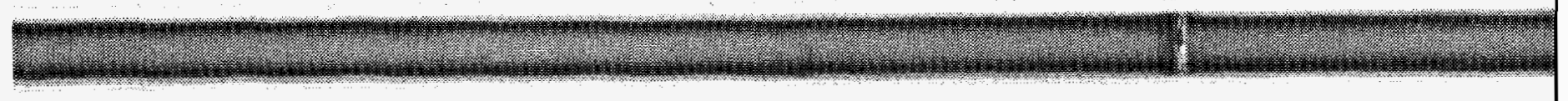

96-147 $0^{\circ} 16$ in. WL-26, 10, 01, 03 Thermal (above) and Epithermal (below) 

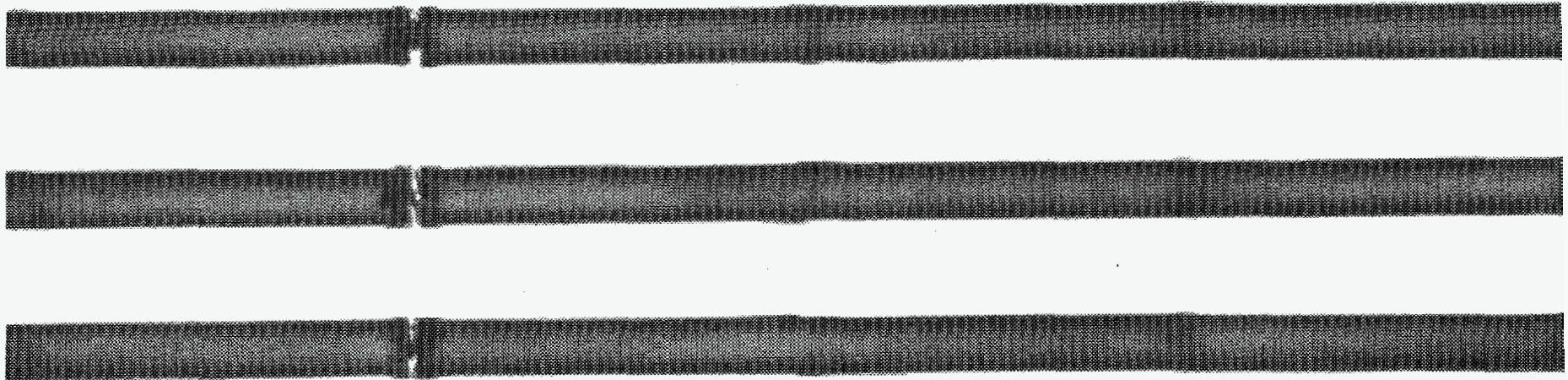

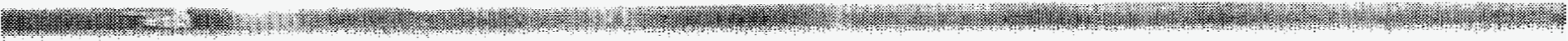
\&:
1:
(1)
譆
s
2
0
管
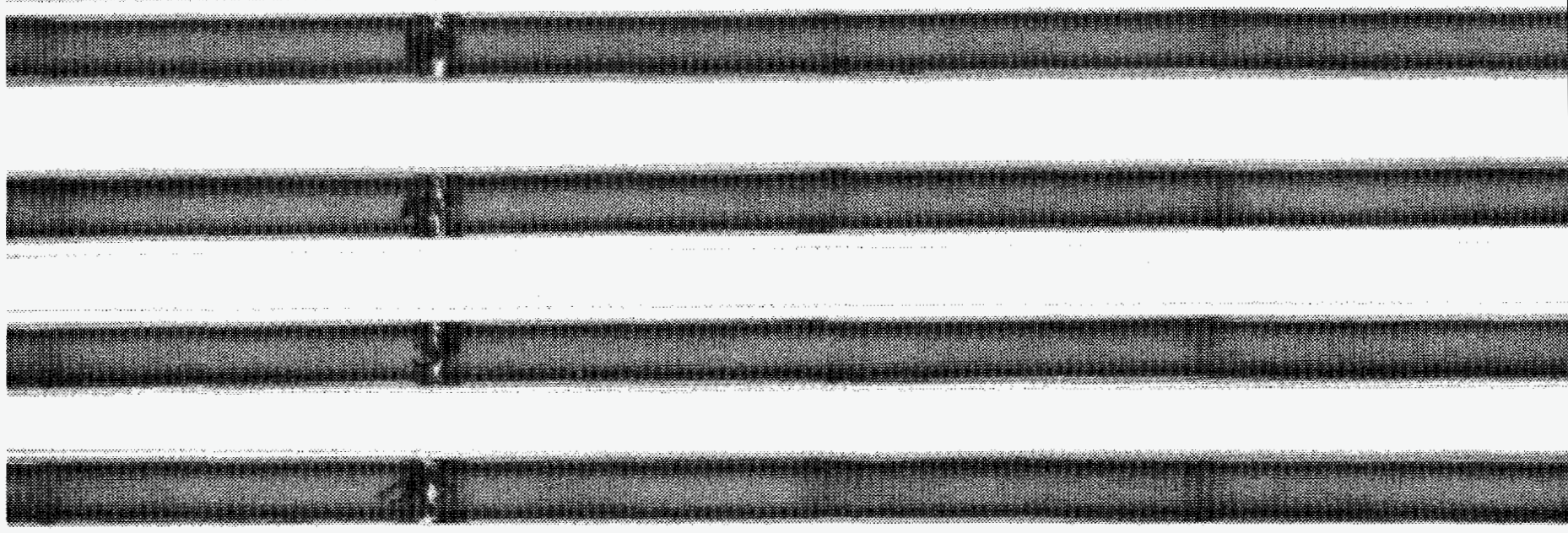

96-146 $0^{\circ} 32$ in. WL-26, 10, 01, 03 Thermal (above) and Epithermal (below) 

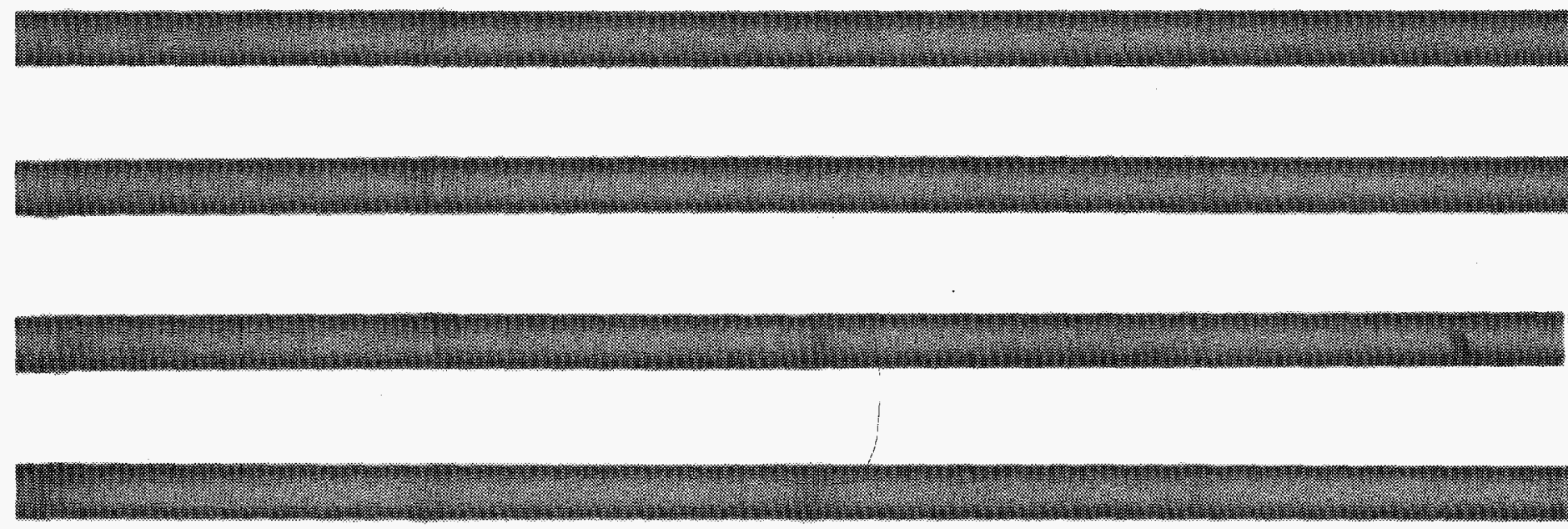

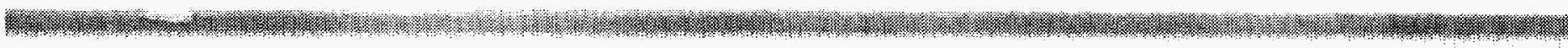
61
(*)
11
(1)
T.
1
0.
$\$$
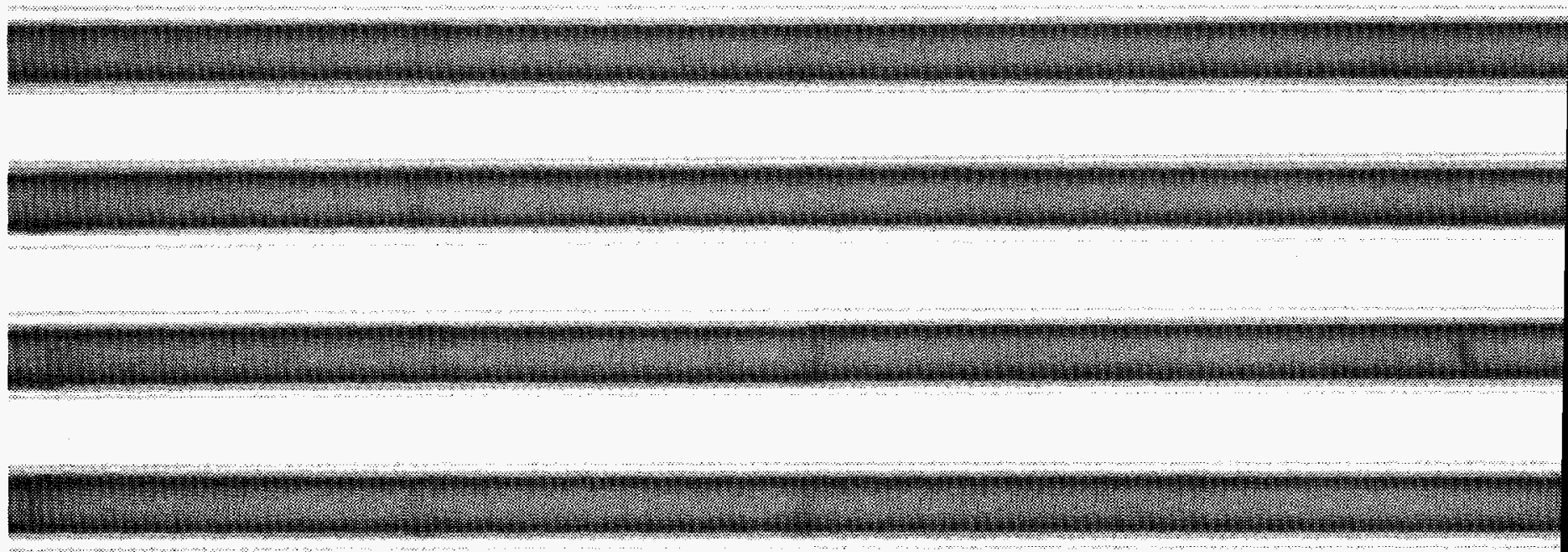

96-145 $0^{\circ} 48$ in. WL-26, 10, 01, 03 Thermal (above) and Epithermal (below) 

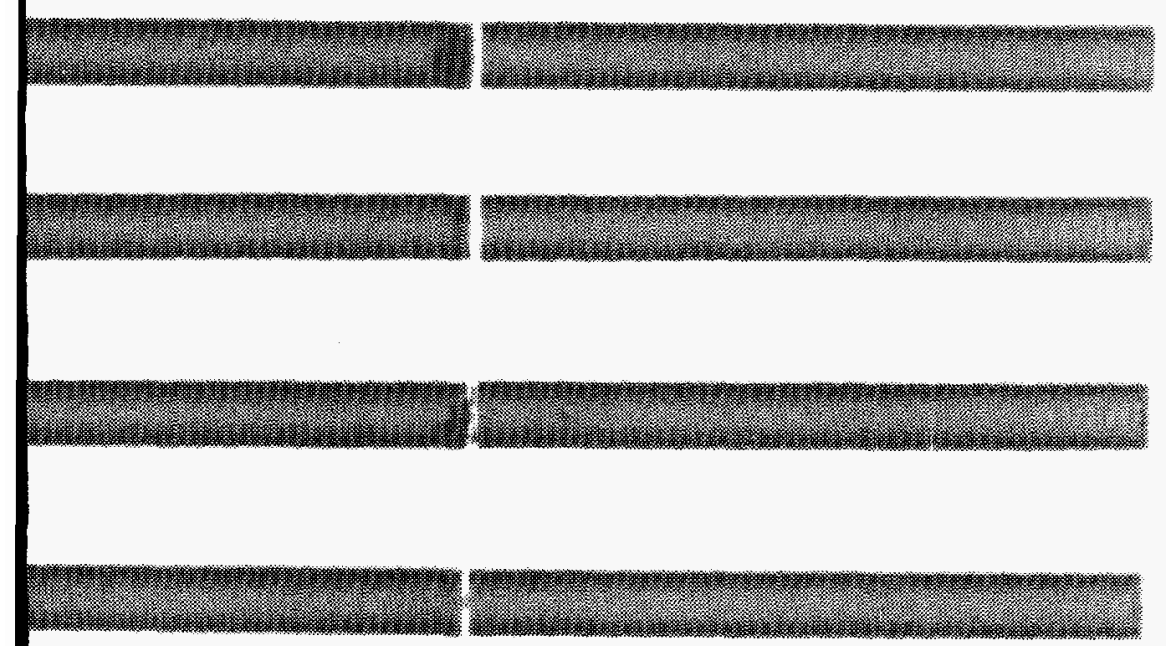

F.

8

24

s.:

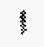

t:

?

s.

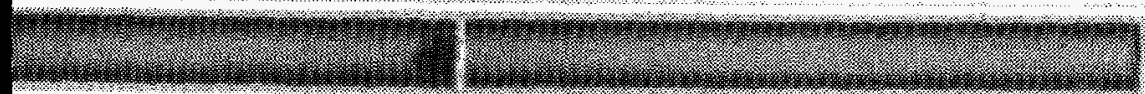

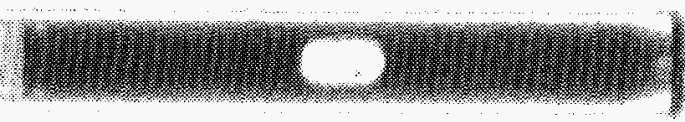

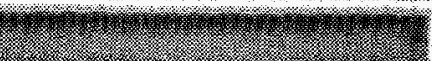

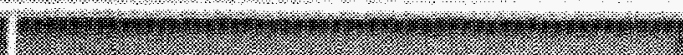

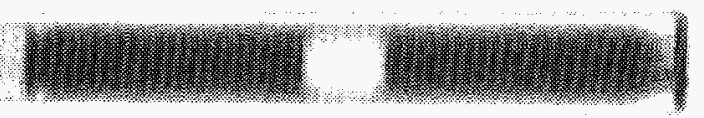

.

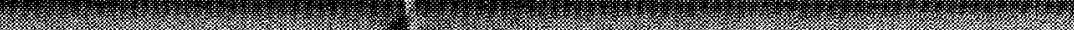

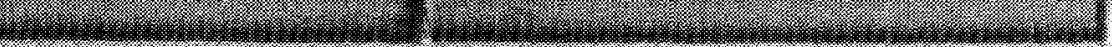

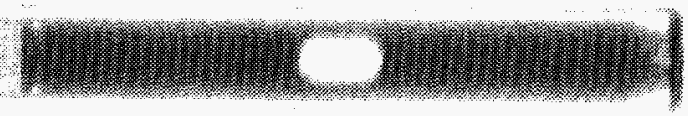

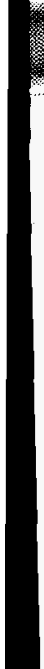

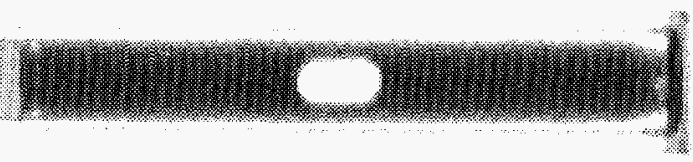

3) 

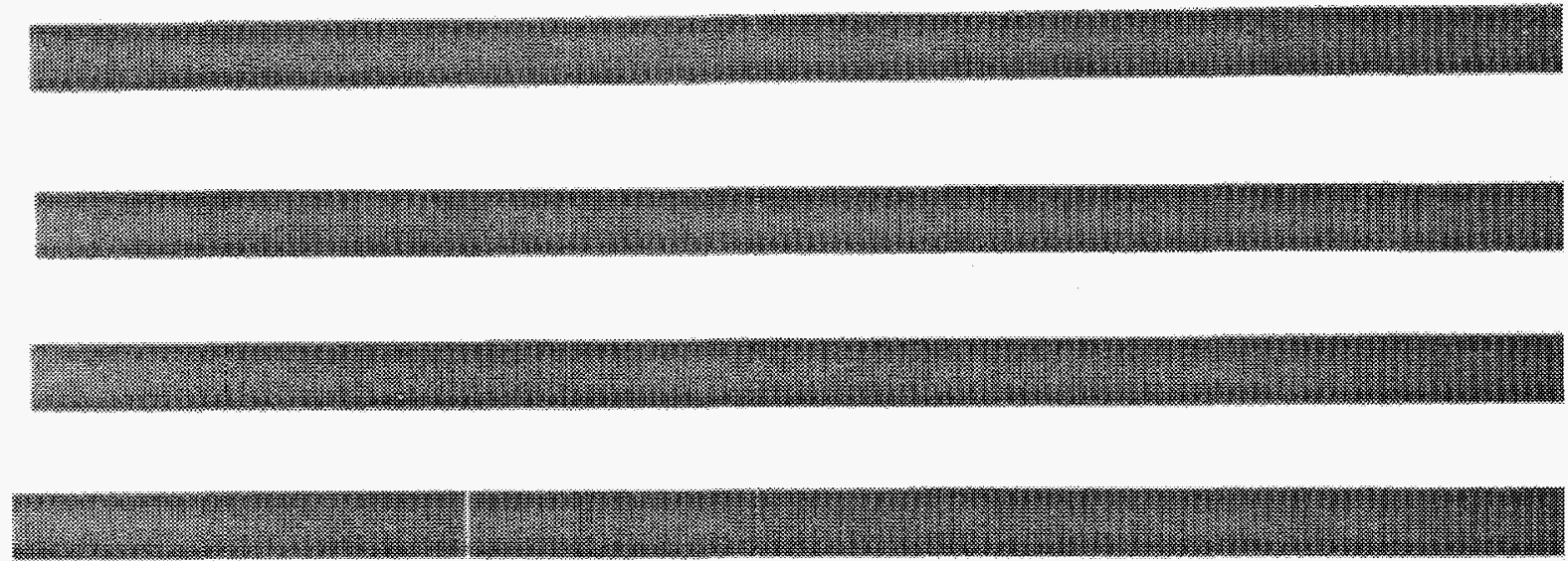

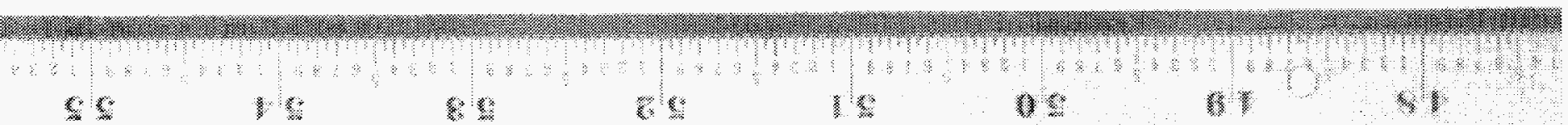

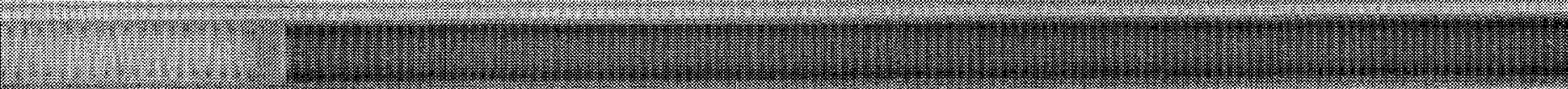
(3)

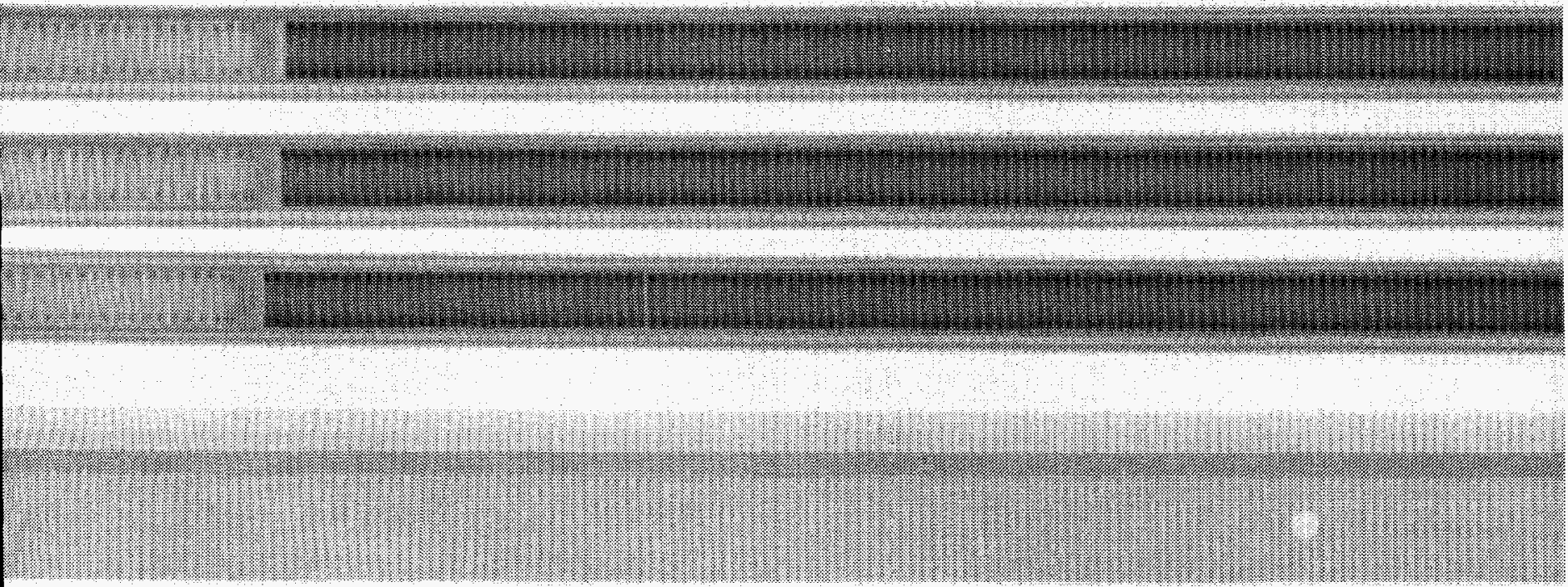



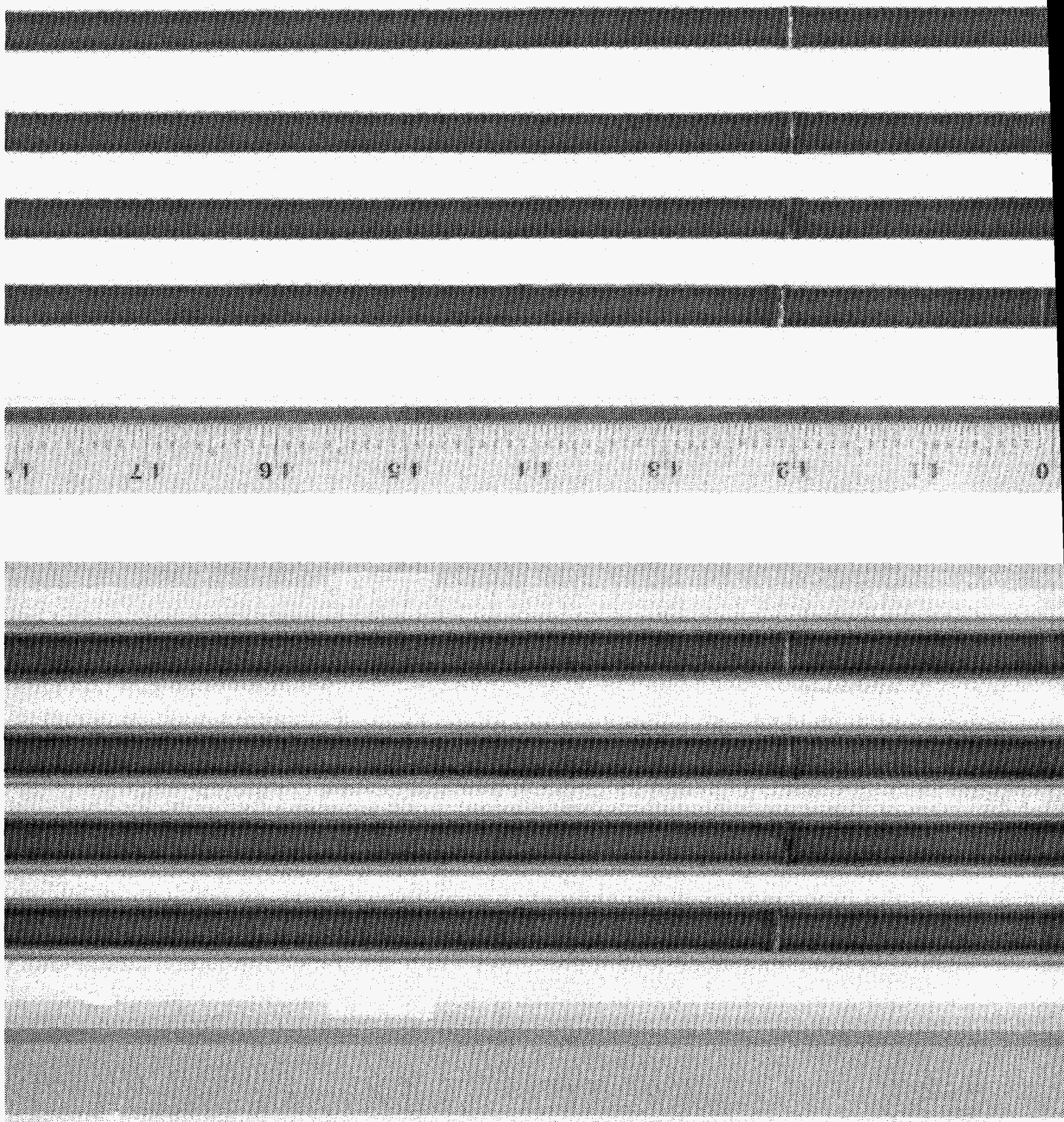

96-151 $90^{\circ} 16$ in. WL-26, 10, 01, 03 Thermal (above) and Epithermal (below) 
( (1) - (
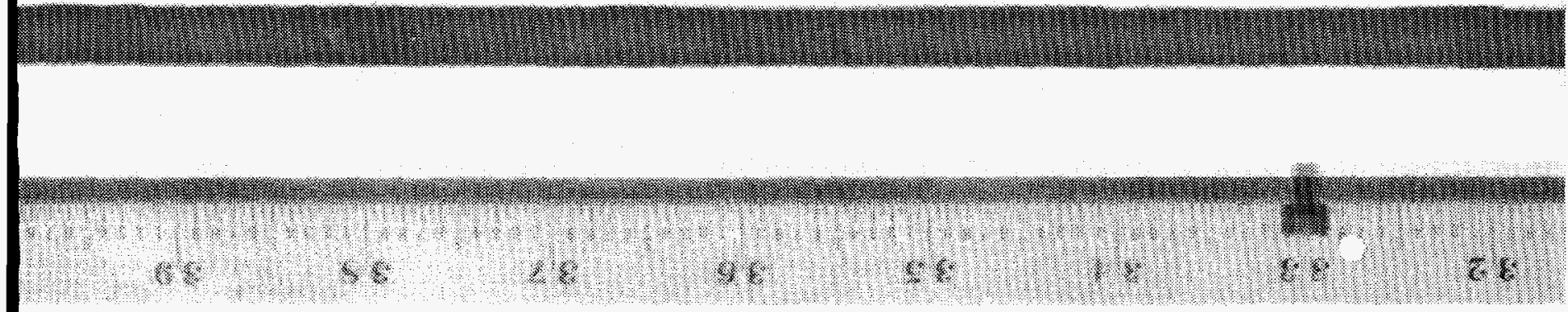

Y

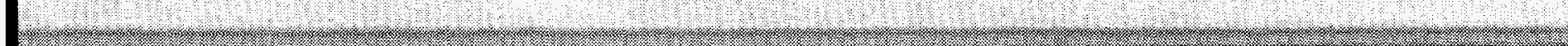
(

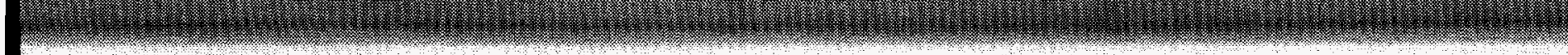
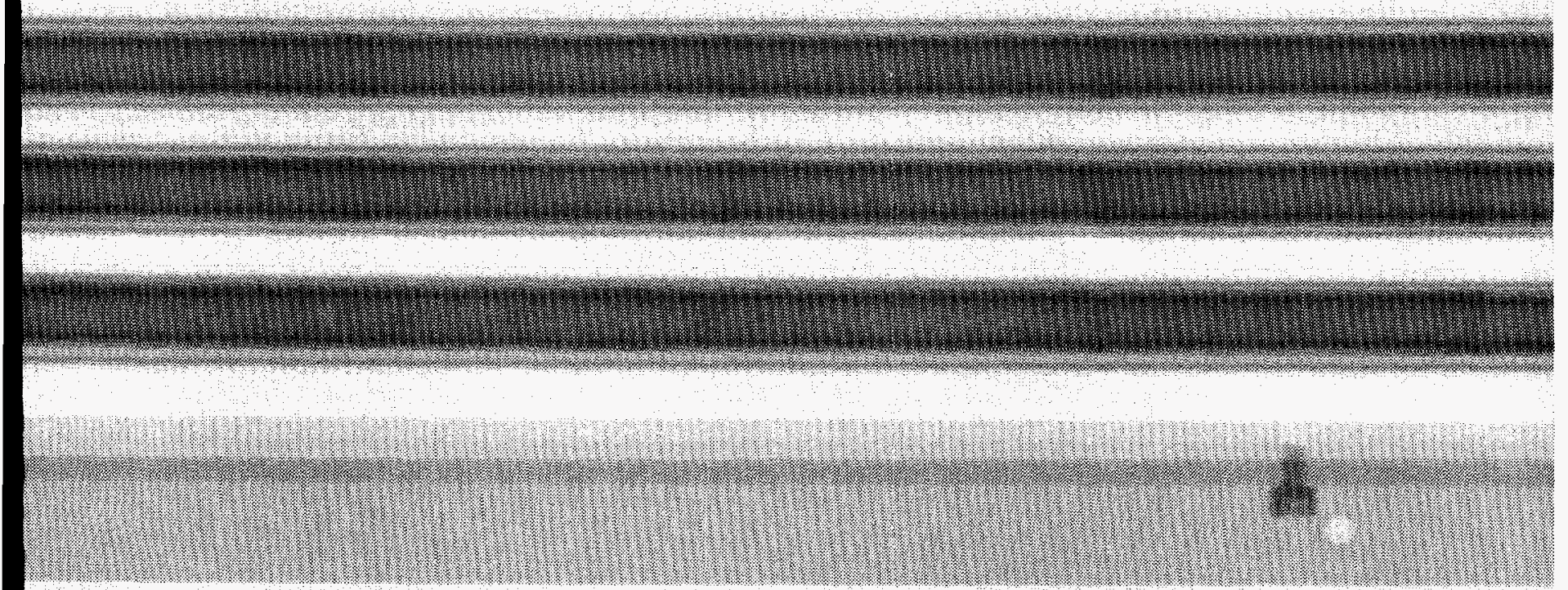

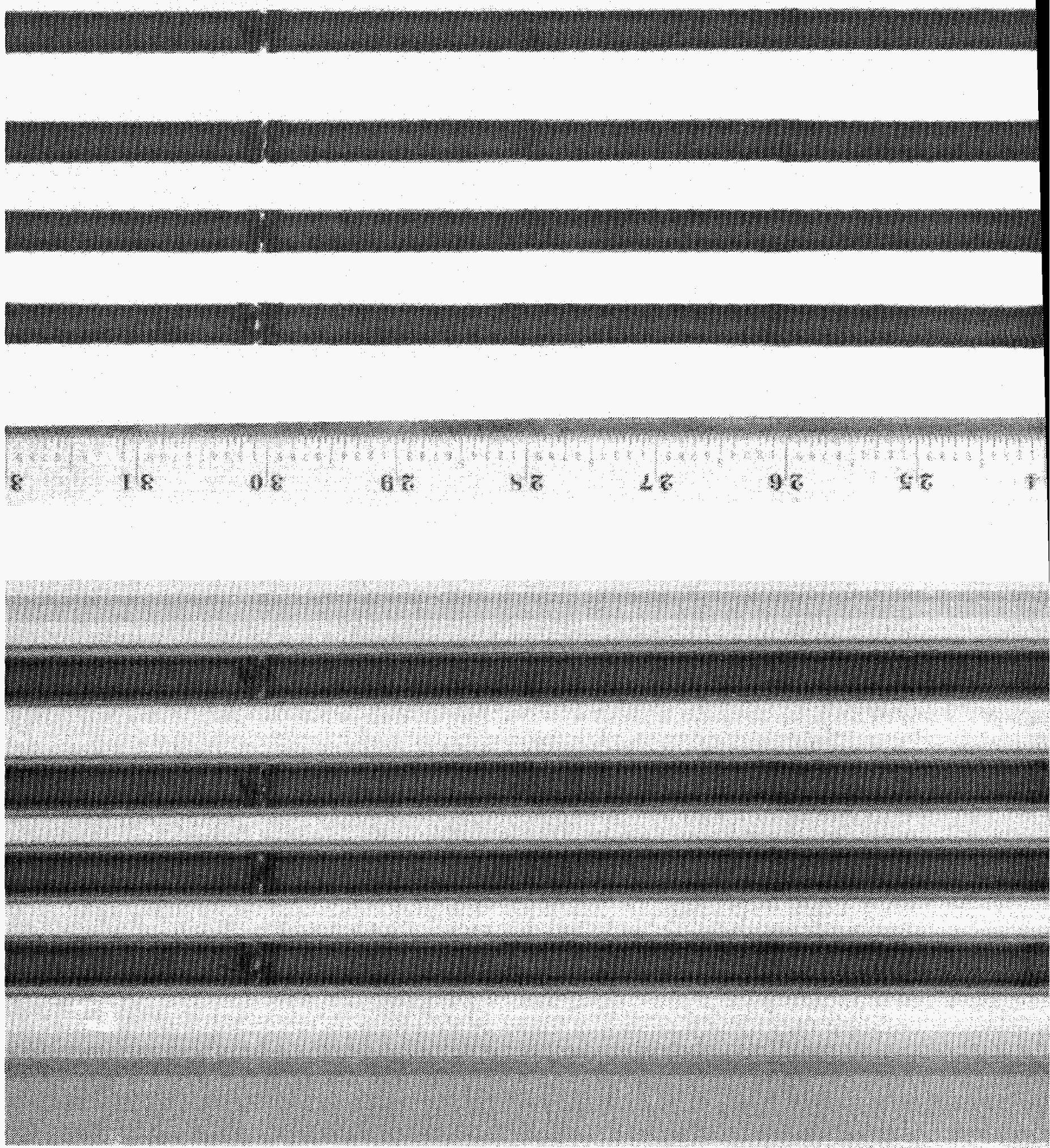

96-150 $90^{\circ} 32$ in. WL-26, 10, 01, 03 Thermal (above) and Epithermal (below) 


\section{अ.}

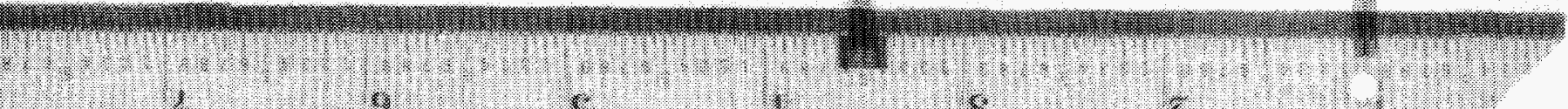

(1)

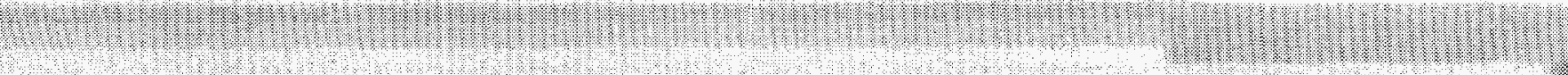



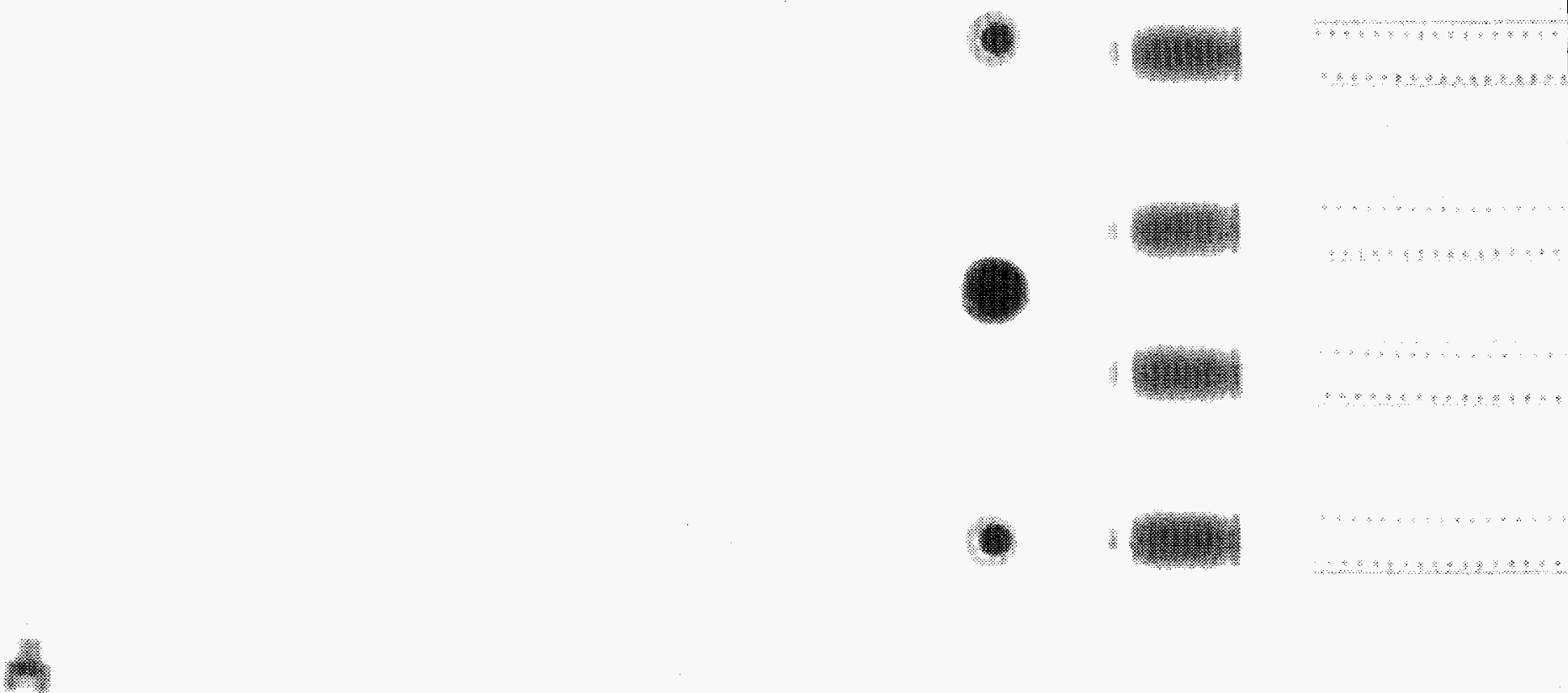

96-140 $0^{\circ} \quad 0$ in. WL-09, 18, 22, 27 Thermal (above) and Epithermal (below) 

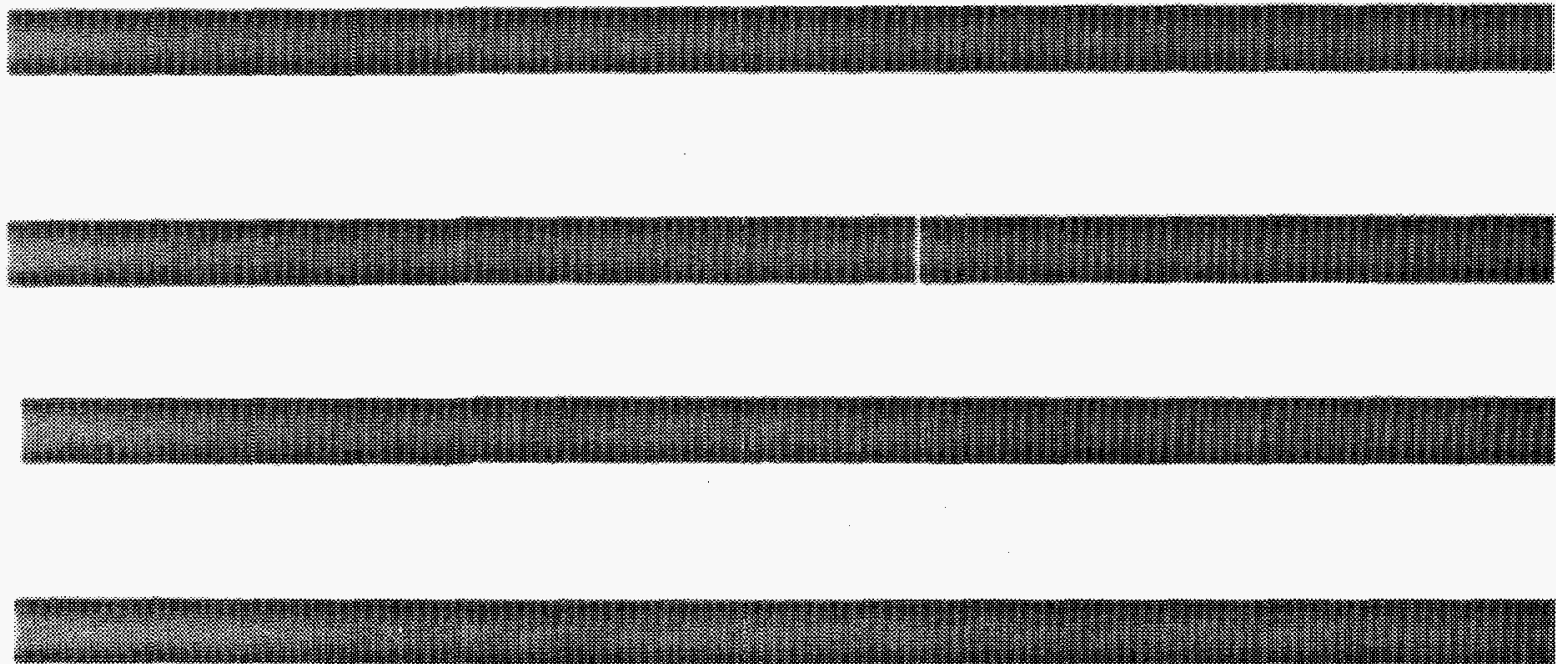

W.

SO
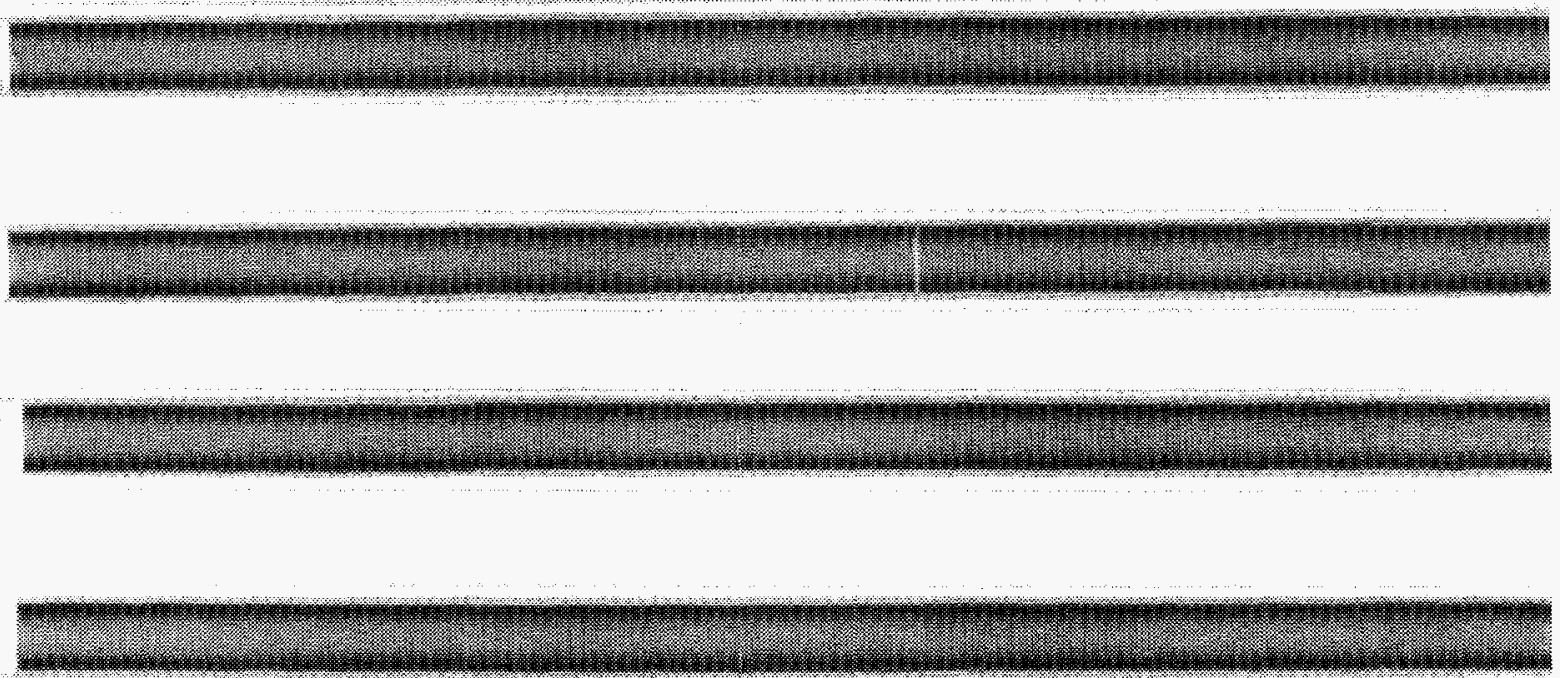

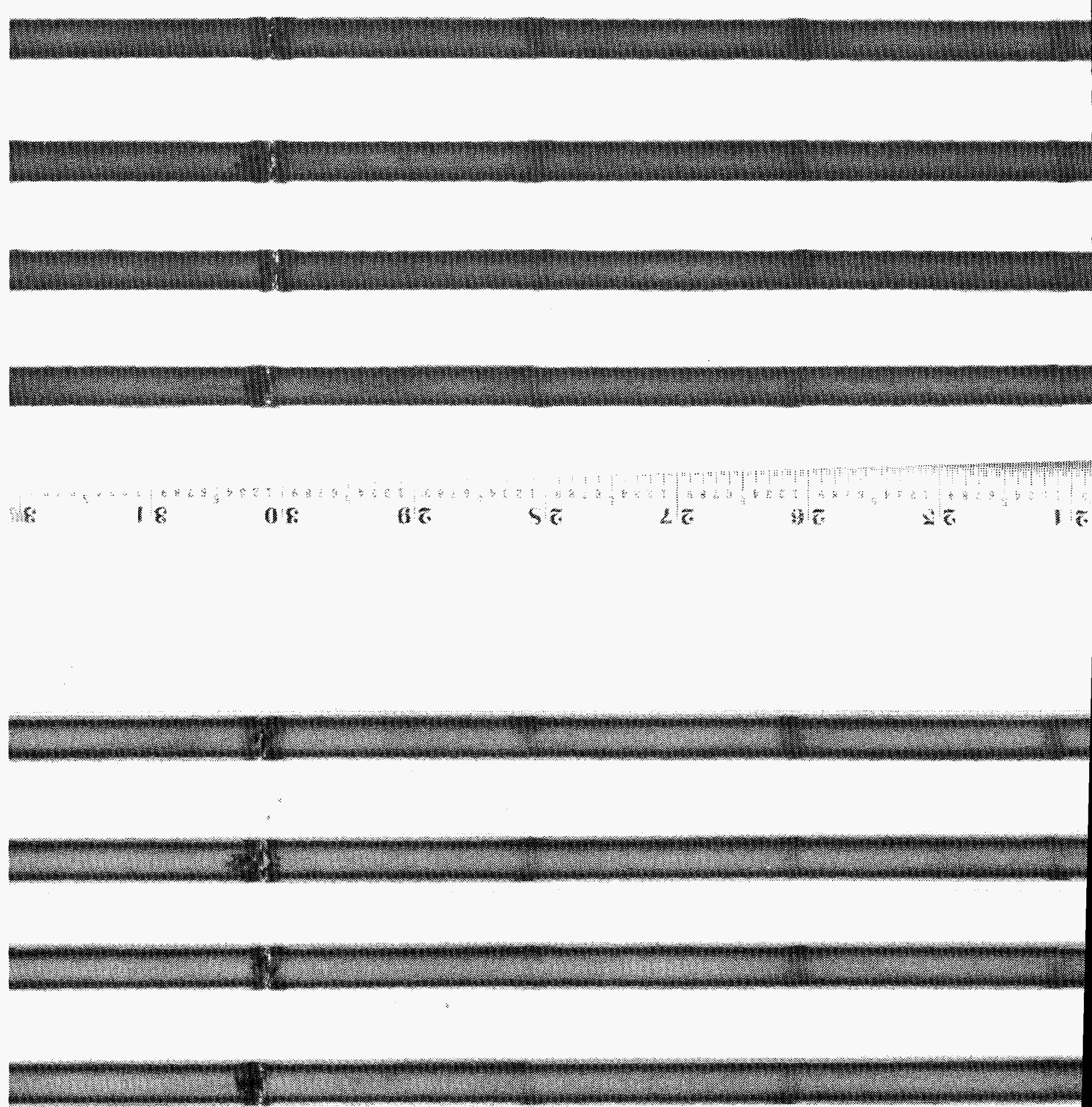

96-138 $0^{\circ} 32$ in. WL-09, 18, 22, 27 Thermal (above) and Epithermal (below) 


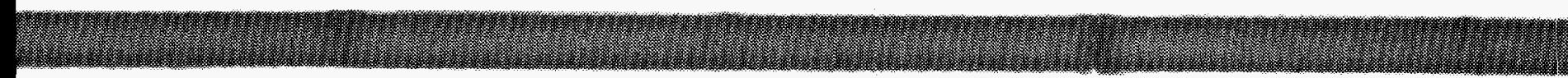
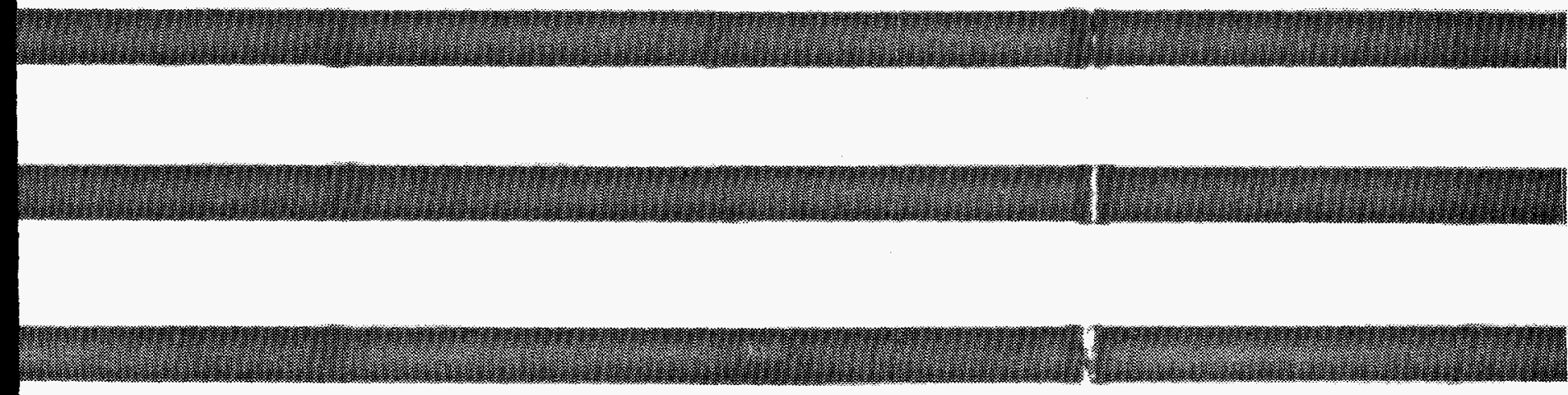

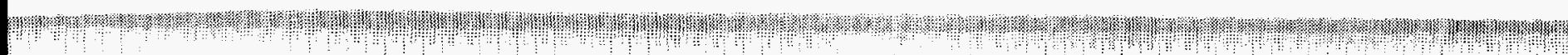

\&

(x)

1

a

(1)

b.

21

9

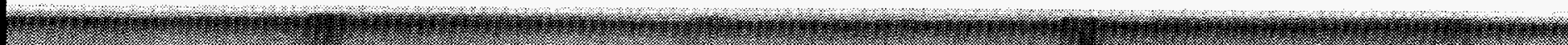

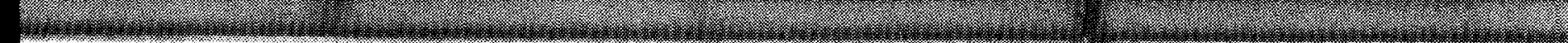

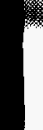

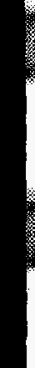

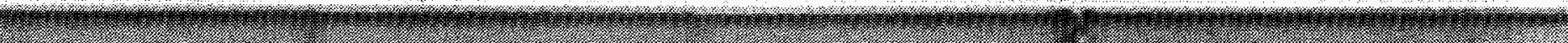

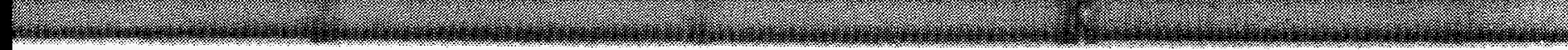

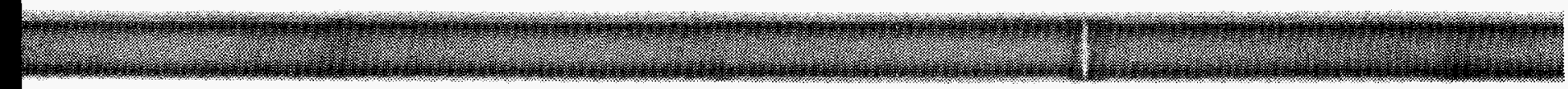

of 

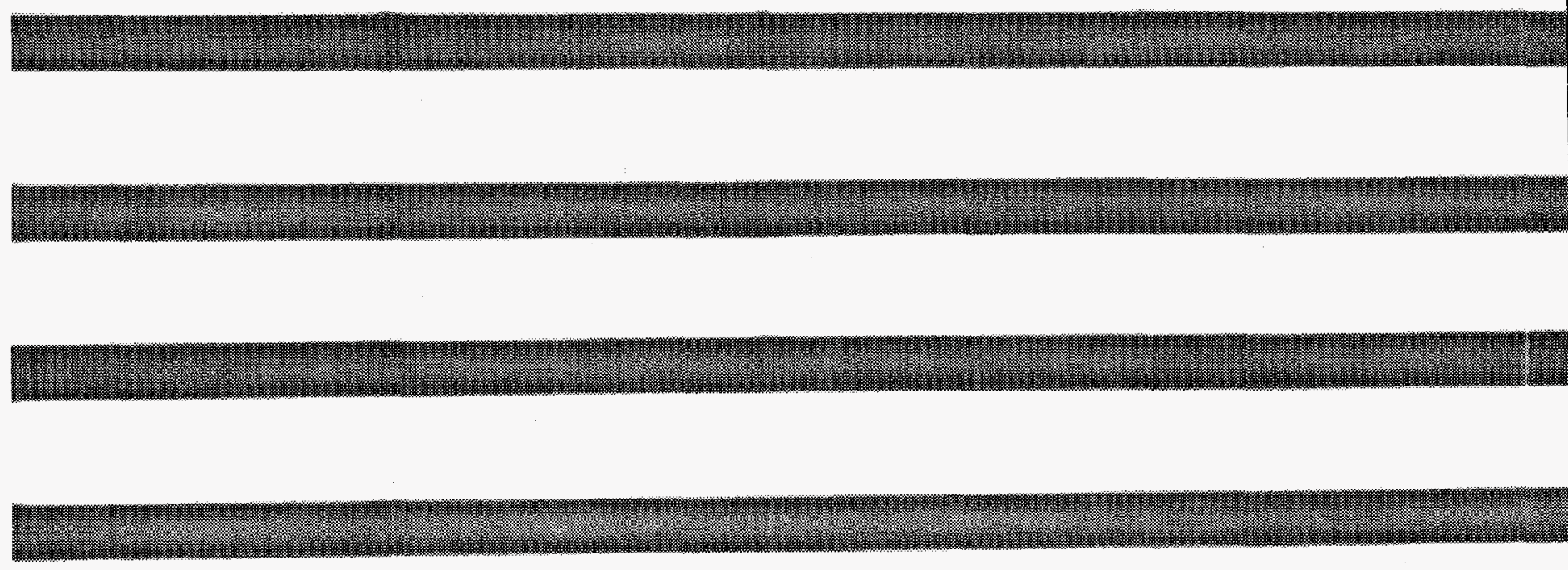

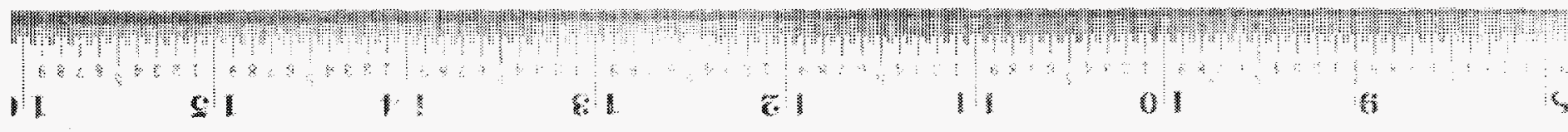
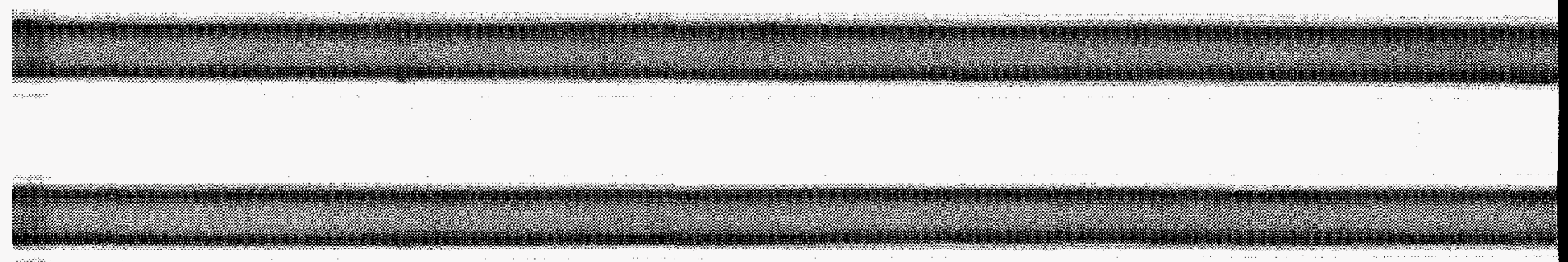
(2).

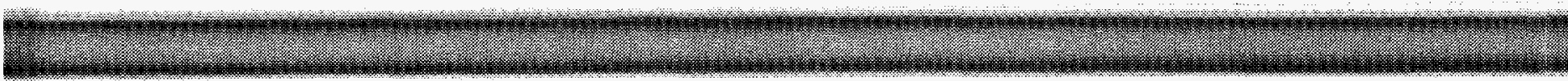

96-137 $0^{\circ} 48$ in. WL-09, 18, 22, 27 Thermal (above) and Epithermal (below) 

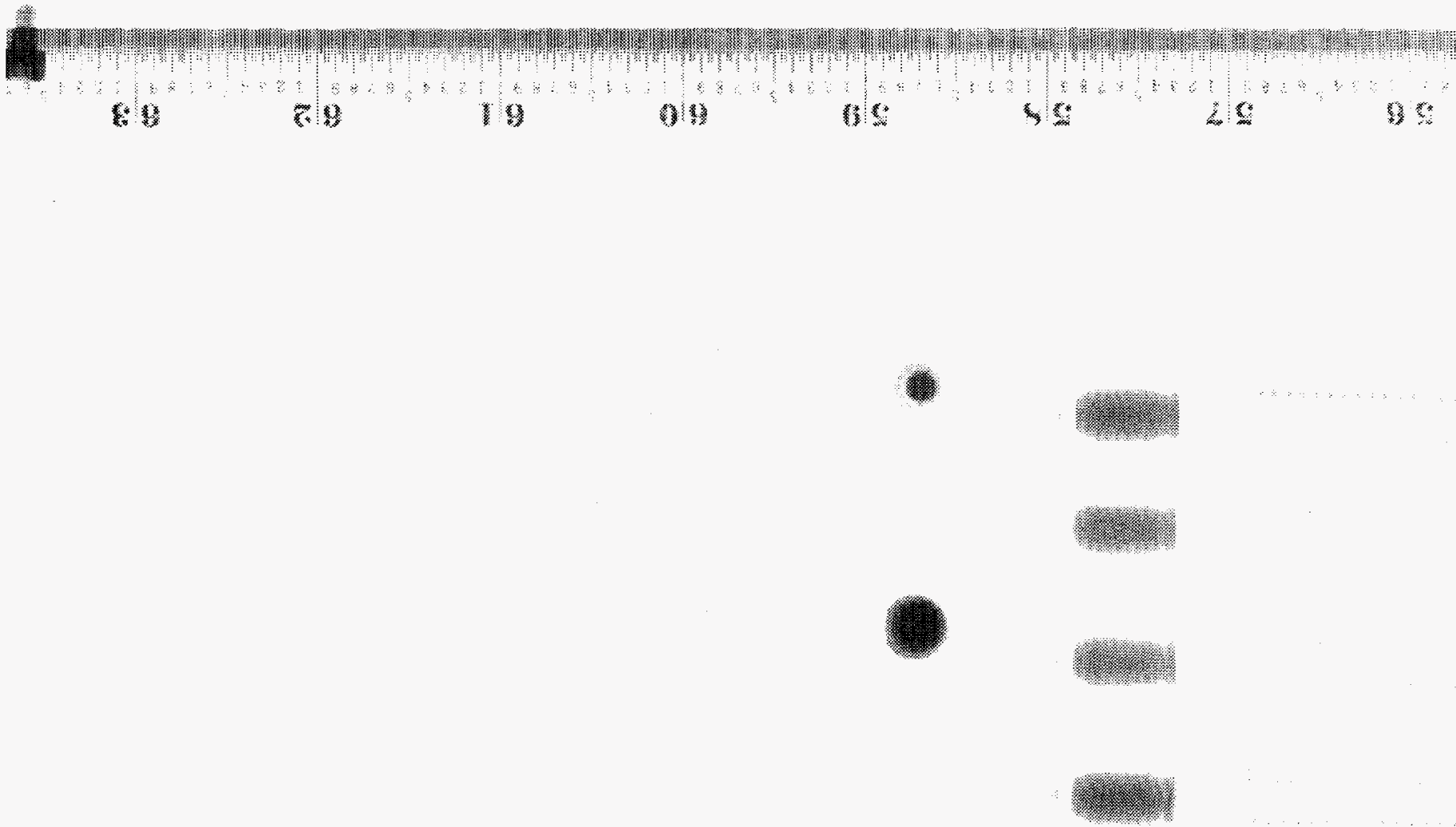

)

96-144 $90^{\circ} 0$ in. WL-09, 18, 22, 27 Thermal (above) and Epithermal (below) 

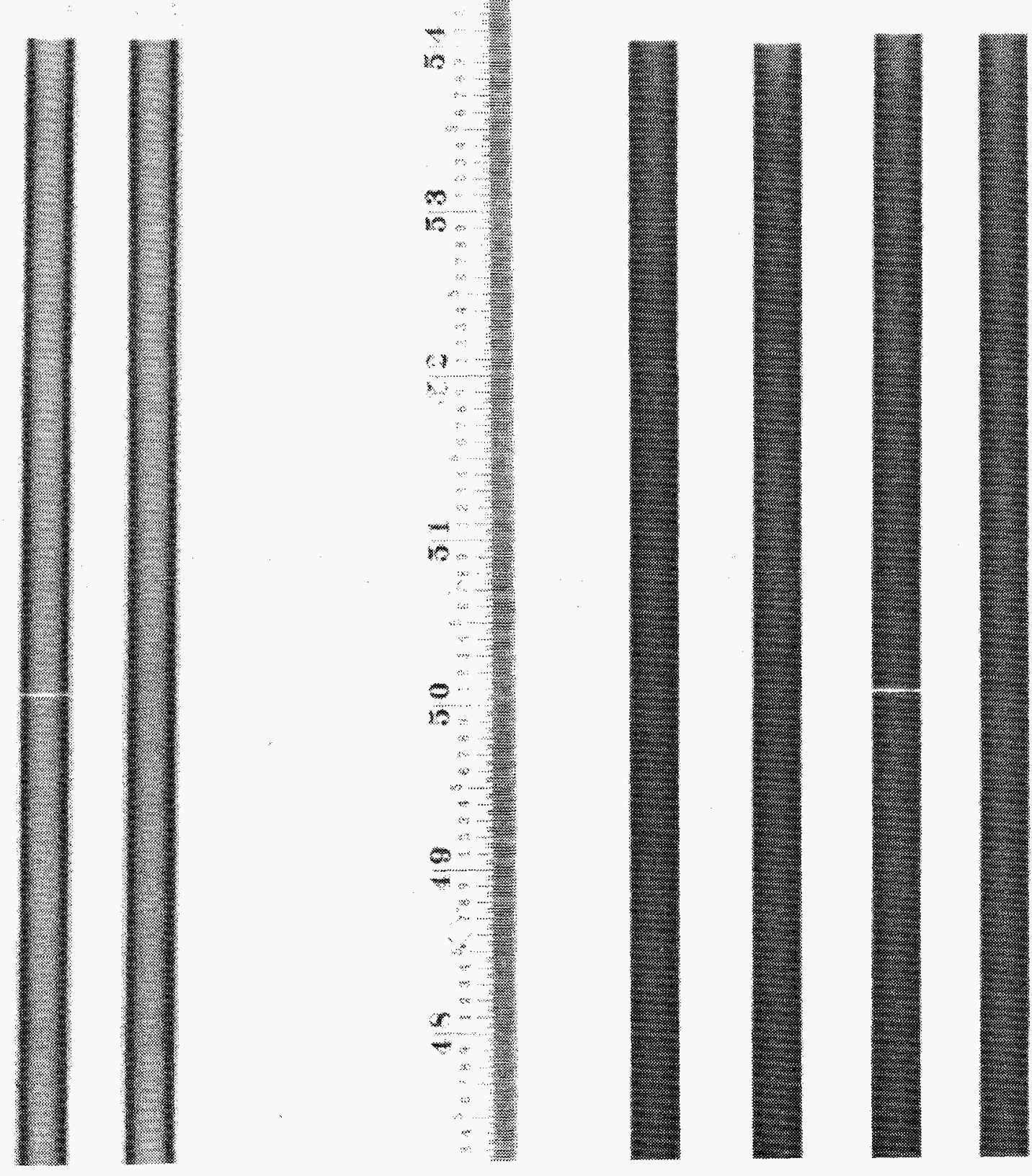

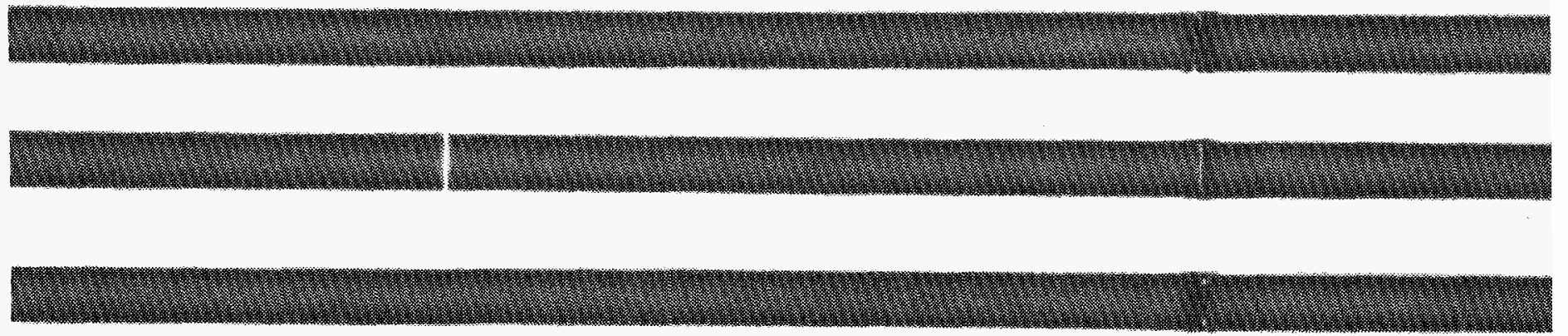

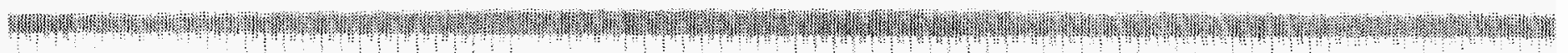
\%
2
.
o
(1)
(5)
;
(

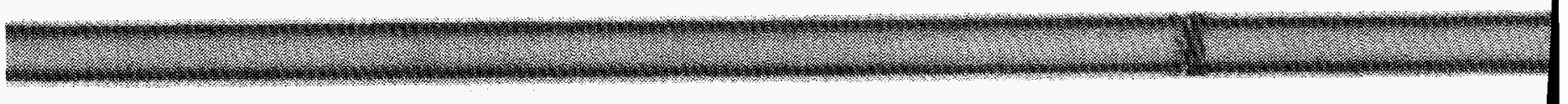

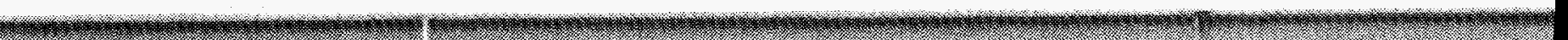

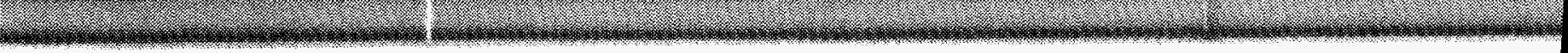
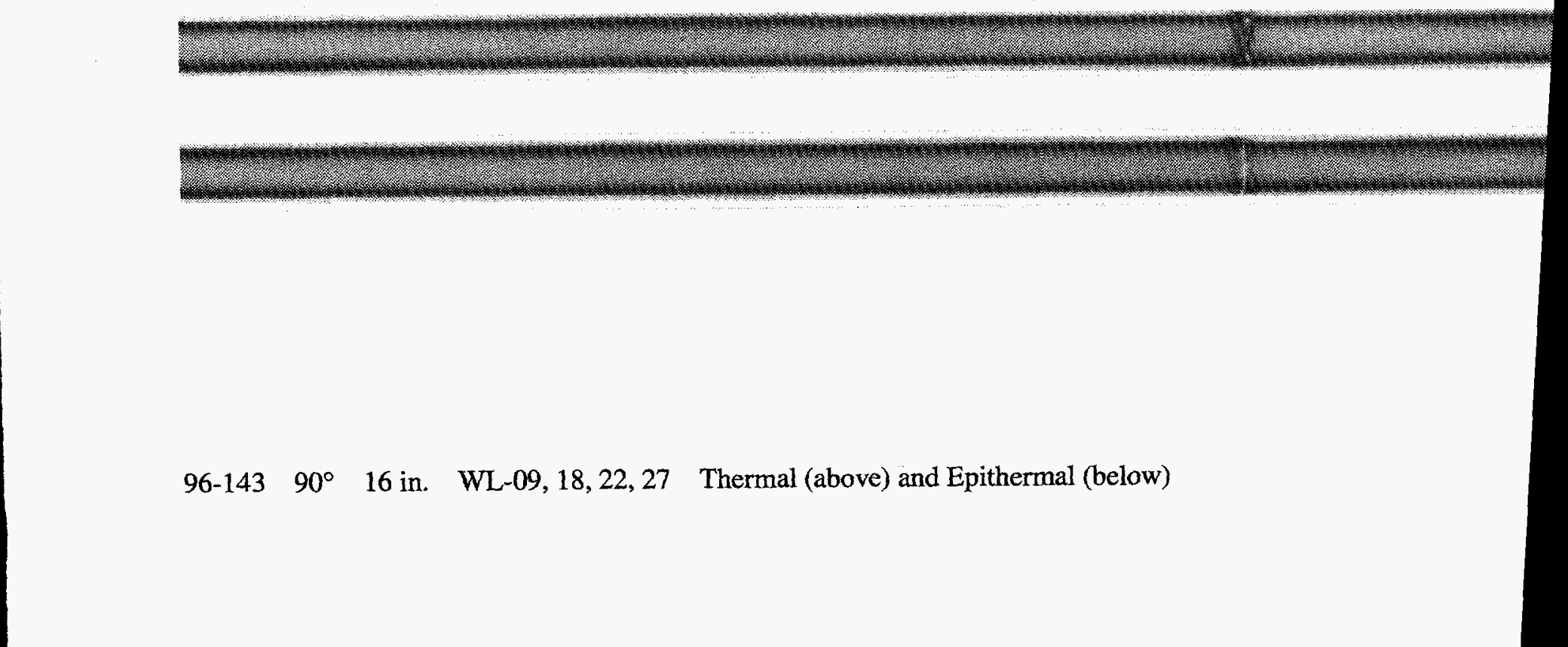

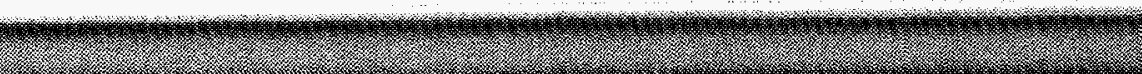

96-143 $90^{\circ} \quad 16$ in. WL-09, 18, 22, 27 Thermal (above) and Epithermal (below) 

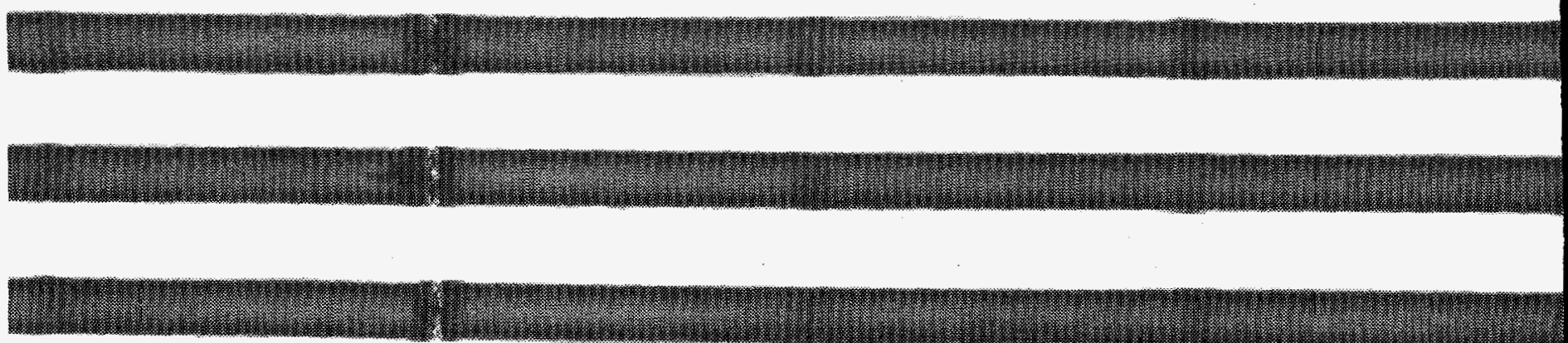

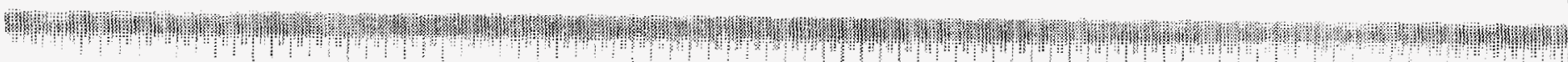

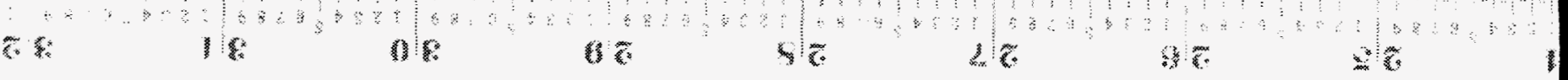

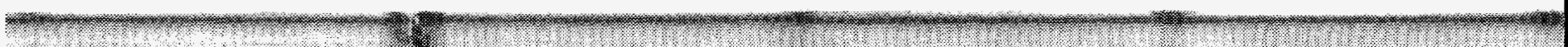

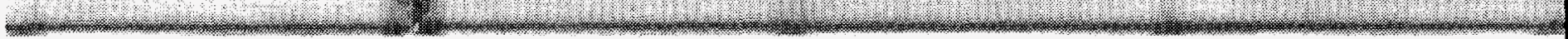

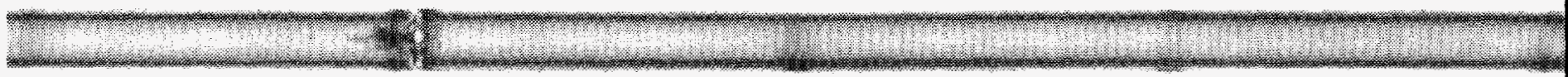

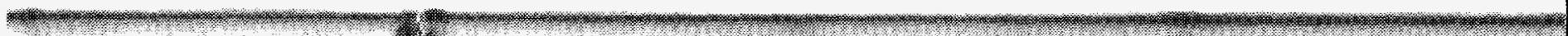

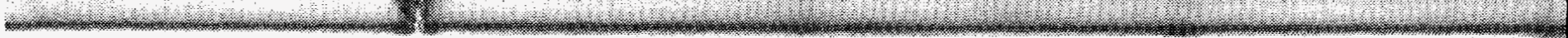

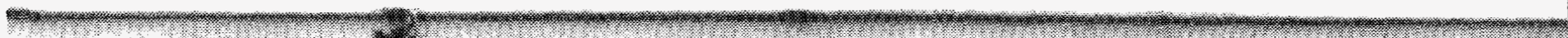

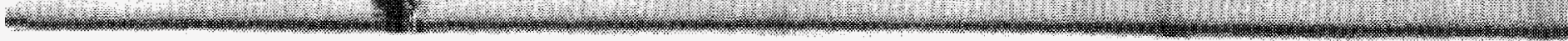

96-142 $90^{\circ} 32$ in. WL-09, 18, 22, 27 Thermal (above) and Epithermal (below) 

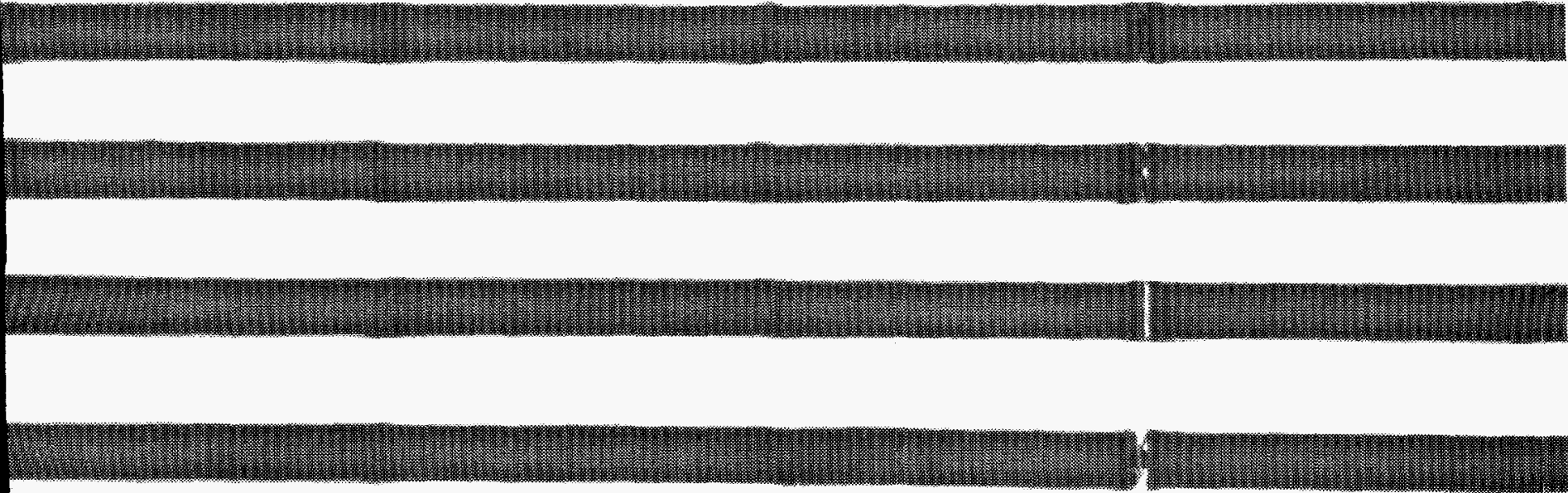

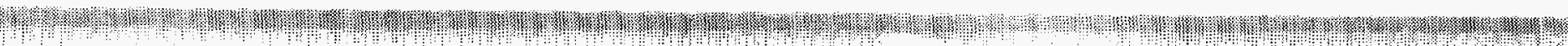

(5)

:

(a)

ro

(6)

(i)

\%

Lox

$\$$

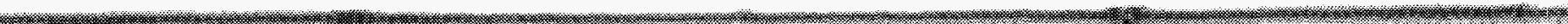

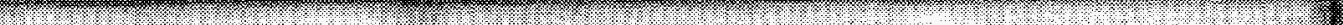

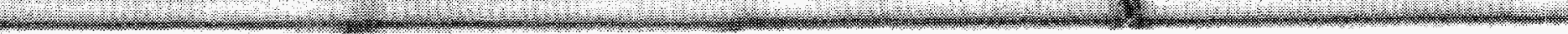

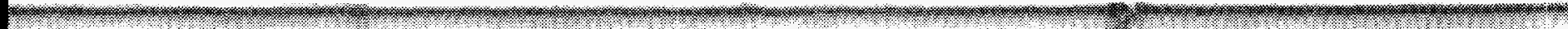

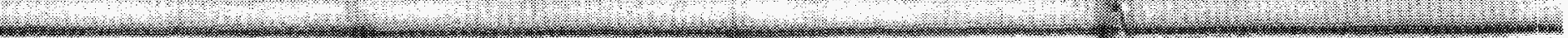

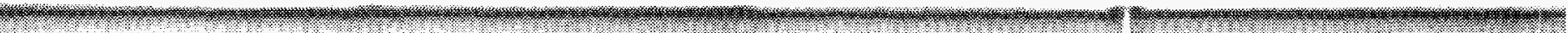

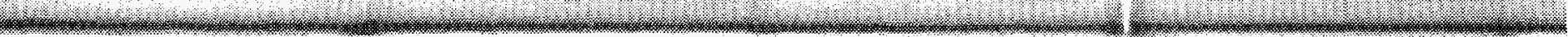

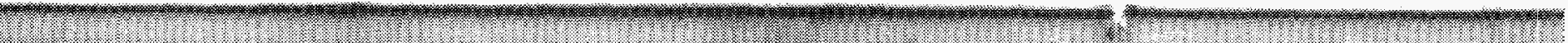

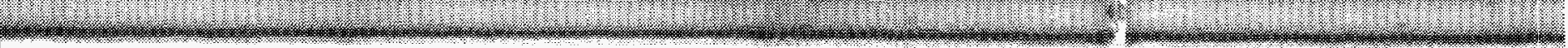



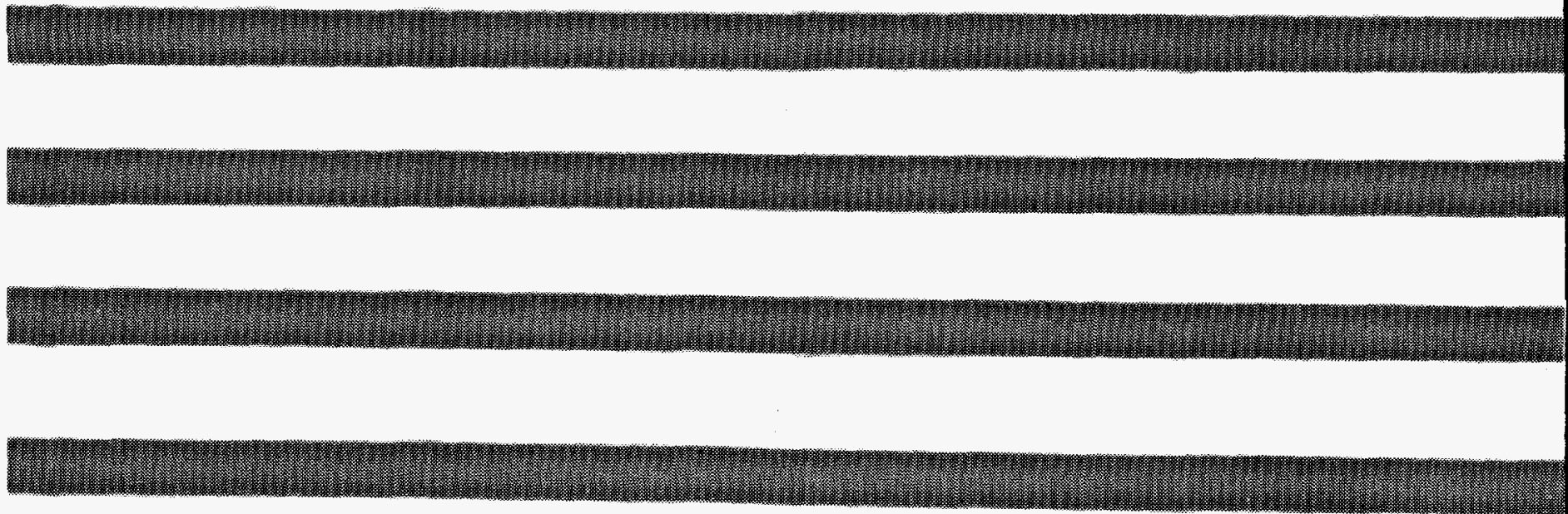

18)
I)
(4)
4
*
*
1
(1)
8

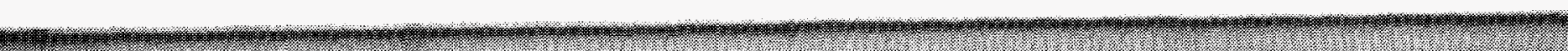

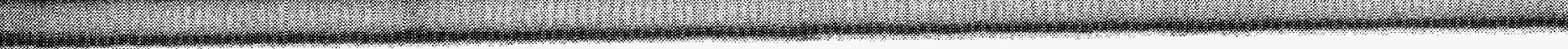

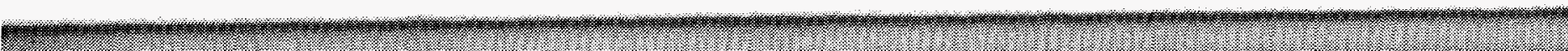

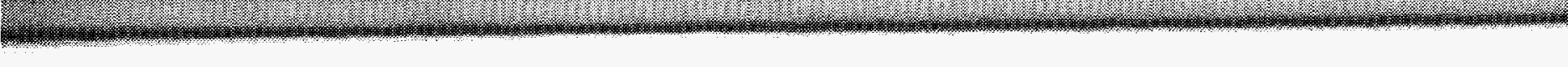

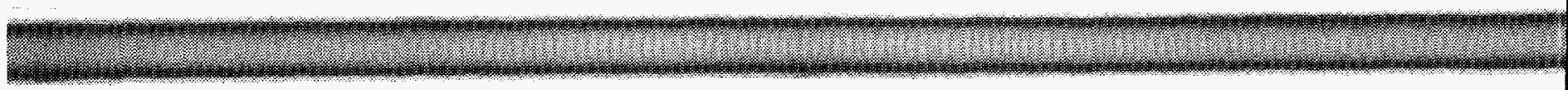

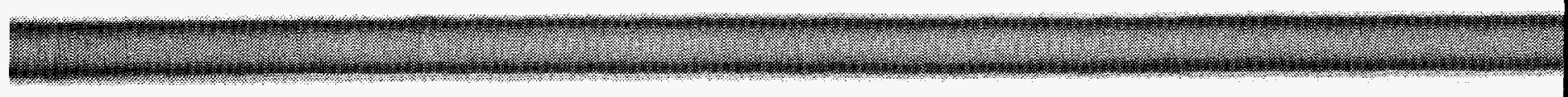

96-141 $90^{\circ} 48$ in. WL-09, 18, 22, 27 Thermal (above) and Epithermal (below) 


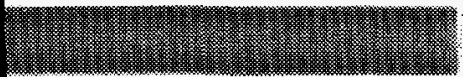
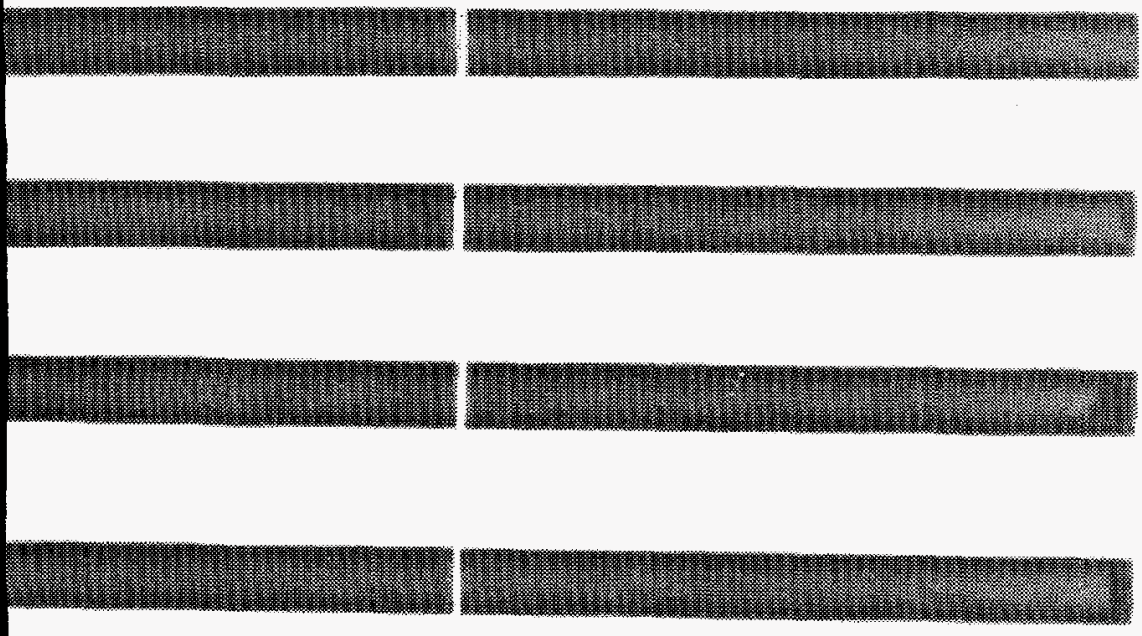

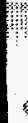

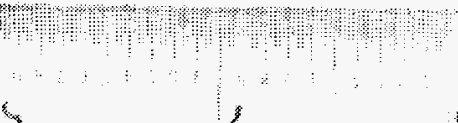

9

3

落

r.

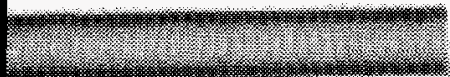

.

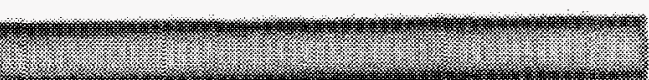

(

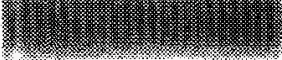

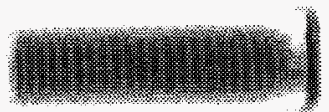

.

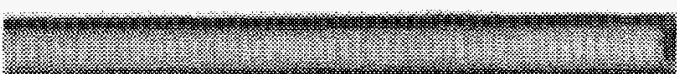

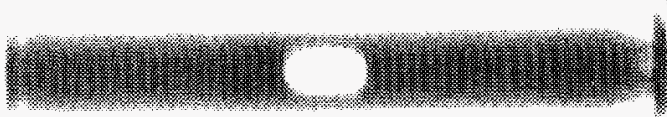

(3.2:

.
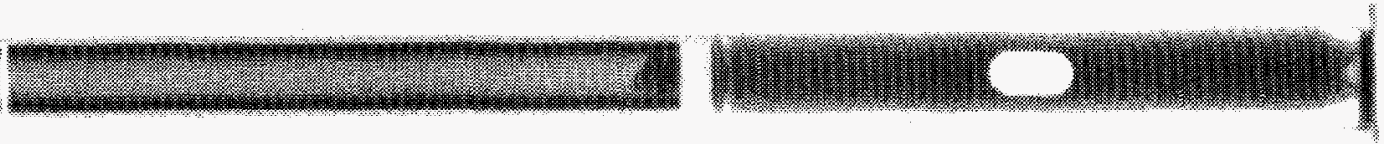

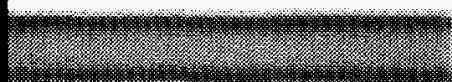

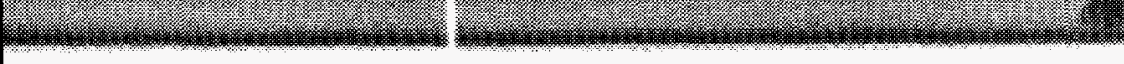

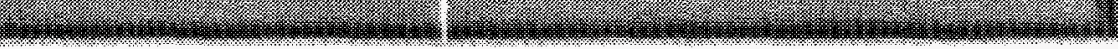

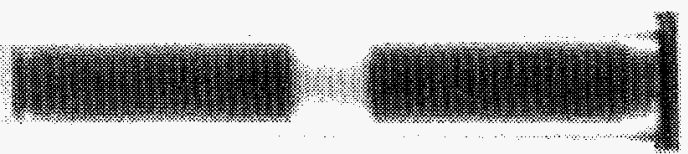



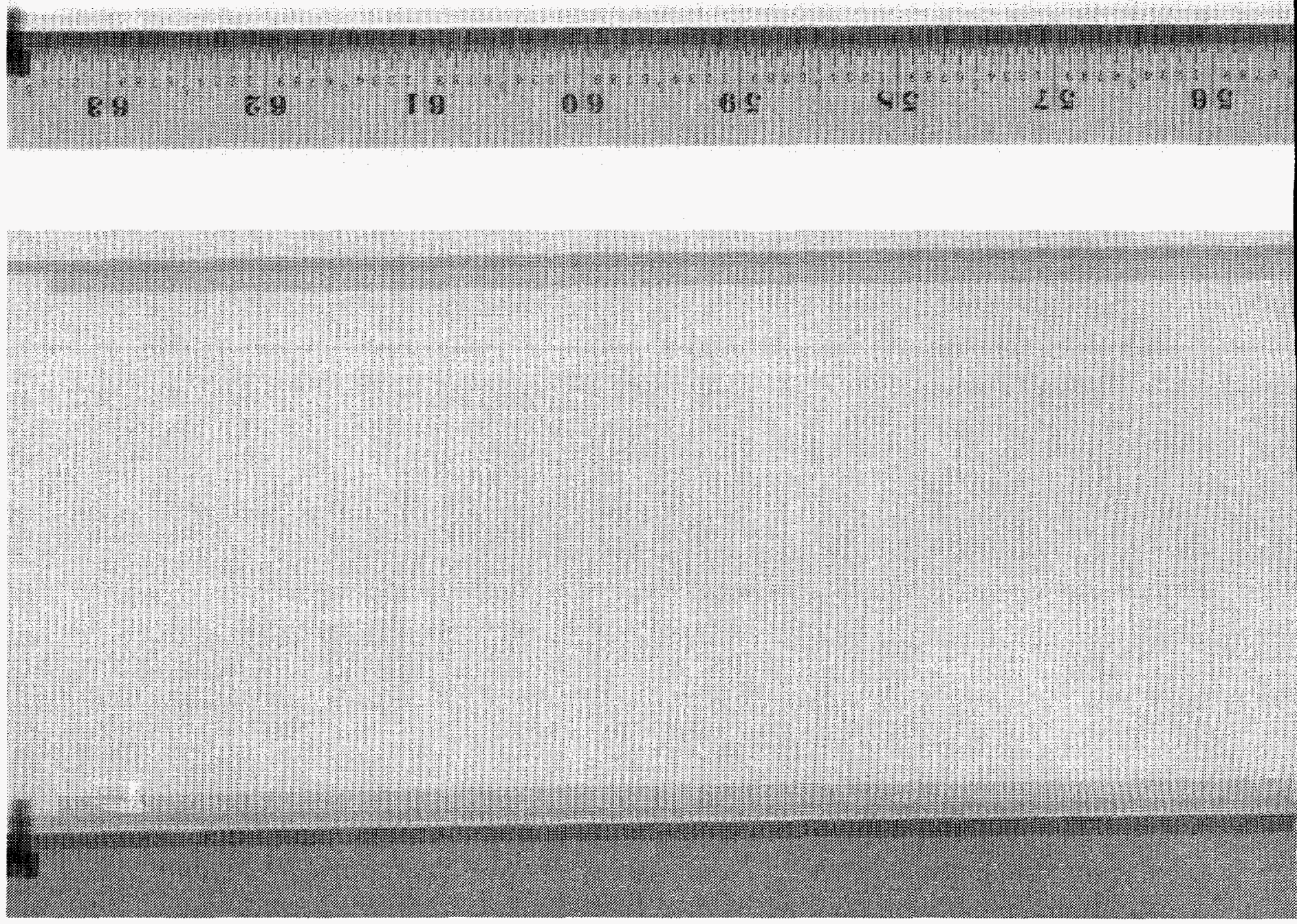

96-171 $0^{\circ} \quad 0$ in. B1, S1 Thermal (above) and Epithermal (below) 


\section{6}
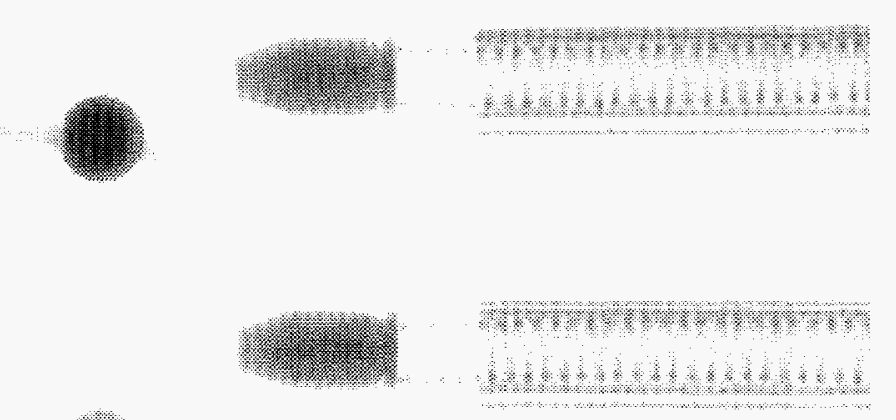

\section{c.}

(

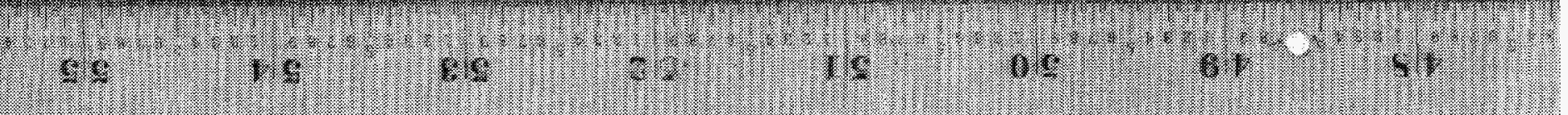

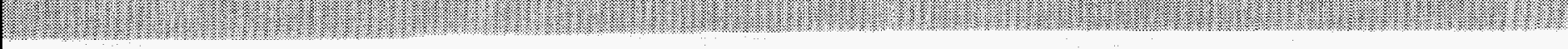

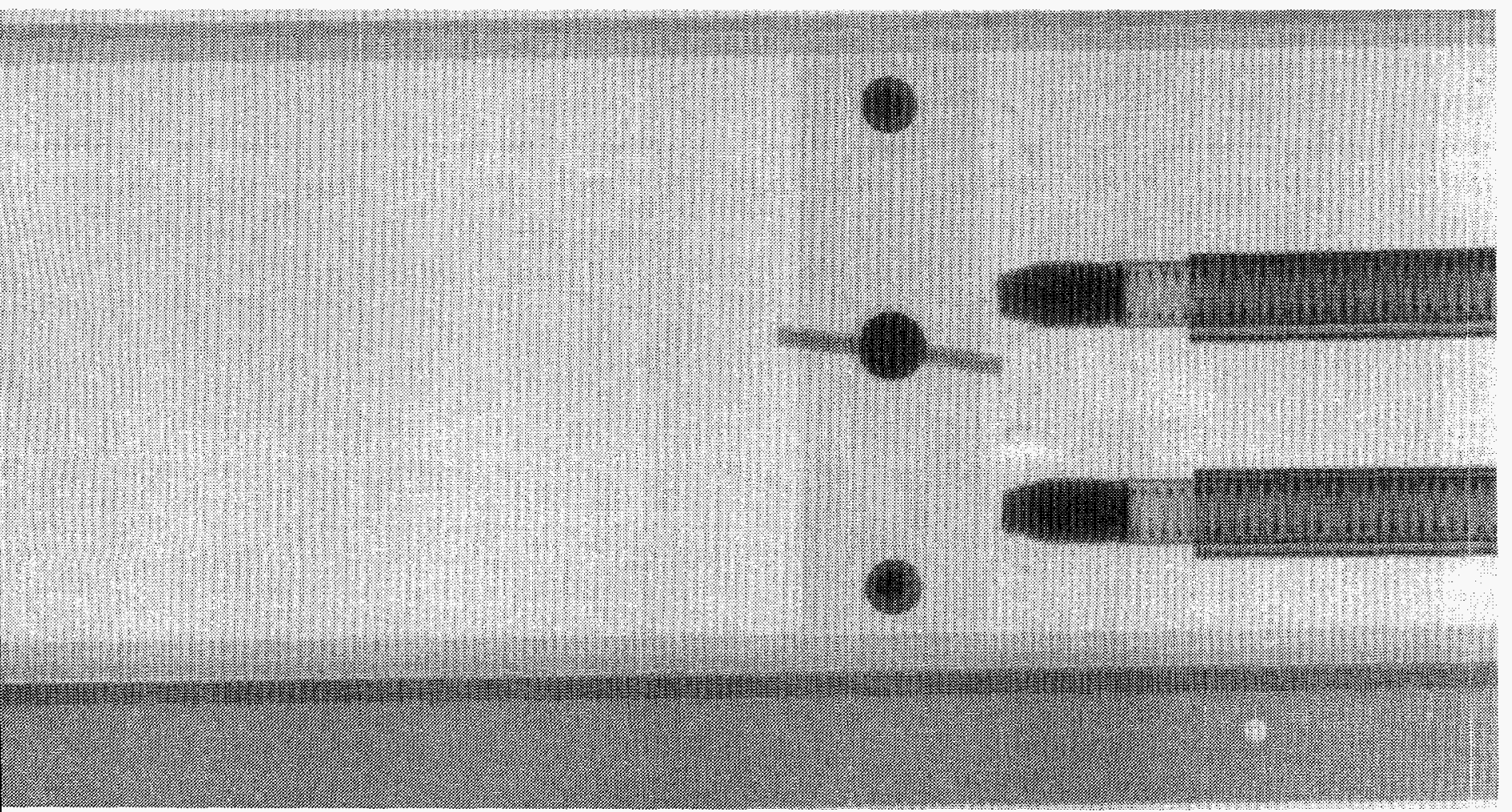



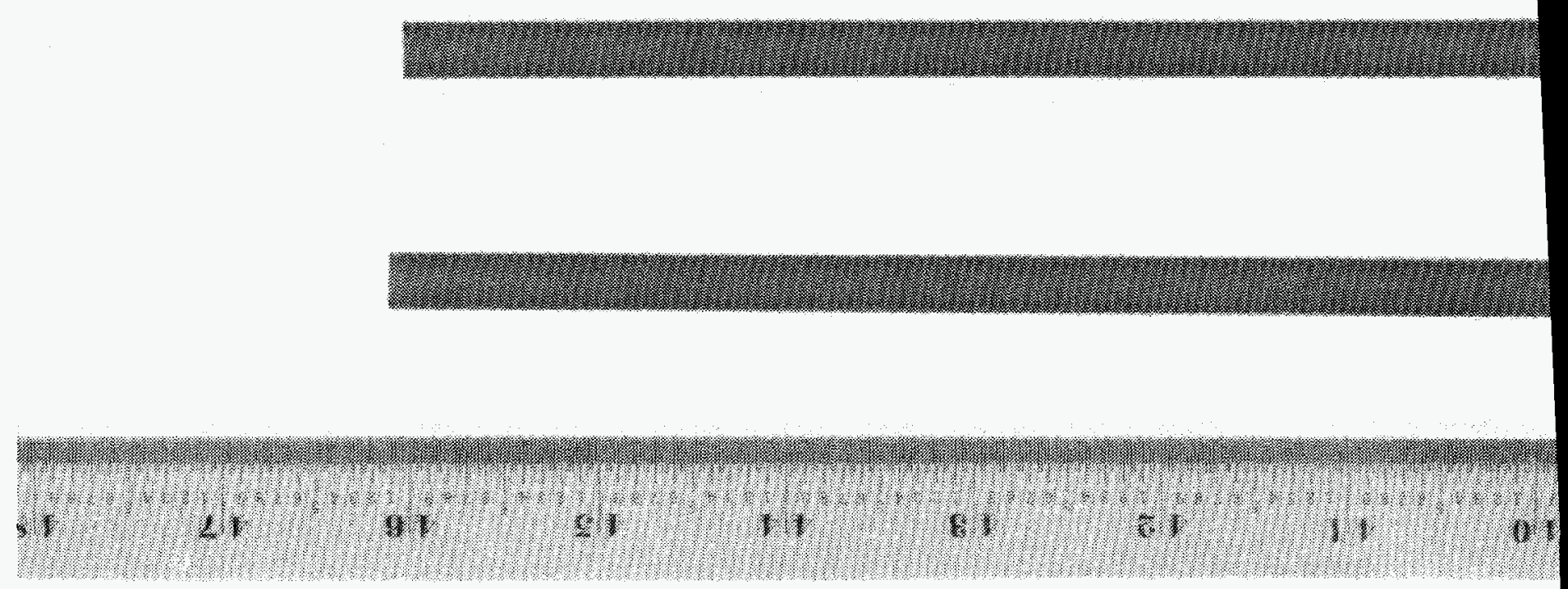

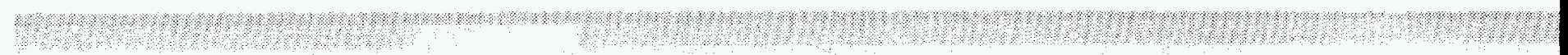
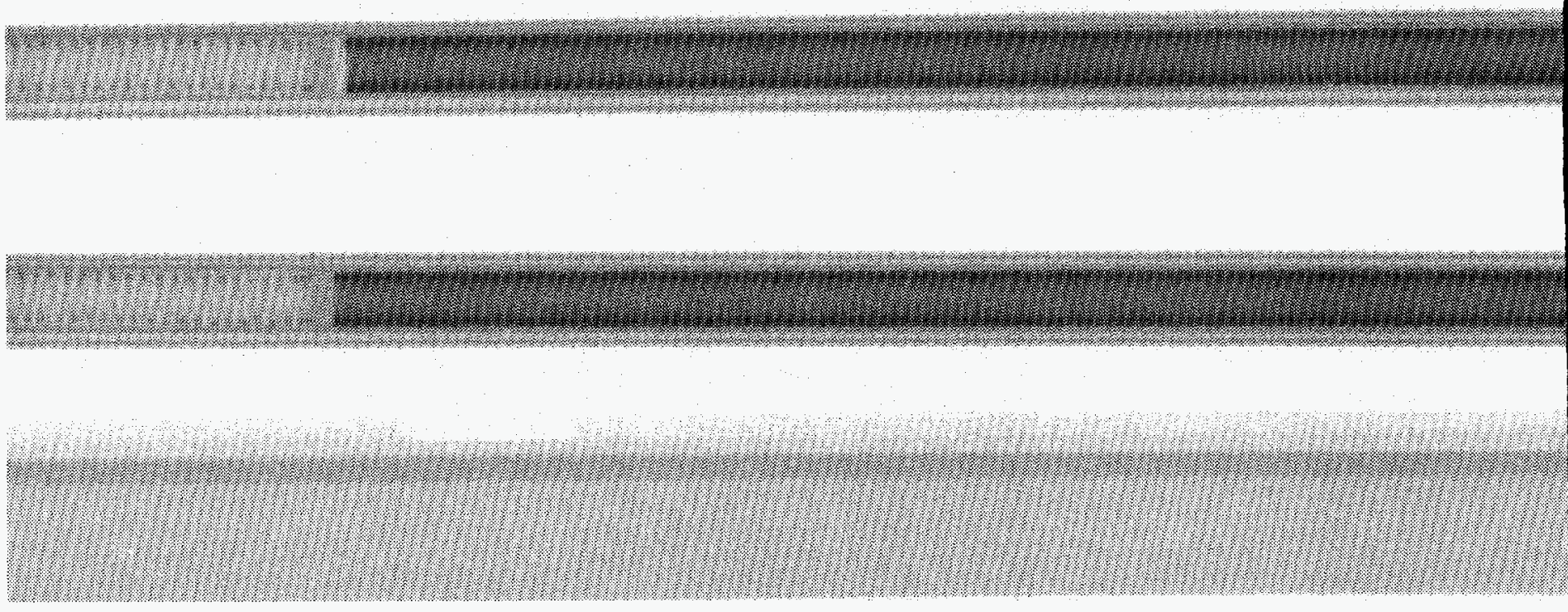

96-170 $0^{\circ} 16$ in. B1, S1 Thermal (above) and Epithermal (below) 

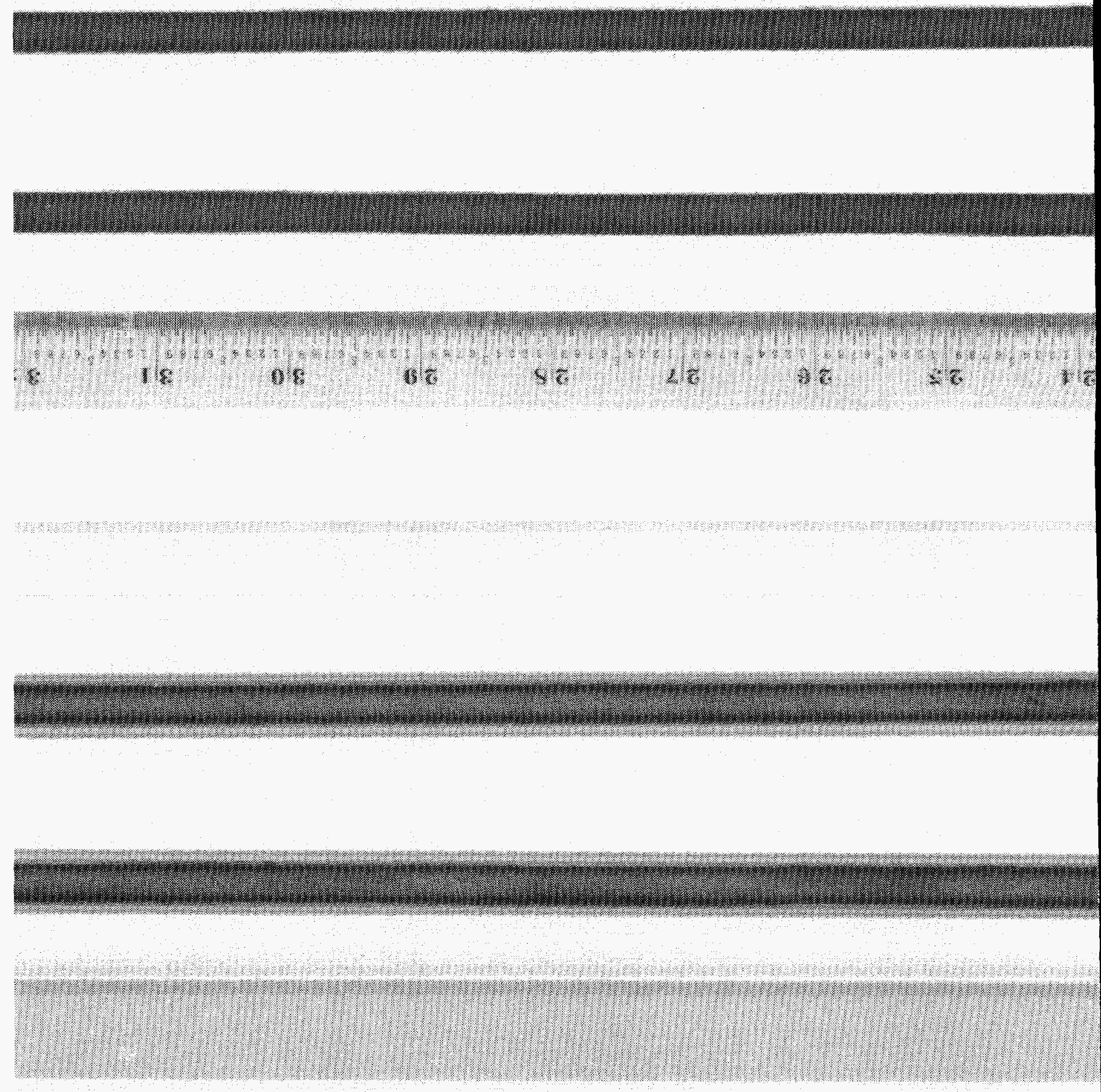

96-169 $0^{\circ} 32$ in. B1, S1 Thermal (above) and Epithermal (below) 

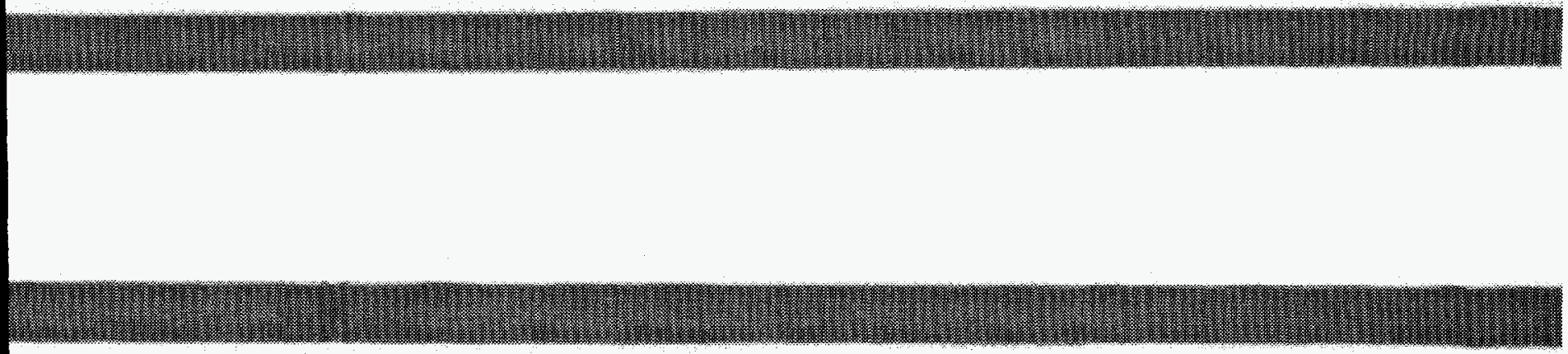

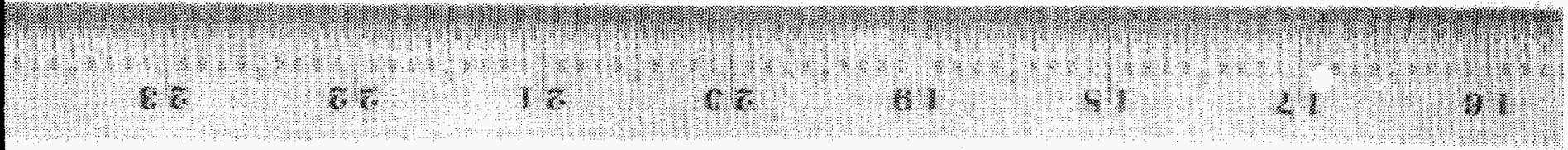

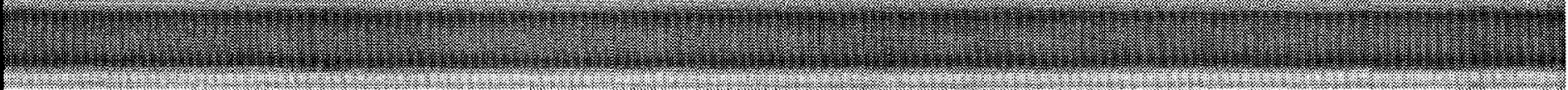

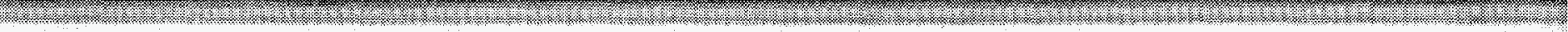

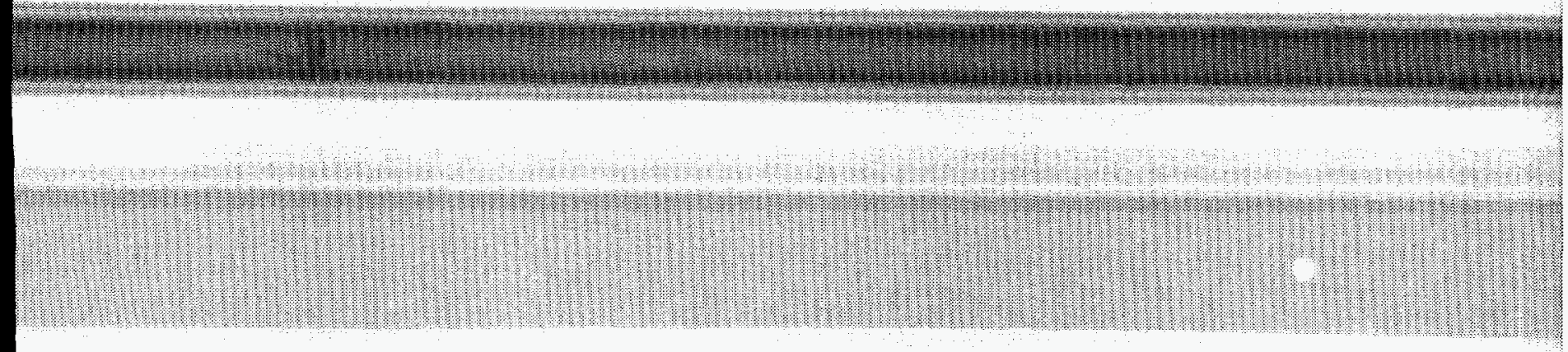



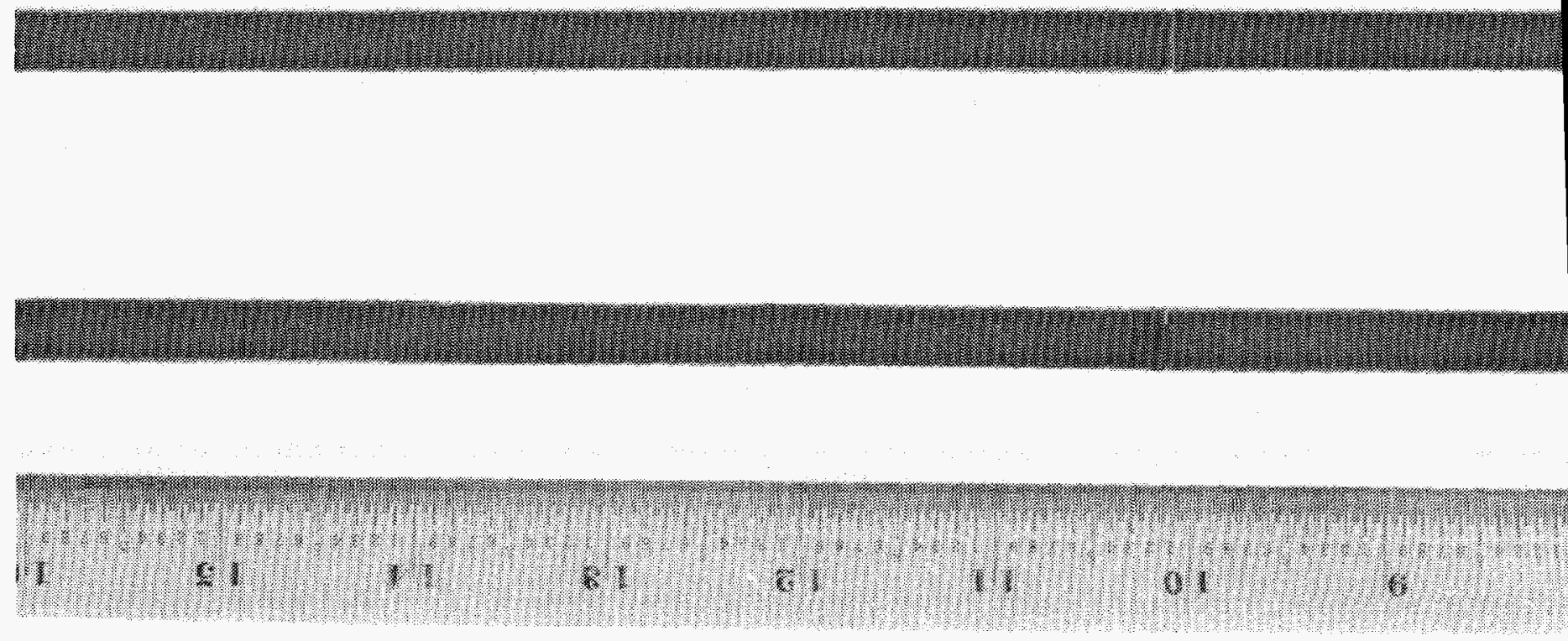

W
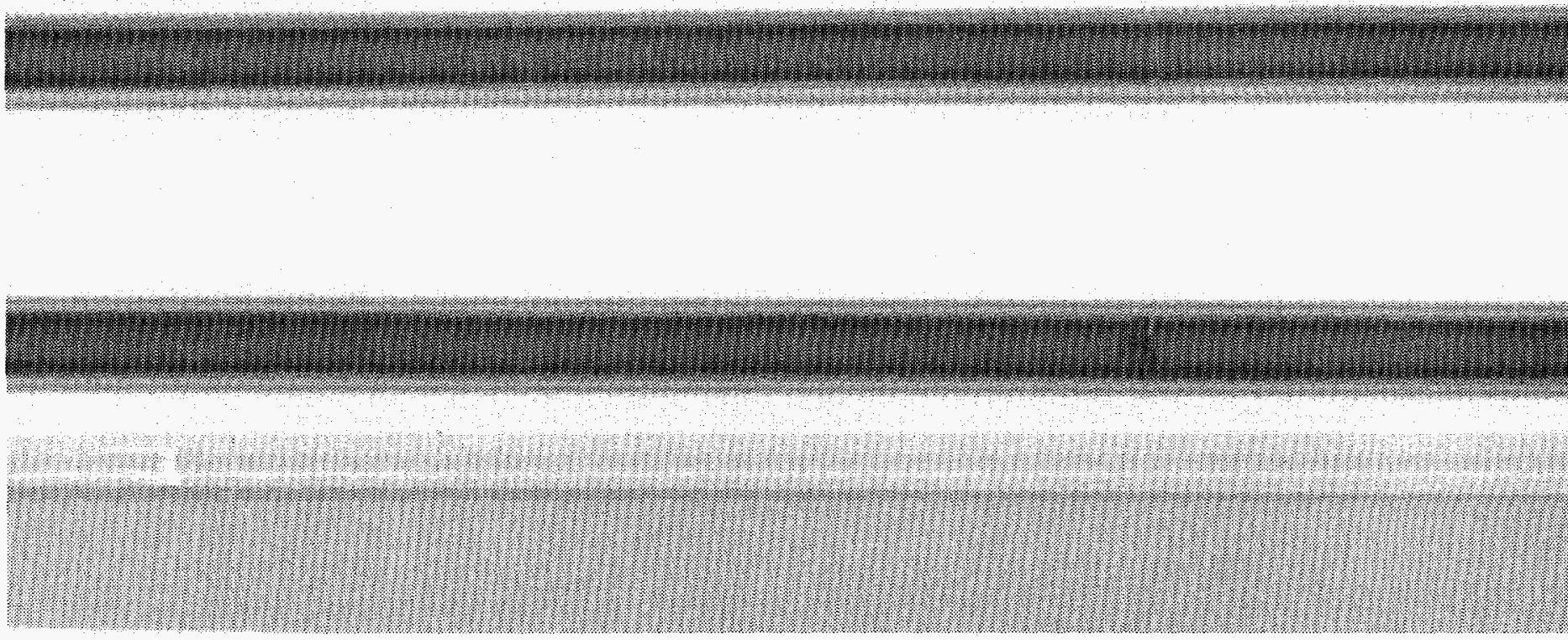

96-168 $0^{\circ} 48$ in. B1, S1 Thermal (above) and Epithermal (below) 


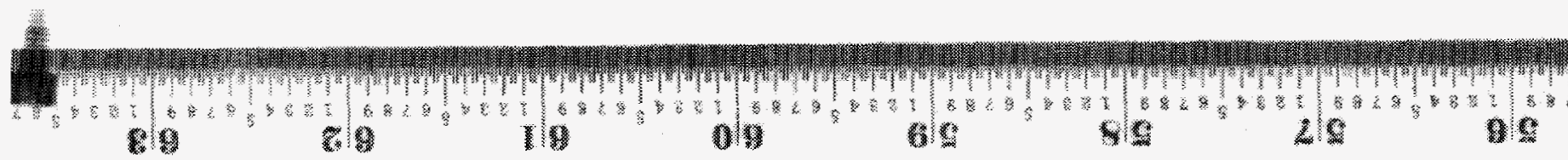

96-175 $90^{\circ} \quad 0$ in. B1, S1 Thermal (above) and Epithermal (below) 


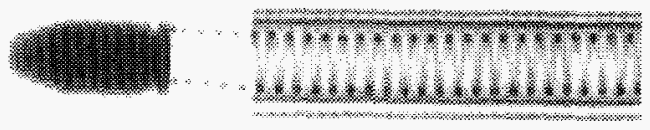

.
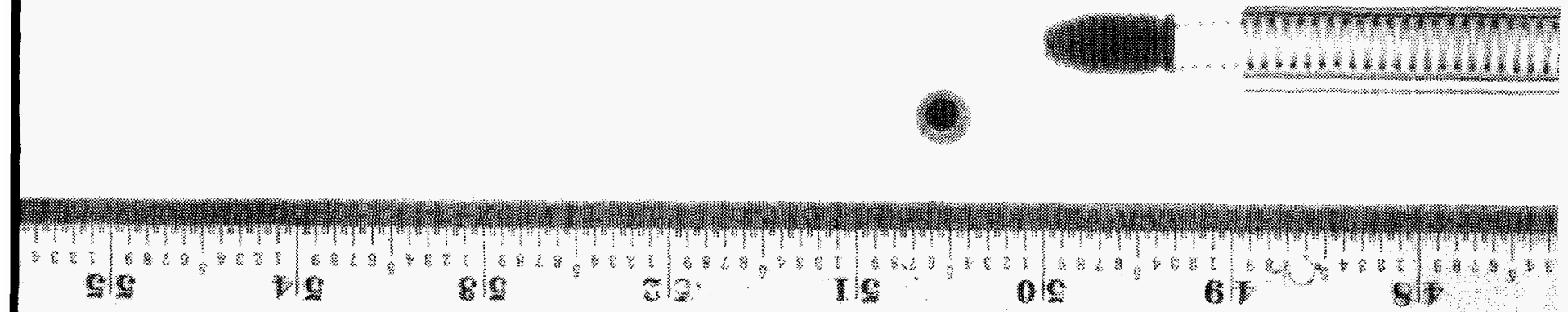

s/x

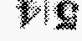

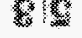

(2)

I.s. 


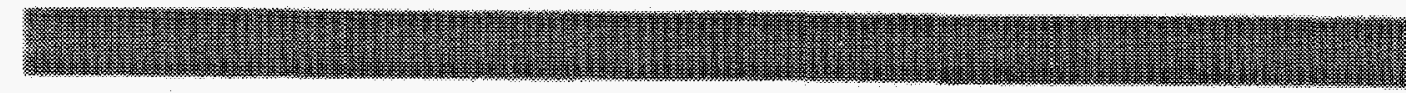

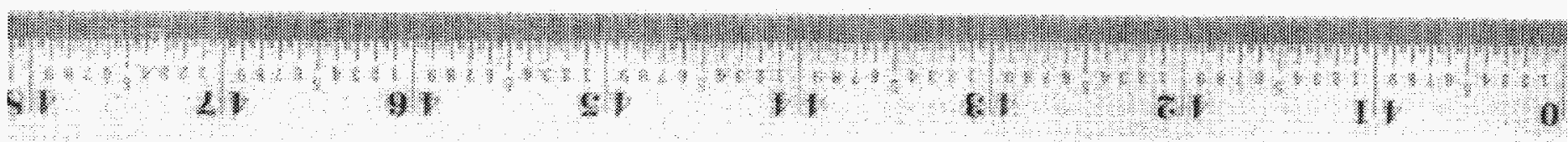
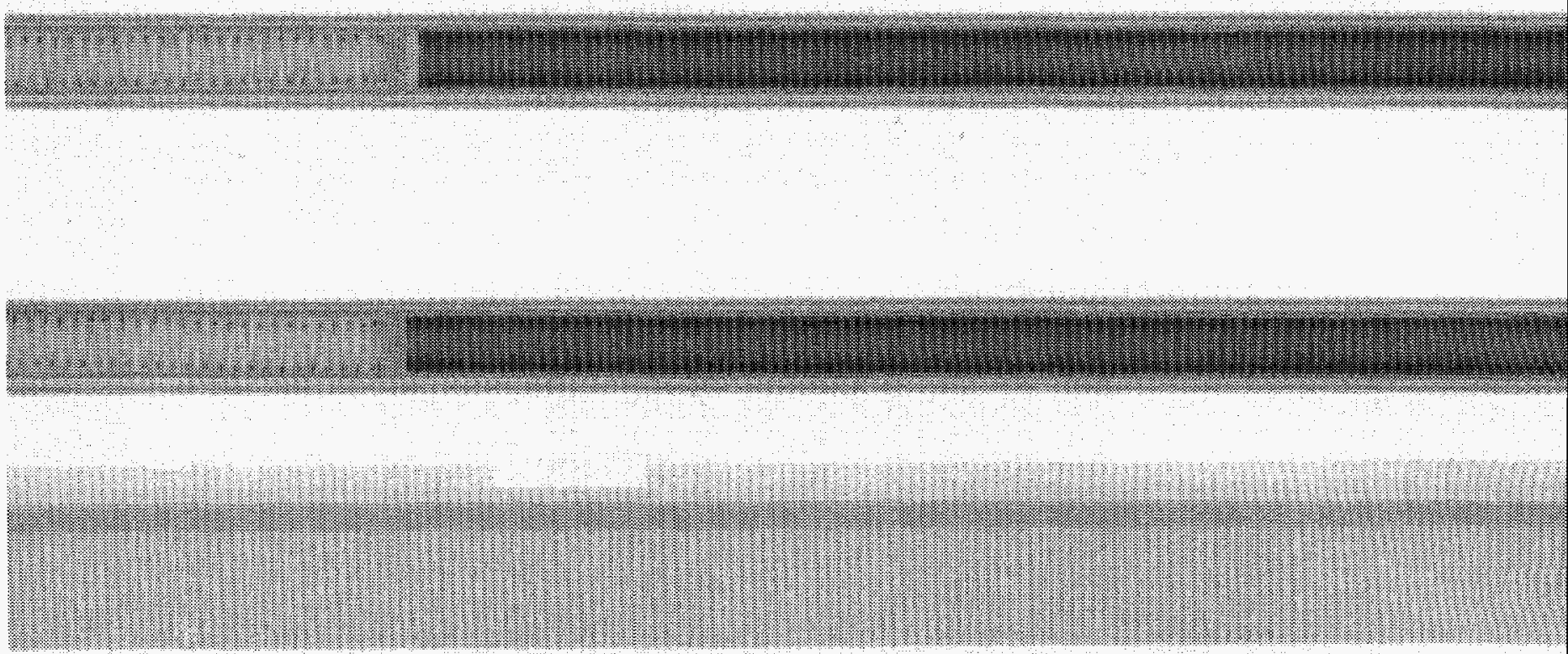

\section{6-174 $90^{\circ} \quad 16$ in. B1, S1 Thermal (above) and Epithermal (below)}



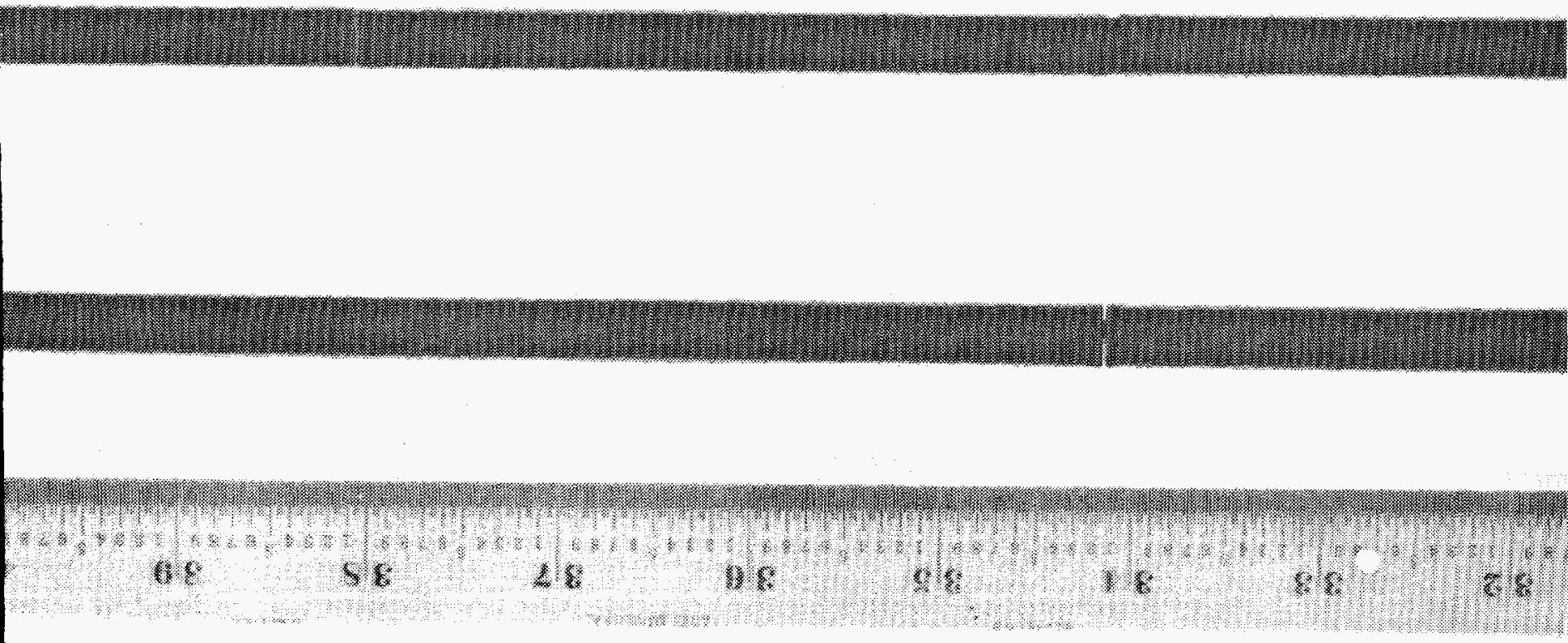

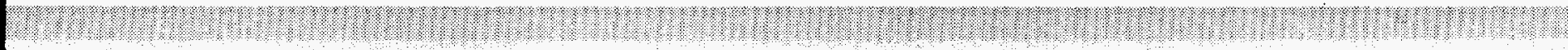
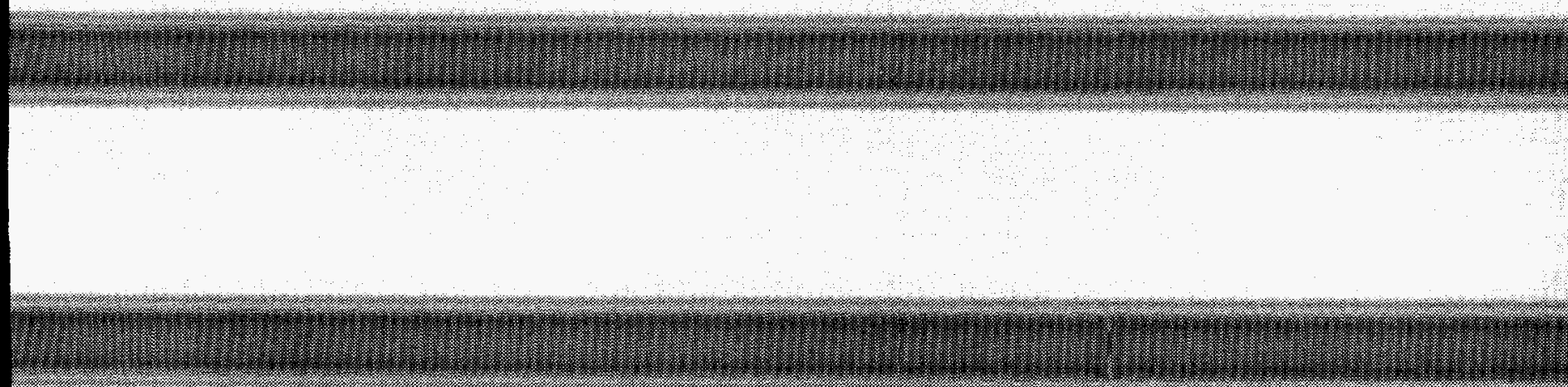

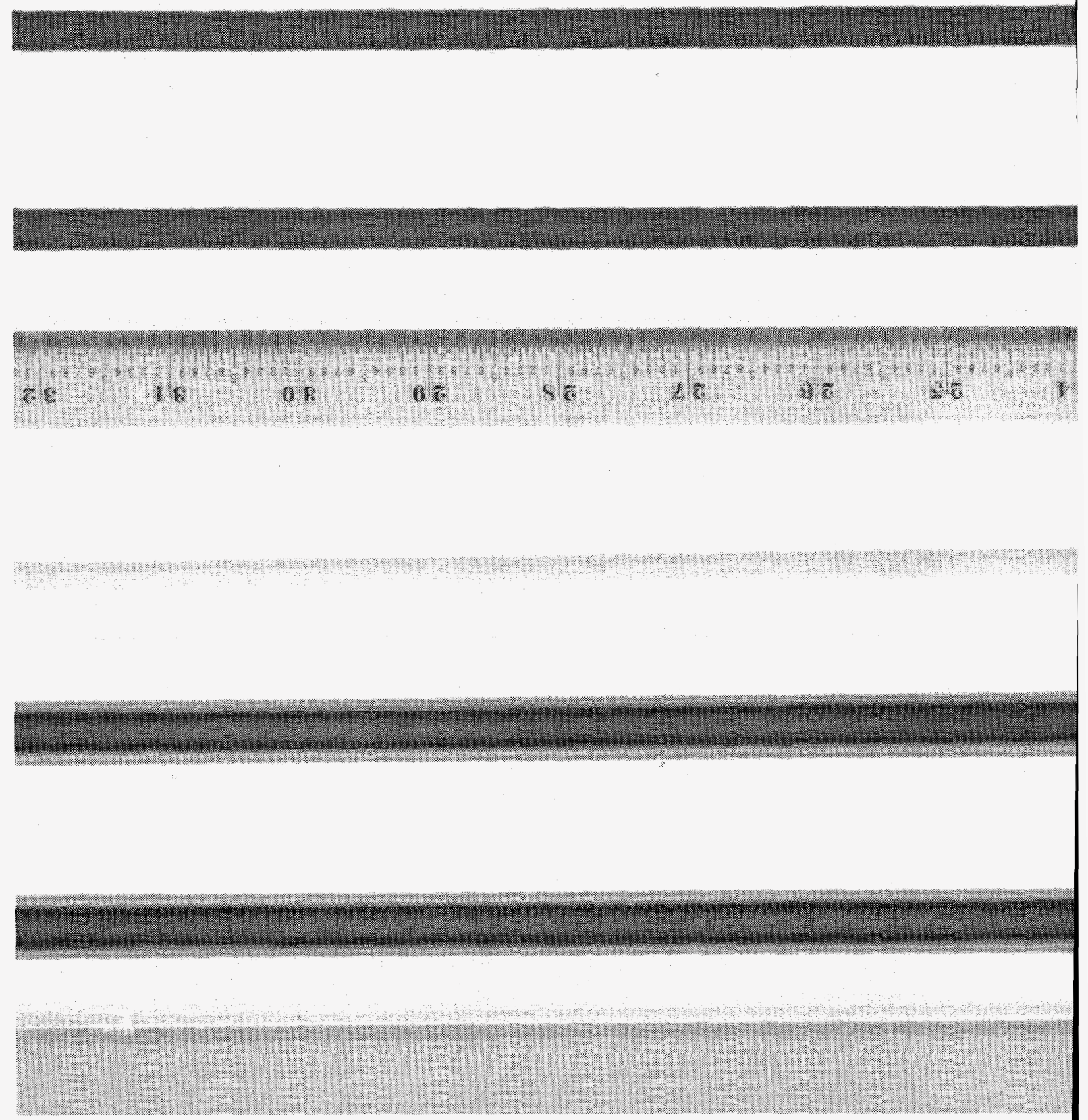

96-173 $90^{\circ} 32$ in. B1, S1 Thermal (above) and Epithermal (below) 

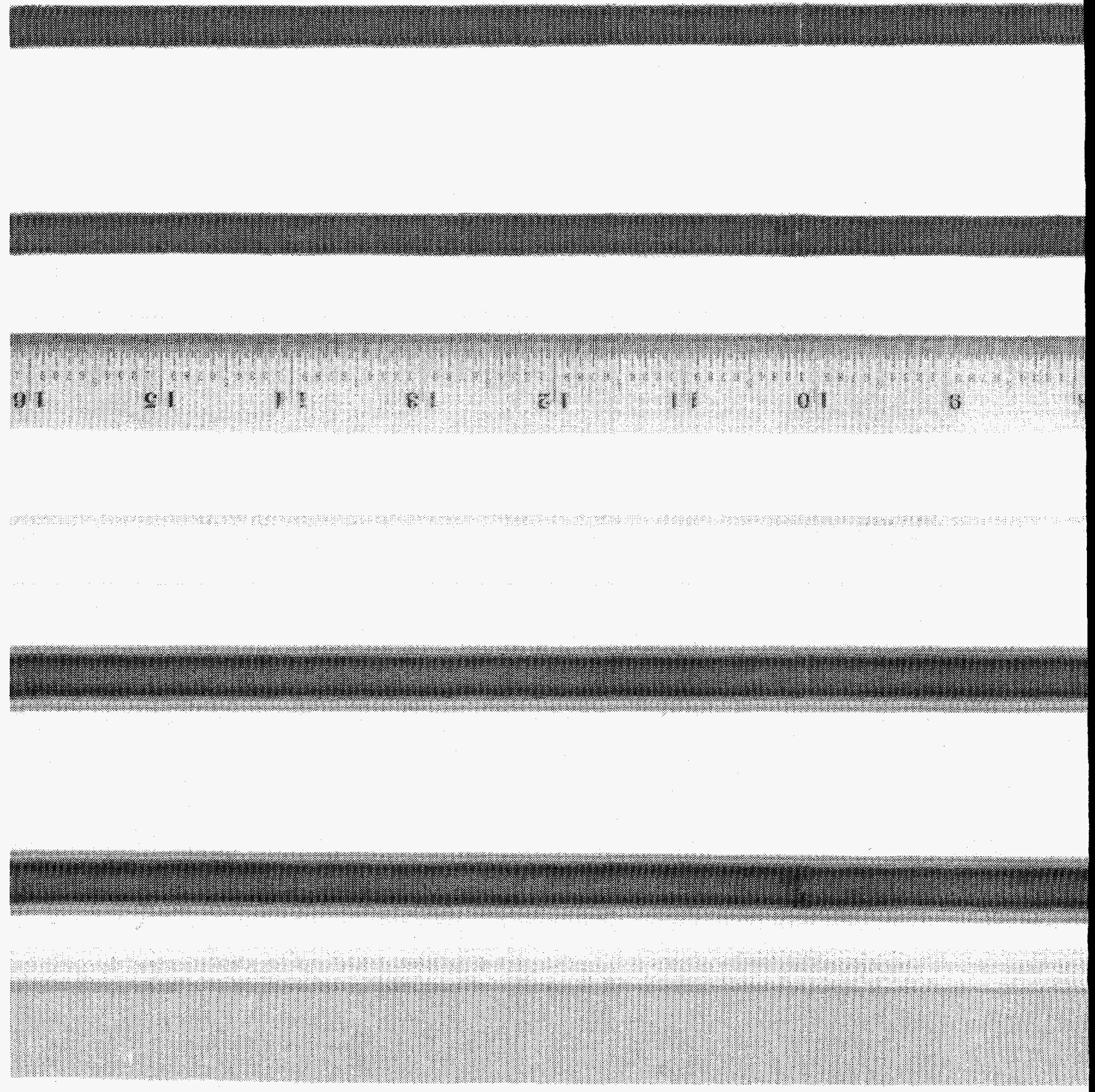

96-172 $90^{\circ} 48$ in. B1, S1 Thermal (above) and Epithermal (below) 


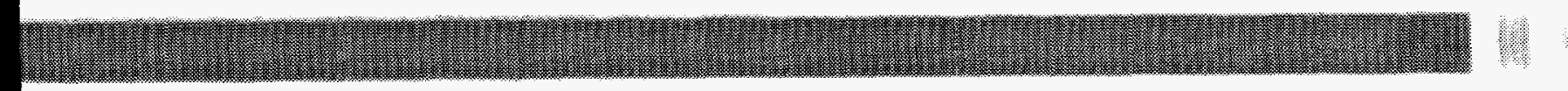

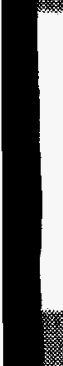

6.

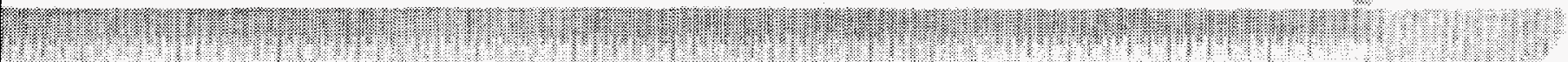

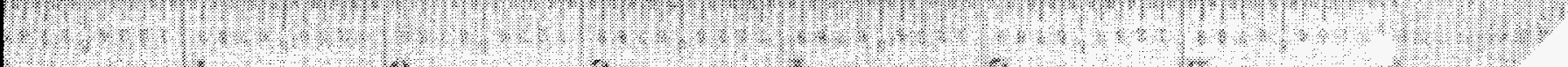

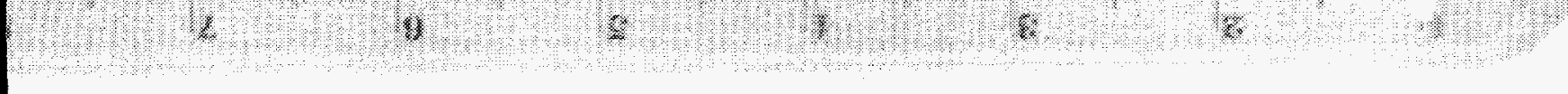

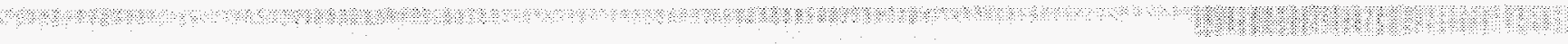

(2):

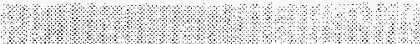
( (

3:

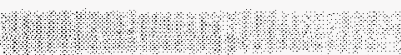


Appendix D

Gamma Scan 


\title{
Appendix D
}

\section{Table of Contents}

\begin{abstract}
WL-01 Gross
WL-01 $\mathrm{Mn}^{54}$

WL-01 $\mathrm{Co}^{60}$

WL-03 Gross

WL-03 $\mathrm{Mn}^{54}$

WL-03 $\mathrm{Co}^{60}$

WL-09 Gross

WL-09 $\mathrm{Mn}^{54}$

WL-09 $\mathrm{Co}^{60}$

WL-10 Gross

WL-10 $\mathrm{Mn}^{54}$

WL-10 $\mathrm{Co}^{60}$

WL-18 Gross

WL-18 $\mathrm{Mn}^{54}$

WL-18 $\mathrm{Co}^{60}$

WL-22 Gross

WL-22 $\mathrm{Mn}^{54}$

WL-22 $\mathrm{Co}^{60}$

WL-26 Gross

WL-26 $\mathrm{Mn}^{54}$

WL-26 $\mathrm{Co}^{60}$

WL-27 Gross

WL-27 $\mathrm{Mn}^{54}$

WL-27 $\mathrm{Co}^{60}$

WT-11 Gross

WT-11 $\mathrm{Mn}^{54}$

WT-11 Co ${ }^{60}$

WT-12 Gross

WT-12 $\mathrm{Mn}^{54}$

WT- $12 \mathrm{Co}^{60}$
\end{abstract}




\section{ARGONNE NATIONAL LABORATORY - WEST \\ P. O. Box 2528, Idaho Falls, Idaho 83403-2528}

\section{BNGITRERLE DVISTOI}

(AL, TREAT, ZPPR, and HFEF)
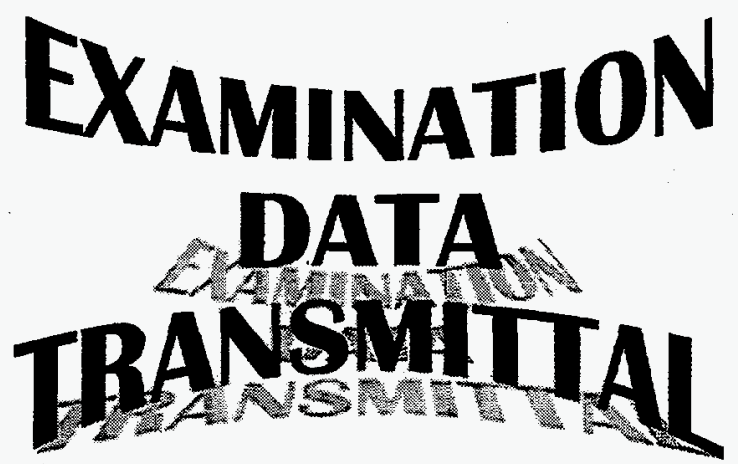

DATE::

January 9, 1997

MATERIAL EXAMINED:

CLWR Loop-1 Target Rods (8)

TYPE OF DATA::

\section{Gamma Scan}

FORM OF DATA

Tables, Charts, User Notes, and CD-ROM's (2)

NOTES AND CONDITIONS:

CC: wiAttachments

None

CC: w/o Attachments

CLWR Loop-1 file

Questions regarding this data transmittal should be directed to the Cognizant Engineer at extension (208) $533-7773$ 


\section{ARGONNE NATIONAL LABORATORY \\ P. O. Box 2528, Idaho Falls, Idaho $83403-2528$}

\section{ENGINEERING DIVISION}

(TREAT, ZPPR, and HFEF)

EXAminatiON

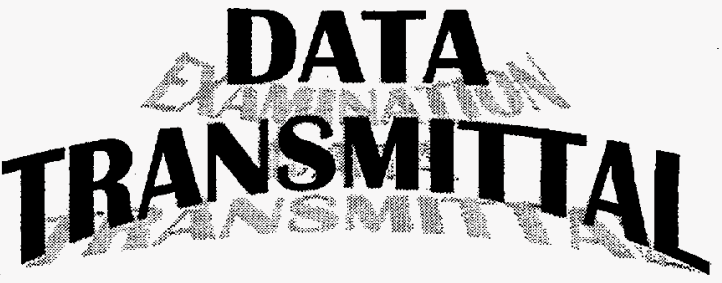

TO:

ADDRESS:

DATE:

Lockheed Martin Idaho Technologies, Co. EROB MS-3760

Idaho Falls, ID 834

March 14, 1997

R. L. Bratton

MATERIAL EXAMINED:

Two Target Rods from CLWR S1/B1 Test

TYPE OF EXAMINATION:

\section{Isotopic Gamma Scans}

FORM OF DATA:

Charts, User Notes, and CD-ROM (2)

NOTES:

CC: w/Attachments

S1/B1 file (charts only)

CC: w/o Altachments

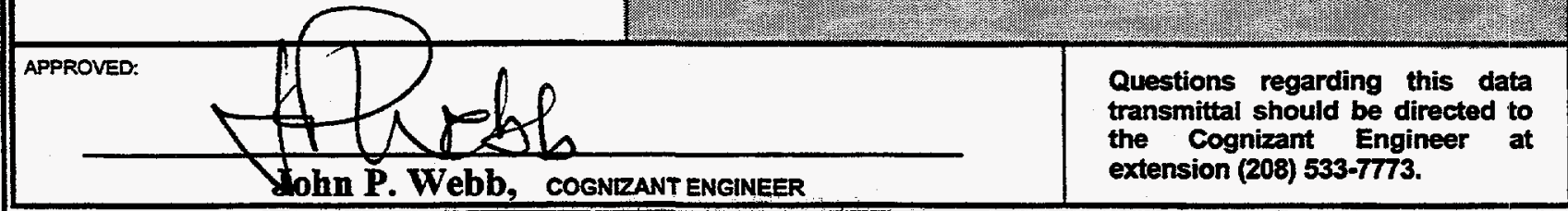




\title{
ARGONNE NATIONAL LABORATORY Hot Fuel Examination Facility PRECISION GAMMA SCANNING
}

\author{
USER NOTES
}

1. The Precision Gamma Scanner - II (PGS-II) is designed to intermittently traverse rod-like objects in front of a small-solid-angle collimator for collection of gamma spectra at a series of positions. Usually the increment of traverse is set equal to the collimator slit opening to provide complete object coverage. This process is called scanning.

2. The gamma-ray spectroscopy sub-system consists of an ultra-pure germanium (UPG) detector with a nominal efficiency of $10 \%$ (relative to a 2" $\times 2 " \mathrm{Nal}$ detector). Active Compton-suppression is provided by detecting the time-coincidence of Compton events from a large volume NaI guard detector surrounding the UPG. A background suppression ratio of 6-10 (based on Co-60 peaks) is achieved.

3. The PGS-II is designed and calibrated to position the center of the collimator slit (with respect to the upper tip of the scanned object) to an accuracy of $\pm 0.005 \mathrm{in}$. for each positional measurement.

4. Data can be acquired in either gross or spectral mode. Gross mode counts gammas without regard to their energies. Spectral mode maps gamma energies (in the range of 50-1600 Kev) into 4096 channels (memory locations).

5. The collimator slit is adjustable from $0-100$ mils, and is generally fixed for any given scan. Slit opening is reported in all data tables and charts.

6. The live time of each positional count is infinitely adjustable, but is usually fixed for any given scan. The live time used for a scan is reported in all data tables and charts.

7. Spectral analysis is performed by post-processing the collected data. The analysis is based on a fixed Channel-to-Energy calibration where:

$$
\text { Channel }=2.5 E_{\gamma}(\mathrm{Kev})
$$

Selected isotopic peaks of interest are located within each spectrum, and a gross peak area is calculated by simple trapezoidal integration. Background for each peak is estimated by fitting (least squares) a straight line to the regions immediately above and below the peak. This background is subtracted from the gross peak area to generate the net peak area (in counts).

8. The isotopic count rate (net peak area divided by the live time) is usually reported at each position in chart and tabular form.

9. If requested, all isotopic data can be corrected for decay using the standard equation:

$$
A_{t}=A_{0} e^{-\lambda t}
$$

This correction is only required when short-lived isotopes are being measured, and it removes the effect of decay during a given scan and between scans of sibling rods.

10. A measure of gross activity is obtained by summing the counts in all 4096 channels, and dividing by the live time. These data are usually reported in chart and tabular form.

11. Spectral data are sometimes provided in digital form on CD-ROM's or floppy disks. The data are stored as plain ASCII data in DOS file format (i.e., records are terminated by $<C R>L L F>$ pairs). The first 10 records contain self-explanatory header information, including the units of the data fields. Below the header records, spectral activity data are stored in tab-delimited fields as follows:

$$
<\text { Position }>\langle t a b><\text { Gross }>\langle t a b<\text { Isotope-1 }>\langle t a b>\text {... }<\text { Isotope-N }>C R><L F>
$$

12. Gross-mode data are not available (at this time) in digital form. 


$$
\text { D-4 }
$$




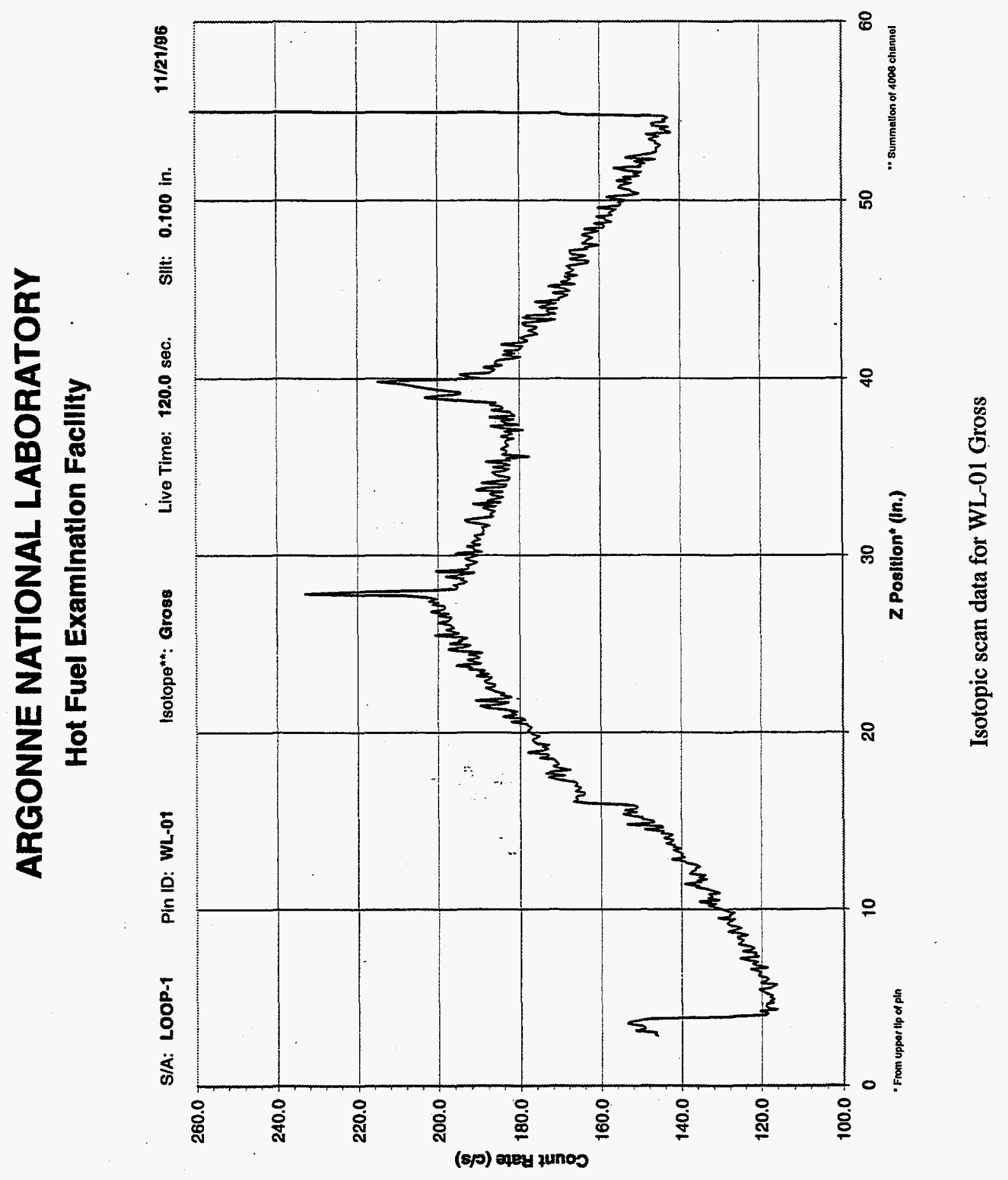

D-5 


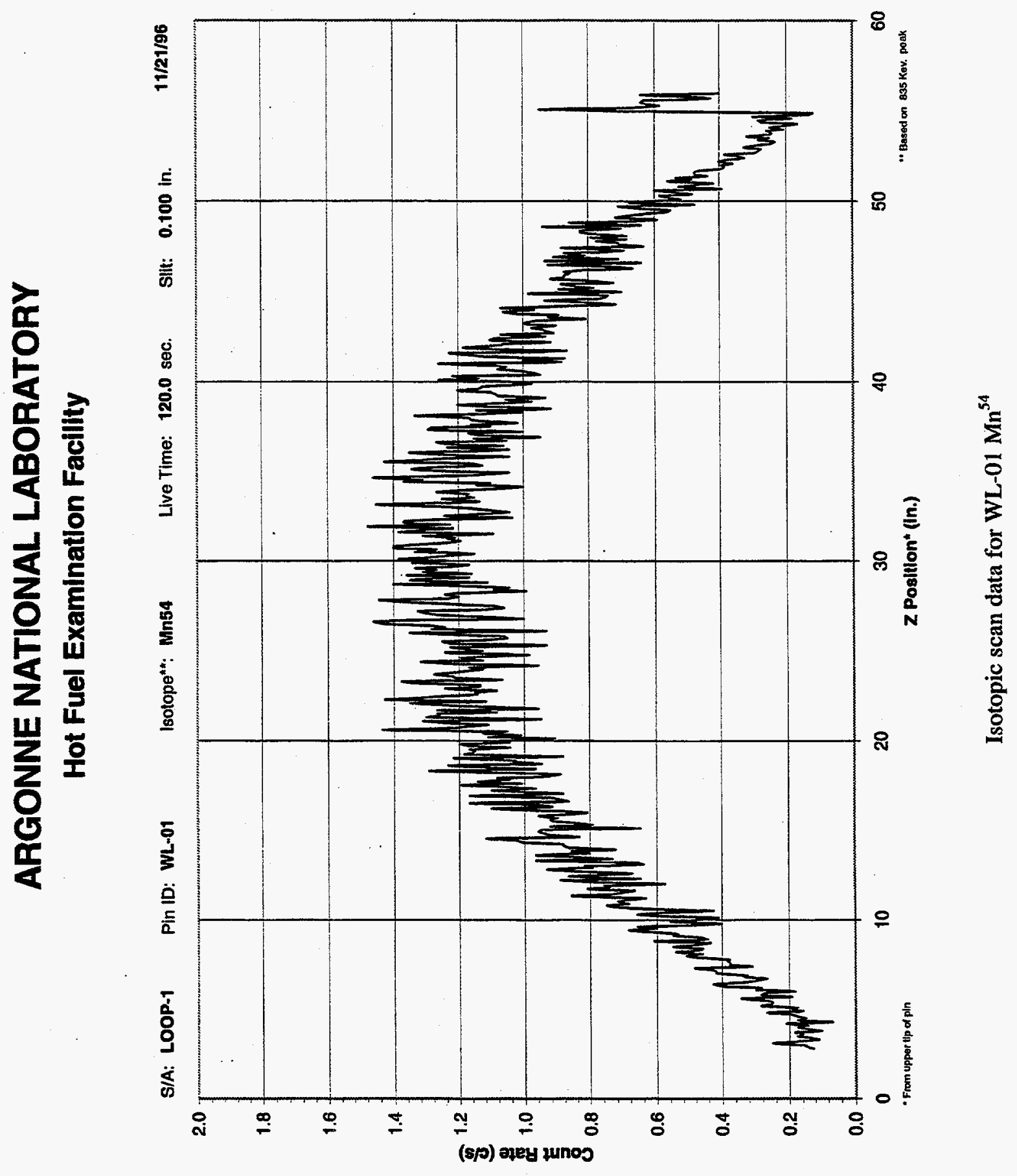




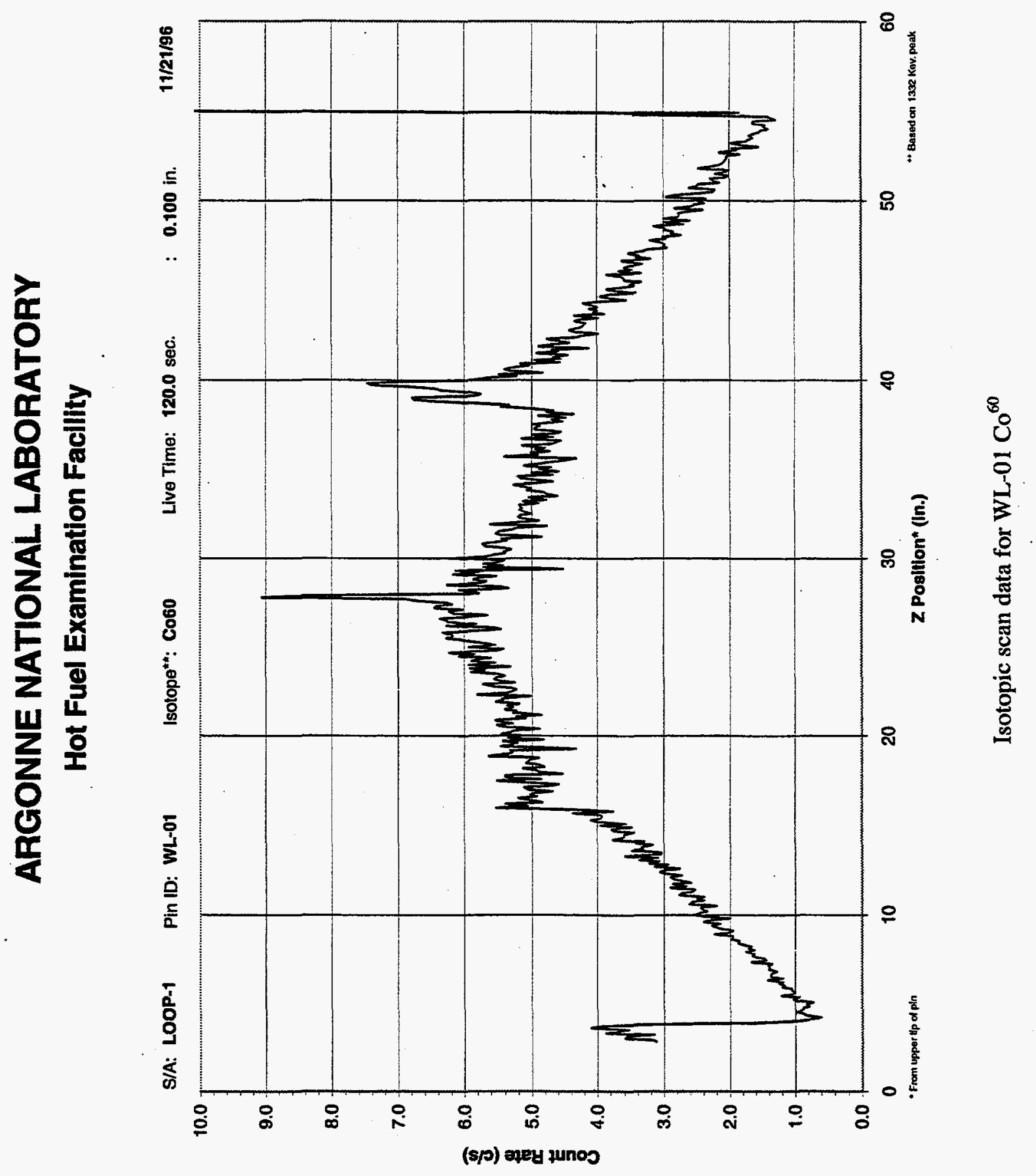




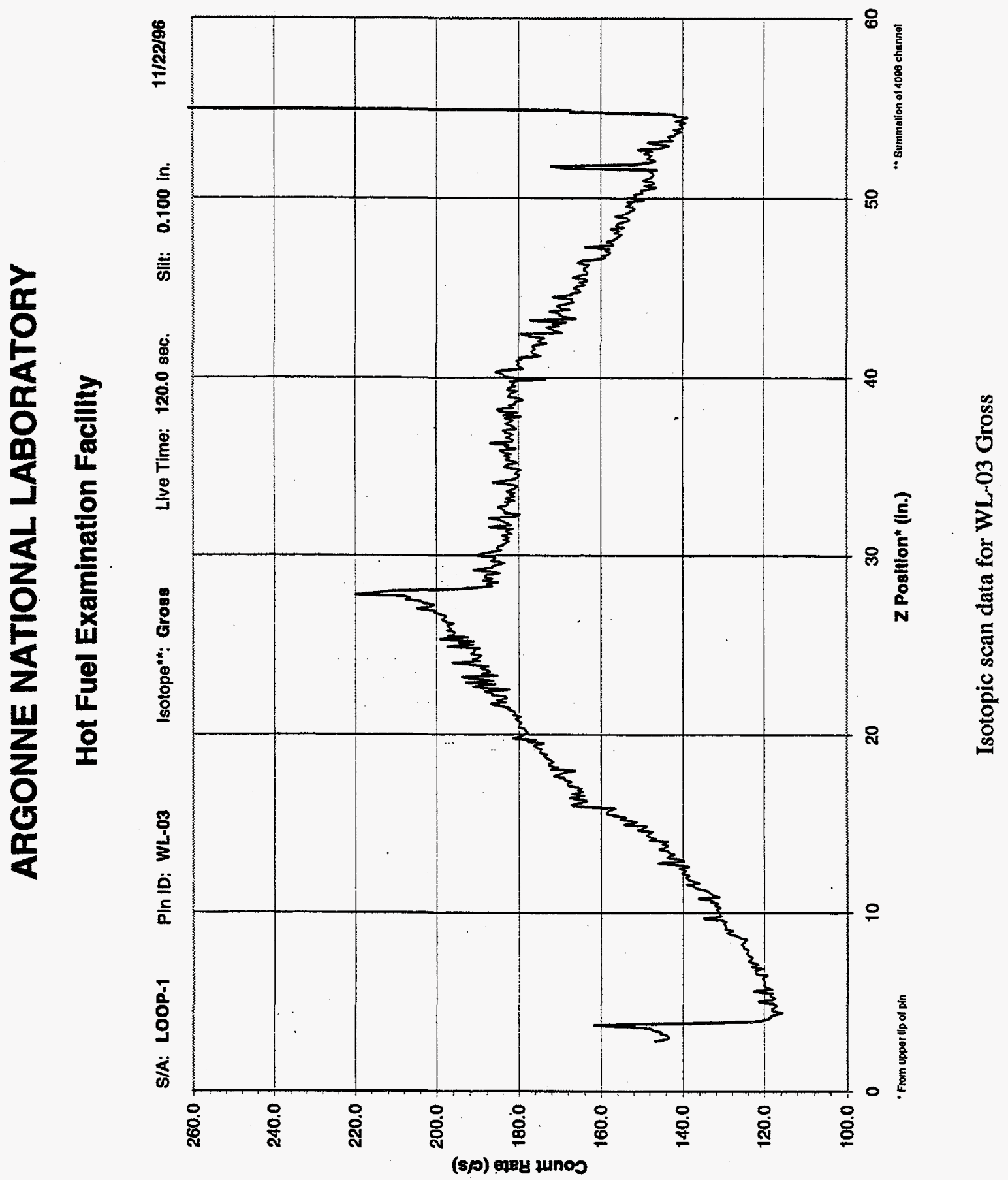

D-8 


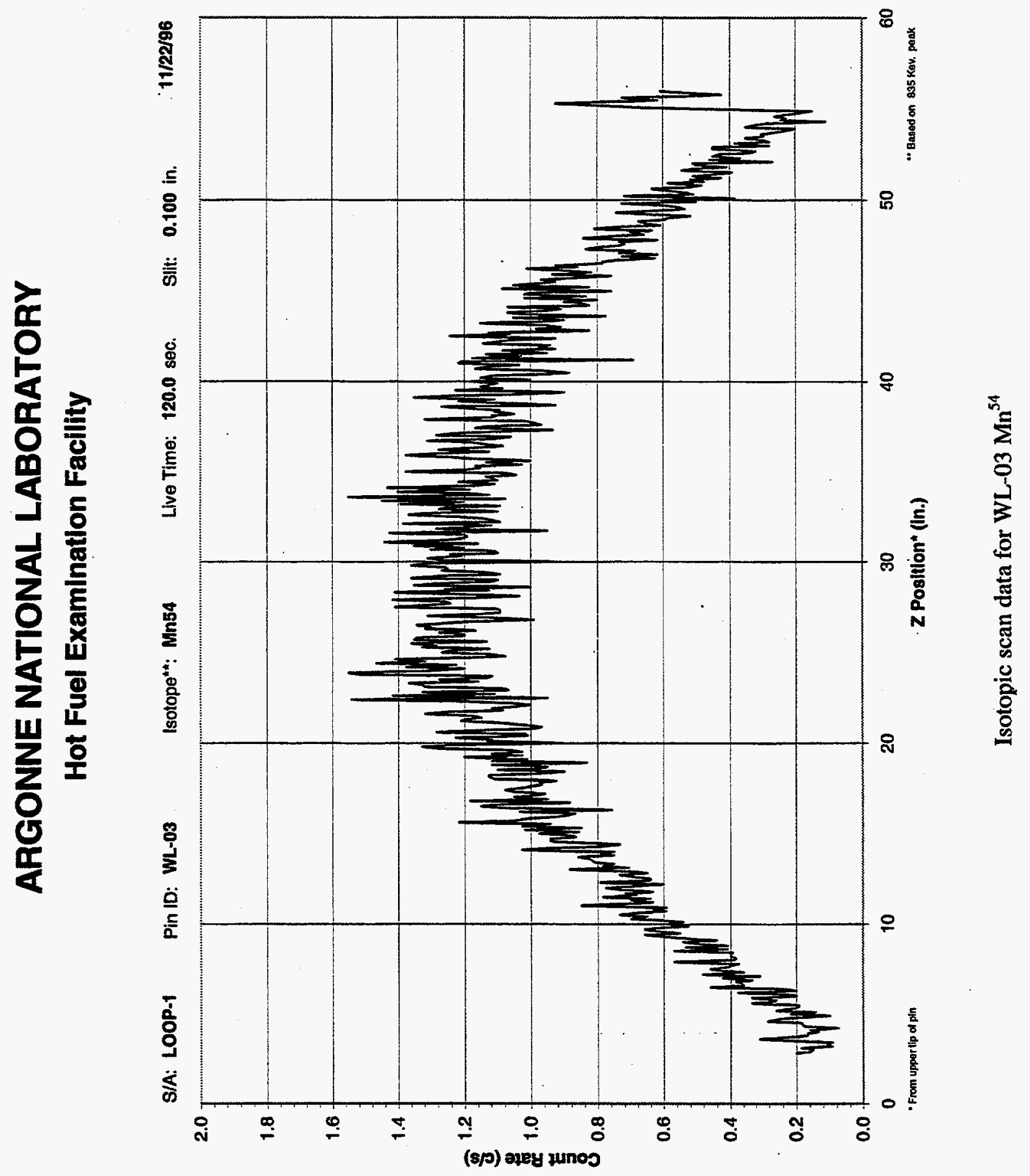




\section{ARGONNE NATIONAL LABORATORY}

\section{Hot Fuel Examination Facility}

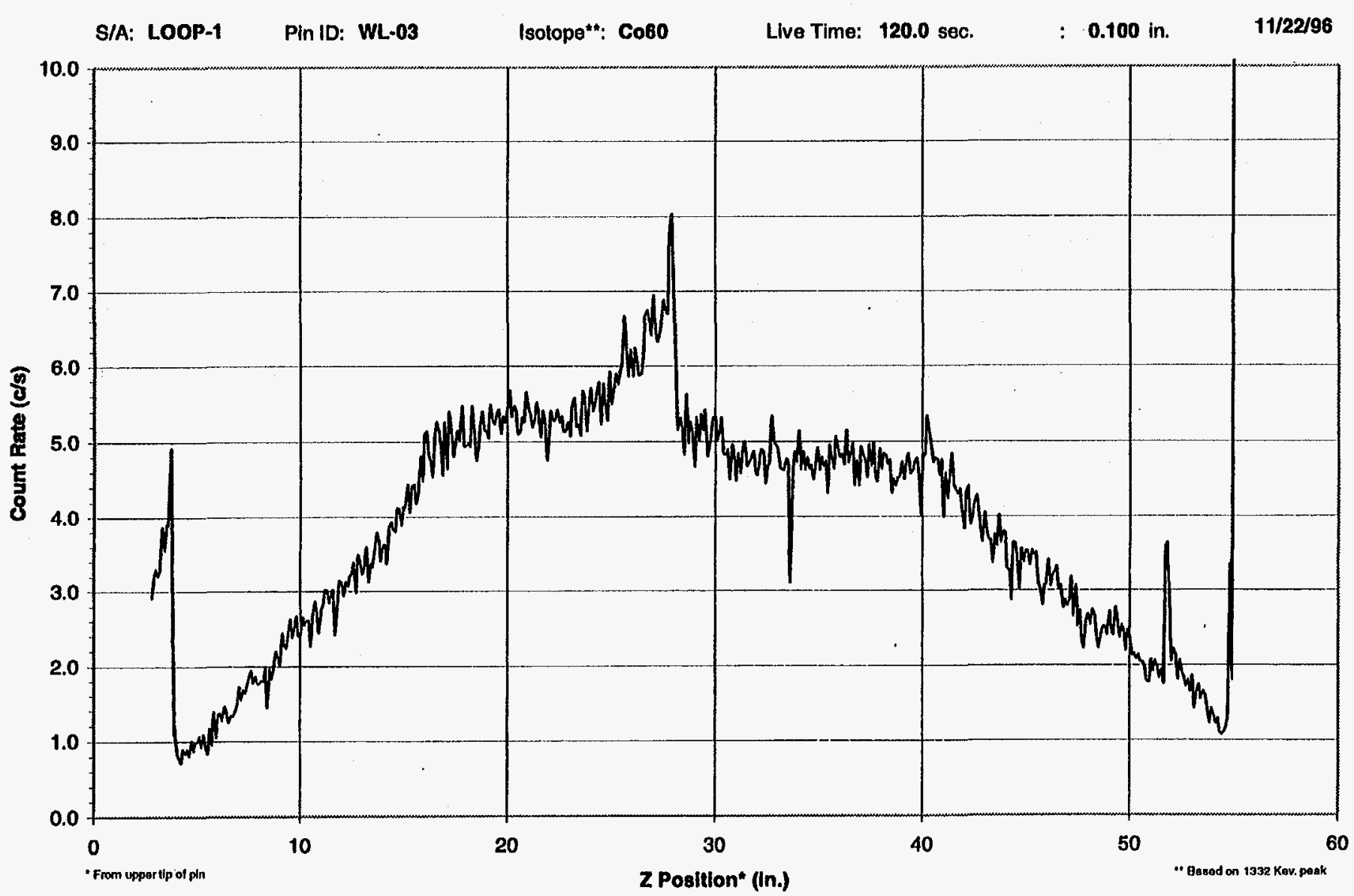

Isotopic scan data for WL-03 $\mathrm{Co}^{60}$ 


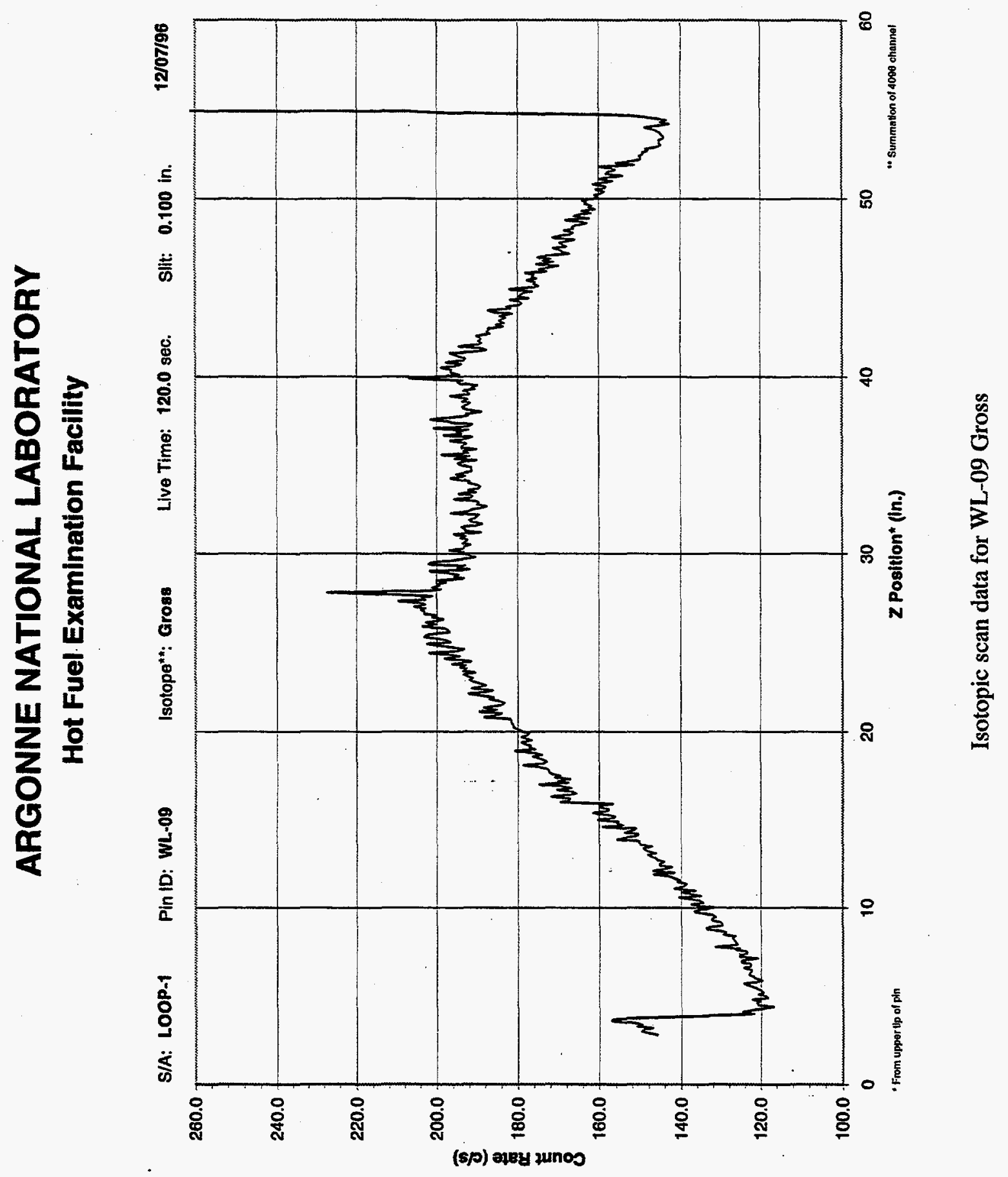




\section{ARGONNE NATIONAL LABORATORY}

\section{Hot Fuel Examination Facility}

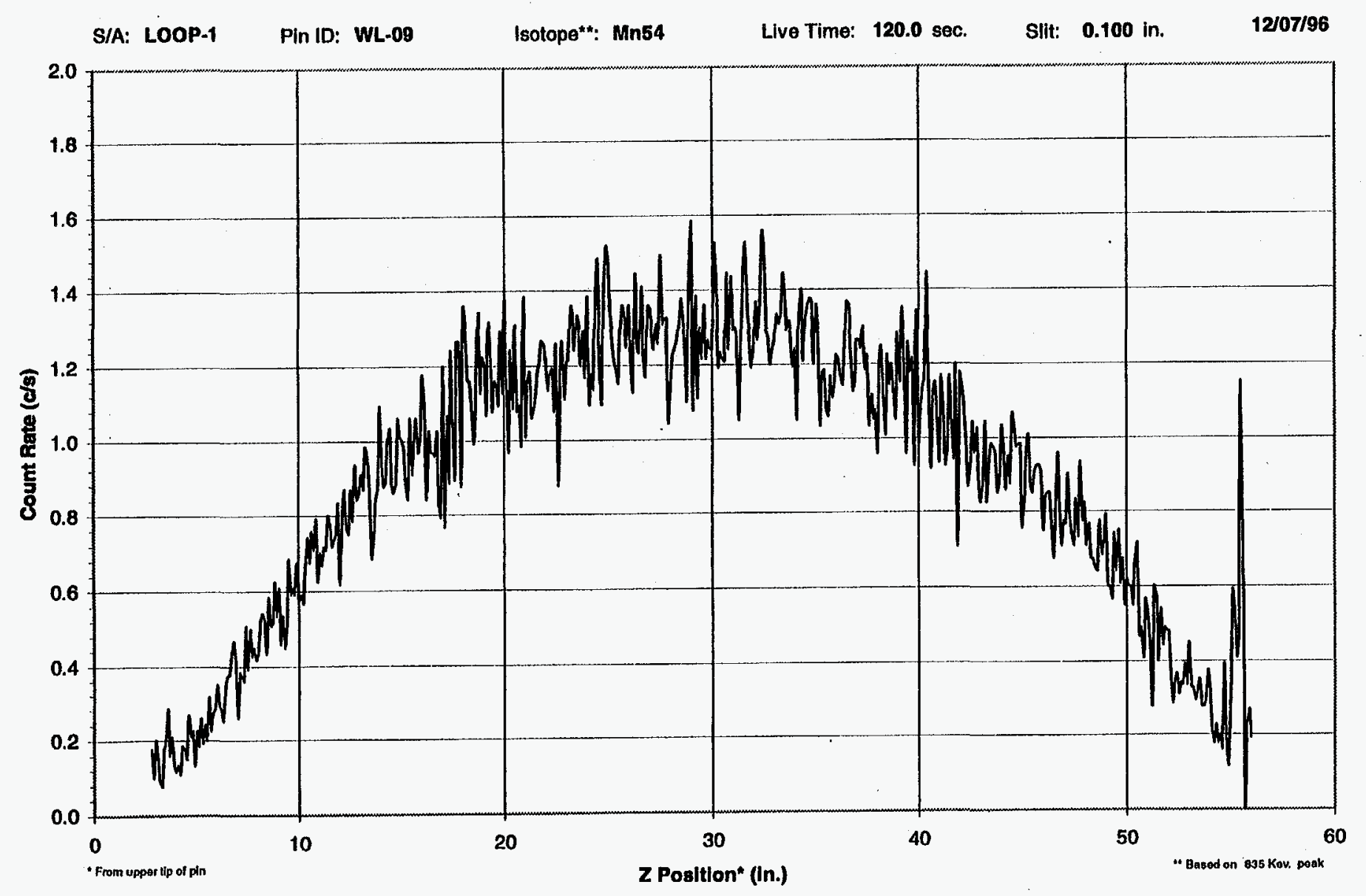

Isotopic scan data for WL-09 $\mathrm{Mn}^{54}$ 


\section{ARGONNE NATIONAL LABORATORY}

Hot Fuel Examination Facility

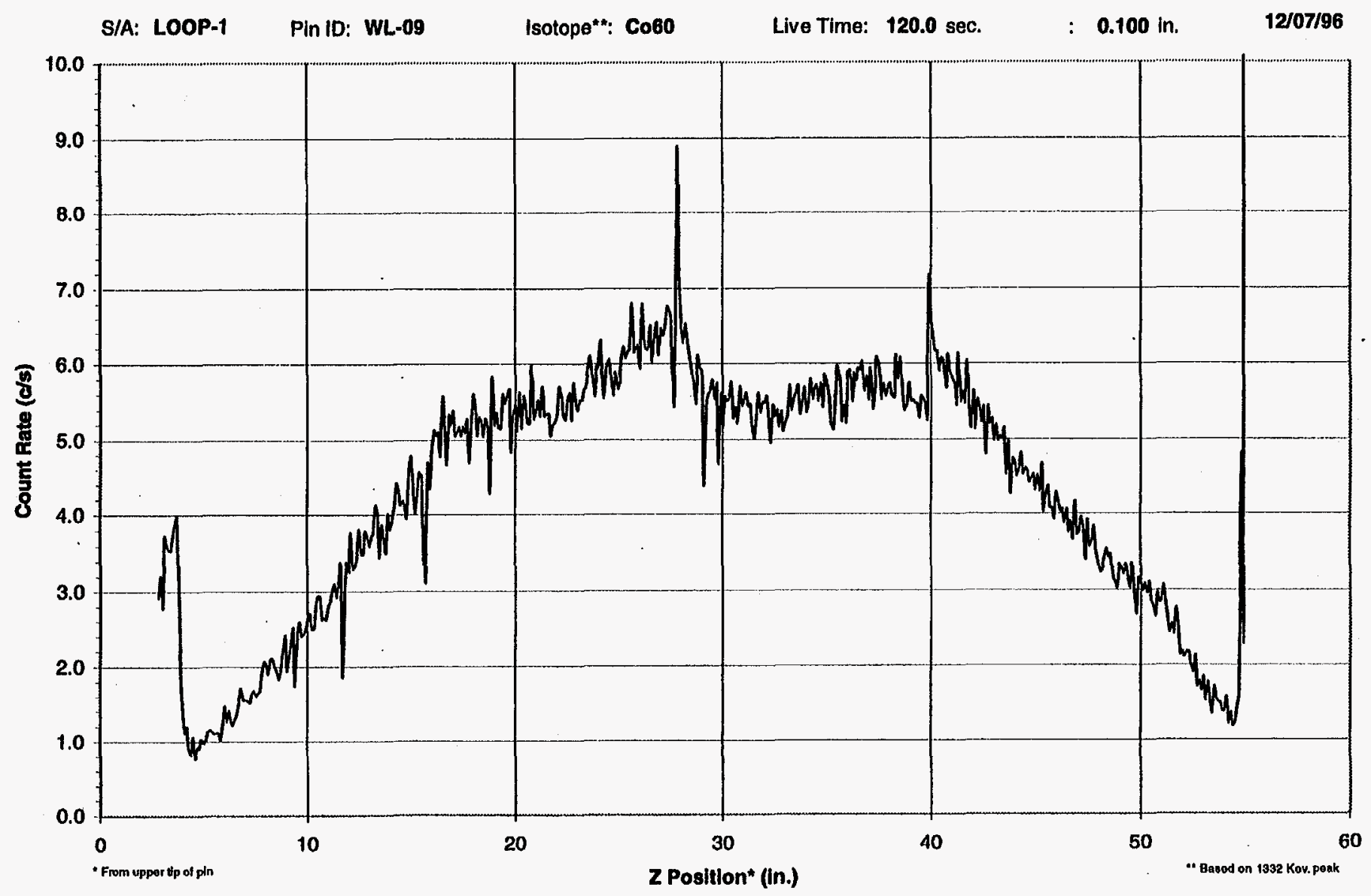

Isotopic scan data for WL-09 $\mathrm{Co}^{60}$ 


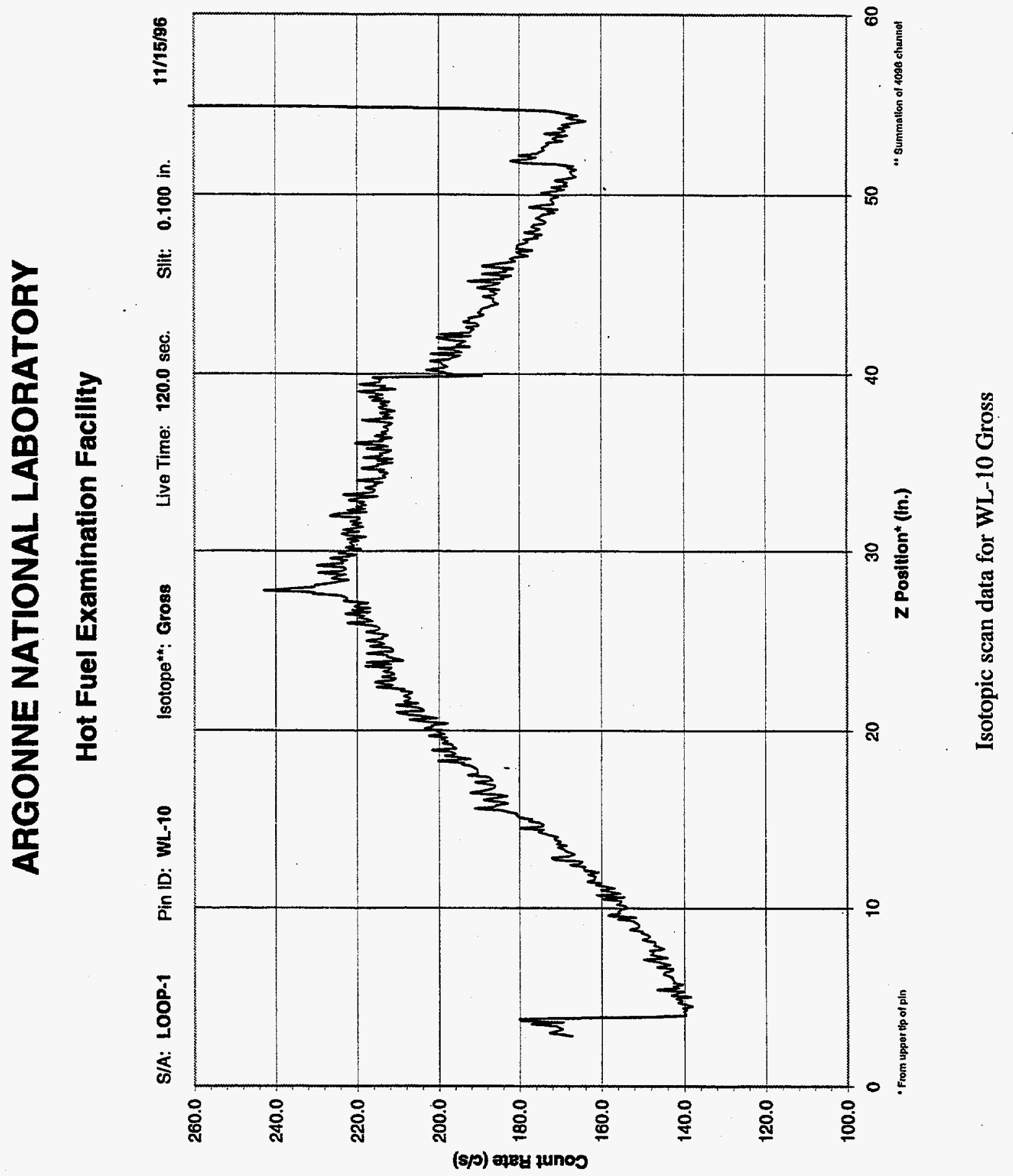




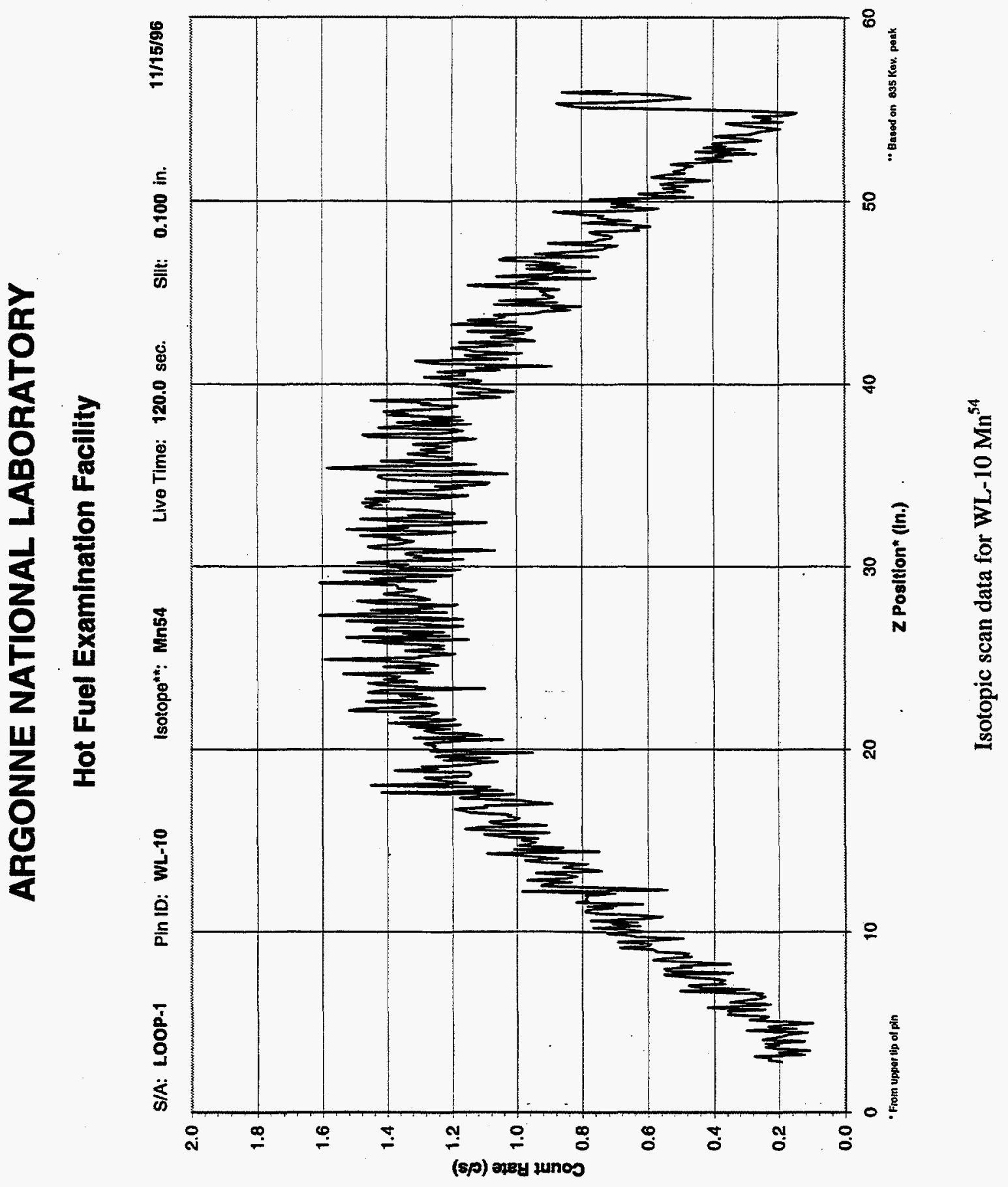

D-15 


\section{ARGONNE NATIONAL LABORATORY}

Hot Fuel Examination Facility

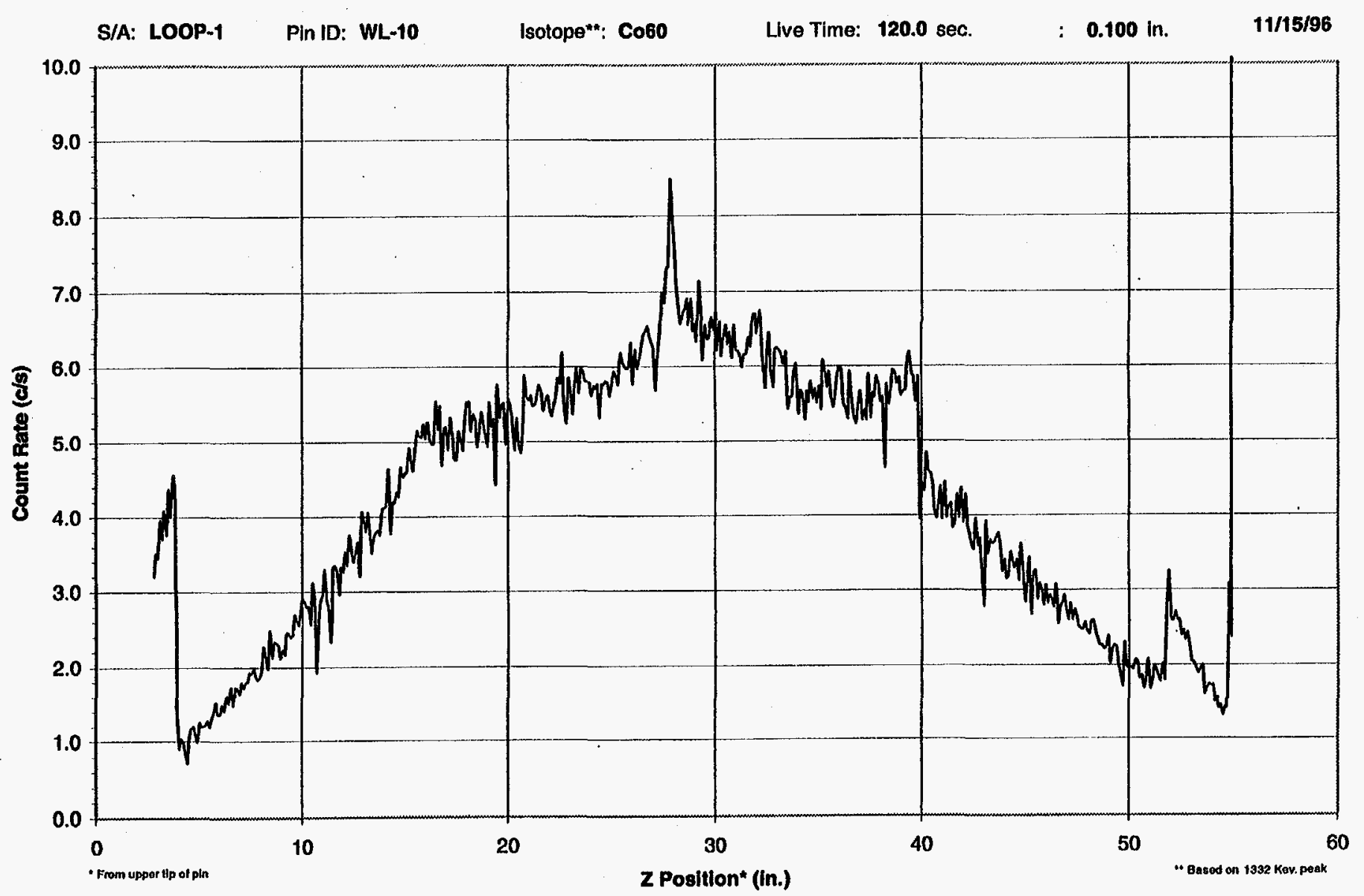

Isotopic scan data for WL-10 $\mathrm{Co}^{60}$ 


\section{ARGONNE NATIONAL LABORATORY}

\section{Hot Fuel Examination Facility}

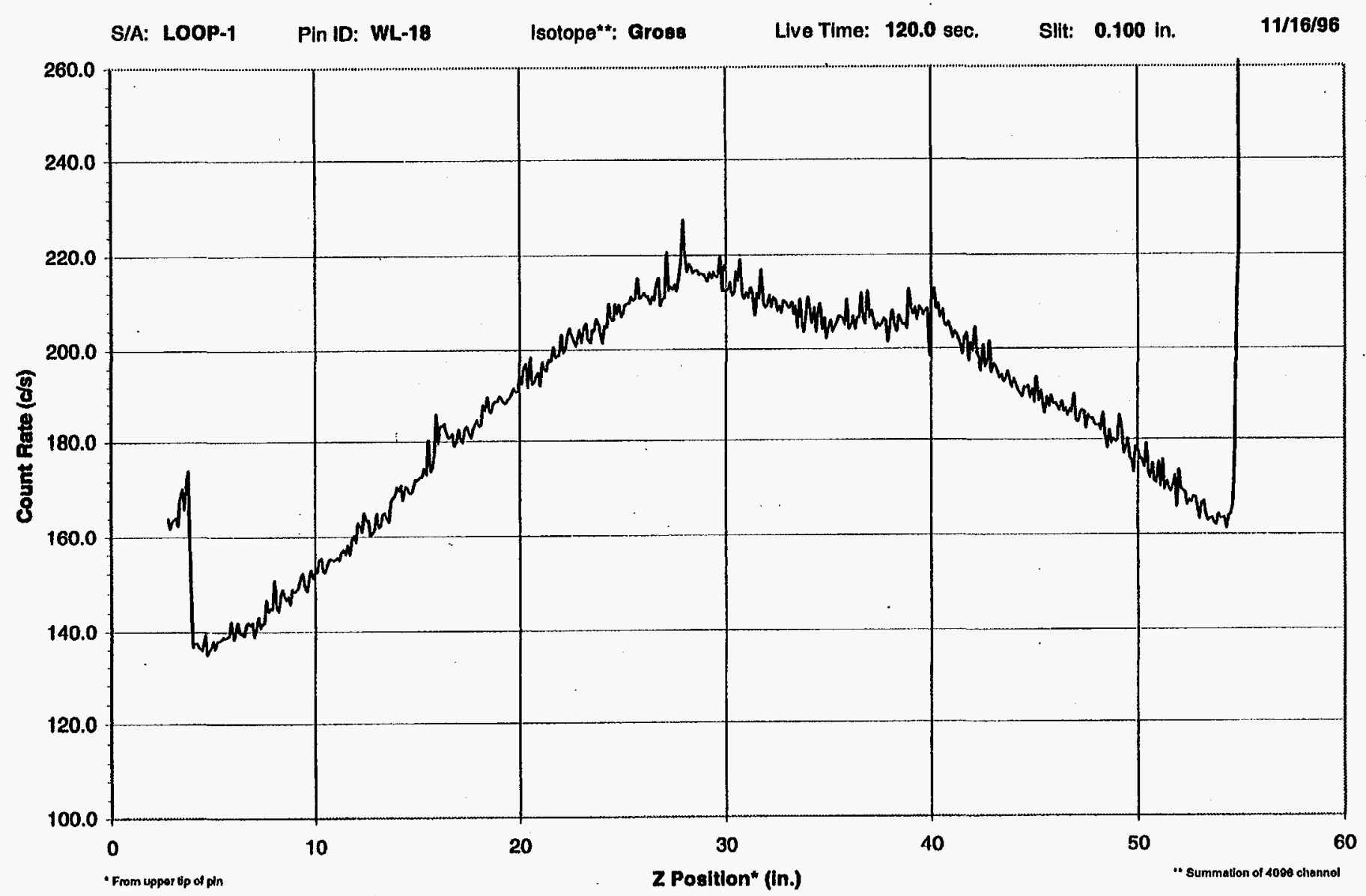

Isotopic scan data for WL-18 Gross 


\section{ARGONNE NATIONAL LABORATORY}

Hot Fuel Examination Facility

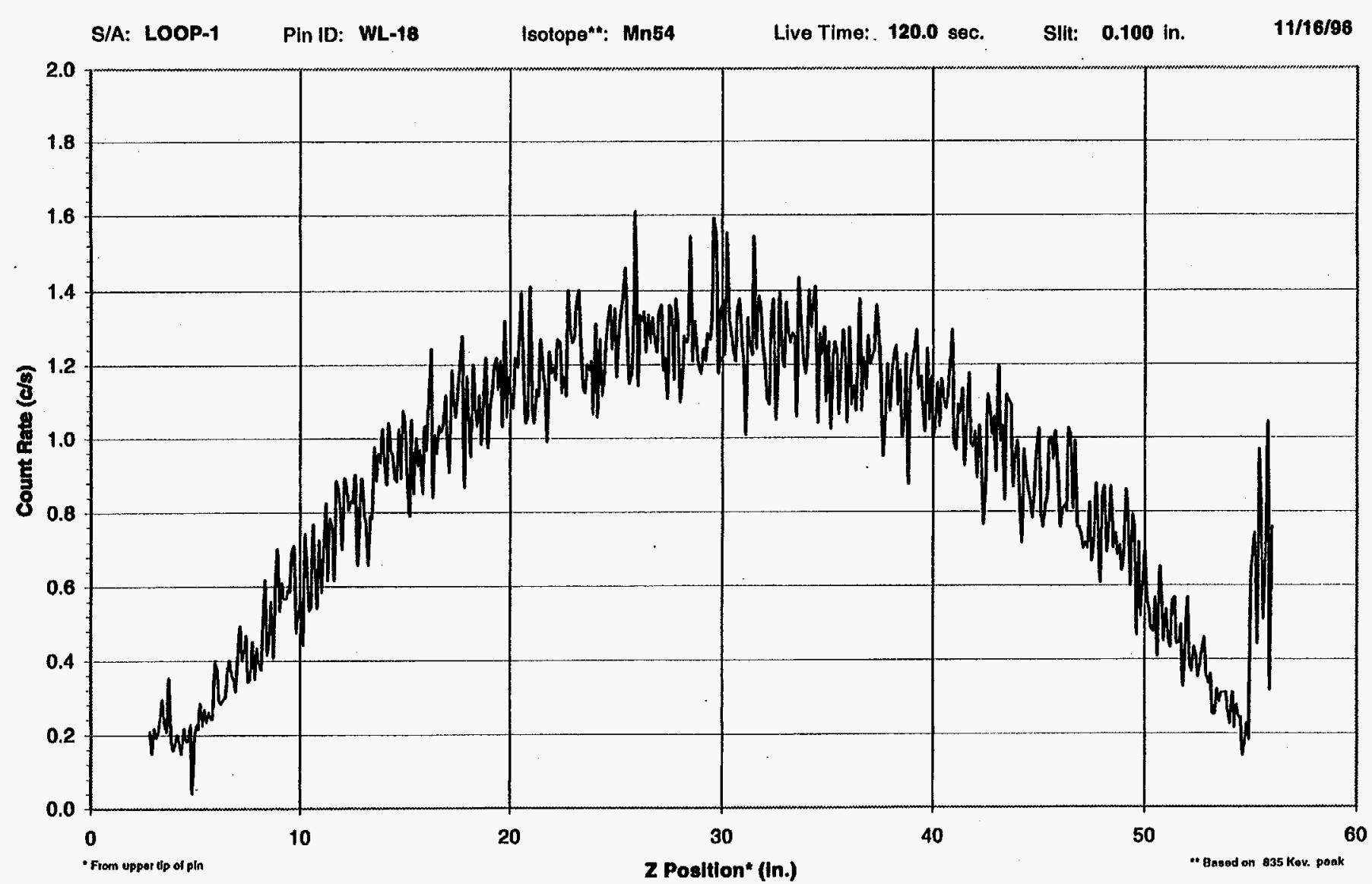

Isotopic scan data for WL-18 $\mathrm{Mn}^{54}$ 


\section{ARGONNE NATIONAL LABORATORY}

Hot Fuel Examination Facility

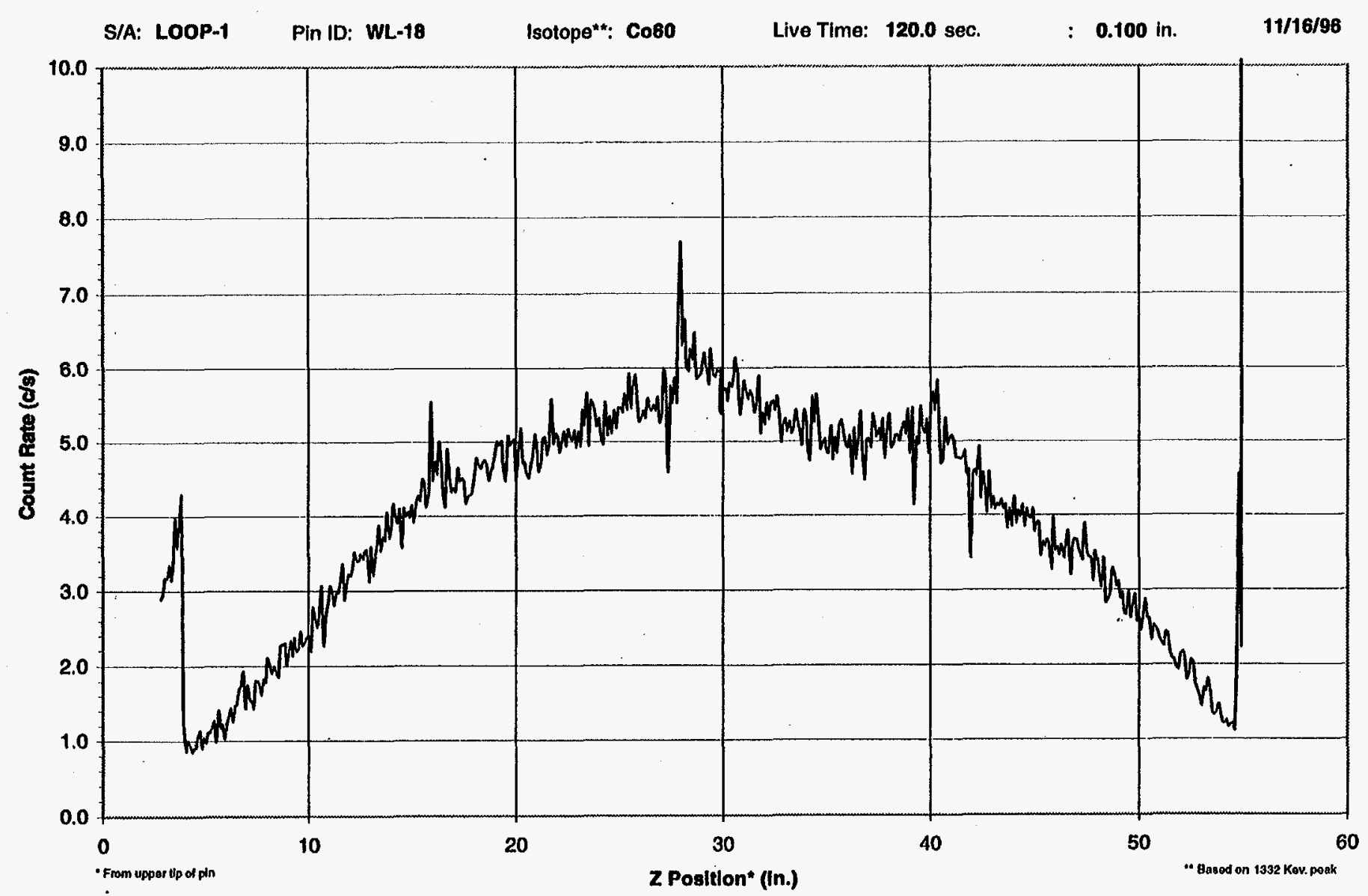

Isotopic scan data for WL-18 $\mathrm{Co}^{60}$ 


\section{ARGONNE NATIONAL LABORATORY}

\section{Hot Fuel Examination Facility}

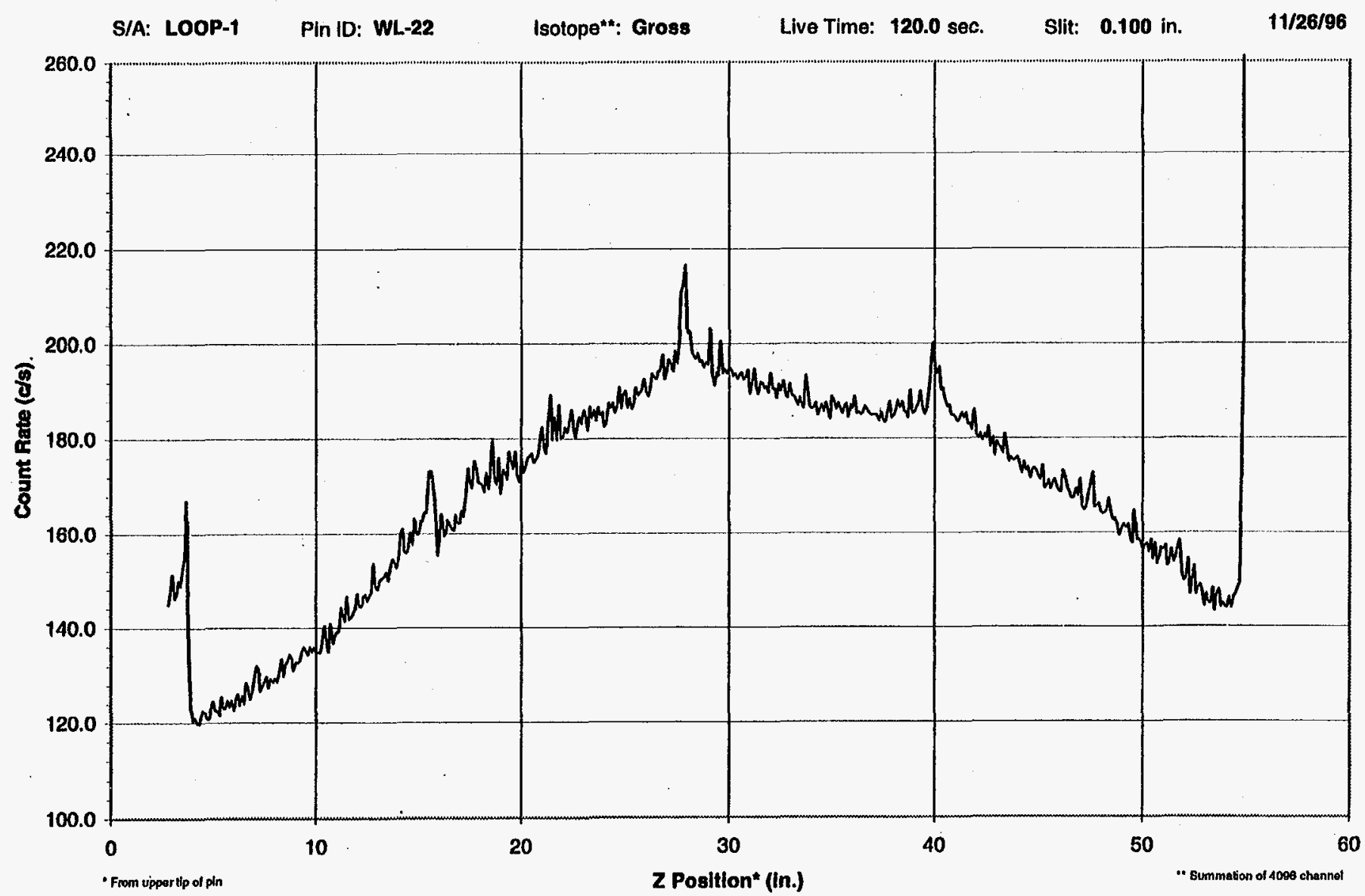

Isotopic scan data for WL-22 Gross 


\section{ARGONNE NATIONAL LABORATORY}

\section{Hot Fuel Examination Facility}

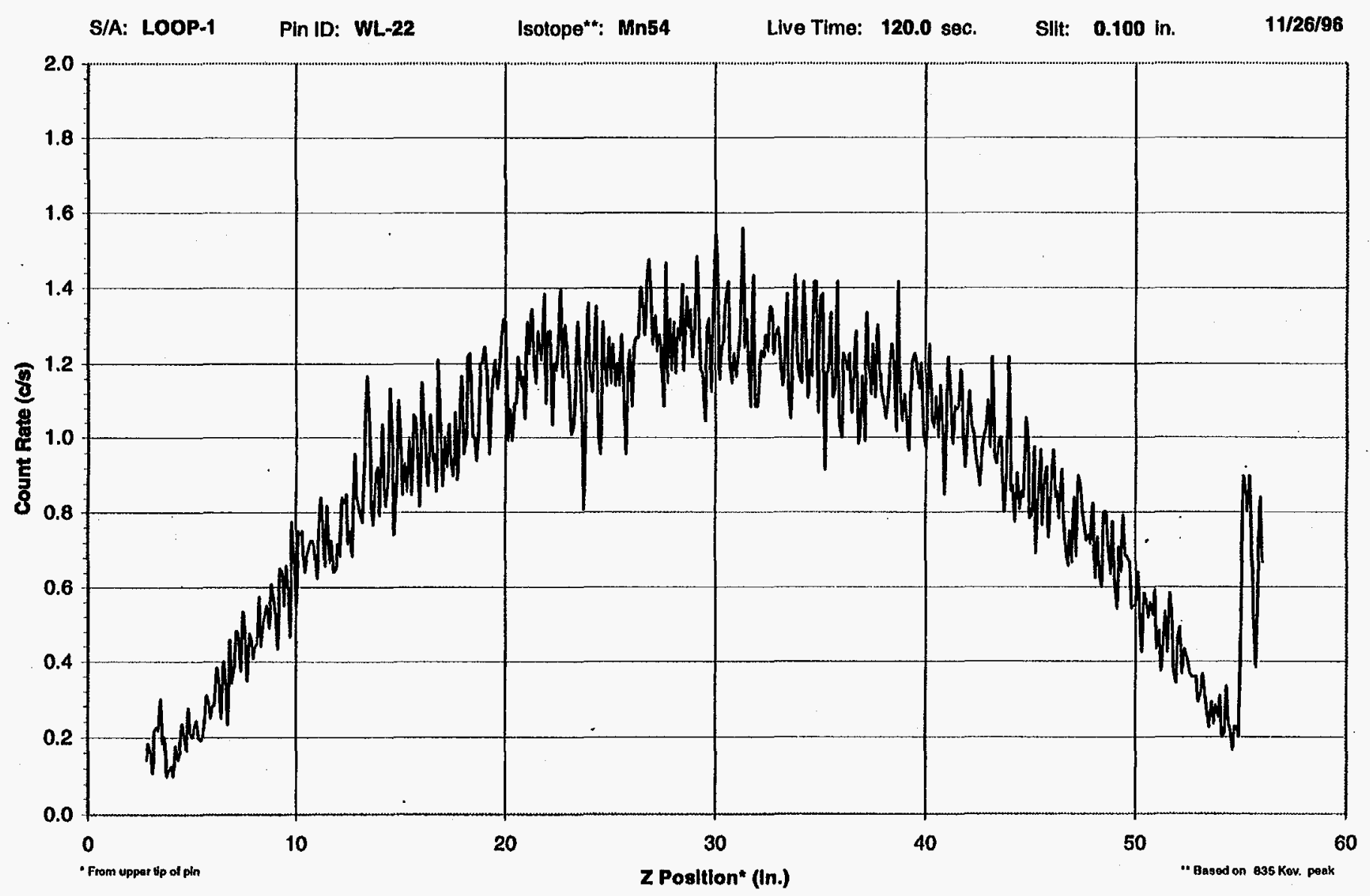

Isotopic scan data for WL-22 $\mathrm{Mn}^{54}$ 


\section{ARGONNE NATIONAL LABORATORY}

Hot Fuel Examination Facility

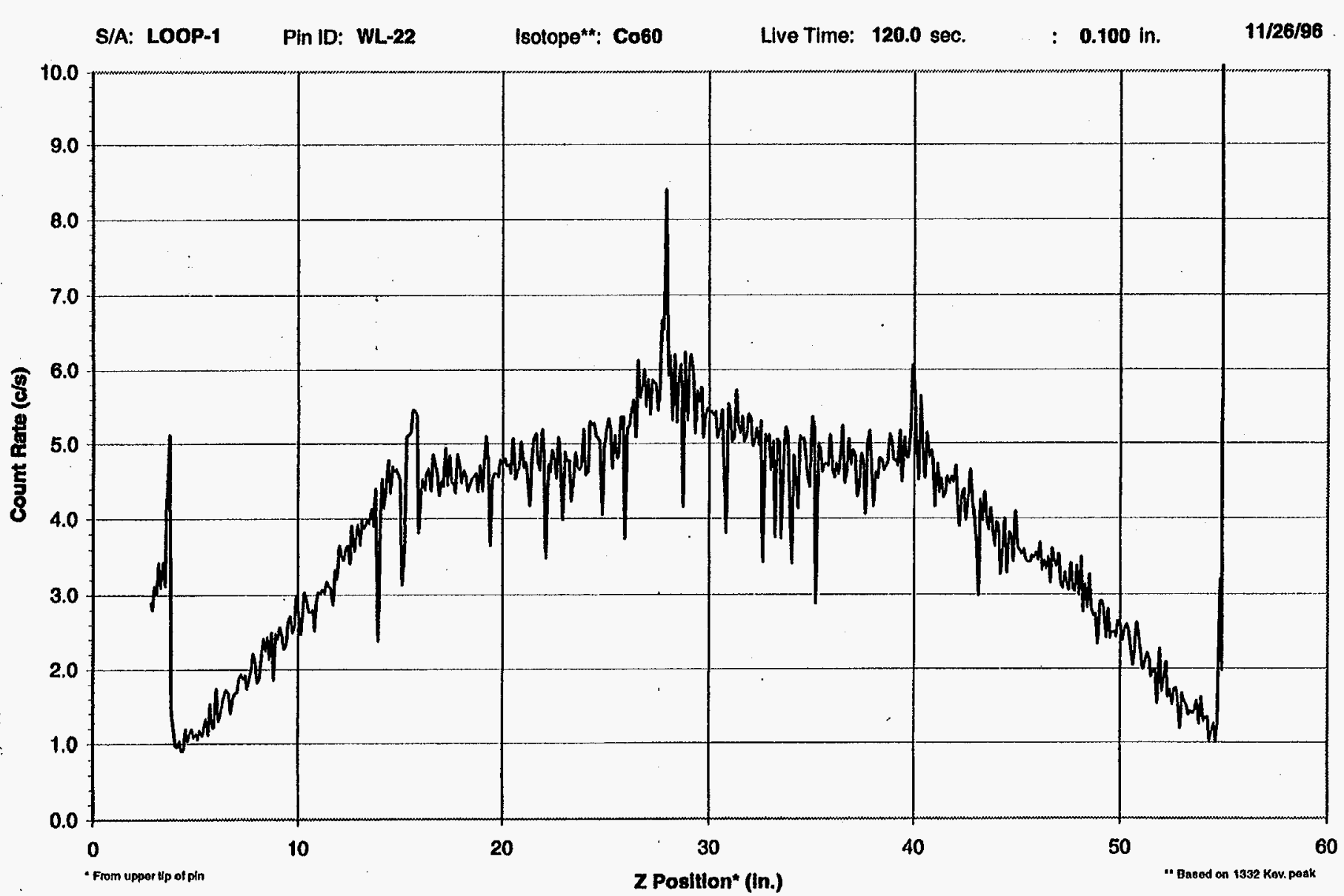

Isotopic scan data for WL-22 $\mathrm{Co}^{60}$ 


\section{ARGONNE NATIONAL LABORATORY}

Hot Fuel Examination Facility

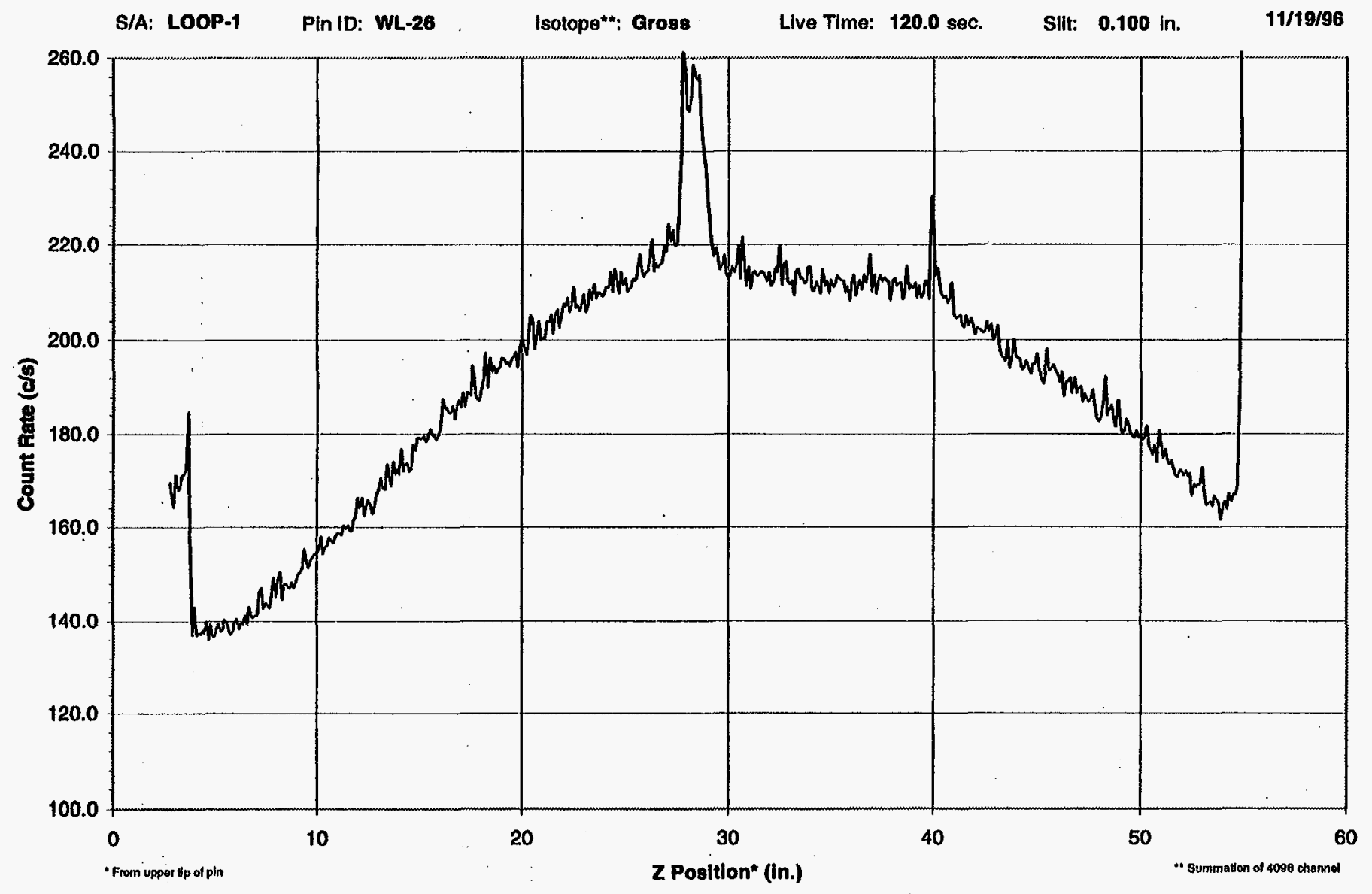

Isotopic scan data for WL-26 Gross 


\section{ARGONNE NATIONAL LABORATORY}

Hot Fuel Examination Facility

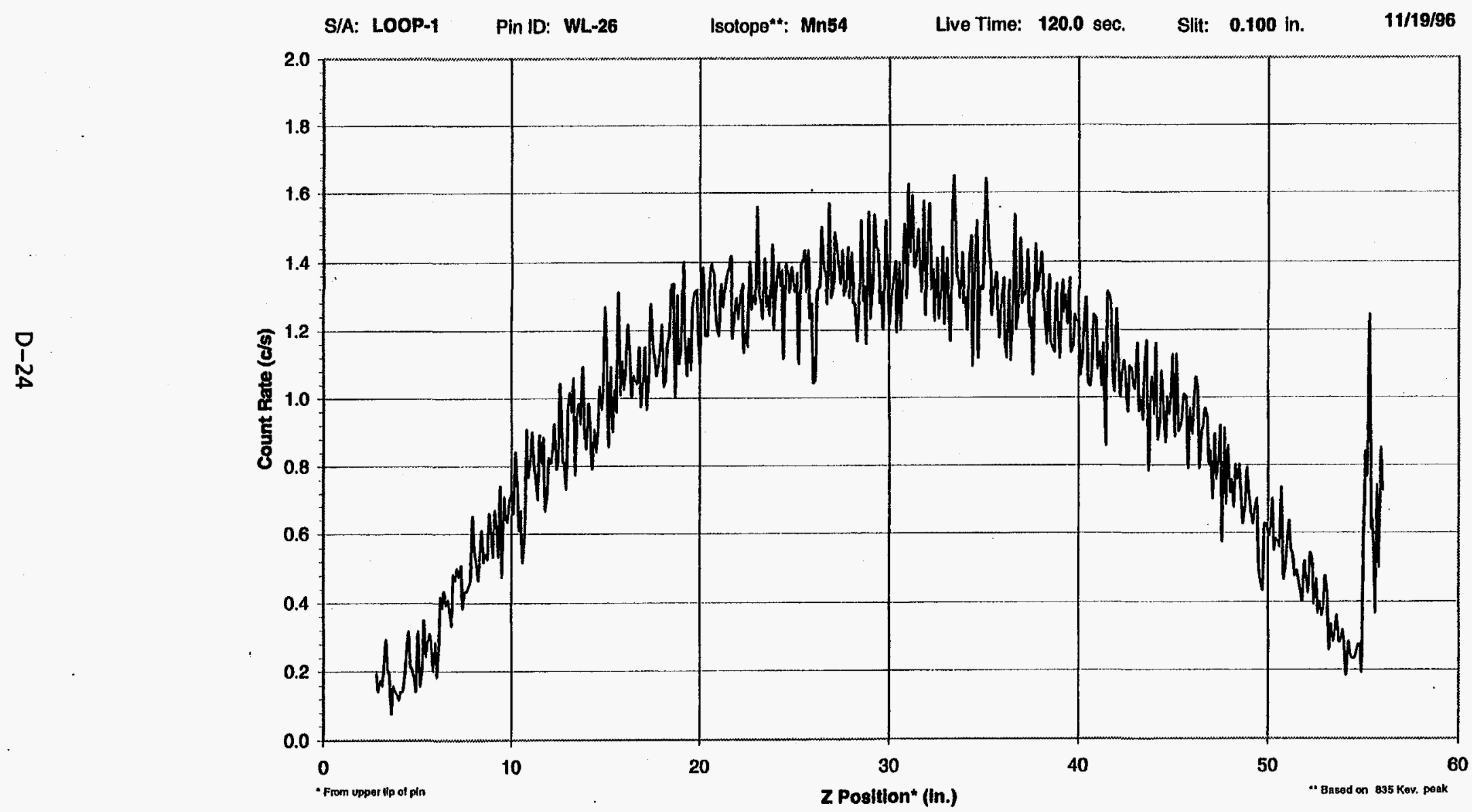

Isotopic scan data for WL-26 $\mathrm{Mn}^{54}$ 


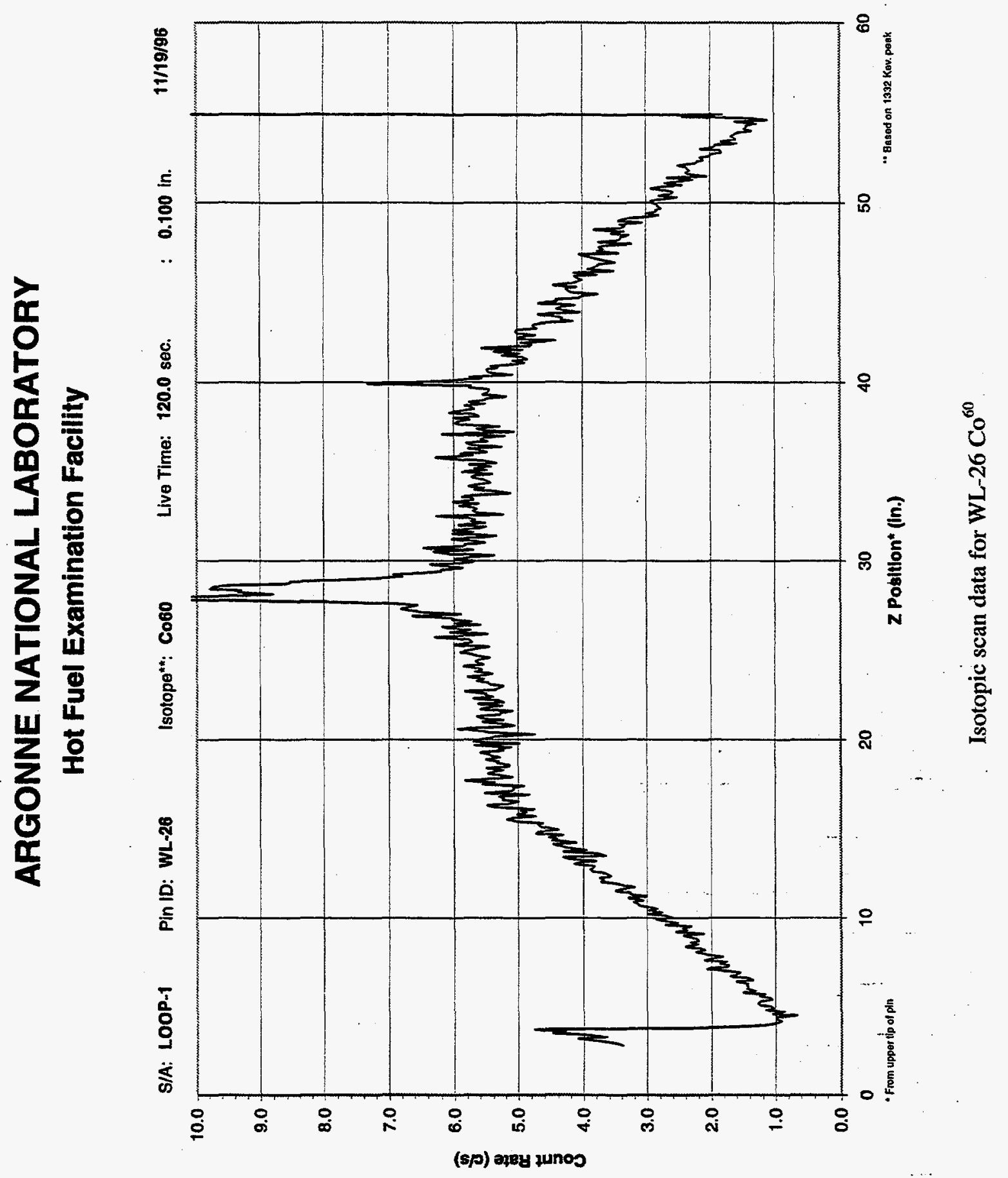




\section{ARGONNE NATIONAL LABORATORY}

\section{Hot Fuel Examination Facility}

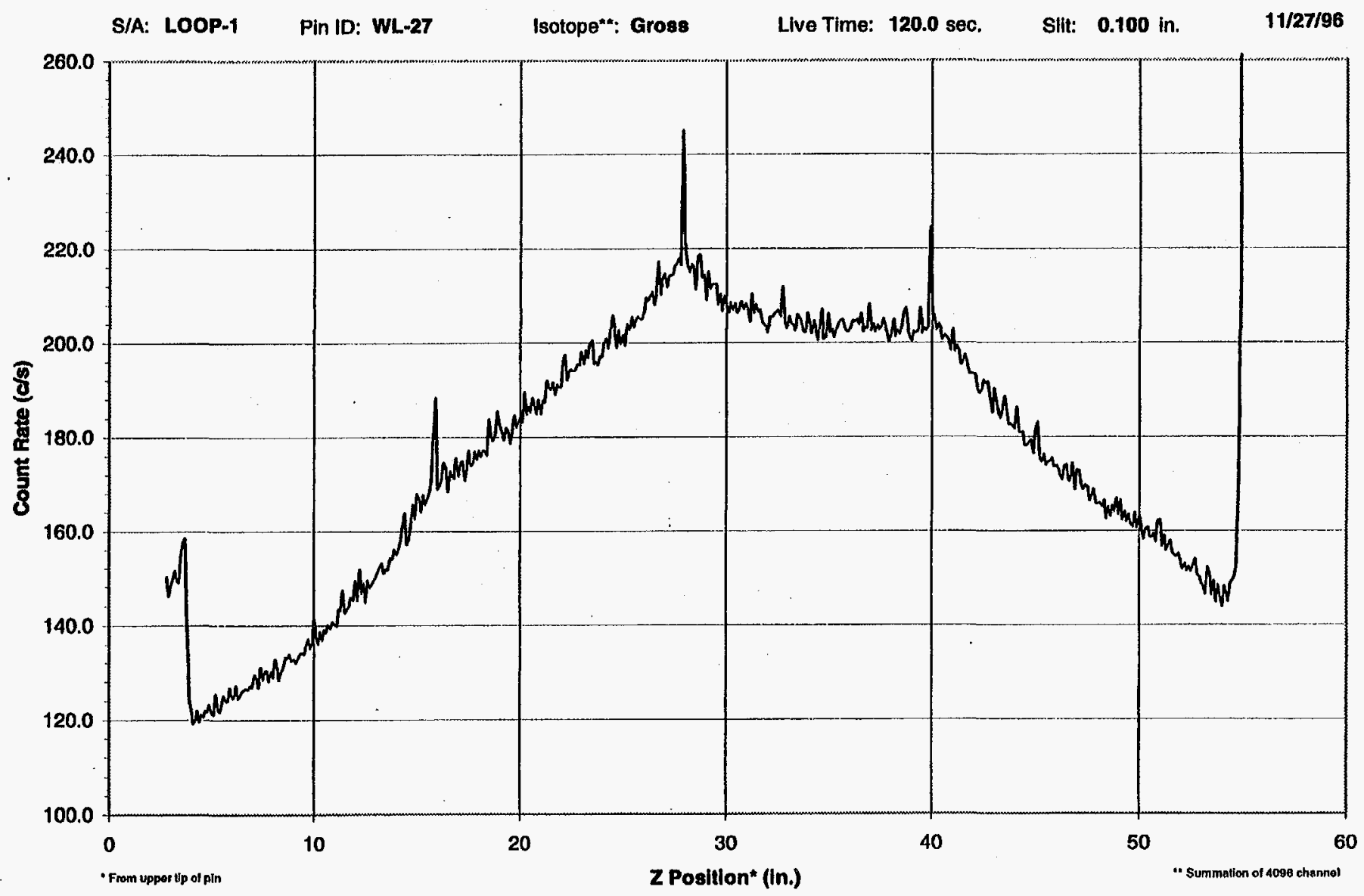

Isotopic scan data for WL-27 Gross 


\section{ARGONNE NATIONAL LABORATORY}

Hot Fuel Examination Facility

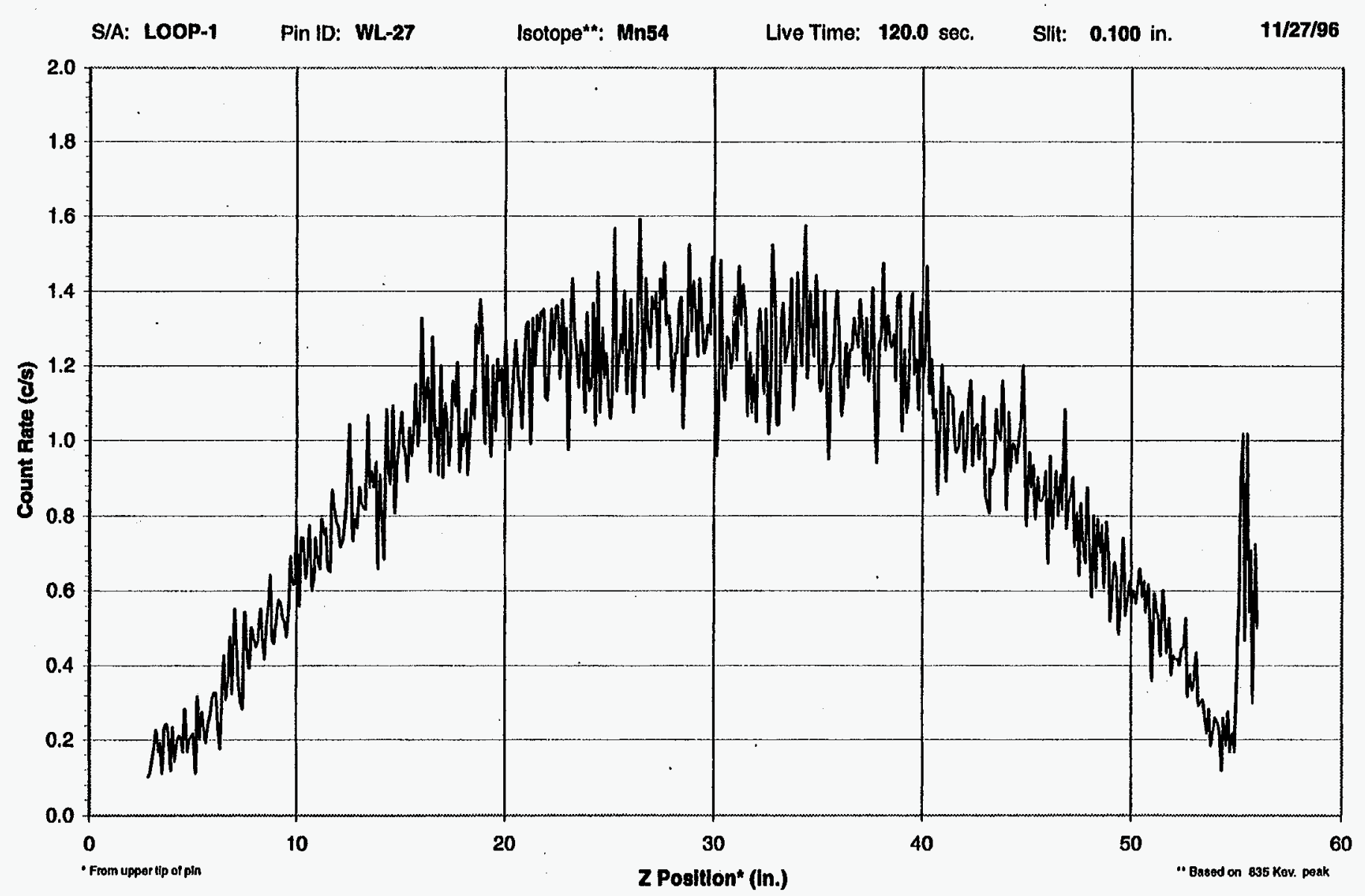

Isotopic scan data for WL-27 $\mathrm{Mn}^{54}$ 


\section{ARGONNE NATIONAL LABORATORY}

\section{Hot Fuel Examination Facility}

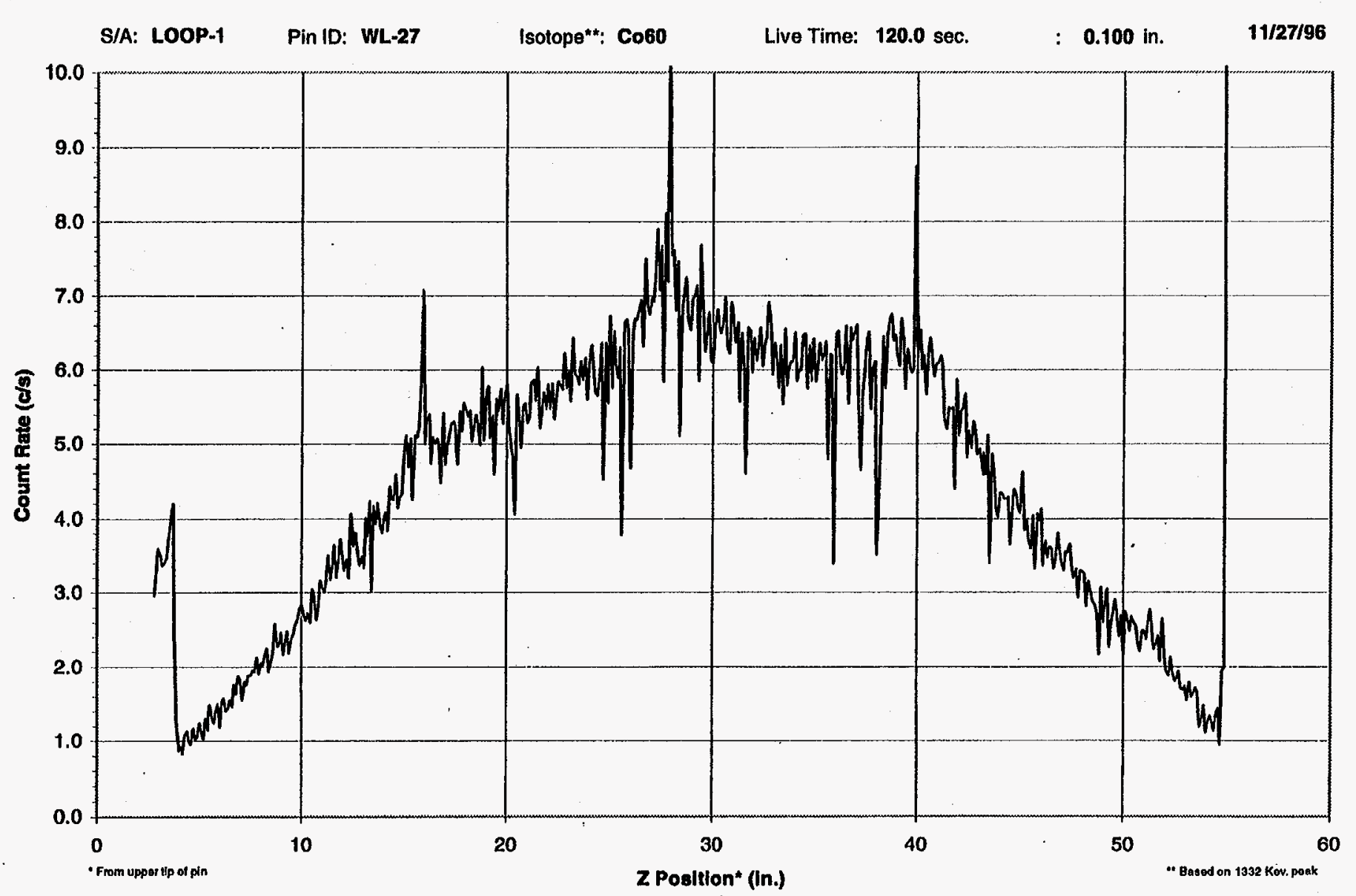

Isotopic scan data for WL-27 Co ${ }^{60}$ 


\section{ARGONNE NATIONAL LABORATORY \\ P. O. Box 2528, Idaho Falls, Idaho $83403-2528$}

\section{ENGINEERING DIVISION}

(TREAT, ZPPR, and HFEF)
EXAminatiON

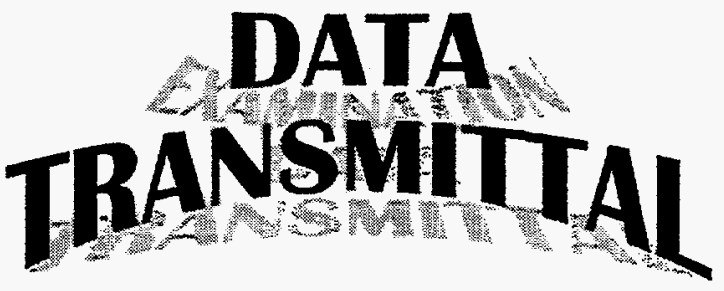

DATE:

March 14, 1997
R. L. Bratton
ADDRESS:

Lockheed Martin Idaho Technologies, Co.

EROB MS-3760

Idaho Falls, ID 834

MATERIAL EXAMINED:

Two Target Rods from CLWR S1/B1 Test

TYPE OF EXAMINATION:

\section{Isotopic Gamma Scans}

FORM OF DATA:

Charts, User Notes, and CD-ROM (2)

NOTES:

CC: wAdtachments

S1/B1 file (charts only)

Cc: w/o Atachments

APPROVED:

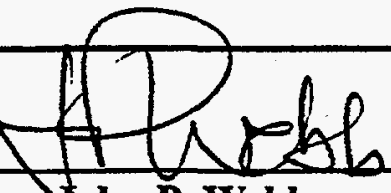

tohn P. Webb, cognzant engineER
Questions regarding this data transmittal should be directed to the Cognizant Engineer at extension (208) 533-7773. 


\title{
ARGONNE NATIONAL LABORATORY Hot Fuel Examination Facility \\ PRECISION GAMMA SCANNING
}

\author{
USER NOTES
}

1. The Precision Gamma Scanner - II (PGS-I) is designed to intermittently traverse rod-like objects in front of a small-solid-angle collimator for collection of gamma spectra at a series of positions. Usually the increment of traverse is set equal to the collimator slit opening to provide complete object coverage. This process is called scanning.

2. The gamma-ray spectroscopy sub-system consists of an ultra-pure germanium (UPG) detector with a nominal efficiency of $10 \%$ (relative to a 2" $\times 2$ " NaI detector). Active Compton-suppression is provided by detecting the time-coincidence of Compton events from a large volume $\mathrm{NaI}$ guard detector surrounding the UPG. A background suppression ratio of 6-10 (based on Co-60 peaks) is achieved.

3. The PGS-II is designed and calibrated to position the center of the collimator slit (with respect to the upper tip of the scanned object) to an accuracy of \pm 0.005 in. for each positional measurement.

4. Data can be acquired in either gross or spectral mode. Gross mode counts gammas without regard to their energies. Spectral mode maps gamma energies (in the range of 50-1600 Kev) into 4096 channels (memory locations).

5. The collimator slit is adjustable from $0-100$ mils, and is generally fixed for any given scan. Slit opening is reported in all data tables and charts.

6. The live time of each positional count is infinitely adjustable, but is usually fixed for any given scan. The live time used for a scan is reported in all data tables and charts.

7. Spectral analysis is performed by post-processing the collected data. The analysis is based on a fixed Channel-to-Energy calibration where:

$$
\text { Channel }=2.5 E_{\gamma}(\mathrm{Kev})
$$

Selected isotopic peaks of interest are located within each spectrum, and a gross peak area is calculated by simple trapezoidal integration. Background for each peak is estimated by fitting (least squares) a straight line to the regions immediately above and below the peak. This background is subtracted from the gross peak area to generate the net peak area (in counts).

8. The isotopic count rate (net peak area divided by the live time) is usually reported at each position in chart and tabular form.

9. If requested, all isotopic data can be corrected for decay using the standard equation:

$$
A_{t}=A_{0} e^{-\lambda t}
$$

This correction is only required when short-lived isotopes are being measured, and it removes the effect of decay during a given scan and between scans of sibling rods.

10. A measure of gross activity is obtained by summing the counts in all 4096 channels, and dividing by the live time. These data are usually reported in chart and tabular form.

11. Spectral data are sometimes provided in digital form on CD-ROM's or floppy disks. The data are stored as plain ASCII data in DOS file format (i.e., records are terminated by $<C R>L F>$ pairs). The first 10 records contain self-explanatory header information, including the units of the data fields. Below the header records, spectral activity data are stored in tab-delimited fields as follows:

$$
<\text { Position }>\langle t a b><\text { Gross }><t a b<\text { Isotope-1 }>\langle t a b>\text {... }<\text { Isotope-N }>C C R>L F>
$$

12. Gross-mode data are not available (at this time) in digital form. 


\section{ARGONNE NATIONAL LABORATORY}

\section{Hot Fuel Examination Facility}

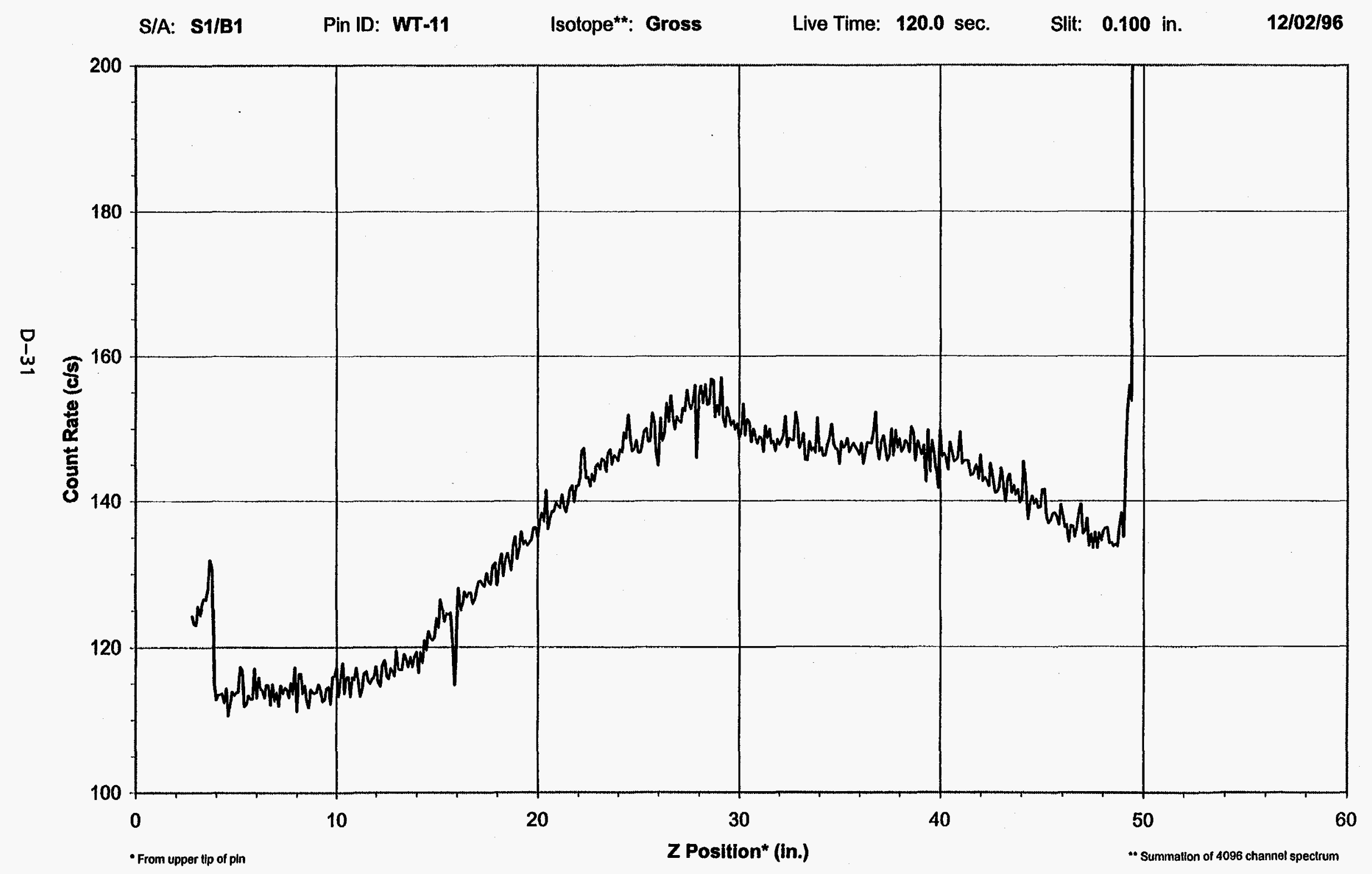



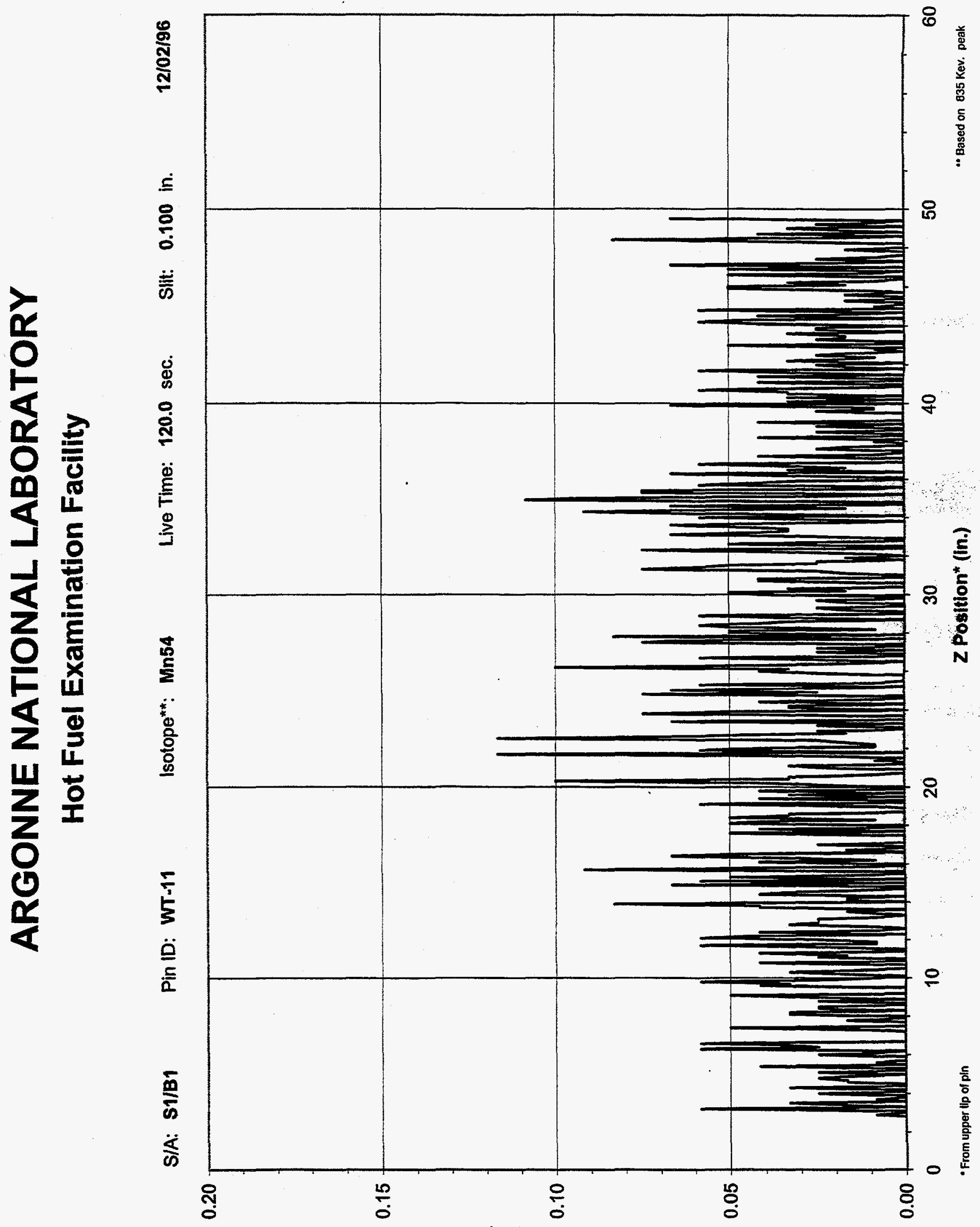

(sp) eqey zunos 


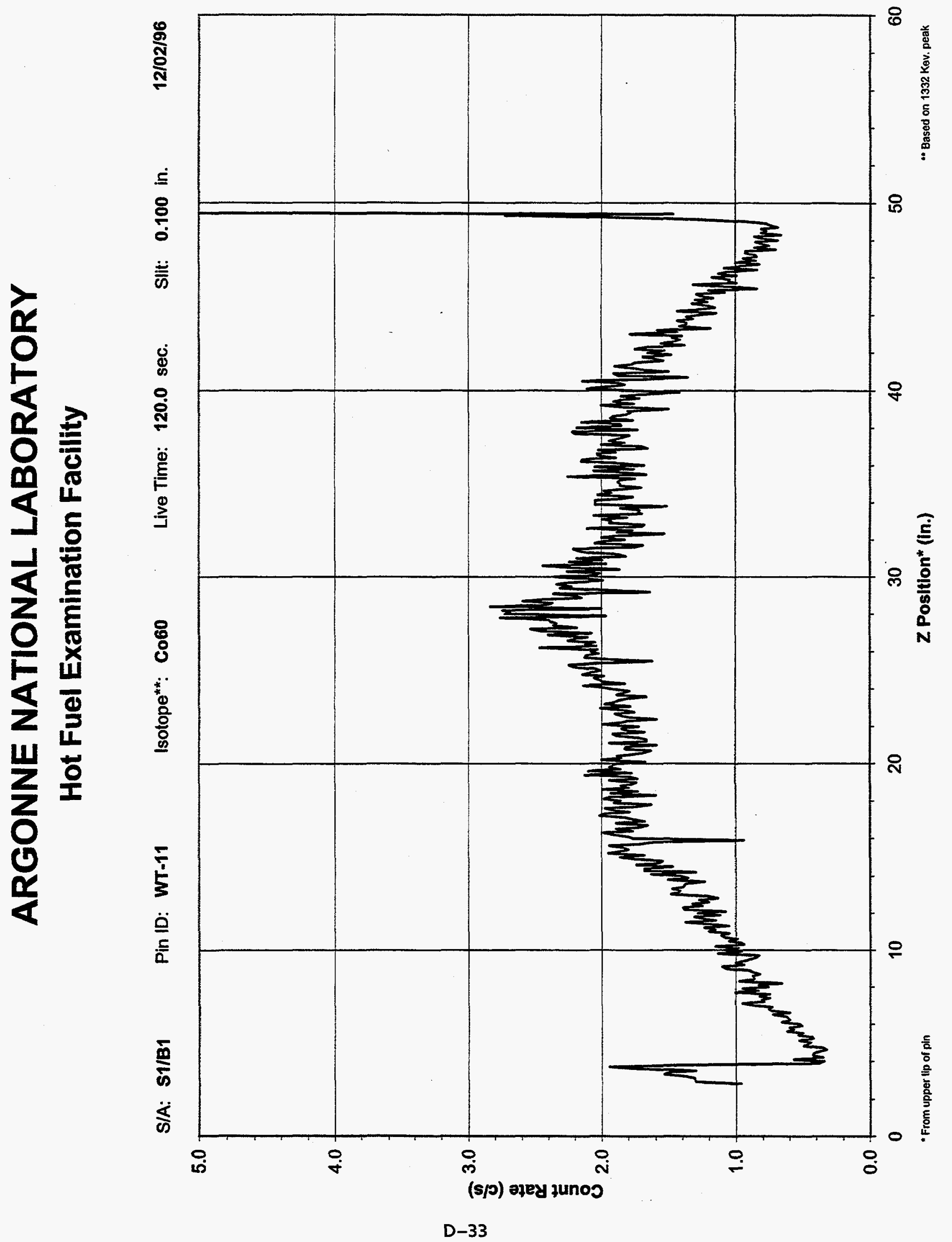




\section{ARGONNE NATIONAL LABORATORY}

\section{Hot Fuel Examination Facility}

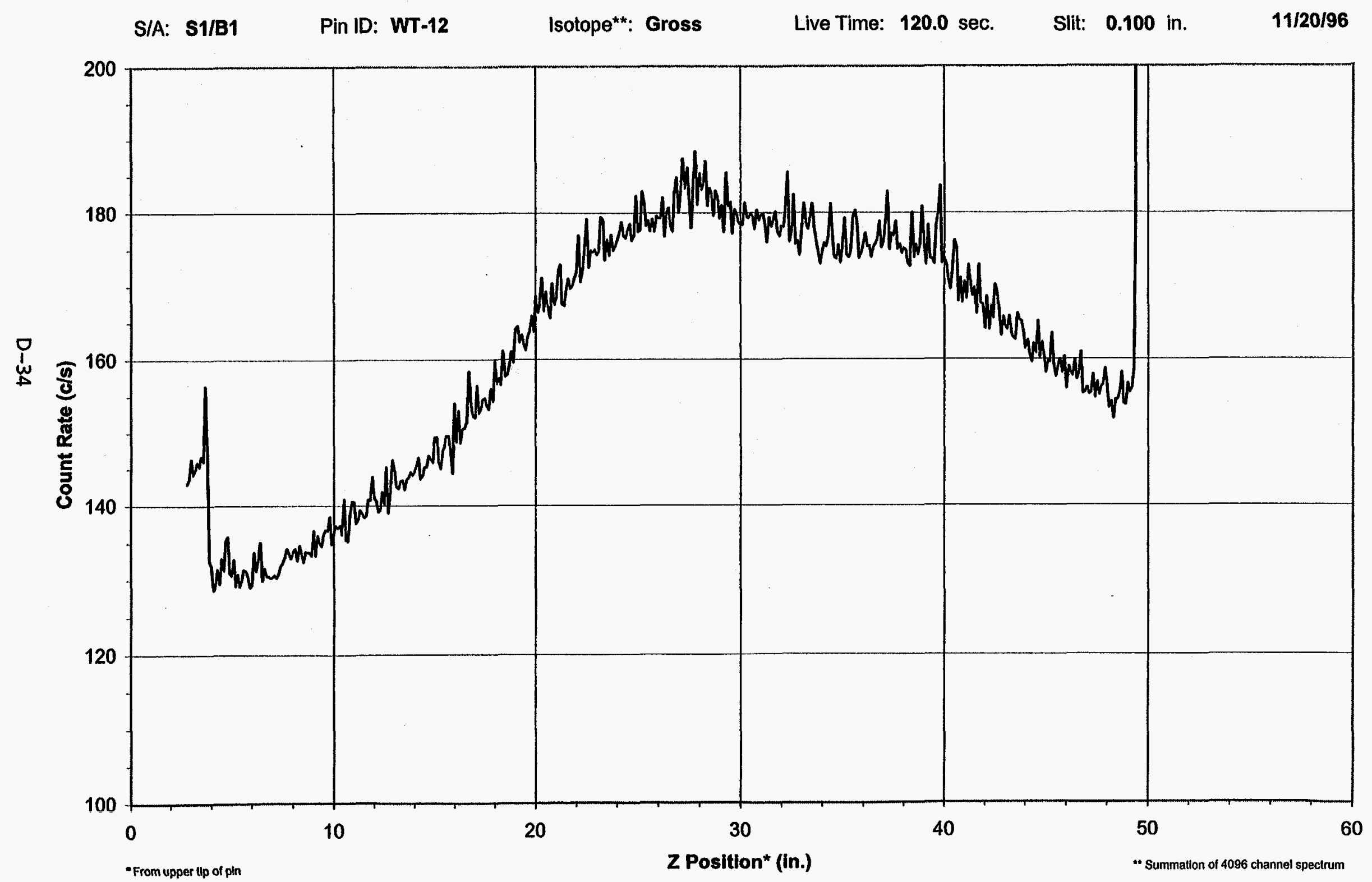




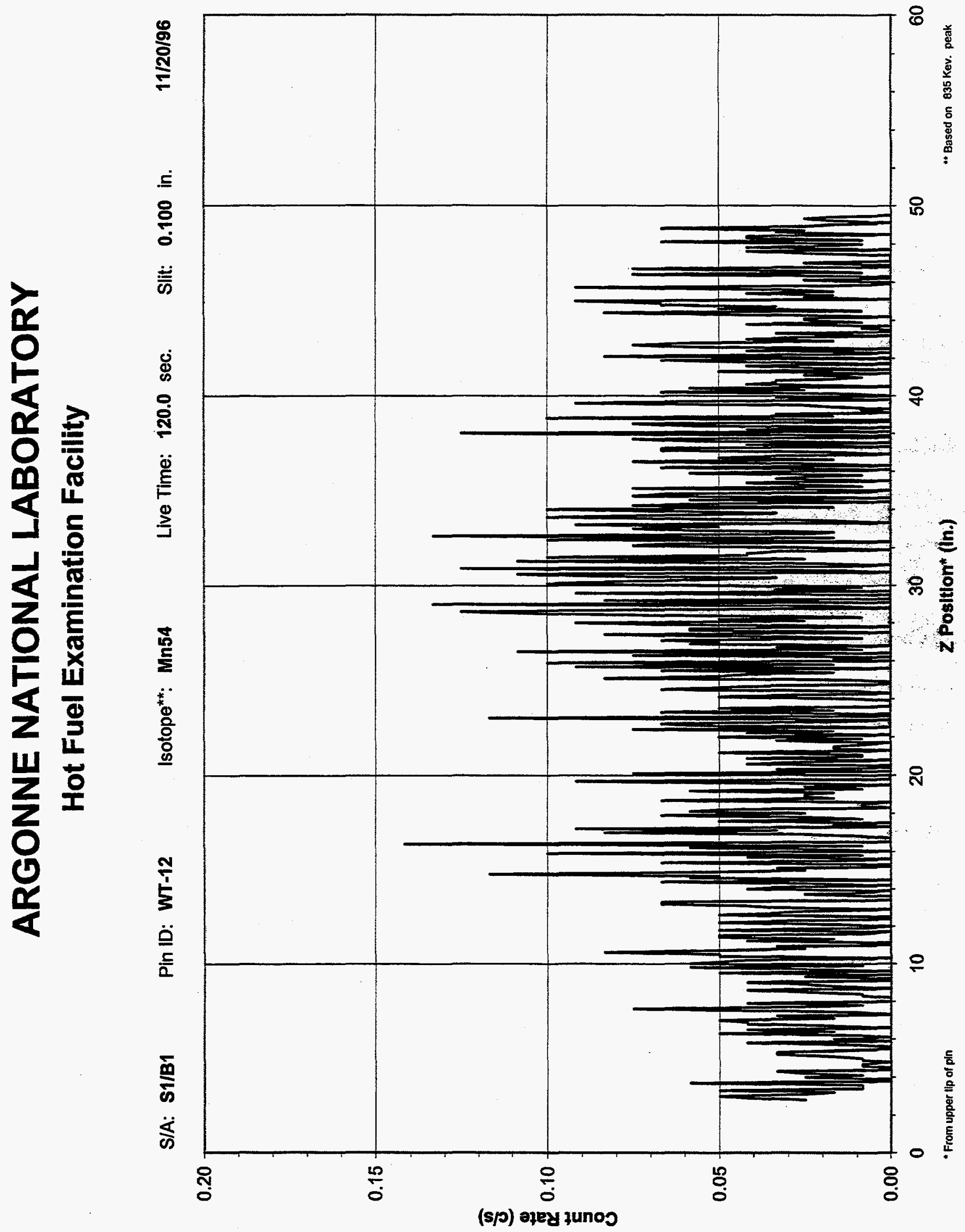

D-35 


\section{ARGONNE NATIONAL LABORATORY}

Hot Fuel Examination Facility

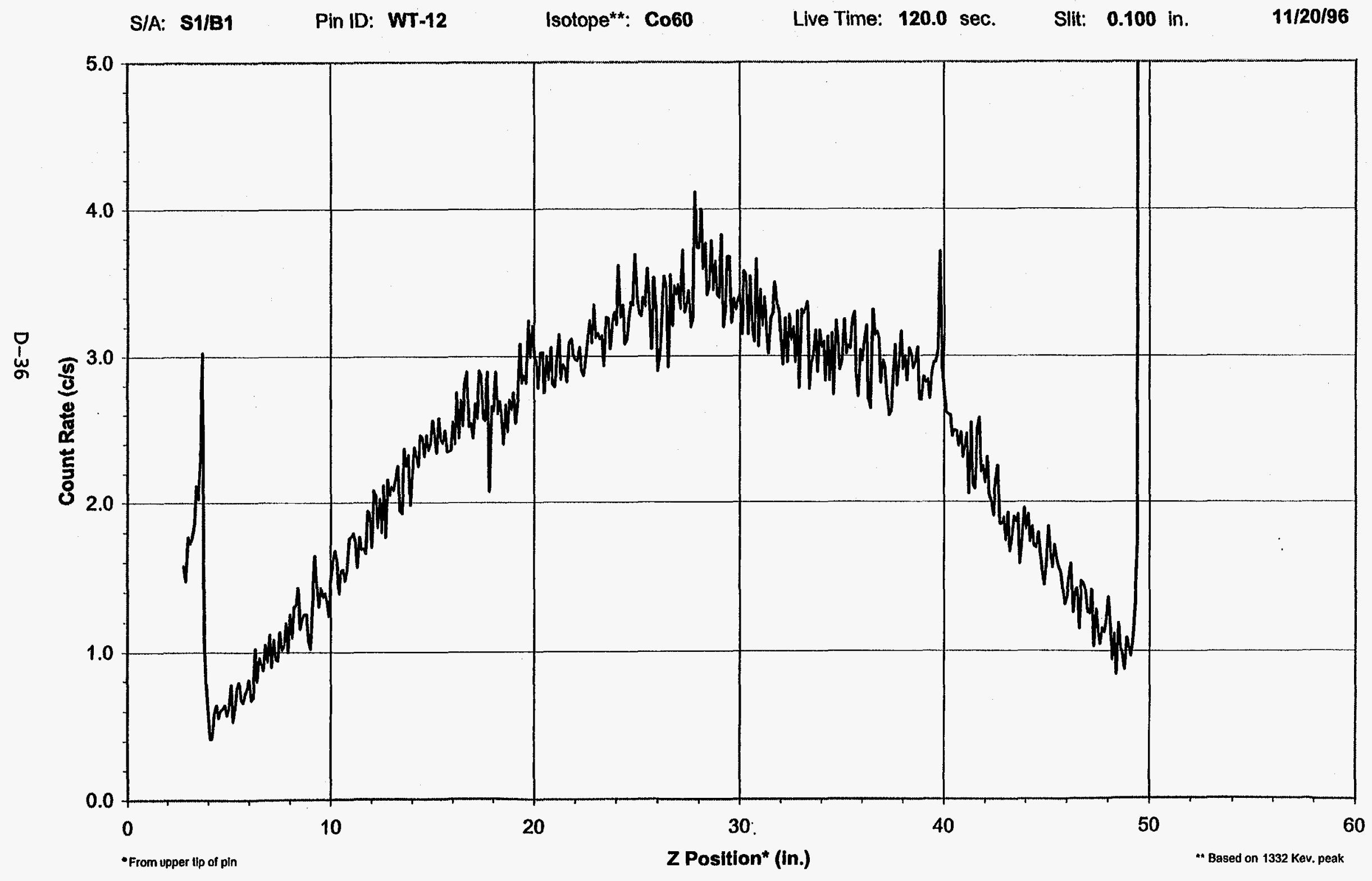


Appendix E

\section{Profilometry Plots}




\section{Appendix $E$}

\section{Table of Contents}

WL-01 Profilometry traces at $0^{\circ}$

WL-01 Profilometry traces at $45^{\circ}$

WL-01 Profilometry traces at $90^{\circ}$

WL-01 Profilometry traces at $135^{\circ}$

WL-03 Profilometry traces at $0^{\circ}$

WL-03 Profilometry traces at $45^{\circ}$

WL-03 Profilometry traces at $90^{\circ}$

WL-03 Profilometry traces at $135^{\circ}$

WL-09 Profilometry traces at $0^{\circ}$

WL-09 Profilometry traces at $45^{\circ}$

WL-09 Profilometry traces at $90^{\circ}$

WL-09 Profilometry traces at $135^{\circ}$

WL-18 Profilometry traces at $0^{\circ}$

WL-18 Profilometry traces at $45^{\circ}$

WL-18 Profilometry traces at $90^{\circ}$

WL-18 Profilometry traces at $135^{\circ}$

WL-22 Profilometry traces at $0^{\circ}$

WL-22 Profilometry traces at $450^{\circ}$

WL-22 Profilometry traces at $90^{\circ}$

WL-22 Profilometry traces at $135^{\circ}$

WL-27 Profilometry traces at $0^{\circ}$

WL-27 Profilometry traces at $45^{\circ}$

WL-27 Profilometry traces at $90^{\circ}$

WL-27 Profilometry traces at $135^{\circ}$

WT-11 Profilometry traces at $0^{\circ}$

WT-11 Profilometry traces at $45^{\circ}$

WT-11 Profilometry traces at $90^{\circ}$

WT-11 Profilometry traces at $135^{\circ}$

WT-12 Profilometry traces at $0^{\circ}$

WT-12 Profilometry traces at $45^{\circ}$

WT-12 Profilometry traces at $90^{\circ}$

WT-12 Profilometry traces at $135^{\circ}$ 
E-ii 


\section{ARGONNE NATIONAL LABORATORY \\ P. O. Box 2528, Idaho Falls, Idaho $83403-2528$}

\section{ENGINEERING}

DIVISION

(TREAT, ZPPR, and HFEF)

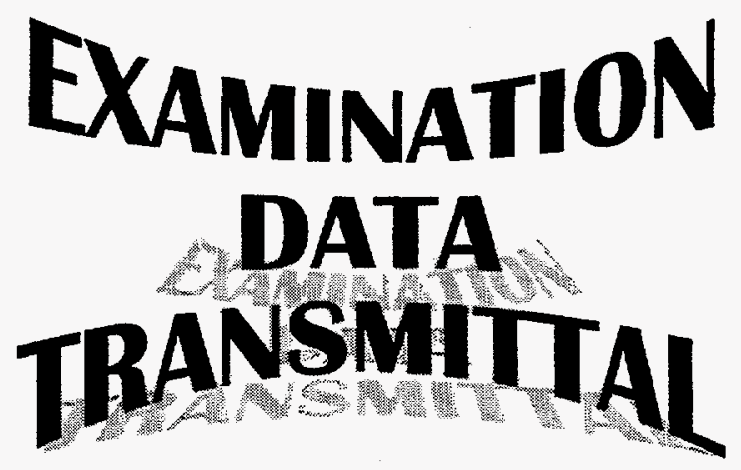

TO:

ADDRESS:

Lockheed Martin Idaho Technologies, Co.

R. L. Bratton EROB MS-3760

Idaho Falls, ID 834

March 12, 1997

(

MATERIAL EXAMINED:

Six Target Rods from CLWR Loop-1 Bundle

TYPE OF EXAMINATION:

Contact Profilometry

FORM OF DATA:

Charts, User Notes, and CD-ROM (2)

NOTES:

CC: w/Attachments

Loop-1 file

CC: wo Attachments

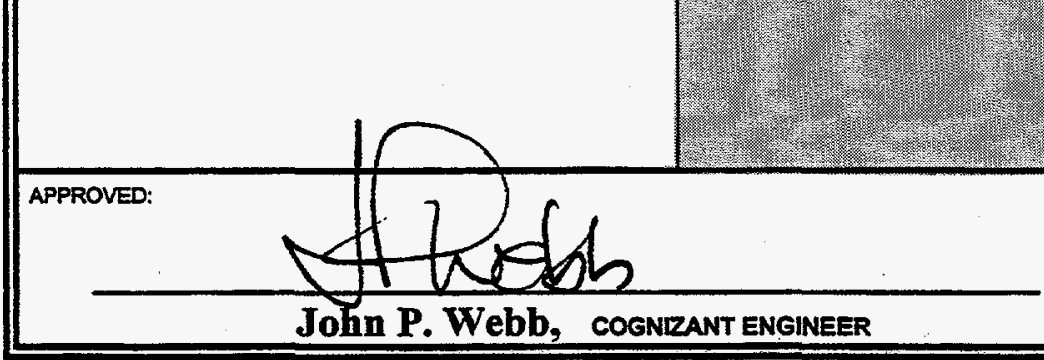

Questions regarding this data transmittal should be directed to the Cognizant Engineer at extension (208) 533-7773. 


\section{ARGONNE NATIONAL LABORATORY \\ Hot Fuel Examination Facility}

\section{Element Contact Profilometry \\ USER NOTES}

1. When fuel elements (pins) or capsules are significantly bowed, a situation can arise when the lower tip of the element or capsule exits the bottom guide roller near the end of the measurement. Depending on the direction and magnitude of the bow, the portion of the element or capsule below the upper guide roller can assume an angle with respect to the measuring probes causing an erroneously high reading. This is an inherent feature of the system that cannot be corrected, and the resultant data must be interpreted with caution.

2. Full-length scans usually start as high as possible on the element or capsule (but no higher than the upper cladding weld) and end at the lower cladding weld. Because of interference from the upper roller and the collet applied to the element or capsule, the topmost four inches cannot be measured.

3. The "Z-Position" reported on ANL-supplied plots is the raw position $\left(Z_{s}\right.$, in inches) of the in-cell positioning stage, and has no fixed or known offset from the top or bottom of the element or capsule. Note that the stage Z-Position is higher toward the bottom of an element or capsule.

4. The "Diameter" reported on ANL-supplied plots is the profile dimension (in inches) perpendicular to the azimuthal position, $\phi_{e}$ (in degrees), as shown in the diagram below.

5. By convention, azimuthal positions increase counter-clockwise (CCW) from the azimuthal reference feature when viewed from the top of the element or capsule as shown in the diagram below.

6. During measurement of the profile, recorded data are sometimes corrupted by electronic noise. Until the source of this noise can be discovered and corrected, obvious data errors may have been removed from the attached data reports.

7. Copies of the profilometry data are provided on floppy disks or CD-ROM's. By convention, the data from each azimuthal angle is contained in a separate file with the name:

\section{filename ext}

where:

filename $=$ Element/Capsule $\mathrm{ID}$

ext $=$ Azimuthal Angle (eg., 045)

8. Within each file, the data are recorded as plain ASCII text with each line terminated by a $\langle C R\rangle L F\rangle$. The first three lines of data contain descriptive text information (such as S/A ID and PLN ID) that is seif explanatory. Below this are three columns of tab-delimited numeric data corresponding (left-toright) to: "Z-Position (in.)", " $\phi_{s}\left({ }^{\circ}\right)$ and "Diameter (in.)". The end of data are sometimes flagged by large contrasting numbers, usually a string of 9 's (eg., "999.999").

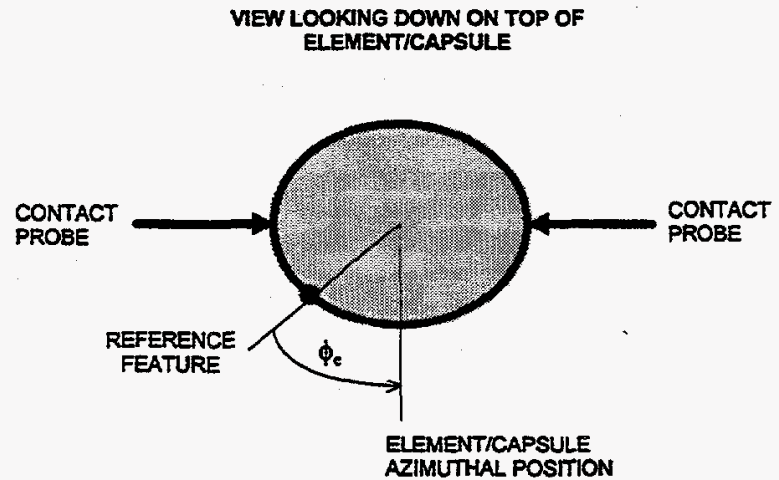




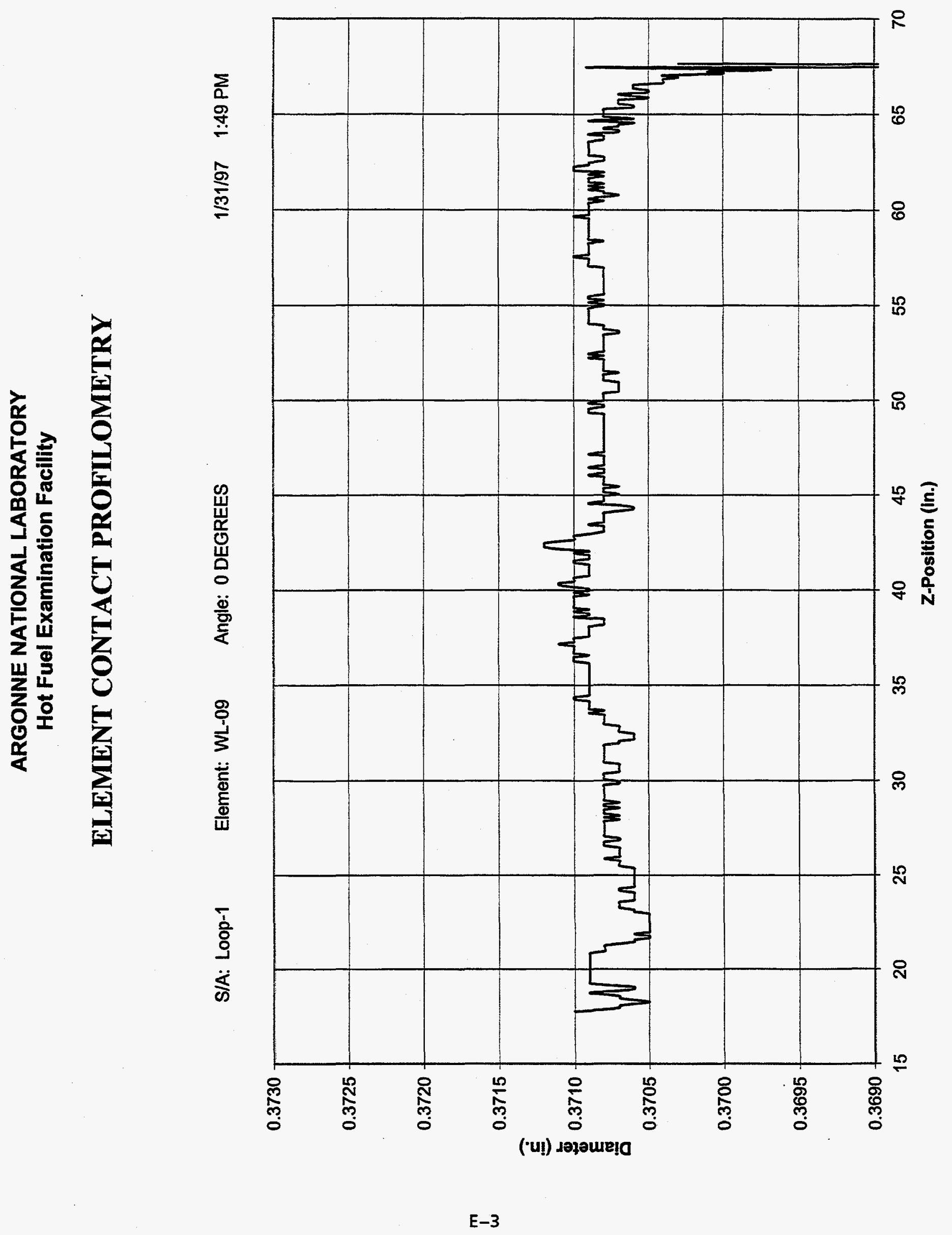


ARGONNE NATIONAL LABORATORY

Hot Fuel Examination Facility

\section{ELEMENT CONTACT PROFILOMETRY}

$\begin{array}{llll}\text { S/A: Loop-1 Element: WL-09 Angle: 45DEGREES } & 1 / 31 / 97 & 1: 53 \text { PM }\end{array}$

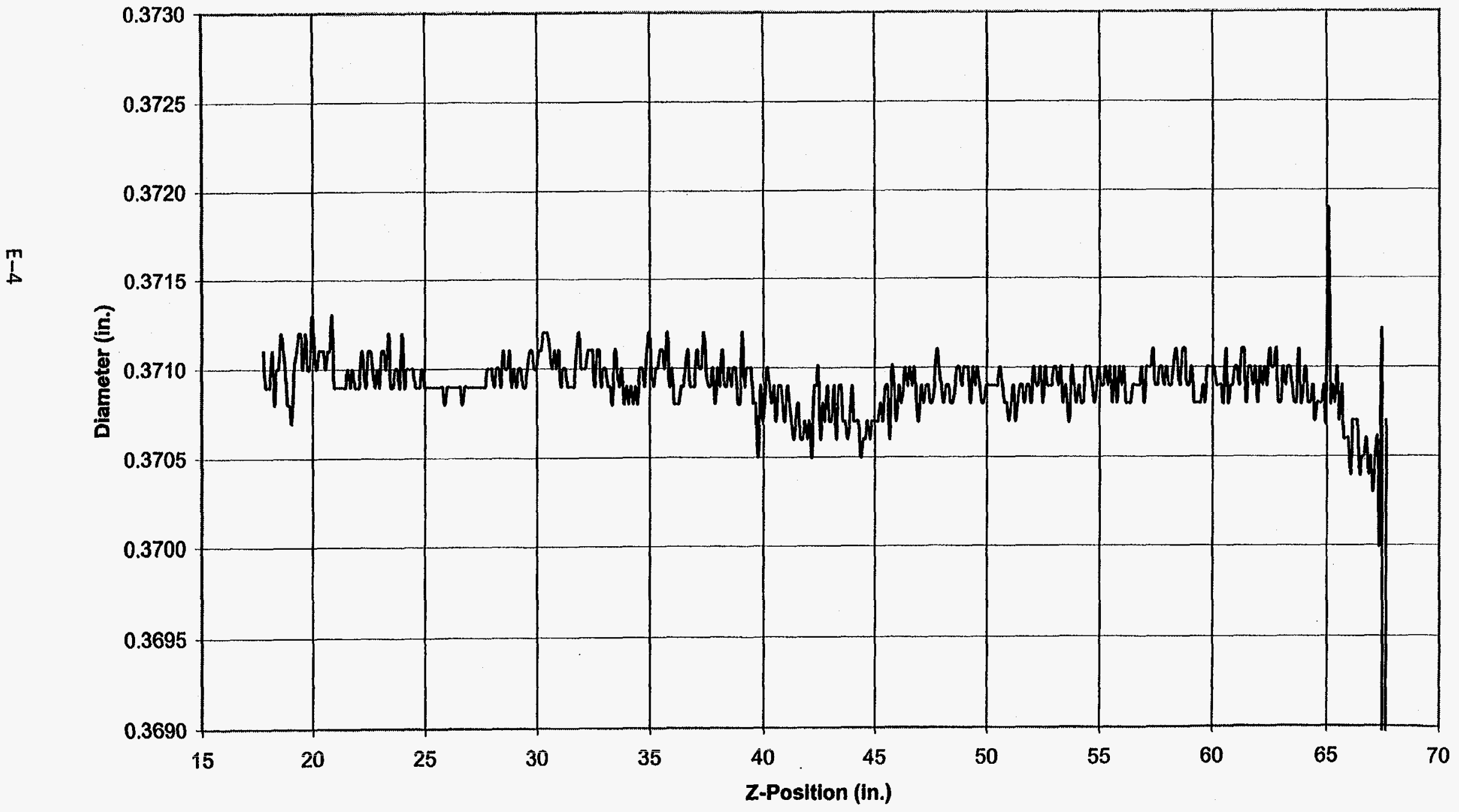

Page 2 

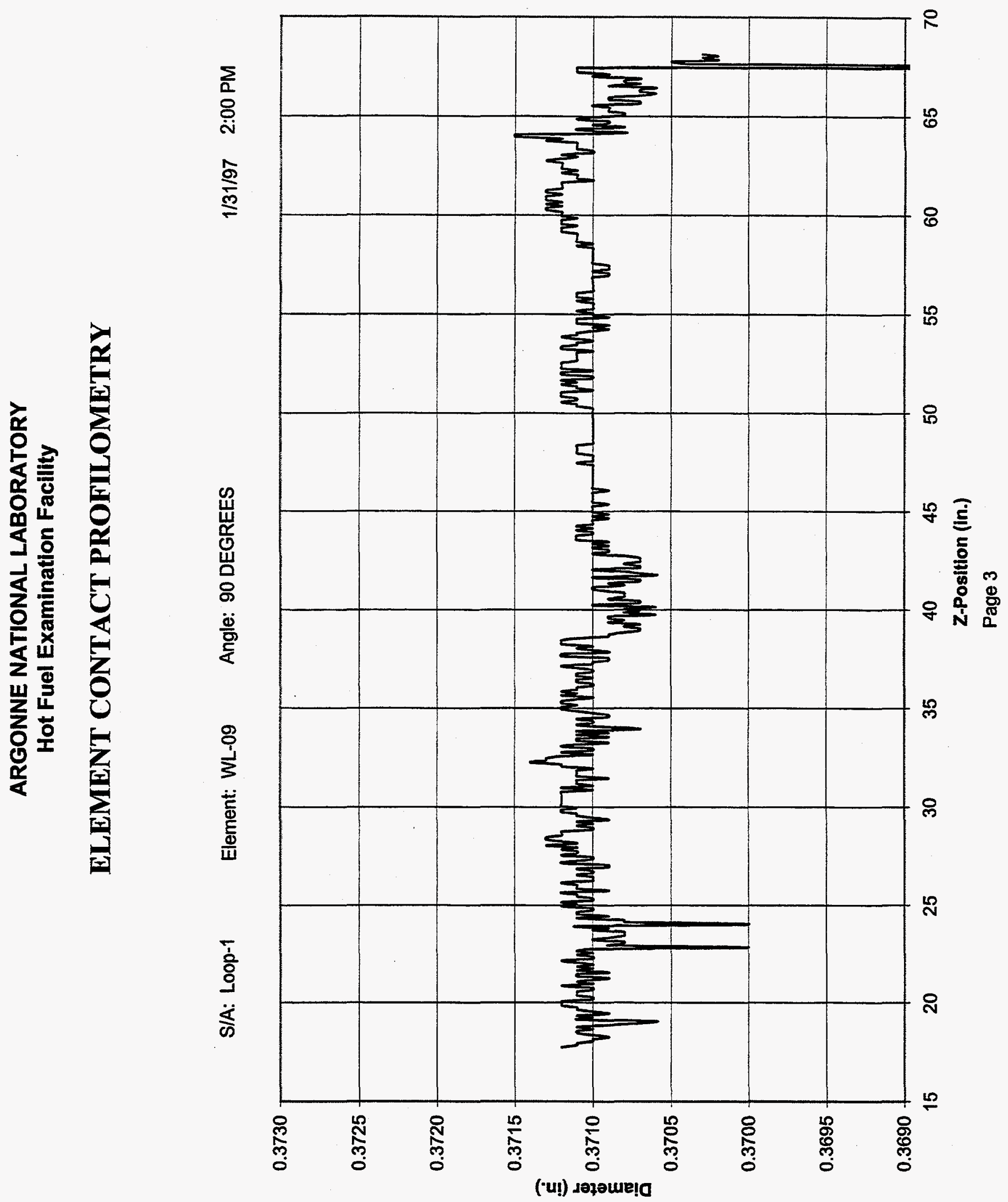

E-5 
ARGONNE NATIONAL LABORATORY

Hot Fuel Examination Facility

\section{ELEMENT CONTACT PROFILOMETRY}

$\begin{array}{llll}\text { S/A: Loop-1 Element: WL-09 Angle: } 135 \text { DEGREES } & \text { 1/31/97 2:06 PM }\end{array}$

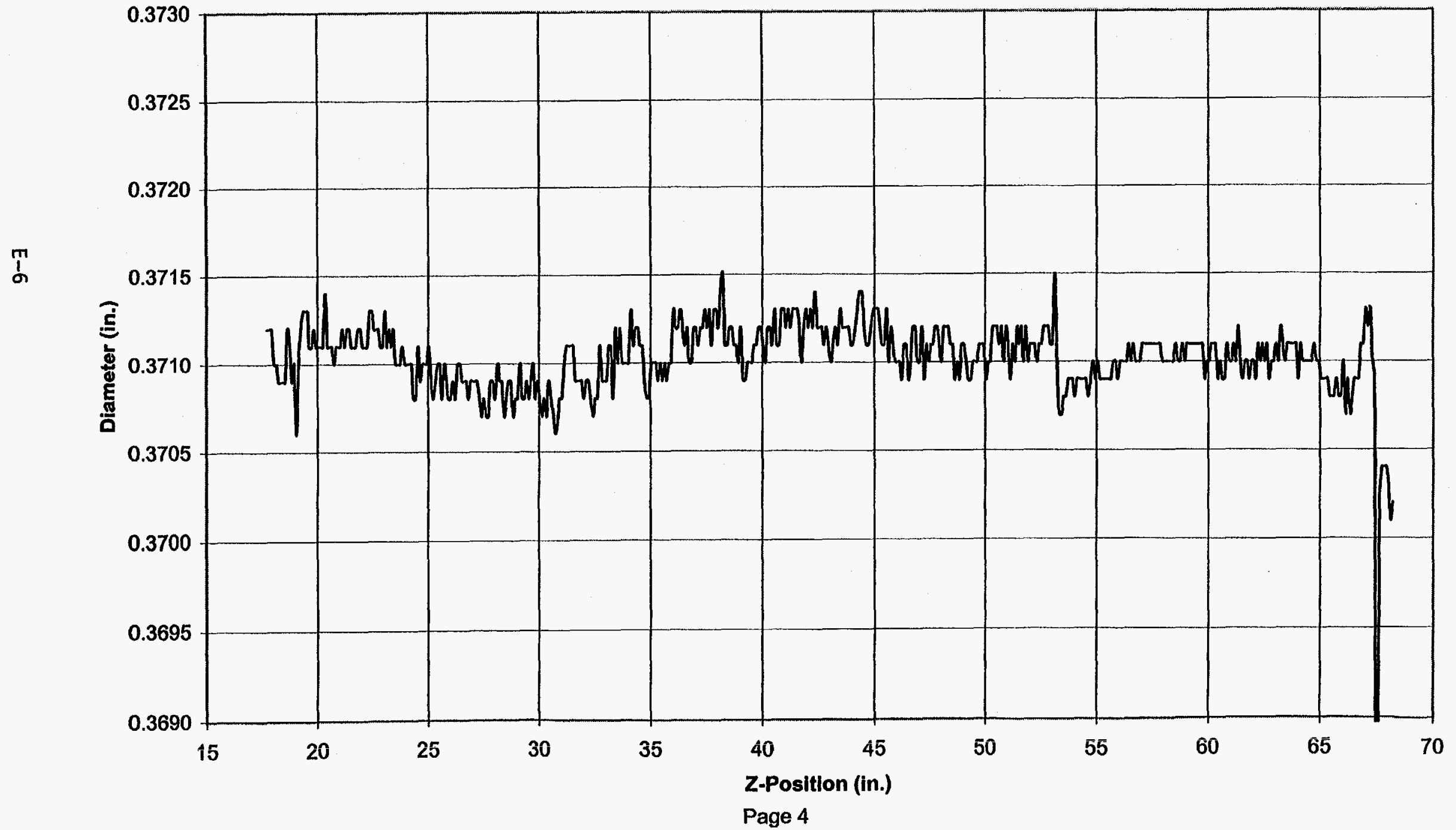




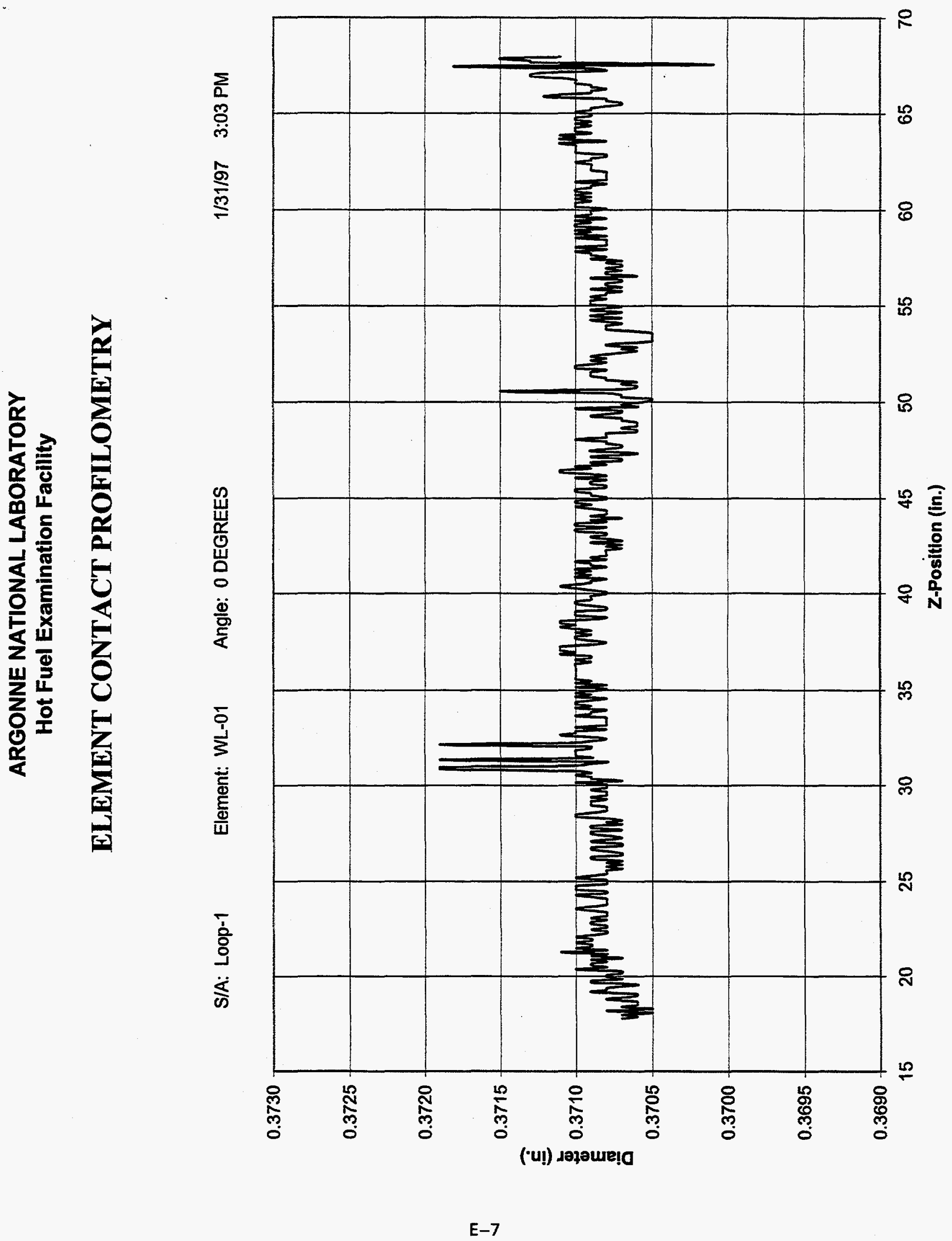


ARGONNE NATIONAL LABORATORY

Hot Fuel Examination Facility

\section{ELEMENT CONTACT PROFILOMETRY}

S/A: Loop-1 Element: WL-01 Angle: 45 DEGREES

1/31/97 3:07 PM

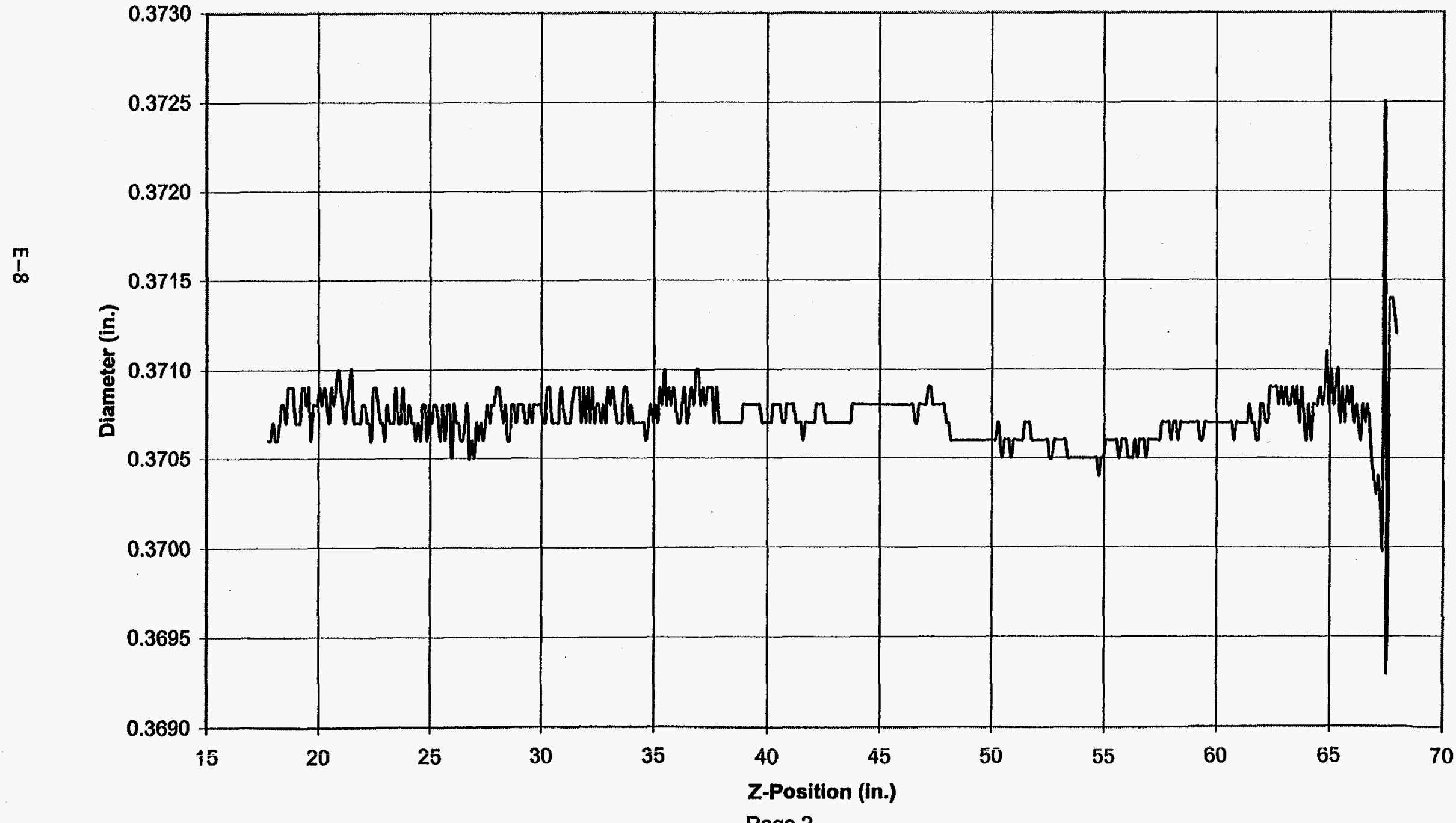

Page 2 

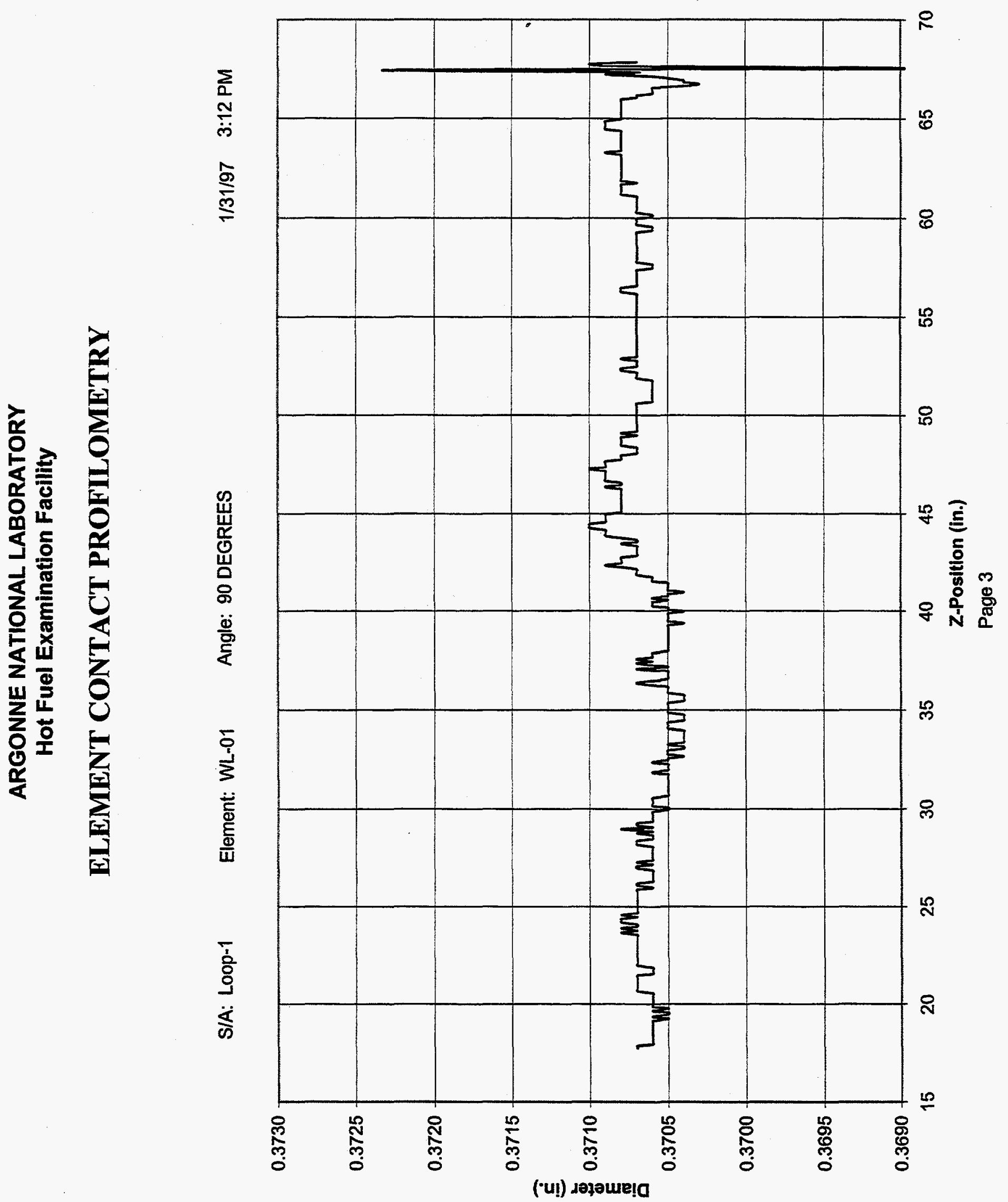
ARGONNE NATIONAL LABORATORY

Hot Fuel Examination Facility

\section{ELEMENT CONTACT PROFILOMETRY}

S/A: Loop-1

Element: WL-01

Angle: 135 DEGREES

1/31/97 3:20 PM

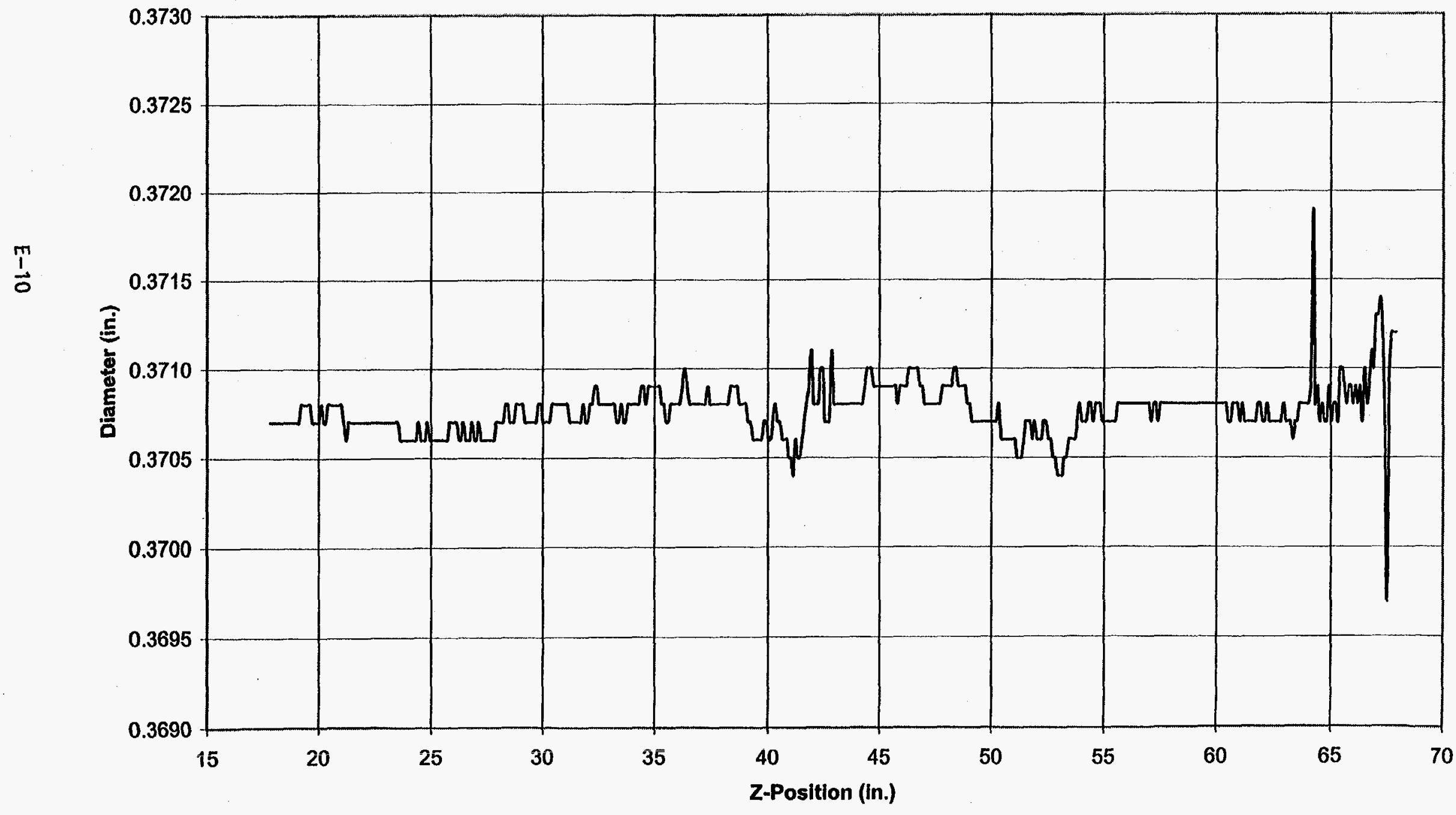

Page 4 


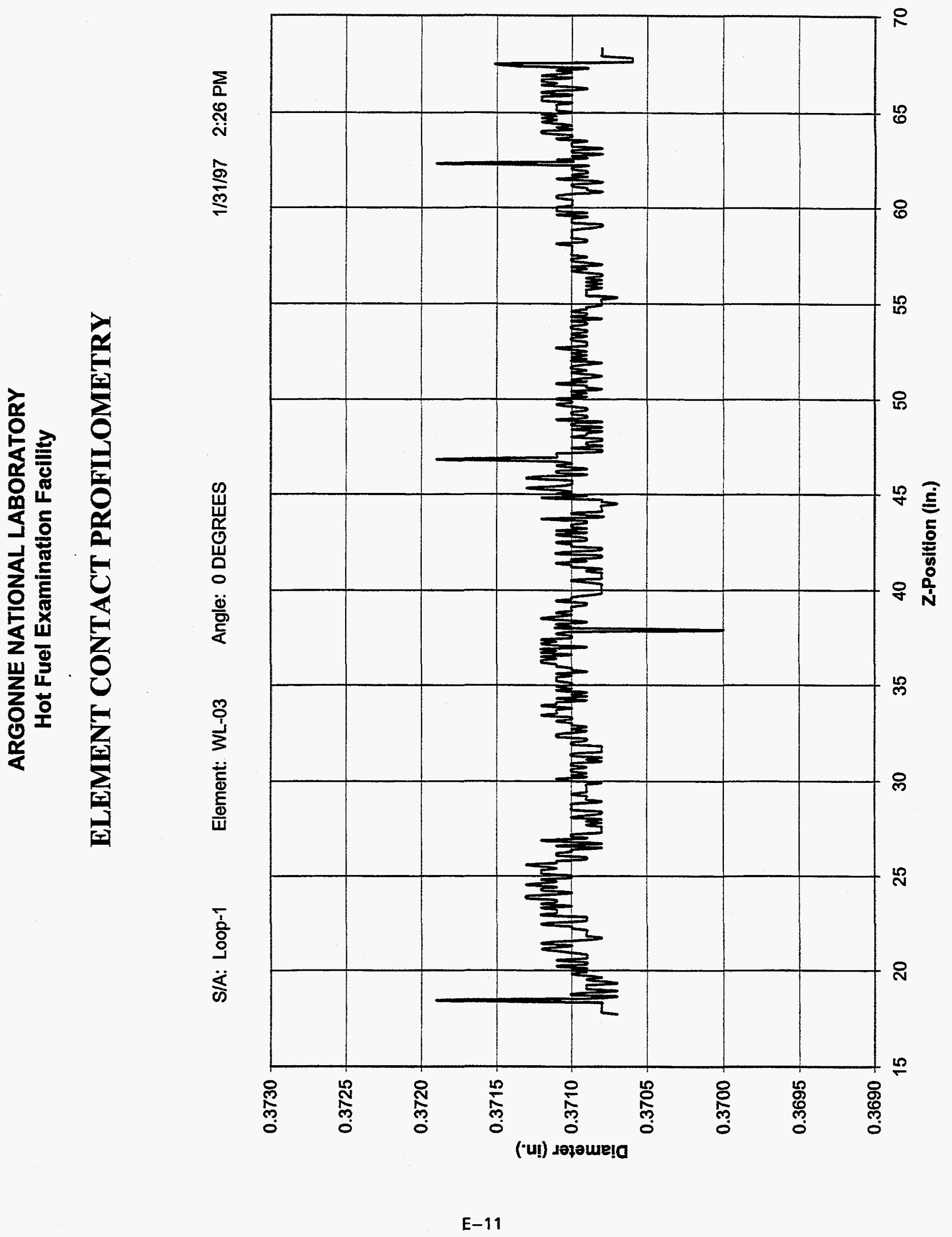


ARGONNE NATIONAL LABORATORY

Hot Fuel Examination Facility

\section{ELEMENT CONTACT PROFILOMETRY}

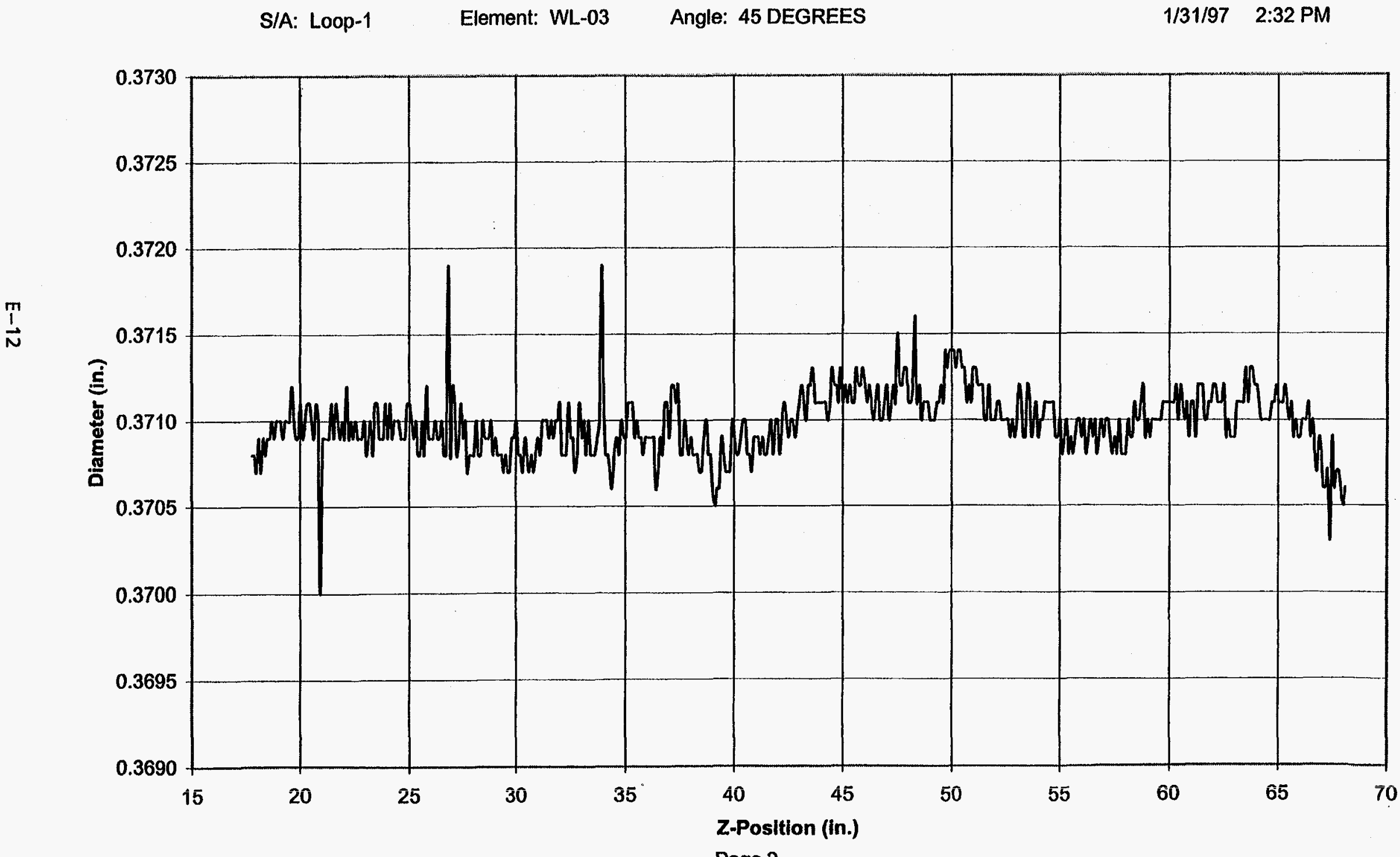

Page 2 

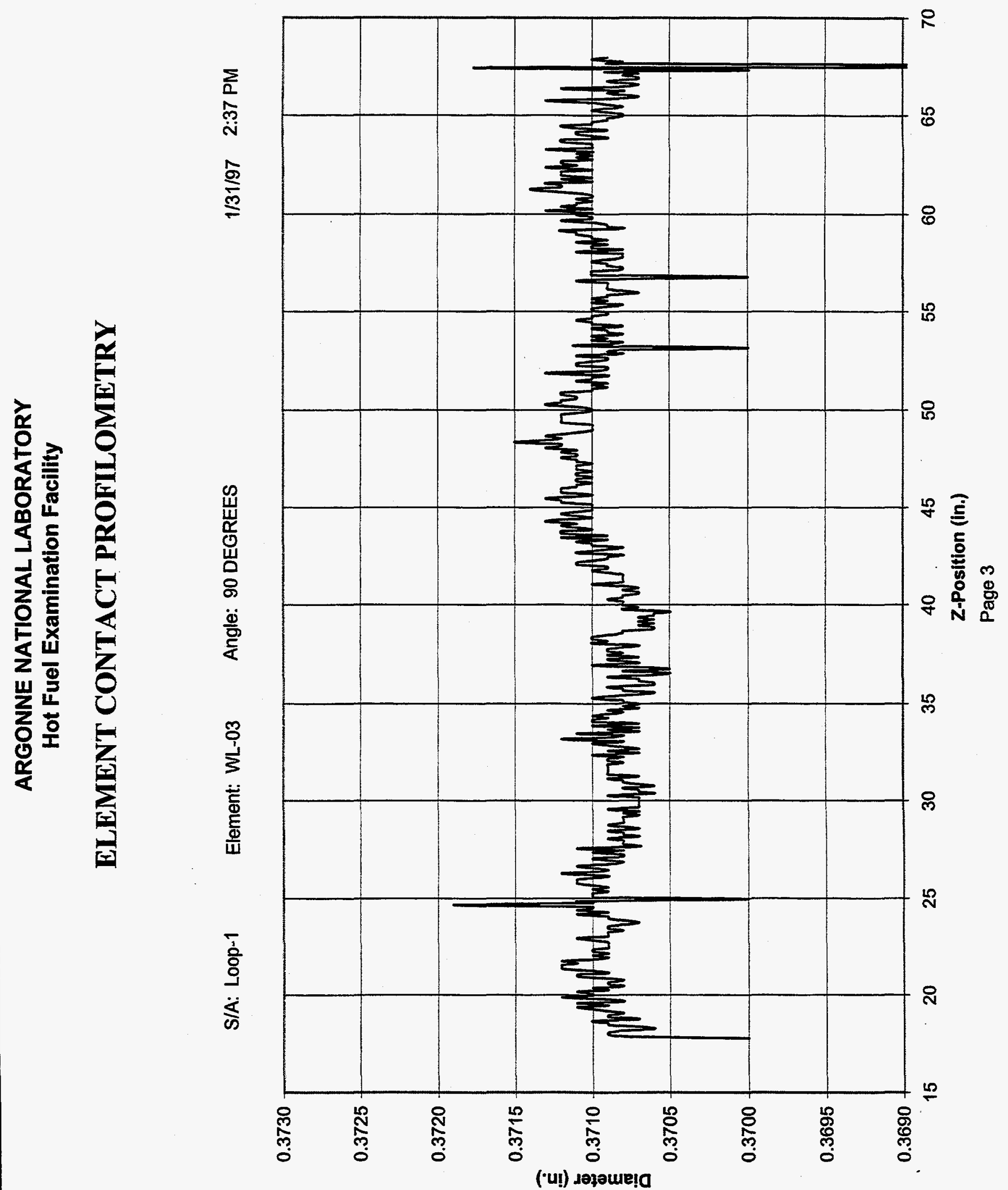
ARGONNE NATIONAL LABORATORY

Hot Fuel Examination Facility

\section{ELEMENT CONTACT PROFILOMETRY}

S/A: Loop-1

Element: WL-03

Angle: 135 DEGREES

1/31/97 2:43 PM

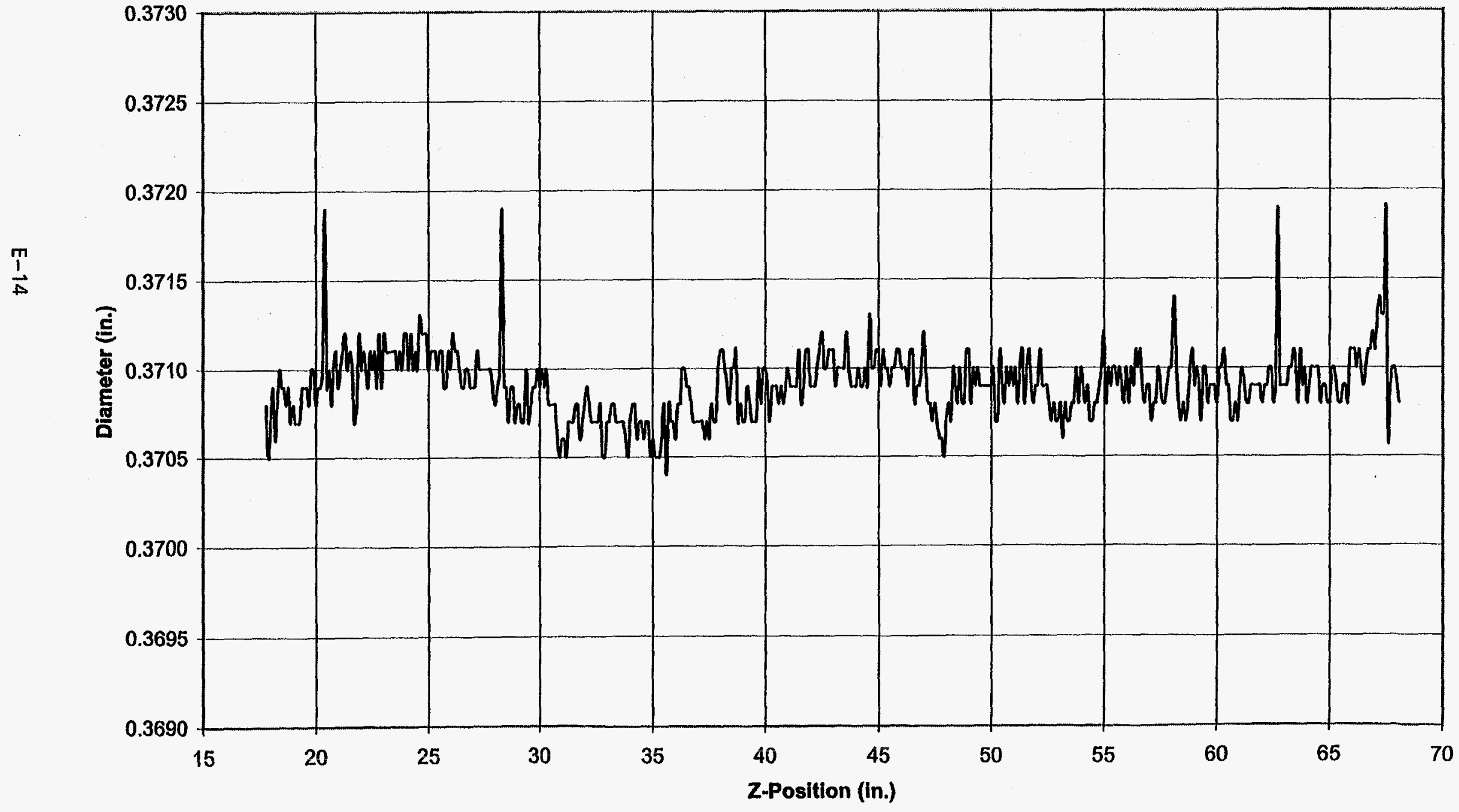

Page 4 


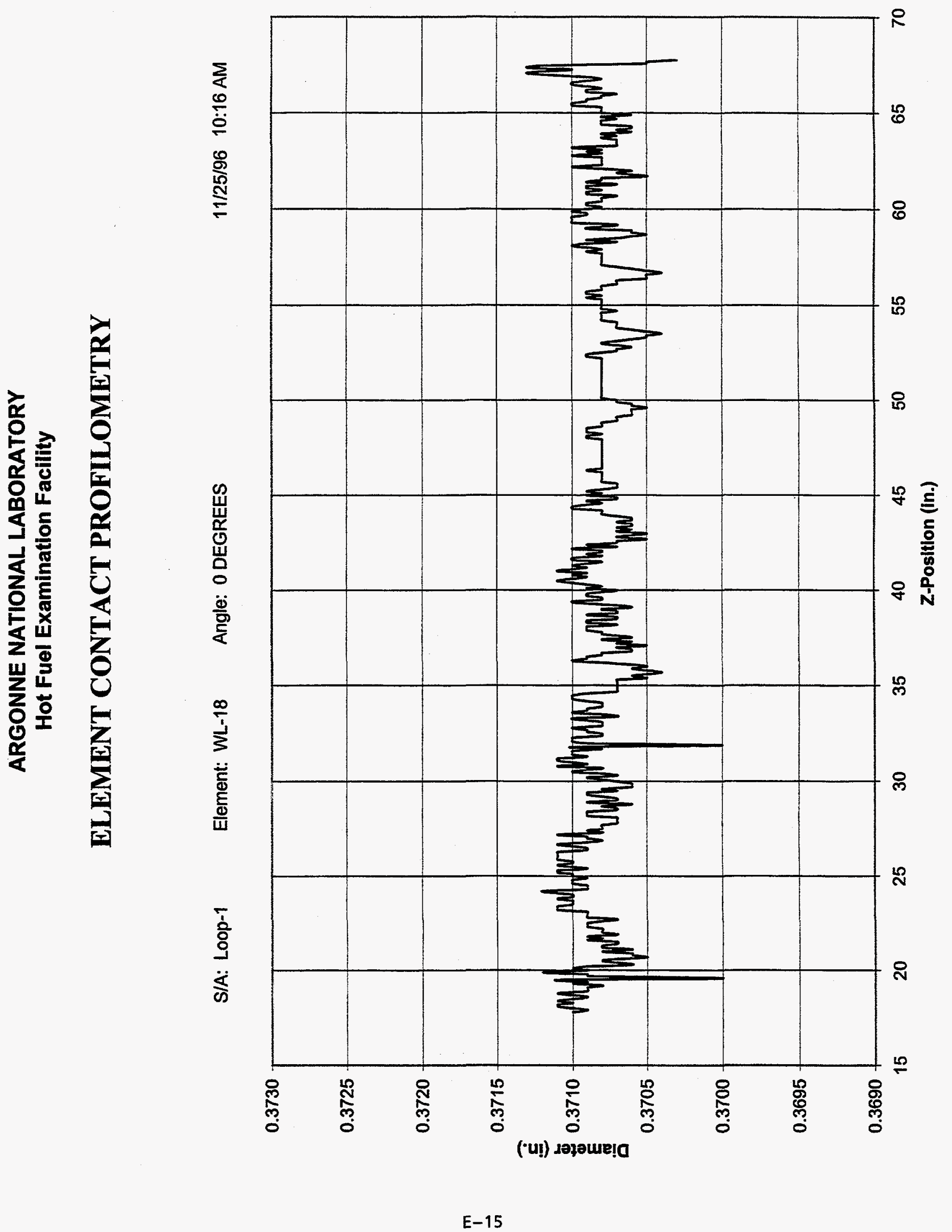


ARGONNE NATIONAL LABORATORY

Hot Fuel Examination Facility

\section{ELEMENT CONTACT PROFILOMETRY}

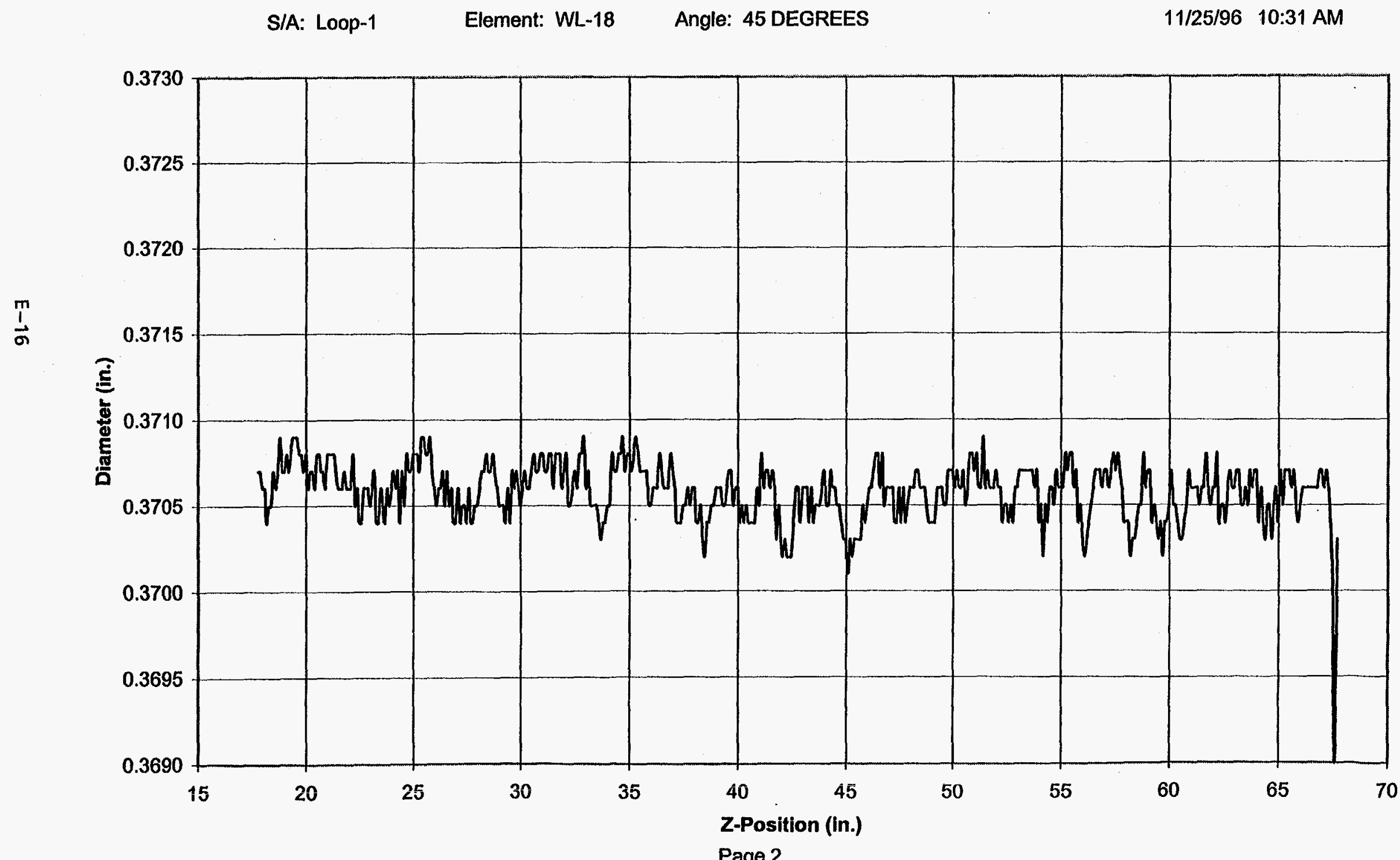


ARGONNE NATIONAL LABORATORY

Hot Fuel Examination Facility

\section{ELEMENT CONTACT PROFILOMETRY}

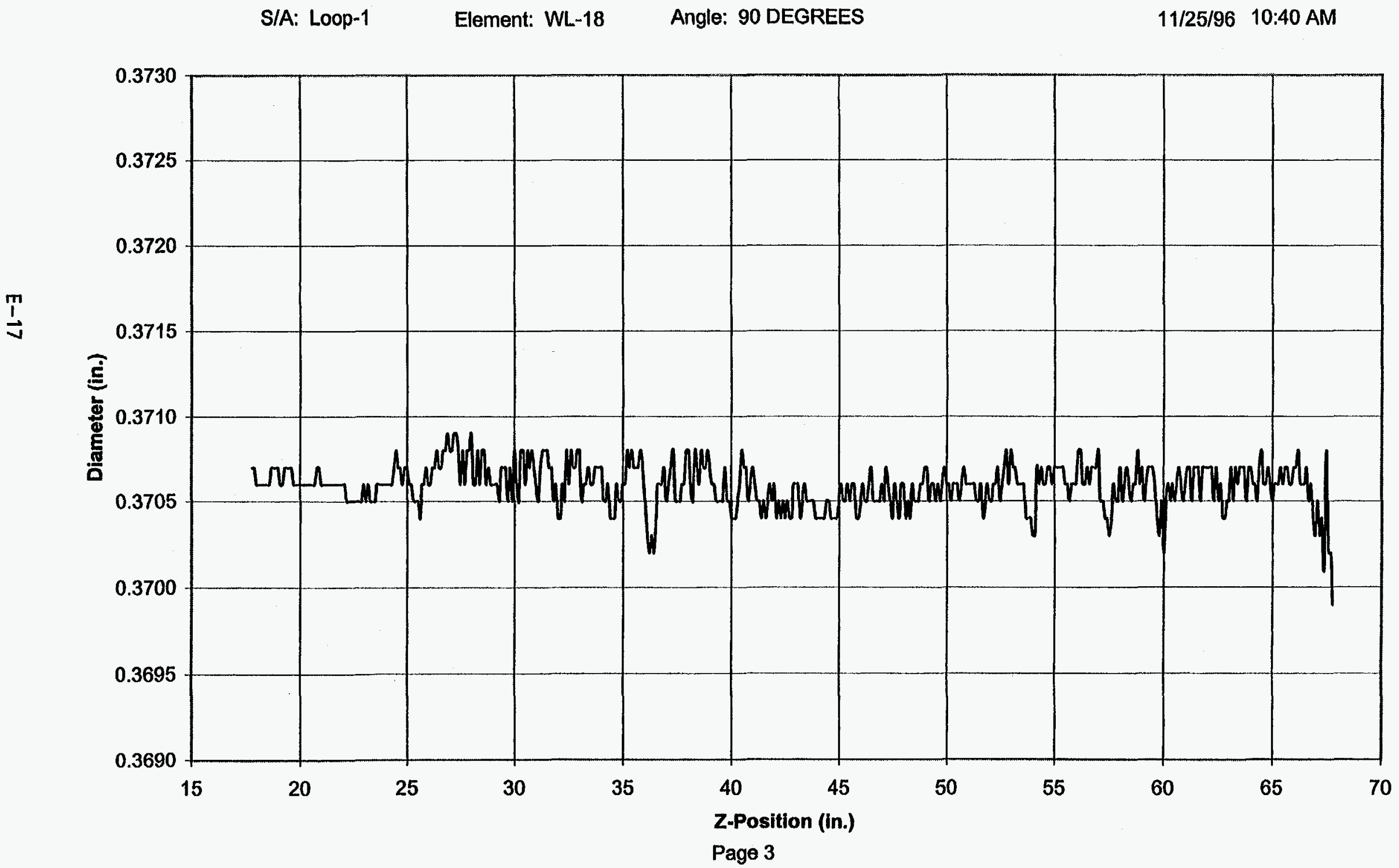


ARGONNE NATIONAL LABORATORY

Hot Fuel Examination Facility

\section{ELEMENT CONTACT PROFILOMETRY}

S/A: Loop-1

Element: WL-18

Angle: 135 DEGREES

11/25/96 10:50 AM

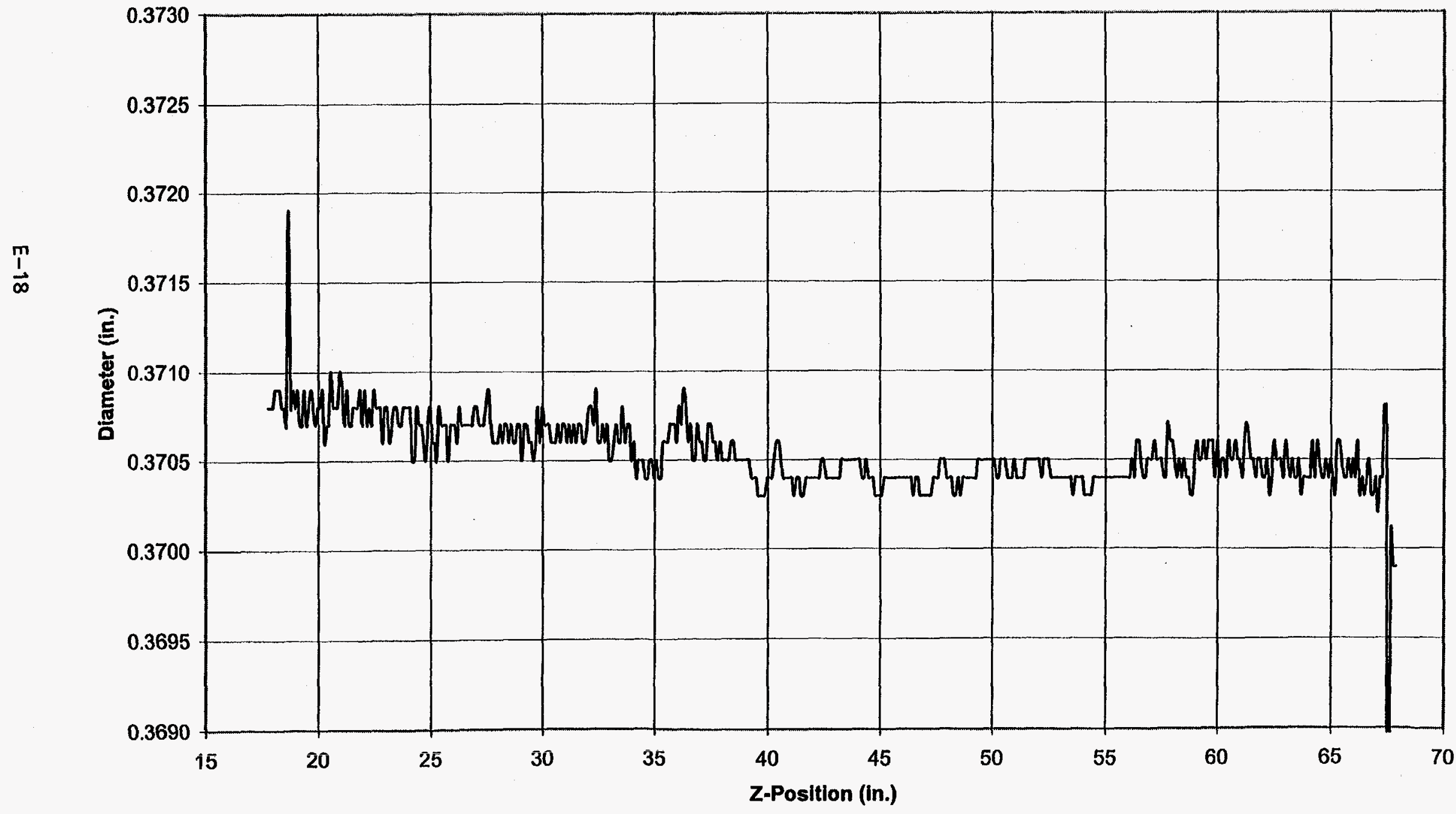

Page 4 

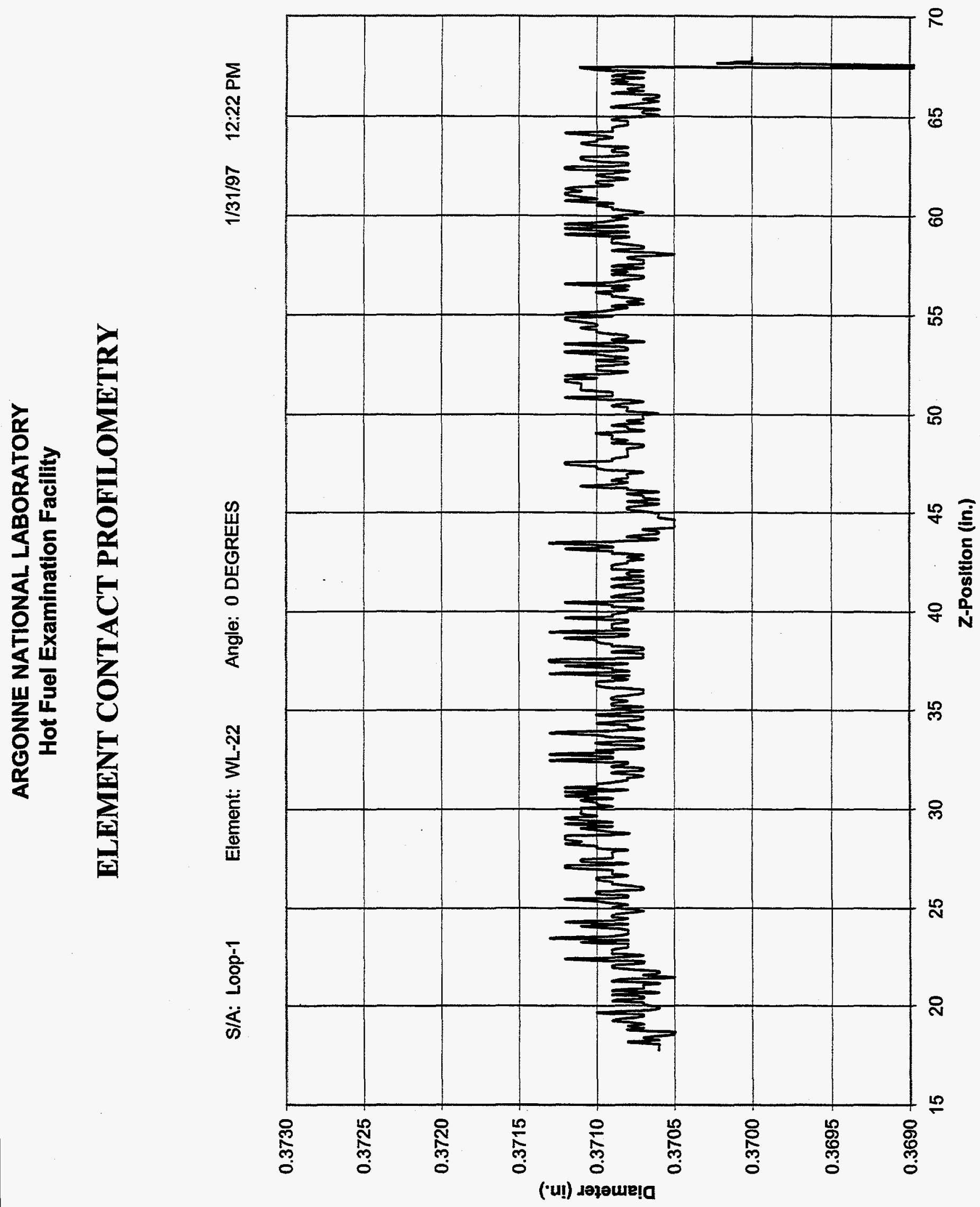
ARGONNE NATIONAL LABORATORY

Hot Fuel Examination Facility

\section{ELEMENT CONTACT PROFILOMETRY}

S/A: Loop-1

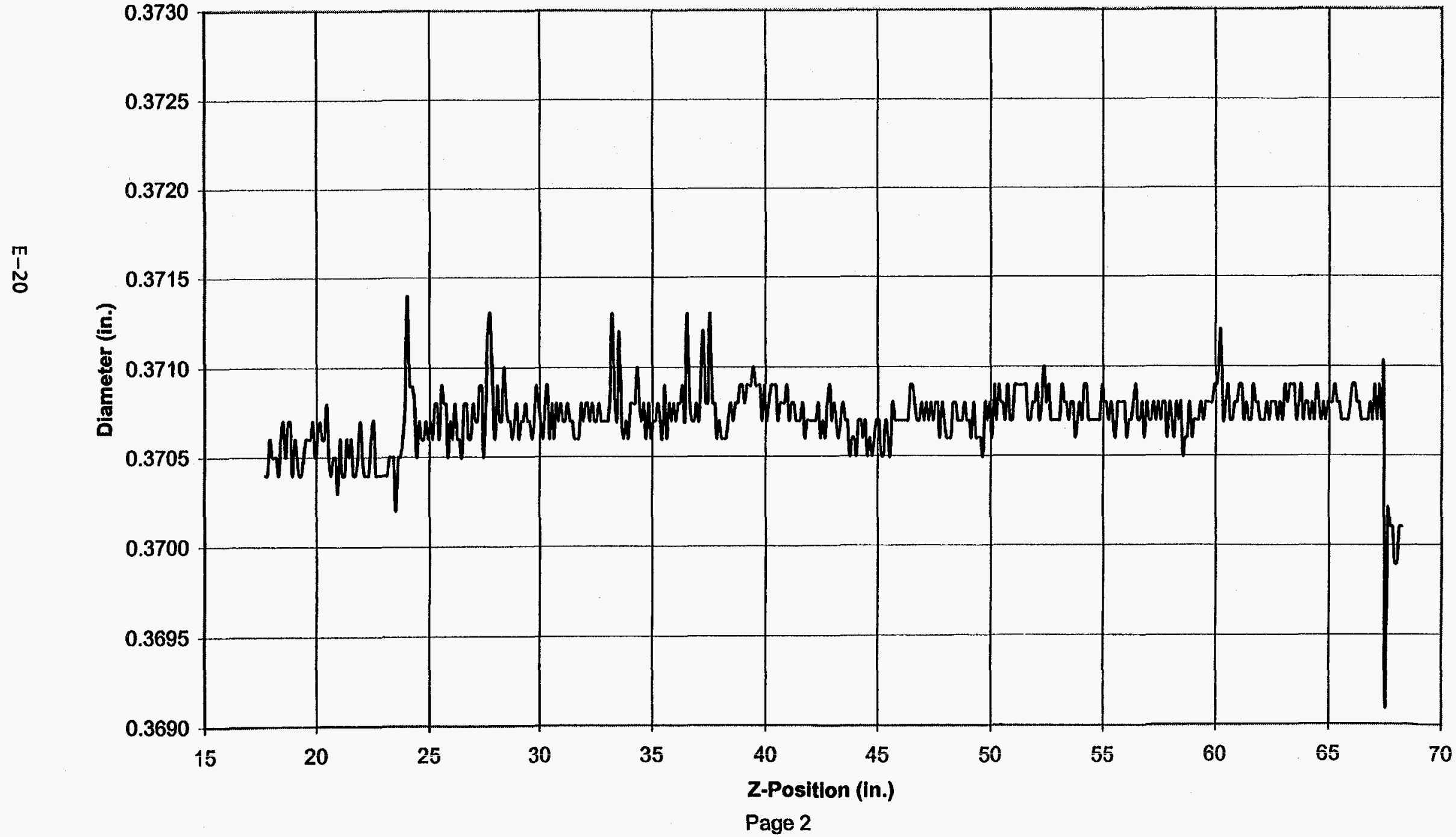




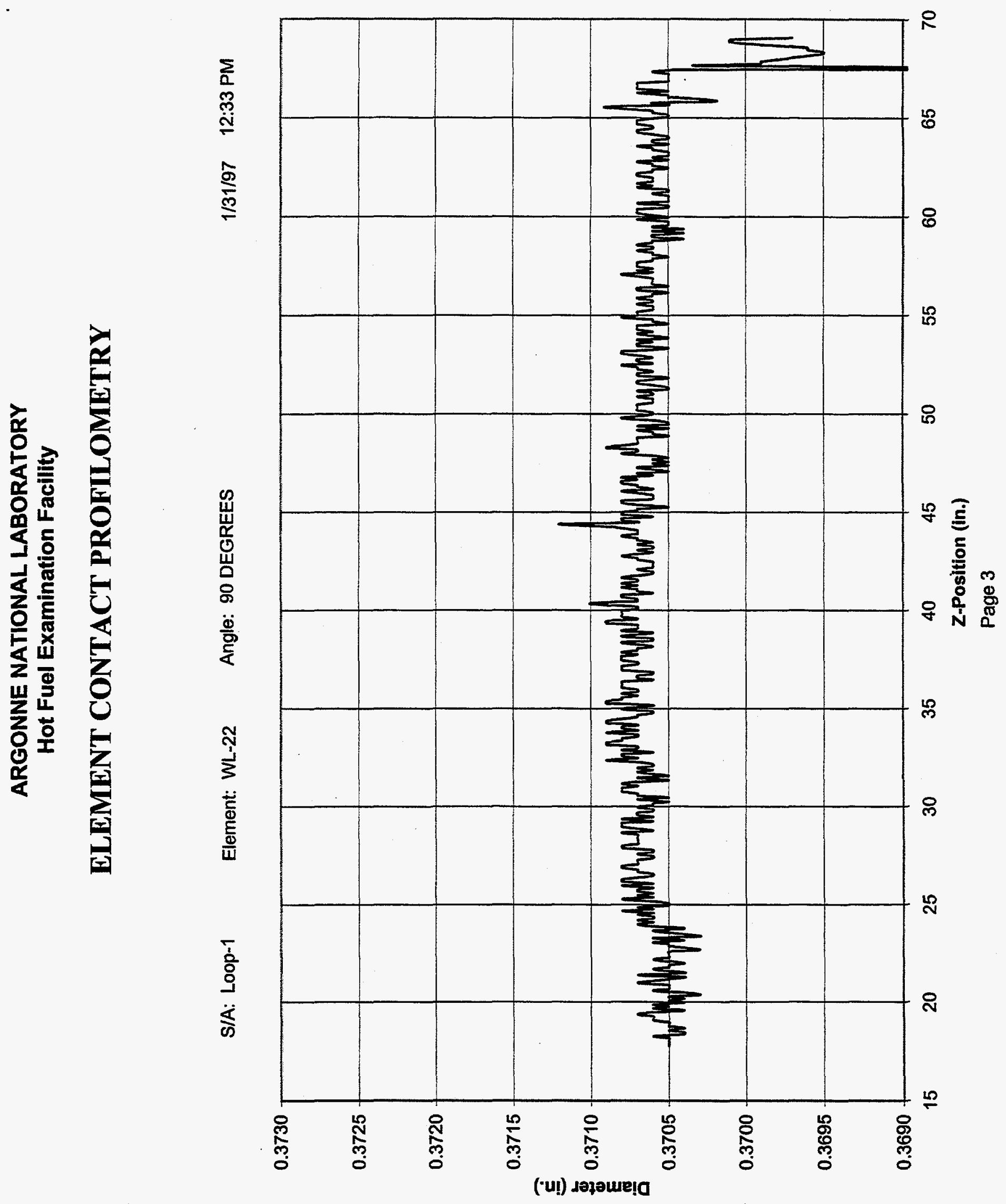




\section{ARGONNE NATIONAL LABORATORY}

Hot Fuel Examination Facility

\section{ELEMENT CONTACT PROFILOMETRY}

S/A: Loop-1

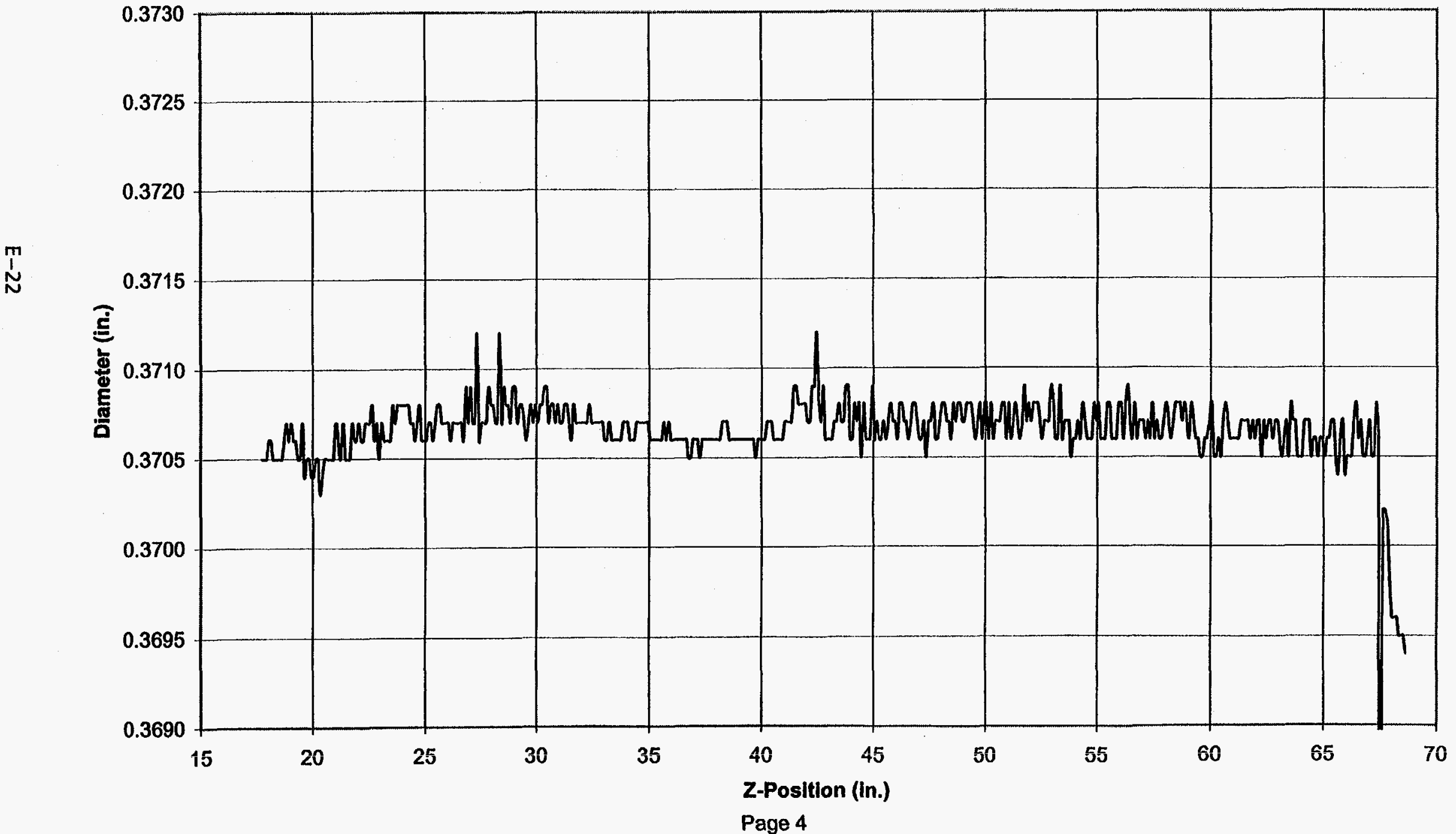



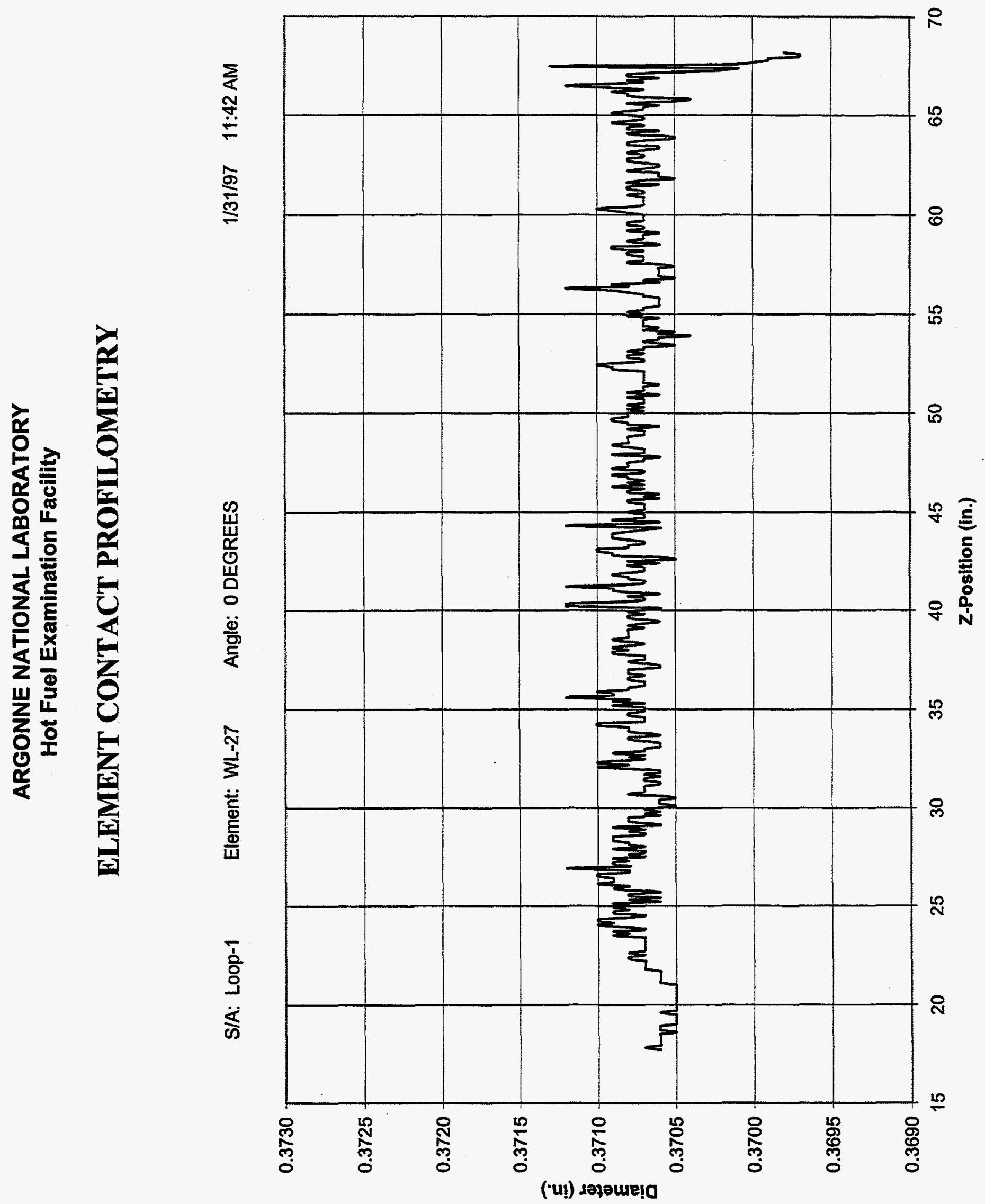
ARGONNE NATIONAL LABORATORY

Hot Fuel Examination Facility

\section{ELEMENT CONTACT PROFILOMETRY}

SIA: Loop-1

Element: WL-27

Angle: 45 DEGREES

1/31/97 11:47 AM

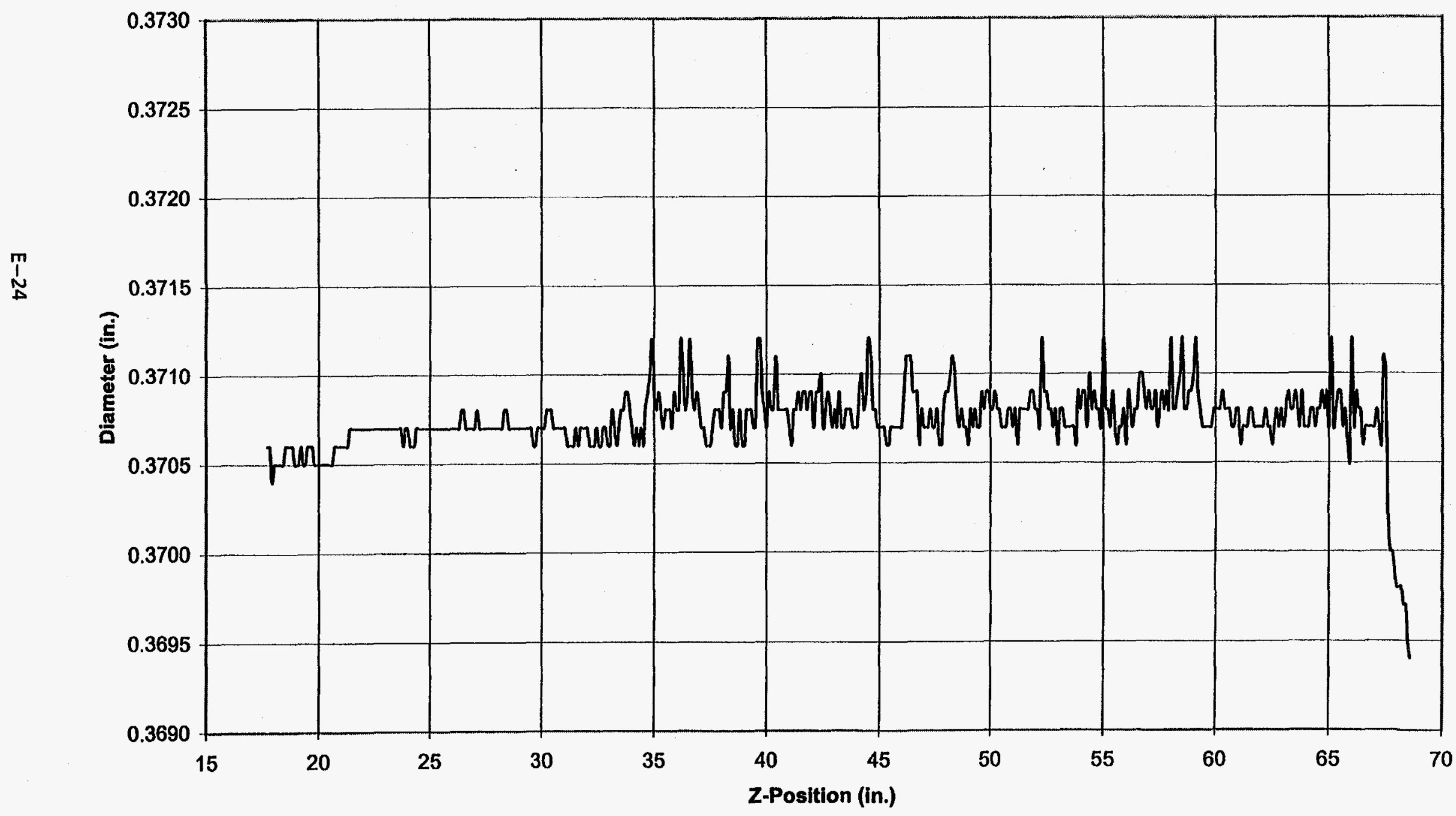

Page 2 

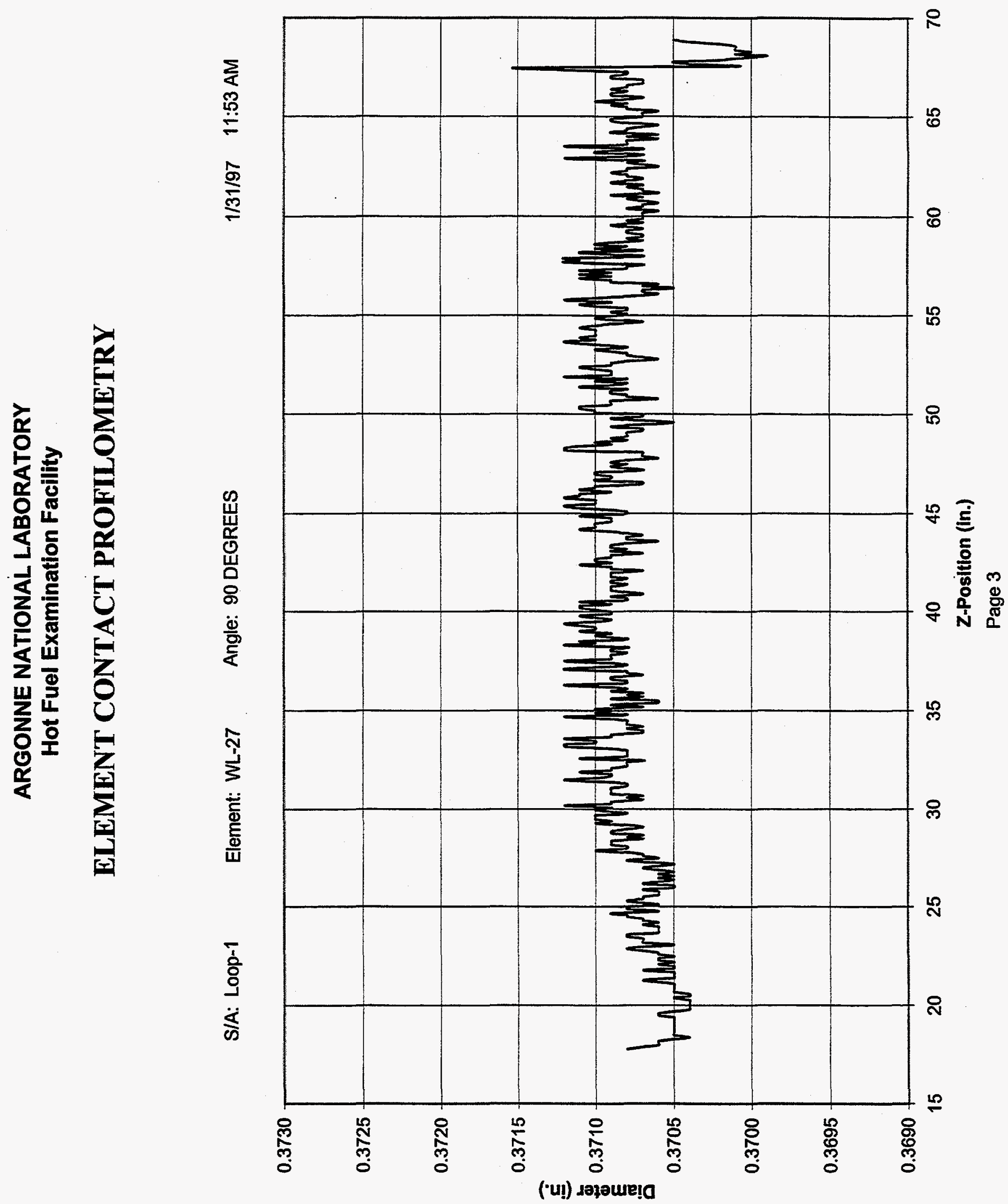
ARGONNE NATIONAL LABORATORY

Hot Fuel Examination Facility

\section{ELEMENT CONTACT PROFILOMETRY}

S/A: Loop-1 Element: WL-27 Angle: 135 DEGREES

$1 / 31 / 97 \quad 12: 00 \mathrm{PM}$

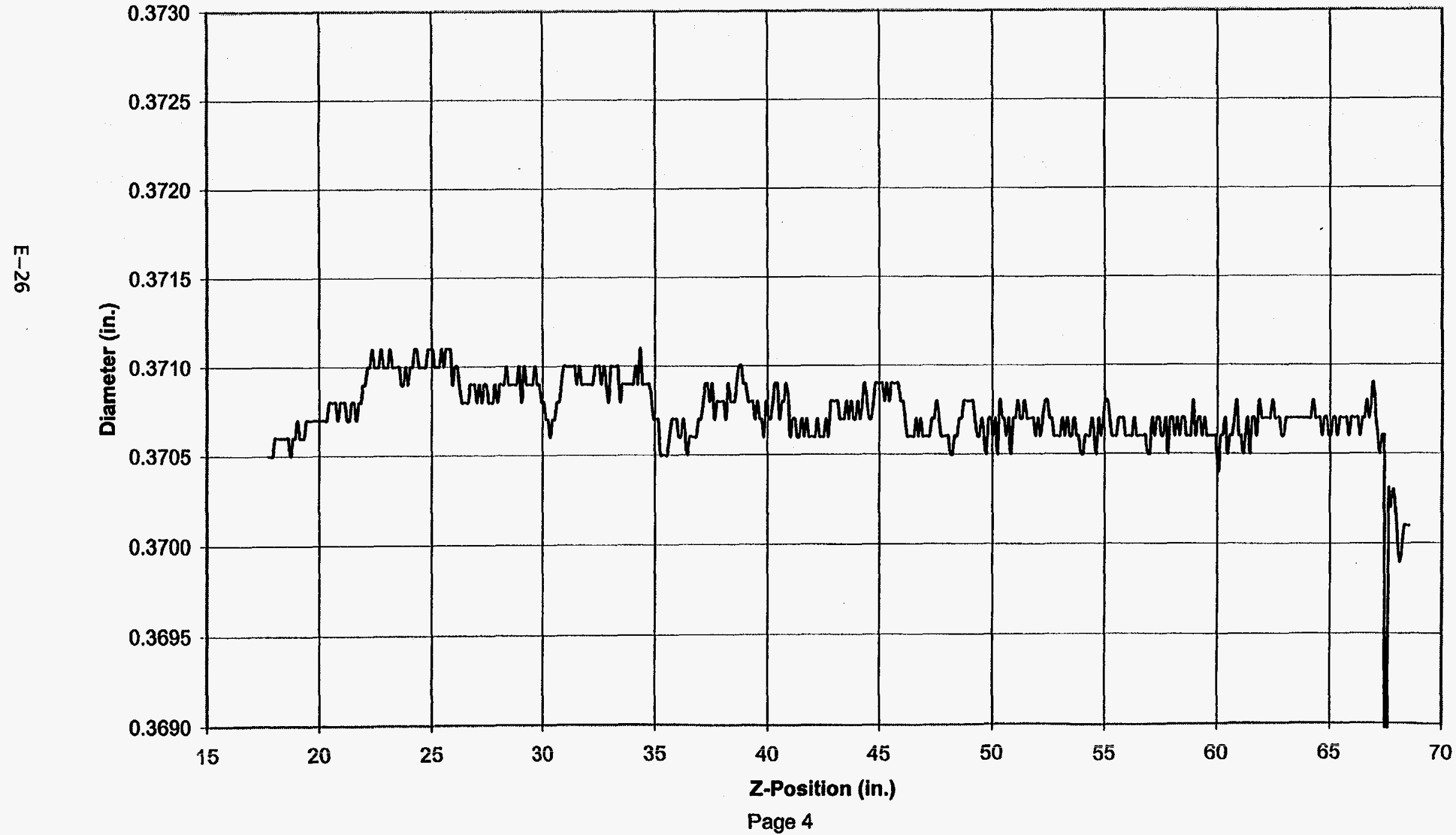




\section{ARGONNE NATIONAL LABORATORY}

P. O. Box 2528, Idaho Falls, Idaho 83403-2528

ENGINEERING DIVISION

(TREAT, ZPPR, and HFEF)
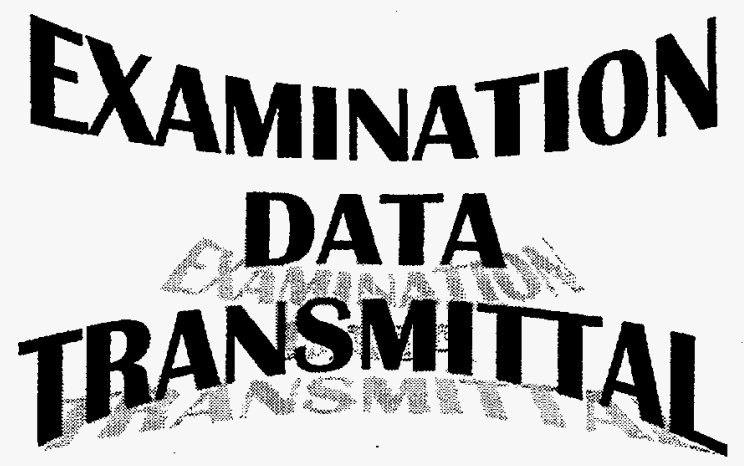

TO:

R. L. Bratton

MATERIAL EXAMINED:

Two Target Rods from CLWR S1/B1 Test

TYPE OF EXAMINATION:

\section{Contact Profilometry}

FORM OF DATA:

Charts, User Notes, and CD-ROM (2) EROB MS-3760

Idaho Falls, ID 834

March 12, 1997

CC: w/Attachments

S1/B1 file

CC: w/o Attachments

NOTES:

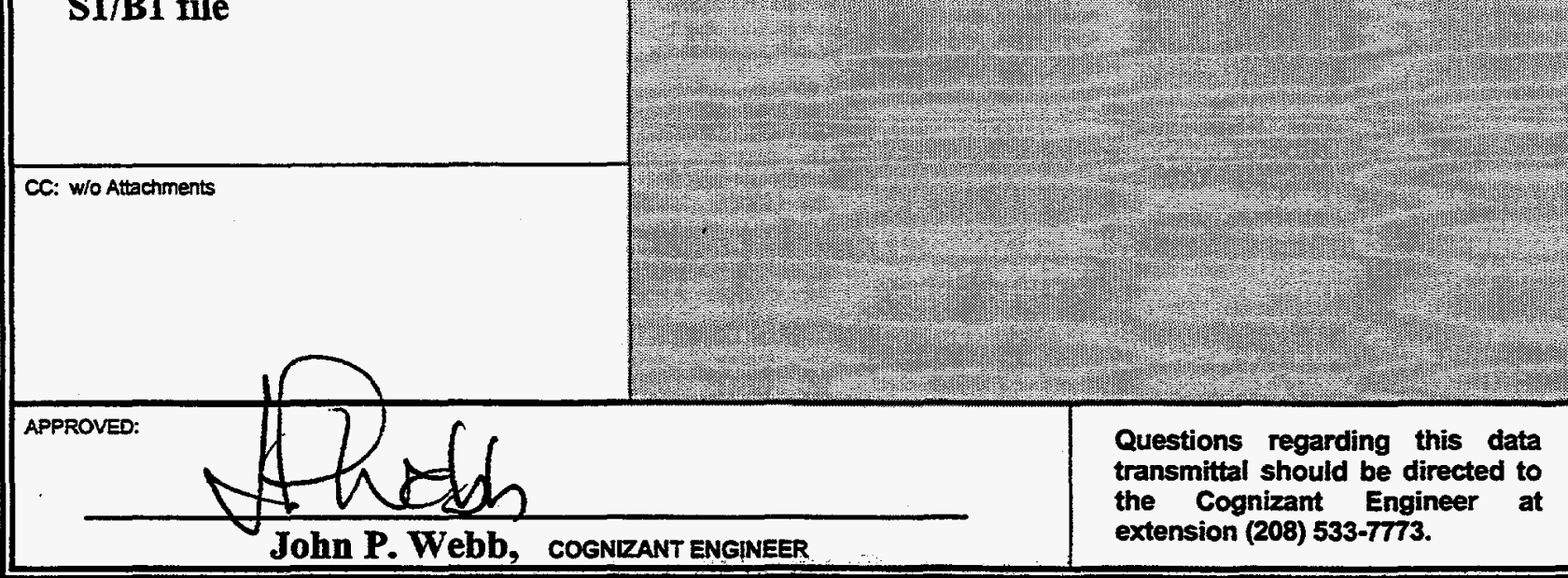

Revised: 2/11/97 
ARGONNE NATIONAL LABORATORY

Hot Fuel Examination Facility

\section{ELEMENT CONTACT PROFILOMETRY}

$\begin{array}{llll}\text { S/A: S1/B1 Element: WT-11 Angle: ODEGREES } & 1 / 31 / 97 & 10: 56 \text { AM }\end{array}$

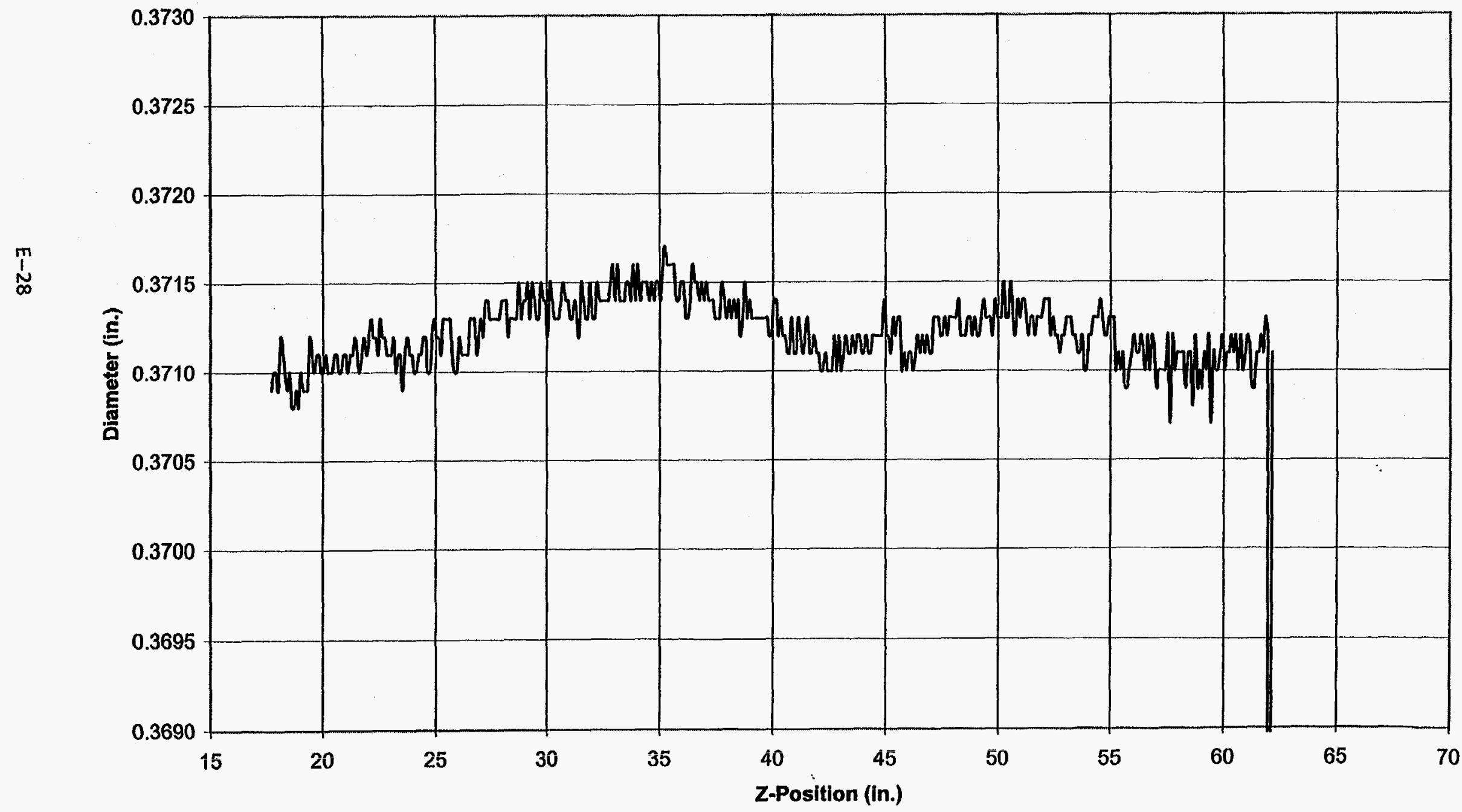



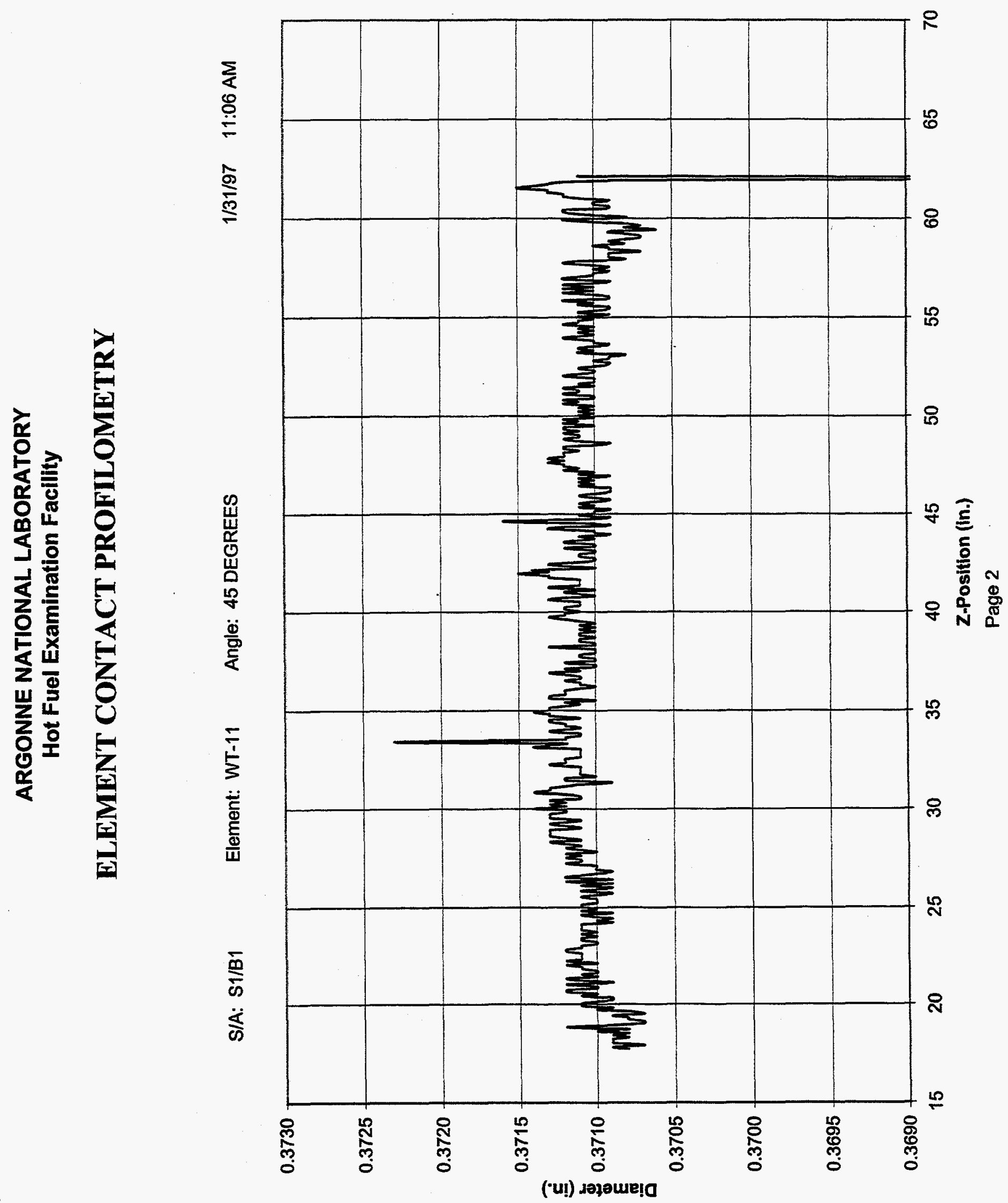
ARGONNE NATIONAL LABORATORY

Hot Fuel Examination Facility

\section{ELEMENT CONTACT PROFILOMETRY}

S/A: S1/B1

Element: WT-11

Angle: 90 DEGREES

1/31/97 11:13 AM

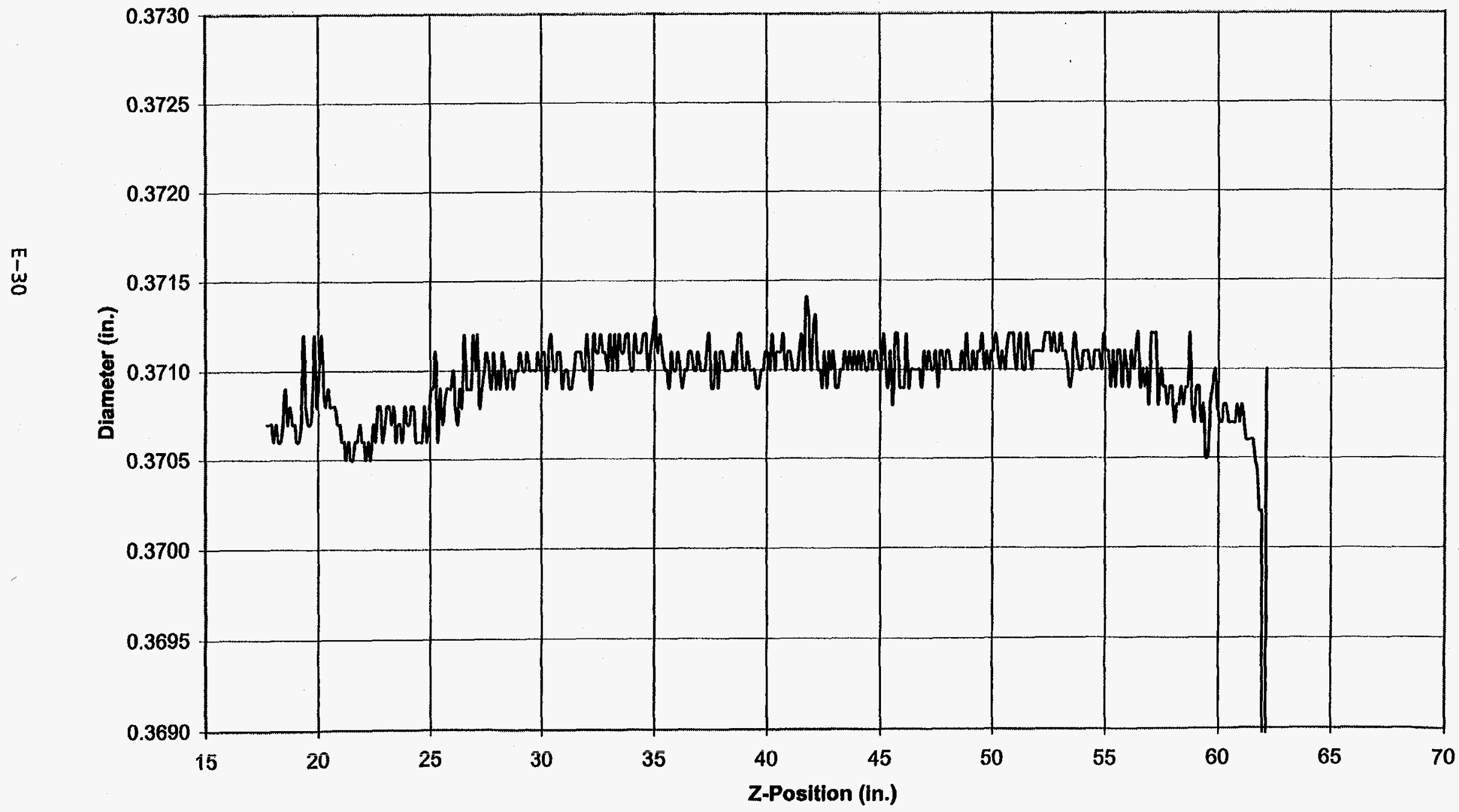

Page 3. 

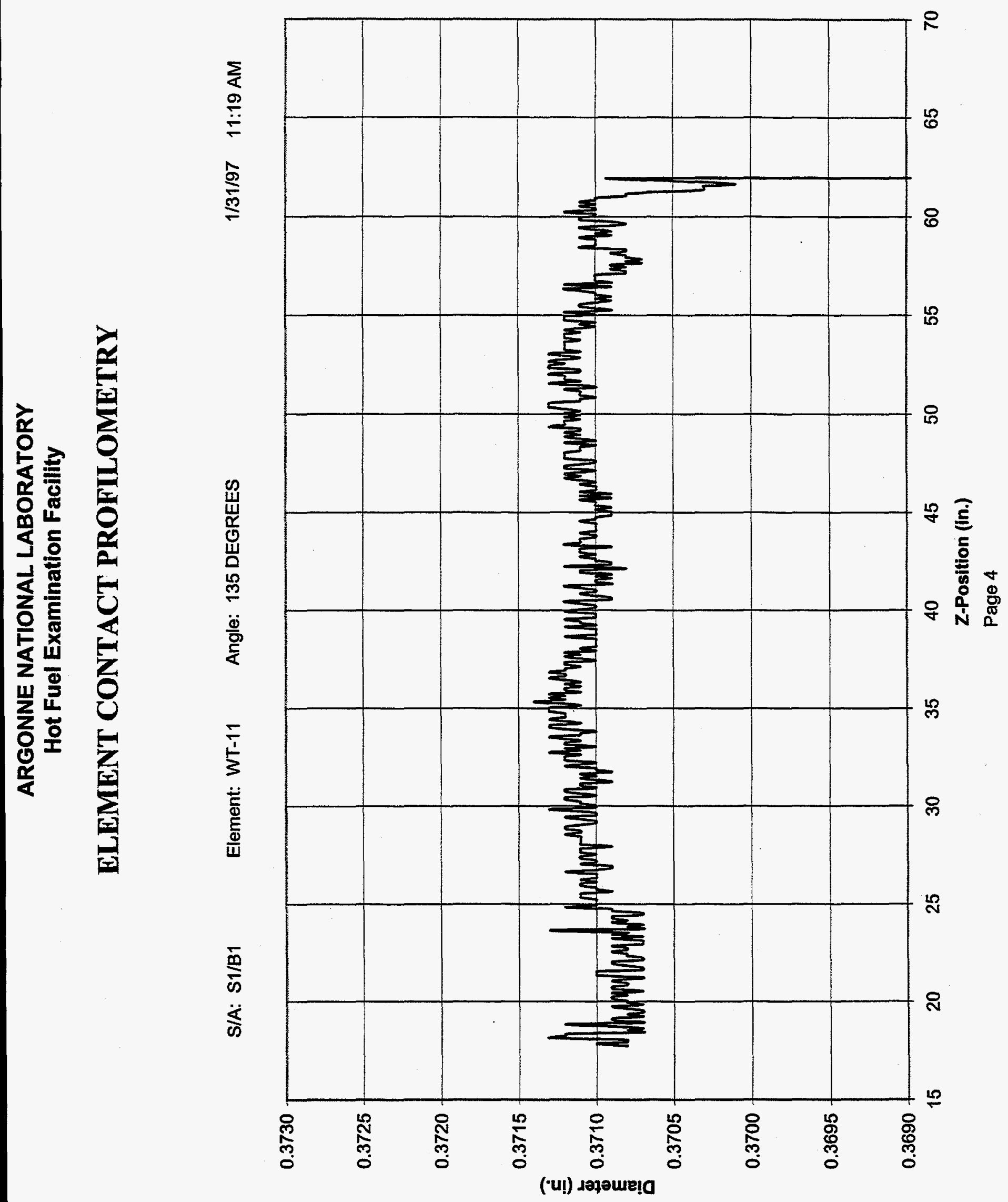


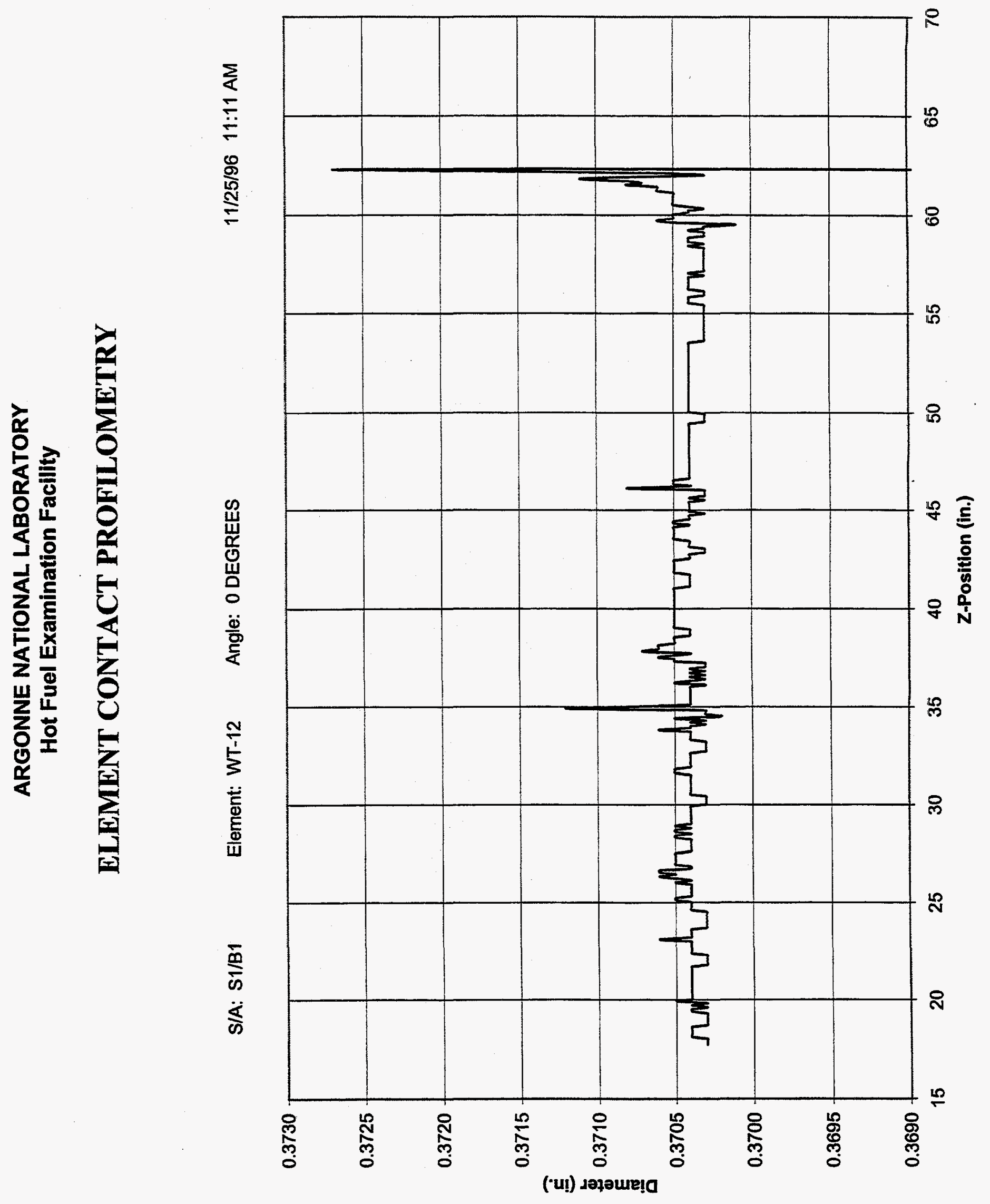



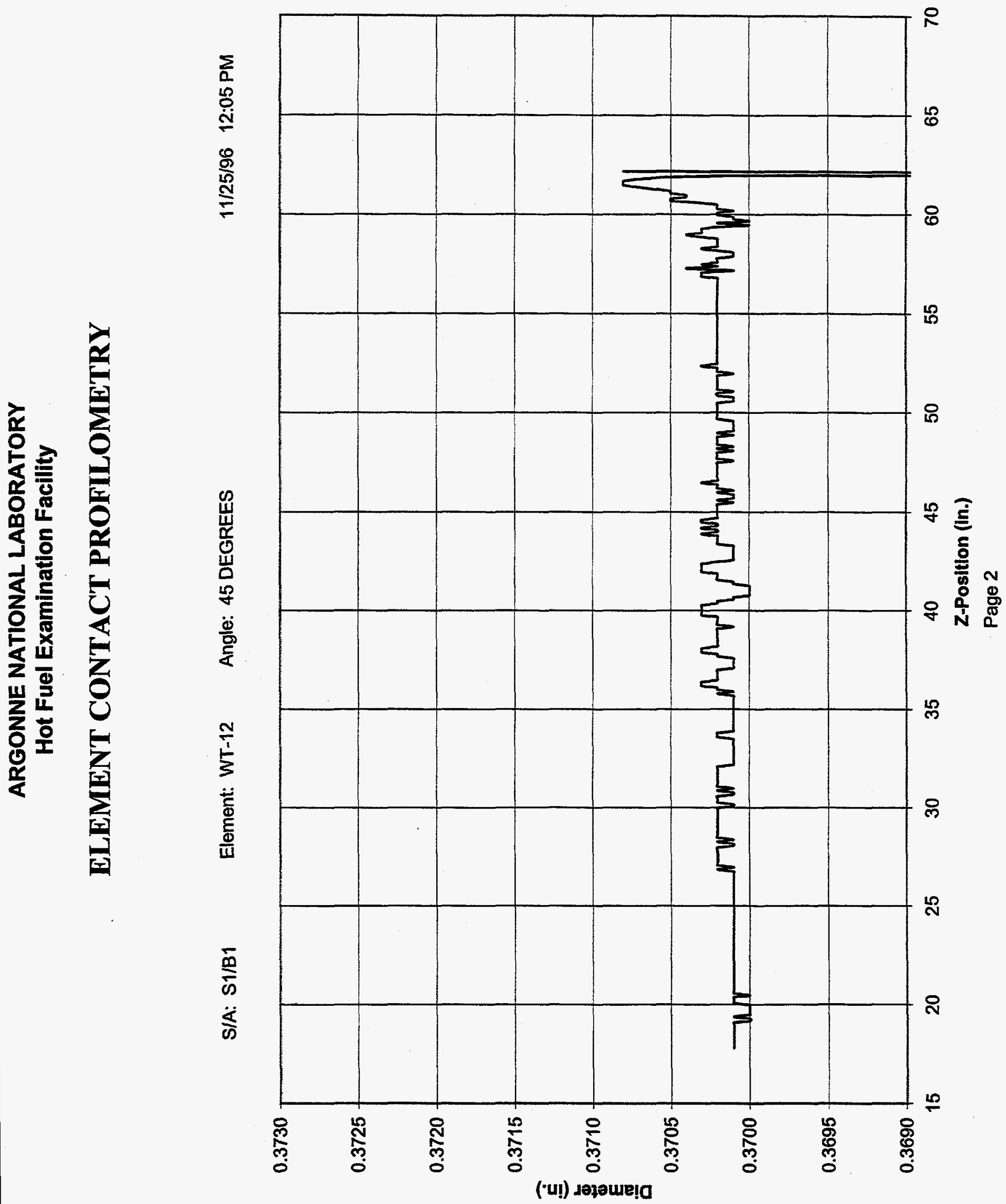
ARGONNE NATIONAL LABORATORY

Hot Fuel Examination Facility

\section{ELEMENT CONTACT PROFILOMETRY}

S/A: S1/B1 Element: WT-12 Angle: 90 DEGREES 11/25/96 12:21 PM

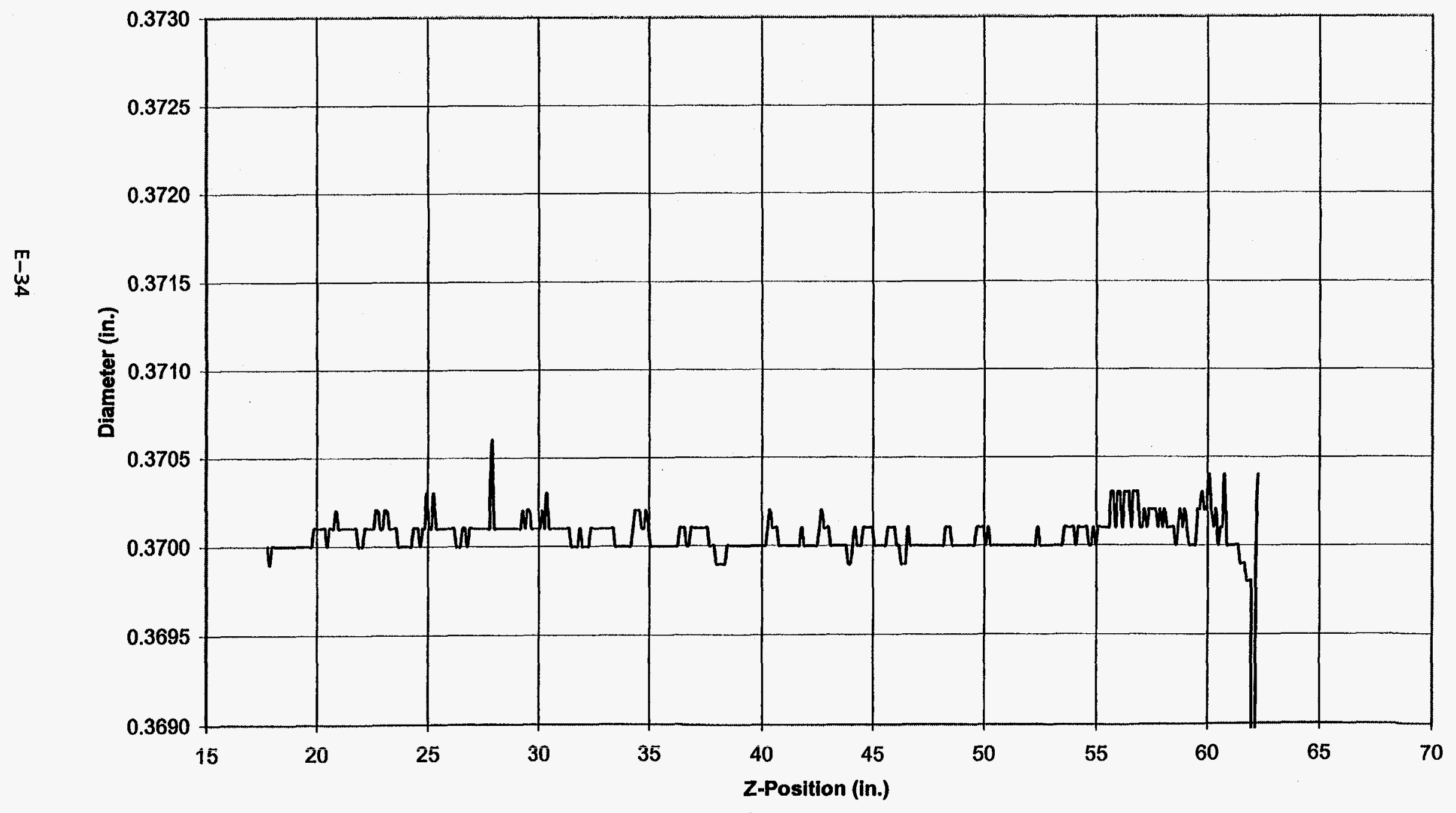

Page 3 


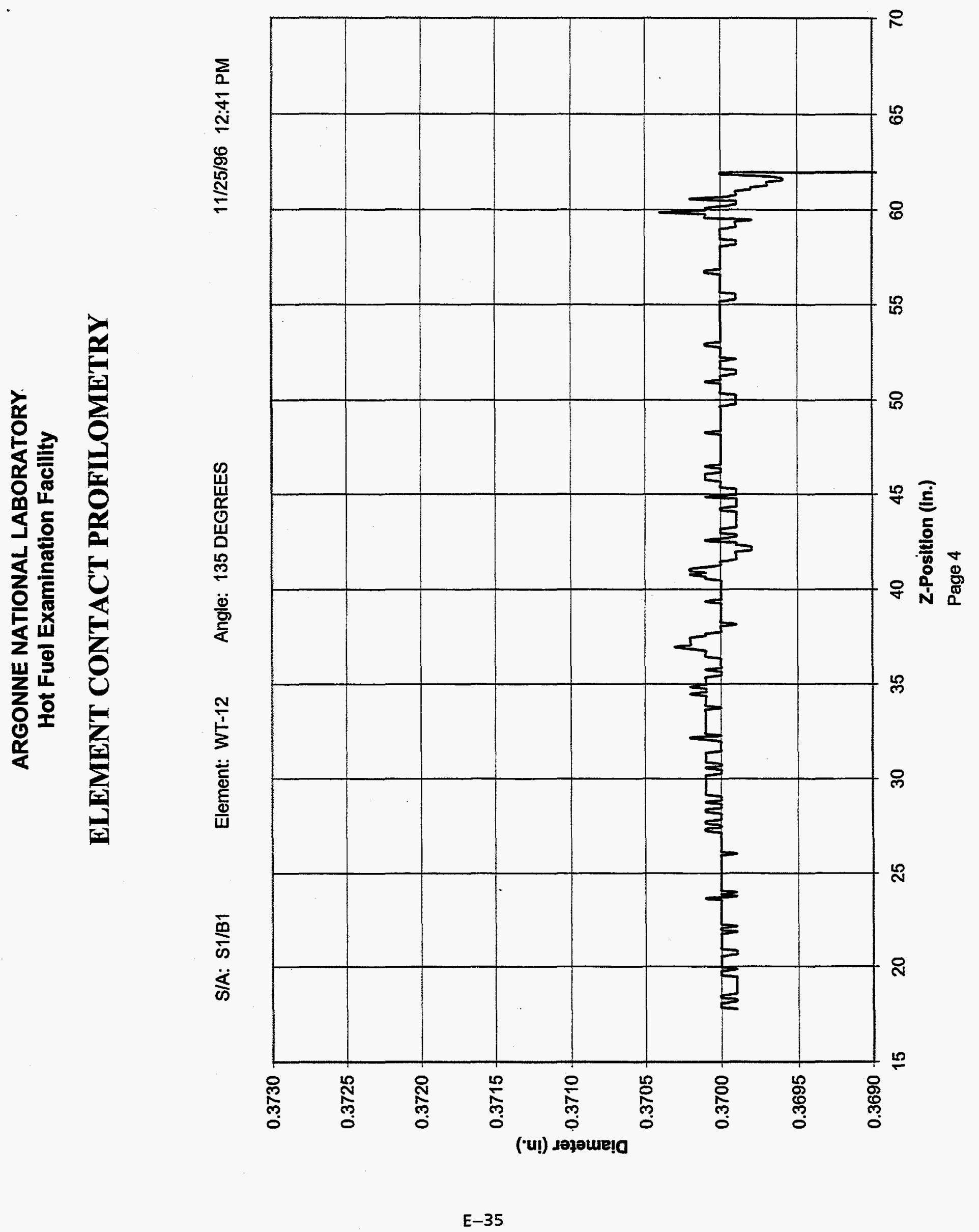


Appendix F

Bow and Length 


\section{Appendix F}

\section{Table of Contents}

Number

WL-01

WL-03

WL-09

WL-10

WL-18

WL-22

WL-26

WL-27

WT-11

WT-12

Bow and Length

Bow and Length

Bow and Length

Bow and Length

Bow and Length

Bow and Length

Bow and Length

Bow and Length

Bow and Length

Bow and Length 
F-ii 


\section{ARGONNE NATIONAL LABORATORY}

P. O. Box 2528, Idaho Falls, Idaho $83403-2528$

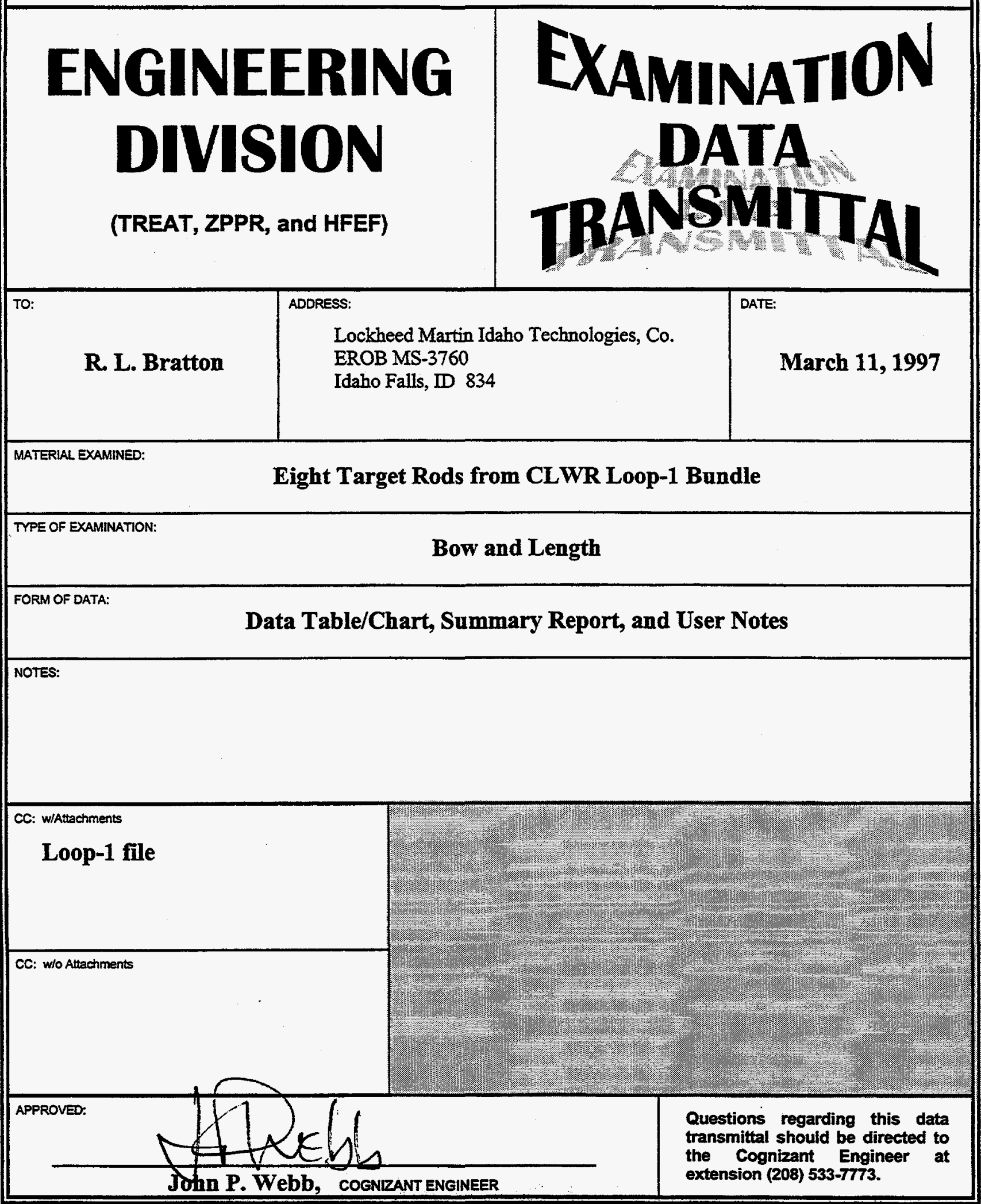




\section{ARGONNE NATIONAL LABORATORY Hot Fuel Examination Facility}

\section{Bow and Length Measurement USER NOTES}

\section{MEASUREMENT PROCESS*}

1. Normal operating procedures for the Bow \& Length (B\&L) System are contained in HFEF Operating Instruction OI-4325.

2. Bow and length measurements are made with the element (or capsule) hanging freely from a collet-style end-fitting applied to it's top-end plug. The end-fitting is held rigidly in the gripper attached to the vertical carriage of the B\&I examination stage.

3. The position of the element is measured in 3D space $(x, y, z)$ at a number of axial locations (typically at 2-in. increments) along it's length.

4. The hanging length of the element is measured by touching the bottom tip of the element to a conductive plate on the B\&L gage assembly, and comparing that $Z$-position to the Z-position of a length standard similarly positioned.

\section{STANDARD CALCULATION*}

- A custom computer program uses the bow and length data acquired in steps 3 and 4 above to calculate bow, bow direction, and corrected length for each element measured.

- Bow and bow direction are reported for each elevation measured in step 3, along with the location and direction of the maximum bow.

- Bow is defined as the distance from the theoretical tip-to-tip centerline to the element's centerline at any axial position along the element. Bow direction is the azimuthal angle of the centerline of the element relative to an angular reference feature on the element, measured counter-clockwise around the tip-to-tip centerline.

- The tip-to-tip centerline is inferred from the bow and length data obtained in steps 3 and 4 by extrapolating the two extreme bow measurements at each end of the element to the theoretical axial locations of the tips based on the hanging length data.

- Corrected length is calculated by summing the segment lengths (from tip to tip) between the calculated 3D positions of the element centerline (i.e., the length along the bowed shape of the element).

\section{STANDARD DATA REPORT}

- The computer program that performs the data reduction described above provides a standard data report.

- Data are reduced in groups of similar elements that share a common length calibration.

- Included in this report is a table of bow data for each element, a table of maximum bow (and bow direction) for each element, and a table of the corrected length of each element.

- This report contains ASCII text only, with no plots or graphics of any kind. It is formatted for printer output, but can be captured to a file and provided to the end user in electronic form if requested.

\section{SPECIAL REPORTS}

- By importing the above standard data report into appropriate computer applications (like Excel), custom reports can be generated that include graphics and other enhancements.

- Because they are not standardized, such reports cannot be described here. Every effort is made to ensure that such special reports self-explanatory.

- If you have any questions about a special report, contact the Cognizant Engineer as described on the Data Transmittal cover sheet.

\section{SPECIAL PROCEDURES}

- Special measurement procedures may sometimes be employed that deviate from the standard methods described above. Such procedures are described in the appropriate HFEF work-control documentation, such as Non-Routine Procedures (NRP's) or Process Work Sheets (PWS's). 


\section{ARGONNE NATIONAL LABORATORY \\ Hot Fuel Examination Facility}

TEST ID: Loop-1

\begin{tabular}{|c|c|c|}
\hline $\begin{array}{c}\text { Z-Pos } \\
\text { (in.) }\end{array}$ & $\begin{array}{c}\text { BOW } \\
\text { (in.) }\end{array}$ & $\begin{array}{c}\text { ANGLE } \\
\text { (") }\end{array}$ \\
\hline 0.00 & 0.000 & \\
\hline 3.41 & 0.011 & 32 \\
\hline 6.41 & 0.021 & 32 \\
\hline 9.41 & 0.036 & 38 \\
\hline 12.42 & 0.050 & 40 \\
\hline 15.41 & 0.061 & 37 \\
\hline 18.42 & 0.074 & 38 \\
\hline 21.41 & 0.081 & 40 \\
\hline 24.41 & 0.079 & 37 \\
\hline 27.41 & 0.076 & 38 \\
\hline 30.41 & 0.075 & 38 \\
\hline 33.41 & 0.075 & 35 \\
\hline 36.41 & 0.071 & 33 \\
\hline 39.41 & 0.068 & 35 \\
\hline 42.41 & 0.062 & 36 \\
\hline 45.42 & 0.054 & 36 \\
\hline 48.41 & 0.044 & 35 \\
\hline 51.41 & 0.031 & 33 \\
\hline 54.41 & 0.019 & 28 \\
\hline 55.51 & 0.013 & 28 \\
\hline 57.87 & 0.000 & \\
\hline & & \\
\hline
\end{tabular}
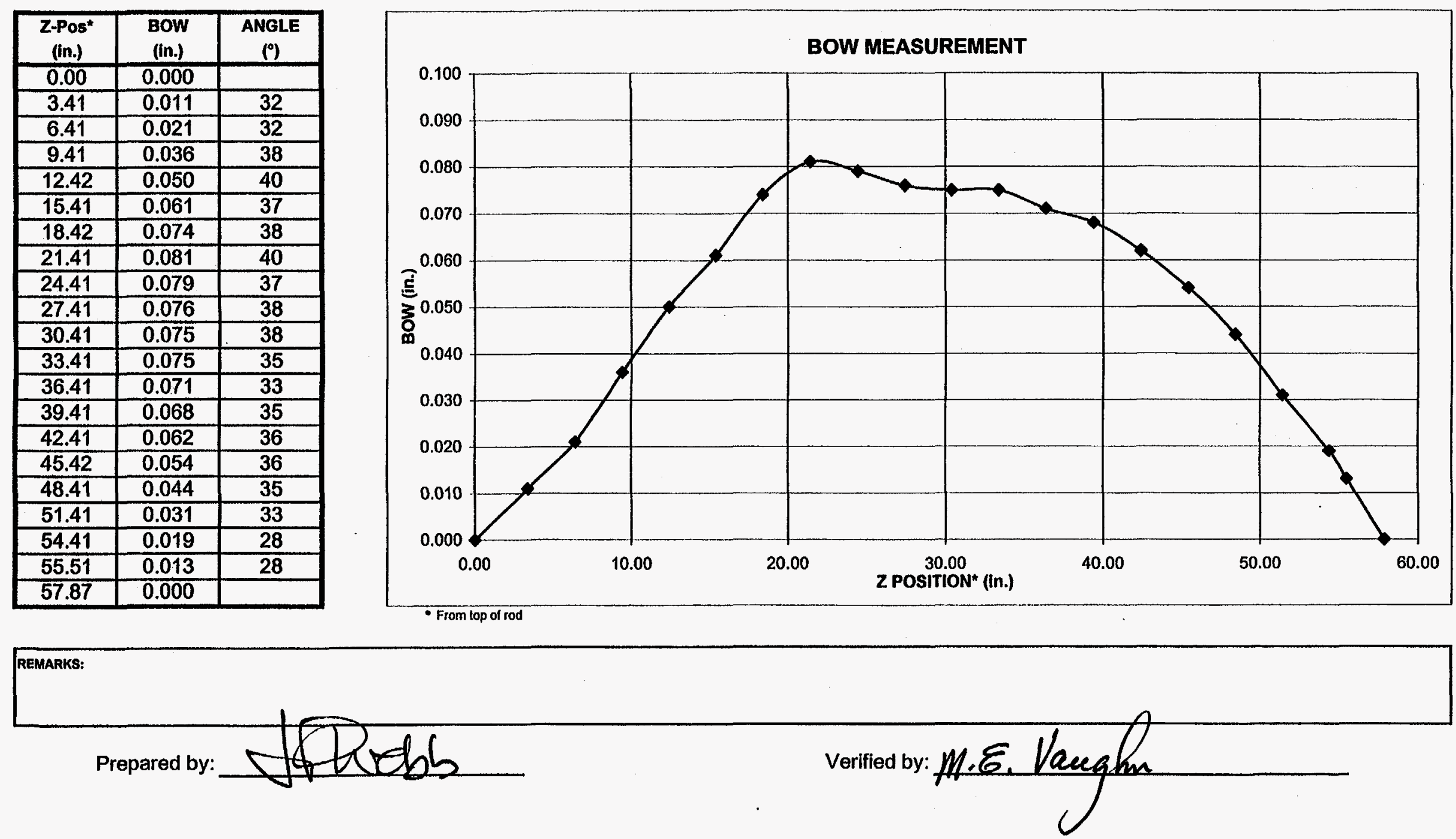

Page 1 of 1 


\section{ARGONNE NATIONAL LABORATORY \\ Hot Fuel Examination Facility}

TEST ID: Loop-1

\begin{tabular}{|c|c|c|}
\hline $\begin{array}{c}\text { Z-POs* } \\
(\mathbf{I n} .)\end{array}$ & $\begin{array}{c}\text { BOW } \\
(\mathbf{I n} \text {.) }\end{array}$ & $\begin{array}{c}\text { ANGLE } \\
\left({ }^{\circ}\right)\end{array}$ \\
\hline 0.00 & 0.000 & \\
\hline 3.39 & 0.004 & 245 \\
\hline 6.38 & 0.008 & 245 \\
\hline 9.38 & 0.012 & 223 \\
\hline 12.38 & 0.014 & 223 \\
\hline 15.39 & 0.016 & 232 \\
\hline 18.38 & 0.015 & 234 \\
\hline 21.38 & 0.013 & 231 \\
\hline 24.39 & 0.011 & 237 \\
\hline 27.38 & 0.014 & 250 \\
\hline 30.39 & 0.014 & 260 \\
\hline 33.39 & 0.010 & 251 \\
\hline 36.39 & 0.002 & 278 \\
\hline 39.38 & 0.001 & 257 \\
\hline 42.38 & 0.002 & 292 \\
\hline 45.38 & 0.002 & 303 \\
\hline 48.38 & 0.001 & 263 \\
\hline 51.38 & 0.001 & 212 \\
\hline 54.38 & 0.003 & 236 \\
\hline 55.49 & 0.002 & 236 \\
\hline 57.82 & 0.000 & \\
\hline & & \\
\hline
\end{tabular}

ROD ID: WL-03

DATE: $\quad \mathbf{1} / \mathbf{1 7 / 9 7}$
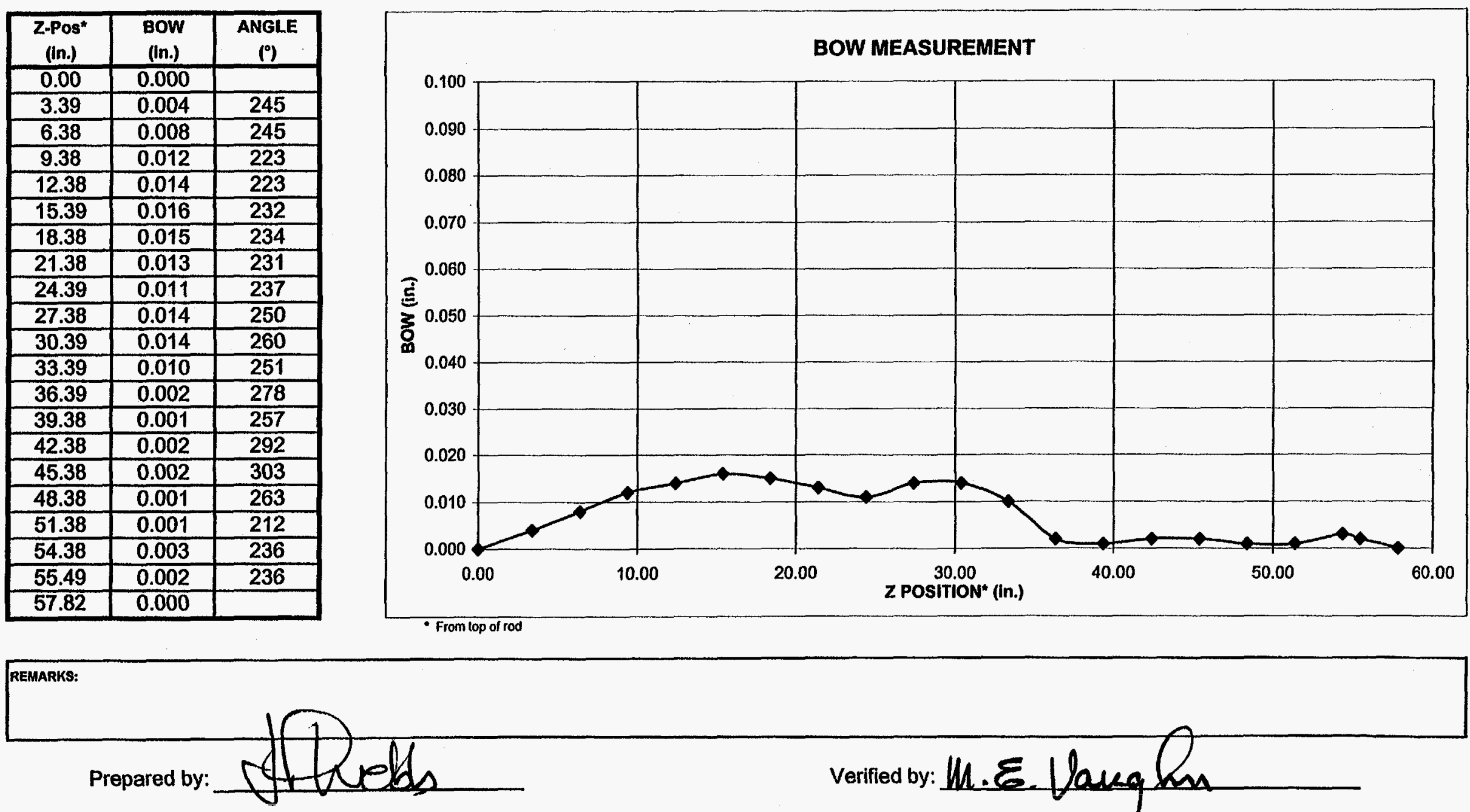

verified by: ML.E. Naug ha

Page 1 of 1 


\section{ARGONNE NATIONAL LABORATORY \\ Hot Fuel Examination Facility}

TEST ID: Loop-1

\begin{tabular}{|c|c|c|}
\hline $\begin{array}{c}\text { Z-Pos } \\
(\text { in. })\end{array}$ & $\begin{array}{c}\text { BOW } \\
(\text { in.) }\end{array}$ & $\begin{array}{c}\text { ANGLE } \\
\left({ }^{\circ}\right)\end{array}$ \\
\hline 0.00 & 0.000 & \\
\hline 3.40 & 0.008 & 313 \\
\hline 6.40 & 0.014 & 313 \\
\hline 9.40 & 0.016 & 299 \\
\hline 12.39 & 0.020 & 282 \\
\hline 15.39 & 0.023 & 287 \\
\hline 18.40 & 0.025 & 272 \\
\hline 21.40 & 0.026 & 260 \\
\hline 24.39 & 0.028 & 257 \\
\hline 27.39 & 0.027 & 248 \\
\hline 30.40 & 0.027 & 244 \\
\hline 33.39 & 0.027 & 238 \\
\hline 36.40 & 0.028 & 232 \\
\hline 39.39 & 0.029 & 224 \\
\hline 42.40 & 0.024 & 221 \\
\hline 45.40 & 0.019 & 217 \\
\hline 48.40 & 0.014 & 215 \\
\hline 51.40 & 0.011 & 210 \\
\hline 54.40 & 0.004 & 196 \\
\hline 55.50 & 0.003 & 196 \\
\hline 57.84 & 0.000 & \\
\hline
\end{tabular}

ROD ID: WL-09

DATE: $\quad$ 1/17/97

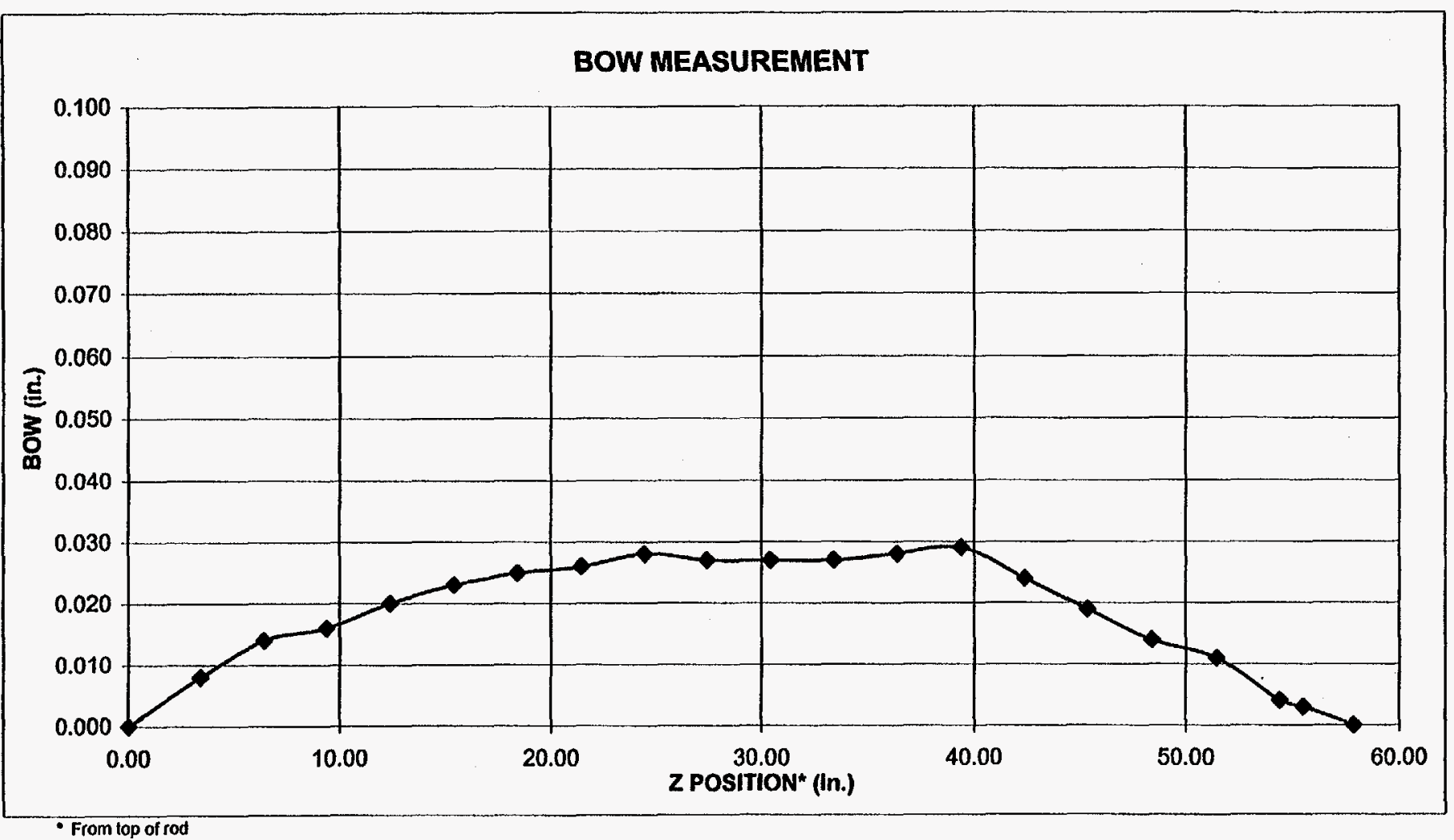

REMARKS:

Prepared by:

trobh

verifled by: M.E. Mesg Kan

Page 1 of 1 


\section{ARGONNE NATIONAL LABORATORY \\ Hot Fuel Examination Facility}

TEST ID: Loop-1

ROD ID: WL-10

DATE: $\quad \mathbf{1 / 1 7 / 9 7}$

\begin{tabular}{|c|c|c|}
\hline $\begin{array}{c}\text { Z-Pos } \\
\text { (in.) }\end{array}$ & $\begin{array}{c}\text { BOW } \\
\text { (in.) }\end{array}$ & $\begin{array}{c}\text { ANGLE } \\
\left({ }^{\circ}\right)\end{array}$ \\
\hline 0.00 & 0.000 & \\
\hline 3.41 & 0.008 & 258 \\
\hline 6.40 & 0.016 & 258 \\
\hline 9.41 & 0.025 & 259 \\
\hline 12.40 & 0.031 & 253 \\
\hline 15.41 & 0.038 & 257 \\
\hline 18.40 & 0.046 & 259 \\
\hline 21.41 & 0.054 & 260 \\
\hline 24.40 & 0.057 & 263 \\
\hline 27.41 & 0.062 & 262 \\
\hline 30.41 & 0.065 & 263 \\
\hline 33.41 & 0.065 & 263 \\
\hline 36.41 & 0.064 & 263 \\
\hline 39.40 & 0.058 & 262 \\
\hline 42.41 & 0.051 & 262 \\
\hline 45.40 & 0.044 & 263 \\
\hline 48.40 & 0.034 & 264 \\
\hline 51.41 & 0.024 & 264 \\
\hline 54.40 & 0.013 & 261 \\
\hline 55.51 & 0.009 & 261 \\
\hline 57.83 & 0.000 & \\
\hline & & \\
\hline
\end{tabular}
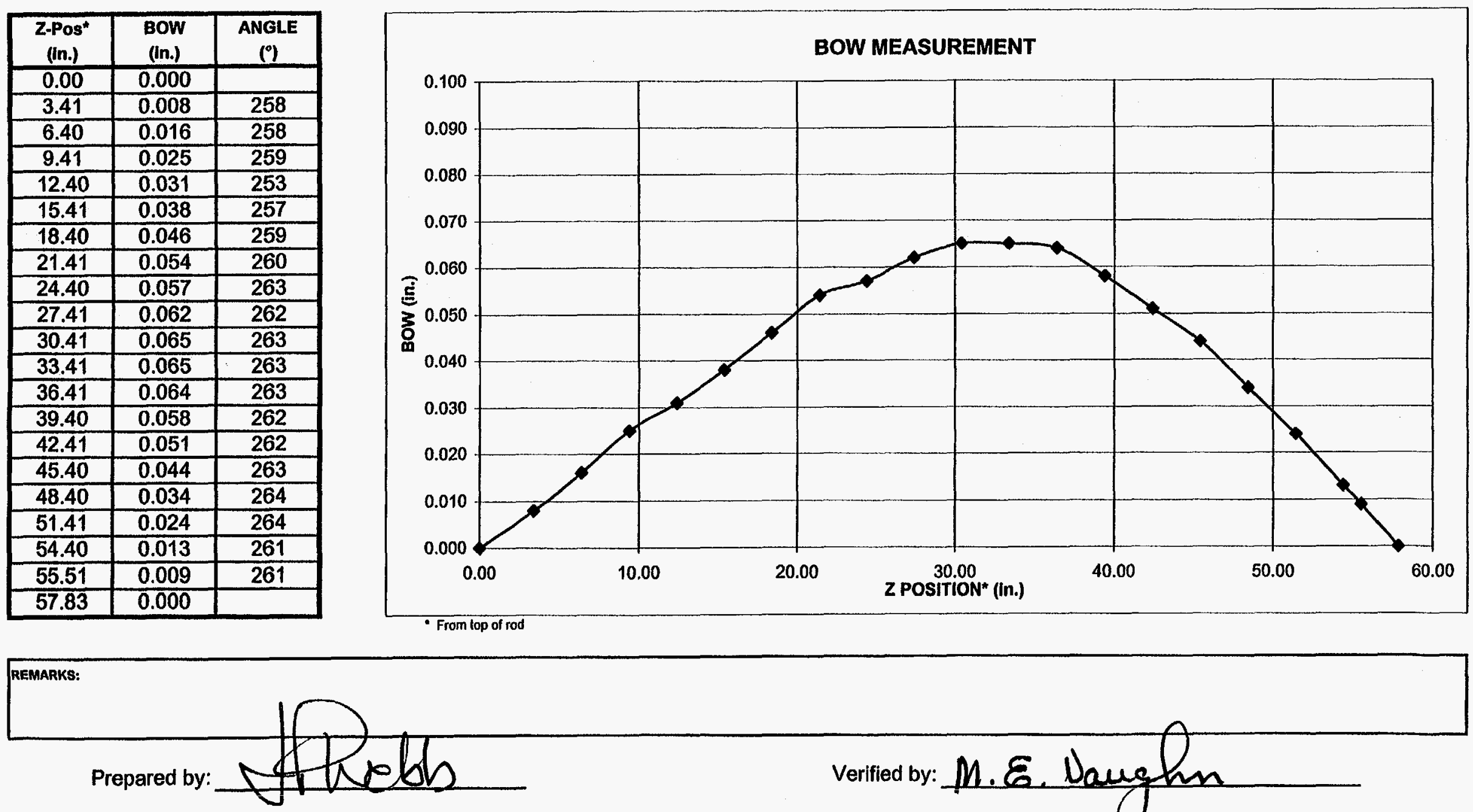

Verified by: M.E. Naughn 


\section{ARGONNE NATIONAL LABORATORY \\ Hot Fuel Examination Facility}

TEST ID: Loop-1

\begin{tabular}{|c|c|c|}
\hline $\begin{array}{c}\text { Z-Pos } \\
\text { (in.) }\end{array}$ & $\begin{array}{c}\text { BOW } \\
\text { (in.) }\end{array}$ & $\begin{array}{c}\text { ANGLE } \\
\left({ }^{\text {( ) }}\right.\end{array}$ \\
\hline 0.00 & 0.000 & \\
\hline 3.33 & 0.001 & 135 \\
\hline 6.33 & 0.003 & 135 \\
\hline 9.33 & 0.007 & 116 \\
\hline 12.33 & 0.009 & 121 \\
\hline 15.34 & 0.010 & 140 \\
\hline 18.33 & 0.012 & 141 \\
\hline 21.34 & 0.011 & 142 \\
\hline 24.34 & 0.008 & 155 \\
\hline 27.34 & 0.005 & 155 \\
\hline 30.34 & 0.005 & 145 \\
\hline 33.33 & 0.005 & 152 \\
\hline 36.33 & 0.003 & 170 \\
\hline 39.34 & 0.006 & 157 \\
\hline 42.34 & 0.005 & 130 \\
\hline 45.33 & 0.005 & 116 \\
\hline 48.34 & 0.005 & 86 \\
\hline 51.34 & 0.002 & 52 \\
\hline 54.33 & 0.002 & 327 \\
\hline 55.44 & 0.001 & 327 \\
\hline 57.77 & 0.000 & \\
\hline & & \\
\hline
\end{tabular}
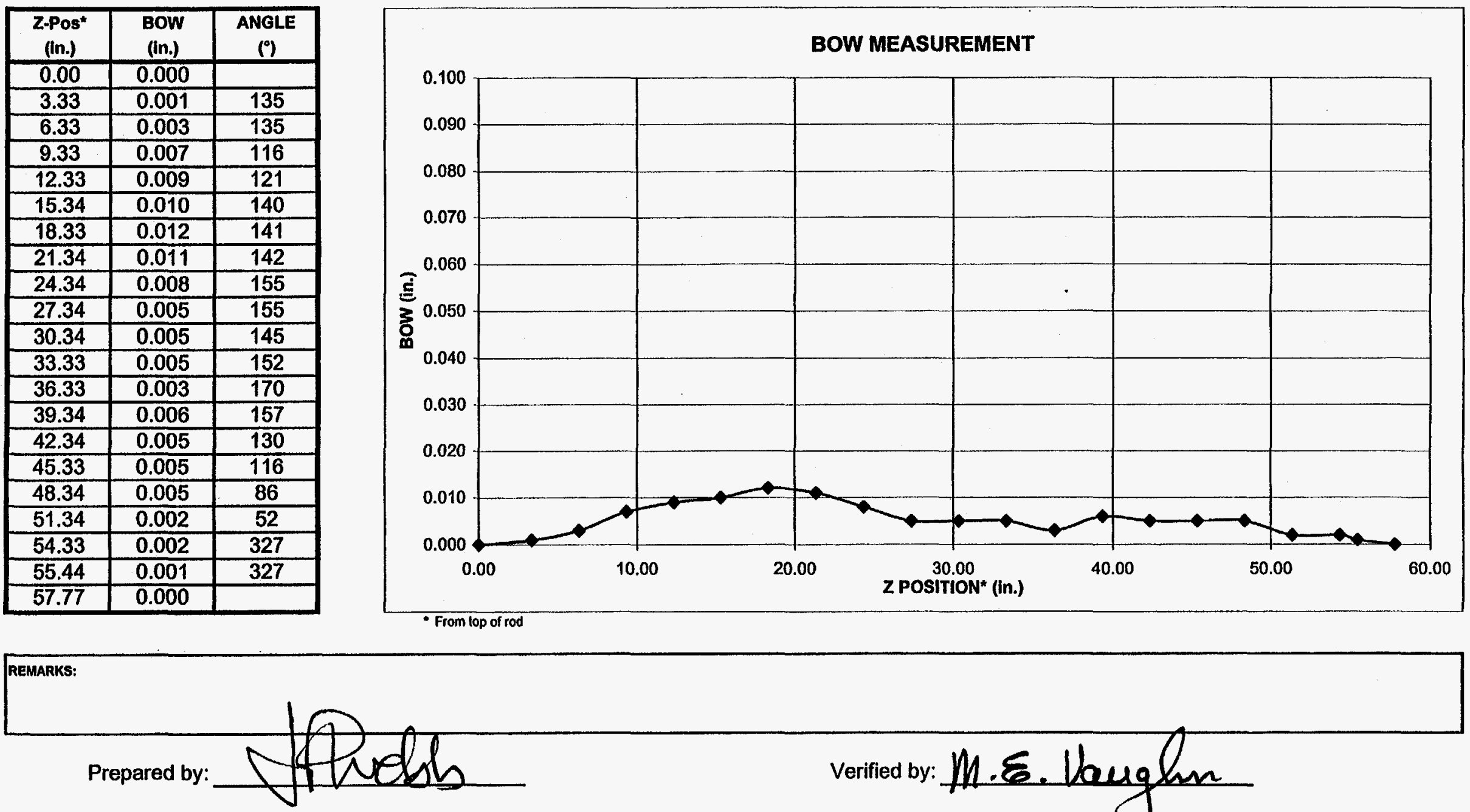

verified by: M.E. Vaughn

Page 1 of 1 


\section{ARGONNE NATIONAL LABORATORY}

Hot Fuel Examination Facility

TEST ID: Loop-1

\begin{tabular}{|c|c|c|}
\hline $\begin{array}{c}\text { Z-Pos* } \\
(\text { in. })\end{array}$ & $\begin{array}{c}\text { BOW } \\
(\text { in. })\end{array}$ & $\begin{array}{c}\text { ANGLE } \\
(?)\end{array}$ \\
\hline 0.00 & 0.000 & \\
\hline 3.39 & 0.008 & 142 \\
\hline 6.38 & 0.015 & 142 \\
\hline 9.38 & 0.024 & 138 \\
\hline 12.38 & 0.030 & 137 \\
\hline 15.38 & 0.044 & 133 \\
\hline 18.38 & 0.051 & 131 \\
\hline 21.39 & 0.056 & 129 \\
\hline 24.38 & 0.057 & 131 \\
\hline 27.38 & 0.057 & 131 \\
\hline 30.38 & 0.063 & 126 \\
\hline 33.39 & 0.063 & 125 \\
\hline 36.38 & 0.061 & 126 \\
\hline 39.38 & 0.056 & 126 \\
\hline 42.39 & 0.048 & 125 \\
\hline 45.38 & 0.041 & 125 \\
\hline 48.38 & 0.032 & 123 \\
\hline 51.38 & 0.021 & 116 \\
\hline 54.38 & 0.012 & 107 \\
\hline 55.49 & 0.008 & 107 \\
\hline 57.83 & 0.000 & \\
\hline & & \\
\hline
\end{tabular}

ROD ID: WL-22

DATE: $\quad 1 / 17 / 97$
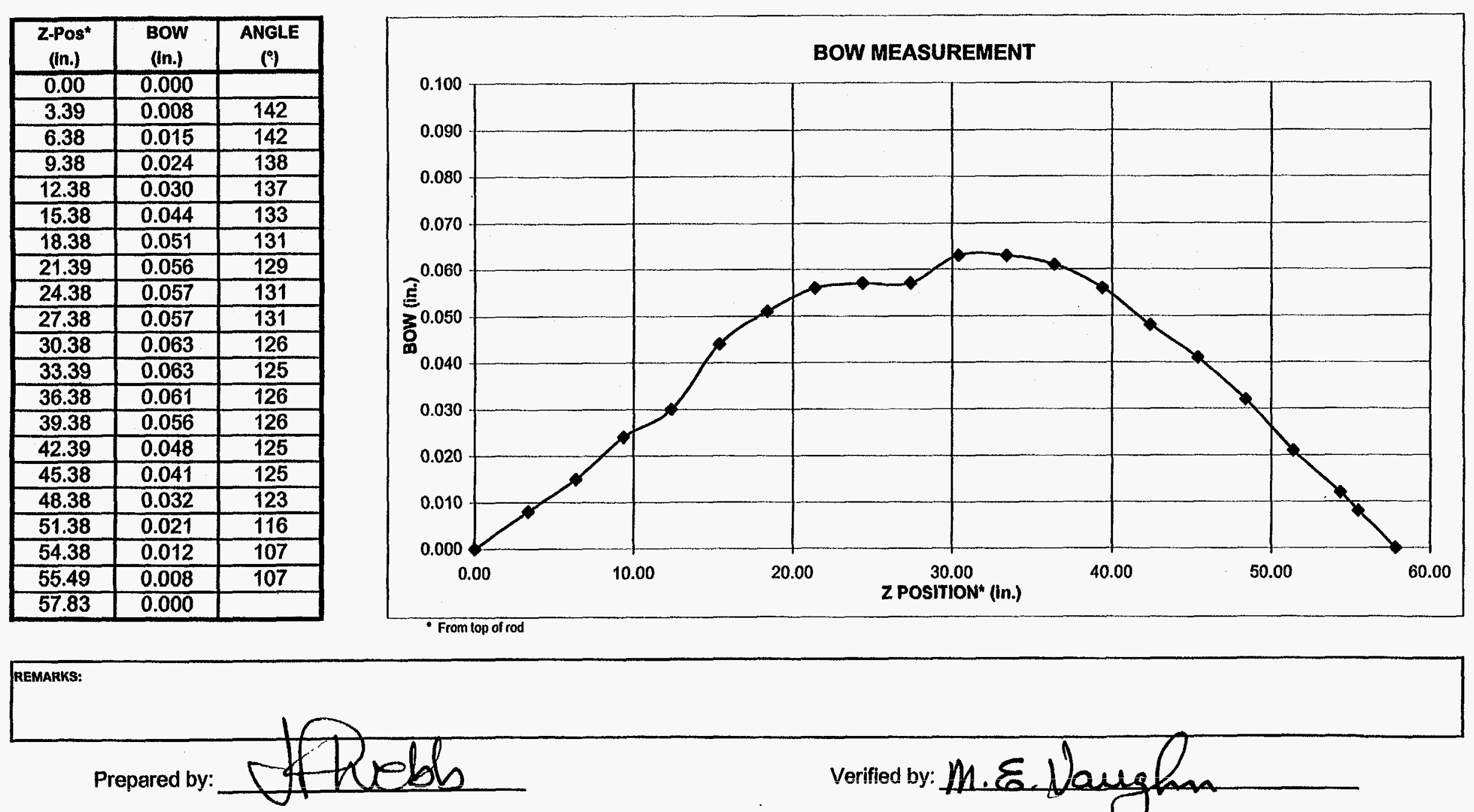

verified by: M.E. Naugha

Page 1 of 1 


\section{ARGONNE NATIONAL LABORATORY \\ Hot Fuel Examination Facility}

TEST ID: Loop-1

\begin{tabular}{|c|c|c|}
\hline $\begin{array}{c}\text { Z-Pos } \\
(\mathbf{( n . )}\end{array}$ & $\begin{array}{c}\text { BOW } \\
(\text { (n.) }\end{array}$ & $\begin{array}{c}\text { ANGLE } \\
\left({ }^{\circ}\right)\end{array}$ \\
\hline 0.00 & 0.000 & \\
\hline 3.35 & 0.017 & 227 \\
\hline 6.35 & 0.032 & 227 \\
\hline 9.35 & 0.045 & 224 \\
\hline 12.35 & 0.062 & 220 \\
\hline 15.35 & 0.071 & 218 \\
\hline 18.35 & 0.082 & 218 \\
\hline 21.35 & 0.088 & 218 \\
\hline 24.35 & 0.092 & 218 \\
\hline 27.35 & 0.094 & 218 \\
\hline 30.35 & 0.094 & 219 \\
\hline 33.35 & 0.089 & 218 \\
\hline 36.35 & 0.083 & 219 \\
\hline 39.35 & 0.073 & 220 \\
\hline 42.35 & 0.065 & 218 \\
\hline 45.35 & 0.055 & 217 \\
\hline 48.35 & 0.045 & 216 \\
\hline 51.35 & 0.032 & 215 \\
\hline 54.35 & 0.018 & 214 \\
\hline 55.45 & 0.012 & 214 \\
\hline 57.76 & 0.000 & \\
\hline
\end{tabular}

ROD ID: WL-26

DATE: $\quad 1 / 17 / 97$
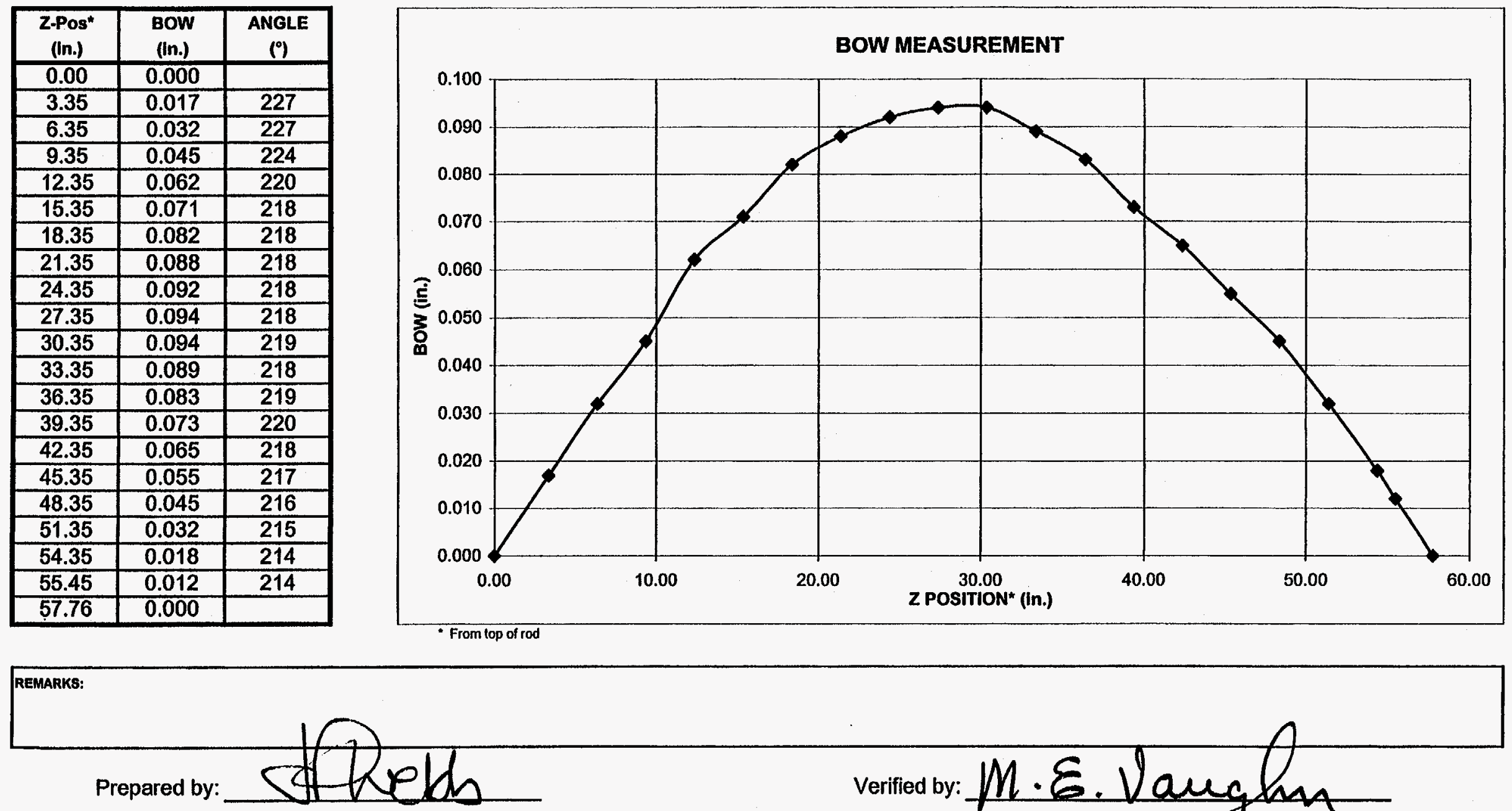

Page 1 of 1 


\section{ARGONNE NATIONAL LABORATORY Hot Fuel Examination Facility}

TEST ID: Loop-1

\begin{tabular}{|c|c|c|}
\hline $\begin{array}{c}\text { Z-Pos } \\
(\text { (n.) }\end{array}$ & $\begin{array}{c}\text { BOW } \\
(\text { (n.) }\end{array}$ & $\begin{array}{c}\text { ANGLE } \\
\left({ }^{\circ}\right)\end{array}$ \\
\hline 0.00 & 0.000 & \\
\hline 3.39 & 0.014 & 157 \\
\hline 6.39 & 0.027 & 157 \\
\hline 9.39 & 0.042 & 156 \\
\hline 12.39 & 0.052 & 156 \\
\hline 15.39 & 0.063 & 156 \\
\hline 18.39 & 0.073 & 154 \\
\hline 21.39 & 0.082 & 154 \\
\hline 24.39 & 0.085 & 152 \\
\hline 27.39 & 0.089 & 152 \\
\hline 30.39 & 0.091 & 150 \\
\hline 33.39 & 0.090 & 151 \\
\hline 36.39 & 0.084 & 149 \\
\hline 39.39 & 0.076 & 148 \\
\hline 42.39 & 0.066 & 146 \\
\hline 45.39 & 0.056 & 145 \\
\hline 48.39 & 0.041 & 146 \\
\hline 51.39 & 0.027 & 147 \\
\hline 54.39 & 0.013 & 148 \\
\hline 55.49 & 0.009 & 148 \\
\hline 57.84 & 0.000 & \\
\hline & & \\
\hline
\end{tabular}

ROD ID: WL-27

DATE: $\quad \mathbf{1 / 1 7 / 9 7}$

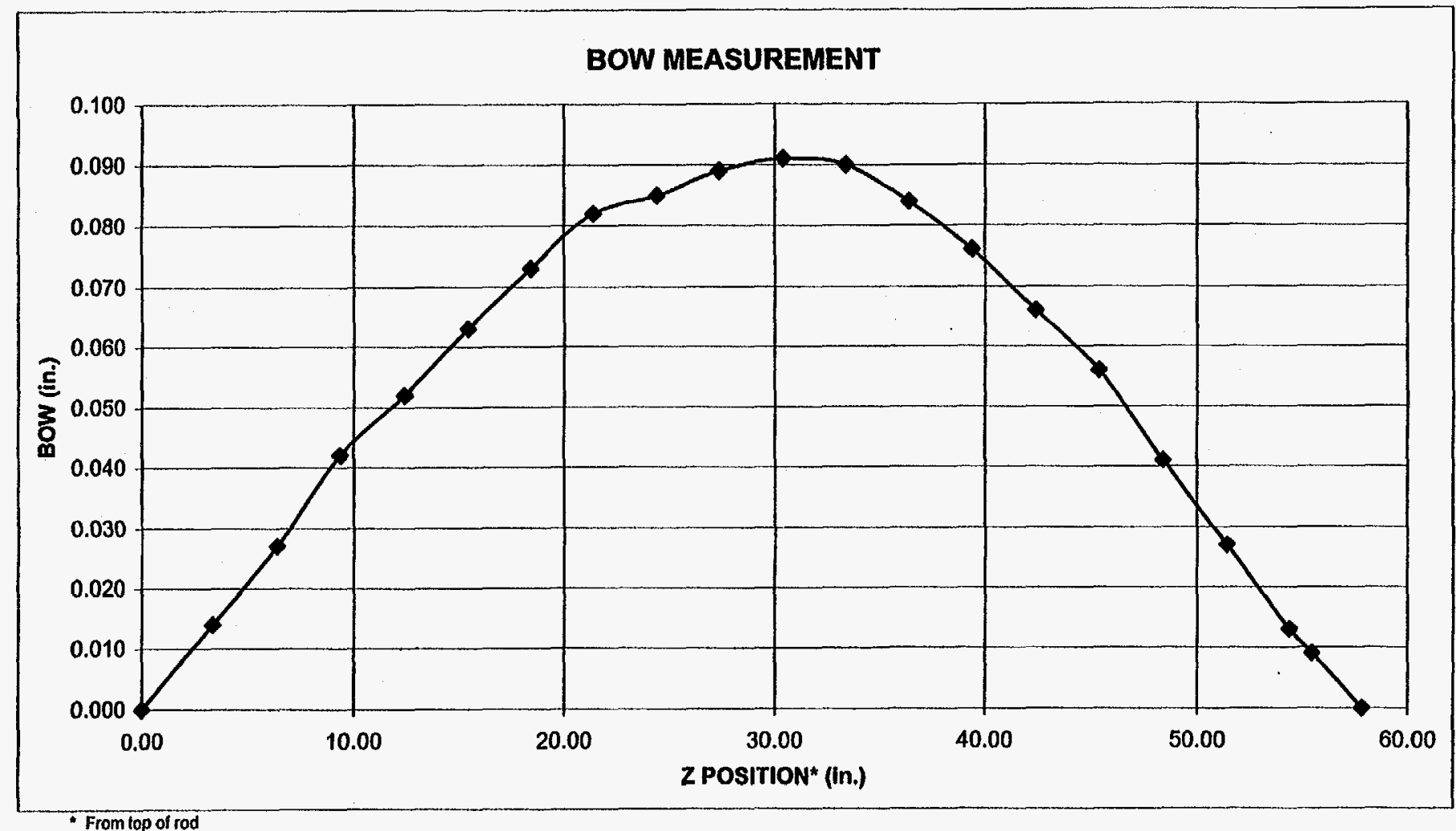

REMARKS:

Prepared by:

47

stavan

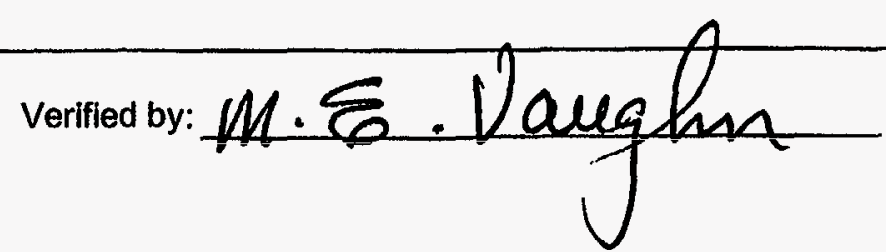




\section{ARGONNE NATIONAL LABORATORY \\ Hot Fuel Examination Facility}

\section{BOW AND LENGTH \\ SUMMARY REPORT}

\section{CLWR Loop-1}

\begin{tabular}{|c|c|c|c|c|c|}
\hline \multirow[b]{2}{*}{ ROD ID } & \multirow{2}{*}{$\begin{array}{l}\text { CORRECTED } \\
\text { LENGTH } \\
\text { (in.) }\end{array}$} & \multicolumn{3}{|c|}{ MAXIMUM BOW } & \multirow[b]{2}{*}{ REMARKS } \\
\hline & & $\begin{array}{l}\text { BOW } \\
\text { (in.) }\end{array}$ & $\begin{array}{l}\text { Zpos } \\
\text { (in.) }\end{array}$ & $\begin{array}{l}\text { Phi } \\
\text { (०) }\end{array}$ & \\
\hline WL-01 & 57.88 & 0.081 & 21.41 & 40 & \\
\hline WL-03 & 57.82 & 0.016 & 15.39 & 232 & \\
\hline WL-09 & 57.85 & 0.029 & 39.39 & 224 & \\
\hline$\overline{\mathrm{WL}}-10$ & 57.83 & 0.065 & 33.41 & 263 & \\
\hline WL-18 & 57.80 & 0.012 & 18.33 & 141 & \\
\hline$\overline{\mathrm{WL}}-22$ & 57.83 & 0.063 & 33.39 & 125 & \\
\hline WL-26 & 57.77 & 0.094 & 30.35 & 219 & \\
\hline WL-27 & 57.85 & 0.091 & 30.39 & 150 & \\
\hline
\end{tabular}

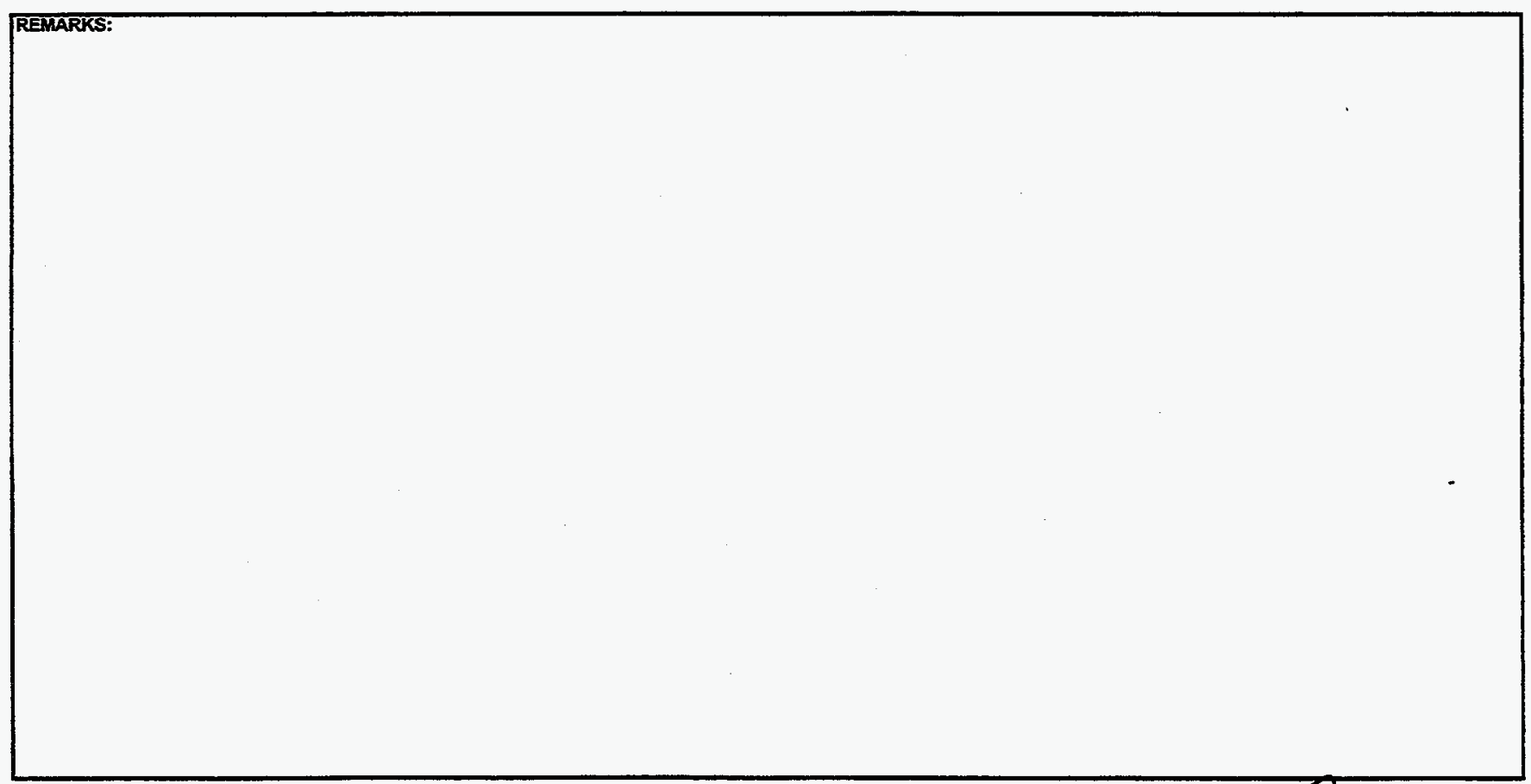

دrepared by:

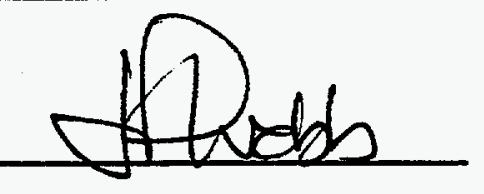




$$
\text { F-12 }
$$




\section{ARGONNE NATIONAL LABORATORY}

P. O. Box 2528, Idaho Falls, Idaho 83403-2528

\section{ENGINEERING DIVISION}

(TREAT, ZPPR, and HFEF)

\section{EXAMination}

DATA

TRANSMITIAL

\begin{tabular}{|c|c|}
\hline R. L. Bratton & $\begin{array}{l}\text { ADDRESS: } \\
\text { Lockheed Martin Idaho Technologies, Co. } \\
\text { EROB MS-3760 } \\
\text { Idaho Falls, ID } 834\end{array}$ \\
\hline MATERIAL EXAMINED: & Two Target Rods from CLWR S1/B \\
\hline
\end{tabular}

TYPE OF EXAMINATON:

Bow and Length

FORM OF DATA:

Data Table/Chart, Summary Report, and User Notes

NOTES:

CC: w/Attachments

S1/B1 file

CC: w/o Attachments

APPROVED:

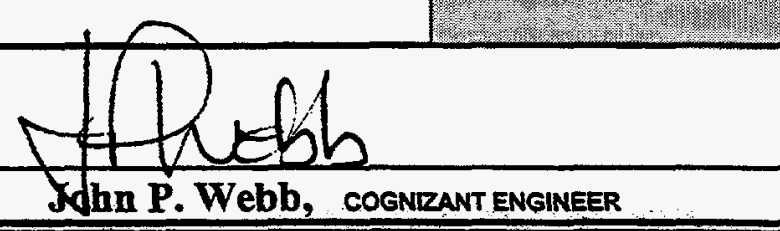

Questions regarding this data transmittal should be directed to the Cognizant Engineer at extension (208) 533-7773. 


\section{ARGONNE NATIONAL LABORATORY \\ Hot Fuel Examination Facility}

\section{Bow and Length Measurement USER NOTES}

\section{MEASUREMENT PROCESS*}

1. Normal operating procedures for the Bow \& Length (B\&L) System are contained in HFEF Operating Instruction OI-4325.

2. Bow and length measurements are made with the element (or capsule) hanging freely from a collet-style end-fitting applied to it's top-end plug. The end-fitting is held rigidly in the gripper attached to the vertical carriage of the $B \& L$ examination stage.

3. The position of the element is meaśured in 3D space $(x, y, z)$ at a number of axial locations (typically at 2-in. increments) along it's length.

4. The hanging length of the element is measured by touching the bottom tip of the element to a conductive plate on the B\&L gage assembly, and comparing that Z-position to the Z-position of a length standard similarly positioned.

\section{STANDARD CALCULATION*}

- A custom computer program uses the bow and length data acquired in steps 3 and 4 above to calculate bow, bow direction, and corrected length for each element measured.

- Bow and bow direction are reported for each elevation measured in step 3, along with the location and direction of the maximum bow.

- Bow is defined as the distance from the theoretical tip-to-tip centerline to the element's centerline at any axial position along the element. Bow direction is the azimuthal angle of the centerline of the element relative to an angular reference feature on the element, measured counter-clockwise around the tip-to-tip centerline.

- The tip-to-tip centerline is inferred from the bow and length data obtained in steps 3 and 4 by extrapolating the two extreme bow measurements at each end of the element to the theoretical axial locations of the tips based on the hanging length data.

- Corrected length is calculated by summing the segment lengths (from tip to tip) between the calculated 3D positions of the element centerline (i.e., the length along the bowed shape of the element).

\section{STANDARD DATA REPORT}

- The computer program that performs the data reduction described above provides a standard data report.

- Data are reduced in groups of similar elements that share a common length calibration.

- Included in this report is a table of bow data for each element, a table of maximum bow (and bow direction) for each element, and a table of the corrected length of each element.

- This report contains ASCII text only, with no plots or graphics of any kind. It is formatted for printer output, but can be captured to a file and provided to the end user in electronic form if requested.

\section{SPECIAL REPORTS}

- By importing the above standard data report into appropriate computer applications (like Excel), custom reports can be generated that include graphics and other enhancements.

- Because they are not standardized, such reports cannot be described here. Every effort is made to ensure that such special reports self-explanatory.

- If you have any questions about a special report, contact the Cognizant Engineer as described on the Data Transmittal cover sheet.

\section{SPECIAL PROCEDURES}

- Special measurement procedures may sometimes be employed that deviate from the standard methods described above. Such procedures are described in the appropriate HFEF work-control documentation, such as Non-Routine Procedures (NRP's) or Process Work Sheets (PWS's).

* See "Elenent Bow Measurement and Data Reduction at the Hot Fuel Examination Facility/North", by R W. Keyes, Proceectings of the American Nuclear Society's 26 " Conference on Remote Systems Technolozy, 1978. 


\section{ARGONNE NATIONAL LABORATORY \\ Hot Fuel Examination Facility}

TEST ID: $\mathbf{S - 1}$

\begin{tabular}{|c|c|c|}
\hline $\begin{array}{c}\text { Z.Pos* } \\
\text { (in.) }\end{array}$ & $\begin{array}{l}\text { BOW } \\
\text { (In.) }\end{array}$ & $\begin{array}{c}\text { ANGLE } \\
\left({ }^{\circ}\right)\end{array}$ \\
\hline 0.00 & 0.000 & \\
\hline 1.46 & 0.005 & 76 \\
\hline 4.47 & 0.016 & 76 \\
\hline 7.46 & 0.019 & 89 \\
\hline 10.46 & 0.022 & 109 \\
\hline 13.46 & 0.024 & 125 \\
\hline 16.47 & 0.026 & 134 \\
\hline 19.46 & 0.025 & 138 \\
\hline 22.46 & 0.028 & 137 \\
\hline 25.46 & 0.031 & 132 \\
\hline 28.46 & 0.033 & 132 \\
\hline 31.47 & 0.029 & 136 \\
\hline 34.46 & 0.026 & 133 \\
\hline 37.46 & 0.022 & 134 \\
\hline 40.46 & 0.017 & 139 \\
\hline 43.47 & 0.012 & 139 \\
\hline 46.47 & 0.005 & 143 \\
\hline 47.57 & 0.003 & 143 \\
\hline 49.91 & 0.000 & \\
\hline & & \\
\hline
\end{tabular}

ROD ID: WT-11

DATE: $\quad \mathbf{1 / 1 7 / 9 7}$
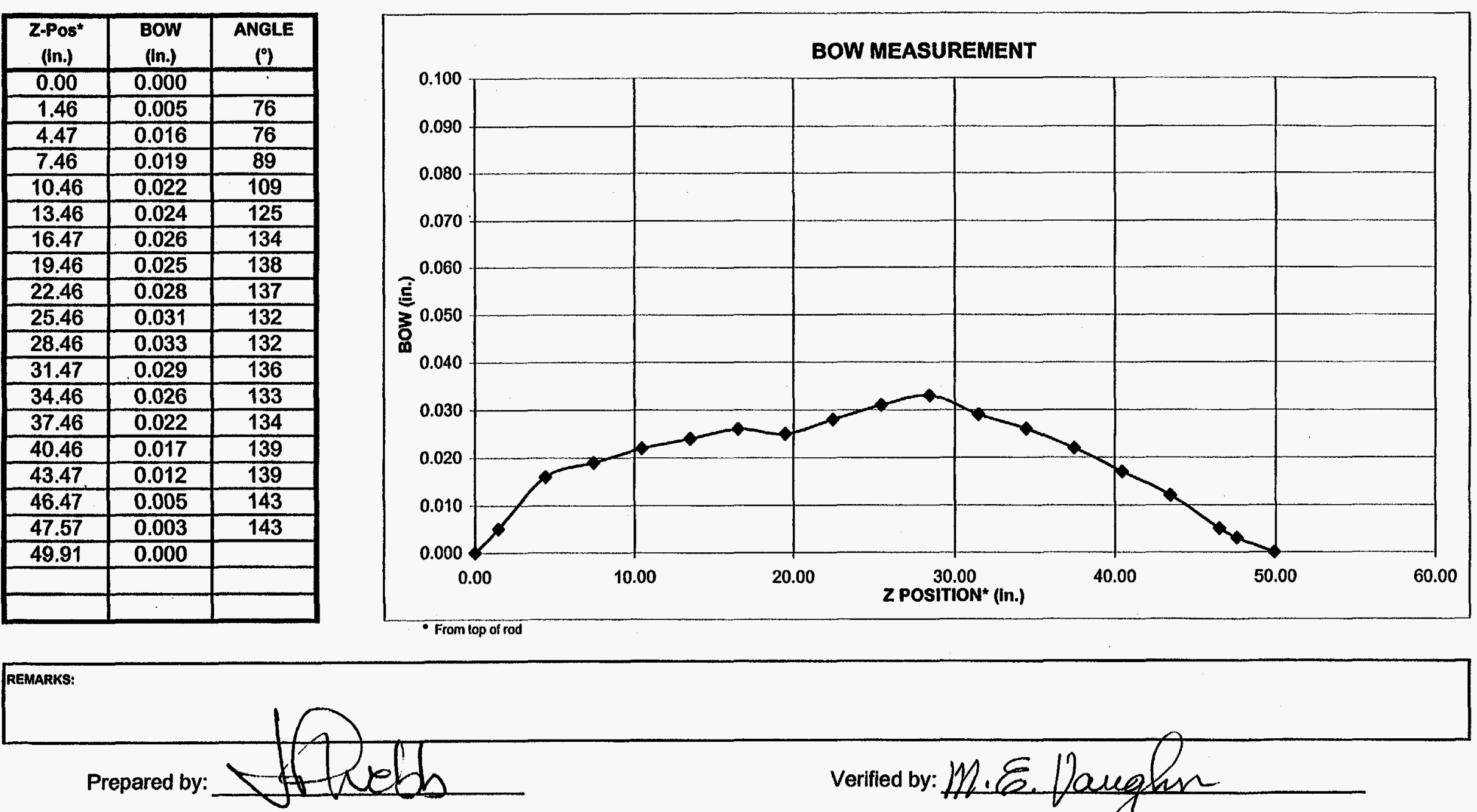

verified by: Wh:E.Haughe 


\section{ARGONNE NATIONAL LABORATORY \\ Hot Fuel Examination Facility}

TEST ID: $\mathbf{B - 1}$

\begin{tabular}{|c|c|c|}
\hline $\begin{array}{c}\text { Z-Pos } \\
\text { (in.) }\end{array}$ & $\begin{array}{c}\text { BOW } \\
(\text { (in.) }\end{array}$ & $\begin{array}{c}\text { ANGLE } \\
\left({ }^{\circ}\right)\end{array}$ \\
\hline 0.00 & 0.000 & \\
\hline 1.42 & 0.004 & 197 \\
\hline 4.42 & 0.013 & 197 \\
\hline 7.42 & 0.020 & 193 \\
\hline 10.42 & 0.024 & 186 \\
\hline 13.42 & 0.026 & 185 \\
\hline 16.42 & 0.028 & 188 \\
\hline 19.32 & 0.033 & 186 \\
\hline 22.42 & 0.037 & 193 \\
\hline 25.42 & 0.042 & 197 \\
\hline 28.42 & 0.044 & 205 \\
\hline 31.42 & 0.043 & 203 \\
\hline 34.42 & 0.040 & 204 \\
\hline 37.42 & 0.036 & 205 \\
\hline 40.42 & 0.030 & 203 \\
\hline 43.42 & 0.023 & 202 \\
\hline 46.42 & 0.011 & 196 \\
\hline 47.52 & 0.008 & 196 \\
\hline 49.83 & 0.000 & \\
\hline & & \\
\hline & & \\
\hline & & \\
\hline
\end{tabular}

ROD ID: WT-12

DATE: $11 / 22 / 96$

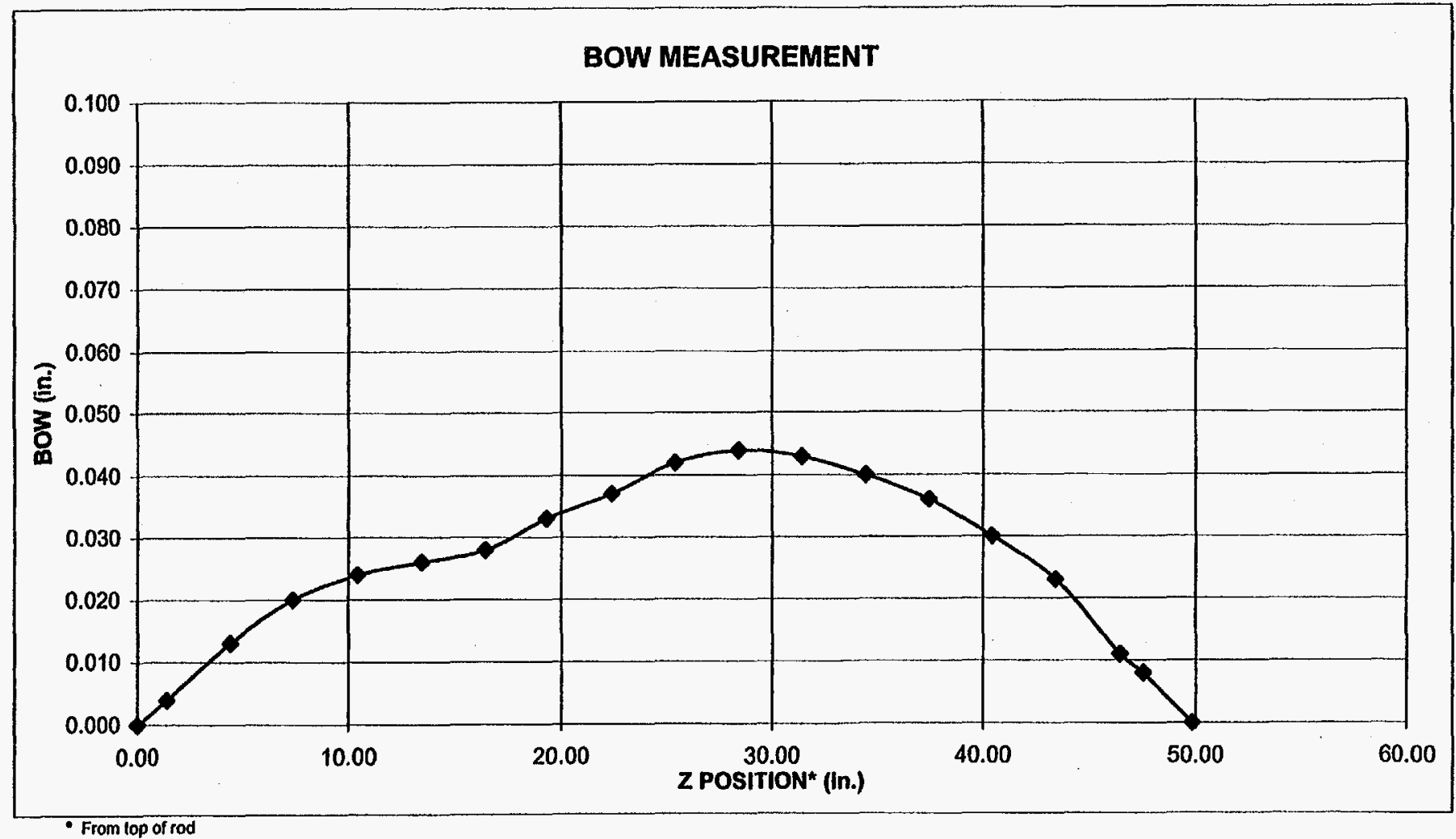

REMARKS:

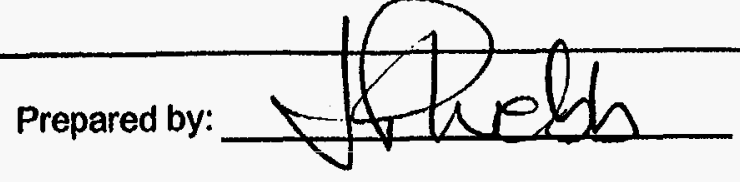

Verified by: $M \cdot[0$. Vaseshas 


\section{ARGONNE NATIONAL LABORATORY \\ Hot Fuel Examination Facility}

\section{BOW AND LENGTH \\ SUMMARY REPORT}

\section{CLWR S1/B1}

\begin{tabular}{|l|c|c|c|c|c|}
\hline \multirow{2}{*}{ ROD ID } & $\begin{array}{c}\text { CORRECTED } \\
\text { LENGTH } \\
\text { (in.) }\end{array}$ & $\begin{array}{c}\text { BOW } \\
\text { (in.) }\end{array}$ & $\begin{array}{c}\text { ZpOS } \\
\text { (in.) }\end{array}$ & $\begin{array}{c}\text { Phi } \\
\left({ }^{\circ}\right)\end{array}$ & \multirow{2}{*}{ REMARKS } \\
\hline WT-11 & 49.91 & 0.033 & 28.46 & 132 & \\
\hline WT-12 & 49.83 & 0.044 & 28.42 & 205 & \\
\hline & & & & & \\
\hline & & & & & \\
\hline & & & & & \\
\hline & & & & & \\
\hline & & & & & \\
\hline
\end{tabular}

REMARKS:

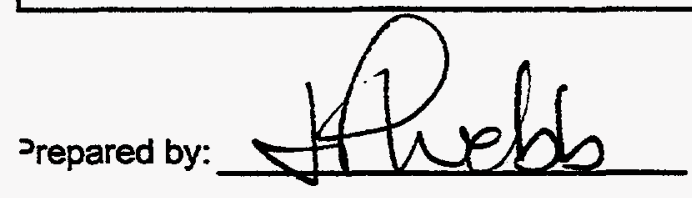

verified by: Af E Vaughes 
Appendix G

Dummy Rod Data 


\section{HFEF GASR TEST \\ CLWR-7 (CRIT2) DUMMY ROD \\ PRESSURE AT TEMPERATURE CERTIFICATION}

Pressure Guage Description: HEISE, temperature compensated, 0-1,000 PSIA, Dial type mirrored ribbon face, tolerance \pm 1 PSI., model 15079.

Calibration no. 705676 , due date $10 / 14 / 97$

Temperature Sensor Description: FLUKE Monitor, $\mathrm{K} / \mathrm{J}$ thermometer, tolerance $\pm 1^{\circ} \mathrm{F}$. Model 52 Calibration no 701703 due date $11 / 25 / 96$

Temperature thermocouple: type K Calibration no. 707477 due date 12/29/96

Tube drawn down to a vacuum of $5 \times 10^{-6} \mathrm{mbar} / \mathrm{s}(\mathrm{cc} / \mathrm{s})$ for 10 Minutes prior to backfilling with helium. Note the tube has been drawn down to $1 \times 10^{-7} \mathrm{mbar} 1 / \mathrm{s}$ for several hours prior to this operation.

Ultra high purity Helium $99.999 \%$ pure used as backfill gas $X$ Yes_No.

Backfill pressure 500-510 PSIA. Actual measured pressure 507.5 PSIA.

Barometric pressure 25.30 Obtained from National Oceanic and Atmospheric Administration (NOAA) date $10 / 10 / 96$ Time 2:35 AM XPM.

(Barometric pressure 25,30 X $14.7 \div 29.92=12.43$ PSIA).

Temperature measured on the outside of the tube approximately $41 / 2$ " from the valved end.

Starting temperature $744^{\circ} \mathrm{F} \quad 2 \cdot 37$ PMTime. After pressurizing and stabilizing for 10 minutes $72.8{ }^{\circ} \mathrm{F} \quad 3.15$ Time

To set the pressure gauge for PSIA, obtain current barometric pressure, convert to PSIA, dial that into the guage and lock it. Draw a vacuum to bring the guage down to Zero. You are now reading PSIA on the dial face.

Welders Name H.J. Hall Welders certification expires 12/3/96 (see attached) Welds helium leak checked down to $1 \times 10^{-6} \mathrm{mbar} \mathrm{V} / \mathrm{s}$, Note the helium leak detector has no calibration sticker. $X$ No leaks. Comments CHECKED fER LEAKS ACrOSS tae valve to $1 \times 10^{-6}$ as well. No Leakace lndoted

Additional Comments: Ress to 507 propped of to 505 After 5 min :03 After ANotwer 5 MIN. PLMPED if to $5: 7.1$ Bumpeo ROO RAISED to 507.7 after ANOTMER FIFTEEN MIN

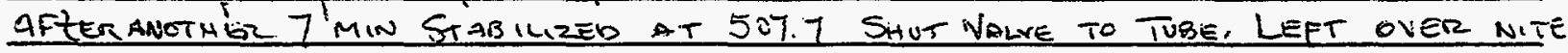
$507.571 .8^{\circ} \mathrm{F} 8.10 \mathrm{Am}$

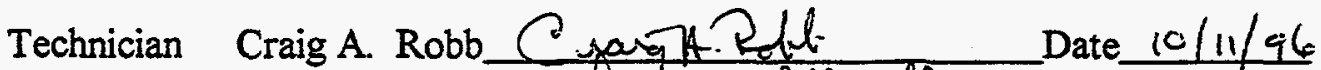
Lead Engineer Larry J. Whitmill Thatuguhitm il _Date 10/11/96 Quality Verification Daniel R. Tonks Lamele Tomb Date $10 / 11 / 96$

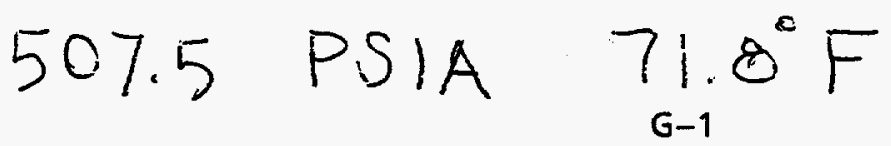


Name

Ball, B. J.

Security Number 24898

Welder Code 103

E $\begin{array}{llll}\text { ASME } & \text { AWS 01.1 } & \text { AWS } 01.2 \text { AWS } 01.3 \text { aWS } 09.1\end{array}$

\begin{tabular}{|c|c|c|c|c|c|}
\hline $\begin{array}{l}\text { WPSIBPS } \\
\text { Number }\end{array}$ & Processs & Position & $\begin{array}{l}\text { Thieksoss } \\
\text { Renste }\end{array}$ & Dismoter & $\begin{array}{l}\text { Expiration } \\
\text { Dote }\end{array}$ \\
\hline 02.8 & Gikh & $12 \mathrm{G} / \mathrm{?}$ & 9.02 & 1.808 & $12 / 63 / 96$ \\
\hline $\operatorname{cs} 2.8$. & GTAR & $12 \mathrm{G} / \mathrm{?}$ & 8.662 & 1.898 & $12 / 03 / 96$ \\
\hline$\$ 2.8$ & GLAR & $12 \mathrm{G} / \mathrm{P}$ & 8.862 & 1.808 & $12 / 83 / 96$ \\
\hline & & & & & \\
\hline & & $r$ & & & \\
\hline & & & & & \\
\hline & & & & & \\
\hline & & & & & \\
\hline & & & & & \\
\hline & & & & & \\
\hline & & & & & \\
\hline & & & & & \\
\hline & & & & & \\
\hline & & & & & \\
\hline & & & & & \\
\hline & & & & & \\
\hline & & & & & \\
\hline & & & & & \\
\hline & & & & & \\
\hline & & & & & \\
\hline
\end{tabular}

(REY. 10-90)

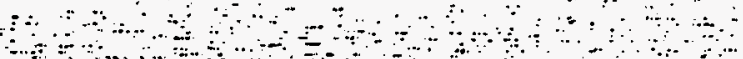

(7) 
OM: EDRA --INELVMI

IO: WIT

--INELVMI I J Whitmill

Date and time

$10 / 02 / 9608: 13: 52$

From: Electronic Forms

Review and Approval system

Subject: CATALOG ORDER

- 96274 PRO2017

Document Form: SYSTEMS SUB-CONTRACTING

Your request has been APPROVED and is being Processed by SYSTEMS CONTRACTING $a$ FINAL Comments:

EDRA 96274 PRO2017 GOOD

DATE: $10-02-96$

TIME: $08: 15: 07$

DELIVER TO: ROBB CRAIG A NHLF NA TOWN

THE FOLIOWING SSR NUMBERS WERE ASSIGNED TO YOUR SYSTEMS SUBCONTRACTING ORDER:

\begin{tabular}{lccccc} 
CONTRACT & SSR & LINE & DESCRIPTION & QTY & UNIT \\
\hline $\mathrm{C} 6$ & B & 68429 & 1 & HEIIUM, UHP, $99.999 \%$ PURITY, & CY
\end{tabular}

WAREHOUSE ORDERS WILL BE ENTERED INTO THE IMMS REQUISITION SYSTEM. SOME SYSTEMS SUBCONTRACTING ORDERS (FURNITURE, ETC.) MAY REQUIRE SPECIAL APPROVAL AND NOT BE IISTED ABOVE. IF YOU HAVE QUESTIONS REGARDING THIS

'DER, PLEASE CALI THE SYSCON ADMINISTRATOR (526-1669).

If you have any questions in the future, please check with them.

NOTE: EDRA is a service ID and not a real person.

Questions should be addressed to SYSTEMS CONTRACTING e 6-1669 


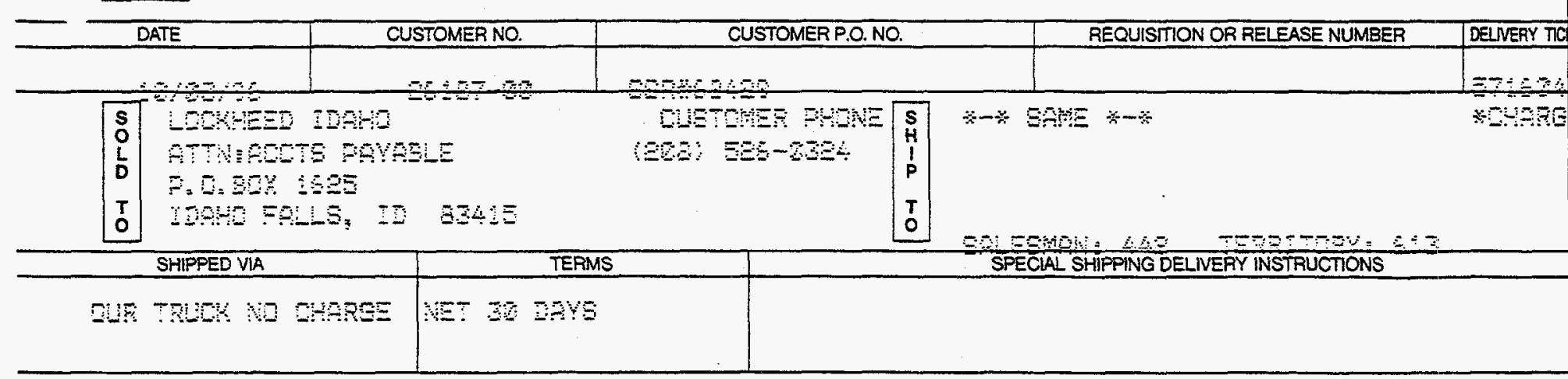

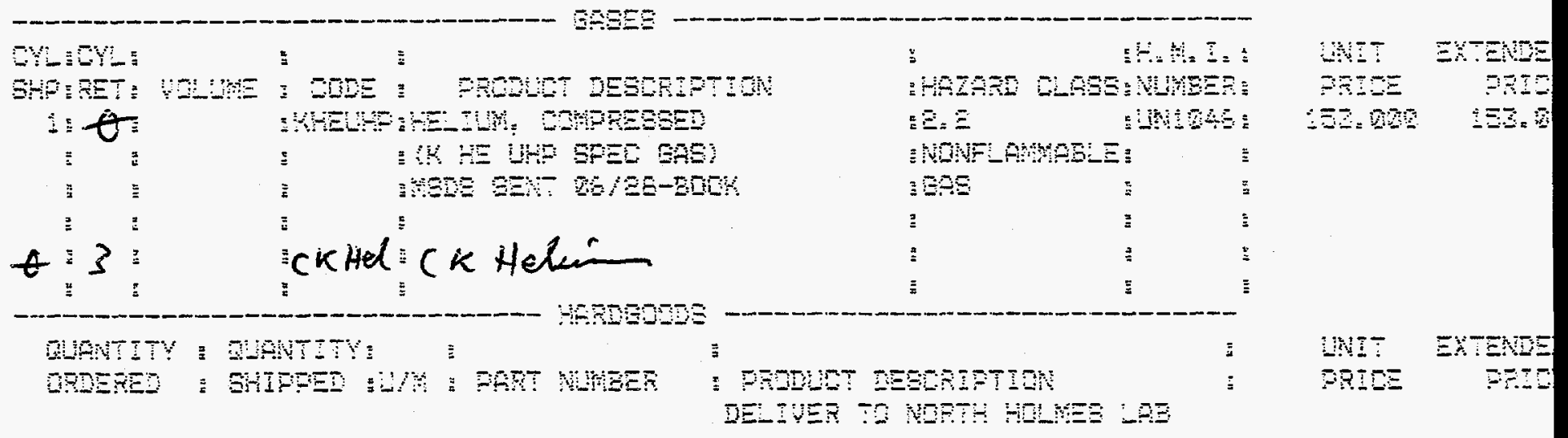
PACKAGED, MARKED, AND LABELED, AND ARE IN PROPER CONDITION FOR TRANSPORTATION, ACCORDING TO APPLICABLE REGULATIONS OF THE DEPARTMENT OF TRANSPORTATION. BY

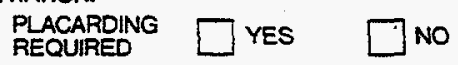
JTE: VERIFY COUNT OF ALI CYUNDERS, REPORT ANY DIFFERENCES IMMEDIATELY. RETURN EMPTY CYLINDERS AND CAPS PROMPTLY. CUSTOMER WIL BE CHARGED FOR LOST CYLNDERS AND CAPS. WARNING: 1 ACKNOWLEDGE THATT I HAVE BEEN INSTRUCTED THAT IT IS HITHLY DANGEROUS AS WELI AS ILLEGAL TO TRANSPORT FLAMMABLE GASES MA CLOSED VEHICLE OR ANY COMPARTMENT THEREON. I HAVE ALSO RECEVED TME PROPEA MSOS FORM. BUYER OR HIS AGENT AGREES TO ALL IERMS AND CONDITIONS OF SALE.

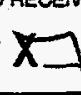


I0540-6

P16CL016
SYSTEMS SUB-CONTRACTING ORDER FORM Total cost $\$ 79.89$

\begin{tabular}{|c|c|c|c|}
\hline Date: $9 / 30 / 96$ & Release No: & Company: & \\
\hline $\begin{array}{l}\text { Employee No: } \\
034778\end{array}$ & $\begin{array}{l}\text { Name: } \\
\text { WHITMILI IARRY } \mathrm{J}\end{array}$ & $\begin{array}{l}\text { Org No: } \\
4130\end{array}$ & $\begin{array}{l}\text { Phone: } \\
6-0357\end{array}$ \\
\hline $\begin{array}{l}\text { Deliver to: } \\
\text { ROBB CRAIG A }\end{array}$ & $\begin{array}{l}\text { Delivery Location: } \\
\text { IF NHLF na }\end{array}$ & $\begin{array}{l}\text { Room/Grid No: } \\
\text { na }\end{array}$ & $\begin{array}{l}\text { Phone: } \\
\text { 5-0684 }\end{array}$ \\
\hline $\begin{array}{l}\text { These items are NOT } \\
1\end{array}$ & $\begin{array}{l}\text { Hazardous Chemicals } \\
\text { hazardous chemicals per } 29 \mathrm{CFR}\end{array}$ & 1910.1200 & \\
\hline
\end{tabular}

dummy target rod, larry

Comments or Justification

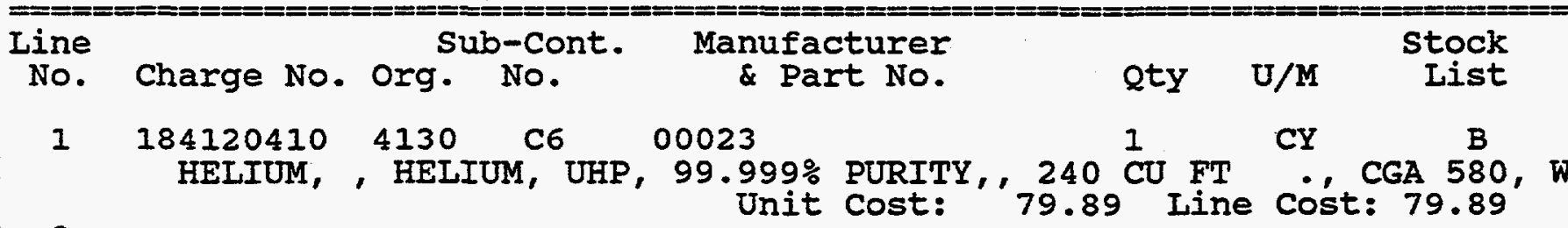
2

3

4

5

6

7

8

9

\$\$DOCNO\$\$

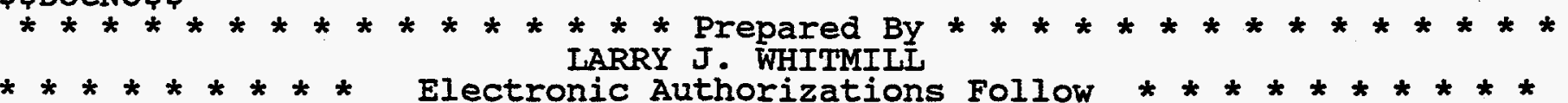




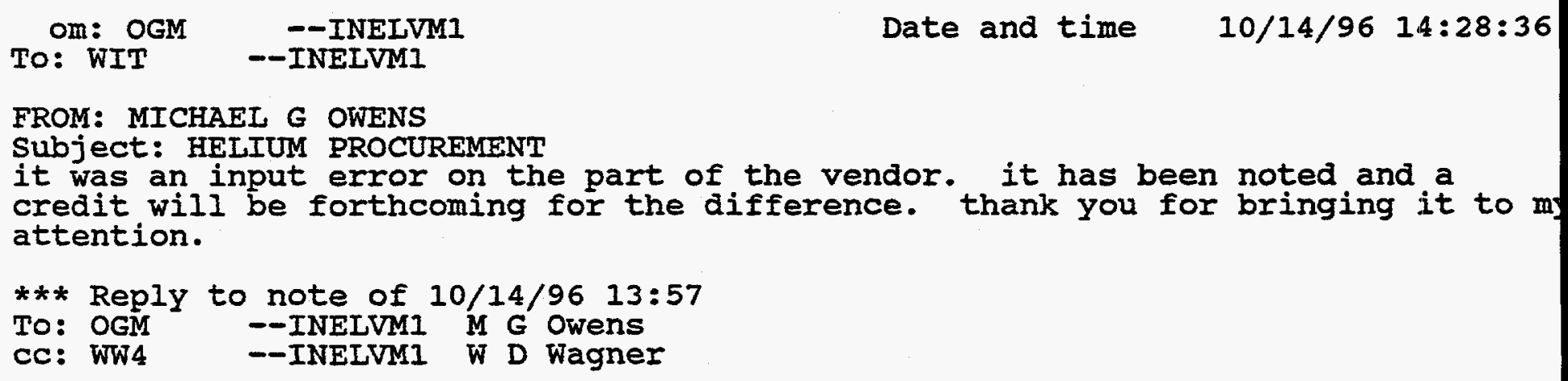

TO: WIT

FROM: MICHAEL G OWENS

Subject: HELIUM PROCUREMENT

it was an input error on the part of the vendor. it has been noted and a credit will be forthcoming for the difference. thank you for bringing it to m attention.

FROM: IARRY J WHITMILI ORG. 4130, EROB-W2-D7

FAX 6-0425, PH. 6-0357, MS 3765

Subject: HELIUM PROCUREMENT

I ordered a bottle of helium on SSR 68429. It was listed at \$79.89. It was delivered by United states welding Inc. with a delivery ticket that says the unit price is $\$ 153.00$. I don't understand, can you

explain.....thanks 
SISP $500-01$

DREP : TOKN

GSH PAGE:
LOCKHEED IDAHO TECHNOLOGIES COMPANYY CO U S WELOINǴ
THE: 16:17:32

PRINT OATE: $10 / 02 / 96$ CUNIRACI PAGE:

T.: $\quad 6429$

LEBRY LOC = NHLF NA NAME: ROJE CRAIG A

MI NG: 34778 NAME = WHITMILL, LARRY \& ORGN=4130 PHONE: 6-C357

THAXISE NO: 184120410 CEL: HSO LOC: IF EROE WE C6 MS: 3165 SITE: T

TNO USE: DUMWY TARGET REQ. LARRY

PRIORITY LEVEL: OO

S.5:3:

A MPRE/ DESCRIPTION

6100023

HELIUH, UHP, $99.999 \%$ PURITY,

¿wi:
$U / 4$

CY
GTY

I
Virit PRICE

73.890

SU' TOTAL
EXT PRICE

79.890

79.890

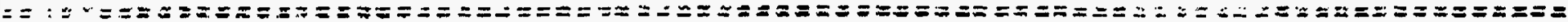
* * REQUESTOK MUST CUURT AHJ INSPECT RECELPTS - NGTIFY WLYCH ON DISCREPANCIES BUYEK: 203 NAME: A K BEKRETY

$3-2723$

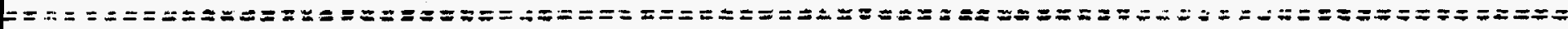

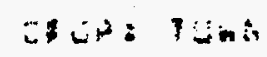

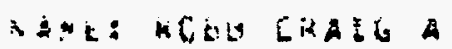

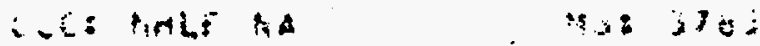

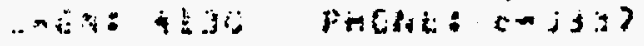

158: :

$\triangle T C K=5$

\section{Exis: Thins}

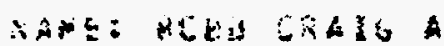

WC: irlte $A$ Ah

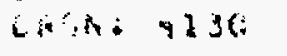

jand extat 4a: 3300

FUतTE:

Lxis $j$ ir I:Ba- + G-7

AANE: NUEII LAALS

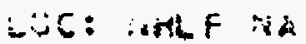

WhE : +30

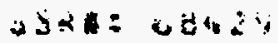

$\therefore P=r r i n$

:40: 3705

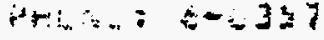

Laid 2 of -

) $\operatorname{lec} x-i$

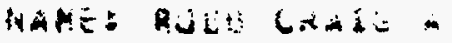
WUC: NintF WA OFAN: 4 i30 iv.75: $=4-2357$

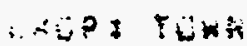
p5: 37 es G-7
\&AEL 4 Dी

$\sim 2 i t-k$ 


\section{Lockheed Martin Idaho Technologies Company}

\section{NOTEGRAM}

Date: Oct. 18,1996

To: Wendell Wagner

From: Larry J. Whitmill Whit

Subject: GASR Test Rod

I have returned the test rod and the spare to Argonne. I have not disclosed to them the condition or contents of the test rod. I have included here the documentation supporting the task I performed for you including the quality certification for the final rod conditions. In addition to labor and the requisitions, there will be a use charge of about $\$ 25$ for each instrument that we got from the instrument pool. Those charges are for three instruments. Total charge for the instruments should be no more than $\$ 75$.

I removed the handle from the valve on the test rod to prevent accidental opening of the valve. It is included here in case you want to keep it.

This notegram serves to close out my activities and forward all project documents to you for your files.

If I can be of any further assistance or you have any questions, feel free to call me at 60357.

R. L. Davison 


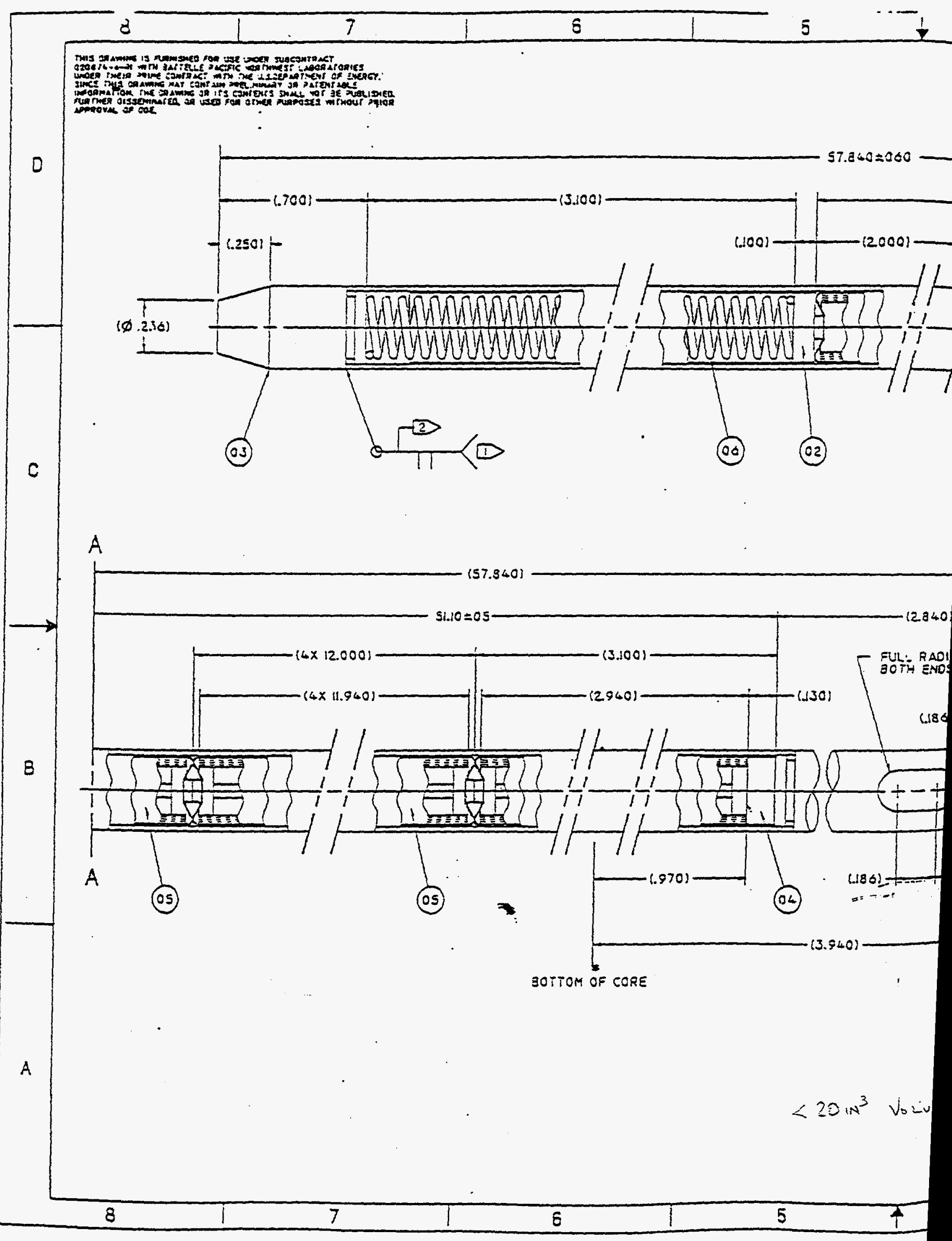




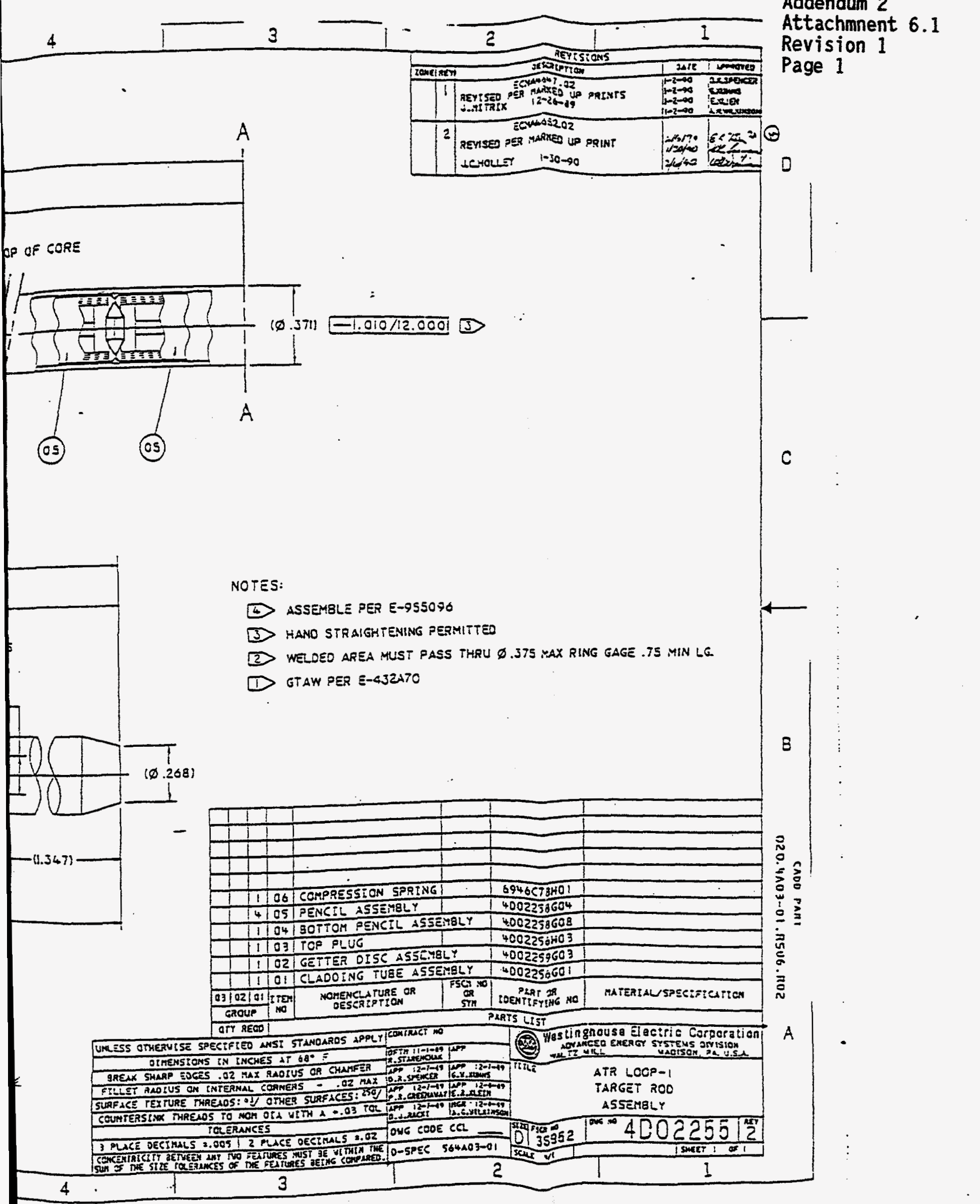

LOOP-1/B-1/S- 1 SOW

CLWR/A-96-001

Addendum 2

Attachmnent 6.1

Revision 1

Page 1

OTES:

(4) ASSEMBLE PER E-955070

3) HANO STRAIGHTEMING PERMITTE

WELOSD AREA MUST PASS THRU $\emptyset .375$ WAX RING GAGE .75 MIN LG

GTAW PER $\leq-432470$ 


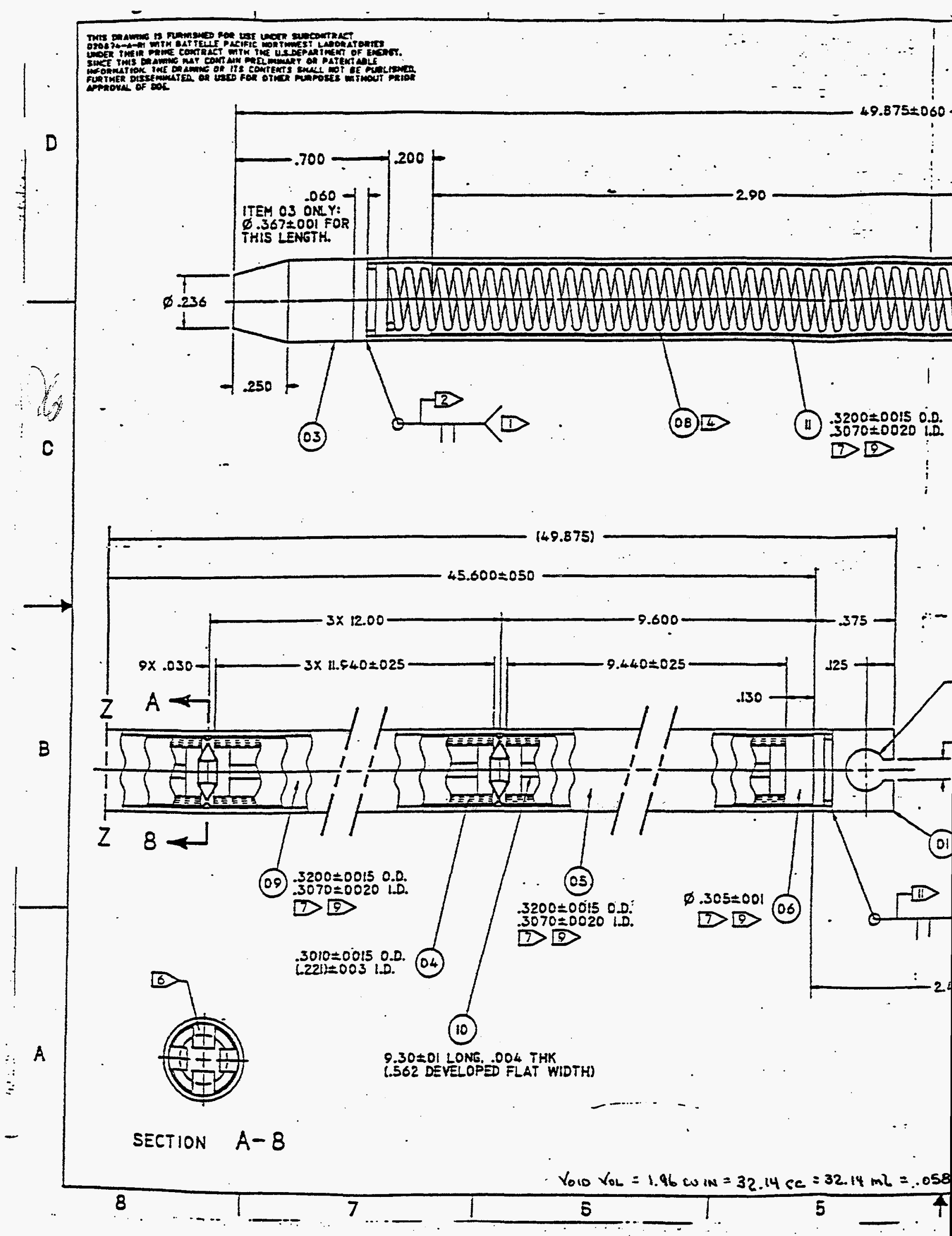


$\underset{\text { FAOM }}{\text { PACKING LIST }}$

CPV MANUFACTURING, INC.

851 N. Preston Street

Philadelphia, PA 19104-1598

Phone (215) 386-6508

Since 1915

Fax (215) 387-9043

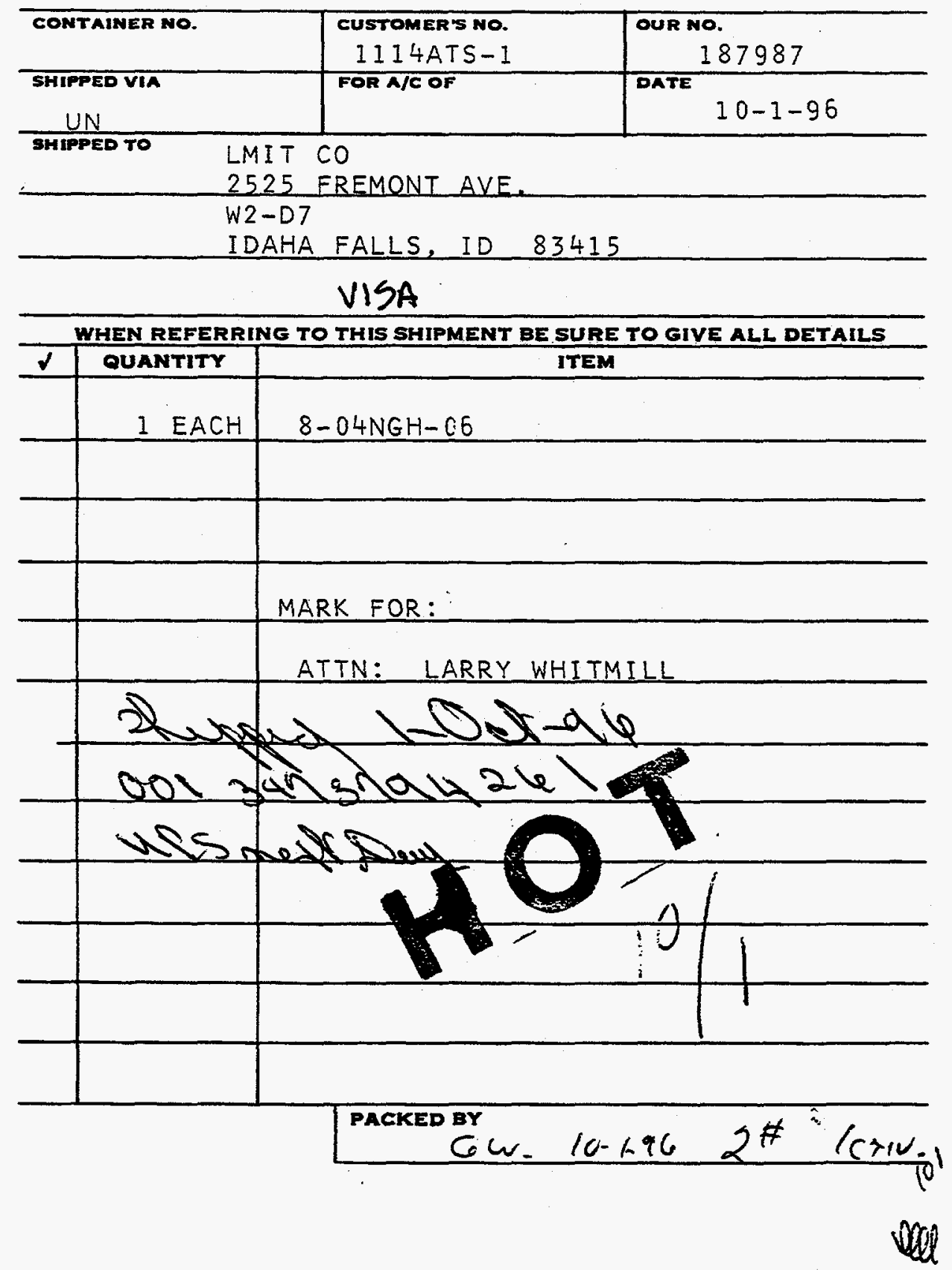




\section{The Ultimate in Positive Fluid Control}

\section{... a unique system of sott-seated valves that slip right in}

Now you can combine the performance advantages of these low torque, soft-seated valves with the superior sealing and unlimited accessibility of Mark VIII O-SEAL fittings.

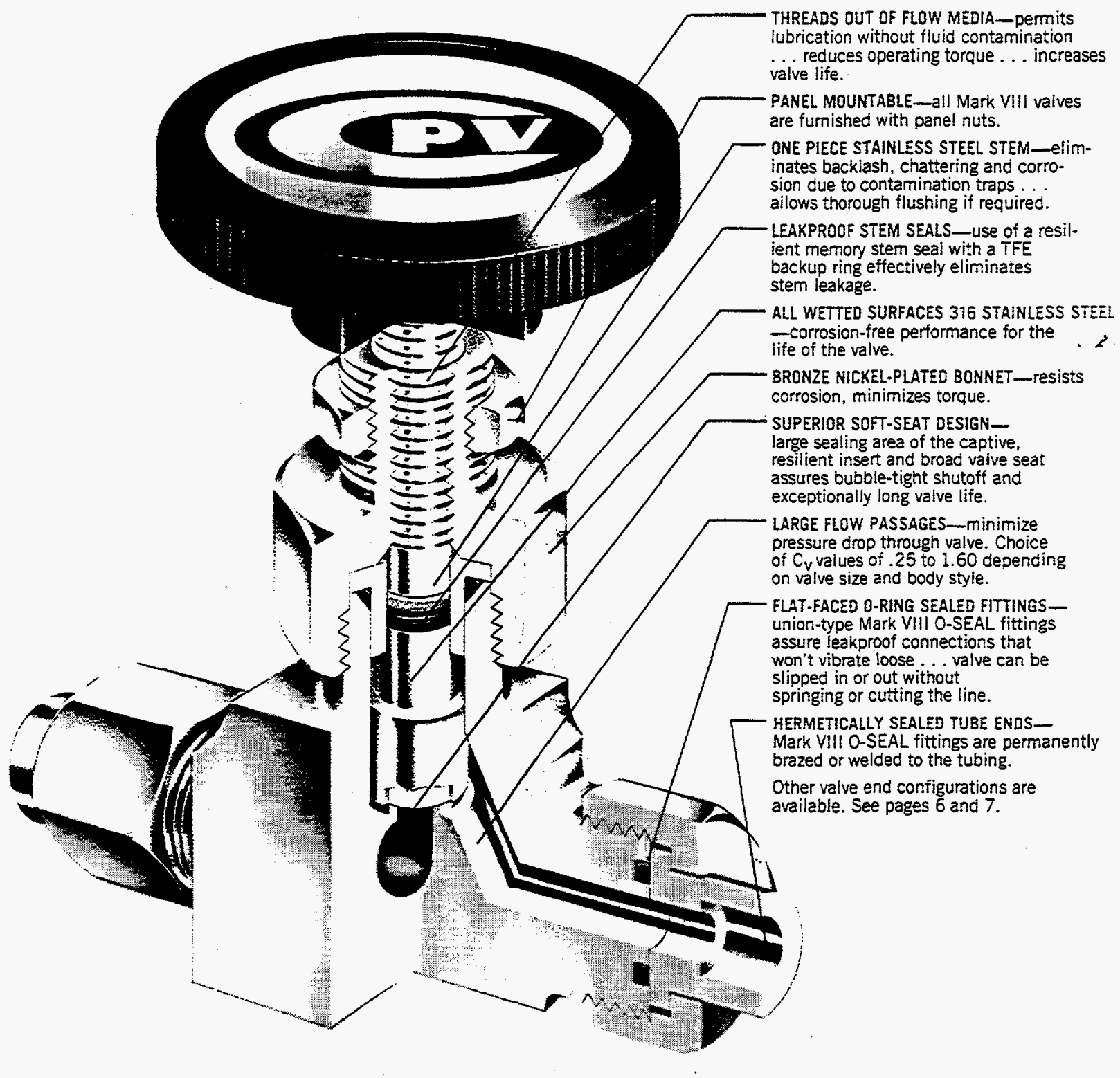

CPV Mark VIII Shutoff and Regulating valves employ a stem with a resilient insert that presses against a raised, flat metal seat to assure "bubble-tight" closure. This Kel-F insert is securely swaged in the valve stem, and the sealing force is distributed over a large circular sealing area to assure long valve life. Two valve configurations are available:

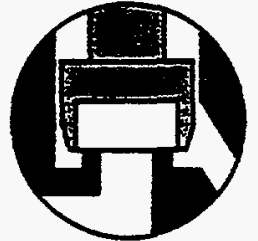

Figure A. Shutoff Valve

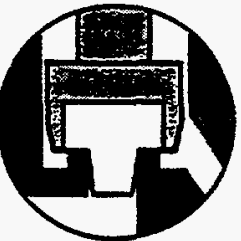

Figure $\mathbf{B}$. Regulating (Needle) Valve 


\section{CPV MARK VIII Shutoff and Regulating Valves Provide Slip-In Installation And Trouble-Free Operation.}

\begin{abstract}
A Proven Design Concept Pioneered by CPV. Many of the key design elements of the Mark VIII valve are based upon CPV's early pioneering work in soft-seated valve design. CPV valves have proven themselves to be the ultimate in positive fluid control in over three decades of use aboard U.S. Navy nuclear submarines and in numerous industrial applications, including chemical process piping and mixed gas diving systems. This same high reliability is now available for your most demanding instrumentation and process piping needs.
\end{abstract}

Valve slips right in, stays leaktight. Only the Mark VIII system of valves and fittings gives you this instant, easy access plus high reliability sealing. The basic Mark VIII system comprises a softseated valve with union-type end connections. An O-ring recessed in a close tolerance groove in each valve end seals against a mating flat-faced fitting.

The result is a valve that you can slip straight in or out without springing or cutting lines-no axial clearance is required. So you can easily remove the valve at will without disturbing other components.

This slip-in feature permits the design of exceptionally compact systems without penalizing the installer. Accessibility to components is assured by the valve's flat-faced design.

And you'll never have to worry about fitting leakage-no matter how often these connections are broken and remade. Once the

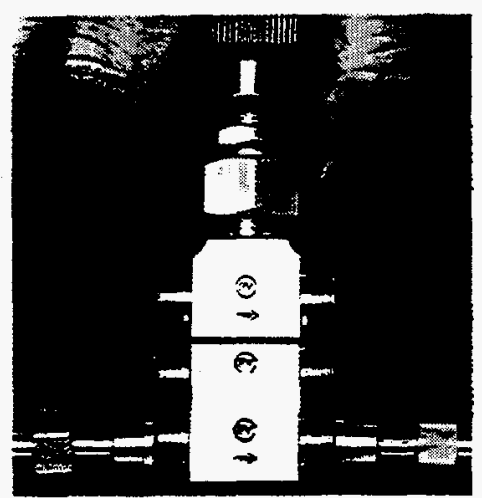

union nuts are tightened, the sealing action of the resilient O-ring assures absolutely leaktight valve connections under all conditions.

Positive Sealing of Liquids and Gases-even elusive helium and hydrogen. All wetted surfaces are of 316 stainless steel to assure contaminant-free operation even after extended service in most aggressive environments. The valves also employ a captive soft seat design that provides absolutely leaktight sealing and exceptionally long valve life. To accommodate almost any fluid media, the valves can be furnished with Buna-N, Viton, Ethylene Propylene, or TFE seals. A TFE backup ring above the stem seal is standard.

Keeps Ultra-Pure Systems Contaminant Free. Valve threads are above the stem seal-completely isolated from the system fluid. This eliminates thread corrosion problems and permits stem thread lubrication without fear of fluid contamination.

Galling-Free, Low Torque Valve Operation. Mark VIII valves are easy to open and close. The stainless steel stem threads into a bronze bonnet that is nickelplated. These dissimilar materials plus external thread lubrication effectively eliminate galling, and subsequently reduce torque requirements.

Non-Clog Operation Even in Severe Service. To ensure reliable performance even under the most demanding service conditions, the valves also feature a one piece stem design that eliminates clogging due to contamination trapping. Because this design has no cavities for corrosive materials to collect in, thorough flushing can be accomplished if desired. This one piece stem also ends backlash problems as well as chatter when used for metering.

Ideal for Instrumentation or Process Systems. These panel-mountable Mark VIII shutoff and regulating valves offer exceptional adaptability. They are available in angle or inline body styles with choice of flow coefficients, and with seals to suit specific requirements. They can be furnished with a variety of valve end configurations including Mark VIII unions, fernale pipe threads, male pipe threads, female straight threads, and direct weid/braze ends in sizes from $1 / 8^{\prime \prime}$ to $5 / 8^{\prime \prime}$. Valve dimensions, ordering information and $C_{v}$ charts are given on the following pages to allow you to select the Mark VIII valves best suited to your particular applications. 


\section{Technical Data}

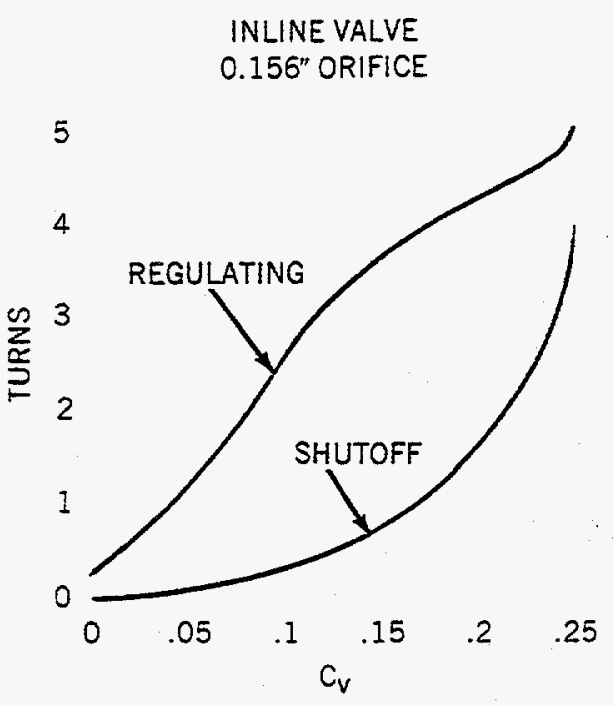

Valve Specifications

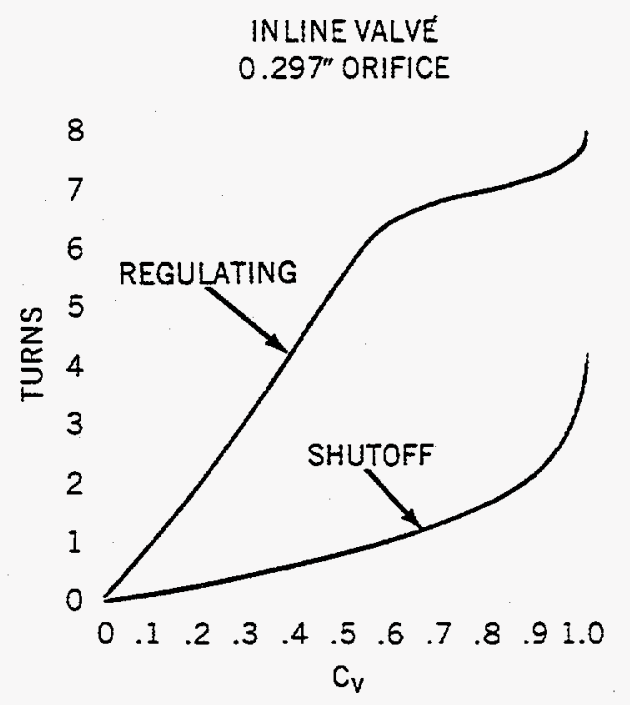

Operating Pressure

$0-3000$ psi

Maximum Temperature Rating

$250^{\circ} \mathrm{F}$.

Flow Coefficient- $\mathrm{C}_{\mathbf{v}}$ :

Inline Body Style:

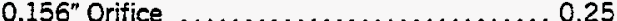

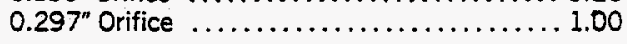

Angle Body Style:

$0.156^{\prime \prime}$ Orifice $\ldots \ldots \ldots \ldots \ldots \ldots \ldots \ldots \ldots .40$

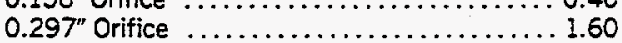

\section{How to Order Mark VIII Valves*}

Select the part number for the valve desired. Add a suffix to the part number to indicate the seal materia! re-

quired. Seal material you specify will be fumished for stem and bonnet seals as well as end seals where applicable.

Typical part number shown broken down:

\begin{tabular}{|c|c|c|c|c|c|c|}
\hline & & & & & & \\
\hline & & 804 & $\mathbf{S}$ & -04 & & \\
\hline $\begin{array}{l}\text { Product } \\
\text { Line }\end{array}$ & Brifice & $\begin{array}{l}\text { Yalre } \\
\text { Type }\end{array}$ & $\begin{array}{l}\text { Body } \\
\text { Style }\end{array}$ & $\begin{array}{l}\text { Body End } \\
\text { Configuration }\end{array}$ & $\begin{array}{l}\text { Tobe } \\
0.0 .\end{array}$ & $\begin{array}{c}\text { Seal } \\
\text { Material * }\end{array}$ \\
\hline 8 & $\begin{array}{ll}04 & 0.156^{\prime \prime} \\
08 & 0.297^{\prime \prime}\end{array}$ & $\begin{array}{ll}\text { S Shutoff } \\
\text { N Regulating }\end{array}$ & $\begin{array}{l}\text { G Inline } \\
\text { A Angle }\end{array}$ & $\begin{array}{l}\text { U } \begin{array}{l}\text { Mark Vlli } \\
\text { union }\end{array} \\
\text { H } \begin{array}{l}\text { Direct } \\
\text { weld/braze }\end{array} \\
\text { T } \begin{array}{l}\text { Straight } \\
\text { thread }\end{array} \\
\text { M Male pipe } \\
\text { NPT } \\
\text { F Female } \\
\text { pipe NPT }\end{array}$ & $\begin{array}{ll}-02 & 1 / 8^{\prime \prime} \\
-03 & 3 / 16 " \\
-04 & 1 / 6^{\prime \prime} \\
-05 & 5 / 16^{\prime \prime} \\
-05 & 3 / 8^{\prime \prime} \\
-08 & 1 / 2^{\prime \prime} \\
-10 & 5 / 8^{\prime \prime}\end{array}$ & $\begin{array}{l}\text { B Buna-N } \\
\text { C Viton } \\
\text { D Ethylene } \\
\text { Propylene } \\
\text { T TFE }\end{array}$ \\
\hline
\end{tabular}

Sil-Braze Rings for Valves with Brazed End Connections

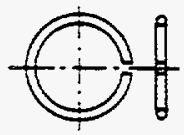

\begin{tabular}{|lc|}
\hline Part No. & Tube O.D. \\
\hline H801-2 & $1 / 8$ \\
\hline$H 801-3$ & $3 / 16$ \\
\hline$H 801-4$ & $1 / 6$ \\
\hline$H 801-5$ & $5 / 16$ \\
\hline$H 801-6$ & $3 / 8$ \\
\hline$H 801-8$ & $1 / 2$ \\
\hline$H 801-10$ & $5 / 8$ \\
\hline
\end{tabular}

Grade VII Sil-8raze Alloy 


\section{CPV Mark VIII Shutoff \& Regulating Valves}
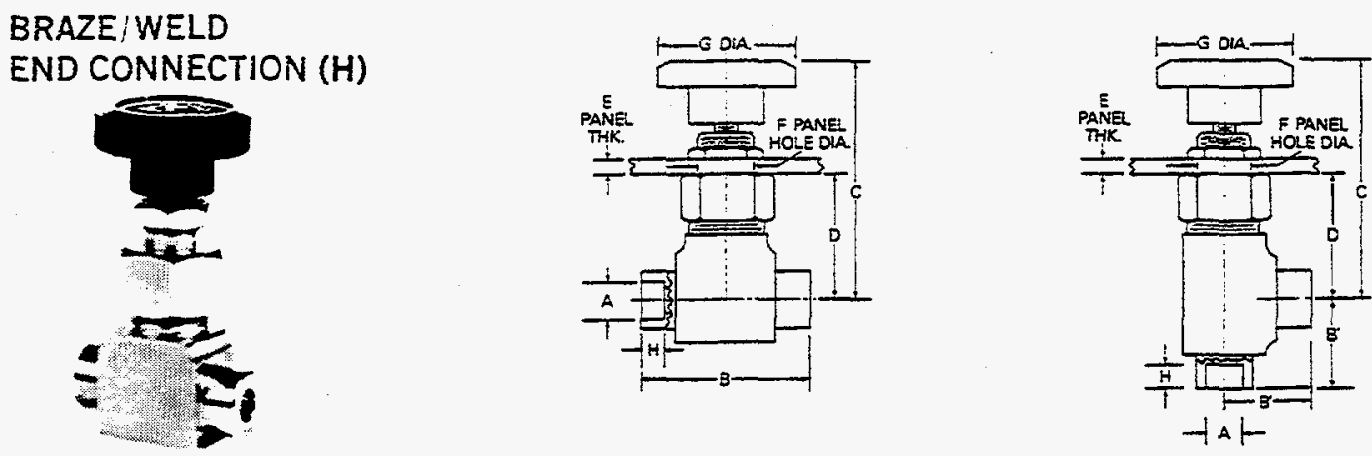

\begin{tabular}{|c|c|c|c|c|c|c|c|c|c|c|c|c|c|}
\hline \multicolumn{2}{|c|}{ PART BUMBER } & \multirow{2}{*}{$\begin{array}{l}\text { VALVE } \\
\text { TYPE }\end{array}$} & & \multicolumn{10}{|c|}{ DIMEMSIOMS (INCHES) } \\
\hline & & & ORIFICE & TUBE'0.D. & B & $\mathbf{B}^{\prime}$ & \multicolumn{2}{|c|}{$\mathrm{C}$} & D & $\stackrel{E}{\text { Ihx }}$ & $\mathbf{F}$ & 6 & $H$ \\
\hline $\begin{array}{l}\text { 804SGH-04 } \\
804 N G H-04\end{array}$ & $\begin{array}{l}\text { 804SAH-O4 } \\
804 N A H-04\end{array}$ & $\begin{array}{l}\text { Shutoff } \\
\text { Regulating }\end{array}$ & \multirow[b]{2}{*}{0.156} & $1 / 4$ & \multirow[b]{2}{*}{$15 / 3$} & \multirow[b]{2}{*}{$13 / 16$} & \multirow[b]{2}{*}{$2^{19 / 32}$} & \multirow[b]{2}{*}{$27 / 16$} & \multirow[b]{2}{*}{$1^{7} / 32$} & \multirow[b]{2}{*}{$1 / 4$} & \multirow[b]{2}{*}{$17 / 32$} & \multirow[b]{2}{*}{$13 / 8$} & $3 / 25$ \\
\hline $\begin{array}{l}8045 G H-06 \\
804 N G H-06\end{array}$ & $\begin{array}{l}\text { 804SAH-06 } \\
\text { 804NAH-06 }\end{array}$ & $\begin{array}{l}\text { Shutoff } \\
\text { Regulating }\end{array}$ & & $3 / 8$ & & & & & & & & & $1 / 4$ \\
\hline $\begin{array}{l}\text { 808SGH-08 } \\
\text { 808NGH-08 }\end{array}$ & $\begin{array}{l}\text { 808SAH-08 } \\
\text { 808NAH-08 }\end{array}$ & $\begin{array}{l}\text { Shutoff } \\
\text { Regulating }\end{array}$ & \multirow[t]{2}{*}{0.297} & $1 / 2$ & \multirow[t]{2}{*}{$23 / 8$} & \multirow[t]{2}{*}{$13 / 16$} & \multirow[t]{2}{*}{$315 / 32$} & \multirow[t]{2}{*}{$33 / 16$} & \multirow[t]{2}{*}{$1^{21 / 32}$} & \multirow[t]{2}{*}{$3 / 8$} & \multirow[t]{2}{*}{$25 / 32$} & \multirow[t]{2}{*}{$17 / 8$} & $5 / 26$ \\
\hline $\begin{array}{l}808 \text { SGH-10 } \\
808 N G H-10\end{array}$ & $\begin{array}{l}\text { 808SAH-10 } \\
\text { 808NAH-10 }\end{array}$ & $\begin{array}{l}\text { Shutoff } \\
\text { Regulating }\end{array}$ & & 58 & & & & & & & & & 洛 \\
\hline
\end{tabular}

STRAIGHT THREAD

END CONNECTION (T)
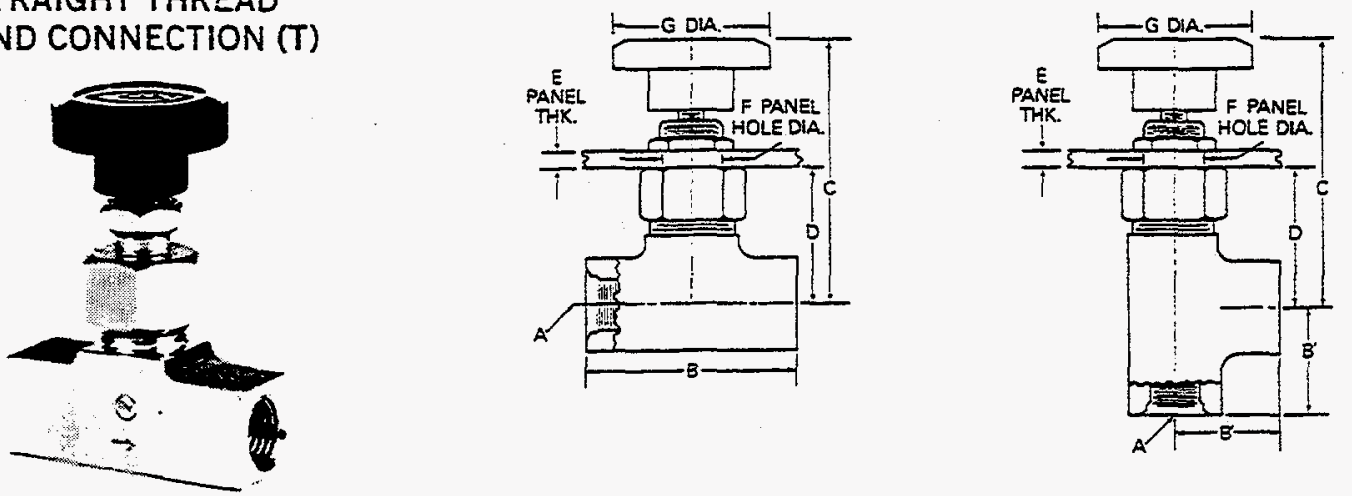

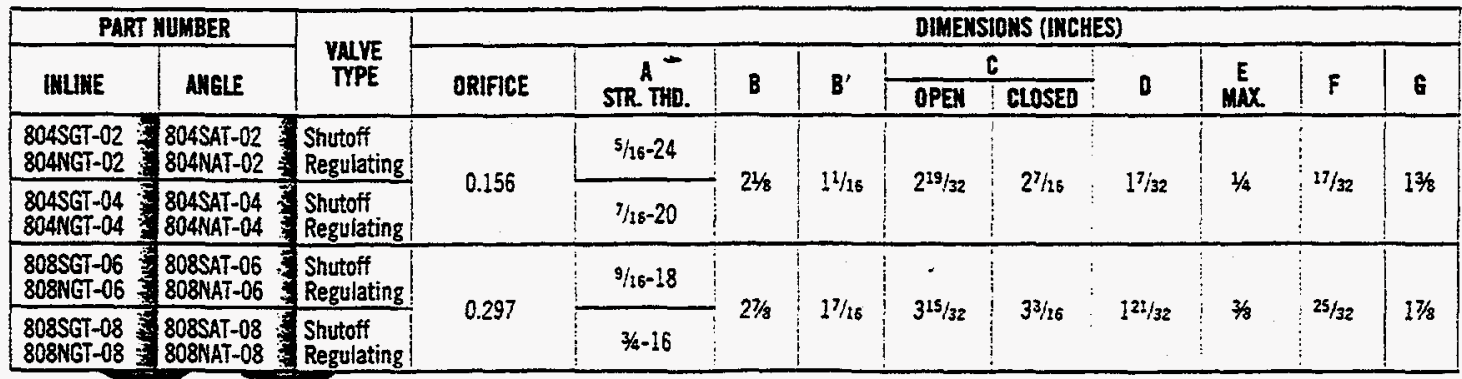

* When ordering, be sure to include the appropriate letter for the seal material desired.

B-Buna-N; C-Viton; D-Ethyiene Propylene; T-TFE (consult factory before ordering). 
CPV 6000 psi O-SEAL Valves and Fittings-a system of sott-seated vaives and fittings in pipe and O.D. tube sizes from $1 / 8$ " to $2^{\prime \prime}$. For positive control and leakproof containment of liquids or gases. Request Catalog 68.

CPV 10,000 psi O-SEAL Valves and Fittings-1/4" pipe size and $9 / 15^{\prime \prime}$ O.D. tube. Request Catalog 80.

CPV MARK VII Tube Fittings-heat-sealed, separable tube fitings for vacuum to 3000 psi. Sizes from $1 / 8^{\prime \prime}$ to $2^{\prime \prime}$. Request Catalog 74 .

CPV MARK VIII Valves-soft-seated regulating and shut-off valves with various end connections. Request Catalog 84.

CPV MARK VIII Hose Fittings-easy connect/disconnect fittings for medium and high pressure hose per SAE 100R5 and 100R2. Request Catalog 75.

CPV MARK VIII Direct Braze/Weld Tube Fittings-couplings, tees, elbows and reducers in sizes from $1 / 4$ " to 2 ' for permanent, hermetically-sealed tube to tube connections. Request Catalog 77.

CPV DYNAIR High Pressure Booster-air-operated pumps for generating liquid pressures to 5000 or 10,000 psi with a regulated air supply of only 80 psi. Request Catalog 42.

CPV Silent Check Valves-spring-actuated; close automatically and silently as soon as forward flow stops. Sizes from $1 / 2$ " to 24 " Pressures from 125 to 2500 psi. Request Catalog 800.

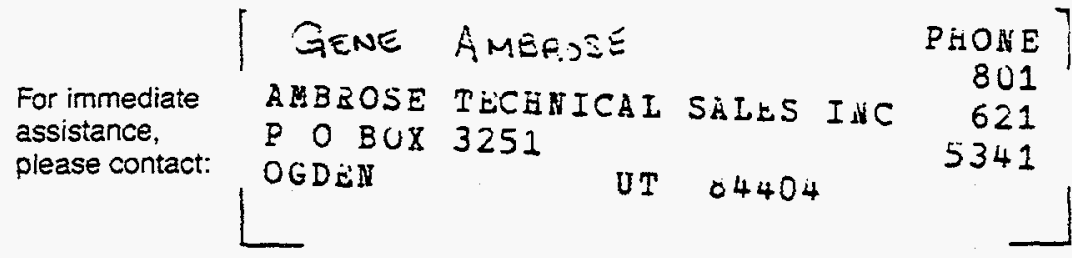

Please fill in and mail the card below. Thank you.

1. What do you want us to send to you?

$\square$ More product data on
$\square$ A quotation on
$\square$ Other

2. What is your application?

3. is your requirement $\square$ immediate $\square 1-3$ months $\square 4-6$ months $\square 7-12$ months $\square$ longer

4. What is your telephone number? Ext.

[I I would like to discuss my requirements with a CPV representative.

\footnotetext{
P 1089041

LAREY WIITHILI

$E G \& G$ IDAHO INC

$P$ O BOX 1025

ODAIO FALIS

ID 83415

is the label correct? Please make any changes right on the label.

Thank you very much for enabling us to be of further service to you.
}

\section{lip right in}

F FLOW MEDIA $\rightarrow$ Dermits rout fluid contamination erating torque.. increases

BLE_-all Mark VIII valves ith panel nuts.

VLESS STEEI STEM-elim, chattering and corrotamination traps...

flushing if required.

M SEALS-use of a resil

Im seal with a TFE

zctively eliminates

RFACES 316 STAINLESS STEEL ? performance for the

PLATED BONNET-resists nizes torque.

SEAT DESIGN-

sa of the captive,

ind broad valve seat

tight shutoff and

ng valve life.

SSAGES-minimize rrough valve. Choice 25 to 1.60 depending $d$ body style.

NG SEALED FITTINGS- Vill O-SEAL fittings

connections that

sse. ... valve can be t without

ting the line.

IEALED TUBE ENDS-

IL fittings are permanently $I$ to the tubing.

configurations are ages 6 and 7 .

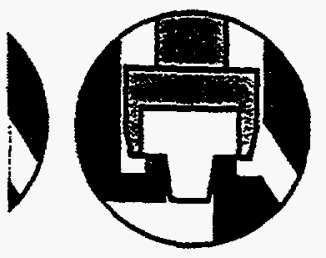

Figure 8.

Regulating (Needle) Valve 

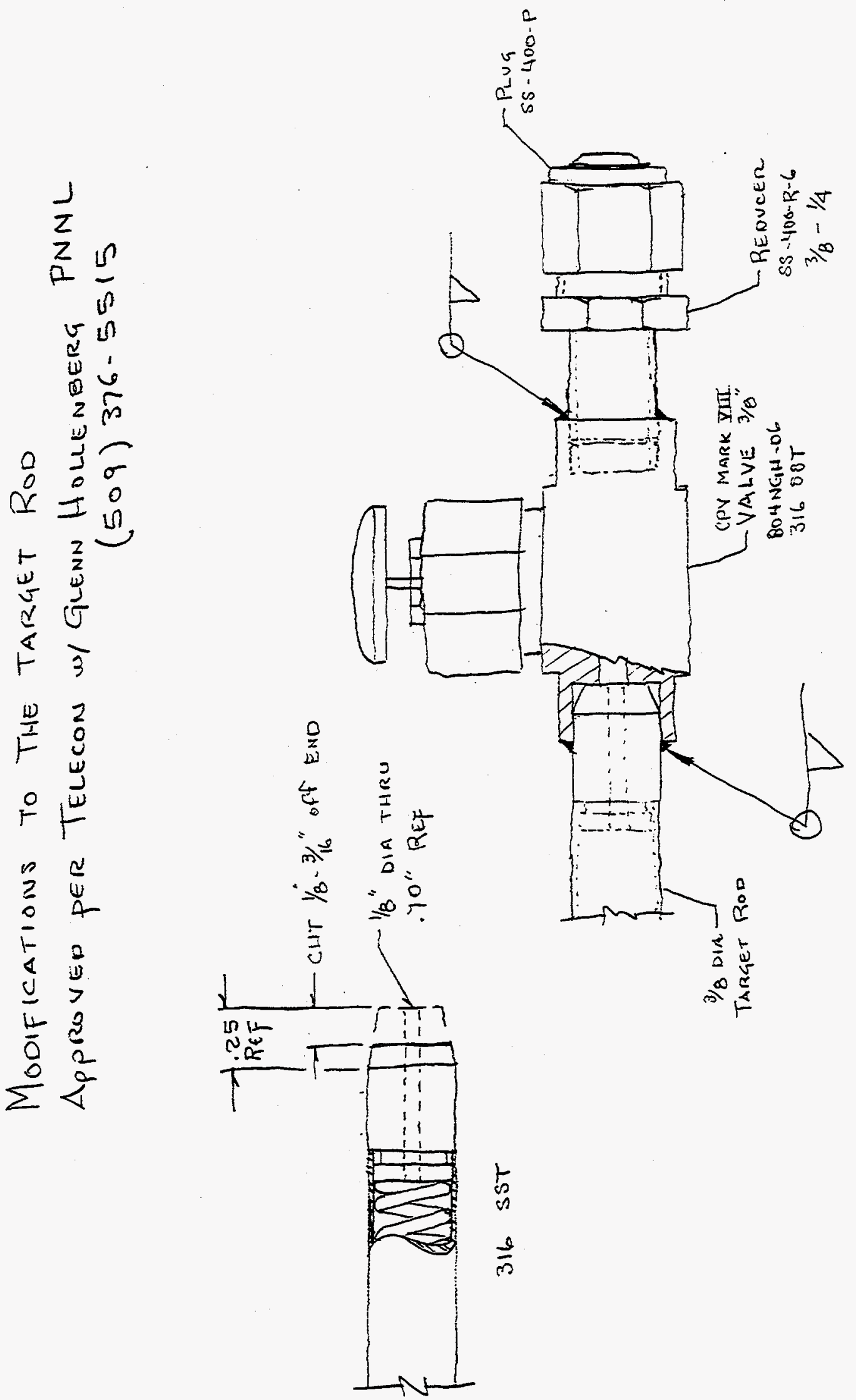


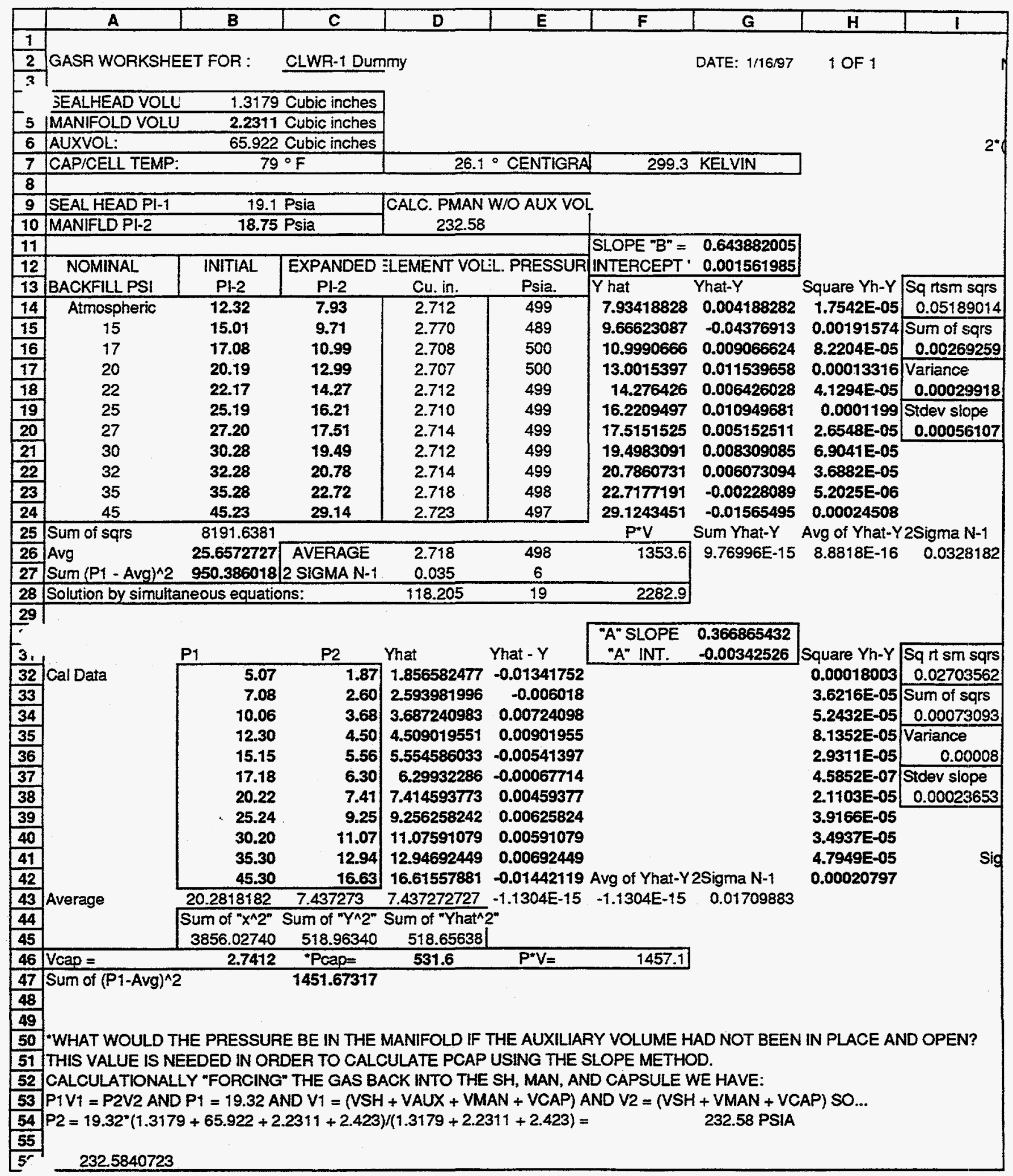




\section{ARCONE NATI ONAL LABORATORY - WEST}

ED

Anal yt $i$ cal Laborat ory

$\Longrightarrow$

Requester: P. WEBB, R. ELLIOTT, R. WISNER, D. HAGAN

Facility: HFEF

Login Name: CLWR PIN GAS (Dummy ROD)

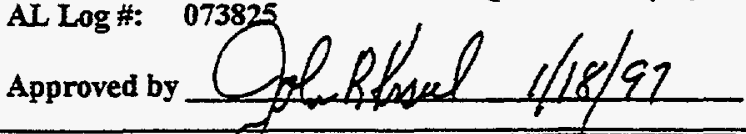

COC \#: 1

Sample \#: 1 of

Sample ID: CYL \#15

Where Taken: HFEF

GROUP

MASS SPEC

\section{ANALYTES}

$\mathrm{H2}$

He

N2

$\mathrm{O} 2$

Ar

$\mathrm{CO} 2$

\section{ANALYTES}

He 3 Isotopic

He 4 Isotopic
FINAL REPORT

$=$
GROUP

ASS SPEC

COMMENT: $\mathrm{ND}=$ Not Detectable.

\begin{tabular}{|c|c|c|}
\hline RESULTS & \multicolumn{2}{|c|}{ ERROR@2 sigma UNITS } \\
\hline 0.02 & $\doteq 25 \%$ & Mole \% \\
\hline 99.46 & $\pm 1.0 \%$ & Mole \% \\
\hline 0.37 & $\pm 25 \%$ & Mole \% \\
\hline 0.06 & $\doteq 25 \%$ & Mole $\%$ \\
\hline 0.09 & $=25 \%$ & Mole \% \\
\hline$<0.01$ & $\mathrm{n} / \mathrm{a}$ & Mole \% \\
\hline
\end{tabular}

RESOLTS

ND

100.0
II/a

a/o
$13: 30$

Date

Date Received: 16 Jan-97
ERROR@2 sigma UNITS

Sampling Date: 16-Jan-97 12:00 Barcode No.: N/A

n/a $\quad \mathrm{N} / \mathrm{a}$


Appendix H

Calibration Sheets and Puncture Data 


\section{Appendix $\mathrm{H}$}

\section{Table of Contents}

\section{Calibration Sheets}

1-21-97

1-27-97

1-30-97

2-03-97

2-04-97

2-05-97

\section{Puncture data}
WL-01
WL-03
WL-09
WL-10
WL-18
WL-22
WL-26
WL-27
WT-11
WT-12

\section{Sample Bottle Data Tables}

Sample Bottle ID

Sample Bottles Used in WL-18 Backfill Analysis

Sample Bottle Volumes

\section{ANL-W Spread Sheets Pressure and Void Volume}

WL-01

WL-03

WL-09

WL-10

WL-18

WL-22

WL-26

WL-27

WT-11

WT-12

\section{GASR System Volumes}

Table 


\section{MATHCAD}

File on pressure and void volume calculation 


\section{Appendix B}

Page 1 of 1

\section{SYSTEM CALIBRATION DATA SHEET}

Date: $1-21-97$

\begin{tabular}{|c|c|c|c|}
\hline \multicolumn{4}{|c|}{ Gauge Calibration Data } \\
\hline $\begin{array}{l}\text { Nominal Pressure } \\
.4 \text { (psia) }\end{array}$ & $\begin{array}{l}\text { Calibrated Gauge } \\
\text { (PI-6) }\end{array}$ & $\begin{array}{r}\text { Seal Head } \\
\text { (PI-1) }\end{array}$ & $\begin{array}{c}\text { Manifold } \\
(\mathrm{PI}-2)\end{array}$ \\
\hline $18 \%$ $5 \%$ & 5.01 & 5,0 & 5.01 \\
\hline (2. & 7.01 & 7. 0 & 7.00 \\
\hline $4 \% 10 \%$ & 10.00 & 10.0 & 10.00 \\
\hline Atmospheric & 11.97 & 12.0 & 11.97 \\
\hline $1 \% 15 \%$ & 15.05 & 15.1 & 15.05 \\
\hline $18 \% 17 \%$ & 17.05 & 17.1 & 17. 04 \\
\hline$\% .20 \%$ & 20.04 & 20,1 & 20.05 \\
\hline $1 \% .1 \% 25 \%$ & 25,06 & 25.1 & 25.06 \\
\hline $1 \% 1 \% 30 \%$ & 30.04 & 30.1 & 30.05 \\
\hline 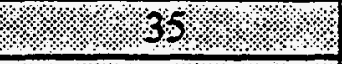 & 35,02 & 35.1 & 35.04 \\
\hline $1.1 .8 .145 .8 \% .8$. & 45.04 & 45.1 & 45.03 \\
\hline
\end{tabular}

\begin{tabular}{|c|c|c|}
\hline \multicolumn{3}{|c|}{ (. Seal HeadAManifoldRatio Data } \\
\hline Nominal Pressure & Initial & $\begin{array}{l}. \\
. \\
\text { Expanged } \\
(\mathbf{P}-\mathbf{2})\end{array}$ \\
\hline \%. $.5 . \% \%$ & 5.04 & 1.84 \\
\hline $9.1 \% 7 \%, \%$ & 7.04 & 2.56 \\
\hline $8 \% 10$ & 10.05 & 3.65 \\
\hline Atmospheric & 12.03 & 4.39 \\
\hline ४। 15 । & 15.13 & 5.51 \\
\hline अ17\% & 17,13 & 6.24 \\
\hline ४ 20 ०ै। & 20.15 & 7.34 \\
\hline ४ै $25 \%$ & 25.19 & 9.18 \\
\hline 30 & 30.20 & 11.02 \\
\hline $35 \quad 3$ & 35,22 & 12.85 \\
\hline 45 & 45.25 & 16.54 \\
\hline
\end{tabular}

Seal Head

Rod Block (Saddle) .375 
Appendix B

Page 1 of 1

\section{SYSTEM CALIBRATION DATA SHEET}

Date: $1-27-97$

\begin{tabular}{|c|c|c|c|}
\hline$\cdots$ & ४ Gauge Calibra & tion Data & - \\
\hline $\begin{array}{l}\text { NominalPressure } \\
\text { (psia) }\end{array}$ & $\begin{array}{l}\text { Calibrated Gauge } \\
\text { (PI-6) }\end{array}$ & $\begin{array}{l}\text { Seal Head } \\
\text { (PI-I) }\end{array}$ & $\begin{array}{l}\text { Manifold } \\
\text { (PI-2) }\end{array}$ \\
\hline - $\$ 5 ४$ & 5.00 & 5.0 & 5.01 \\
\hline अ $7 \%$ & 7.03 & 7.0 & 7.02 \\
\hline 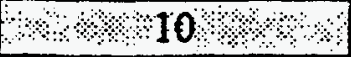 & 10.00 & 10. 0 & 10.00 \\
\hline Atmospheric & 12.24 & 12.2 & 12.23 \\
\hline ४। $15 \%$ । & 15.03 & 15.0 & 15.04 \\
\hline अ $17 \%$ & 17.03 & 17.0 & 17.04 \\
\hline अ ४ $20 \%$ & 20.00 & 20.0 & 20.01 \\
\hline ४ $25 \%$ ४ & 25.02 & 25.0 & 25.01 \\
\hline $3 \bullet 30 \%$ ४ & 30.03 & 30.0 & 30.01 \\
\hline ४४ $235 \%$ & 35.04 & 35.0 & 35.03 \\
\hline $4 \% / 45 \%$ & 45.03 & 45.0 & 45.04 \\
\hline
\end{tabular}

\begin{tabular}{|c|c|c|}
\hline \multicolumn{3}{|c|}{ ০ } \\
\hline $\begin{array}{l}\text { Nominal Pressure } \\
\text { (psia) }\end{array}$ & mitial $(P 12) \%$ & $\begin{array}{l}\text { Expanded } \\
(\mathrm{P} I-2)\end{array}$ \\
\hline 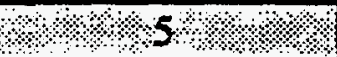 & 5.03 & 1.87 \\
\hline 1..\%\%\%. & 7.05 & 2.60 \\
\hline \%०. $10 \%$ & 10.05 & 3.70 \\
\hline Asmöspherie & 12.28 & 4.52 \\
\hline ४ै। 15 & 15.11 & 5.57 \\
\hline - 17\% ४ & 17.12 & 6.31 \\
\hline \%20 & 20.12 & 2.42 \\
\hline ४ै $25 \%$ & 25.15 & 9.25 \\
\hline $30 \quad 3$ & 30.17 & 11.09 \\
\hline m & 35.21 & 12.94 \\
\hline 45 & 45.26 & 16.64 \\
\hline
\end{tabular}

Seal Head

Rod Block (Saddle) . 375 


\section{Appendix B}

Page 1 of 1

\section{SYSTEM CALIBRATION DATA SHEET}

Date: $1-30-97$

\begin{tabular}{|c|c|c|c|}
\hline $\begin{array}{l}\text { Nominal Pressure } \\
\text { (psia) }\end{array}$ & $\begin{array}{l}\text { Calibrated Gauge } \\
\text { (PI-6) }\end{array}$ & $\begin{array}{l}\text { Seal Head } \\
\text { (PI-1) }\end{array}$ & $\begin{array}{l}\text { Manifold } \\
+(P I-2)\end{array}$ \\
\hline $1.8 \%$ & 5.01 & 5.0 & 5.01 \\
\hline $4.1 \%$ & 7.00 & 7.0 & 200 \\
\hline $1 \%, 110 \%$ & 10.00 & 10.0 & 10.02 \\
\hline Atmospheric & 12.28 & 12.3 & 12.28 \\
\hline $4 \% 15 \%$ & 15.01 & 15,0 & 15.03 \\
\hline $1 \% 17 \%$ & 17.03 & 17.0 & 17.05 \\
\hline $1.120 \%$ & 20.03 & 20.0 & 20.03 \\
\hline $4.4 \% 25 \%$ & 25.01 & 25.0 & 25.03 \\
\hline 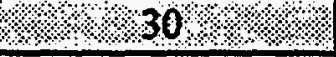 & 30.01 & 30.0 & 30.03 \\
\hline $1.1 .7 .35 \%$ & 35.02 & 350 & 3506 \\
\hline 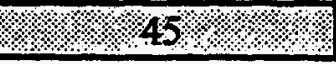 & 45.0 .2 & 45.0 & 45.00 \\
\hline
\end{tabular}

\begin{tabular}{|c|c|c|}
\hline \multicolumn{3}{|c|}{ 1. Seal HeadManifold Ratio Data } \\
\hline $\begin{array}{l}\text { Nominal Pressure } \\
\text { (psia) }\end{array}$ & hitial & $\begin{array}{c}\text { Exparided } \\
(P 1-2)\end{array}$ \\
\hline 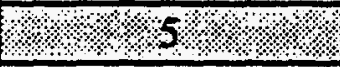 & 5.03 & 1.86 \\
\hline$\% 7 \%$ & 7.03 & 2.59 \\
\hline $1.10 \%$ & 10.06 & 3.69 \\
\hline Atmospherie & 12.34 & 4.53 \\
\hline $1 \% 15 \%$ ४ & 15.10 & 5.54 \\
\hline ४৯17^॰४ & 17.13 & 6.28 \\
\hline $420 \%$ & 20.13 & 7.38 \\
\hline $8 \% \div 25 \%$ & 25.16 & 9.22 \\
\hline $30 \%$ & 30.18 & 11.06 \\
\hline 35 & 35.23 & 12.91 \\
\hline 45 & 45.22 & 16.60 \\
\hline
\end{tabular}

Seal Head

Rod Block (Saddle) .375 


\section{Appendix B}

Page 1 of 1

\section{SYSTEM CALIBRATION DATA SHEET}

Date: $2-3-97$

\begin{tabular}{|c|c|c|c|}
\hline \multicolumn{4}{|c|}{ Gauge Calibration Data } \\
\hline $\begin{array}{l}\text { Nominal Pressure } \\
\text { (psia) }\end{array}$ & $\begin{array}{l}\text { Calibrated Gauge } \\
\text { (PI-6) }\end{array}$ & $\begin{array}{c}\text { Seal Head } \\
(\mathrm{Pl}-1)\end{array}$ & $\begin{array}{c}\text { Manifold } \\
(\text { (PI-2) }\end{array}$ \\
\hline$\% .4 \% \%$ & 5.02 & 5.0 & 5.07 \\
\hline . & 7.01 & 7.0 & 7.02 \\
\hline $1 \% 10 \% 10 \%$ & 10.06 & 10.0 & 10.08 \\
\hline Atmospheric & 12.19 & 12.2 & 12.21 \\
\hline $1 \%, 15 \%$ & $|5,0|$ & 15,0 & 15.05 \\
\hline$\% \% 17 . \% \%$ & 17.00 & 17.0 & 17.03 \\
\hline $28 \% 20 \%$ & 20.03 & 20.0 & 20.08 \\
\hline $1 \% 25 \%$ & 25.02 & 25.0 & 25.04 \\
\hline $4.30 \%$ & 30.00 & 36.0 & 30.01 \\
\hline 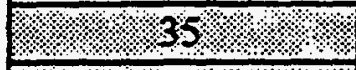 & 35.00 & 35.0 & 35.03 \\
\hline $17.23 \%$ & 45.09 & 45.1 & 45,10 \\
\hline
\end{tabular}

\begin{tabular}{|c|c|c|}
\hline \multicolumn{3}{|c|}{ Seal HeadMarifoldRatio Data } \\
\hline $\begin{array}{l}\text { Nominalpressure } \\
\text { (psia) }\end{array}$ & $\begin{array}{l}7 \text { Initial } \\
(\mathbf{P}(-2)\end{array}$ & $\begin{array}{l}\text { Expanded } \\
(\mathrm{PI}-2)\end{array}$ \\
\hline $1 \% \vee 5 \%$ & 5.11 & 1.90 \\
\hline$\% \% 7 \%$ & 7.06 & 2.61 \\
\hline $1 \% 10 \%$ & 10.13 & 3.73 \\
\hline Atmospheric. & 12.26 & 4.51 \\
\hline ২. $15 \%$ & 15.12 & 5.58 \\
\hline अ17॰ ४४ & 17.11 & 6.31 \\
\hline $2020 \%$ & 20.18 & 7.43 \\
\hline $7425 \%$ & 25.17 & 9.26 \\
\hline 30 & 30.16 & 11.08 \\
\hline 35 & 35.20 & 12.92 \\
\hline 45 & 45,32 & 16.65 \\
\hline
\end{tabular}

Seal Head

Rod Block (Saddle) .375 


\section{Appendix B}

Page 1 of 1

\section{SYSTEM CALIBRATION DATA SHEET}

Date: $2-4-97$

\begin{tabular}{|c|c|c|c|}
\hline$\therefore:$ & Gauge Calibr & & \\
\hline $\begin{array}{l}\text { Nominal Pressure } \\
\text { (psia) }\end{array}$ & $\begin{array}{l}\text { Calibrated Gauge } \\
\text { (PI-6) }\end{array}$ & $\begin{array}{c}\text { Seal Head } \\
\text { (PI:1) }\end{array}$ & $\begin{array}{c}\text { Manifold } \\
(\text { (PI-2) }\end{array}$ \\
\hline \%० 5 Hै। & 5.01 & 50 & 5.03 \\
\hline $7.7 \%$ & $7.0 x$ & 71 & 7.06 \\
\hline$\%, \% 10 \% \%$ & 10.00 & 10.0 & 10.01 \\
\hline Atmospheric & 12.18 & 12.2 & 12.18 \\
\hline $8 \times 15 \%$ & 15.02 & 15.0 & 15.02 \\
\hline $1 \% \% 17 \%$ & 17.02 & 17.0 & 17.04 \\
\hline 2820 & 20.00 & 20.0 & 20.01 \\
\hline $18 \% 425 \% 8 \%$ & 25.04 & 25.0 & 25.02 \\
\hline $4 \times 30 \%$ & 30.03 & 30.0 & 30.01 \\
\hline $5 \times 35 \%$ & 35.05 & 35.0 & 35.07 \\
\hline $1.75 \%$ & 45.05 & 45.0 & 45.01 \\
\hline
\end{tabular}

\begin{tabular}{|c|c|c|}
\hline \multicolumn{3}{|c|}{ 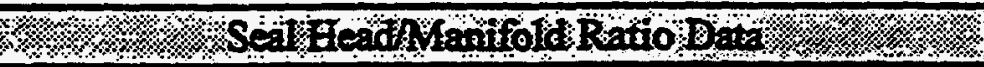 } \\
\hline Nominats resume & (6) & 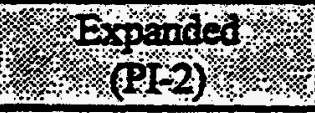 \\
\hline $48 \% 5 \%$ & 5.06 & 1.86 \\
\hline \%.1.7\% \% & 7.10 & 2.61 \\
\hline $18 \% 10 \%$ & 10.06 & 3.62 \\
\hline \%Atmosplatic & 12.24 & 4.49 \\
\hline ४ю15\%४ & 15.09 & 5.54 \\
\hline - $17 \% \%$ & $17 \cdot 13$ & 6.29 \\
\hline $20 \%$ & 20.11 & 7.38 \\
\hline 425 & 25.14 & 9.22 \\
\hline $30 \% 3$ & $30 \cdot 16$ & 11.06 \\
\hline $35 \%$ & 35.24 & 12.92 \\
\hline 45 & 45.24 & 16.60 \\
\hline
\end{tabular}

Seal Head

Rod Block (Saddle) .375 


\section{Appendix B}

Page 1 of 1

\section{SYSTEM CALIBRATION DATA SHEET}

Date: $2-5-97$

\begin{tabular}{|c|c|c|c|}
\hline & \multicolumn{2}{|c|}{ Gauge Calibration Data } & ঝे \\
\hline $\begin{array}{r}\text { Nominal Pressure } \\
\text { (psia) }\end{array}$ & $\begin{array}{l}\text { Calibrated Gauge } \\
\text { (PI- } 6)\end{array}$ & $\begin{array}{l}\text { Seal Head } \\
\%(\mathrm{PI}-1)\end{array}$ & $\begin{array}{l}\text { Manifold } \\
\text { (PI-2) }\end{array}$ \\
\hline \%४४5 ४४। & 5.01 & 5.0 & $5: 02$ \\
\hline 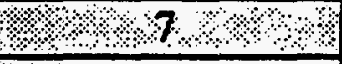 & 2003 & $7 /$ & 7.02 \\
\hline ४ & 10,00 & 10.00 & 10.00 \\
\hline Atuospheric & 12,16 & 2,1 & 12.16 \\
\hline $4 \% 15$ & 15.00 & 15.2 & 15.01 \\
\hline ॥. $17 \%$ & 17,05 & 12.0 & 17.07 \\
\hline \%. $2020 \%$ & 20.02 & 20.0 & 20,2 \\
\hline $1 \% \% 25 \%$ & 25202 & $25: 0$ & 25.01 \\
\hline $2 \% 130 \%$ & 20.01 & 30.0 & 30.00 \\
\hline (... & 35.01 & 35.6 & 35.02 \\
\hline \%... & 45.04 & 45.0 & 45,00 \\
\hline
\end{tabular}

\begin{tabular}{|c|c|c|}
\hline \multicolumn{3}{|c|}{ 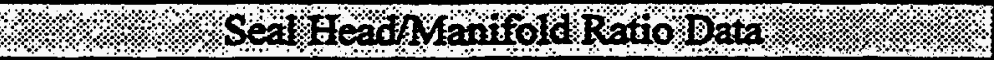 } \\
\hline $\begin{array}{l}\text { Nominal pessure } \\
\text { (psta) }\end{array}$ & \%initar & \%xpandeg \\
\hline $1 \% \%, 5 \% \%$ & 5.05 & 1.85 \\
\hline 1.. & 7.06 & 2,57 \\
\hline \%४. $100 \%$ & 10.05 & 3.67 \\
\hline Atmospheric & $12 \cdot 21$ & 4.46 \\
\hline ४ $15 \bullet$ ४ & $15 \cdot 08$ & 5,52 \\
\hline এ $17 \%$ ॰ & 17.15 & 6,28 \\
\hline ২০20\% \% & 20.12 & $7: 37$ \\
\hline ४२25 ^ै। & 25.14 & 9.20 \\
\hline $30 \mathrm{~m}$ & 30,15 & 11,03 \\
\hline$\because \cdots 35 \quad 3$ & 35,19 & 12.87 \\
\hline 45 & 45.23 & 16,56 \\
\hline
\end{tabular}

Seal Head

Rod Block (Saddle) .325 


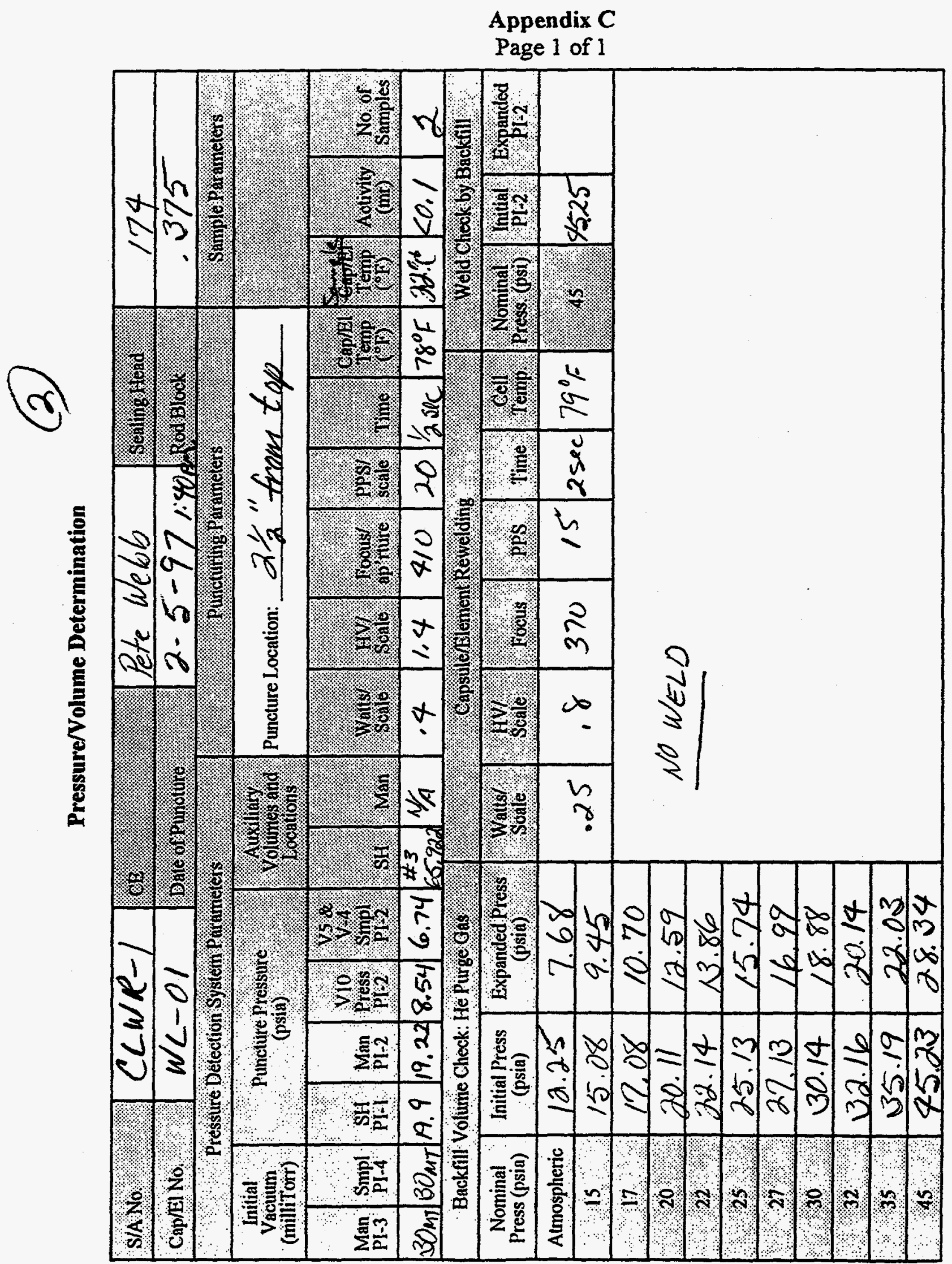


Pressure/Volume Determination

(1)

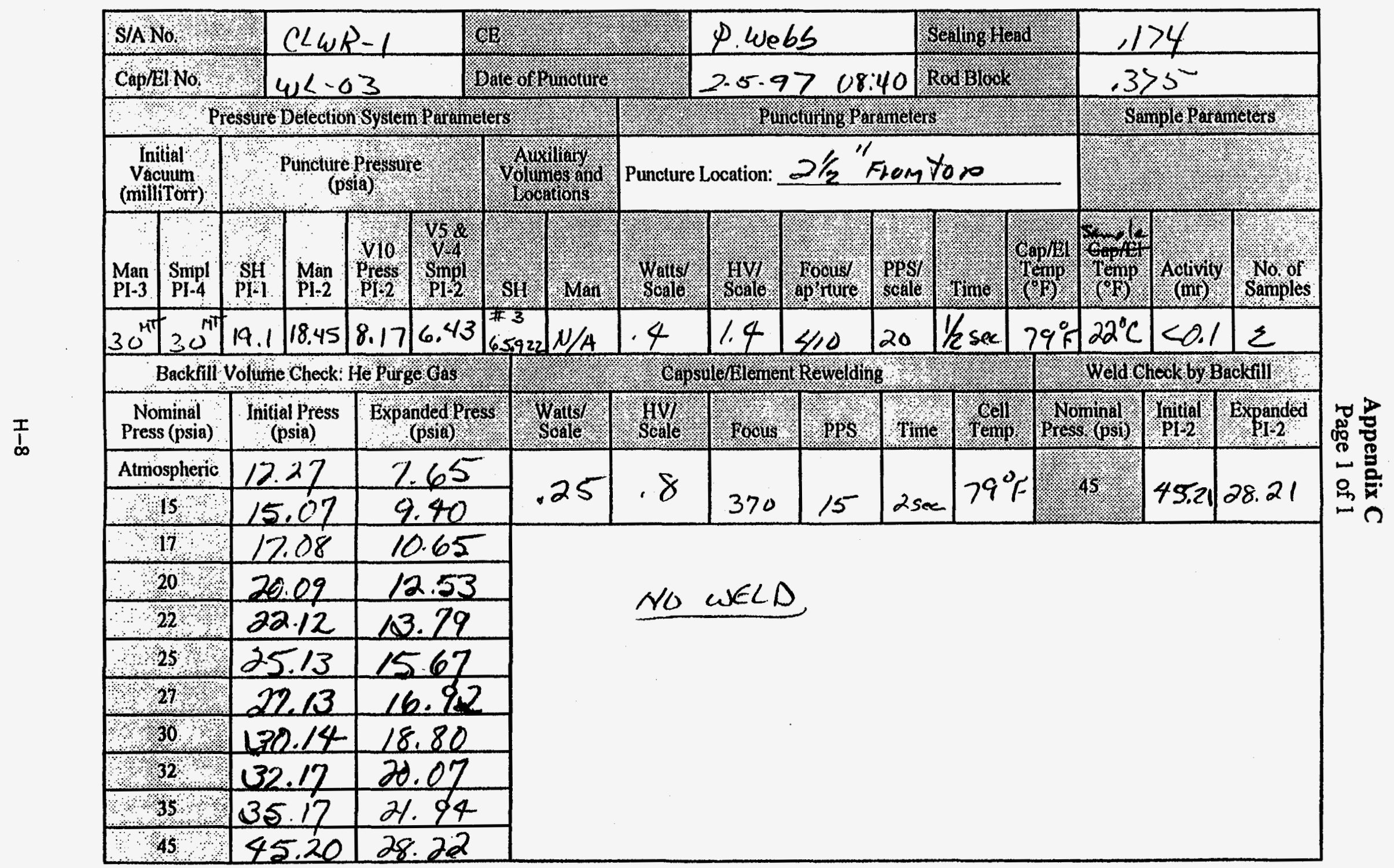




\section{Appendix C}

Page 1 of 1

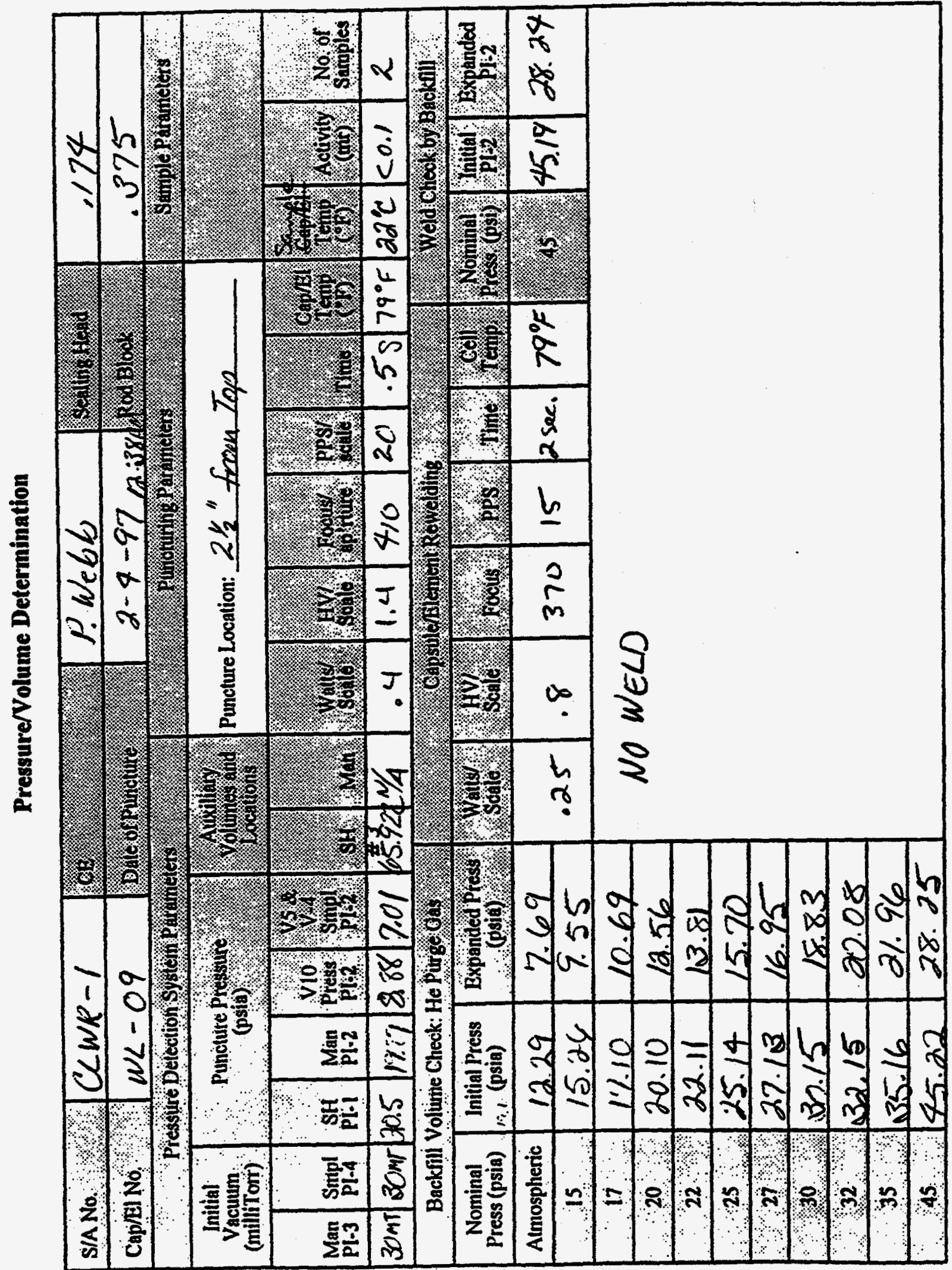


Appendix C

Page 1 of 1

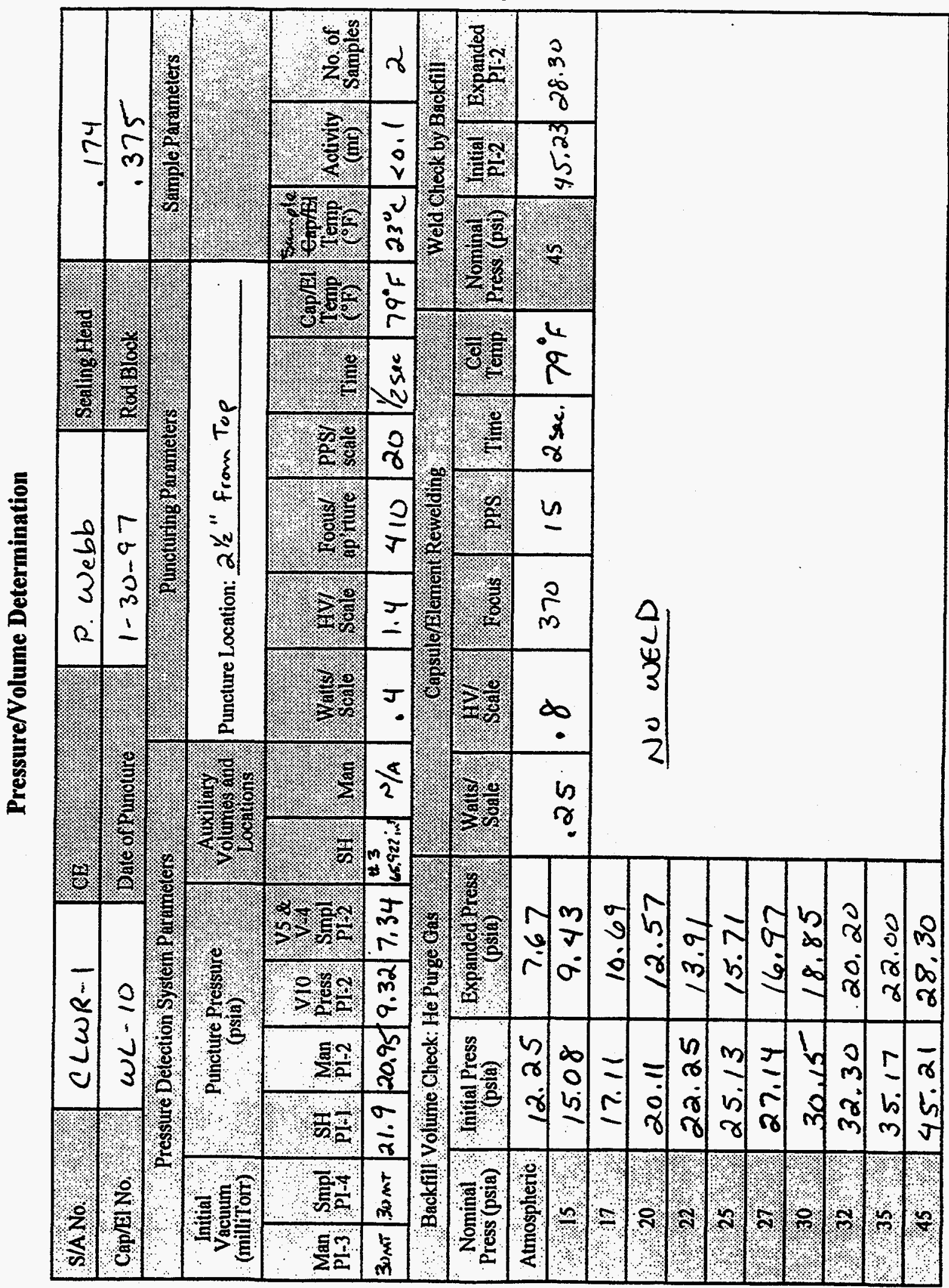


Pressure/Volume Determination

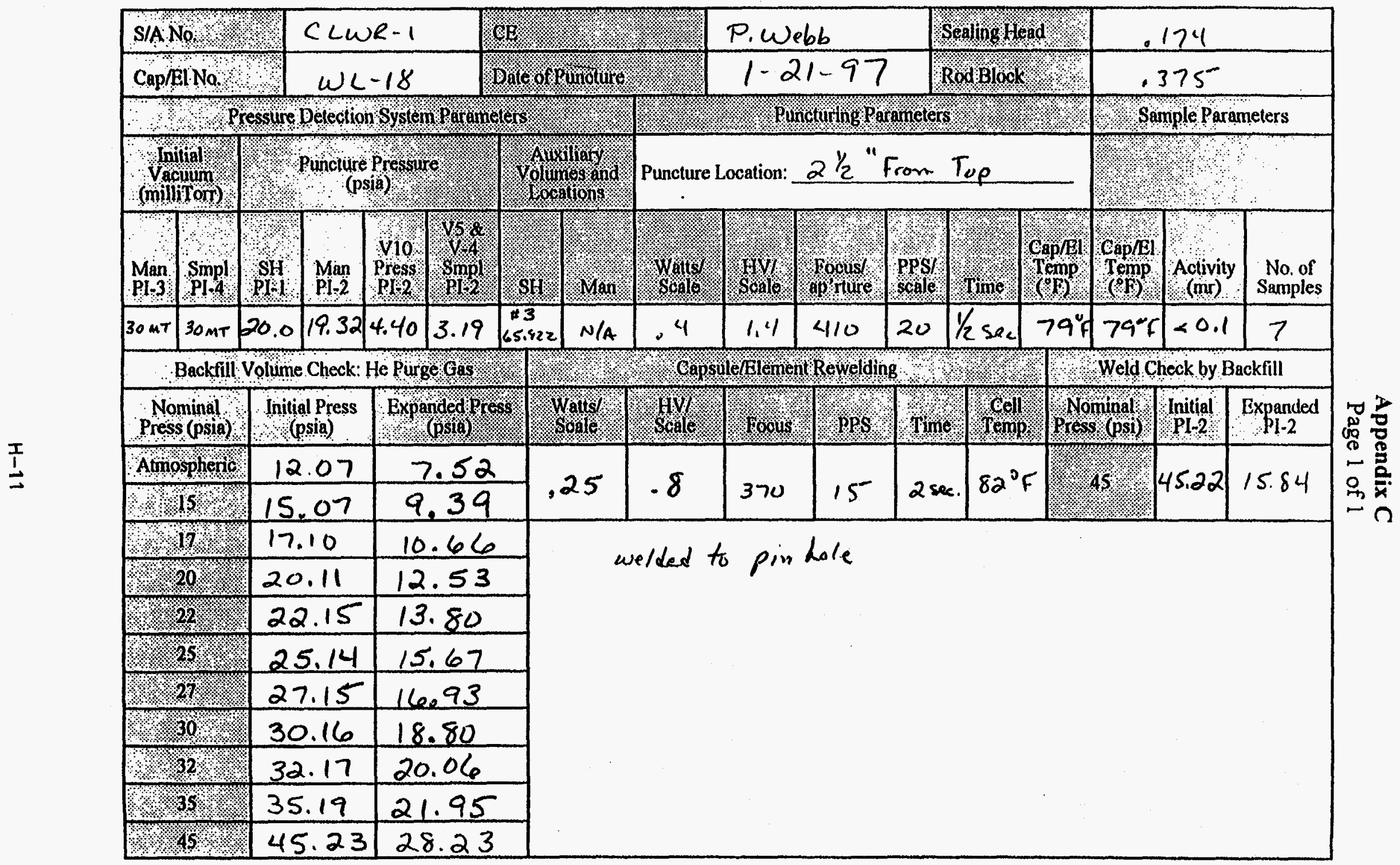


Appendix C

Page l of 1

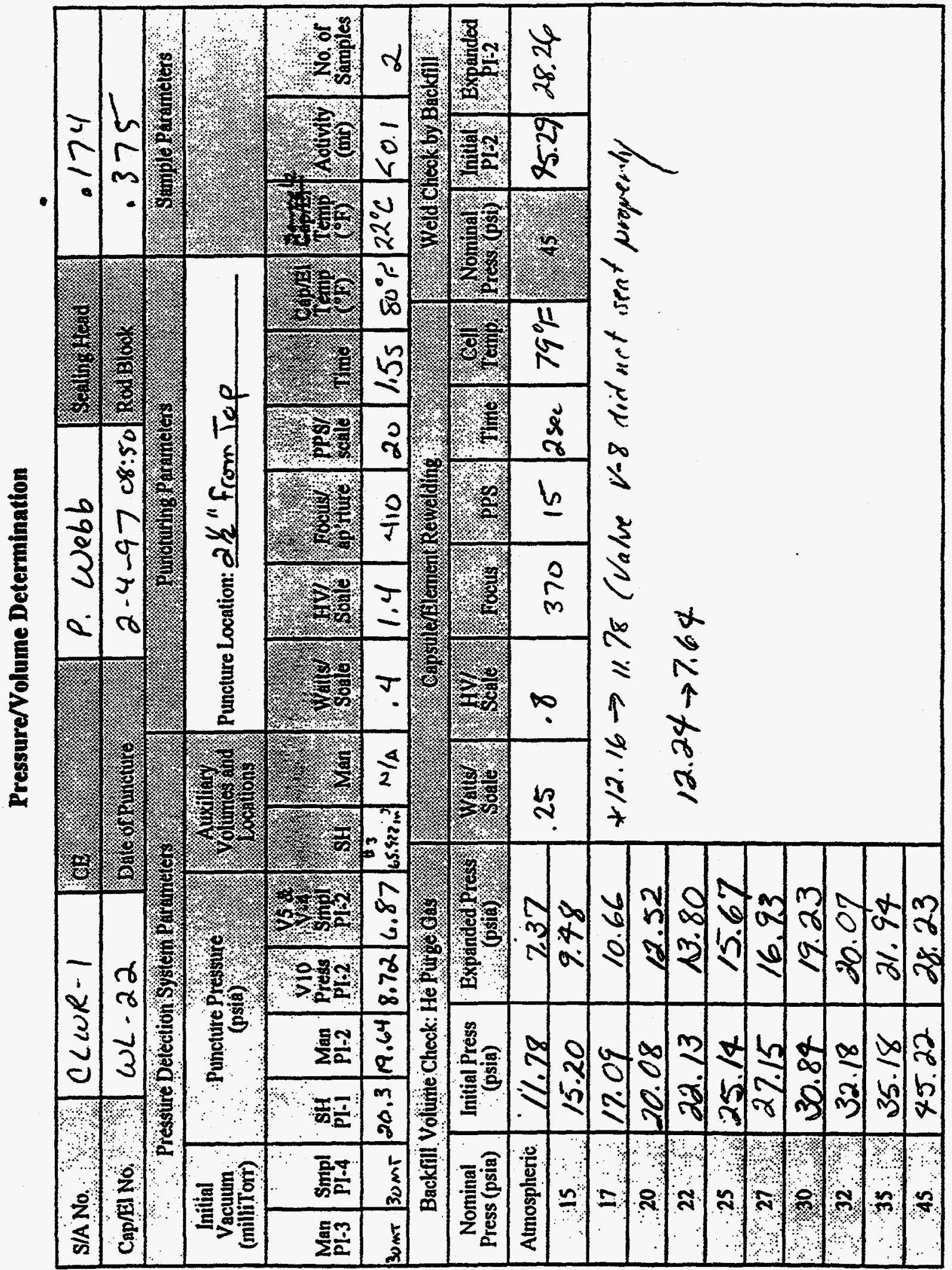




\section{Pressure/Volume Determination}

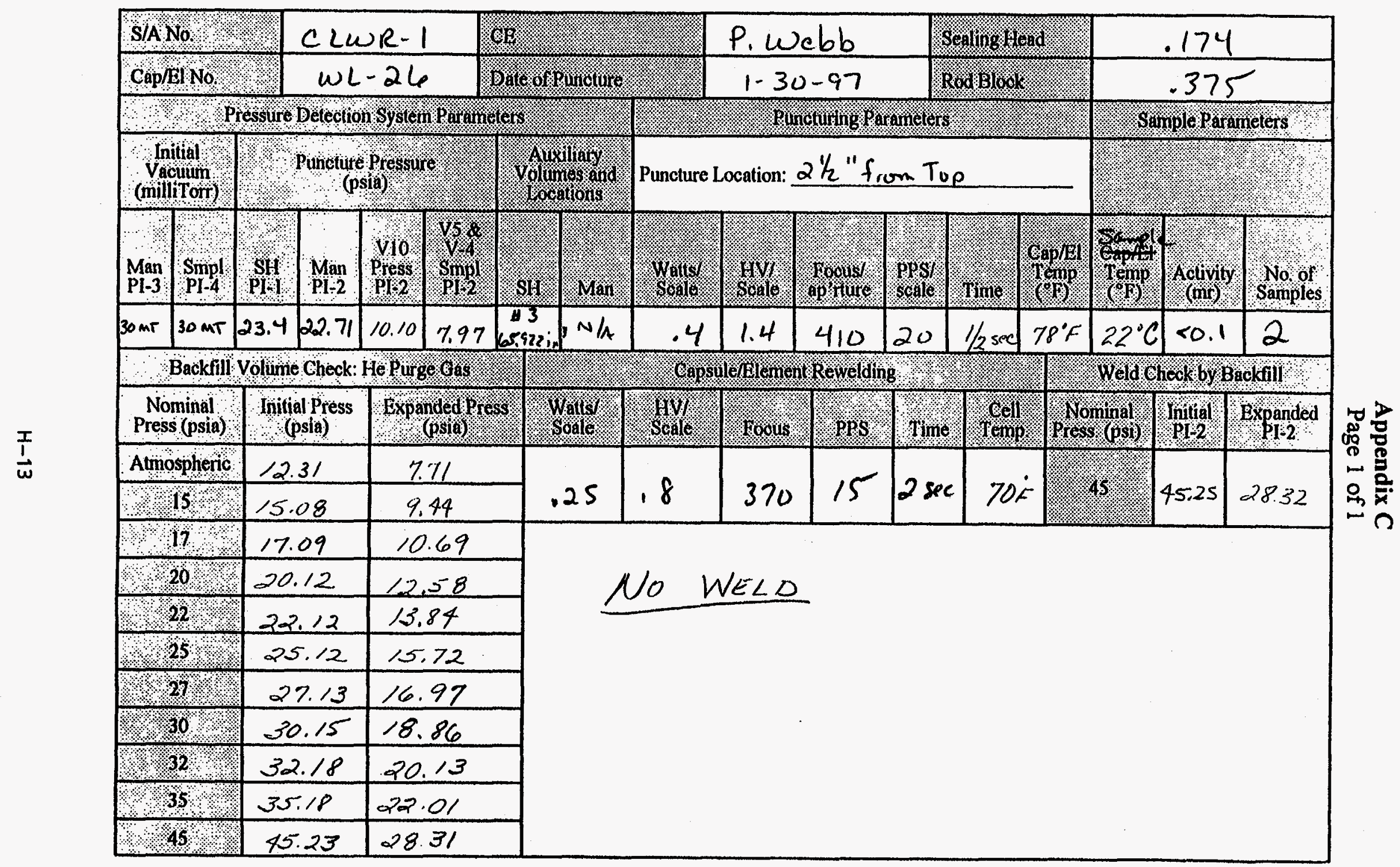


Pressure/Volume Determination

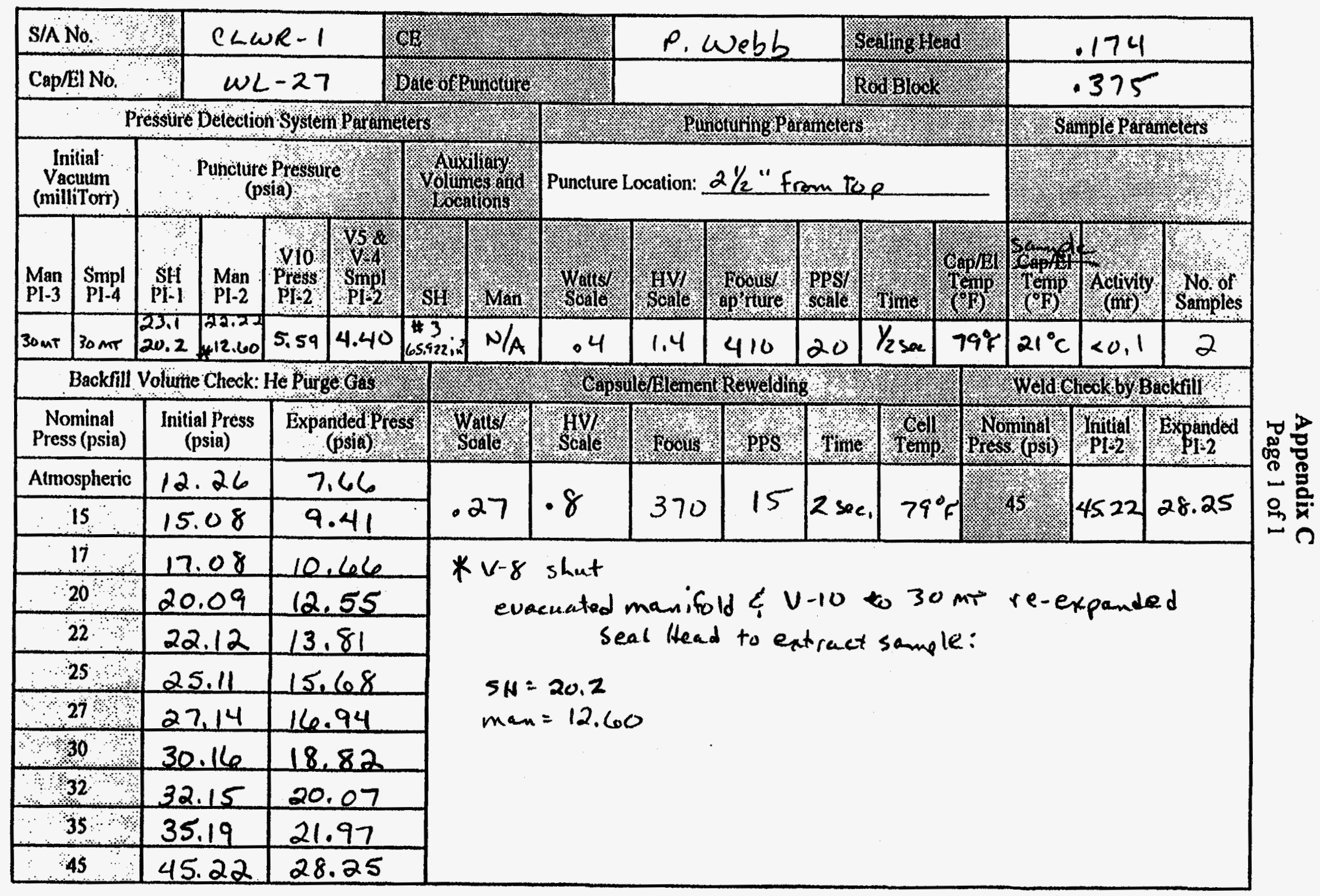


Appendix C

Page 1 of 1

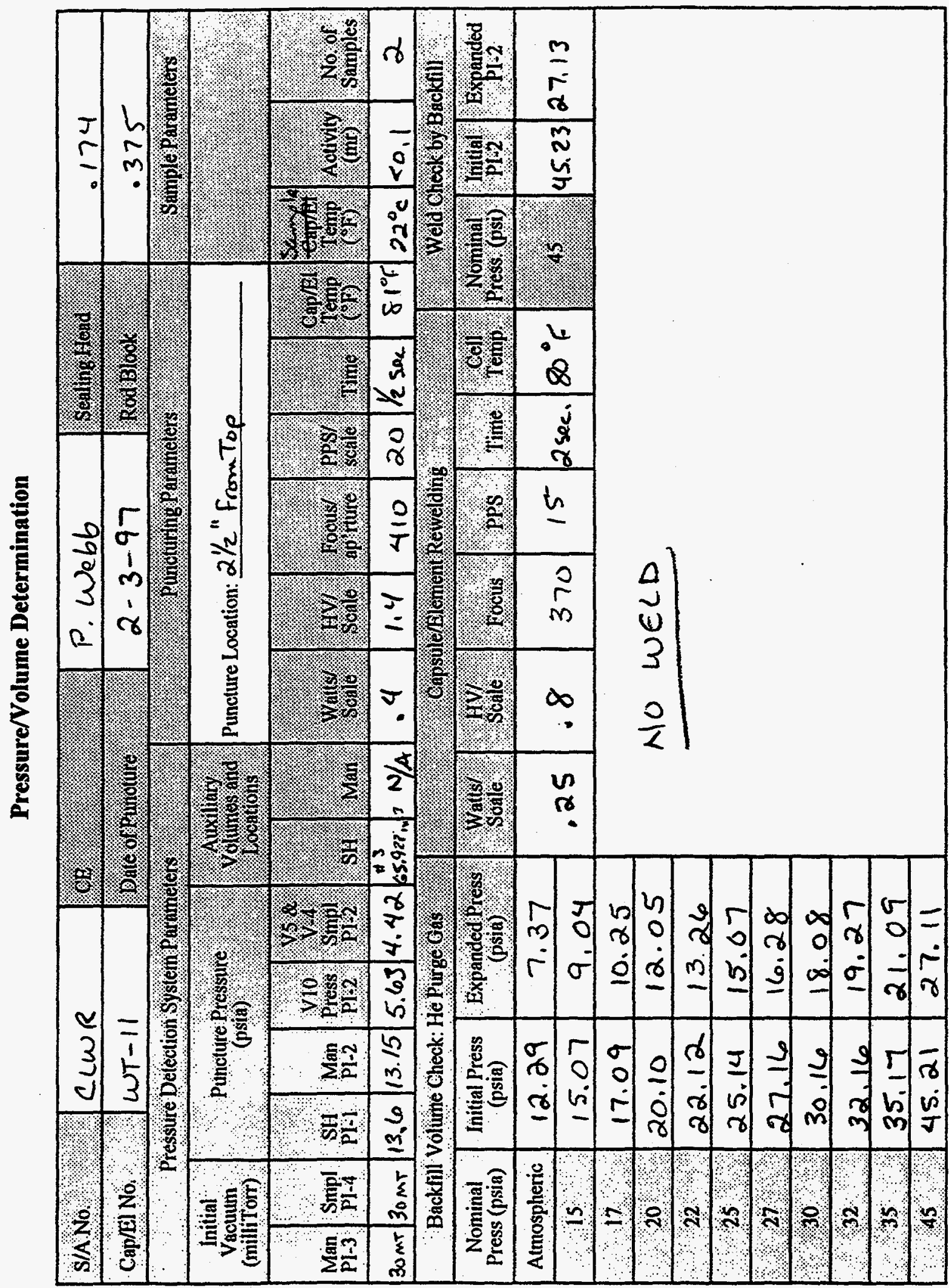


Appendix C

Page 1 of 1

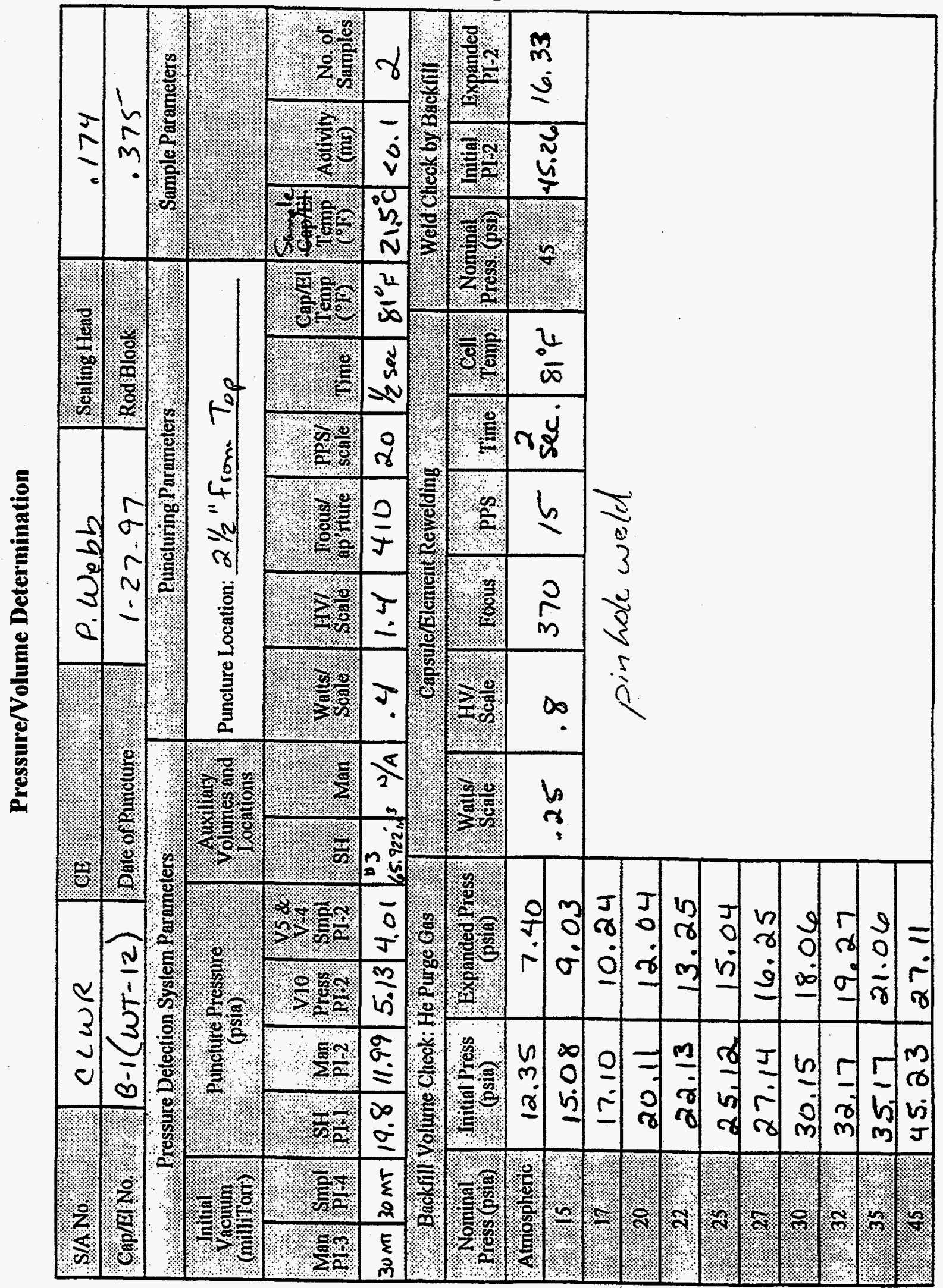




\section{Sample Bottle ID}

\begin{tabular}{|c|c|c|c|}
\hline \hline & Gas Composition & Tritium Analysis & Pressure (psia) \\
\hline Rod & Sample bottle ID & Sample bottle I & at degree Celsius \\
\hline WL-01 & 2 & 5 & $6.74 @ 22$ \\
\hline WL-03 & 1 & 4 & $6.43 @ 22$ \\
\hline WL-09 & 16 & 15 & $7.01 @ 22$ \\
\hline WL-10 & 8 & 7 & $7.34 @ 23$ \\
\hline WL-18 & 10 & 11 & $3.19 @ 22$ \\
\hline WL-22 & 10 & 11 & $6.87 @ 22$ \\
\hline WL-26 & 5 & 6 & $7.97 @, 22$ \\
\hline WL-27 & 12 & 13 & $4.4 @ 21$ \\
\hline WT-12 & 2 & 14 & $4.01 @ 21.5$ \\
\hline WT-11 & 15 & 3 & $4.42 @ 22$ \\
\hline
\end{tabular}


Sample Bottles Used in WL-18 Backfill Analysis

\begin{tabular}{|c|c|c|}
\hline \hline Backfill & Tritium Analysis & Pressure (psia) \\
\hline Pressure & Sample Bottle ID & at degree Celsius \\
\hline Atms. & 16 & $2.93 @ 22$ \\
\hline 15 psia & 9 & $3.65 @ 22$ \\
\hline 17 psia & 13 & $4.15 @ 22$ \\
\hline 35 psia & 12 & $8.55 @ 23$ \\
\hline 45 psia & 15 & $10.99 @ 23$ \\
\hline
\end{tabular}




\begin{tabular}{|c|c|c|c|}
\hline \multicolumn{4}{|c|}{ Sample Bottle Volumes } \\
\hline \hline Sample Bottle \# & Volume (cc) & Sample Bottle \# & Volume (cc) \\
\hline 1 & 26.5 & 9 & 26.2 \\
\hline 2 & 26.3 & 10 & 26.5 \\
\hline 3 & 26.2 & 11 & 26.1 \\
\hline 4 & 26.2 & 12 & 26.3 \\
\hline 5 & 26.2 & 13 & 26.4 \\
\hline 6 & 26.2 & 14 & 26.2 \\
\hline 7 & 26.4 & 15 & 26.5 \\
\hline 8 & 26.1 & 16 & 26.3 \\
\hline
\end{tabular}




$$
\mathrm{H}-20
$$




\section{DRAFT}

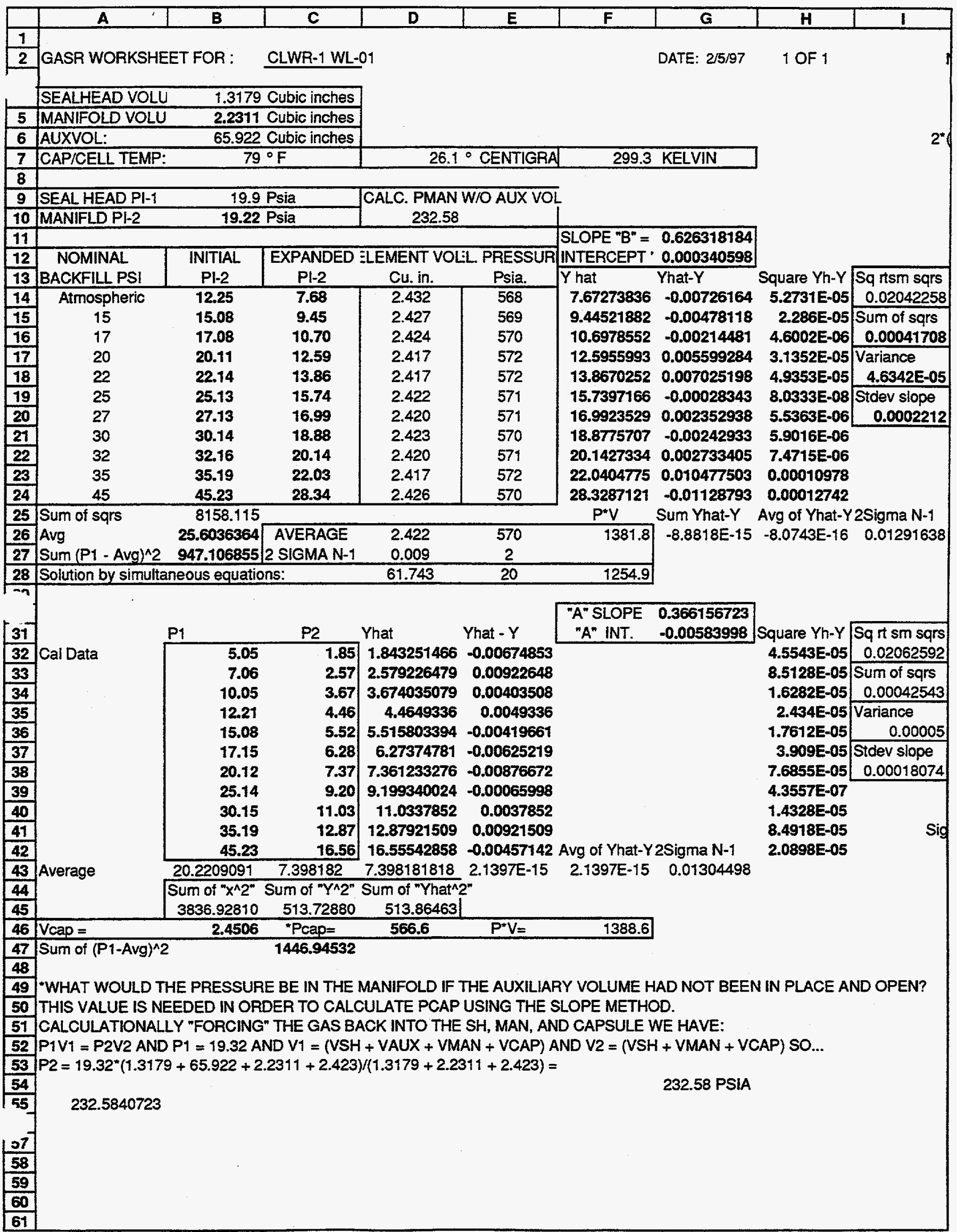




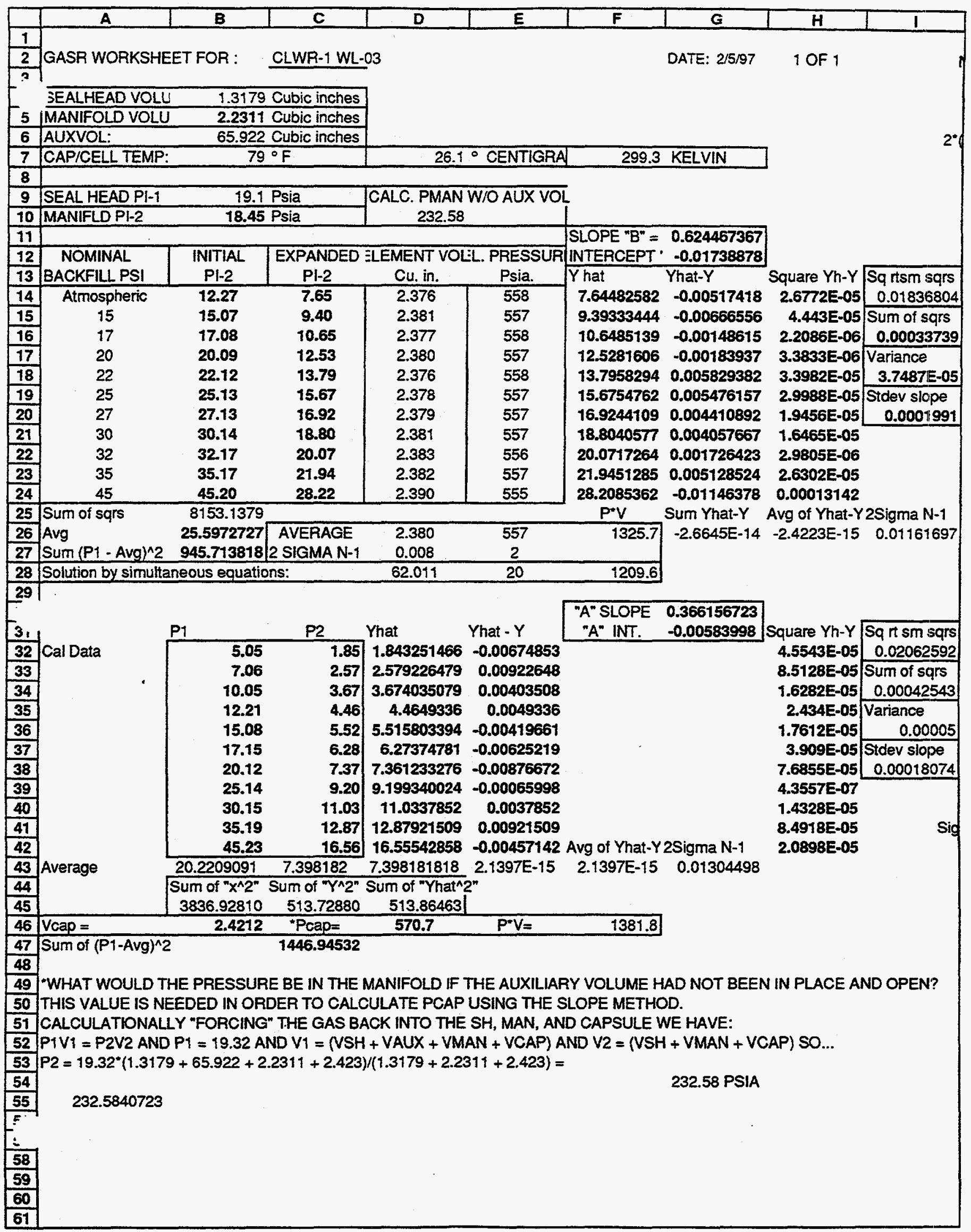




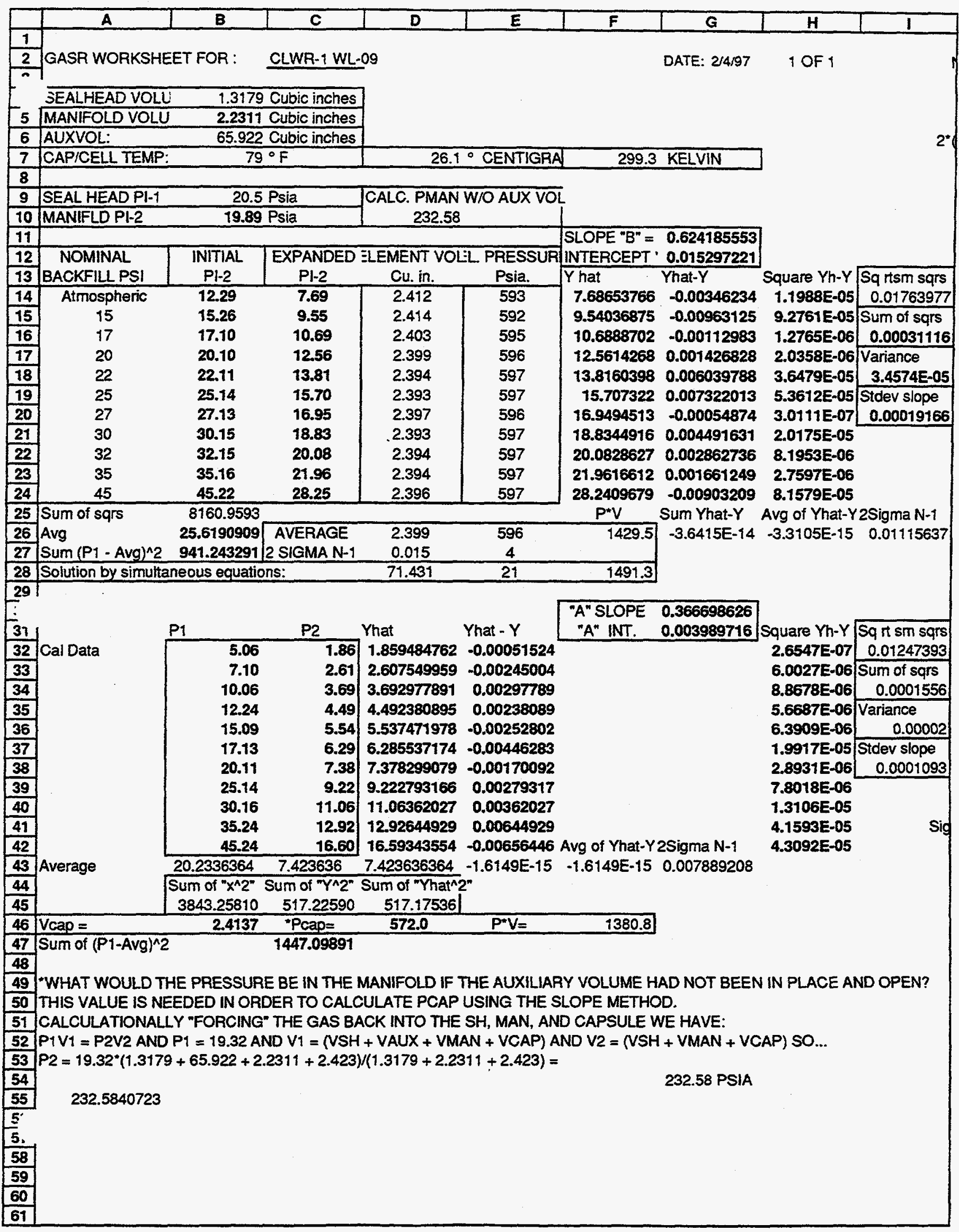




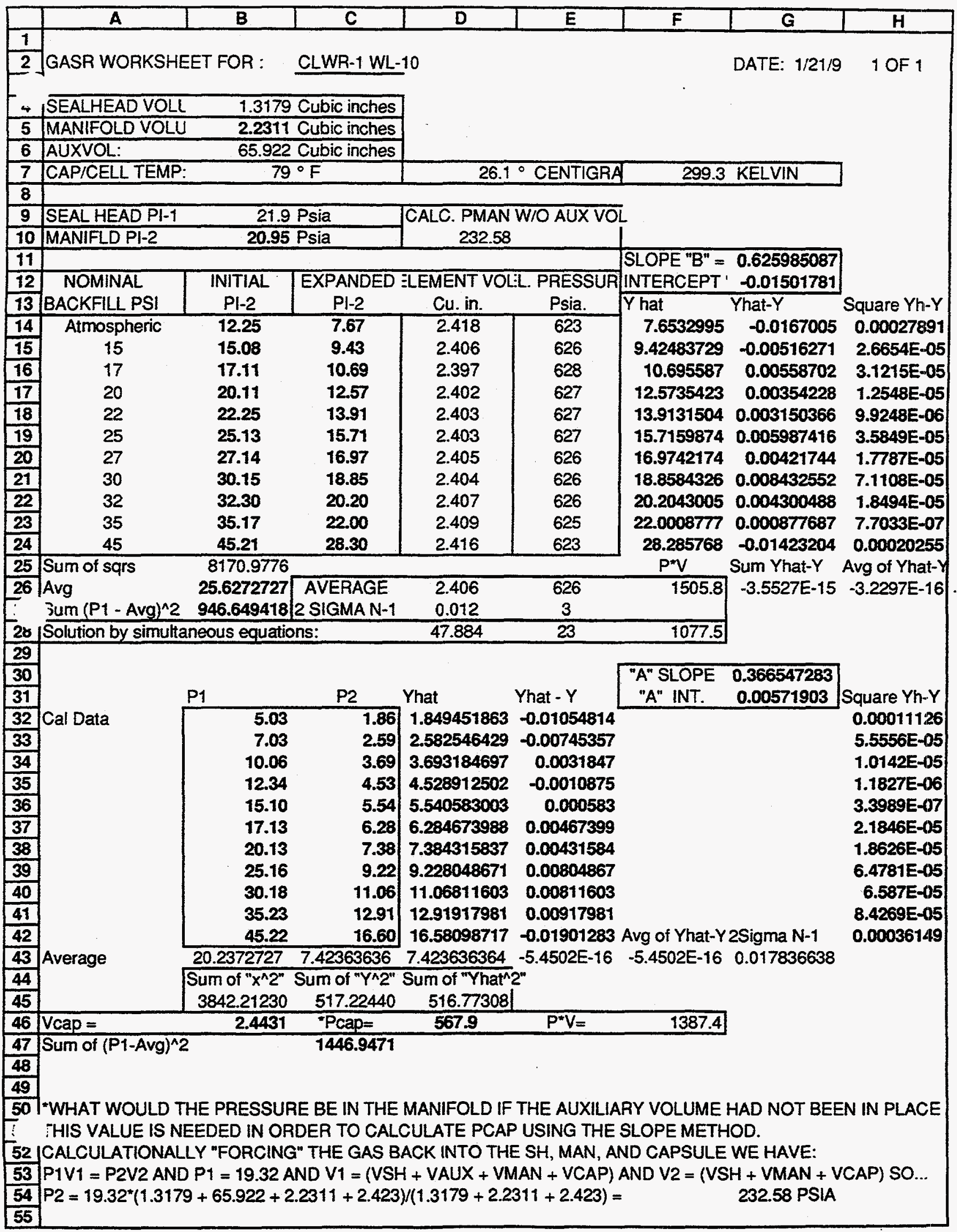




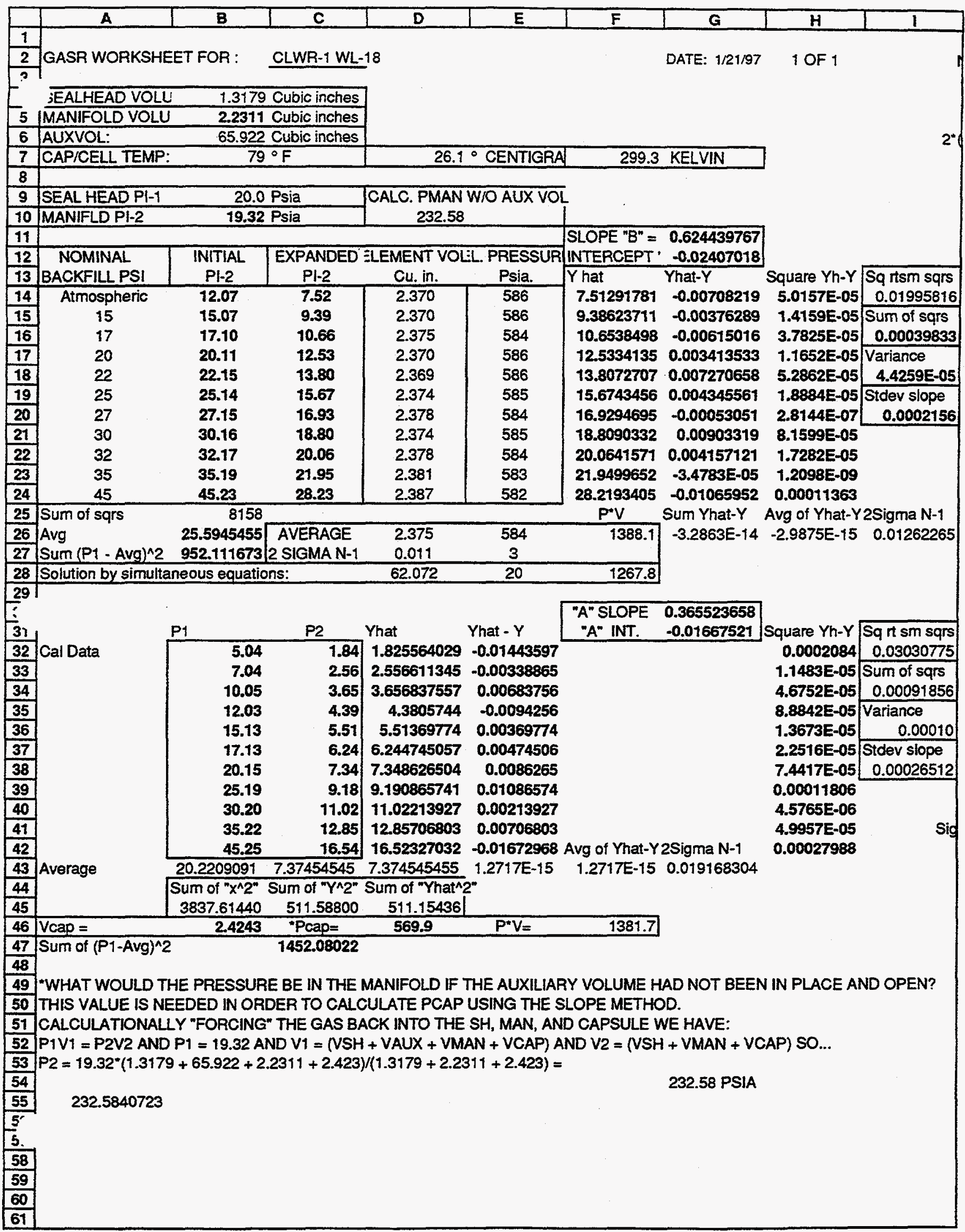




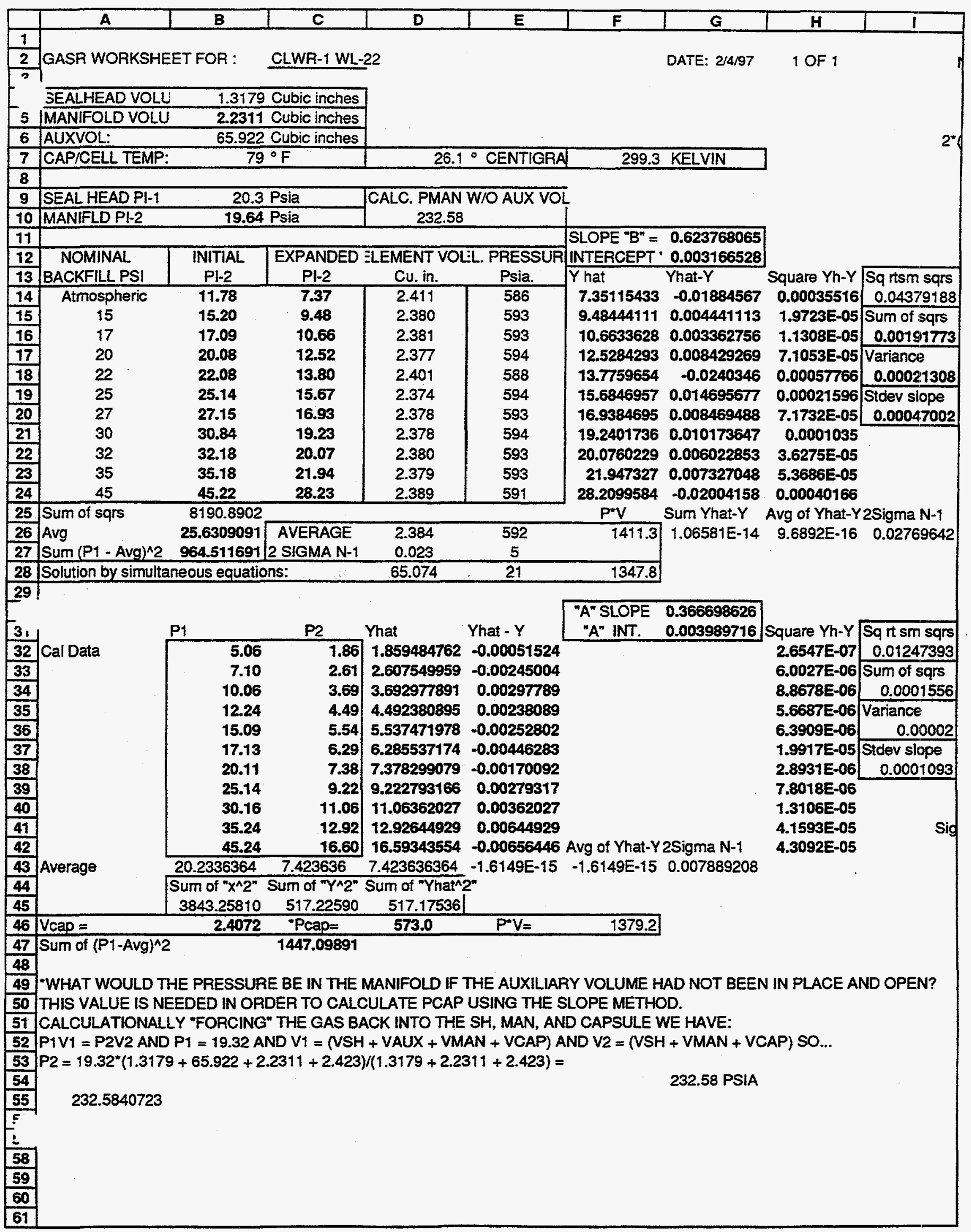




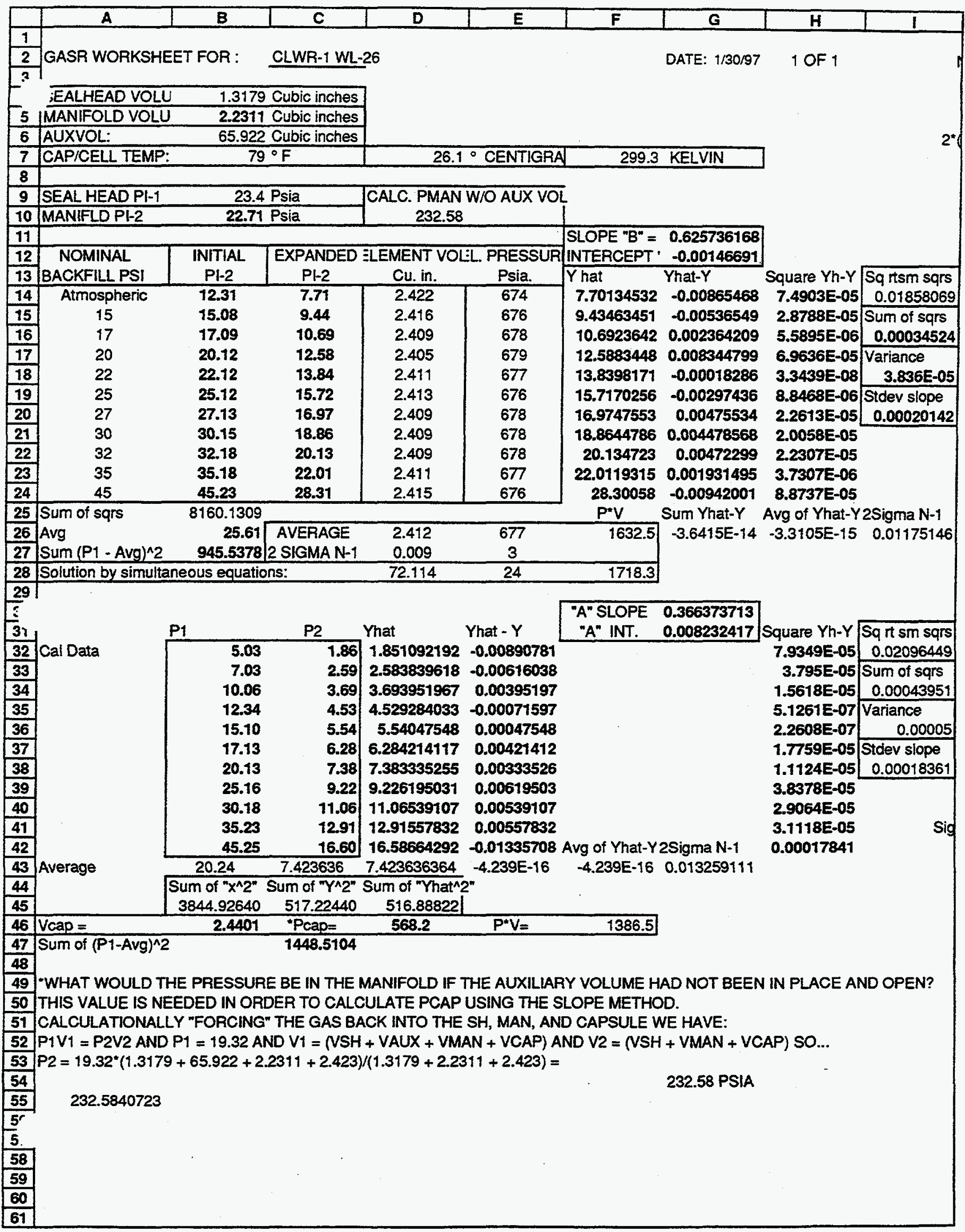




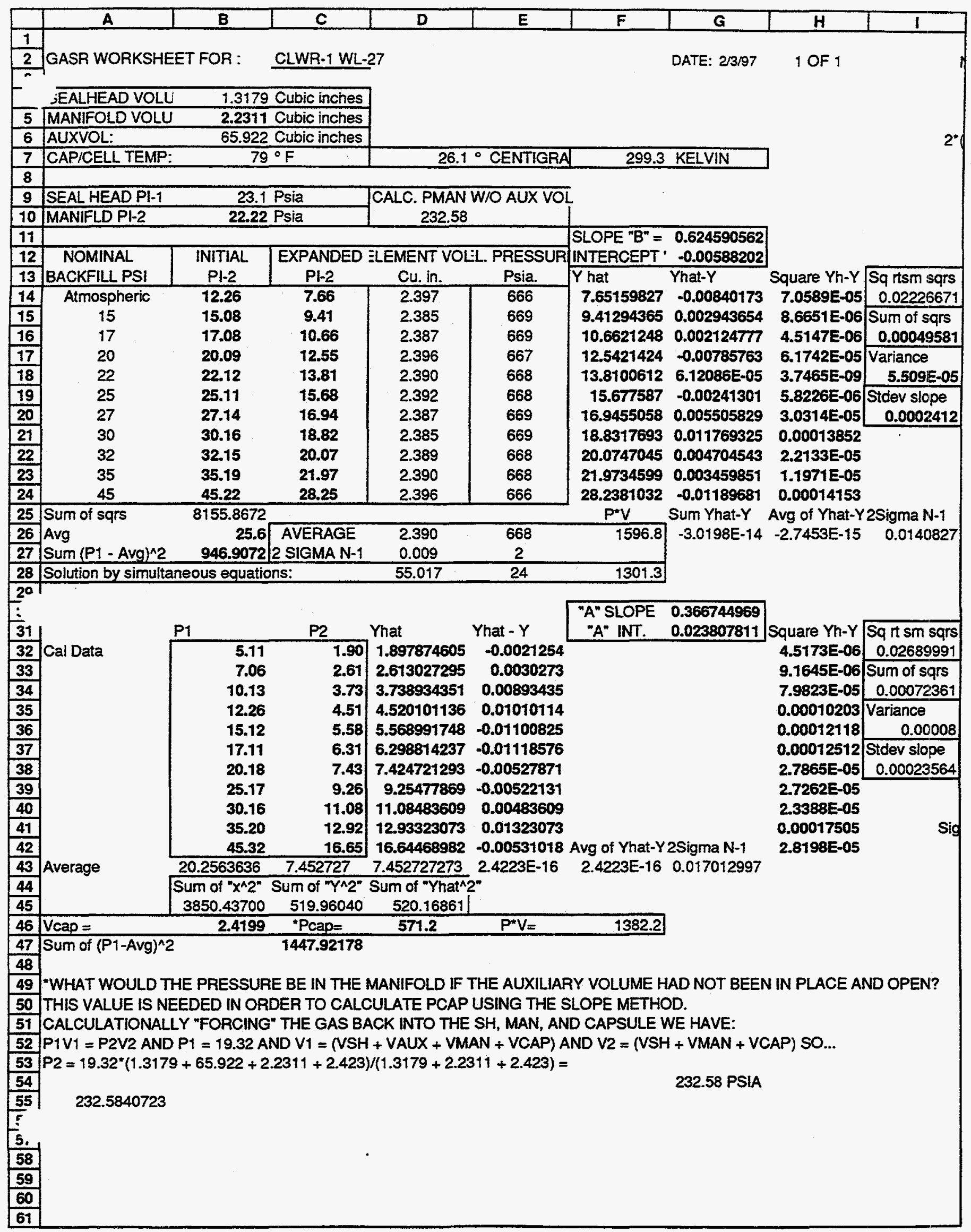




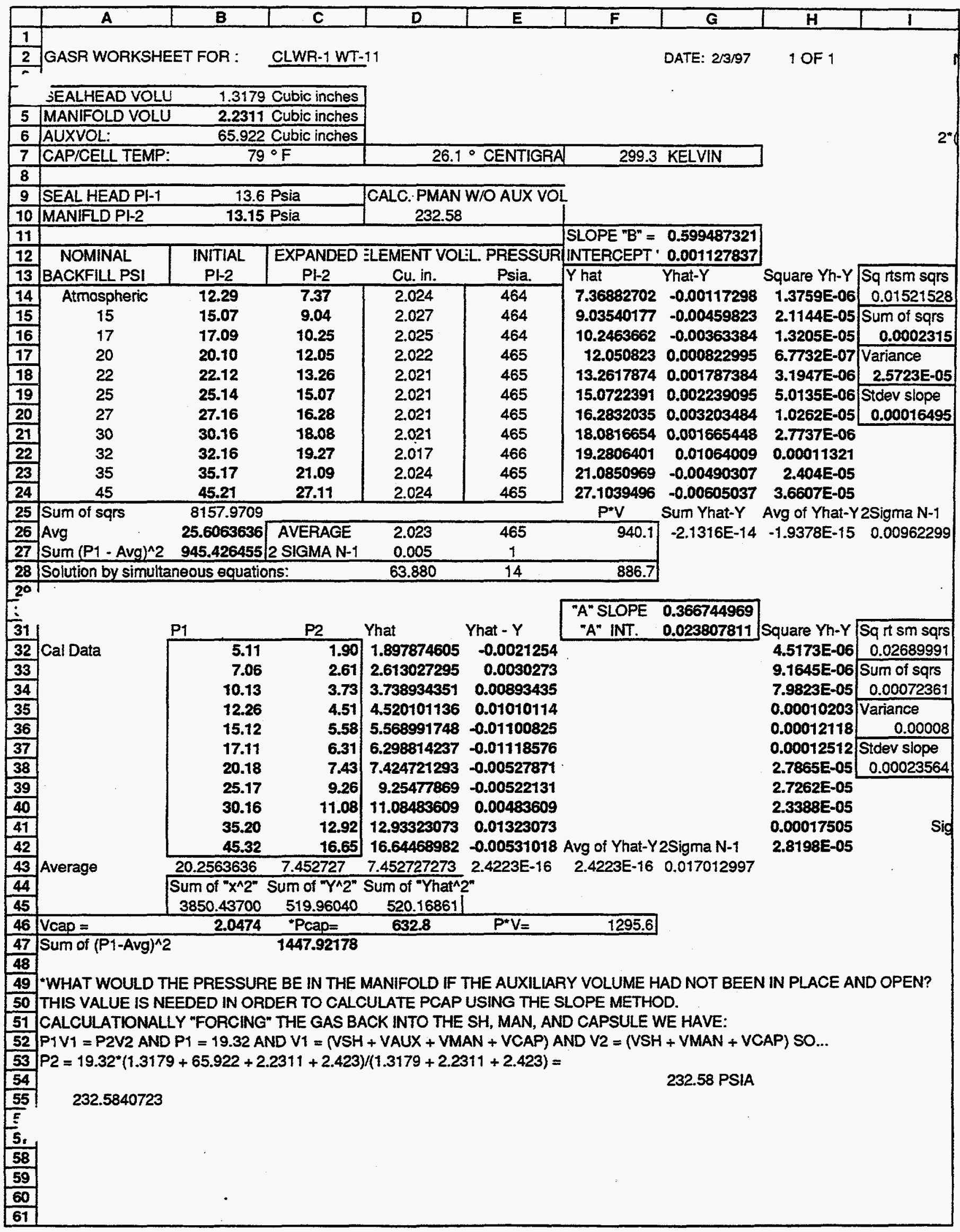




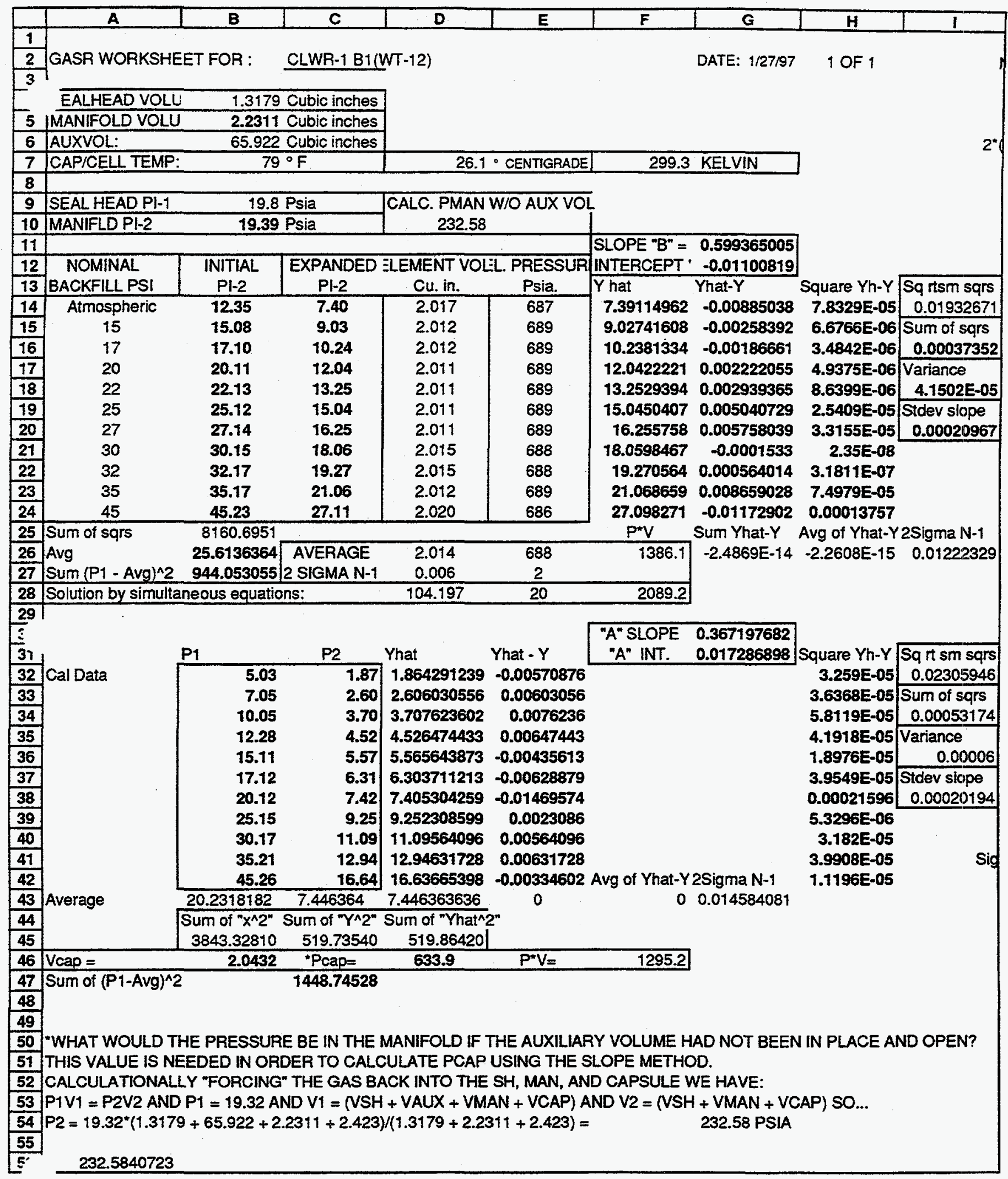




\section{ARGONNE NATIONAL LABORATORY \\ P. O. Box 2528, Idaho Falls, Idaho $83403-2528$}

\section{ENGINEERING DIVISION}

(TREAT, ZPPR, and HFEF)

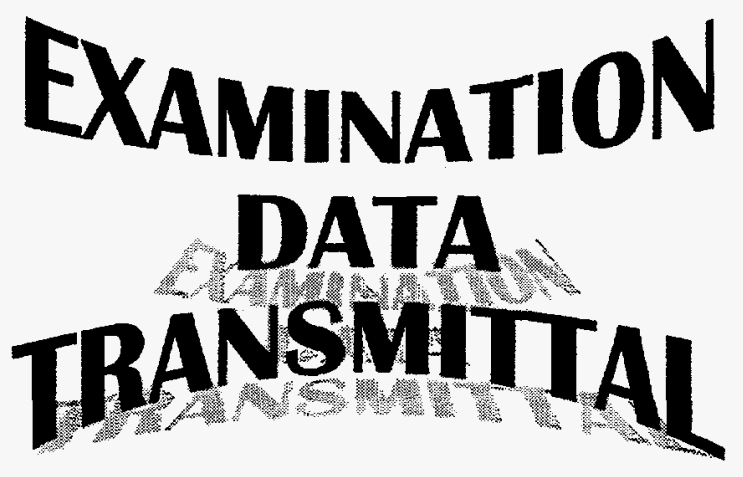

\begin{tabular}{|l|l|}
\hline TO: & $\begin{array}{l}\text { ADDRESS: } \\
\text { R. L. Bratton }\end{array}$ \\
& Lockheed Martin Idaho Technologies, Co. \\
& EROB MS-3760 \\
& Idaho Falls, ID 834 \\
& \\
\hline
\end{tabular}

DATE:

March 11, 1997

MATERIAL EXAMINED:

Eight Target Rods from CLWR Loop-1 Bundle

TYPE OF EXAMINATION:

Gas Analysis, Sample, and Recharge (GASR)

FORM OF DATA:

Summary Report and User Notes

NOTES:

CC: w/Attachments

Loop-1 file

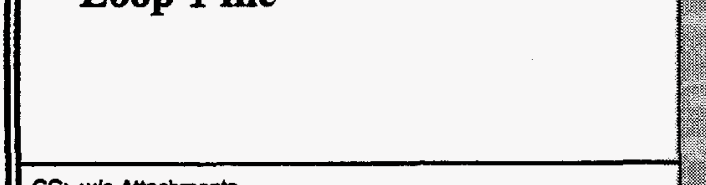

CC: w/o Attachments

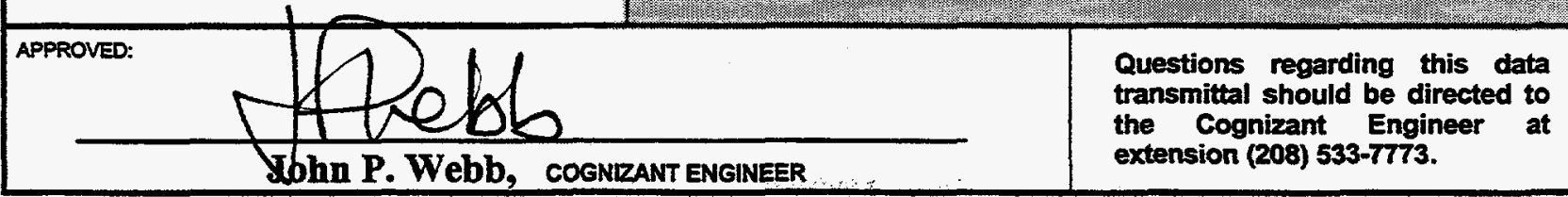

Revised: 2/11/97 


\section{ARGONNE NATIONAL LABORATORY \\ Hot Fuel Examination Facility}

\section{Gas Analysis, Sample, \& Recharge (GASR) System}

\section{USER NOTES}

\section{STANDARD GAS-SAMPLING PROCESS}

1. Normal operating procedures for the GASR System are contained in HFEF Operating Instruction OI-4381.

2. The system is prepared for operation by calibrating and/or checking the calibration of all system instrumentation. If needed, system volumes are augmented by attaching selected chambers of known volume.

3. With the Seal Head (SH) of the GASR system blocked off, a series of back-fills and expansions are performed to establish a pressure ratio (Slope $A$ ) that is used in later check calculations

4. The preferred puncture site on capsules to be punctured is clamped against a saddle-shaped gasket in the Seal Head.

5. The entire volume of the GASR system, including the sample chambers, is evacuated to $<30 \mathrm{mtorr}$.

6. Valves are closed to isolate the Seal Head from the Manifold (MAN) and the sampling chamber(s).

7. The laser is operated to drill a small hole (5-10 mils dia.) in the capsule. Gas from the capsule expands into the Seal Head, where pressure is monitored on a high-range meter $\left(P_{1}\right)$.

8. When pressure in the Seal Head has equilibrated, the valve to the Manifold is opened, and the gas expands into the Manifold, where pressure is monitored on an accurate low-range meter $\left(\mathrm{P}_{2}\right)$, and capsule temperature is monitored on an in-cell thermocouple.

9. The valves to the sample line are opened to allow the gas to expand into the sample chamber(s). Isolation valves integral to the sample chamber(s) are closed to capture the gas sample(s), which are hand-carried to the Analytical Laboratory for analysis of the gas. Sample pressure is monitored on $\mathrm{P}_{2}$, and sample temperature is monitored on a thermometer inside the sampling cabinet.

10. A series of back-fills and expansions are performed to accurately determine the capsule void volume (and establish Slope B). Helium is used to back-fill the capsule to selected target pressures (in the range 5-45 psia, as monitored on $\mathrm{P}_{2}$ ). The Seal Head is isolated, and the Manifold is re-evacuated to $<30$ mtorr. The helium in the Capsule/Seal Head is then expanded into the manifold where the pressure is monitored on $P_{2}$.

11. If desired, the capsule may be back-filled with any gas of choice and the puncture site plugged by de-focusing the laser beam to create a "puddle" weld. No assurance of leak-tightmess is available.

\section{STANDARD CALCULATION}

- Using the Ideal Gas Law, the capsule void volume $\left(V_{\text {cap }}\right)$ is calculated from the backfill/expansion data acquired in step 10 above. A statistical estimate of error is derived from the replicated readings.

- Again using the Ideal Gas Law, the capsule initial pressure $\left(P_{\text {cap }}\right)$ is calculated from $V_{c a p}$ and data acquired in step 8 above.

\section{CHECK CALCULATIONS}

- Using the Ideal Gas Law, $V_{\text {cap }}$ and $P_{\text {eap }}$ can be calculated directly from the data acquired in steps 7 and 8 above. This quality of this result is poor because of inaccuracy in the $P_{1}$ observation.

- Using Slope $A$ and Slope $B, V_{\text {eap }}$ and $P_{\text {cap }}$ can be calculated without apriori knowledge of the volumes of the G.ASR system. While potentially better than the standard calculation, this method has not been validated, nor has it received peer review.

\section{SPECLAL PROCEDURES}

- Special procedures may sometimes be employed that deviate from the standard methods described above. Such procedures are described in the appropriate HFEF work-control documentation, such as Non-Routine Procedures (NRP's) or Process Work Sheets (PWS's). 


\section{ARGONNE NATIONAL LABORATORY Hot Fuel Examination Facility}

\section{Gas Analysis, Sample, and Recharge (GASR) System \\ SUMMARY REPORT}

S/A or TEST: Loop-1

3/11/97

\begin{tabular}{|c|c|c|c|c|c|c|c|c|}
\hline $\begin{array}{c}\text { ROD } \\
\text { ID }\end{array}$ & \multicolumn{2}{|c|}{ VOLUME* $^{*}$} & \multicolumn{2}{c|}{ PRESSURE $^{*}$} & TEMPERATURE & $\begin{array}{c}\text { REWELD } \\
\text { STATUS }\end{array}$ & $\begin{array}{c}\text { SAMPLE } \\
\text { DATE }\end{array}$ & REMARKS \\
\hline WL-01 & 2.442 & 0.009 & 570 & 2 & 78 & fail & $2 / 5 / 97$ & placed in Swagelok sealed tube \\
\hline WL-03 & 2.380 & 0.008 & 557 & 2 & 79 & fail & $2 / 5 / 97$ & placed in Swagelok sealed tube \\
\hline WL-09 & 2.399 & 0.015 & 596 & 4 & 79 & fail & $2 / 4 / 97$ & placed in Swagelok sealed tube \\
\hline WL-10 & 2.406 & 0.012 & 626 & 3 & 79 & fail & $1 / 30 / 97$ & placed in Swagelok sealed tube \\
\hline WL-18 & 2.375 & 0.011 & 584 & 3 & 79 & pinhole & $1 / 21 / 97$ & placed in Swagelok sealed tube \\
\hline WL-22 & 2.382 & 0.020 & 592 & 5 & 80 & fail & $2 / 4 / 97$ & placed in Swagelok sealed tube \\
\hline WL-26 & 2.412 & 0.009 & 677 & 3 & 78 & fail & $1 / 30 / 97$ & placed in Swagelok sealed tube \\
\hline WL-27 & 2.390 & 0.009 & 668 & 2 & 79 & fail & $2 / 3 / 97$ & placed in Swagelok sealed tube \\
\hline
\end{tabular}

" Determined by the backfillexpansion method.

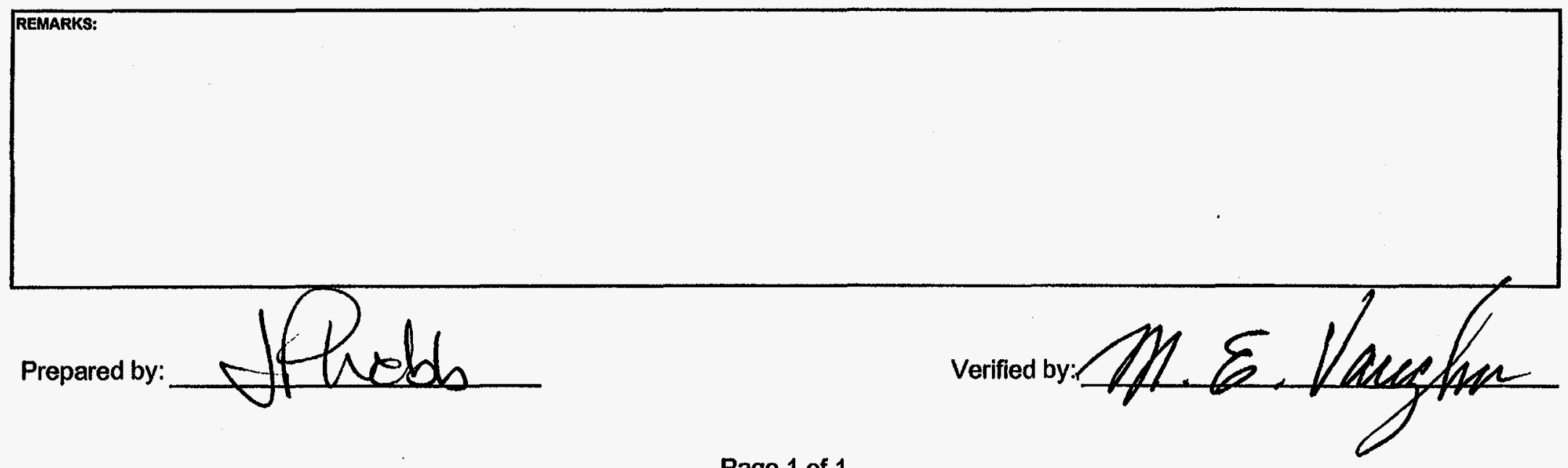




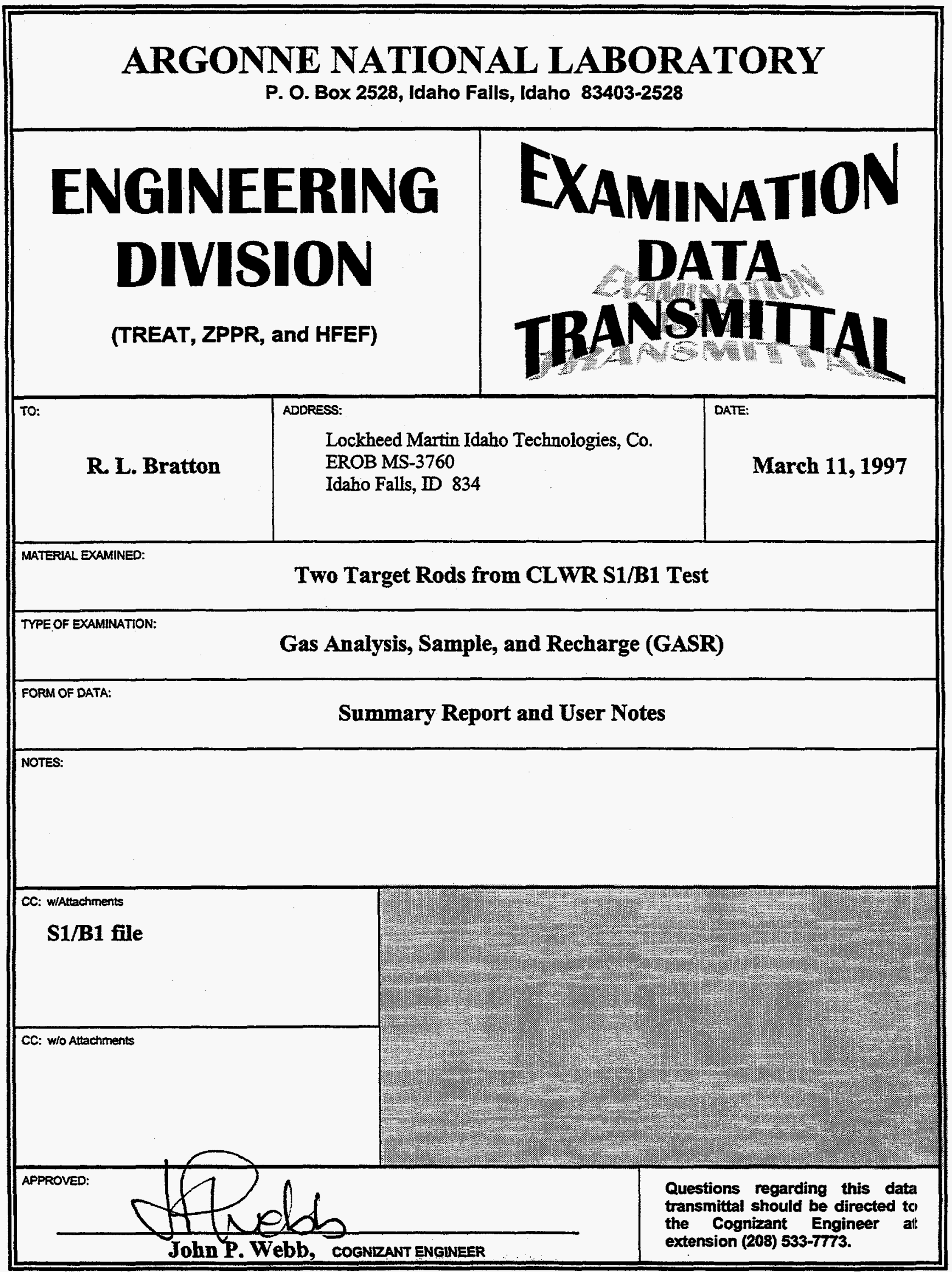

Revised: 2/11/97 


\section{ARGONNE NATIONAL LABORATORY}

Hot Fuel Examination Facility

\section{Gas Analysis, Sample, and Recharge (GASR) System SUMMARY REPORT}

S/A or TEST: S1/B1

3/11/97

\begin{tabular}{|c|c|c|c|c|c|c|c|c|}
\hline \multirow{2}{*}{$\begin{array}{c}\text { ROD } \\
\text { ID }\end{array}$} & \multicolumn{2}{|c|}{ VOLUME* } & \multicolumn{2}{|c|}{ PRESSURE* $^{*}$} & \multirow{2}{*}{$\begin{array}{l}\text { TEMPERATURE } \\
\text { (' } \mathrm{F})\end{array}$} & \multirow{2}{*}{$\begin{array}{l}\text { REWELD } \\
\text { STATUS }\end{array}$} & \multirow{2}{*}{$\begin{array}{l}\text { SAMPLE } \\
\text { DATE }\end{array}$} & \multirow[t]{2}{*}{ REMARKS } \\
\hline & $\left(\ln ^{3}\right)$ & 2Sigma & (psia) & 2 Sigma & & & & \\
\hline WT-11 & 2.032 & 0.005 & 465 & 1 & 81 & fail & $2 / 3 / 97$ & placed in Swagelok sealed tube \\
\hline WT-12 & 2.014 & 0.006 & 688 & 2 & 81 & pinhole & $1 / 27 / 97$ & placed in Swagelok sealed tube \\
\hline & & & & & & & & \\
\hline & & & & & & & & \\
\hline & & & & & & & & \\
\hline & & & & & & & & \\
\hline & & & & & & & & \\
\hline & & & & & & & & \\
\hline
\end{tabular}

Determined by the backilltexpansion melhod.
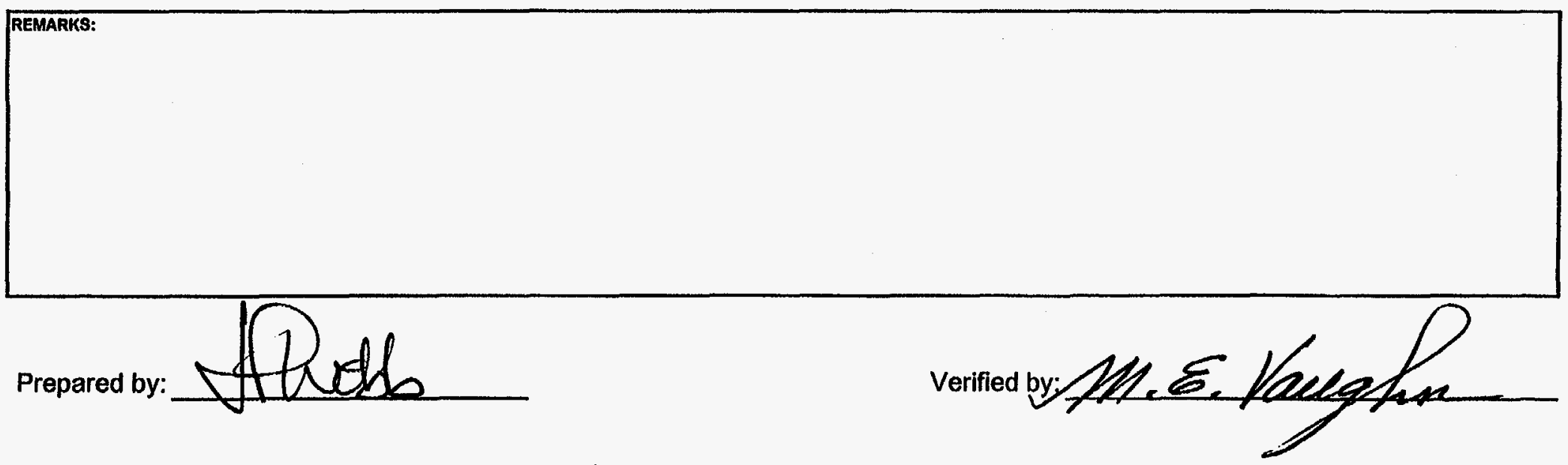


\section{GASR System Volumes}

\begin{tabular}{ll}
\multicolumn{1}{c}{ Component } & Volumes (in. ${ }^{3}$ ) \\
\hline Manifold & 2.2311 \\
Driver seal head & 1.3179 \\
Auxiliary volume 1 and piping & 65.922 \\
\hline
\end{tabular}


This analysis is of the gas puncture for the dummy and 10 CLWR rods. The rods were punctured in Argonne National Laboratory-West Hot Fuel Examination Facility during the time period of January 21 through February 5, 1997.

Varibles used to describe the GASR system are the seal head volume $\left(N_{\text {seal }}\right)$, the manifold volume $\left(N_{m a n}\right)$, and the auxilary volume $\left(N_{a u x}\right)$.

$$
\mathrm{V}_{\text {seal }}:=1.3179 \cdot \mathrm{in}^{3} \quad \mathrm{~V}_{\text {aux }}:=65.922 \cdot \mathrm{in}^{3} \quad \mathrm{~V}_{\operatorname{man}}:=2.2311 \cdot \mathrm{in}^{3}
$$

The dummy rod was constructed from a critical assembly rod used to mock up the Loop-1 experiment. A valve was weled to the upper end of the rod and high purity helium was used to pressurized the rod to a pressure known only to LMITCO. This was a blind test for Argonne to benchmark the gas puncture system and analysis at Argonne-West Below is a calculation of the void volume and pressure for the dummy rod using data from the puncture

$$
\mathrm{P}_{\mathrm{di}}:=\left[\begin{array}{c}
12.33 \\
15.01 \\
17.08 \\
20.19 \\
22.17 \\
25.19 \\
27.20 \\
30.28 \\
32.28 \\
35.28 \\
45.23
\end{array}\right] \quad \mathrm{P}_{\mathrm{de}}:=\left[\begin{array}{c}
7.93 \\
9.71 \\
10.99 \\
12.99 \\
14.27 \\
16.21 \\
17.51 \\
19.49 \\
20.78 \\
22.72 \\
29.14
\end{array}\right]
$$

$$
\begin{gathered}
\text { Dummy Rod Data } \\
\text { Void }:=\frac{\frac{P_{\text {man }}:=18.75 \cdot \frac{\mathrm{lb}}{\text { in }^{2}}}{\left(\mathrm{P}_{\mathrm{de}}-\mathrm{P}_{\mathrm{di}}\right) \cdot \mathrm{V}_{\text {seal }}+\mathrm{P}_{\mathrm{de}} \cdot \mathrm{V}_{\text {man }}}}{\left(\mathrm{P}_{\mathrm{di}}-\mathrm{P}_{\mathrm{de}}\right)} \\
\text { Pressure }:=\frac{\frac{\mathrm{P}_{\text {man }} \cdot\left(\mathrm{Void}+\mathrm{V}_{\text {seal }}+\mathrm{V}_{\text {man }}+\mathrm{V}_{\text {aux }}\right)}{\text { Void }}}{}
\end{gathered}
$$

$$
\text { Void }=\frac{2.703}{2.77}
$$

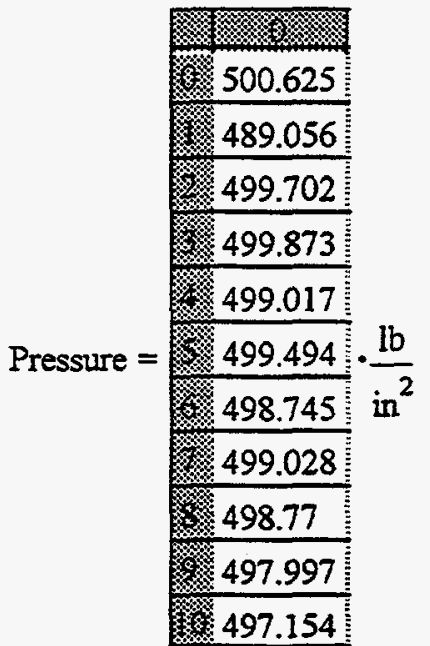

Mean of the dummy's void volume is

$$
\text { Void_Mean }:=\operatorname{mean}(\text { Void }) \text { Void_Mean }=2.717 \cdot \mathrm{in}^{3}
$$

Standard Deviation of the void volume is

$$
\text { Void_Std }:=\operatorname{stdev}\left(\text { Void) } \quad \text { Void_Std }=0.017 \cdot \mathrm{in}^{3}\right.
$$

Mean of the dummy's capsule pressure is Pressure_Mean := mean(PressurePressure_Mean $=498.133 \cdot \frac{1 \mathrm{~b}}{\mathrm{in}^{2}}$

Standard Deviation of the dummy's pressure is Pressure_Std $:=\operatorname{stdev}$ (Pressure) Pressure_Std $=3.004 \cdot \frac{\mathrm{lb}}{\mathrm{in}^{2}}$ 
WL-18 Loop Rod

This rod was punctured with V8 open.

$P_{\text {int }}:=\left[\begin{array}{c}12.07 \\ 15.07 \\ 17.10 \\ 20.11 \\ 22.15 \\ 25.14 \\ 27.15 \\ 30.16 \\ 32.17 \\ 35.19 \\ 45.23\end{array}\right] \quad P_{\text {exp }}:=\left[\begin{array}{c}7.52 \\ 9.39 \\ 10.66 \\ 12.53 \\ 13.80 \\ 15.67 \\ 16.93 \\ 18.80 \\ 20.06 \\ 21.95 \\ 28.23\end{array}\right]$

$$
\begin{aligned}
& \text { WL-18 Manifold Pressure } \\
& \mathrm{P}_{\operatorname{man}}:=19.32 \cdot \frac{\mathrm{b} b}{\mathrm{in}^{2}}
\end{aligned}
$$

Void $:=\frac{\overline{\left(P_{\exp }-P_{\text {int }}\right) \cdot V_{\text {seal }}+P_{\text {exp }} \cdot V_{\text {man }}}}{\left(P_{\text {int }}-P_{\text {exp }}\right)}$

Pressure $:=\frac{\overrightarrow{P_{\text {man }}\left(V_{\text {oid }}+V_{\text {seal }}+V_{\text {man }}+V_{\text {aux }}\right)}}{\text { Void }}$

$$
\text { Void }=\frac{2.37}{2.37} \frac{2.375}{2.37}
$$

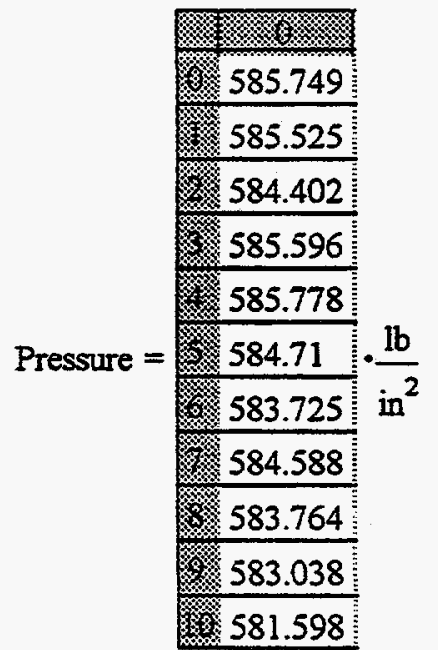

Mean of the dummy's void volume is

Void_Mean $:=\operatorname{mean}\left(\right.$ Void) Void_Mean $=2.375 \cdot$ in $^{3}$

Standard Deviation of the void volume is

Void_Std $:=\operatorname{stdev}($ Void $) \quad$ Void_Std $=5.251 \cdot 10^{-3} \cdot$ in $^{3}$

Mean of the dummy's capsule pressure is Pressure_Mean := mean (PressurePressure_Mean $=584.407 \cdot \frac{\text { Ib }}{\text { in }^{2}}$

Standard Deviation of the dummy's pressure is Pressure_Std $:=\operatorname{stdev}\left(\right.$ Pressure) Pressure_Std $=1.247 \cdot \frac{\mathrm{lb}}{\text { in }^{2}}$ 


\section{B-1 Rod (WT-12)}

This rod was punctured with V8 closed. This requires different equations to be used to calculate the capsule pressure.

$\mathrm{P}_{\text {int }}:=\left[\begin{array}{c}12.35 \\ 15.08 \\ 17.10 \\ 20.11 \\ 22.13 \\ 25.12 \\ 27.14 \\ 30.15 \\ 32.17 \\ 35.17 \\ 45.23\end{array}\right] \quad \mathrm{P}_{\exp }:=\left[\begin{array}{c}7.40 \\ 9.03 \\ 10.24 \\ 12.04 \\ 13.25 \\ 15.04 \\ 16.25 \\ 18.06 \\ 19.27 \\ 21.06 \\ 27.11\end{array}\right]$

$$
\begin{aligned}
& \text { WT-12 Manifold Pressure } \\
& \text { Void }:=\frac{\frac{\mathrm{P}_{\text {man }}:=11.99 \cdot \frac{\mathrm{lb}}{\text { in }^{2}}}{\left(\mathrm{P}_{\exp }-\mathrm{P}_{\text {int }}\right) \cdot \mathrm{V}_{\text {seal }}+\mathrm{P}_{\text {exp }} \cdot \mathrm{V}_{\text {man }}}}{\left(\mathrm{P}_{\text {int }}-\mathrm{P}_{\text {exp }}\right)} \\
& \text { Pressure }:=\frac{\frac{\mathrm{P}_{\text {man }}\left(\mathrm{V}_{\text {oid }}+\mathrm{V}_{\text {seal }}+\mathrm{V}_{\text {aux }}\right) \cdot\left(\mathrm{V}_{\text {oid }}+\mathrm{V}_{\text {seal }}+\mathrm{V}_{\text {man }}\right)}{V_{\text {oid }} \cdot\left(\mathrm{Void}_{\mathrm{d}}+\mathrm{V}_{\text {seal }}\right)}}{}
\end{aligned}
$$

$$
\text { Void }=\frac{2.017}{2.012}
$$

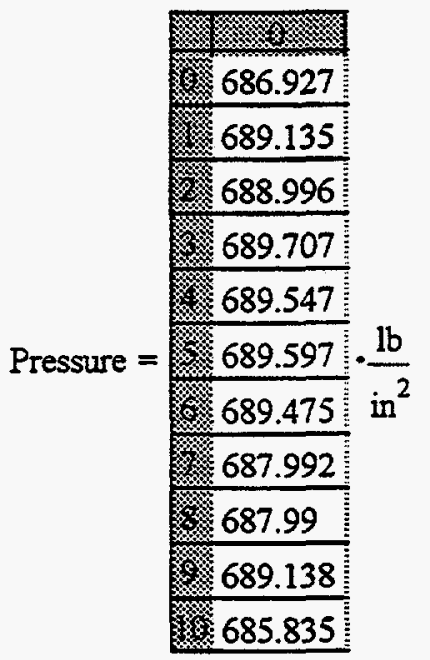

Mean of the dummy's void volume is

Void_Mean $:=\operatorname{mean}\left(\right.$ Void) Void_Mean $=2.014 \cdot$ in $^{3}$

Standard Deviation of the void volume is

$$
\text { Void_Std }:=\operatorname{stdev}(\text { Void }) \quad \text { Void_Std }=2.893 \cdot 10^{-3} \cdot \text { in }^{3}
$$

Mean of the dummy's capsule pressure is Pressure_Mean $:=$ mean (Pressure Pressure_Mean $=688.576 \cdot \frac{\mathrm{lb}}{\mathrm{in}^{2}}$

Standard Deviation of the dummy's pressure is Pressure_Std := stdev(Pressure) Pressure_Std $=1.198 \cdot \frac{\mathrm{lb}}{\mathrm{in}^{2}}$ 
WL-10 Loop Rod

This rod was punctured with V8 open.

$$
P_{\text {int }}:=\left[\begin{array}{c}
12.25 \\
15.08 \\
17.11 \\
20.11 \\
22.25 \\
25.13 \\
27.14 \\
30.15 \\
32.30 \\
35.17 \\
45.21
\end{array}\right] \quad P_{\exp }:=\left[\begin{array}{c}
7.67 \\
9.43 \\
10.69 \\
12.57 \\
13.91 \\
15.71 \\
16.97 \\
18.85 \\
20.20 \\
22.00 \\
28.30
\end{array}\right]
$$

WL-10 Manifold Pressure

$$
P_{\operatorname{man}}:=20.95 \cdot \frac{\mathrm{lb}}{\mathrm{in}^{2}}
$$

Void $:=\frac{\overline{\left(P_{\text {exp }}-P_{\text {int }}\right) \cdot V_{\text {seal }}+P_{\text {exp }} \cdot V_{\text {man }}}}{\left(P_{\text {int }}-P_{\text {exp }}\right)}$

$$
\text { Pressure }:=\frac{\overrightarrow{P_{\text {man }} \cdot\left(V_{\text {oid }}+V_{\text {seal }}+V_{\text {man }}+V_{\text {aux }}\right)}}{\text { Void }}
$$

$$
\text { Void }=\frac{2.418}{2.406}
$$

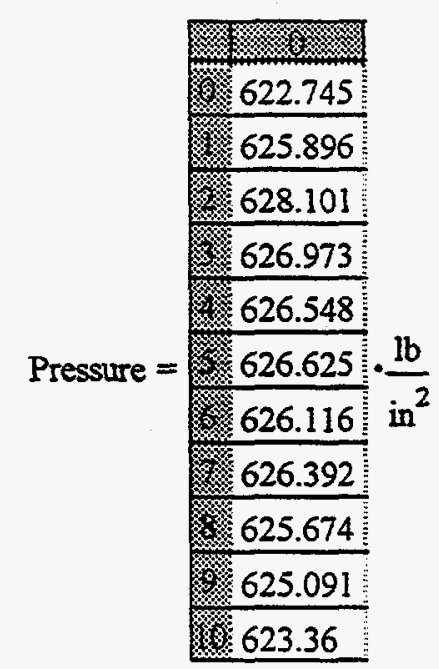

Mean of the dummy's void volume is

Standard Deviation of the void volume is
Void_Mean $:=\operatorname{mean}$ (Void) Void_Mean $=2.406 \cdot$ in $^{3}$

Void_Std $:=\operatorname{stdev}\left(\right.$ Void) Void_Std $=5.909 \cdot 10^{-3} \cdot \mathrm{in}^{3}$

Mean of the dummy's capsule pressure is Pressure_Mean := mean(Pressure Pressure_Mean $=625.775 \cdot \frac{\mathrm{lb}}{\mathrm{in}^{2}}$

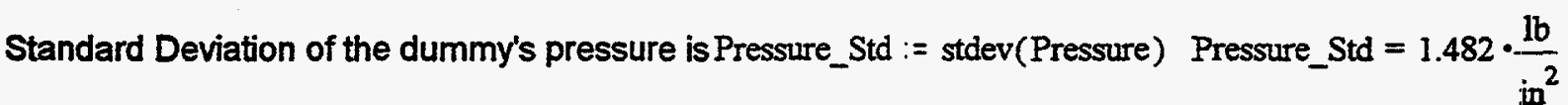


WL-26 Loop Rod

This rod was punctured with V8 open.

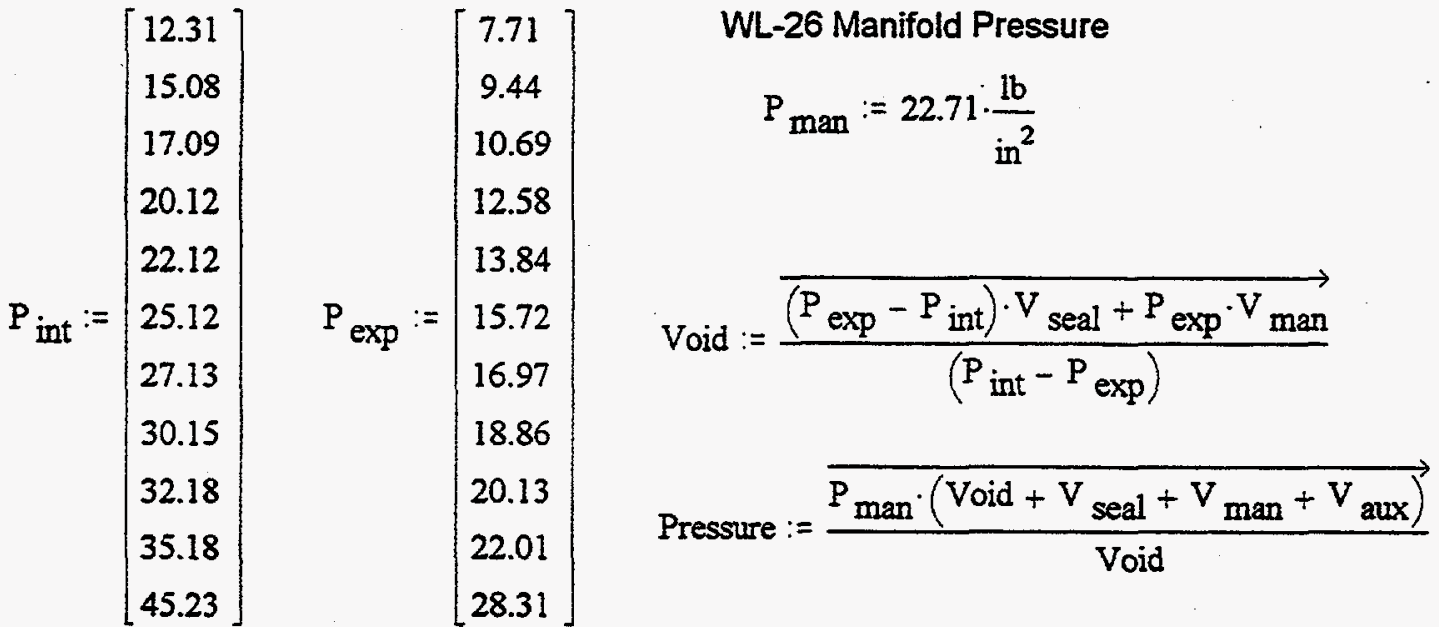

$$
\begin{aligned}
& \text { Void }=\frac{2.422}{2.413}
\end{aligned}
$$

Mean of the dummy's void volume is

Void_Mean $:=\operatorname{mean}($ Void $)$ Void_Mean $=2.412 \cdot$ in $^{3}$

Standard Deviation of the void volume is

Void_Std $:=\operatorname{stdev}($ Void $) \quad$ Void_Std $=4.446 \cdot 10^{-3} \cdot$ in $^{3}$

Mean of the dummy's capsule pressure is Pressure_Mean $:=$ mean (PressurePressure_Mean $=676.888 \cdot \frac{1 \mathrm{~b}}{\text { in }^{2}}$

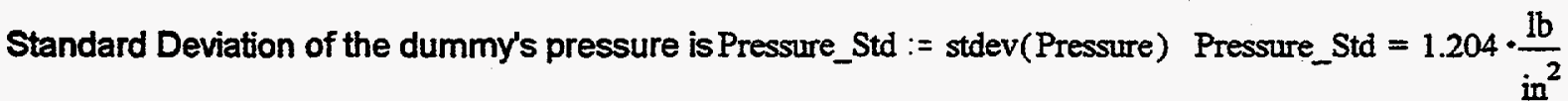


WT-11 (S1) Loop Rod

This rod was punctured with V8 open.

$\mathrm{P}_{\text {int }}:=\left[\begin{array}{c}12.29 \\ 15.07 \\ 17.09 \\ 20.10 \\ 22.12 \\ 25.14 \\ 27.16 \\ 30.16 \\ 32.16 \\ 35.18 \\ 45.21\end{array}\right] \quad \mathrm{P}_{\exp }:=\left[\begin{array}{c}7.37 \\ 9.04 \\ 10.25 \\ 12.05 \\ 13.26 \\ 15.07 \\ 16.28 \\ 18.08 \\ 19.27 \\ 21.09 \\ 27.11\end{array}\right] \quad$ Void $:=\frac{\mathrm{WT}-11(\mathrm{P}-1) \text { Manifold Pressure }}{\left(\mathrm{P}_{\operatorname{man}}:=13.15 \cdot \frac{\mathrm{b}}{\mathrm{in}^{2}}\right.}$

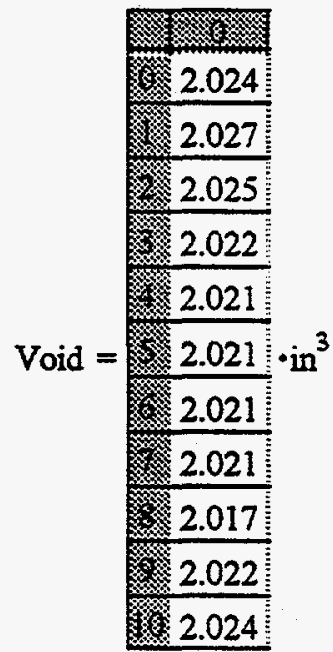

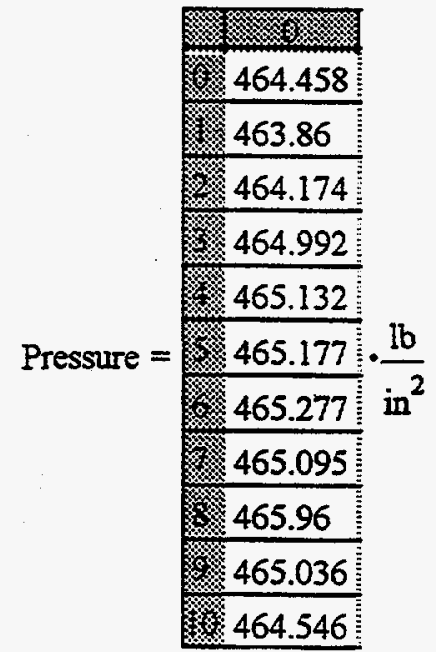

Mean of the dummy's void volume is Void_Mean $:=\operatorname{mean}($ Void $)$ Void_Mean $=2.022 \cdot \mathrm{in}^{3}$

Standard Deviation of the void volume is Void_Std $:=\operatorname{stdev}($ Void $) \quad$ Void_Std $=2.485 \cdot 10^{-3} \cdot$ in $^{3}$ Mean of the dummy's capsule pressure is Pressure_Mean $:=\operatorname{mean}$ (Pressure Pressure_Mean $=464.882 \cdot \frac{1 \mathrm{~b}}{\text { in }^{2}}$

Standard Deviation of the dummy's pressure is Pressure_Std $:=\operatorname{stdev}\left(\right.$ Pressure) Pressure_Std $=0.555 \cdot \frac{\text { lb }}{\text { in }^{2}}$ 


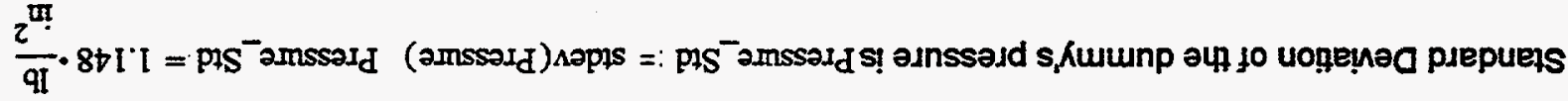

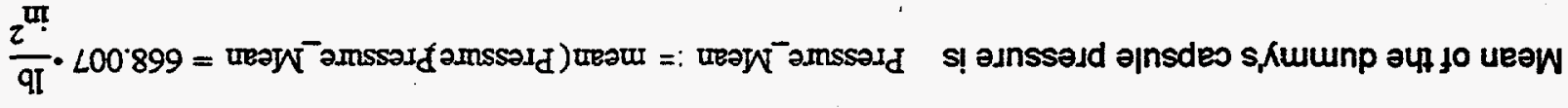

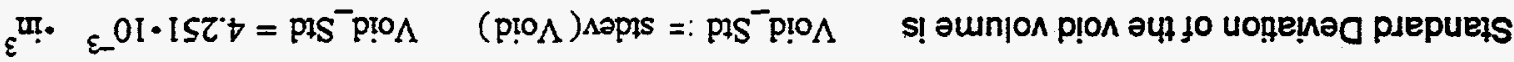

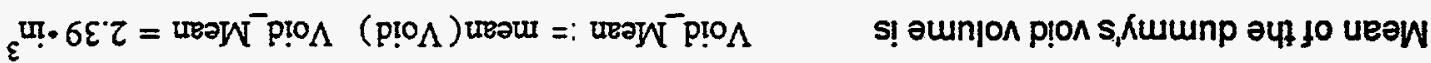

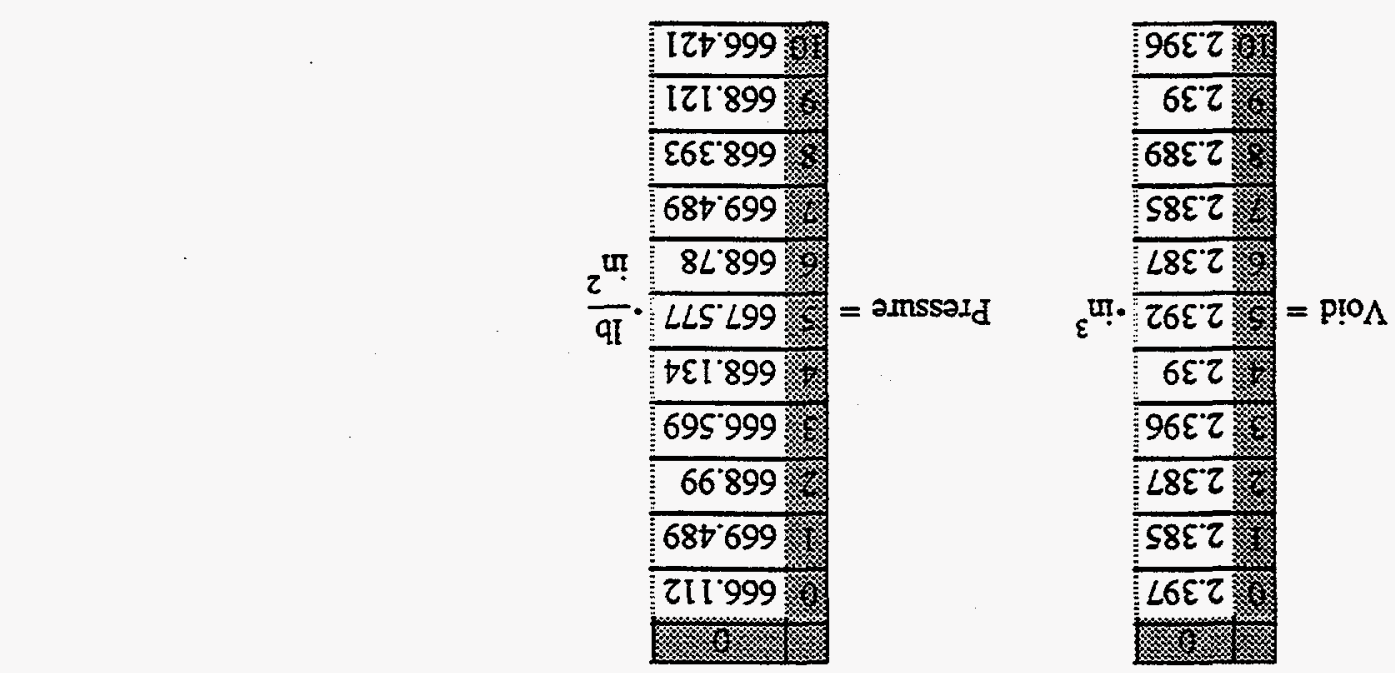

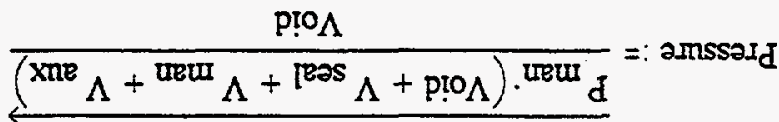

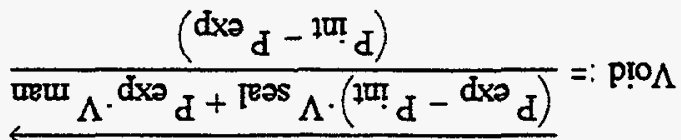

$$
\frac{z^{\mathrm{un}}}{\mathrm{ql}} \cdot \tau \tau \cdot \tau \tau=:{ }^{\mathrm{uru}} \mathrm{d}
$$

annssadd P|O!!UEW LZ-7M

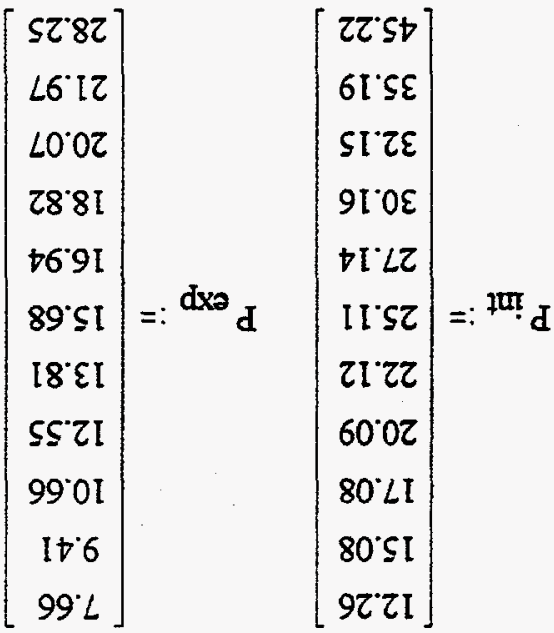

-uədo $8 \wedge$ 4!M pasnound sem pos s!4 $\perp$ 


\section{WL-22 Loop Rod}

This rod was punctured with V8 open.

$$
P_{\text {int }}:=\left[\begin{array}{c}
12.24 \\
15.2 \\
17.09 \\
20.08 \\
22.13 \\
25.14 \\
27.15 \\
30.84 \\
32.18 \\
35.18 \\
45.22
\end{array}\right] \quad P_{\exp }:=\left[\begin{array}{c}
7.64 \\
9.48 \\
10.66 \\
12.52 \\
13.81 \\
15.67 \\
16.93 \\
19.23 \\
20.07 \\
21.94 \\
28.23
\end{array}\right]
$$

WL-22 Manifold Pressure

$$
\mathrm{P}_{\operatorname{man}}:=19.64 \cdot \frac{\mathrm{lb}}{\mathrm{in}^{2}}
$$

Void $:=\frac{\overrightarrow{\left(P_{\text {exp }}-P_{\text {int }}\right) \cdot V_{\text {seal }}+P_{\text {exp }} \cdot V_{\text {man }}}}{\left(P_{\text {int }}-P_{\text {exp }}\right)}$

Pressure $:=\frac{\overrightarrow{P_{\text {man }} \cdot\left(V_{\text {oid }}+V_{\text {seal }}+V_{\text {man }}+V_{\text {aux }}\right)}}{\text { Void }}$

$$
\text { Void }=\frac{2.388}{2.381}
$$

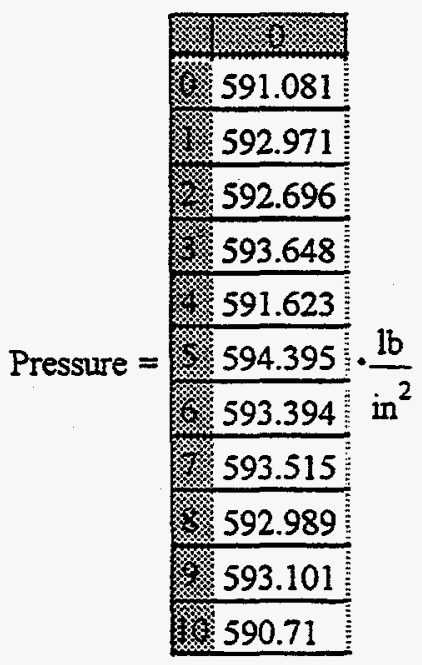

Mean of the dummy's void volume is

Standard Deviation of the void volume is
Void_Mean $:=$ mean (Void) Void_Mean $=2.381 \cdot$ in $^{3}$

Void_Std $:=\operatorname{stdev}($ Void $) \quad$ Void_Std $=4.514 \cdot 10^{-3} \cdot$ in $^{3}$

Pressure_Mean $:=\operatorname{mean}\left(\right.$ Pressure Pressure_Mean $=592.738 \cdot \frac{\mathrm{lb}}{\mathrm{in}^{2}}$

Standard Deviation of the dummy's pressure is Pressure_Std $:=\operatorname{stdev}\left(\right.$ Pressure) Pressure_Std $=1.085 \cdot \frac{\mathrm{lb}}{\text { in }^{2}}$ 
WL-09 Loop Rod

This rod was punctured with V8 open.

$\mathrm{P}_{\text {int }}:=\left[\begin{array}{c}12.29 \\ 15.26 \\ 17.1 \\ 20.1 \\ 22.11 \\ 25.14 \\ 27.13 \\ 30.15 \\ 32.15 \\ 35.16 \\ 45.23\end{array}\right] \quad \mathrm{P}_{\mathrm{exp}}:=\left[\begin{array}{c}7.69 \\ 9.55 \\ 10.69 \\ 12.56 \\ 13.81 \\ 15.7 \\ 16.95 \\ 18.83 \\ 20.08 \\ 21.96 \\ 28.25\end{array}\right]$

WL-09 Manifold Pressure

$$
P_{\text {man }}:=19.89 \cdot \frac{\mathrm{lb}}{\text { in }^{2}}
$$

Void $:=\frac{\overline{\left(P_{\exp }-P_{\text {int }}\right) \cdot V_{\text {seal }}+P_{\text {exp }} \cdot V_{\text {man }}}}{\left(P_{\text {int }}-P_{\text {exp }}\right)}$

Pressure $:=\frac{\overrightarrow{P_{\text {man }} \cdot\left(V_{\text {oid }}+V_{\text {seal }}+V_{\text {man }}+V_{\text {aux }}\right)}}{\text { Void }}$

$$
\text { Void }=\frac{2.312}{2.314}
$$

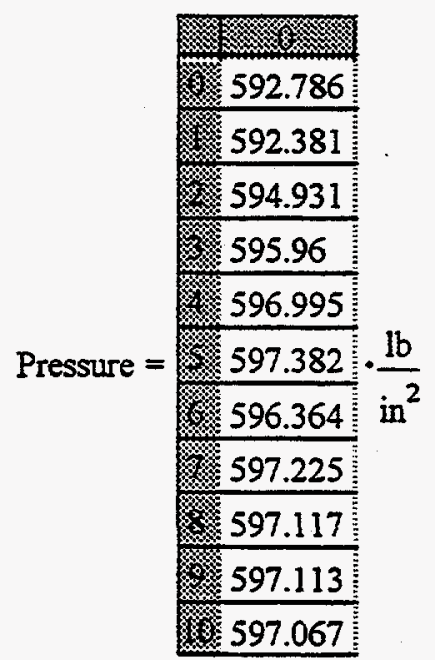

Mean of the dummy's void volume is

Standard Deviation of the void volume is

Mean of the dummy's capsule pressure is
Void_Mean $:=$ mean (Void) Void_Mean $=2.399 \cdot$ in $^{3}$

Void_Std $:=\operatorname{stcev}\left(\right.$ Void) Void_Std $=7.203 \cdot 10^{-3} \cdot$ in $^{3}$

Pressure_Mean $:=$ mean (Pressure Pressure_Mean $=595.938 \cdot \frac{\mathrm{lb}}{\text { in }^{2}}$

Standard Deviation of the dummy's pressure is Pressure_Std $:=\operatorname{stdev}\left(\right.$ Pressure) Pressure_Std $=1.724 \cdot \frac{1 \mathrm{~b}}{\text { in }^{2}}$ 
WL-03 Loop Rod

This rod was punctured with V8 open.

$$
\mathrm{P}_{\text {int }}:=\left[\begin{array}{l}
12.27 \\
15.07 \\
17.08 \\
20.09 \\
22.12 \\
25.13 \\
27.13 \\
30.14 \\
32.17 \\
35.17 \\
45.20
\end{array}\right] \quad \mathrm{P}_{\exp }:=\left[\begin{array}{c}
7.65 \\
9.40 \\
10.65 \\
12.53 \\
13.79 \\
15.67 \\
16.92 \\
18.80 \\
20.07 \\
21.94 \\
28.22
\end{array}\right] \quad \text { Woid }:=\frac{\mathrm{P}_{\text {man }}:=18.45 \cdot \frac{\mathrm{lb}}{\text { in }^{2}}}{\left(\mathrm{P}_{\exp }-\mathrm{P}_{\text {int }}\right) \cdot \mathrm{V}_{\text {seal }}+\mathrm{P}_{\text {exp }} \cdot \mathrm{V}_{\text {man }}}
$$

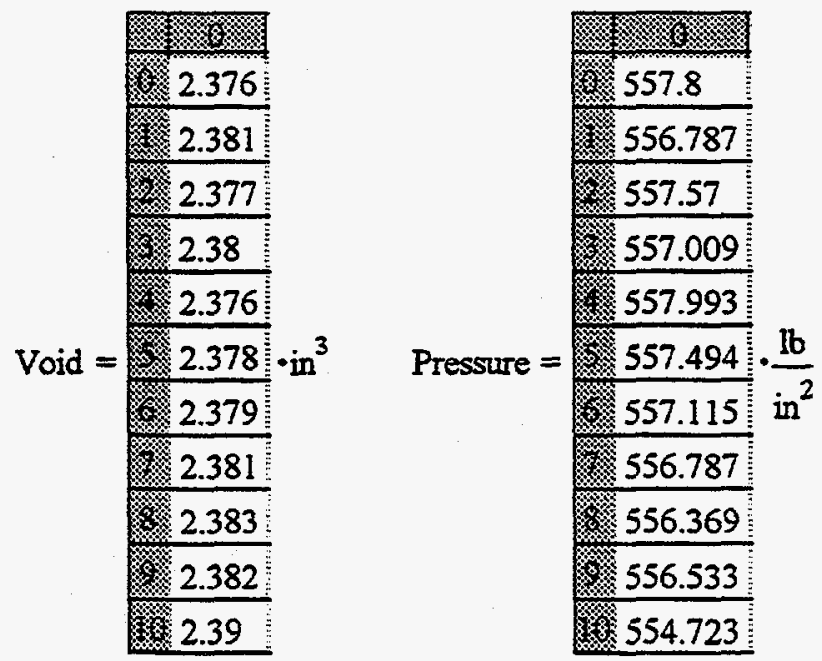

Mean of the dummy's void volume is Standard Deviation of the void volume is
Void_Mean $:=\operatorname{mean}\left(\right.$ Void) Void_Mean $=2.38 \cdot$ in $^{3}$

Void_Std $:=\operatorname{stdev}($ Void $) \quad$ Void_Std $=3.787 \cdot 10^{-3} \cdot$ in $^{3}$

Mean of the dummy's capsule pressure is Pressure_Mean $:=$ mean (PressurePressure_Mean $=556.925 \cdot \frac{1 \mathrm{~b}}{\text { in }^{2}}$

Standard Deviation of the dummy's pressure is Pressure_Std $:=\operatorname{stdev}$ (Pressure) Pressure_Std $=0.855 \cdot \frac{\mathrm{lb}}{\text { in }^{2}}$ 


\section{WL-01 Loop Rod}

This rod was punctured with V8 open.

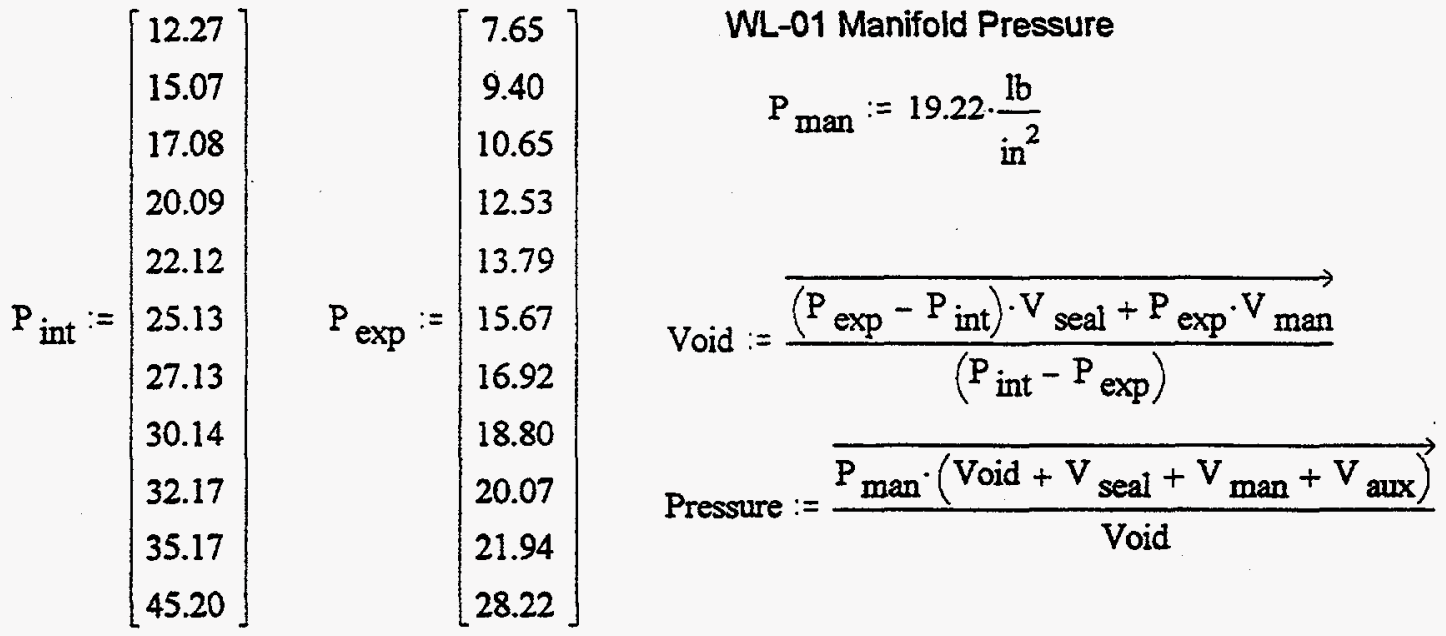

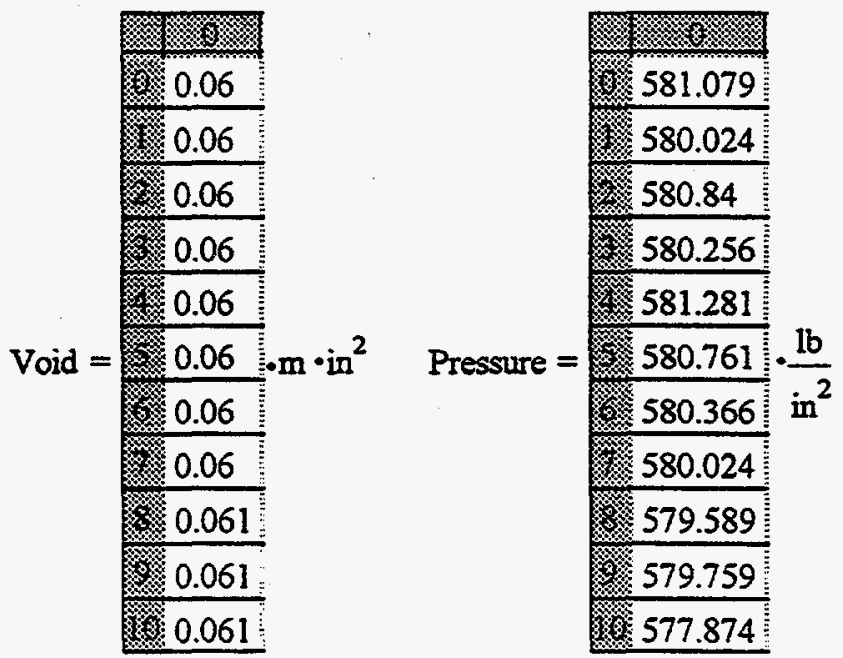

Mean of the dummy's void volume is Void_Mean $:=\operatorname{mean}\left(\right.$ Void) Void_Mean $=2.38 \cdot$ in $^{3}$

Standard Deviation of the void volume is Void_Std $:=\operatorname{stdev}$ (Void) Void_Std $=3.787 \cdot 10^{-3} \cdot$ in $^{3}$

Mean of the dummy's capsule pressure is Pressure_Mean := mean (PressurePressure_Mean $=580.168 \cdot \frac{\mathrm{lb}}{\text { in }^{2}}$

Standard Deviation of the dummy's pressure is Pressure_Std := stdev(Pressure) Pressure_Std $=0.891 \cdot \frac{1 \mathrm{~b}}{\mathrm{in}^{2}}$ 


\section{Appendix I}

Plenum Gas Analysis 


\section{Appendix I}

\section{Table of Contents}

- CLWR Test Loop-1 Gas Analysis for WL-01 through WL-27

- CLWR Test Gas Analysis for WL-11 and WL-12 


\section{ARGONNE NATIONAL LABORATORY}

P. O. Box 2528, Idaho Falls, Idaho $83403-2528$

\section{ENGINEERING DIVISION}

(TREAT, ZPPR, and HFEF)

EXAMination

DATA

TRANSMITAL

\begin{tabular}{|l|l|}
\hline TO: & ADDRESS: \\
& Lockheed Martin Idaho Technologies Co. \\
& P. O. Box 1625 \\
& EROB MS- 3760 \\
& Idaho Falls, ID 83415 \\
& \\
\hline
\end{tabular}

DATE:

March 14, 1997

Eight Rods from CLWR Test Loop-1

TYPE OF EXAMINATION:

Gas Analysis (AL)

FORM OF DATA:

Revision 1 of Summary Tables (2)

NOTES:

This is an augmented re-transmittal of the gas analysis data. Additional information has been added in the form of: sample container data; dates of radiochemical analyses; and tritium measurement of five backfill/expansion samples. The original transmittal (dated 2/25/97) may be discarded in its entirety.

CC: w/Aftachments

Loop-1 file

CC: w/O Attactrments

Questions, regarding this data transmittal should be directed to the Cognizant Engineer at extension (208) 533-7773. 


\section{ARGONNE NATIONAL LABORATORY \\ Hot Fuel Examination Facility}

SUMMARY OF RADIOCHEMICAL TRITIUM ANALYSES

PROGRAM: CLWR

TEST: . Loop-1

DATE:

3/13/97

\begin{tabular}{|c|c|c|c|c|c|c|c|c|c|c|}
\hline \multirow{3}{*}{$\begin{array}{c}\text { ROD } \\
\text { ID } \\
\#\end{array}$} & \multicolumn{6}{|c|}{ SAMPLE CONTAINER } & \multicolumn{3}{|c|}{ TRITIUM } & \multirow{3}{*}{ REMARKS } \\
\hline & \multirow{2}{*}{$\begin{array}{l}\text { ID } \\
\#\end{array}$} & \multirow{2}{*}{$\begin{array}{l}\text { BACKFILL } \\
\text { (psia) }\end{array}$} & \multicolumn{2}{|c|}{ Volume } & \multirow{2}{*}{$\begin{array}{c}\text { Temperature } \\
\left({ }^{\circ} \mathrm{C}\right)\end{array}$} & \multirow{2}{*}{$\begin{array}{c}\text { Pressure } \\
\text { (psia) }\end{array}$} & \multicolumn{3}{|c|}{ Activity } & \\
\hline & & & (cc) & (Error*\%) & & & $(\mu \mathrm{Cl})$ & (Error*\%) & DATE & \\
\hline WL-01 & 05 & $3=0$ & 26.2 & \pm 1 & 22 & 6.74 & 0.022 & \pm 30 & $2 / 5 / 97$ & \\
\hline WL-03 & 04 & 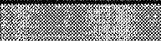 & 26.2 & \pm 1 & 22 & 6.43 & 0.024 & \pm 30 & $2 / 5 / 97$ & \\
\hline WL-09 & 15 & 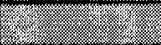 & 26.5 & \pm 1 & 22 & 7.01 & 0.023 & \pm 30 & $2 / 4 / 97$ & \\
\hline WL-10 & 07 & $\sqrt{2.2}$ & 26.4 & \pm 1 & 23 & 7.34 & 0.034 & \pm 30 & $1 / 30 / 97$ & \\
\hline WL-18 & 11 & 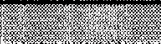 & 26.1 & \pm 1 & 22 & 3.19 & 0.022 & \pm 30 & $1 / 21 / 97$ & \\
\hline WL-18 & 16 & atmos. & 26.3 & \pm 1 & 22 & 2.93 & 0.005 & \pm 30 & $1 / 21 / 97$ & first backfill/expansion sample \\
\hline WL-18 & 13 & 15 & 26.4 & \pm 1 & 22 & 3.65 & 0.011 & \pm 30 & $1 / 21 / 97$ & second backfill/expansion sample \\
\hline WL-18 & 09 & 17 & 26.2 & \pm 1 & 22 & 4.15 & 0.005 & \pm 30 & $1 / 21 / 97$ & third backfill/expansion sample \\
\hline$\overline{W L-18}$ & 12 & 35 & 26.3 & \pm 1 & 23 & 8.55 & 0.022 & \pm 30 & $1 / 21 / 97$ & next-to-last backfill/expansion sample \\
\hline WL-18 & 15 & 45 & 26.5 & \pm 1 & 23 & 10.99 & 0.056 & \pm 30 & $1 / 21 / 97$ & last backfill/expansion sample \\
\hline WL-22 & 11 & $x=2$ & 26.1 & \pm 1 & 22 & 6.87 & 0.028 & \pm 30 & $2 / 4 / 97$ & \\
\hline$\overline{\text { WL-26 }}$ & $\overline{06}$ & (3) & 26.2 & \pm 1 & 22 & 7.97 & 0.021 & $\pm \mathbf{3 0}$ & $1 / 30 / 97$ & \\
\hline WL-27 & $\overline{13}$ & 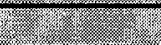 & 26.4 & \pm 1 & 21 & 4.40 & 0.017 & \pm 30 & $2 / 3 / 97$ & \\
\hline
\end{tabular}

REMARKS:

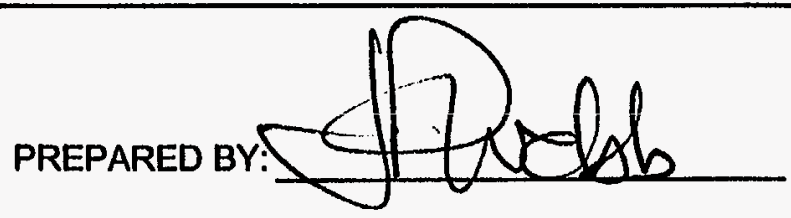




\section{ARGONNE NATIONAL LABORATORY Analytical Laboratory}

\section{SUMMARY OF GAS MASS-SPECTROSCOPY ANALYSES}

PROGRAM: CLWR

TEST: Loop-1

DATE:

3/13/97

\begin{tabular}{|c|c|c|c|c|c|c|c|c|c|c|c|c|c|c|c|}
\hline \multirow{3}{*}{ ROD ID } & \multirow{3}{*}{$\begin{array}{c}\text { SAMPLE } \\
\text { CONT. } \\
\text { ID }\end{array}$} & \multicolumn{8}{|c|}{ Gas Composition (Mole \%) } & \multicolumn{6}{|c|}{ Isotoplc Abundance (Atom \%) } \\
\hline & & \multirow{2}{*}{$\begin{array}{c}\mathrm{H}_{2} \\
(\mathrm{~m} / \mathrm{o})\end{array}$} & \multicolumn{2}{|r|}{ He } & \multirow{2}{*}{$\begin{array}{c}N_{2} \\
(\mathrm{~m} / \mathrm{o})\end{array}$} & \multirow{2}{*}{$\begin{array}{c}\mathrm{O}_{2} \\
(\mathrm{~m} / \mathrm{o})\end{array}$} & \multicolumn{2}{|c|}{$\mathbf{A r}$} & \multirow{2}{*}{$\begin{array}{c}\mathrm{CO}_{2} \\
(\mathrm{~m} / \mathrm{o})\end{array}$} & \multicolumn{2}{|c|}{$\mathrm{He}^{3}$} & \multicolumn{2}{|c|}{$\mathrm{He}^{9}$} & \multicolumn{2}{|c|}{$\mathrm{He3/ \textrm {He } 4}$} \\
\hline & & & $(\mathbf{m} / \mathbf{o})$ & (Error \%) & & & $(\mathrm{m} / \mathrm{o})$ & (Error \%) & & $(\mathrm{a} / \mathrm{o})$ & (Error \%) & (a/o) & (Error \%) & (Ratio) & (Error \%) \\
\hline WL-01 & 02 & $<0.01$ & 99.93 & \pm 1.0 & $<0.01$ & $<0.01$ & 0.07 & \pm 10 & $<0.01$ & 1.56 & \pm 10 & 98.44 & \pm 1 & 0.0159 & \pm 10 \\
\hline WL-03 & 01 & $<0.01$ & 99.91 & \pm 1.0 & $<0.01$ & $<0.01$ & 0.09 & \pm 10 & $<0.01$ & 1.69 & \pm 10 & 98.31 & \pm 1 & 0.0171 & \pm 10 \\
\hline WL-09 & 16 & $<0.01$ & 99.92 & \pm 1.0 & $<0.01$ & $<0.01$ & 0.08 & \pm 10 & $<0.01$ & 1.57 & \pm 10 & 98.43 & \pm 1 & 0.0160 & \pm 10 \\
\hline WL-10 & 08 & $<0.01$ & 99.92 & \pm 1.0 & $<0.01$ & $<0.01$ & 0.08 & \pm 25 & $<0.01$ & 1.43 & \pm 10 & 98.57 & \pm 1 & 0.0145 & \pm 10 \\
\hline WL-18 & 10 & $<0.01$ & 99.80 & \pm 1.0 & $<0.01$ & $<0.01$ & 0.20 & \pm 25 & $<0.01$ & 1.55 & \pm 10 & 98.45 & \pm 1 & 0.0157 & \pm 10 \\
\hline WL-22 & 10 & $<0.01$ & 99.92 & \pm 1.0 & $<0.01$ & $<0.01$ & 0.08 & \pm 10 & $<0.01$ & 1.57 & \pm 10 & 98.43 & \pm 1 & 0.0159 & \pm 10 \\
\hline WL-26 & 05 & $<0.01$ & 99.92 & \pm 1.0 & $<0.01$ & $<0.01$ & 0.08 & \pm 10 & $<0.01$ & 1.32 & \pm 10 & 98.68 & \pm 1 & 0.0134 & \pm 10 \\
\hline WL-27 & 12 & $<0.01$ & 99.91 & \pm 1.0 & $<0.01$ & $<0.01$ & 0.09 & \pm 10 & $<0.01$ & 1.37 & \pm 10 & 98.63 & \pm 1 & 0.0139 & \pm 10 \\
\hline
\end{tabular}

* At 2 sigma

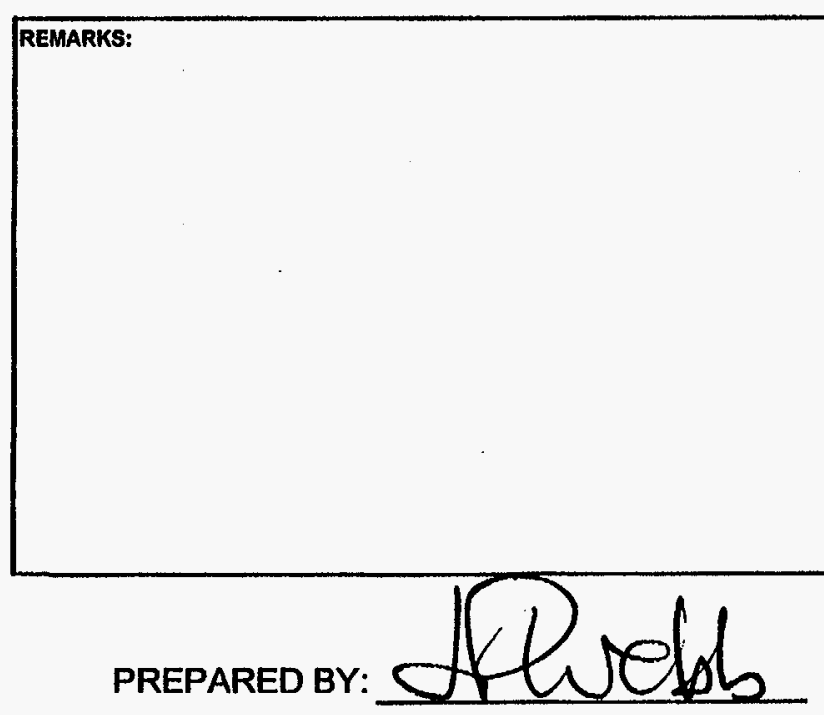

VERIFIED BY:

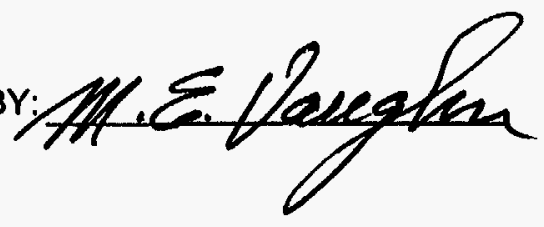




$$
\text { 1-4 }
$$




\section{ARGONNE NATIONAL LABORATORY \\ P. O. Box 2528, Idaho Falls, Idaho 83403-2528}

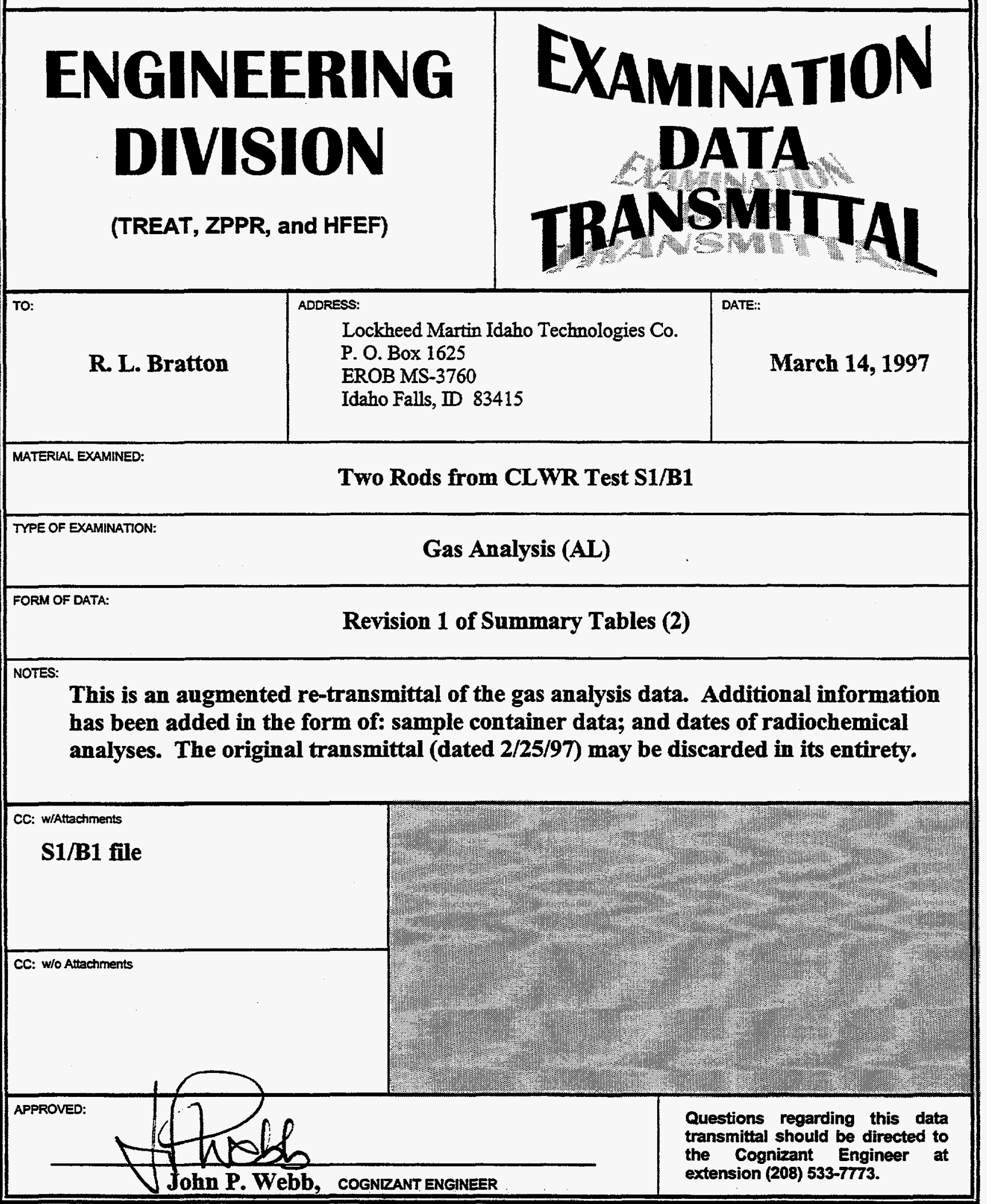




\section{ARGONNE NATIONAL LABORATORY Analytical Laboratory}

SUMMARY OF GAS MASS-SPECTROSCOPY ANALYSES

PROGRAN CLWR

TEST: $\mathbf{S 1 / B 1}$

DATE: $\quad 3 / 14 / 97$

\begin{tabular}{|c|c|c|c|c|c|c|c|c|c|c|c|c|c|c|c|c|}
\hline \multirow{3}{*}{ ROD ID } & \multirow{3}{*}{$\begin{array}{c}\text { SAMPLE } \\
\text { CONT. } \\
\text { ID }\end{array}$} & \multicolumn{9}{|c|}{ Gas Composition (Mole \%) } & \multicolumn{6}{|c|}{ Isotopic Abundance (Atom \%) } \\
\hline & & \multicolumn{2}{|c|}{$\mathrm{H}_{2}$} & \multicolumn{2}{|c|}{$\mathrm{He}$} & \multirow{2}{*}{$\begin{array}{c}\mathrm{N}_{2} \\
(\mathrm{~m} / \mathrm{o})\end{array}$} & \multirow{2}{*}{$\begin{array}{c}\mathrm{O}_{2} \\
(\mathrm{~m} / \mathrm{o})\end{array}$} & \multicolumn{2}{|c|}{$\mathrm{Ar}$} & \multirow{2}{*}{$\begin{array}{c}\mathrm{CO}_{2} \\
(\mathrm{~m} / \mathrm{o})\end{array}$} & \multicolumn{2}{|c|}{$\mathrm{He}^{3}$} & \multicolumn{2}{|c|}{$\mathrm{He}^{9}$} & \multicolumn{2}{|c|}{ He3/He4 } \\
\hline & & $(\mathrm{m} / \mathrm{d})$ & Г(Error \%) & (m/o) & (Error \%) & & & $(\mathrm{m} / \mathrm{a})$ & (Error \%) & & (a/o) & (Error\%) & (a/0) & (Error \%) & (Ratio) & (Error\%) \\
\hline WT-11 & 02 & $<0.01$ & $\mathbf{n} / \mathbf{a}$ & 99.87 & \pm 1.0 & $<0.01$ & $<0.01$ & 0.13 & \pm 10 & $<0.01$ & 1.14 & \pm 10 & 98.86 & \pm 1 & 0.0116 & \pm 10 \\
\hline WT-12 & 01 & 0.01 & \pm 25 & 99.82 & \pm 1.0 & $<0.01$ & $<0.01$ & 0.17 & \pm 25 & $<0.01$ & 0.78 & \pm 10 & 99.22 & \pm 1 & 0.0078 & \pm 10 \\
\hline & & & & & & & & & & & & & & & & \\
\hline & & & & & & & & & & & & & & & & \\
\hline & & & & & & & & & & & & & & & & \\
\hline & & & & & & & & & & & & & & & & \\
\hline & & & & & & & & & & & & & & & & \\
\hline & & & & & & & & & & & & & & & & \\
\hline
\end{tabular}

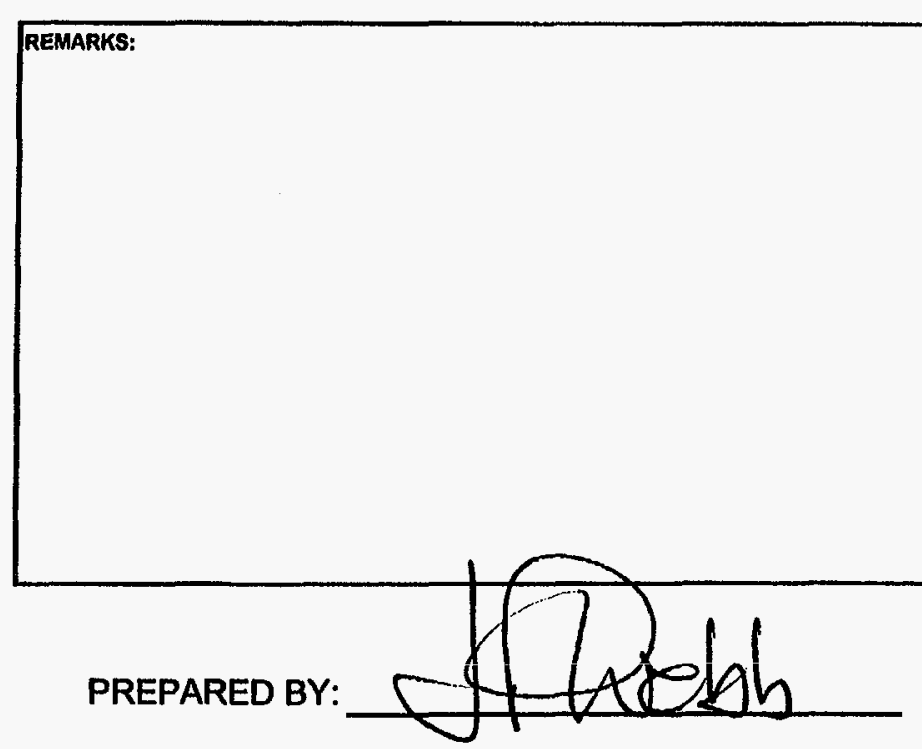

VERIFIED BY: 


\section{ARGONNE NATIONAL LABORATORY}

\section{Hot Fuel Examination Facility}

\section{SUMMARY OF RADIOCHEMICAL TRITIUM ANALYSES}

PROGRAM: CLWR

TEST: $\quad \mathbf{S 1 / B 1}$

DATE:

$3 / 14 / 97$

\begin{tabular}{|c|c|c|c|c|c|c|c|c|c|c|}
\hline \multirow{3}{*}{$\begin{array}{c}\text { ROD } \\
\text { ID } \\
\#\end{array}$} & \multicolumn{6}{|c|}{ SAMPLE CONTAINER } & \multirow{2}{*}{\multicolumn{3}{|c|}{$\frac{\text { TRITIUM }}{\text { Activity }}$}} & \multirow{3}{*}{ REMARKS } \\
\hline & \multirow{2}{*}{$\begin{array}{l}\text { ID } \\
\#\end{array}$} & \multirow{2}{*}{$\begin{array}{l}\text { BACKFILL } \\
\text { (psia) }\end{array}$} & \multicolumn{2}{|c|}{ Volume } & \multirow{2}{*}{$\begin{array}{c}\text { Temperature } \\
\left({ }^{\circ} \mathrm{C}\right)\end{array}$} & \multirow{2}{*}{$\begin{array}{c}\text { Pressure } \\
\text { (psia) }\end{array}$} & & & & \\
\hline & & & (cc) & (Error \%) & & & $(\mu \mathrm{Cl})$ & (Error*\%) & DATE & \\
\hline Wt-11 & 03 & -2 & 26.2 & \pm 1 & 22.0 & 4.42 & 0.006 & \pm 35 & $2 / 5 / 97$ & \\
\hline$\overline{W t-12}$ & 14 & 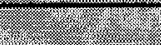 & 26.2 & \pm 1 & 21.5 & 4.01 & 0.025 & \pm 30 & $2 / 5 / 97$ & \\
\hline & & 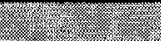 & & & & & & & & \\
\hline & & 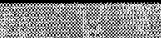 & & & & & & & & \\
\hline & & का & & & & & & & & \\
\hline & & 5 & & & & & & & & \\
\hline & & $=27.0$ & & & & & & & & \\
\hline & & 5 & & & & & & & & \\
\hline & & 2.25 & & & & & & & & \\
\hline & & 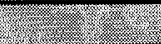 & & & & & & & & \\
\hline & & 28 & & & & & & & & \\
\hline & & 200 & & & & & & & & \\
\hline & & 5 & & & & & & & & \\
\hline
\end{tabular}

REMARKS:

PREPARED BY: S4tacds 